\title{
Representative ways to analyze and survey changes in long-term electrocardiographic recordings
}

\author{
Simona Gabriela Crihalmeanu \\ West Virginia University
}

Follow this and additional works at: https://researchrepository.wvu.edu/etd

\section{Recommended Citation}

Crihalmeanu, Simona Gabriela, "Representative ways to analyze and survey changes in long-term electrocardiographic recordings" (2000). Graduate Theses, Dissertations, and Problem Reports. 1069. https://researchrepository.wvu.edu/etd/1069

This Thesis is protected by copyright and/or related rights. It has been brought to you by the The Research Repository @ WVU with permission from the rights-holder(s). You are free to use this Thesis in any way that is permitted by the copyright and related rights legislation that applies to your use. For other uses you must obtain permission from the rights-holder(s) directly, unless additional rights are indicated by a Creative Commons license in the record and/ or on the work itself. This Thesis has been accepted for inclusion in WVU Graduate Theses, Dissertations, and Problem Reports collection by an authorized administrator of The Research Repository @ WVU. For more information, please contact researchrepository@mail.wvu.edu. 


\title{
Representative ways to analyze and survey changes in long-term electrocardiographic recordings
}

\author{
Simona Gabriela Crihalmeanu
}

\author{
Thesis submitted to \\ The College of Engineering and Mineral Resources \\ at West Virginia University \\ in partial fulfillment of the requirements \\ for the degree of \\ Master of Science \\ in \\ Electrical Engineering
}

Stephanie Schuckers, Ph.D., Chair

Wils L. Cooley, Ph.D.

Biswajit A.Das, Ph.D.

Kristine Krajnak, Ph.D.

Department of Computer Science and Electrical Engineering

Morgantown, West Virginia

2000

Keywords: long-term recordings, heart rate variability, rabbit, electrocardiogram 


\title{
ABSTRACT \\ Representative ways to analyze and survey changes in long-term electrocardiographic recordings
}

\author{
Simona Gabriela Crihalmeanu
}

The goal of this research is to explore techniques with which long-term physiologic timeseries data can be analyzed, so that relevant changes in physiological signals, particularly the electrocardiogram signal, can be captured, processed, quantified and stored. A new experimental model was developed such that the electrocardiogram can be monitored continuously over thirteen weeks. Cardiotoxicity was progressively induced with doxorubicin in a rabbit model, and electrocardiographic progressions from normal state to diseased state were continuously tracked. Automated methods for analyzing the data were developed to manage and control the extensive electrocardiogram dataset. A significant challenge to this work is the sheer mass of data. This experiment generated 180 megabytes per day per rabbit, totaling around 66 gigabytes for the entire study. Classical ECG parameters significant for the evaluation of heart rate variability were calculated by computer for the entire period of the recordings, and visualized with six different methods. 


\section{ACKNOWLEDGEMENTS}

I would like to express my deepest gratitude to my advisor Dr. Stephanie Schuckers, who guided me during the course of this work. I offer my sincerest thanks for her suggestions and enlightened discussions on both theoretical and experimental aspects of the problems encountered, and for her constant interest and encouragement.

I would like also to thank Dr. Wils L. Cooley, Dr. Biswajit A. Das, and Dr. Kristine Krajnak, for serving on my committee.

I am grateful to Dr. Mitch Finkel for his guidance and assistance in medical area, and for his help in understanding and interpreting the electrocardiograms and laboratory results.

I wish to thank my colleagues with whom I shared the work and knowledge in the Biomedical Signal Analysis Laboratory, especially Xueyan Xu - Sherry, who developed individual heart rate variability modules, and Pisut Raphisak, who developed the trigger point program and participated in the rabbit surgeries.

A special thank to my husband, Musat, for the moral support and encouragement, and to my parents and parents in law for the love and care for my children while I was far away from home.

This work was supported by grants from the American Heart Association and the National Science Foundation. 


\section{TABLE OF CONTENTS}

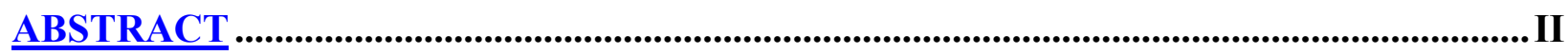

ACKNOWLEDGEMENTS.................................................................................................................... III

TABLE OF CONTENTS.......................................................................................................................IV

TABLE OF FIGURES .............................................................................................................................VI

TABLE OF TABLES .................................................................................................................. VIII

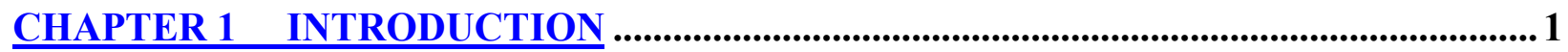

CHAPTER 2 LITERATURE SURVEY ……...........................................................................

2.1 Long-term studies of the electrocardiogram ………….............................................. 3

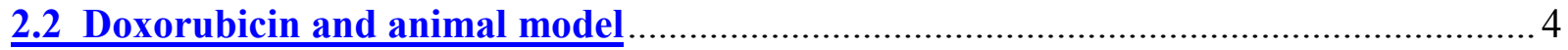

2.3 Circadian rhythm of heart rate variability in normal subjects .................................. 6

2.4 Heart rate variability and heart diseases ....................................................................

2.4.1 Heart rate variability and heart failure .................................................................. 7

2.4.2 Heart rate variability and sudden cardiac death ..................................................... 8

2.4.3 Circadian rhythm of heart rate variability in disease................................................ 9

CHAPTER 3 BACKGROUND ............................................................................11

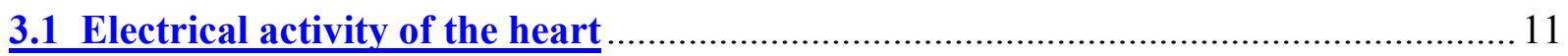

3.2 Influences on cardiac heart rate ……………………............................................ 14

3.3 Heart rate variability, measurement, interpretation .................................................. 16

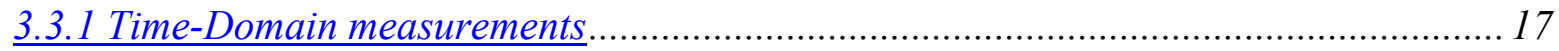

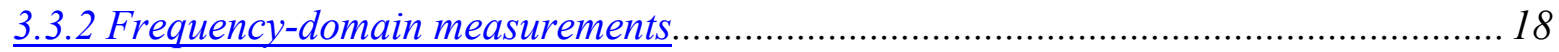

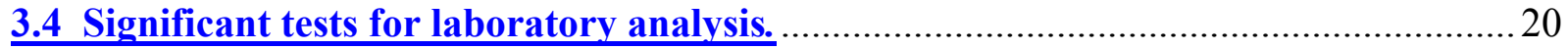

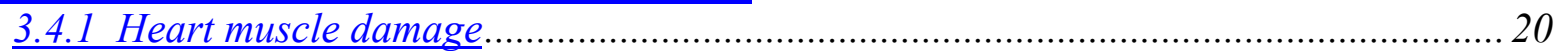

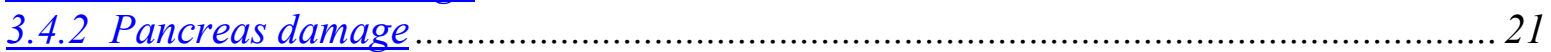

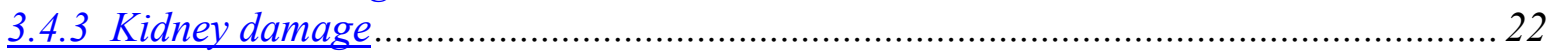

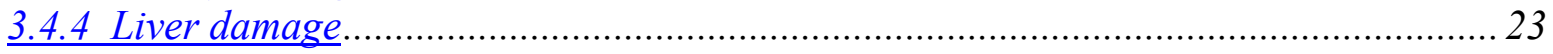

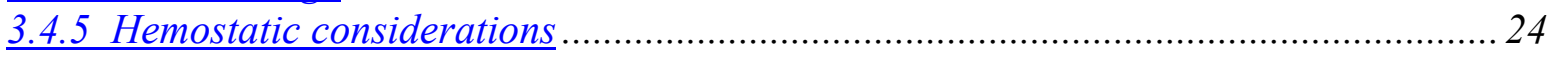

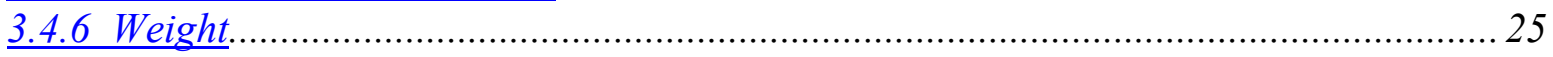

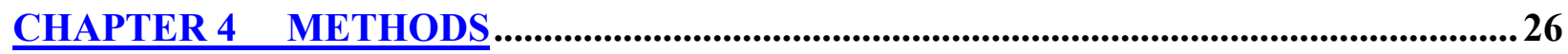

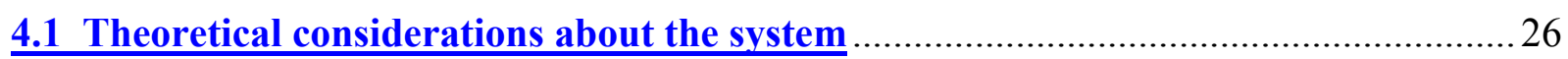

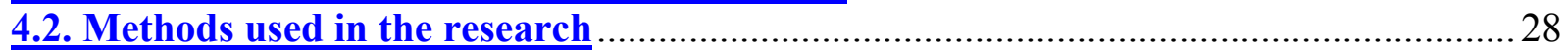

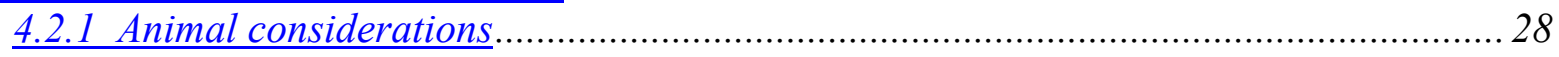

4.2.2 Signals. The hardware and the software used …………..................................... 28 


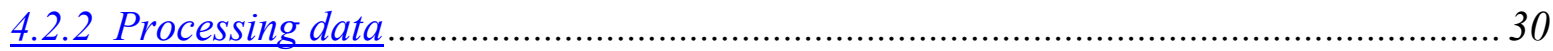

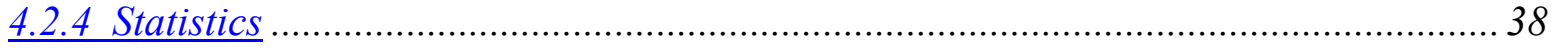

CHAPTER 5 RESULTS .......................................................................................................39

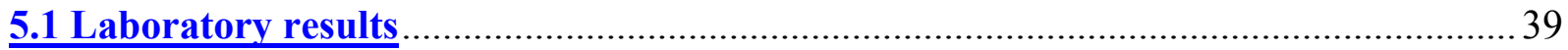

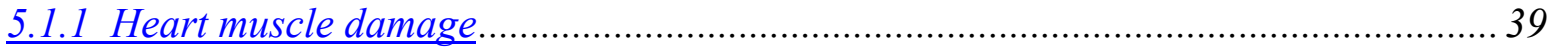

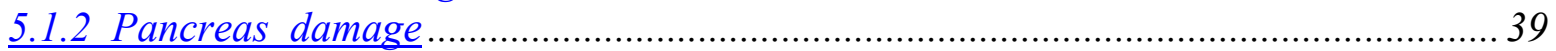

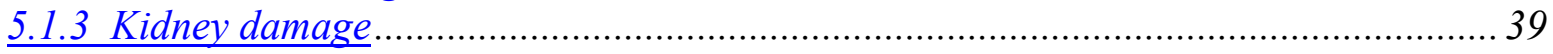

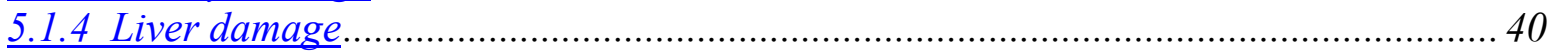

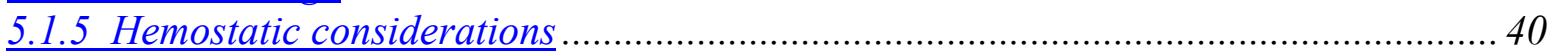

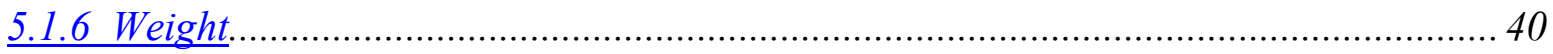

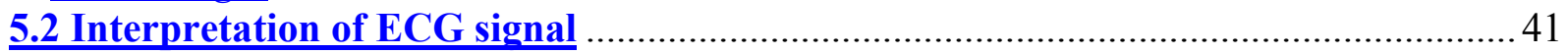

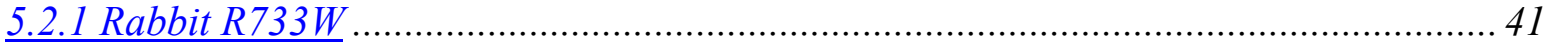

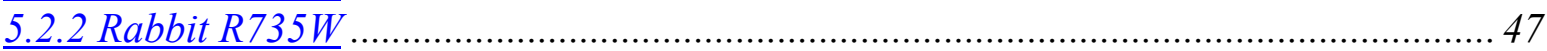

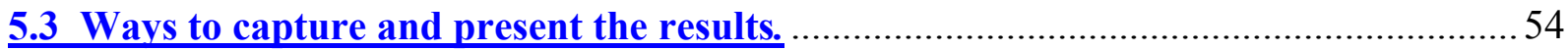

5.3.1 Individual plots, from averaged data, hourly and weekly ...........................................54

5.3.2 Individual plots where each 5 minute segment is averaged weekly.............................60

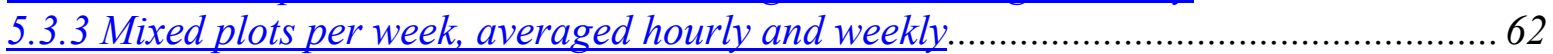

5.3.4 Individual and mixed plots, from averaged data per day and night ........................... 64

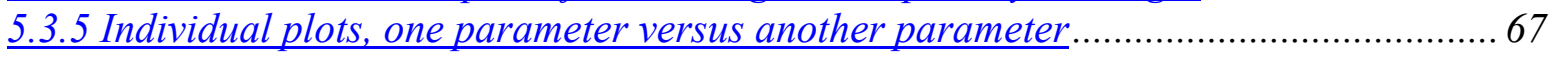

5.3.6 Individual plots, all data from five weeks plotted versus hour................................... 72

CHAPTER 6 CONCLUSIONS .............................................................................................76

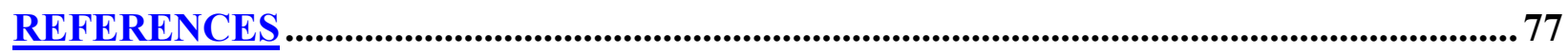

Appendix A Individual plots, from averaged data, hourly and weekly ............................. 82

Appendix B Individual plots where each 5 minute segment is averaged weekly................ 94

Appendix C Mixed plots per week, averaged hourly and weekly .................................... 106

Appendix D Individual and mixed plots from averaged data per day and night ................ 126

Appendix E Individual plots, one parameter versus another parameter ............................ 133

Appendix F Individual plots, all data from five weeks plotted versus hour........................ 143

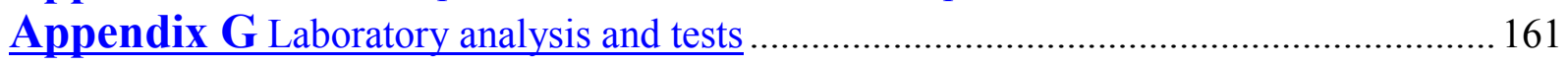

Appendix H ECG signal, injected rabbit R735W, intracardiac channel ........................... 191 


\section{TABLE OF FIGURES}

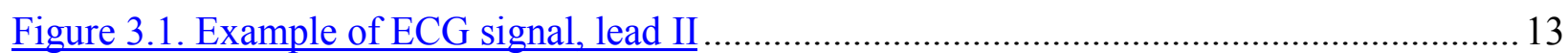

Figure 3.2 Representative changes in ECG signal for a diseased heart ....................................... 14

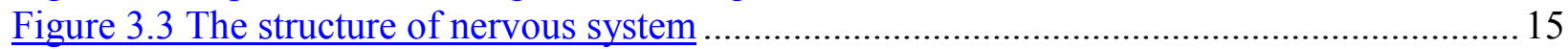

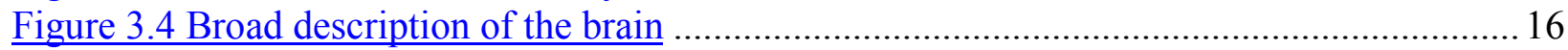

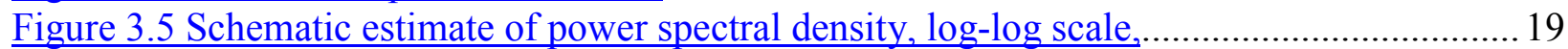

obtained from the entire 24-hour of long-term recordings in humans ........................................... 19

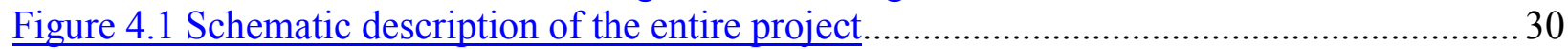

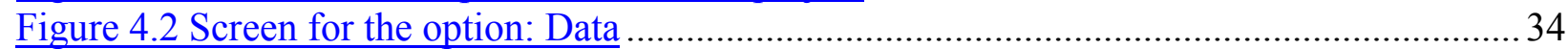

Figure 4.3 Screen for option: One five-minute segment from each hour ……………………..... 35

Figure 4.4 Screen for option: Continuous five minute segments for each hour.............................36

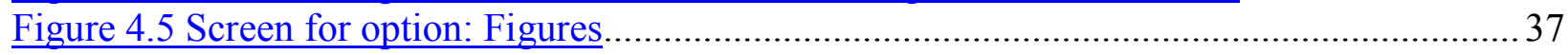

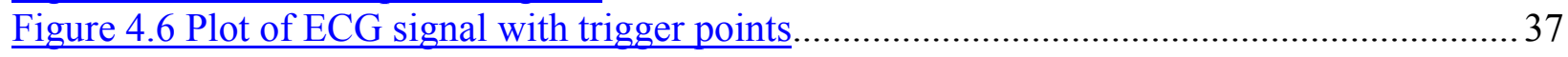

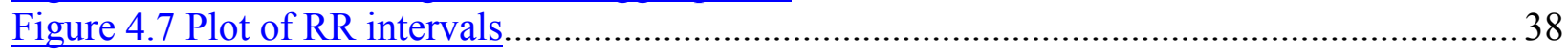

Figure 5.1 ECG signal before the first injection, one second per screen ..................................... 42

Figure 5.2 ECG signal before the first injection, four seconds per screen..................................... 42

Figure 5.3 ECG signal on March $10^{\text {th }}$, four seconds per screen................................................... 43

Figure 5.4 ECG signal on March $20^{\text {th }}$, four seconds per screen.................................................. 43

Figure 5.5 ECG signal on March 20 $0^{\text {th }}$, four seconds per screen ................................................... 44

Figure 5.6 ECG signal on March $20^{\text {th }}$, one second per screen .................................................... 44

Figure 5.7 ECG signal; the beginning of bradycardia, four seconds per screen ........................... 45

Figure 5.8 ECG signal, bradycardia, four seconds per screen ................................................... 45

Figure 5.9 ECG signal, ventricular bigeminy, four seconds per screen ........................................4 46

Figure 5.10 ECG signal, ventricular bigeminy, twelve seconds per screen...................................4 46

Figure 5.11 ECG signal before the first injection, four seconds per screen...................................4 48

Figure 5.12 ECG signal on February $11^{\text {th }}$, four seconds per screen.............................................48

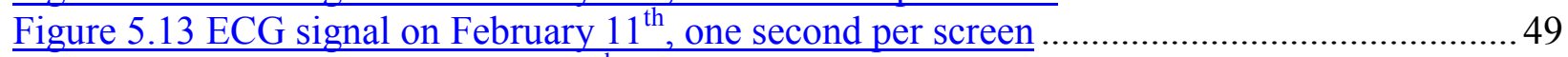

Figure 5.14 ECG signal on March $2^{\text {nd }}$, four seconds per screen ................................................... 49

Figure 5.15 ECG signal, the moment of myocardial infarction, for seconds per screen ...............50

Figure 5.16 ECG signal on March $6^{\text {th }}$, before myocardial infaction, one second per screen.........50

Figure 5.17 ECG signal on March $6^{\text {th }}$, after myocardial infarction, one second per screen ..........51

Figure 5.18 ECG signal, bradycardia, four seconds per screen ................................................ 51

Figure 5.19 ECG signal on March $6^{\text {th }}$, bradycardia, four seconds per screen..............................52

Figure 5.20 ECG signal on March $6^{\text {th }}$, bradycardia, fourty seconds per screen..............................52

Figure 5.21 ECG signal, bradycardia, before the death, fourty seconds per screen .....................53

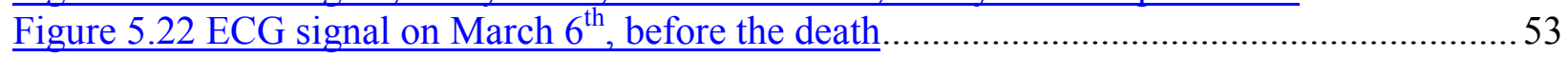

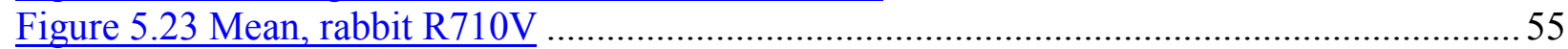

Figure 5.24 Coefficient of covariance, rabbit R710V ............................................................5

Figure 5.25 High frequency, rabbit R710V........................................................................56 
Figure 5.26 Root mean square successive differences, rabbit R710V …................................56

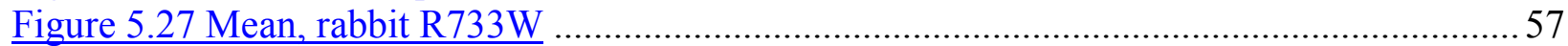

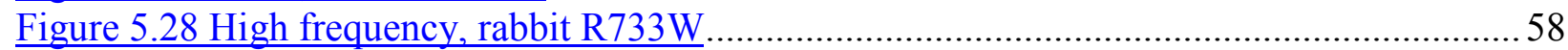

Figure 5.29 Ratio low frequency/high frequency, rabbit R733W .......................................... 58

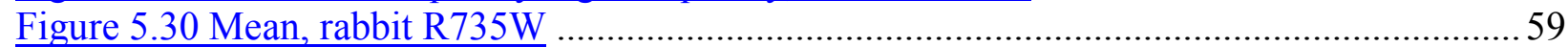

Figure 5.31 Standard deviation of RR intervals, rabbit R735W ............................................ 59

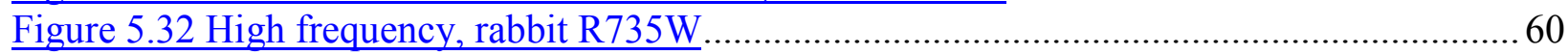

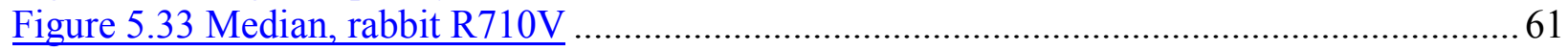

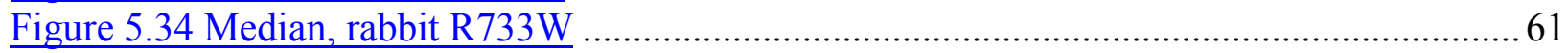

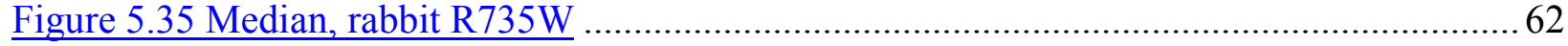

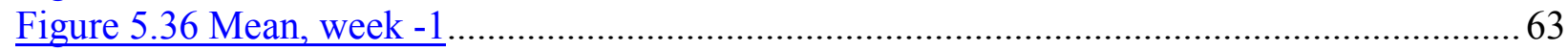

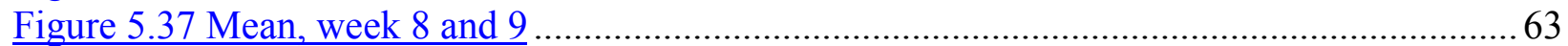

Figure 5.38 Variation of interquartile range of variation of RR intervals, week -1 ................... 64

Figure 5.39 Variation of interquartile range of variation of RR intervals, week 5 .................... 64

Figure 5.40 Variation of standard deviation between day and night ....................................... 65

Figure 5.41 Variation of high frequency between day and night.............................................66

Figure 5.42 Variation of low frequency between day and night .............................................66

Figure 5.43 Variation of interquartile range of RR intervals between day and night ..................67

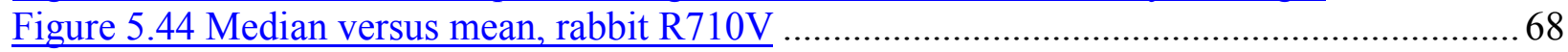

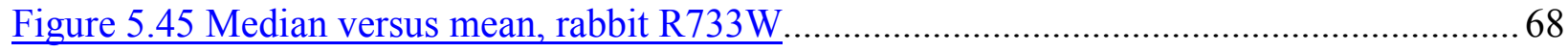

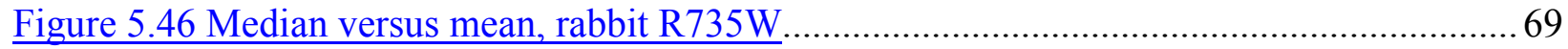

Figure 5.47 Interquartile range of RR intervals versus mean, rabbit R710 V...........................69

Figure 5.48 Interquartile range of RR intervals versus mean, rabbit R733W ............................. 70

Figure 5.49 Interquartile range of RR intervals versus mean, rabbit R735W........................... 70

Figure 5.50 Interquartile range of variation of RR intervals versus interquartile range of RR

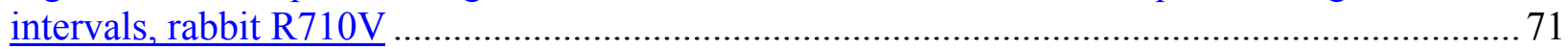

Figure 5.51 Interquartile range of variation of RR intervals versus interquartile range of RR intervals, rabbit R733W …........................................................................................... 71

Figure 5.52 Interquartile range of variation of RR intervals versus interquartile range of RR

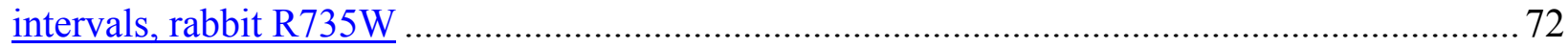

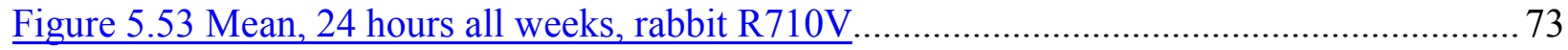

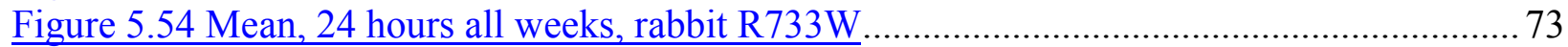

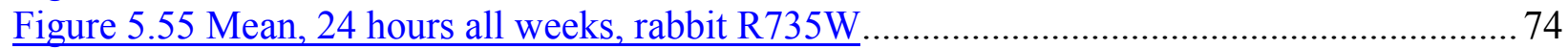

Figure 5.56 Coefficient of variance, 24 hours all weeks, rabbit R710V ..................................... 74

Figure 5.57 Coefficient of variance, 24 hours all weeks, rabbit R733W ................................. 75

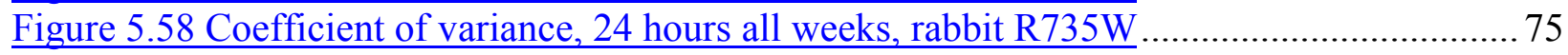




\section{TABLE OF TABLES}

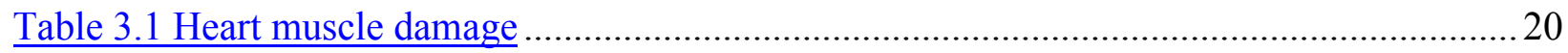

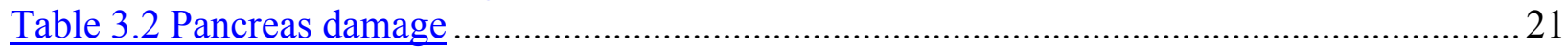

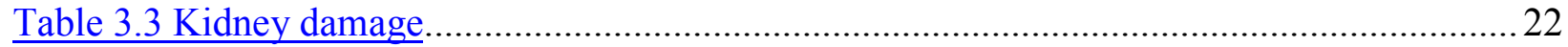

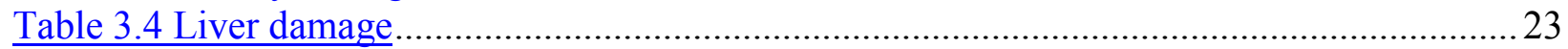

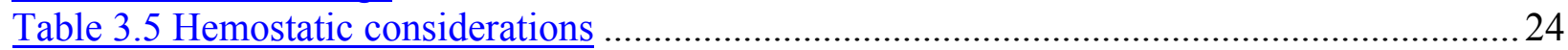

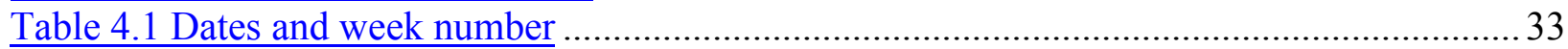




\section{Chapter 1 INTRODUCTION}

The rapid advances in computing power, miniature radio-transmitters, and the relative inexpensiveness of data storage, makes it now possible to perform long-term physiologic monitoring where the data is recorded for many months, even years, to document physiologic changes. Despite these advances, little or no work of this type has been performed in the biomedical area to discover insight into physiologic mechanisms and disease. Tools and methods for handling and processing must be developed and utilized, so that significant and relevant changes in physiological signals can be captured, analyzed, quantified and stored. In comparison with short-term recordings, long- term recordings are very large, and complex data sets.

The quality of the long-term recordings, provided by the most recent technology, opens new doors for the study and prediction of heart diseases. This is especially important because one to two million adults are affected in the United States by heart failure with 200,000 deaths annually [1,2]. In addition, $30-50 \%$ of these deaths are sudden and have been attributed to ventricular tachyarrhythmias, bradycardia, and electro-mechanical dissociation [1,2].

Longitudinal tracking of ECG changes could impact understanding of the disease and the changes that occur over time as the disease develops. Furthermore, little work has been done to analyze the electrocardiogram in the weeks, days, and hours before a spontaneous event, in order to establish a short-term electrophysiologic predictor of sudden cardiac death, heart failure and other heart disease. If a ventricular fibrillation (VF) episode could be predicted, long- and shortterm interventions could be attempted to prevent the event [3].

The goal of this research is to explore techniques with which long-term physiologic timeseries data can be analyzed. For the first time, long-term studies, particularly electrocardiographic progressions from normal state to diseased state have been continuously tracked. In this thesis, a new experimental model was developed such that the electrocardiogram can be monitored continuously, that is, a 1000 samples per second, over thirteen weeks. In this model cardiotoxicity was progressively induced with doxorubicin in a rabbit model, and the ECG was continuously tracked. A significant challenge to this work is the sheer mass of data. This experiment generated 180 megabytes per day per rabbit, totaling 66 gigabytes for the study 
reported in this thesis. For five weeks, classical ECG parameters were calculated by computer and monitored before and during the development of heart disease. Unique, automated methods for analyzing the data were developed to manage and control the extensive electrocardiogram dataset. 


\section{Chapter 2 LITERATURE SURVEY}

It is presently accepted that long term ECGs (typically over 24 hours derived from ambulatory or Holter monitoring) are a very useful clinical tool to gather information on a variety of ECG features in order to predict heart disease (see ECG signal, figure 3.1). Much research has been performed to determine the changes in ECG features for different types of heart diseases, most of them being performed on a population of humans that were tracked for heart disease. But the difficulty of such studies is that changes over time of these features are not known. The advantage of animal models is that more elaborate, time-dependent, and controlled studies can be performed. An animal model provides a means to track ECG changes, which allows better evaluation of their relevance, their relationship in time to events, and their reproducibility.

\section{$\underline{\text { 2.1 Long-term studies of the electrocardiogram }}$}

Several studies have utilized long-term electrocardiographic recordings, usually around 24 or 48 hours recordings. In one study, high frequency ECG changes in dogs were monitored for 24 hours to evaluate myocardial ischemia and recovery [23]. In another study, the time course of spontaneous and induced ventricular arrhythmias were monitored in dogs after myocardial infarction [24]. Analysis was based on a 24-hour continuous recording the day of the operation and 10 different two-hour recordings in the 29 days following operation. The major aim of the study was to determine the inducibility of ventricular arrhythmia following infarction through classic ECG features (PR, QRS, QT, QTc, RR). None of the measurements were determined statistically significant.

In another study using rats, continuous ECG recording were made in the two days following ligation [25]. This study used telemetric monitoring to provide an untethered setting for the rat. The incidence of ventricular arrhythmias and ectopic beats was measured, but no actual ECG measurements were made to determine their presence and relationship to the event. Another study with the rat modeled cardiac hypertrophy and senescence. In this case, 
spontaneous rhythms were monitored for 24 hours and were comprised primarily of supraventricular and atrioventricular block [26].

In other studies, rabbits were selected since they develop similar morphologic changes to those described in humans. The aim of one study was to examine right ventricular electrophysiological changes in an animal model of chronic cardiac failure [27]. Continuous ambulatory ECG monitoring was undertaken using a bipolar signal from the right ventricular permanent pacing electrode, and at least one complete 24 hour ECG was attempted in each animal between weeks 8 and 10 of the study [27]. The conclusion was that shortening of ventricular repolarisation and refractory period in heart failure may represent an intrinsic mechanism predisposing to arrhythmia and sudden death.

In summary, while many animal models have been developed and several have been used for a longer term, no studies have monitored the progression of the ECG for long-term (over 48 hours) changes with the progression of the disease.

\section{$\underline{\text { 2.2 Doxorubicin and animal model }}$}

Doxorubicin [21], also called adriamycin, is effective treatment in humans for acute leukemias and malignant lymphomas, and is also active in a number of solid tumors, breast cancer - particularly carcinoma of the breast, carcinoma of the ovary and small carcinoma of the lung. Special attention must be given to the adverse reactions of adriamycin among which can be enumerated:

- Cutaneous reactions. If adriamycin is not administrated intravenously, severe local tissue necrosis occurs. Adriamycin must not be given by the intramuscular or subcutaneous route.

- Gastrointestinal reactions. Acute nausea, vomiting, stomatitis, gastrointestinal disturbances occurs frequently and may be severe, but they are reversible.

- Cardiotoxicity reaction. Irreversible myocardial toxicity, manifested in its most severe form, potentially fatal congestive heart failure may occur either during therapy or months to years after termination of therapy. Severe cardiac toxicity may occur precipitously without antecedent ECG changes. Two kinds of cardiomyopathies may occur. An acute form is characterized by abnormal electrocardiographic changes, including ST-T wave alterations and arrhythmias. An exaggerated manifestation of acute myocardial damage 
may be characterized by severe disturbances in impulse conduction and frank congestive heart failure.

The second type of cardiomyopathie is chronic, cumulative dose-related toxicity, manifested by congestive heart failure.

- Other reactions.

The purpose of the research is to track changes in the electrocardiogram (ECG) using doxorubicin to induce heart disease in rabbit model. Besides the conclusions related to changes of ECG signal and heart disease, the study is relevant for cancer patients who utilize doxorubicin as a treatment $[28,29,30,31]$. Doxorubicin treatment is limited by the risk of cardiotoxicity; therefore predictors for the beginning of cardiotoxicity must be developed.

The model is created by regular injections for eight to ten weeks, once or twice a week, on the order of $2 \mathrm{mg} / \mathrm{kg}$ per week. The injections can be performed intravenously or through dedicated ear ports. Studies have shown that it takes approximately eight weeks for heart failure to develop and most deaths have been reported to occur after eight weeks. Various studies have reported the following number of deaths: 18 out of 27 animals [33], 4/9 [32], 22/46 [34], 40/104 [35], and 2/12 [36, 37] over eight to twelve weeks. Several studies have described these deaths as "presumably of arrhythmia" and "no apparent cause" [36, 27], but none have had electrocardiograms at the time of death. Little work has been performed regarding characterization of spontaneous arrhythmias in the model [27]. In one study, twenty-four hour ambulatory recordings were attempted in 40 animals, but only twelve recording were successful (due to animals chewing through the cable) [27]. The study did not publish when the one-time recordings were made in the development of heart failure. No ventricular tachyarrhythmias were seen during the time of the recording, but several animals had ectopic beats. This study did not have ability to record continuous over the development of the model and could have very easily "missed" any arrhythmic events.

Studies of the animal model have been utilized to understand the mechanism of heart failure. One of the studies examines the haemodynamic and humoral responses to graded treadmill exercise, serially during the development of congestive heart failure [32]. A low output congestive cardiomyopathy was induced with adriamycin. Another study investigates the effect of doxorubicin induced cardiomyopathy on ventricular repolarization and refractoriness, spontaneous arrhythmias and neurohormonal activation in order to characterize possible 
mechanisms for the predisposition to malignant ventricular arrhythmias in congestive heart failure [27]. Sudden cardiac deaths have been reported in both humans and animal models [32, 33, 34, 35, 36, 37]. Doxorubicin was also used in studies of cellular, electrophysiologic and autonomic changes in the animal model $[34,35]$.

Using doxorubicin in an animal models to induce heart failure, sudden cardiac death, or arrhythmias is not only a relevant way to study the heart diseases but also a representative way to examine the gradual development of disease in humans.

\subsection{Circadian rhythm of heart rate variability in normal subjects}

Studies about circadian rhythm and its reproducibility have captured the attention of scientists. Research in this area was done in humans or in animal models, in healthy or diseased subjects. In humans [38], after 24-hour electrocardiographic recordings a reproducible circadian rhythm of heart rate variability (HRV), a method of assessing the autonomic nervous system described in chapter 3 was observed in normal subjects with a maximum occurring early in the morning before waking up [38]. The heart rate variability decreased abruptly during the hours after waking, perhaps because of inhibition of the parasympathetic cardioinhibitory center at the time of waking [38]. The objective of the studies in the case of healthy patients was to assess the influence of day-night cycle, and sleep-awake period on the circadian pattern of HRV [39]. The conclusion is that sympathetic activity is dominant during the day and in the early waking hours, while parasympathetic activity is dominant during the night [39]. Stability over time of circadian rhythm of heart rate variability was also studied in patients with coronary artery disease [40], sudden cardiac death [42], in ventricular arrhythmias for patients with implantable cardioverter defibrillators [41].

The causes of circadian changes in heart rate variability are not fully elucidated, and require more and new studies and work. In order to accomplish this need, rabbits were used as animal model in another study [43]. In addition, circadian rhythm in rabbits is relevant since rabbits are the models used in this thesis. Radio-telemetry along with the implantable transmitters enable the free movement of the animal in the cage, and many parameters like heart rate, blood pressure, temperature, and others may be recorded. A study on rabbits, which has 
used 24-hour recordings of heart rate, blood pressure and behavioral activity by telemetry, showed that there is a rise of heart rate in the morning period [43]. Changes were expressed as mean, its standard deviation and coefficient of variance. The main finding of the study was that changes in circadian rhythm of heart rate are due to behavioral activity rather than to the daynight cycle. Other studies were done to assess changes in heart rate and blood pressure [44, 45, 46]. The variabilities were assessed for 24 hours for circadian rhythm and one hour for shortterm variability, and results were compared between rabbits and rats [44]. The conclusion was that short- term variability can be classified in two major components. One is behavioral change including eating, and drinking, and the other is emotional or neuronal changes which are independent of body movement [44]. The unique characteristics of short-term variability must be taken in consideration when the circadian rhythm is focused on. Important conditions such as stress and environmental conditions as auditory stimuli must be also taken in consideration. Little work was done for circadian rhythm in rabbits in case of heart diseases, and little is known about factors, which influences these variations.

\section{$\underline{\text { 2.4 Heart rate variability and heart diseases }}$}

\subsubsection{Heart rate variability and heart failure}

The clinical relevance of HRV was first appreciated in 1965 when Hon and Lee noted that fetal distress was preceded by alterations in interbeat intervals before any appreciable change occurred in heart rate itself [4]. Since than, researchers focused their attention on the study of heart rate variability, and tried to prove relationships between heart rate variability and heart diseases. Because HRV monitors changes in autonomic activity, and because diseases like acute myocardial infarction, heart failure, sudden cardiac death and others are associated with altered autonomic activity, HRV is a promising marker in assessment of a healthy heart.

Heart failure includes in the broadest sense all those patients both symptomatic and asymptomatic having left and/or right ventricular failure, and systolic and/or diastolic dysfunction [5]. Now it is generally accepted that heart rate variability (HRV) is reduced in the case of heart failure. Normal subjects are able to modulate heart rate in a wide range of different levels and these levels can be modified very rapidly. Heart failure subjects, on the contrary, are not able to modify heart rate to the same extent, nor as rapidly as normal subjects. There are 
different interesting issues that can be exploited. One is to speculate whether a disease causing heart failure influences HRV. In this case HRV can describe the severity of the underlying heart disease instead of being an effect of heart failure. Studies of Gang et al and Dadoun et al on subjects whose heart failure was of ischemic and cardiomyopathy etiology were done recently and no differences in HRV were found [6,7]. On the other hand, other studies have shown HRV is decreased in heart failure, and there is a demonstrated relationship between death and decreased HRV. Hence HRV could be a powerful predictor of mortality. Up to $80 \%$ of heart failure patients die suddenly with an average $60 \%$ survival at 4 years [8]. Time domain studies of subjects with heart failure showed marked depression of HRV consistent with a major reduction in parasympathetic activity, but do not provide necessary insights into sympathetic changes. Frequency domain studies revealed a reduction in total power. Casolo et al [9] found a tenfold decrease in absolute power of all spectral components between 0.021 and $0.32 \mathrm{~Hz}$ (an evident decrease in high frequency power), but showed the low frequency power (LF) band to be unchanged relative to normal. Circadian changes in LF power and LF/HF ratio were depressed also, except during the morning hours (1:00 AM to noon). Several experimental investigations [5] show that cardiac neurotransmitter activity is altered in heart failure, and the alteration responsible for the low HR variation in heart failure is partially reversible with treatment and should be viewed like a functional autonomic denervation.

\subsubsection{Heart rate variability and sudden cardiac death}

Death due to paroxysmal dysrhythmia (usually ventricular fibrillation), termed SCD, is the most common form of sudden death in the Western world, with more than 400,000 cases per year occurring in the United States alone, usually in the setting of coronary artery disease [10]. Researchers have proved that autonomic imbalance is a determinant in predisposition to ventricular tachyarrhymias and fibrillations. Sympathetic activity seems to facilitate such a predisposition, while parasympathetic activity seems to protect against it. Being relevant for fluctuations in autonomic nervous system balance, heart rate variability (HRV) is a good predictor of risk of SCD compared with other measures used previously, like left ventricular ejection fraction, standard electrocardiographic indices, the response to exercise or signalaveraged electrocardiogram. Time domain studies of Martin GJ et al $[11,12,13]$ showed that heart rate variability (HRV) was significantly lower in SCD survivors and in patients 
experiencing SCD during ambulatory monitoring than for non-SCD patients with structural heart disease and normal control subjects. This research supports suggestions that this method may be useful in identifying individuals at high risk of sudden death. Long-term (to 8 years) follow-up of SCD survivors with mortality as the end point confirms the strong predictive value of low HRV for recurrence of major arrhythmic events. Of seven patients exhibiting two or more major arrhythmic events, five died during the observation period. Of these, four exhibited very low HRV [10]. Preliminary observations suggest that it is possible to define cut off points for time domain measures that separate SCD survivors from non-SCD patients with heart disease with an $80 \%$ sensitivity and $66 \%$ specificity [10]. A great attention has to be taken with respect to aging, where studies suggest that age can contribute to the lowering HRV and must be taken in account when establishing the cutoff points. In frequency domain measures, Meyers et al [16], found that changes in HF component is well established and draw the line between SCD survivors, nonSCD patients with other heart disease and normal individuals. The LF component of spectral analysis was not relevant and was not useful in delimitation of SCD, although values for ill subjects were lower compared with those of healthy ones. At great significance is also the fact that low vagal tone was found in subjects with noncardiac disease states, like diabetes and alcoholic neuropathy. Much more work has to be done in this area; patterns and standards must be issued for an accurate assessment of SCD.

\subsubsection{Circadian rhythm of heart rate variability in disease}

Each biophysiological system presents physiological rhythms that oscillate at specific frequencies. A circadian rhythm, strictly speaking, refers to a time event series with a principal frequency of one cycle in 24 to 26 hours. Because of this, the maximum length data series has to be at least 48 hours in order to capture the cycle length.

Neural regulation of the cardiovascular system in healthy individuals, which refers to autonomic activity, shows a distinct circadian rhythm with prevalence of sympathetic activity during the day and a marked increase in parasympathetic activity during the night. The onset of acute cardiovascular events does not occur randomly, and it is reflected in changes in circadian rhythm of heart rate variability (HRV). For this reason, studies of circadian rhythm of HRV were made in healthy subjects and patients with coronary artery disease, in patients with sudden cardiac death after myocardial infarction and in other heart diseases. It is accepted that onset of 
acute cardiovascular events occurs in a characteristic diurnal pattern with a prominent peak in the morning hours between 6 am and 12 noon [5]. Triggers of increased vascular tone during the morning, like cortisol secretion, platelets aggregability, plasma catecholamines, and fibrinogen activity present a circadian pattern with the maximum during the same period, between 6 am and 12 noon [5]. These together with the rapid change in heart rate during the morning and the change in blood pressure has lead the researchers to look for a relation between sleep-awake cycle and the fluctuations of the autonomic nervous system, respectively with the imbalanced sympathetic and parasympathetic activity. Because of sleep-awake cycle and external factors coincident with arousal, the peaks of circadian rhythm at the event onset can hardly be distinguished [14,15]. Studies have found that HR and HRV during the night are decreased in healthy subjects, although there is an increase in parasympathetic activity.

In the case of heart failure, HRV is decreased and as a consequence the pattern of diurnal variations of HRV is reduced also. In his study, Caloso et al [16] used mean hourly HR standard deviation and marked the depression of HRV throughout the day in 20 cardiac heart failure patients. A decrease in absolute power of spectral components, including a decrease of LF representative for sympathetic activity, was also found. This study showed that neurohormonal activity correlated with the increase of some potential triggers, like catecholamines or angiotensin II and the decrease of barorecepter sensitivity, can explain the suppression of normal autonomic activity.

Circadian variation of HRV is important and useful to characterize abnormalities of neurocardiac function. Research has to be continued to fully understand the basis of many interacting physiological processes. 


\section{Chapter 3 BACKGROUND}

\subsection{Electrical activity of the heart}

It is well known that cardiac muscle is capable of generating action potentials without any nervous stimulation. When one of the cardiac cells spontaneously undergoes an action potential, the electrical impulse spreads to all the other cells, which become excited. The action potential in turn stimulates the contraction of the cardiac muscle cell. The synchronous contraction of the muscle cells, which are part of the four chambers of the heart, two atria and two ventricles, produces the necessary force to push the enclosed blood. The property of generating an action potential by itself is known as autorhythmicity. This is due to a small number of cardiac cells, which form a specialized conducting system that ensures the synchronization between the contraction of atria and ventricles. The remaining numbers of cells, which represent $99 \%$ of the heart, are contractile cells, which do not typically initiate their own action potential, but instead perform the mechanical work of a contraction [20].

The cardiac cells that form the conducting system can be found in four specific locations and have various rates at which they are capable of generating action potentials, because of their different rates of slow depolarization to threshold

1. The sinoatrial node (SA node) in the right atrial wall near the opening of the superior vena cava, has a rate of action potentials around $70-80$ beats per minute.

2. The atrioventricular node (AV node) at the base of the right atrium near the septum, above the junction of the atria and ventricles, has a rate of action potentials around $40-$ 60 beats per minute. The AV node is the electrical connection between the atrium and ventricles.

3. The bundle of His, a tract of cells that originates in the AV node, goes down along the ventricular septum, divided in the left branch and right branch of bundle of His, then each branch makes a turn at the bottom of the ventricles and goes back to atria along the outer walls, has a rate of action potentials around 
$20-40$ beats per minute. The bundle of His and Purkinje fibers are a fast conduction system for the ventricles.

4. The Purkinje fibers, very small branches that start at the end of His bundle and are spread all over the ventricular myocardium, has a rate of action potential around 20 - 40 beats per minute [20].

The pacemaker of the heart is the SA node, which drives the heart at its rate of 70 to 80 beats per minute, in normal conditions, being the fastest rate. The remaining autorhythmic cardiac cells cannot assume their own rates because they are activated by action potentials of the SA node first. If the SA node is damaged and becomes nonfunctional, the AV node drives the heart, at a slower rate, at about 50 beats per minute. If the AV node is damaged, the impulse between atria and ventricles is blocked and the atria continue at the rate imposed by the SA node, but the ventricles contract at a much slower rate, at about 30 beats per minute, imposed by the bundle of His and Purkinje fibers. This is known as complete heart block [20].

Another situation is when a small area of the heart called an ectopic focus, depolarizes at a faster rate than the SA node and initiates an action potential before the action potential issued by the SA node. The heart rate becomes suddenly greatly accelerated until the ectopic focus disappears. One cycle of this is called a premature beat or extrasystole and multiple cycles are called tachycardias. A random contraction of isolated ventricles' cardiac muscle fibers, due to an uncoordinated excitation, is called fibrillation. Ventricular fibrillation causes a rapid death, because the ventricles are no longer capable to pump the blood into the arteries.

During an action potential, or polarization and depolarization of the cardiac muscle, the electrical currents are also spread into the tissue adjacent to the heart and a part of this electrical activity reaches the body surface, which can be recorded. The recording is called electrocardiogram with the abbreviation ECG. 


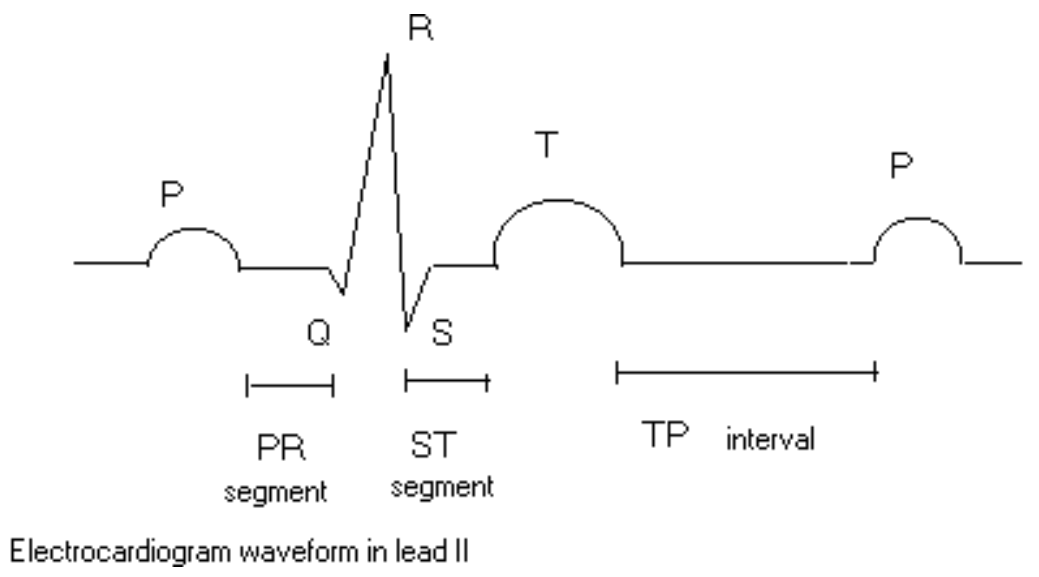

Figure 3.1. Example of ECG signal, lead II

A normal ECG has four different waveforms (Figure 3.1):

1. The baseline, where the ECG remains flat, because no current flows through the heart:

- PR segment represents AV nodal delay.

- ST segment represents the time in which ventricles are contracting and emptying.

- TP segment when ventricles are relaxing and filling.

2. The $\mathbf{P}$ wave, which represents atrial depolarization.

3. The QRS complex, which represents ventricular depolarization.

4. The $\mathbf{T}$ wave, which represents ventricular repolarization.

Because various components of the ECG can be correlated to specific cardiac events, this recording is useful in diagnosing heart diseases (Figure 3.2), like:

1. Abnormalities in rate:

- Tachycardia: characterized by a fast heart rate, more than 100 beats per minute.

- Bradycardia: characterized by a slow heart rate, less than 60 beats per minute.

2. Abnormalities in rhythm: - arrhythmia, characterized by any variation from the normal rhythm and sequence of excitation.

3. Cardiac myopathies: represents the damage of the cardiac muscle, and is characterized by abnormal QRS waveforms [20]. 

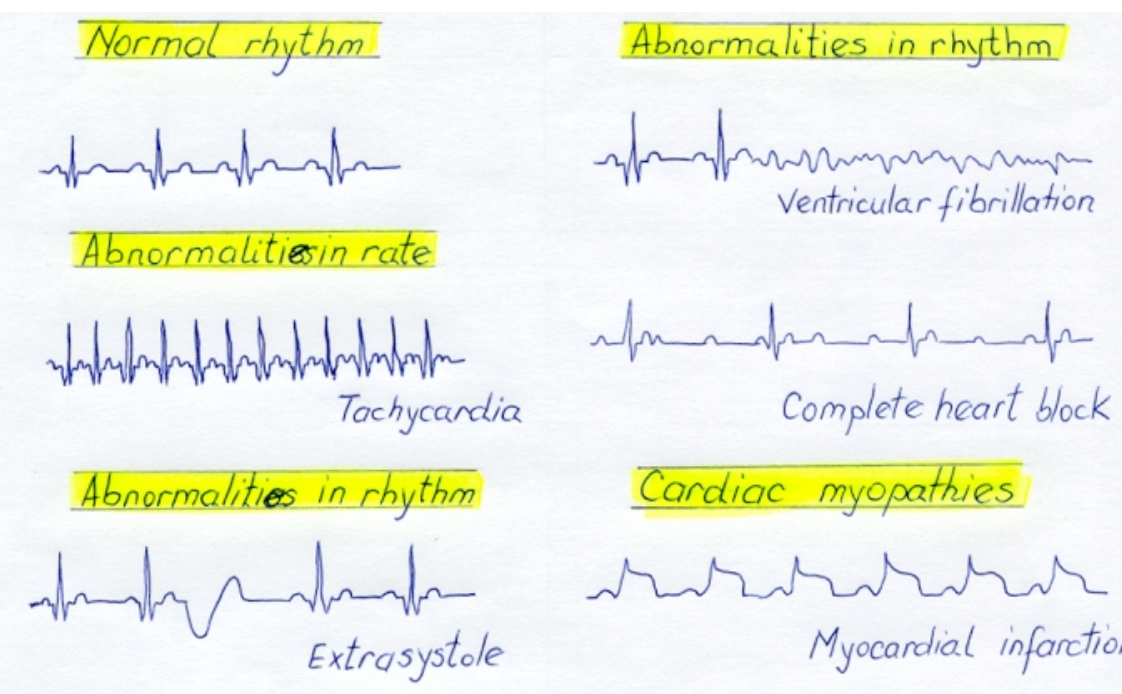

Cardiac myopathies

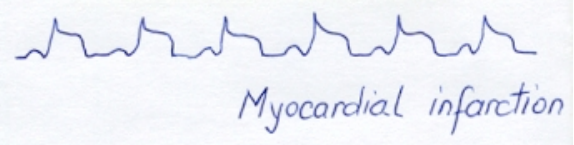

Figure 3.2 Representative changes in ECG signal for a diseased heart

\subsection{Influences on cardiac heart rate}

Even though intrinsic heart rate is normally determined by the rate of depolarization of the cardiac pacemaker cells, it is well known that, at any moment in time, heart rate represents the effects of factors including the autonomic nervous system, respiratory system and reflexes like baroreceptors, chemoreceptors, atrial receptors, etc.

Heart rate is determined primarily by the influence of the autonomic nervous system and can be used like a quantitative marker of autonomic activity. The heart is innervated by both sections of the autonomic nervous system: sympathetic and parasympathetic, which can modify the rate or the strength of contraction.

The parasympathetic nervous system innervates the atrium, SA node and AV node, but there is no significant innervation of the ventricles. It controls the heart in quiet, relaxed situations by decreasing the rate of slow depolarization to threshold of the SA node and decreasing the excitability of the AV node. The heart beats less rapidly and the contraction is weaker.

The sympathetic nervous system also innervates the SA node and AV node, but there is a very rich innervation of the ventricles. It controls the heart in situations in which a greater 
cardiac output is needed, in exercise and tense situations, by increasing the rate of depolarization of the SA node, so that the SA node fires more frequently. Sympathetic nervous system also reduces the AV nodal delay, so that the actions potentials are much more rapidly spread in the cardiac muscle of the ventricles.

Sympathetic and parasympathetic nervous system has antagonistic effects, their actions oppose one to each other, and at any moment in time there is a balance between the inhibitory effect of the parasympathetic nervous system, also called vagus nerve and stimulatory effect of the sympathetic nervous system (Figure 3.3).

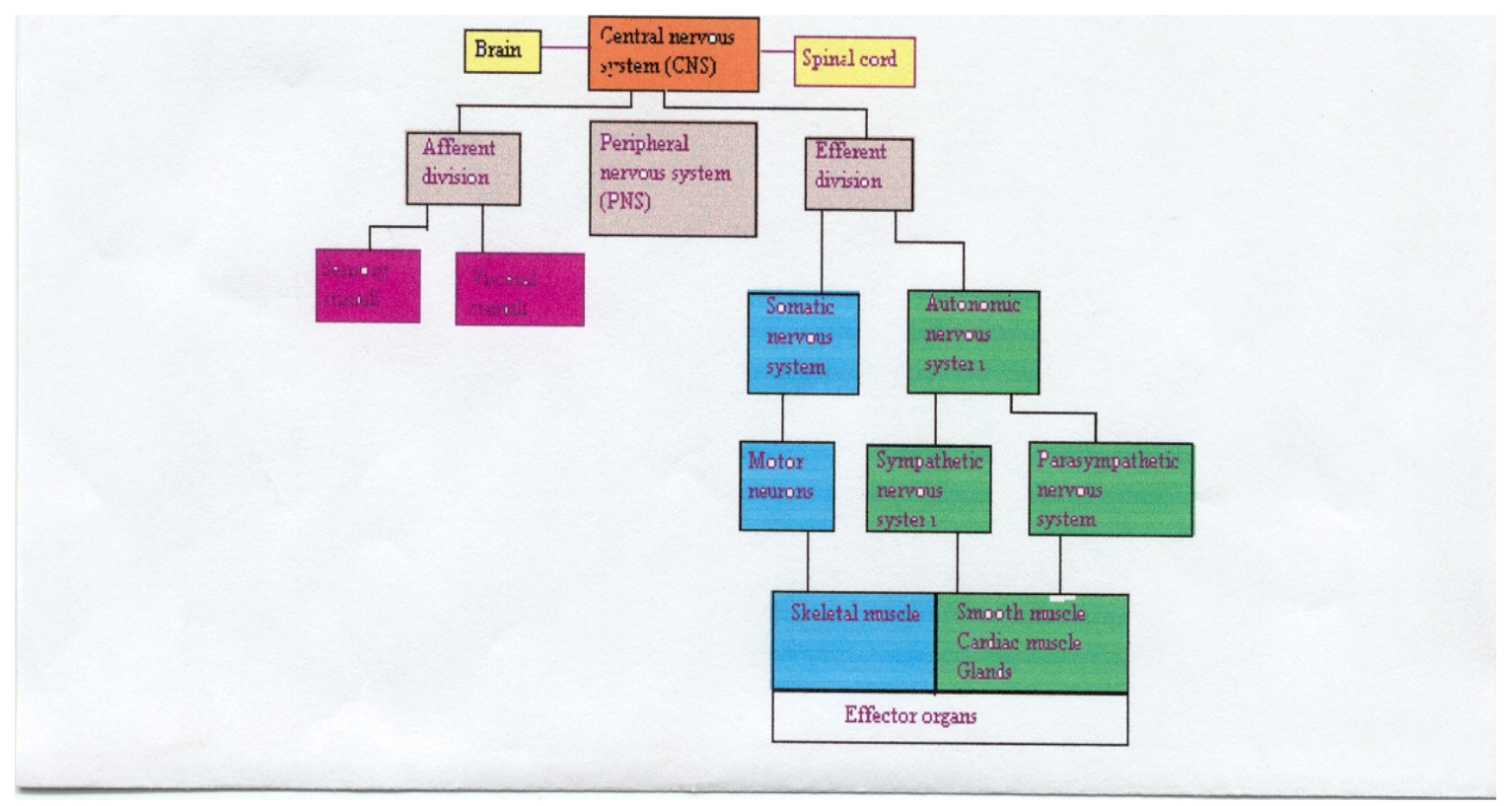

Figure 3.3 The structure of nervous system

The activity of the autonomic nervous system is coordinated by the cardiovascular control center located in the brain stem (Figure 3.4) 


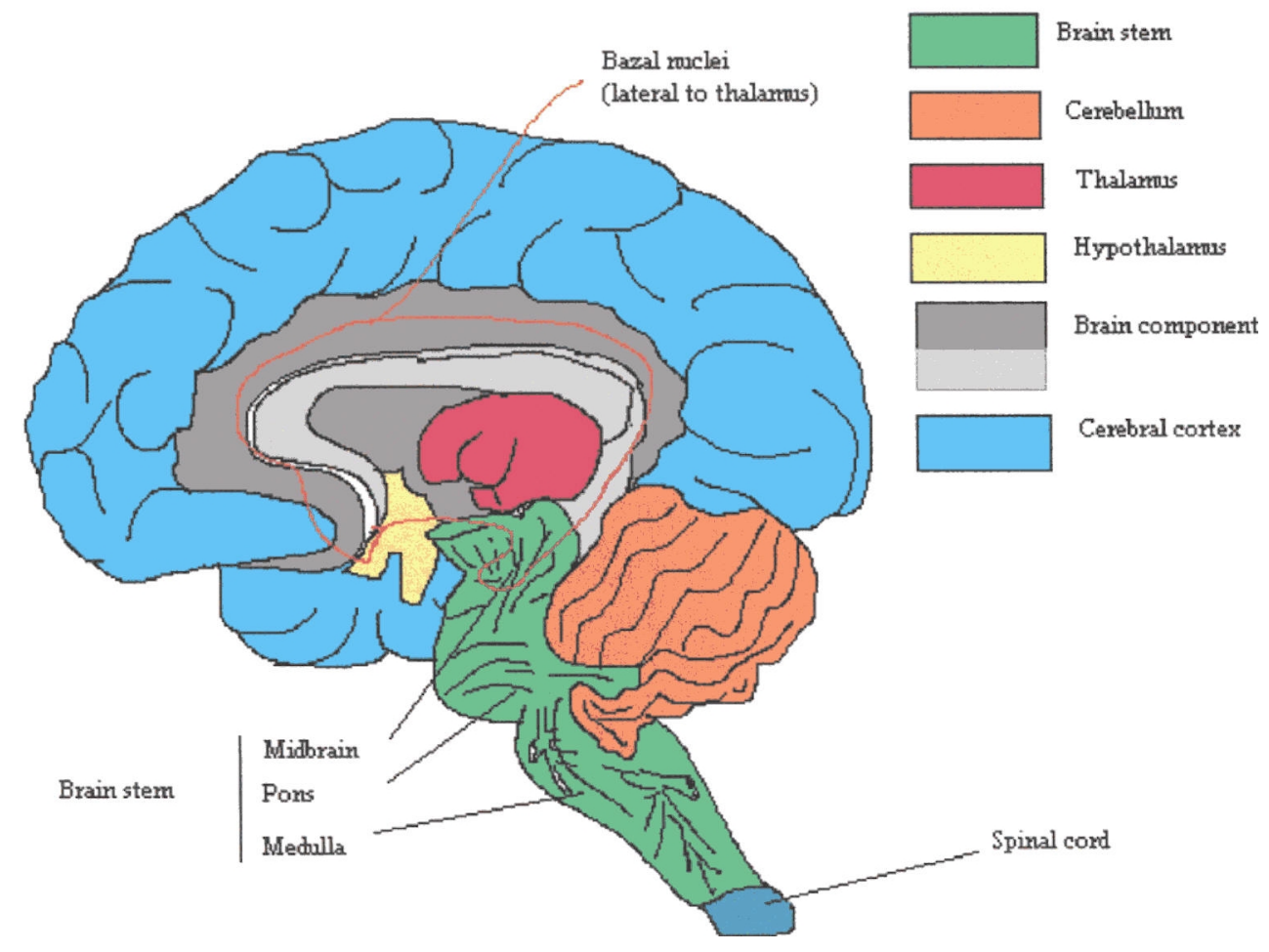

Figure 3.4 Broad description of the brain

Another important factor that influences the heart rate is the respiratory system. During inspiration, heart rate increases, and during expiration heart rate decreases. Heart rate presents a complex cyclical variation with respiration and involves both central and reflex interactions. In addition very slow circadian changes occur throughout the day and night that are mediated by neural and hormonal influences.

\section{$\underline{\text { 3.3 Heart rate variability, measurement, interpretation }}$}

The fact that the physiological cardiac rhythm in a healthy heart is not regular has been shown many years ago [18]. The recognition of this fact has led the people involved in this area of research to try to understand the clinical importance of heart rate variability and to develop appropriate standards and nomenclature, develop definitions of terms, specify standard methods of measurement, and define physiological and pathophysiological correlates. Heart rate variability (HRV) is a term used to describe variations of both instantaneous heart rate and RR intervals. HRV has the potential to provide valuable insight into the physiological and 
pathological conditions of the heart, and to provide information about the general health of the heart.

The variation of heart rate may be evaluated by a number of methods [18]:

- Time domain measurements

- Frequency domain measurements

- Nonlinear methods

In all the methods, analysis of heart rate variability is done from:

- Short-term recordings, which means segments of 5 minutes recordings. The major advantage of such method is the limited duration of ECG monitoring. This method allows also the comparison of different parameters or heart rate variability to be made during different activities like sleep or rest, tilt and upright position. The major disadvantage is that such short recordings may fail to detect long-term trends or diurnal influences in heart rate or parameters computed, which may have clinical importance. The stationarity of the signal is likely.

- Long-term recordings, which means a 24-hour continuous recording. This method has been proven to be more sensitive in detecting abnormalities in autonomic function, but despite the improvements made in collecting and computer processing of recordings, has the disadvantage of using a signal that is perturbed by artifacts, ectopic beats, premature beats, very short periods of time without signal, and requires human editing to detect erroneously labeled beats. The difficulty with this technique is that the signal is most likely not stationary.

From a continuous ECG recording, each QRS complex is determined, intervals between successive complexes are computed, and the normal-to-normal beat $\mathrm{NN}$ or the RR interval and the instantaneous heart rate are obtained. Ectopic beats, premature beats or artifacts are excluded from analysis.

\subsubsection{Time-Domain measurements}

Once the set of beat-to-beat intervals is obtained, a large number of HRV parameters can be measured. In the time-domain, measurements include:

* Statistical methods 
- Time-domain measures based on beat-to-beat intervals

Among the simplest variables to calculate, there are the MEAN and MEDIAN, and the standard deviation of the heart period for the whole-recording period. If the last is computed for a 24hour recording, it can be referred as cycle length variability with abbreviation CLV. Others are standard deviation of $\mathrm{NN}$ intervals (SDNN), which represents the square root of the variance, and the standard deviation of the 5-minute mean cycle lengths over the entire recording (SDANN).

- Time-domain measures based on the difference between adjacent cycle lengths.

These include the square root of the mean squared differences of successive NN intervals (RMSSD), the number of interval differences of successive NN intervals greater than $50 \mathrm{~ms}$ (NN50), and pNN50, which is obtained by dividing NN50 with the total number of NN intervals.

* Geometric methods

This method uses the sequence of RR intervals to construct a specific geometrical form:

- The density histogram of RR interval durations

- The density histogram of differences between successive RR intervals

- The duration of each RR interval against the duration of the immediately preceding RR interval

After the plots are done, HRV is evaluated using different methods:

- HRV triangular index

- Triangular interpolation of RR interval histogram.

\subsubsection{Frequency-domain measurements}

Frequency-domain measurements, also called power spectral analysis, decomposes the heart rate signal into its frequency components, and evaluates them in terms of quantity, and provides the basic information of how power distributes as a function of frequency. The research in frequency domain has showed that changes in heart rate variability and parameters computed reflect the oscillations in sympathetic and parasympathetic balance. There is an association 
between low frequency (LF) and sympathetic nervous activity on one hand and an association between high frequency (HF) and parasympathetic nervous activity on the other hand.

There are two ways for calculation of power spectral density:

- $\quad$ Parametric, which utilizes model estimation technique.

The result is a continuous smooth spectrum of activity.

- Non-parametric, using fast Fourier transformation

Data to be processed must be stationary and sufficiently long. In humans, there are two major frequency bands within the range from 0 to $0.5 \mathrm{~Hz}[18]$ (Figure 3.5)

- High frequency band approximately from $0.15-0.4 \mathrm{~Hz}$ reflects parasympathetic activity, with the peak around $0.25 \mathrm{~Hz}$.

- Low frequency band approximately from $0.04-0.15 \mathrm{~Hz}$ reflects both the activity of parasympathetic and sympathetic activity, but predominantly the latter.

Another frequency band is the very low frequency component (VLF), which is much less defined, and is proved to be a non-harmonic component, and has no coherent properties. The range of the very low frequency component is from $0.003 \mathrm{~Hz}$ to $0.04 \mathrm{~Hz}$.

The ratio LF/HF is considered important because it reflects the balance between the two branches of autonomic nervous system. The evaluation of HF and LF is calculated in absolute values of power or measured in normalized units.

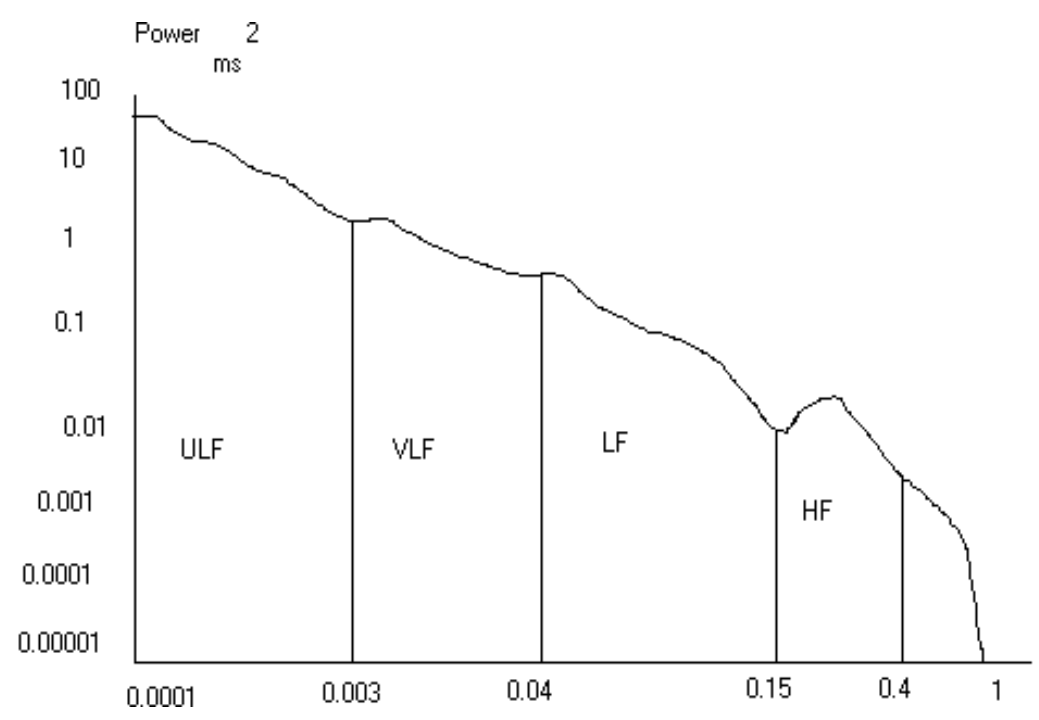

Figure 3.5 Schematic estimate of power spectral density, log-log scale, obtained from the entire 24-hour of long-term recordings in humans 


\subsection{Significant tests for laboratory analysis.}

Laboratory tests are used on one hand to demonstrate the damage of different parts of the body like heart muscle damage, and on the other hand to assess the effect of doxorubicin and its adverse reactions.

\subsubsection{Heart muscle damage}

Significant ECG abnormalities are not always found in acute myocardial injury (AMI). Analysis of creatine kinase (CK) and creatine kinase MB (CK-MB) combined from a blood sample are presently the major available standards to assess injury to the myocardium [19].

Table 3.1 Heart muscle damage

\begin{tabular}{|c|c|c|c|}
\hline Name & Additional information & Use & Limitations \\
\hline $\begin{array}{l}\text { CREATINE } \\
\text { KINASE }\end{array}$ & $\begin{array}{l}\text { Released by the muscle cells } \\
\text { (skeletal muscle and heart } \\
\text { muscle), brain cells } \\
\text { Three fractions of CK } \\
\text { normally may be found: } \\
\text { - } \quad \text { MM is present in normal } \\
\text { serum } \\
\text { - } \quad \text { MB is the myocardial } \\
\text { fraction } \\
\text { - } \quad \text { BB is rarely present. }\end{array}$ & $\begin{array}{l}\text { Test for acute myocardial infarct } \\
\text { and other heart diseases, and for } \\
\text { skeletal muscular disease or } \\
\text { damage, for brain cells damage. } \\
\text { High CK is found after trauma, } \\
\text { surgery, and exercise; but these } \\
\text { entities are not accompanied by } \\
\text { elevation of CK-MB. }\end{array}$ & $\begin{array}{l}\text { Intramuscul } \\
\text { ar injections } \\
\text { increase } \\
\text { serum CK } \\
\text { activity. }\end{array}$ \\
\hline CK-MB & $\begin{array}{l}\text { Released by the heart muscle } \\
\text { cells. } \\
\text { CK-MB normally is much } \\
\text { less than } 0.6 \text { of the total CK }\end{array}$ & $\begin{array}{l}\text { CK-MB is used for the detection } \\
\text { of AMI, and damage of the heart } \\
\text { muscle. } \\
\text { CK-MB increases have been } \\
\text { reported with other entities, } \\
\text { which cause damage to the } \\
\text { myocardium such as } \\
\text { myocarditis, some instances of } \\
\text { cardiomyopathy. CK does not } \\
\text { generally rise and fall in such } \\
\text { nonacute myocardial infarct } \\
\text { settings, as it does in acute } \\
\text { myocardial infarct. } \\
\text { CK-MB is not elevated in case } \\
\text { of injection into muscle, brain } \\
\text { disorders in which total CK may } \\
\text { be increased. }\end{array}$ & $\begin{array}{l}\text { CK-MB is } \\
\text { increased } \\
\text { with cardiac } \\
\text { surgery. }\end{array}$ \\
\hline
\end{tabular}




\subsubsection{Pancreas damage}

Table 3.2 Pancreas damage

\begin{tabular}{|c|c|c|c|}
\hline Name & $\begin{array}{l}\text { Additional } \\
\text { information }\end{array}$ & Use & Limitations \\
\hline AMYLASE & $\begin{array}{l}\text { One of the } \\
\text { pancreatic enzymes, } \\
\text { produced by the } \\
\text { exocrine pancreas, } \\
\text { which contributes to } \\
\text { carbohydrate } \\
\text { digestion. Converts } \\
\text { polysaccharides into } \\
\text { disaccharides. }\end{array}$ & $\begin{array}{l}\text { Is usually a sensitive and } \\
\text { useful diagnostic method for } \\
\text { acute pancreatitis. Serum } \\
\text { amylase is used to work up } \\
\text { abdominal pain, epigastric } \\
\text { tenderness, nausea, and } \\
\text { vomiting, which characterize } \\
\text { acute pancreatitis, as well as } \\
\text { gastrointestinal perforation, } \\
\text { intestinal obstruction or } \\
\text { bowel infarct, and common } \\
\text { duct stones. }\end{array}$ & $\begin{array}{l}\text { Poor specificity. } \\
\text { The entire } \\
\text { pancreas can be } \\
\text { destroyed in } \\
\text { pancreatitis and } \\
\text { still the amylase } \\
\text { will derive from } \\
\text { other structures. } \\
\text { Salivary amylase } \\
\text { makes up about } \\
60 \% \text { of the serum } \\
\text { enzyme, while the } \\
\text { pancreatic } \\
\text { fraction is of } \\
\text { clinical interest. }\end{array}$ \\
\hline TRIGLYCERIDES & $\begin{array}{l}\text { TG is a family of } \\
\text { complex lipids } \\
\text { composed of glycerol } \\
\text { esterified with three } \\
\text { fatty acids. Are not } \\
\text { soluble in blood and } \\
\text { constitute almost all } \\
\text { of tissue storage fat. }\end{array}$ & $\begin{array}{l}\text { Test of triglycerides is } \\
\text { required for determination of } \\
\text { lipid risk factors of coronary } \\
\text { artery diseases, to evaluate } \\
\text { turbid samples of blood, } \\
\text { plasma and serum. } \\
\text { Extremely high triglyceride } \\
\text { levels suggest the possibility } \\
\text { of pancreatitis. Triglycerides } \\
\text { may also increase with } \\
\text { chronic renal and liver } \\
\text { disease. }\end{array}$ & \\
\hline
\end{tabular}




\subsubsection{Kidney damage}

Table 3.3 Kidney damage

\begin{tabular}{|c|c|c|c|}
\hline Name & Additional information & Use & Limitations \\
\hline $\begin{array}{l}\text { BLOOD } \\
\text { UREA } \\
\text { NITROGEN }\end{array}$ & $\begin{array}{l}\text { BUN is an end product } \\
\text { of body metabolism, } \\
\text { resulting from the } \\
\text { breakdown of protein. } \\
\text { Urea is easily filtered } \\
\text { by the renal glomeruli } \\
\text { and is partially } \\
\text { reabsorbed by the renal } \\
\text { tubules. Urea nitrogen } \\
\text { reflects the ratio } \\
\text { between urea } \\
\text { production and } \\
\text { clearance. Increase } \\
\text { urea nitrogen is caused } \\
\text { by increased } \\
\text { production or decrease } \\
\text { excresion. }\end{array}$ & $\begin{array}{l}\text { This lab test is useful to assess } \\
\text { renal function. High BUN occurs } \\
\text { in renal diseases like } \\
\text { glomerulonephritis, } \\
\text { acute renal failure, urinary tract } \\
\text { obstruction etc. It is dependent on } \\
\text { the renal blood flow and urine } \\
\text { flow rates. } \\
\text { Increased BUN includes also } \\
\text { among other causes severe } \\
\text { congestion of heart failure. } \\
\text { Low BUN occurs with low protein } \\
\text { intake, with some antibiotics and } \\
\text { with liver damage. }\end{array}$ & \\
\hline CREATININE & $\begin{array}{l}\text { Creatinine is also an } \\
\text { end product of bodily } \\
\text { metabolism, which } \\
\text { accumulated in the } \\
\text { body is toxic. }\end{array}$ & $\begin{array}{l}\text { Is the most common clinical } \\
\text { function test, which provides } \\
\text { information on glomerular } \\
\text { filtration. The renal diseases, } \\
\text { urinary tract obstruction, reduced } \\
\text { renal blood flow as a consequence } \\
\text { of heart failure and dehydration } \\
\text { cause high level of creatinine. } \\
\text { Low creatinine is present in liver } \\
\text { disease, from decreased hepatic } \\
\text { production of creatinine or an } \\
\text { inadequate protein intake. }\end{array}$ & $\begin{array}{l}\text { Concentration } \\
\text { of creatinine } \\
\text { only becomes } \\
\text { abnormal } \\
\text { when half or } \\
\text { more of the } \\
\text { neuphrons } \\
\text { have stopped } \\
\text { functioning. } \\
\text { Increased } \\
\text { values for } \\
\text { creatinine } \\
\text { may result } \\
\text { also from } \\
\text { noncreatinine } \\
\text { substances } \\
\text { such as } \\
\text { glucose,uric } \\
\text { acid etc. }\end{array}$ \\
\hline
\end{tabular}


Table 3.4 Liver damage

\begin{tabular}{|c|c|c|c|}
\hline Name & Additional information & Use & Limitations \\
\hline CHOLESTEROL & $\begin{array}{l}\text { Cholesterol is a lipid, so is not soluble } \\
\text { in the blood, unless is attached to the } \\
\text { specific plasma protein carriers in the } \\
\text { form of lipoprotein complexes: } \\
\text { - HDL, most proteins, less cholesterol } \\
\text { - LDL, less proteins, more cholesterol } \\
\text {-VLDL, least proteins, most } \\
\text { cholesterol } \\
\text { The liver has the primary role in } \\
\text { cholesterol metabolism, in } \\
\text { determining total blood cholesterol } \\
\text { levels. The liver may be } \\
\text { manufacturing new cholesterol, } \\
\text { extracting old one from the blood and } \\
\text { secreting it into the bile, or converting } \\
\text { old cholesterol into bile salts, which } \\
\text { also are secreted into the bile. }\end{array}$ & $\begin{array}{l}\text { Evaluate risk of } \\
\text { coronary artery } \\
\text { occlusion, } \\
\text { atherosclerosis, } \\
\text { myocardial } \\
\text { infarction. } \\
\text { Cholesterol } \\
\text { values are } \\
\text { altered by } \\
\text { weight loss, } \\
\text { acute illness, as } \\
\text { well as by acute } \\
\text { myocardial } \\
\text { infarct. }\end{array}$ & $\begin{array}{l}\text { There are also } \\
\text { other risk } \\
\text { factors for } \\
\text { coronary } \\
\text { arterial } \\
\text { disease like: } \\
\text { hypertension, } \\
\text { family } \\
\text { history, } \\
\text { smoking, } \\
\text { obesity, but } \\
\text { still test of } \\
\text { cholesterol } \\
\text { provide } \\
\text { reliable } \\
\text { information. }\end{array}$ \\
\hline BILIRUBIN & $\begin{array}{l}\text { Bilirubin is the primary bile pigment } \\
\text { derived from the breakdown of worn- } \\
\text { out red cells, is the end product } \\
\text { resulting from the hemoglobin } \\
\text { contained within these old red blood } \\
\text { cells. Bilirubin does not play a role in } \\
\text { digestion at all, but instead is one of } \\
\text { the few waste products, which is } \\
\text { extracted from the blood by the } \\
\text { hepatocytes and is excreted in the bile. } \\
\text { The intestine normally reabsorbs a } \\
\text { small amount back into the blood and } \\
\text { the kidneys excrete the rest only after } \\
\text { it has been modified during its } \\
\text { passage through the liver and } \\
\text { intestine. }\end{array}$ & $\begin{array}{l}\text { Evaluate liver } \\
\text { and biliary } \\
\text { disease, like: } \\
\text { hepatitis, } \\
\text { cholecystitis, } \\
\text { cirrhosis, biliary } \\
\text { obstructions etc. }\end{array}$ & $\begin{array}{l}\text { Differential } \\
\text { diagnosis of } \\
\text { liver diseases } \\
\text { requires total } \\
\text { and direct } \\
\text { bilirubin } \\
\text { values, as } \\
\text { well as other } \\
\text { tests. }\end{array}$ \\
\hline
\end{tabular}




\subsubsection{Hemostatic considerations}

Table 3.5 Hemostatic considerations

\begin{tabular}{|c|c|c|c|}
\hline Name & Additional information & Use & Limitation \\
\hline PLATELETS & $\begin{array}{l}\text { Platelets, also called thrombocytes, are } \\
\text { tiny, disk-shaped structures that play a } \\
\text { critical role in the process of blood } \\
\text { coagulation. They are manufactured in } \\
\text { the bone marrow and usually survive } \\
\text { for about ten days. Are a part of } \\
\text { cellular elements with } 2-3 \text { microns in } \\
\text { diameter, but large forms called } \\
\text { megathrombocytes appear when } \\
\text { production is increased. Platelets are } \\
\text { subject to circadian periodicity; } \\
\text { highest platelets count occurs during } \\
\text { midday. }\end{array}$ & $\begin{array}{l}\text { Evaluate and monitor } \\
\text { bleeding disorders, drug } \\
\text { induced } \\
\text { thrombocytopenia, } \\
\text { disseminated } \\
\text { intravascular } \\
\text { coagulation, leukemia, } \\
\text { and chemotherapeutic } \\
\text { management of } \\
\text { malignant disease. } \\
\text { In case of increased } \\
\text { platelets count, clots } \\
\text { named emboli, that } \\
\text { obstruct blood vessels } \\
\text { can develop. }\end{array}$ & $\begin{array}{l}\text { Clumping } \\
\text { may cause } \\
\text { false low } \\
\text { count. } \\
\text { A variety } \\
\text { of factors } \\
\text { affect the } \\
\text { distributio } \\
\text { n of } \\
\text { platelets, } \\
\text { so } \\
\text { interpretat } \\
\text { ion of the } \\
\text { results } \\
\text { must be } \\
\text { done } \\
\text { carefully. }\end{array}$ \\
\hline HGB & $\begin{array}{l}\text { The red blood cells carry oxygen and } \\
\text { CO2 through the body, because of } \\
\text { presence of hemoglobin, the pigment } \\
\text { that is formed when the red blood cells } \\
\text { develop in the bone marrow. Each } \\
\text { hemoglobin molecule is made up of a } \\
\text { protein molecule called globin and } \\
\text { four pigmented molecules of a } \\
\text { compound called heme. Each heme } \\
\text { molecule has one atom of iron, and } \\
\text { there are four heme molecules in a } \\
\text { single molecule of hemoglobin. This } \\
\text { structure makes it possible for one } \\
\text { hemoglobin molecule to join with four } \\
\text { oxygen molecules. This reaction is } \\
\text { reversible, enabling hemoglobin to } \\
\text { pick up oxygen when in the lungs and } \\
\text { to release oxygen in the cells when the } \\
\text { blood is pumped to the tissue. }\end{array}$ & $\begin{array}{l}\text { Evaluate anemia, blood } \\
\text { loss, hemolysis, } \\
\text { polycythemia etc. }\end{array}$ & \\
\hline HCT & $\begin{array}{l}\text { Hematocrit represents the } \\
\text { percent of whole blood that is red } \\
\text { blood cells. }\end{array}$ & $\begin{array}{l}\text { Evaluate anemia, blood } \\
\text { loss, hemolytic anemia, } \\
\text { polycythemia etc. }\end{array}$ & \\
\hline
\end{tabular}




\begin{tabular}{|c|c|c|}
\hline $\mathrm{RBC}$ & $\begin{array}{l}\text { There are three cellular elements in the } \\
\text { blood manufactured by the bone } \\
\text { marrow and visible only under the } \\
\text { microscope: red blood cells, white } \\
\text { blood cells and platelets. Red blood } \\
\text { cells carry oxygen from the lungs to } \\
\text { the various body tissues and CO2 from } \\
\text { the body to the lungs, with the help of } \\
\text { hemoglobin. }\end{array}$ & $\begin{array}{l}\text { Evaluate anemia, } \\
\text { polycythemia } \\
\text { Decrease in RBC count } \\
\text { may be the result of the } \\
\text { red cell loss by bleeding } \\
\text { or hemolysis, failure of } \\
\text { marrow production. } \\
\text { Increase in RBC count } \\
\text { may be the result of } \\
\text { primary polycythemia } \\
\text { or secondary } \\
\text { polycythemia which } \\
\text { includes cardiovascular } \\
\text { disease and stress } \\
\text { polycythemia which } \\
\text { represents } \\
\text { hemoconcentration } \\
\text { associated with } \\
\text { exercise, fright, stress }\end{array}$ \\
\hline
\end{tabular}

\subsubsection{Weight}

Weight is another important test used to assess the healthiness of the subject. 


\section{Chapter 4 METHODS}

In this thesis, a unique experimental setup was designed such that an animal can be monitored continuously for several months. Many aspects needed to be considered including the animal model, computers, hardware, interfaces, servers and connections, and logical system, represented by software for different levels, needs, and scopes. Lastly, automated analysis tools were developed to extract manageable representations of the data in order to draw conclusions.

\subsection{Theoretical considerations about the system}

This large project is a system with multiple stages, where decisions need to be made at each stage. After the system is organized, results were obtained at each level. The stages for this project are as follows:

\section{Animal considerations}

○ Kind of animal: size, lifetime, cost, if they approximate humans

○ Sensors: how are attached, animal rejection, power, longevity

○ Environment: captivity, light/dark cycles, care options

- Implanted device: size, bandwidth, radio-frequency, reusability, recharge battery

- Sources of noise: sensor' s interaction, motion, other electrical signals

- Model: how to induce cardiac disease, response to captivity, how to protect them from other disease, number and type of controls (like blood analysis, X-ray, etc.)

\section{Collection}

o The hardware needed so that the information from the implanted device arrives to the computer

o The software needed for the acquisition of the data, which among others encapsulates the type of recordings (analog, digital)

o Continuous or periodic recordings

o The location of the transmitters on the cages

o Collection of the environmental data like temperature, feeding patterns, cleaning cages, etc. 


\section{Compression:}

○ Which data to compress, real-time data or post-processed data

○ Nondestructive or destructive way for redundancy or losing data

- Initial scan for some events (portions with too much noise)

\section{Mass storage}

o The network, how data from the computer is transferred on the server

o Kind of storage used, like optical disks for huge amount of data

o How to organize data, in flat files or database

o How to access and query data, sequential type versus random access

o Security levels on accessing data.

\section{Processing and Extraction}

o Use of software to build efficient programs in a specific language to accurately process data and determine parameters related to the application

o Features extraction

o Classification according to importance

o How long it takes to process data

o How to store results

\section{Visualization}

o How to visualize

o How to combine information

o How much to visualize

\section{Final analysis}

○ Pattern recognition

○ Decision making

○ Prediction

The last three components of the final analysis refer to methods to link features and events, to standardize nomenclature and develop definitions of terms, specify standard methods of measurement, define physiological and pathophysiological correlates, and establish standard levels for normal and abnormal values for parameters in order to be able to predict feature events. 
The following section describes decisions, which made for this experiment. Not all items listed above were addressed in this thesis, and are the subject of future work.

\subsection{Methods used in the research}

\subsubsection{Animal considerations}

In order to study the progression of ECG from normal to a diseased state of heart failure, three rabbits, New Zeeland breed, and female, were monitored continuously for thirteen weeks. These 13 weeks include 3 weeks of control data and 10 weeks of weekly injections of doxorubicin until death. The control rabbit (R710V) was injected with $3.75 \mathrm{ml}$ saline, and the other two (R733W, R735W) were injected with doxorubicin, at $2 \mathrm{mg} / \mathrm{kg}$ per week. For all three rabbits, blood was collected weekly from left ear and laboratory analysis and tests were done, for blood cell count, blood smear evaluation, routine coagulation, basic chemistry, pancreatic function tests, cardiac markers and other tests at West Virginia University Hospitals Clinical Laboratories.

\subsubsection{Signals. The hardware and the software used}

For the rabbits, R710V and R733W, a CTA-F40 implantable device (Data Sciences Intl., Inc., Minneapolis, MN), was inserted subcutaneously over the scapulae with the leads in a LEAD II configuration for $\mathrm{R} 710 \mathrm{~V}$ and $\mathrm{MX}$ configuration for $\mathrm{R} 733 \mathrm{~W}$. MX is a modified lead position, at the top center of the ribcage and at the sternum. This configuration was shown to be better than Lead II during motion. For the third rabbit, R735W, a TA10CA-F40 intracardiac implantable device was implanted, with the leads in a MX configuration for channel one and in an intracardiac configuration for channel two. The intracardiac channel is a bipolar electrode lead placed in the right ventricular apex through the jugular vein. Implantation was performed with assistance from Garry Linton, director of the HSC Animal Laboratories in accordance with DSI recommendations and ACUC protocol 9901-03. An intracardiac lead was added for the third rabbit since it is completely free of motion artifact and extremely useful for measurements based on the RR interval. A surface lead is still needed to make standard clinical ECG diagnosis. 
Using Dataquest A.R.T. (acquisition and radio-transmitter) biotelemetry system (Data Sciences Intl.,Inc.,Minneapolis, MN), the signal with a sampling frequency of $1000 \mathrm{~Hz}$ is transmitted to a RMC1 type receiver, which is attached to the cage (figure 4.1). The receiver is connected to Data Exchange Matrix and an IBM compatible computer with Data Sciences' acquisition program. The Matrix supplies power to the receivers and provides an interface between the receivers and the A.R.T. System. The system accepts up to 20 inputs: 16 solely for connection to receivers and 4 available for connection to receivers or other Matrices. Up to four animals can be monitored continuously. The Dataquest A.R.T. system detects, collects and analyzes electrical signals from one or several animals, and graphically represents this signal data as waveforms. Each waveform contains specific parameters, which are extracted to provide different types of data for analysis.

Due to the sheer quantity of data, the entire dataset cannot be stored on the Dataquest system permanently. In fact, only one animal generates 180 megabytes per day. To address this, data is transferred to a HP9000 dataserver (Hewlett Packard) via a network connection. Total space usage on the server is 292 gigabytes. Only about 4 gigabytes is on a hard disk; the rest is on magnetic-optical drives for " near-line" storage. The amount of data generated for this study was 55,440 megabytes.

To transfer the ECG data, which is organized in binary files, to a dataserver for storage, custom software was written in REXX language. At a specified time, once a day, data is automatically transferred and sorted according to the code of the rabbit. To sort the data according to the day of recording, a program in $\mathrm{C}++$ was developed, which automatically creates one directory for each day, extracts from each file the day of the recording, and copies the file into appropriate directory. Data sorted on the server can be further processed.

This aspect of the study was designed such that each component performed automatically. After testing the system for several months, the experiment proceeded without loss of data due to system failure. The only loss of data was due to artifact (noise), described in later sections, and times when the rabbit was disconnected from the receiver (during injections and cage cleanings). 


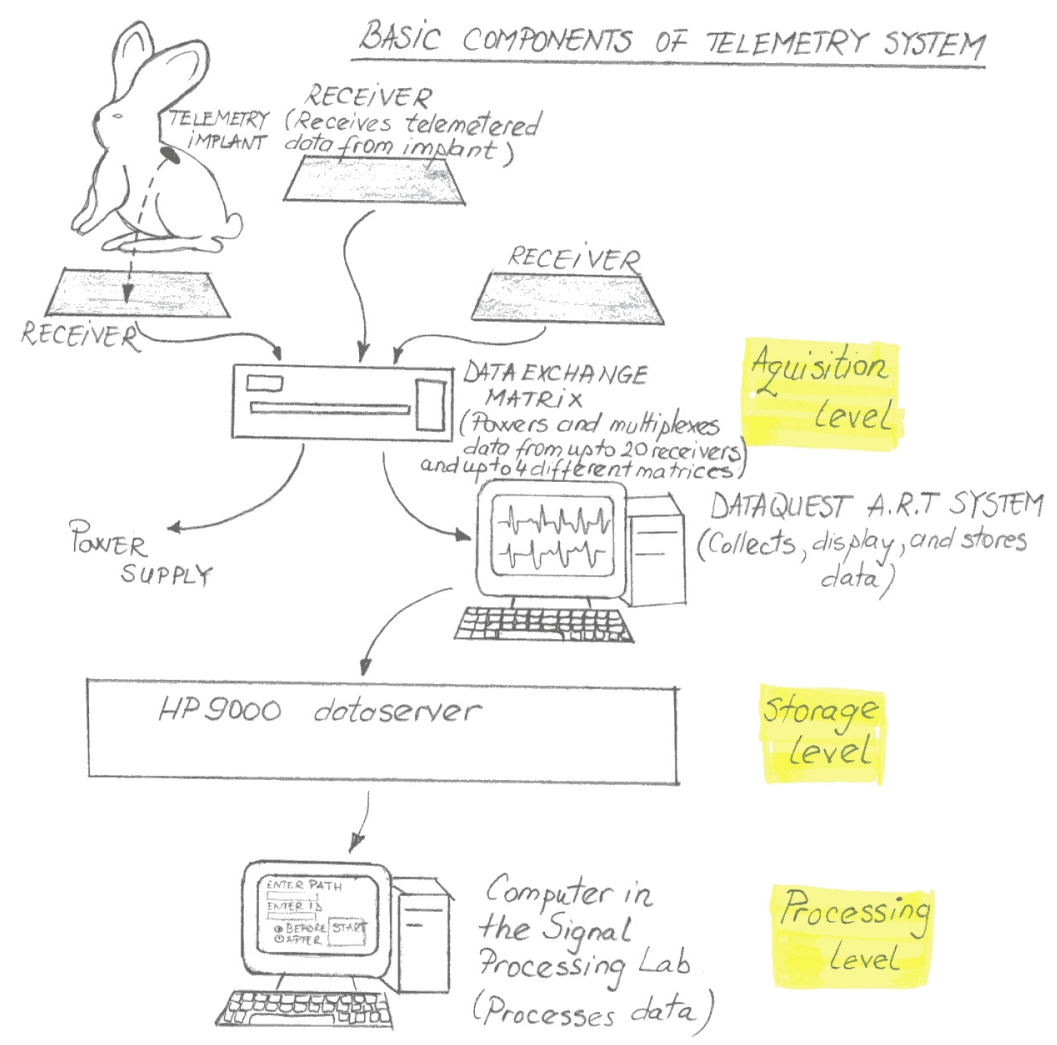

Figure 4.1 Schematic description of the entire project

\subsubsection{Processing data}

In order to process data, a program was written with Matlab 5.3. Unless otherwise specified the program was written by the author of the thesis. In cases where individual modules were written by colleagues on the project, the modules were integrated by the author of the thesis. The program extracts the ECG signal stored in the Datasciences (DSI) binary files, finds location of each beat or trigger points, computes RR intervals, detects artifacts and computes eleven HRV parameters.

There are at least three types of data storage files in a Dataquest A.R.T.system:

- Waveform file, a binary file, which contains waveform data for each animal and channel

- Parameter file, contains all parameters for each animal

- $\quad$ Log file, which is an ASCII file, one for each animal. 
The ECG signal is stored in waveform files, with adjustable size. From the naming convention point of view, every waveform file has a name, which is the animal' s ID or base name, and a three-digit extension. The first number reflects the number of the channel, and the last two represent a number, which is sequentially incremented from 00 though $\mathrm{ZZ}$ to permit a series of files for the same data category. This scheme makes filenames easy recognizable according to the animal and channel. For example the filename R710V.1A6 shows that the data collected is for the rabbit with the combination code $\mathrm{R} 710 \mathrm{~V}$, channel 1 and number of the file in hexadecimal is A6.

The DSI data record file format has five record types:

- 000 (NULL), length 1 byte, used for patching.

- 255 (SYNC), length 8 bytes, for file repair when necessary.

- 030 (TRIDEFINE), has variable length, contains information about tridefine section, tridefine record size, link offset, ID, location, number of bins.

- 031(TRIBURST), has variable length, contains information about waveform burst record, triburst record size, triburst time, state, sampling rate, burst length in bytes, number of samples, compression mode, full scale, and burst data. This is the part that effectively contains ECG data.

- 033 (TRIPARS), length 15 bytes, contain information for parameters.

A Matlab program (written by Pisut Raphisak) converts normal time (year, month, day, hour, minute, second) corresponding to the required ECG in DSI time, and searches through each triburst section in the binary file to find the corresponding DSI time, stored in 4 bytes length, then extracts

- Sampling rate, stored in 2 bytes unsigned,

- Number of samples, stored in 4 byte unsigned,

- Full scale, stored in 4 byte float

- The ECG value stored in 2 bytes each sample, if not compressed.

The ECG signal is stored in Matlab workspace like a column vector and then trigger points are found. In order to detect trigger points, which are R peaks from the PQRST ECG complex, an algorithm was used. The subprogram, which detects trigger points, was coded by my colleague Pisut Raphisak. An initial value for a threshold is calculated from the first 10 peaks detected, using the first maximum values detected. Whenever a value greater than the threshold is 
encountered, a trigger is marked. Throughout the algorithm, the initial threshold value is always updated, following a decreasing exponential curve between beats. After the new beat is located the threshold is updated as a percentage of the peak of the new beat. Positions of trigger points are stored in the Matlab workspace in a column vector, and correspond to the time in miliseconds.

RR intervals, the difference between successive trigger points, were computed. Artifacts are detected, if the RR interval is less or more than $20 \%$ from the previous RR interval. Interpolation is performed between good beats to replace the artifact [48]. Artifact detection is necessary since the ECG is subject to motion artifact (except in the intracardiac lead). Other forms of artifact included "full-scale" when the ECG voltage was above the input range and maintained a fixed value at the maximum voltage, typically due to motion and " no signal" when the voltage was at zero for extended periods when the animal received care or treatment.

After RR intervals are calculated and interpolation is performed, eleven parameters, relevant to heart rate variability and progression of ECG are calculated. My colleague Xueyan $\mathrm{Xu}$, has coded the individual heart rate variability modules.

1.Time domain measurements:

Mean (MEAN), Median (MEDIAN), Standard deviation of the durations of normal-to-normal NN intervals (SDNN), Coefficient of variance (CV), Interquartile range (IQR).

2.Time domain based on difference of consecutive $R R$ intervals $(\Delta R R)$ measurements: Standard deviation of $\Delta \mathrm{RR}$ (SDSD), Interquartile range of $\Delta \mathrm{RR}$ (DDIQR), Root mean square successive difference (RMSSD).

3.Frequency domain measurements:

Low frequency (LF), High frequency (HF), The ratio low frequency/high frequency (LHF).

The coefficient of variance $(\mathrm{CV})$ represents the standard deviation (SDNN) divided by the MEAN. For interquartile range (IQR), the RR interval column vector obtained is sorted, then values in the location at 25 and 75 percentile from the length of the vector are extracted and the parameter is the difference between these two values. RMSSD represents the square root of the mean of the sum of the squares of differences between adjacent normal RR intervals. For high frequency HF, and low frequency LF, power spectrum density is calculated, and then HF and LF are calculated as a sum of values of power spectral within the appropriate range. The ranges for 
rabbit model are from $0.4373 \mathrm{~Hz}$ to $0.5625 \mathrm{~Hz}$ for $\mathrm{HF}$ and from $0.0625 \mathrm{~Hz}$ to $0.1875 \mathrm{~Hz}$ for $\mathrm{LF}$ [47].

Data to be processed is organized in weeks, accordingly to the number of injection for that week. For the data recorded after the day of the first injection, the week always starts the next day after injection, and is considered to be with positive sign. For the data recorded before the day of the first injection, the week ends with one day before the day of the injection, and the week is considered to be with negative sign. Next table shows the days only for the weeks used in the results. The reason data was processed every two weeks is that the clear differences are noticeable only at a two-week interval.

Table 4.1 Dates and week number

\begin{tabular}{|c|c|l|l|l|l|l|}
\hline $\begin{array}{c}\text { \# of the day in the } \\
\text { week }\end{array}$ & Week -1 & Week 3 & Week 5 & Week 7 & Week 8 & Week 9 \\
\hline 1 & $12 / 29 / 99$ & $01 / 21 / 00$ & $02 / 04 / 00$ & $02 / 18 / 00$ & $02 / 25 / 00$ & $03 / 03 / 00$ \\
\hline 2 & $12 / 30 / 99$ & $01 / 22 / 00$ & $02 / 05 / 00$ & $02 / 19 / 00$ & $02 / 26 / 00$ & $03 / 04 / 00$ \\
\hline 3 & $12 / 31 / 99$ & $01 / 23 / 00$ & $02 / 06 / 00$ & $02 / 20 / 00$ & $02 / 27 / 00$ & $03 / 05 / 00$ \\
\hline 4 & $01 / 01 / 00$ & $01 / 24 / 00$ & $02 / 07 / 00$ & $02 / 21 / 00$ & $02 / 28 / 00$ & $03 / 06 / 00$ \\
\hline 5 & $01 / 02 / 00$ & $01 / 25 / 00$ & $02 / 08 / 00$ & $02 / 22 / 00$ & $02 / 29 / 00$ & $03 / 07 / 00$ \\
\hline 6 & $01 / 03 / 00$ & $01 / 26 / 00$ & $02 / 09 / 00$ & $02 / 23 / 00$ & $03 / 01 / 00$ & $03 / 08 / 00$ \\
\hline 7 & $01 / 04 / 00$ & $01 / 27 / 00$ & $02 / 10 / 00$ & $02 / 24 / 00$ & $03 / 02 / 00$ & $03 / 09 / 00$ \\
\hline
\end{tabular}

The results are stored in Matlab files with extension' .mat'. The Matlab program provides two ways of processing data:

- The parameters are processed from 5-minute segments, one segment from each hour, and the result for one week is stored in one file. Parameters are stored in matrices, one column for each day of the week, and one row for each hour (24 rows, 7 columns). Results from this analysis are not shown in this thesis.

- The parameters are processed continuously for each 5-minute segment for the entire 24-hour period of time. For the RR interval the results for one day of data are stored in two files, each 12 hours long. The RR interval is stored in matrices with two columns, one for the value of RR interval and the other column for the 
time corresponding to that RR interval. Parameters are stored in vectors, length 288 ( 24 hours * 12 segments of length 5 minutes each in one hour).

Matlab' s Graphical Interface Tools was used to build a friendly and easy to use interface for the user. The main menu has four options:

- Data

- Programs

- $\quad$ Figures

- Exit

Before working with the program, the user must provide the path for the file named Install.mat, which keeps track of the rabbit' s ID, rabbit' $s$ date for the first injection with doxorubicin, and other parameters for the combo boxes for the screens.

* Choosing option Data from the main menu, a new window is opened in which, the user may add or delete the ID for a rabbit, may register the date for the first injection with doxorubicin or may see all the data, ID and the date of the first injection in a list box.

This option will update the file Install.mat

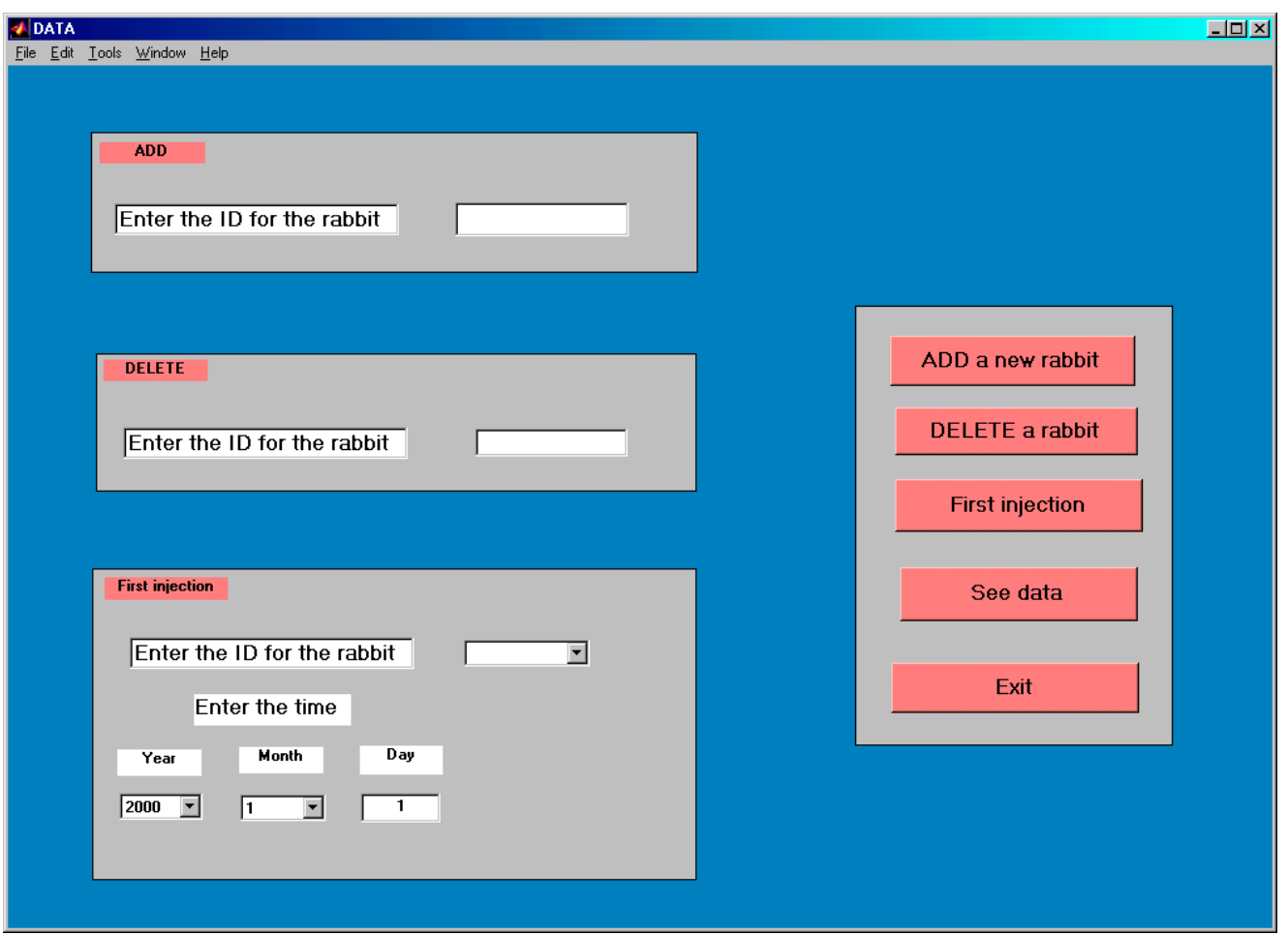

Figure 4.2 Screen for the option: Data 
Choosing option Programs from the main menu, the user may start to process data in two ways:

○ One five minute segment from each hour

○ Continuous five minute segments for each hour

Whichever way is chosen from this popup menu a new window will be opened.

The user has to specify:

- The path to the file, which contains data to process

- The path to the file where the results should be stored

$\circ$ The ID for the rabbit

○ The number of the week

○ The channel to process $(1,2$ or 3$)$

○ The number of the day after injection (this means the number of the day for that week)

○ The hours for the time interval to process

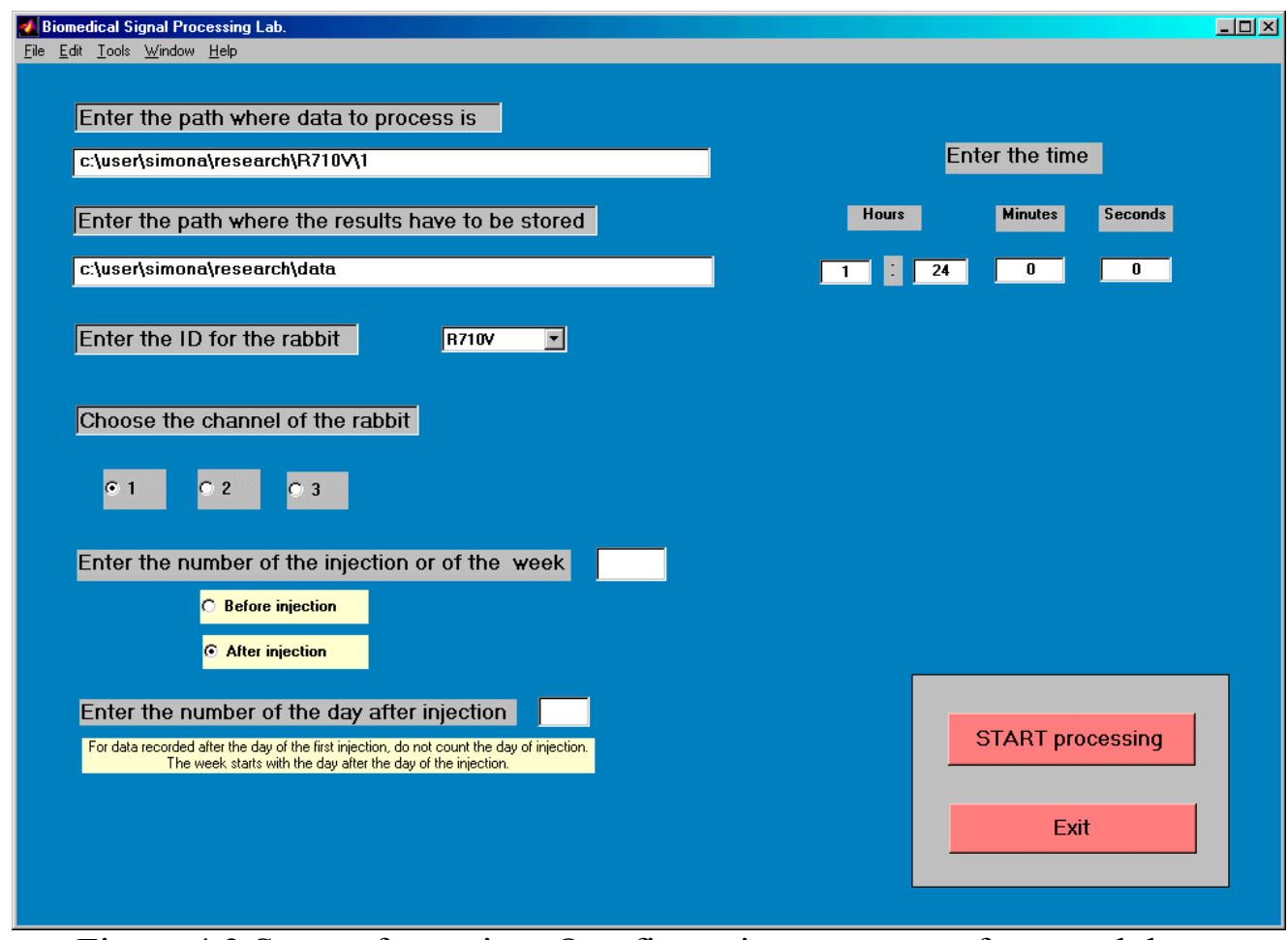

Figure 4.3 Screen for option: One five-minute segment from each hour 


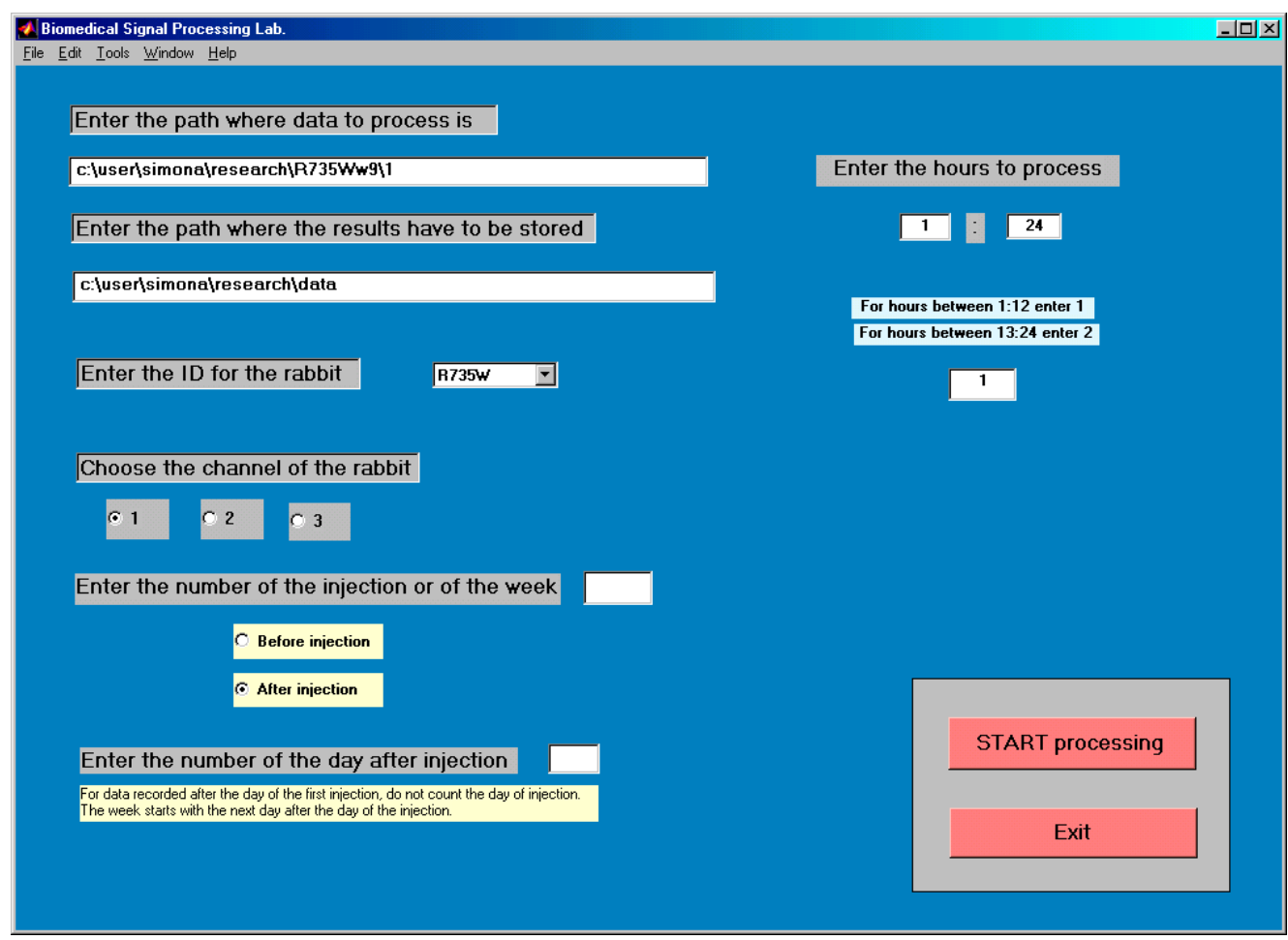

Figure 4.4 Screen for option: Continuous five minute segments for each hour

After the command button "START processing" is pressed, its "Enable' property becomes ' off', untill the processing is over or canceled with CTRL-C command.

* Choosing option Figures from the main menu, the user can plot either the ECG signal with trigger points marked or the RR intervals for a specified five minutes segment (Figure 4.5). In case of ECG signal, the scale for $\mathrm{X}$-axis may be chosen, among 1,4,8,12,20 and $40 \mathrm{sec} / \mathrm{screen}$, so that ECG signal can be viewed in detail (Figure 4.6). The ECG plotting module was written by Steve Hudson. This program was used to verify the accuracy of trigger points. For the plot of RR intervals, the interpolated intervals, which replaced artifacts, are the red points (Figure 4.7). 


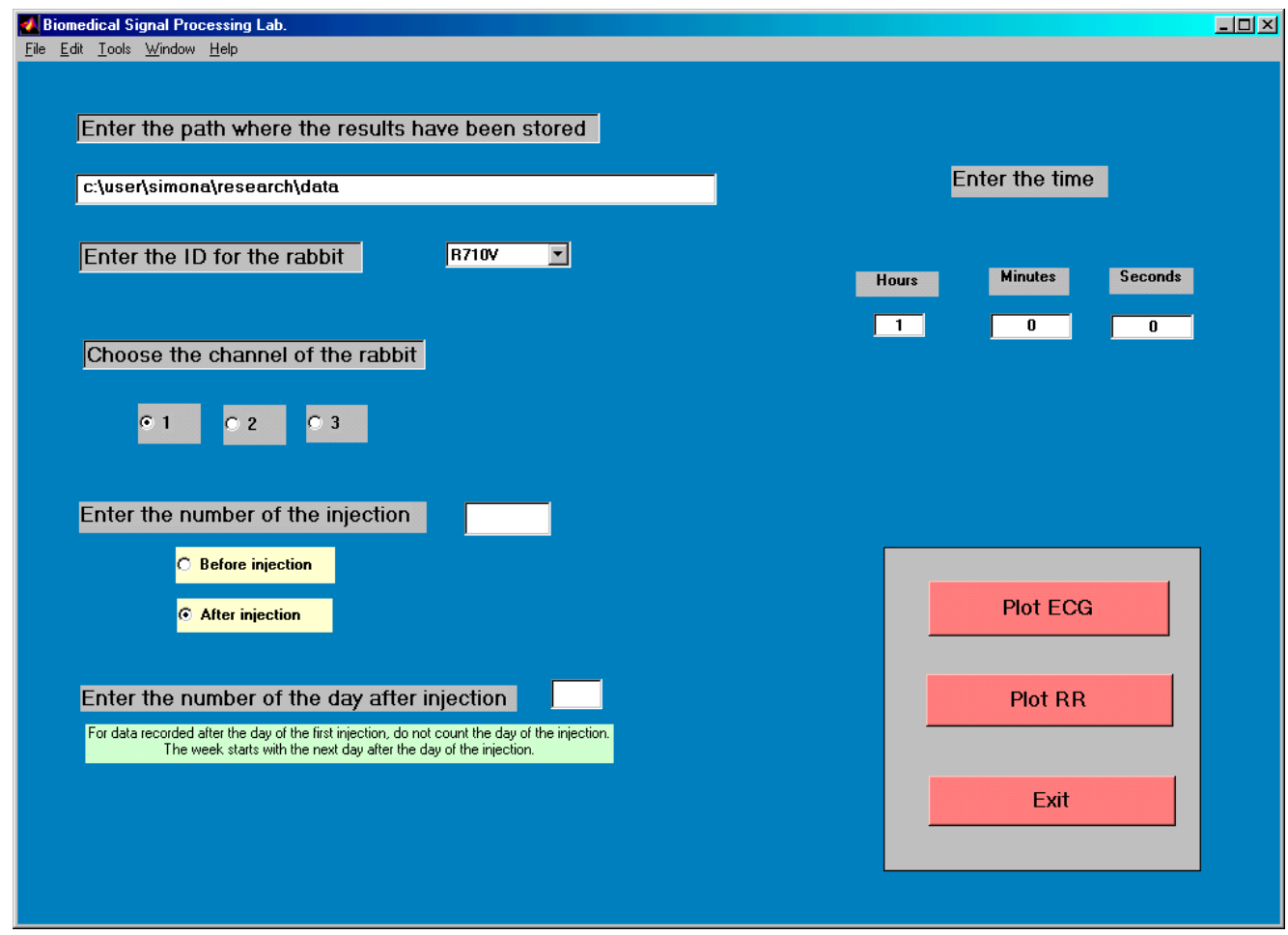

Figure 4.5 Screen for option: Figures

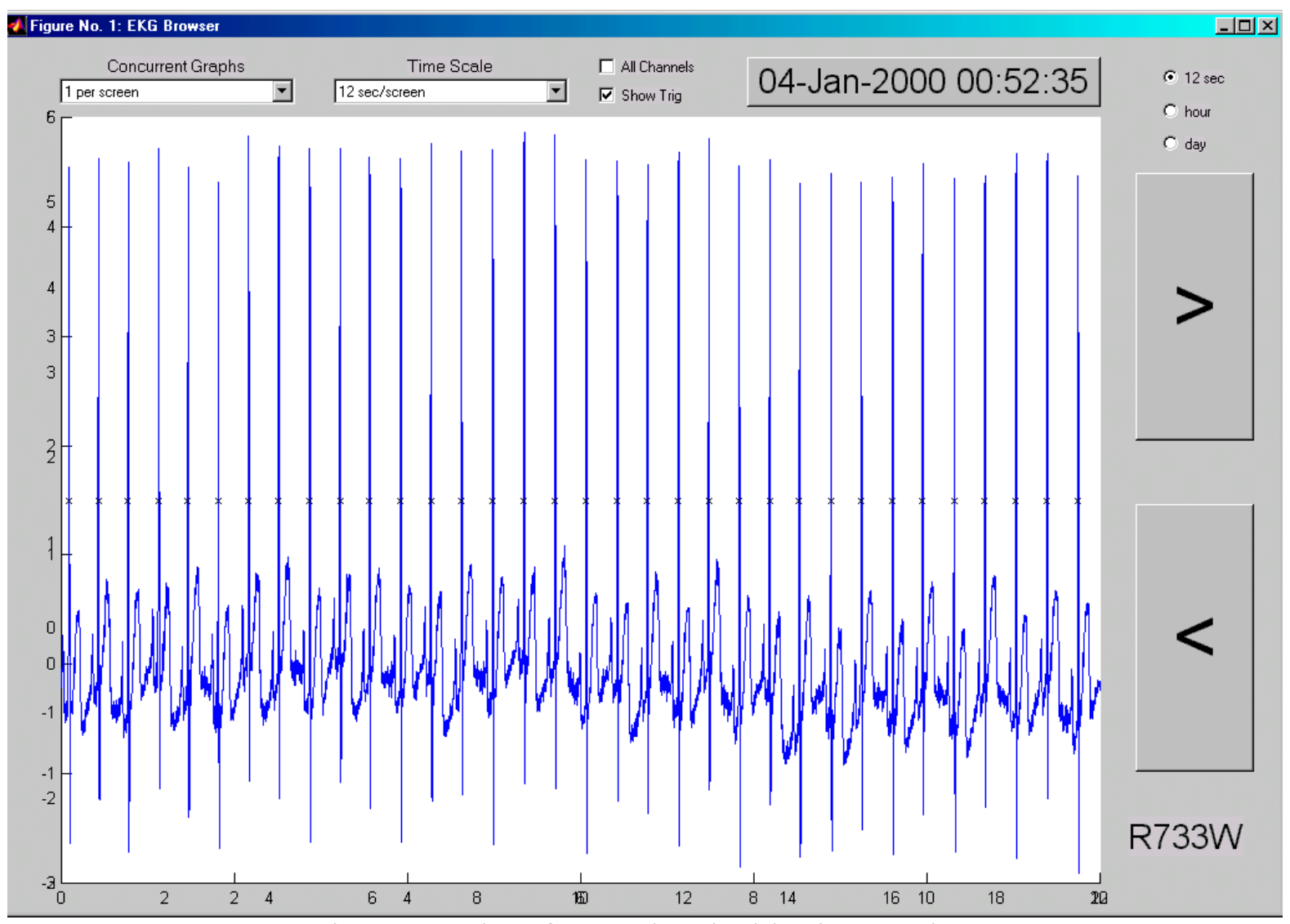

Figure 4.6 Plot of ECG signal with trigger points 


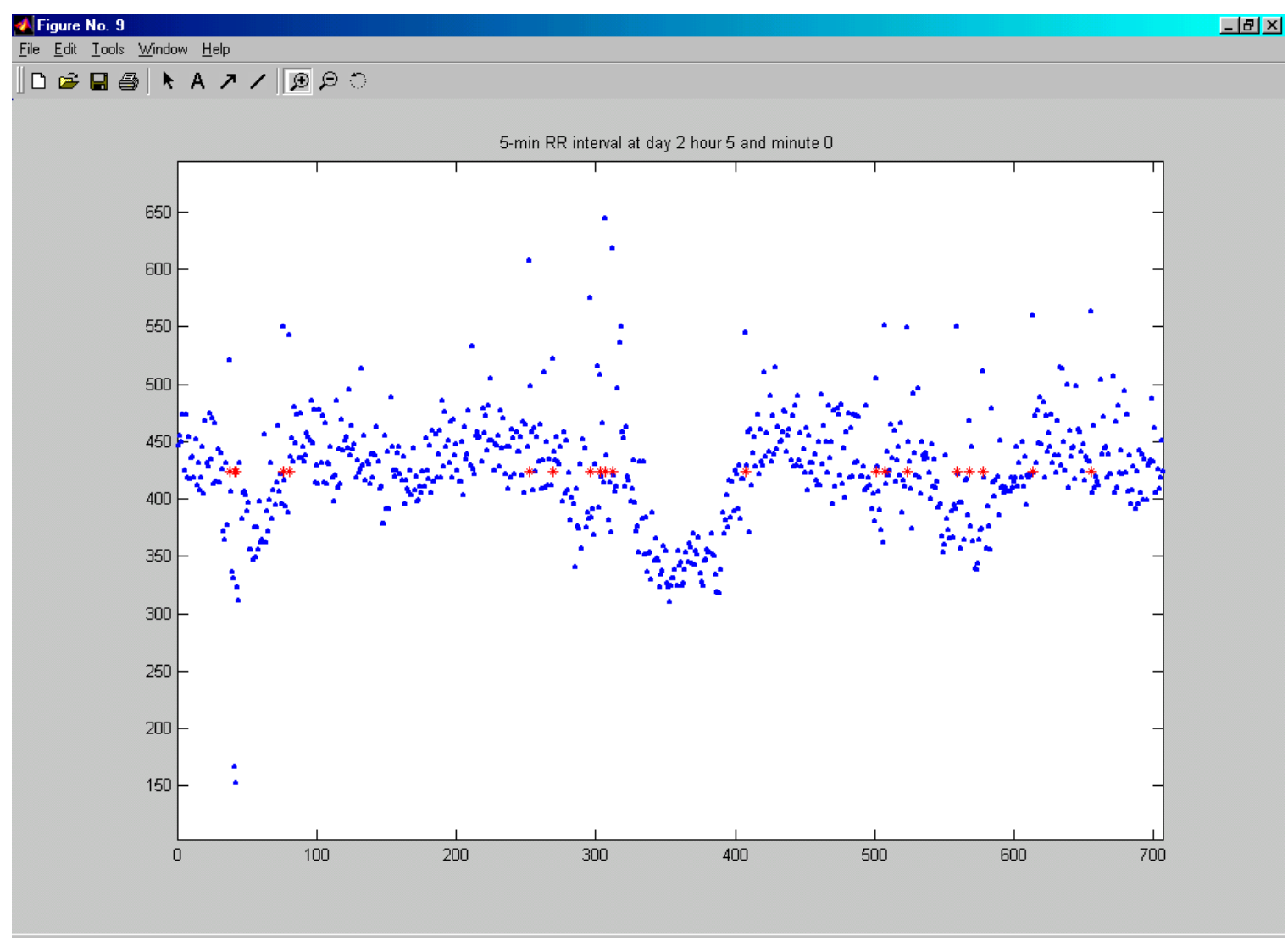

Figure 4.7 Plot of RR intervals

Choosing option Exit from the main menu, the user exits the program.

Dialog boxes are opened to confirm the completion of specific tasks or to ask for permission to continue.

\subsubsection{Statistics}

Unfortunately, even though the amount of data was enormous, there weren' $t$ enough independent subjects in order to use statistical techniques to quantify the differences.

\section{Contributions:}

Xueyan $\mathrm{Xu}$ - Development of the individual heart rate variability modules

Pisut Raphisak - Design and code of the trigger point subprogram and DSI format conversion program

Steve Hudson - Design of ECG plotting program

Simona Crihalmeanu - Design of the interface, REXX language program to transfer the data, $\mathrm{C}++$ program to sort data on the dataserver, process the data, plots of the results. 


\section{Chapter 5 RESULTS}

The following chapter discusses the results of the experiment for the rabbits, one control and two with doxorubicin injections. The first section discusses the results of blood analysis, the second elaborates on the electrocardiogram interpretation at the end of the study. The last sections describe unique methods for presenting results of this massive dataset.

\section{$\underline{5.1 \text { Laboratory results }}$}

A cardiologist, Dr. Mitch Finkel MD, from West Virginia University Hospitals, Cardiology, has agreed to collaborate on the project and has helped to interpret the laboratory results and electrocardiography. Plots for the following blood analysis are in Appendix G.

In future work, when more subjects are obtained, results of blood work will be correlated with changes in HRV with prediction of events the goal.

\subsubsection{Heart muscle damage}

The plots G.48, G.7, G.17, G.27, G.47, G.6, G.16, and G.26 for creatine kinase (CK) and CK-MB for all three rabbits show a dramatic increase on $25^{\text {th }}$ of February of both laboratory tests for rabbit $\mathrm{R} 733 \mathrm{~W}$, which indicates heart muscle damage. For rabbit $\mathrm{R} 735 \mathrm{~W}$, an increase can be noticed for CK but not CK-MB. The control rabbit remains level for CK and CK-MB throughout the experiment.

\subsubsection{Pancreas damage}

The plots G.51, G.1, G.11, and G.21 of amylase and G.53, G.3, G.13, and G.23 for triglycerides for all three rabbits show an increase for the rabbits (R733W and R735W) that were injected with adriamycin. The plot for rabbit R735W reveals an abrupt increase of triglycerides up to the last day of testing, over eight times the value of the first day of testing. Amylase has also a significant increase at almost two times the value of the first day of testing. These considerations indicate pancreas damage for the rabbit R735W.

\subsubsection{Kidney damage}

The plots for creatinine are G.50, G.8, G.18, G.28 and for BUN are G.49, G8, G.18, G.28. Both rabbits, which were injected with adriamycin, present lower values for blood urea nitrogen in the period following the first injection with adriamycin. In the next period of time, 
values raise, especially for rabbit $\mathrm{R} 735 \mathrm{~W}$, which may be caused by bleeding and decreased blood flow. The creatinine lab test is much more relevant in case of $\mathrm{R} 735 \mathrm{~W}$. Values of creatinine rise from the beginning, with an abrupt increase in the last period, which may be interpreted as kidney damage.

\subsubsection{Liver damage}

The plots G.58, G.9, G.19, G.29 for all three rabbits show variations of bilirubin within a small range of values that suggest no important changes for bilirubin. However, the values for cholesterol G.52, G.3, G.13, G.23 raise abruptly at almost 5 times initial value for rabbit R735W which is diagnosed with pancreatitis, and at almost 3 times initial value for the rabbit R733W, which is suspected to have heart muscle damage.Values for the control rabbit remain steady at a value less than $50 \mathrm{mg} / \mathrm{dL}$.

\subsubsection{Hemostatic considerations}

Plots for HGB G.55, G.32, G.37, and G.42, for HCT G.56, G.33, G.38, G.43 and for RBC G.57, G.34, G.39, G.44 were done for all three rabbits. Before the first injection with adriamycin, the values for the injected rabbits (R733W and $\mathrm{R} 735 \mathrm{~W})$ are similar with the values of the control rabbit R710V. For the following period, the plots reveal a constant decrease of all three-laboratory tests to half the initial value. Low values for hemoglobin (HGB) and hematocrit (HCT) could be due to anemia and alteration in cell size (RBC) as with some regenerative anemias - reticulocytosis. While the platelets' curve for control rabbit R710V varies within a small range of values, the platelets' curve for injected rabbits $\mathrm{R} 733 \mathrm{~W}$ and $\mathrm{R} 735 \mathrm{~W}$ decreases after the first injection with adriamycin, and then rises abruptly till the last day of analysis. Increased values for platelets may be due to a response to anemia. The most likely cause for the pattern of lab results is the bone marrow suppression side effects caused by doxorubicin (adriamycin). Plots for platelets are G.54, G.35, G.40, and G.42.

\subsubsection{Weight}

Surprisingly, weight for both injected rabbits, is decreased by almost half kilogram. In case of heart muscle damage, heart failure, an increase in weight is expected. Losing weight can be connected to loss of fluid from the body, which can cause an increase of concentration for body wastes and other substances. Plots G.46, G.10, G.20, G.30. 


\subsection{Interpretation of ECG signal}

\subsubsection{Rabbit R733W}

One of the most important facts in this experiment is that the continuous recording of ECG signal from the rabbit allows us to observe the changes of ECG features, both in terms of the whole ECG and HRV parameters, along the progression of the heart disease.

Figure 5.1 shows the ECG signal before the rabbit has received the injections with adriamycin. There are three beats in one second; the P wave, the complex QRS and the T wave are very clear. Figure 5.2 shows the ECG signal from the same day at a scale of four seconds per screen. On March $10^{\text {th }}$, after nine injections with adriamycin heart rate is increased, and ECG signal is altered (figure 5.3). On March $20^{\text {th }}$, the day in which the rabbit died, the ECG signal is altered in shape, the P wave is not clearly visible, and the ST segment and T wave are very high (figure 5.4 and figure 5.5). ST segment normally is flat, horizontal and at baseline level. If ST segment is elevated or depressed beyond the normal baseline level, this is often an important sign of serious pathology [22]. Figure 5.7 shows the beginning of bradycardia, when the sinus node (SA node) paces the heart at a slower rate. If SA node pacing fails, a slow rate can be also due to atrial automaticity focus or junctional automaticity focus. This focus becomes the dominant pacemaker by overdrive-supressing all lower levels of foci, since they have slower inherent rates [22]. It is important to identify the origin, the location of the irritable automaticity focus. In this subject, it appears to be sinus bradycardia. The phenomenon of ventricular bigeminy is showed in figures 5.9 and 5.10. In case of premature beats, an irritable focus spontaneously fires a single stimulus earlier than expected in the rhythm [22], which can be atrial premature beat, junctional premature beat, or ventricular premature beat. A premature ventricular beat is called premature ventricular contraction. The irritable automaticity focus is in a ventricle and produces a giant ventricular complex on ECG [22]. This is showed in figure 5.10 at twelve seconds per screen. This rabbit was euthanized. 


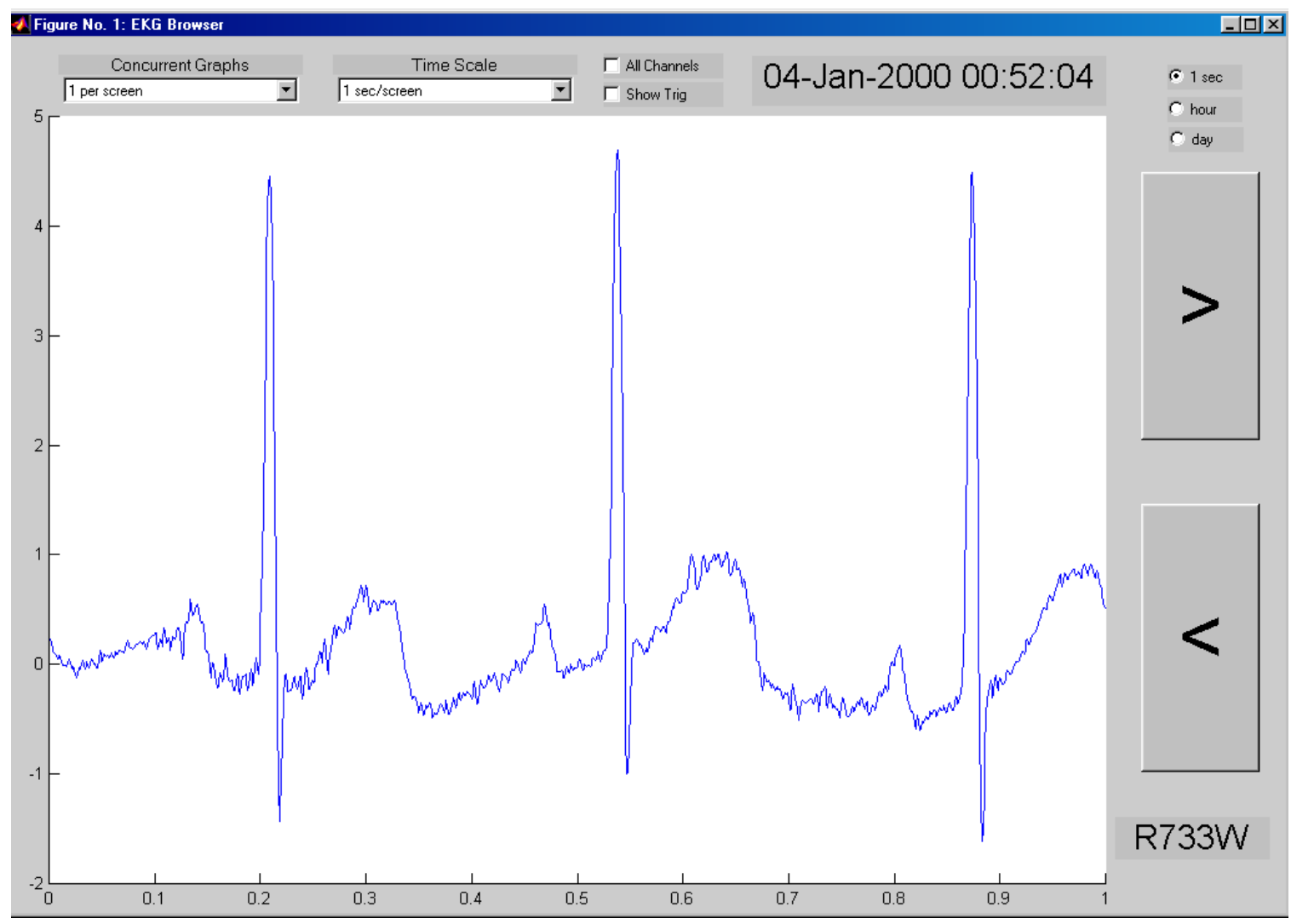

Figure 5.1 ECG signal before the first injection, one second per screen

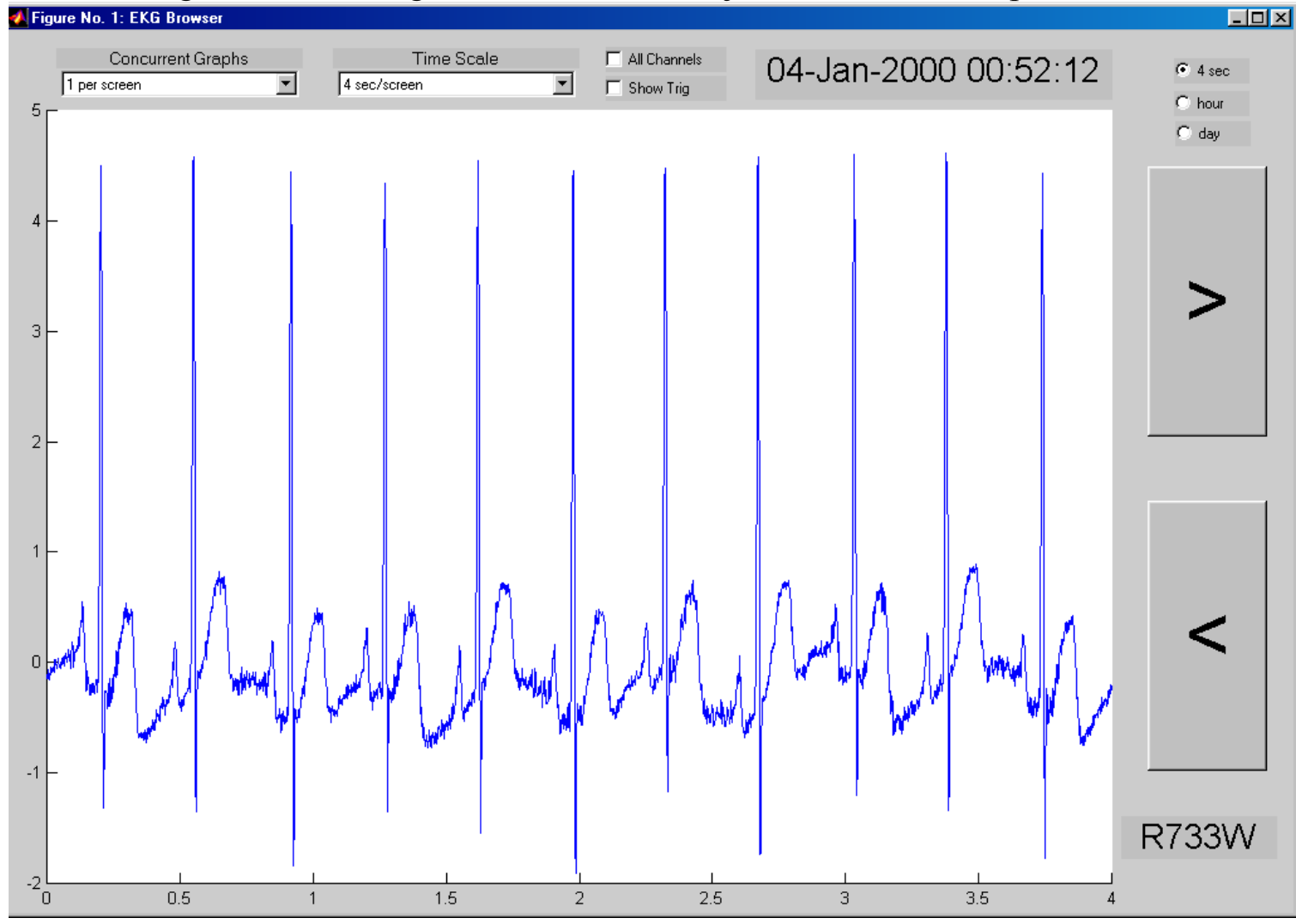

Figure 5.2 ECG signal before the first injection, four seconds per screen 


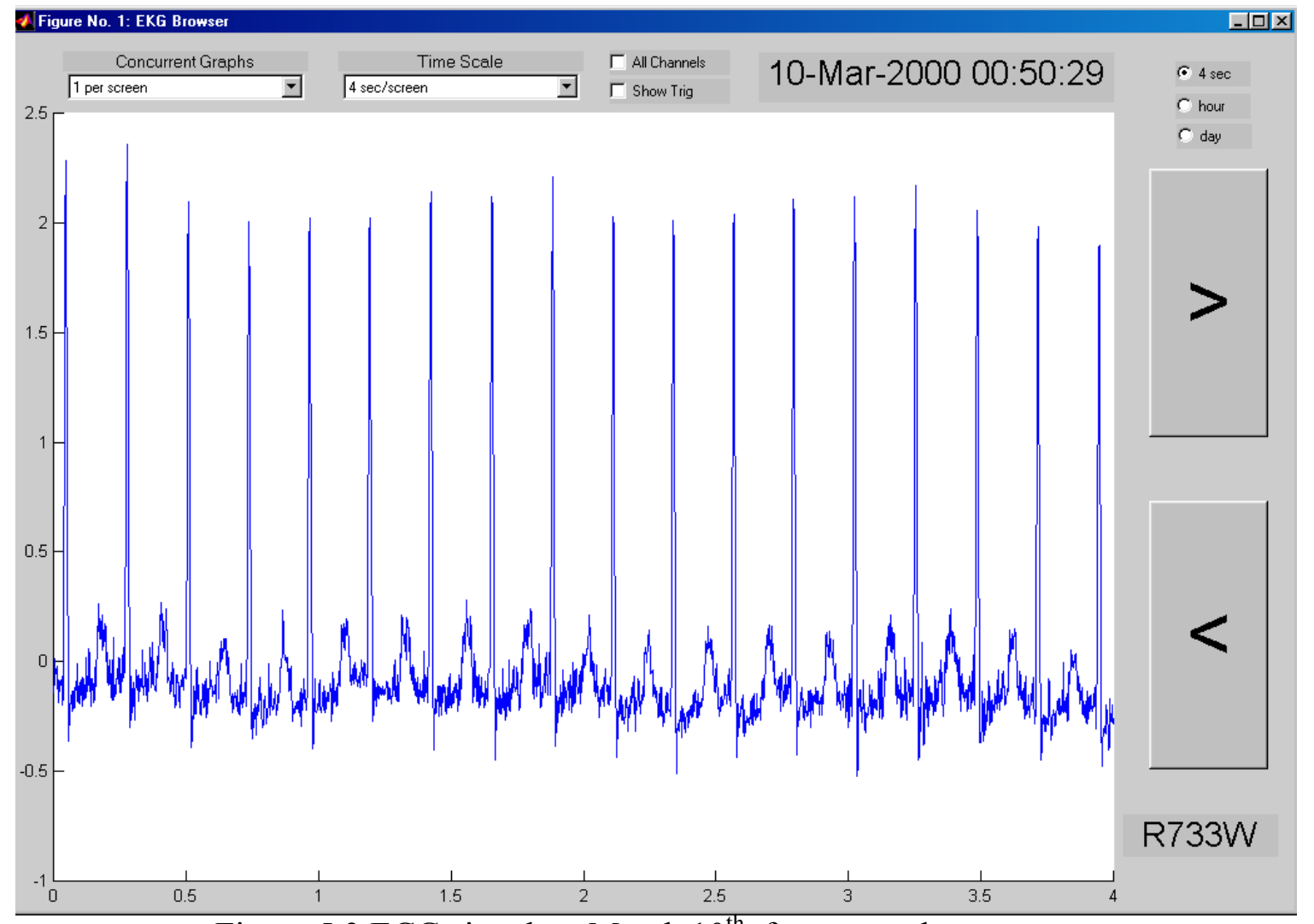

Figure 5.3 ECG signal on March $10^{\text {th }}$, four seconds per screen

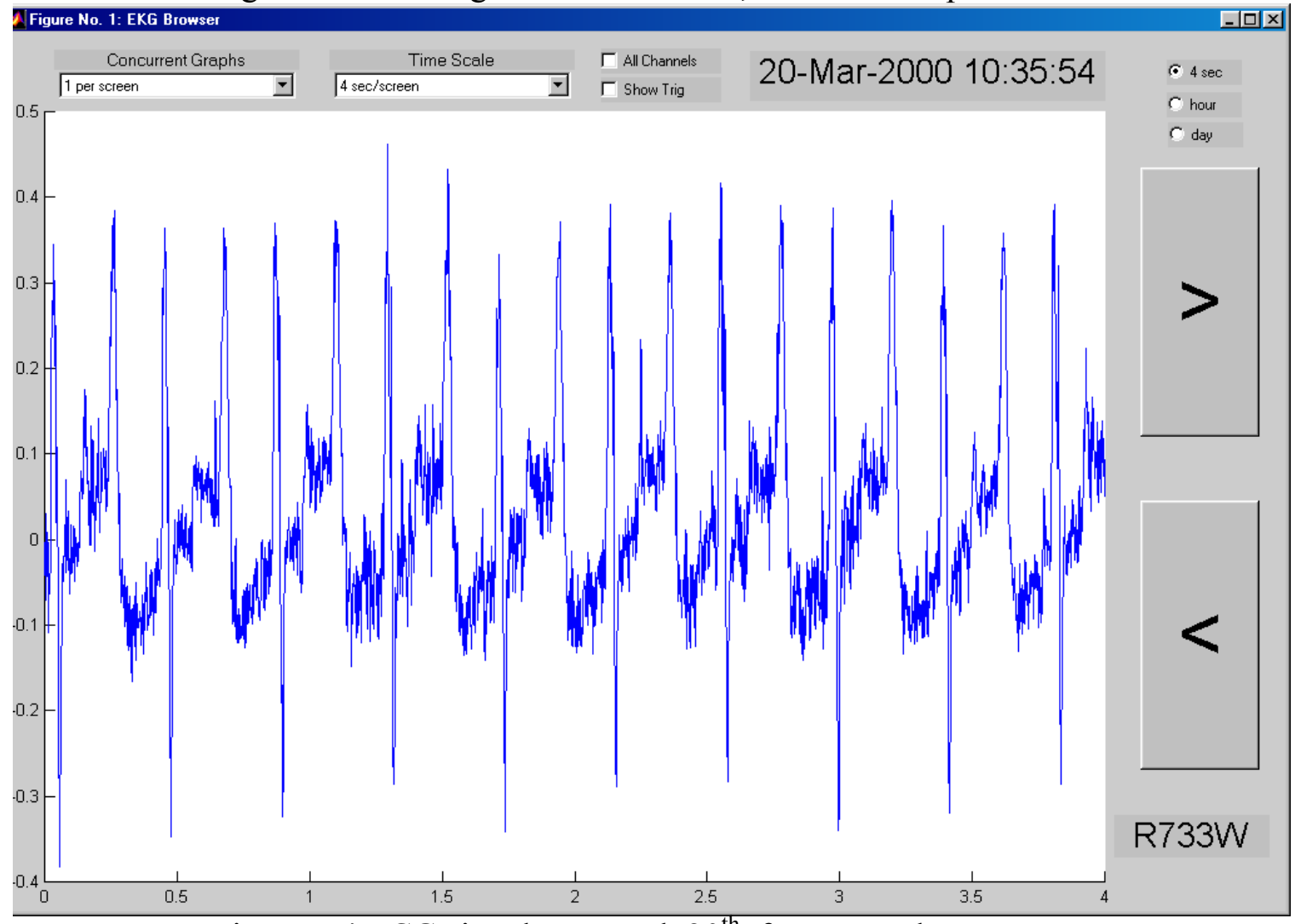

Figure 5.4 ECG signal on March 20 ${ }^{\text {th }}$, four seconds per screen 


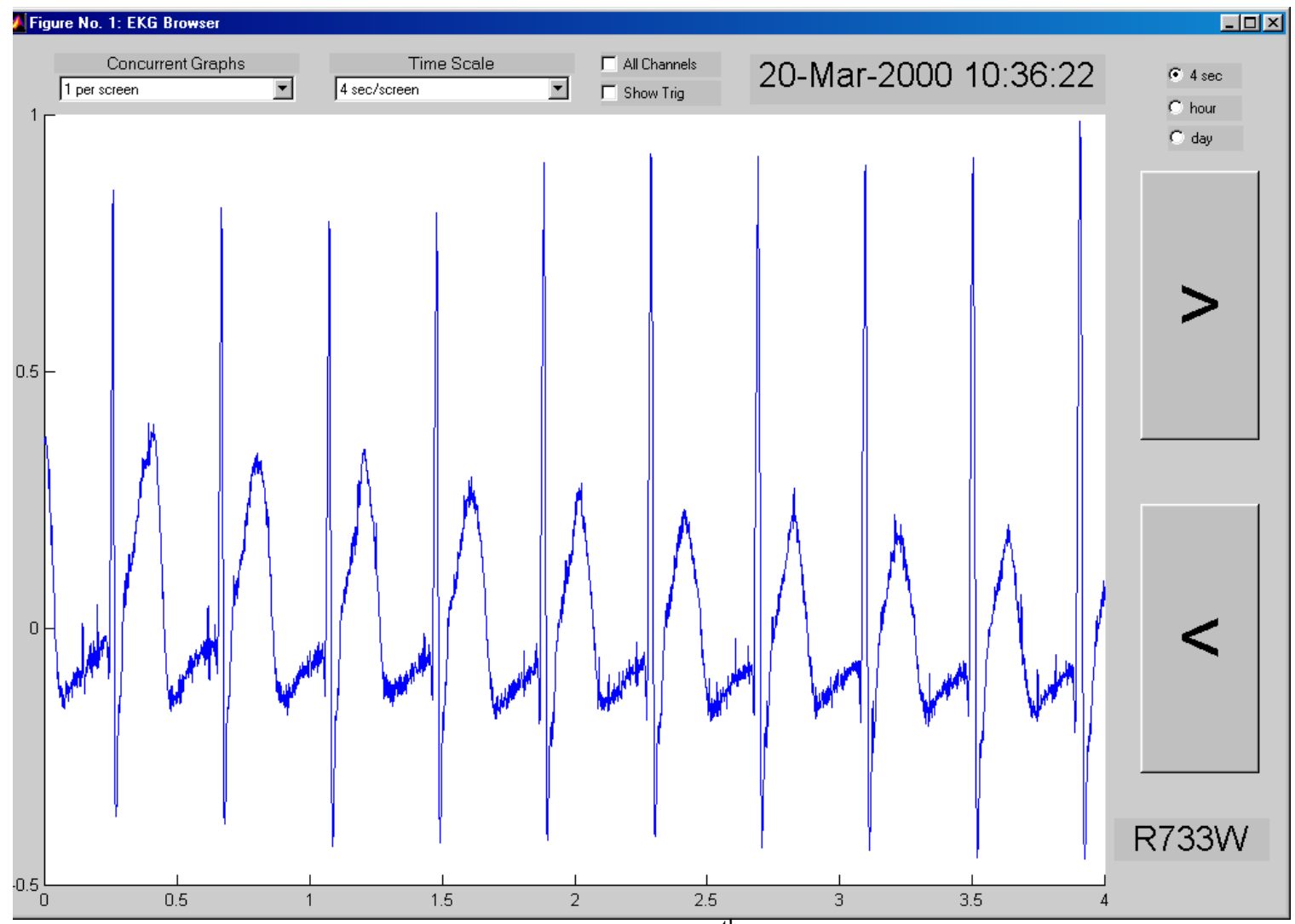

Figure 5.5 ECG signal on March 20 ${ }^{\text {th }}$, four seconds per screen

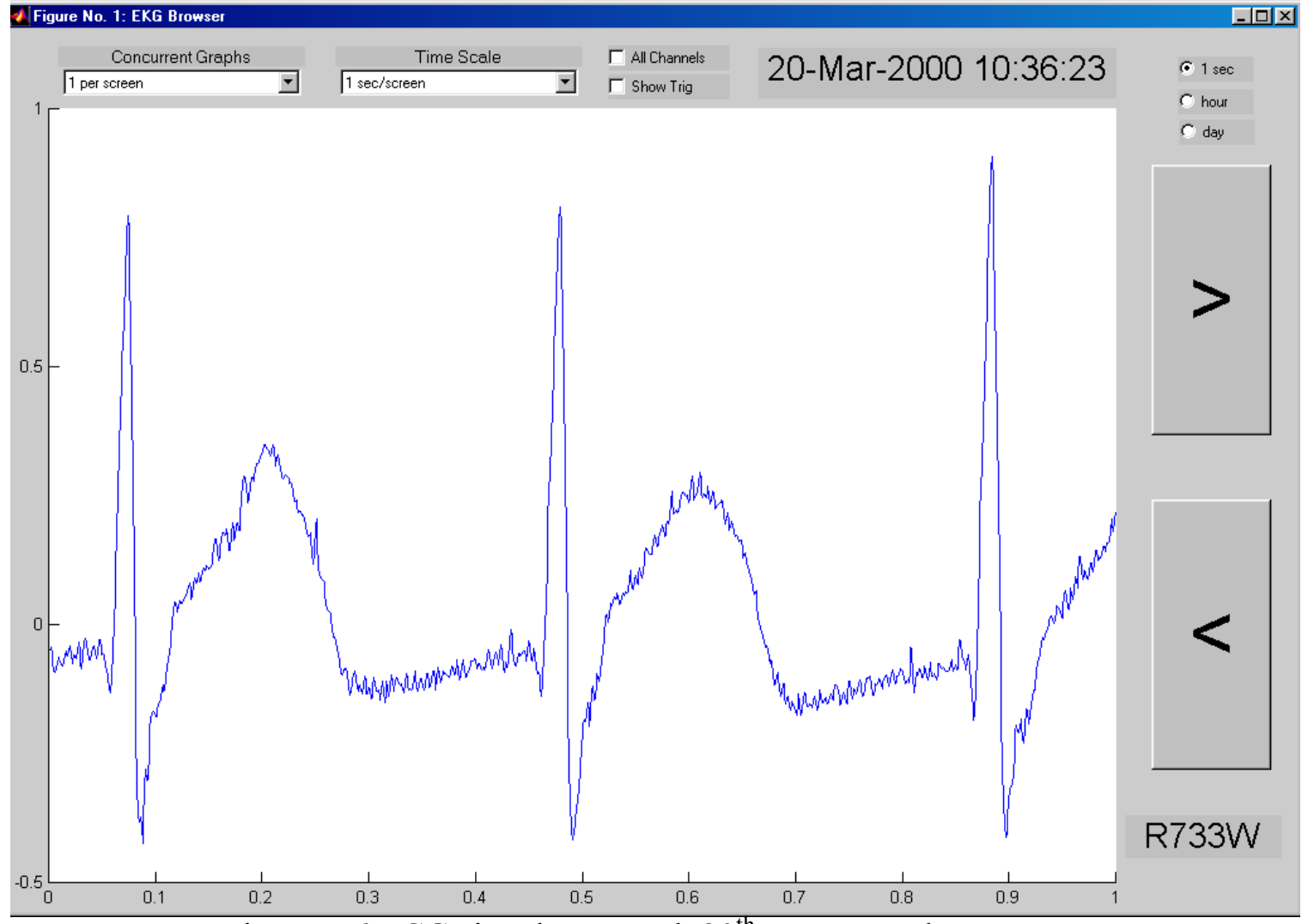

Figure 5.6 ECG signal on March $20^{\text {th }}$, one second per screen 


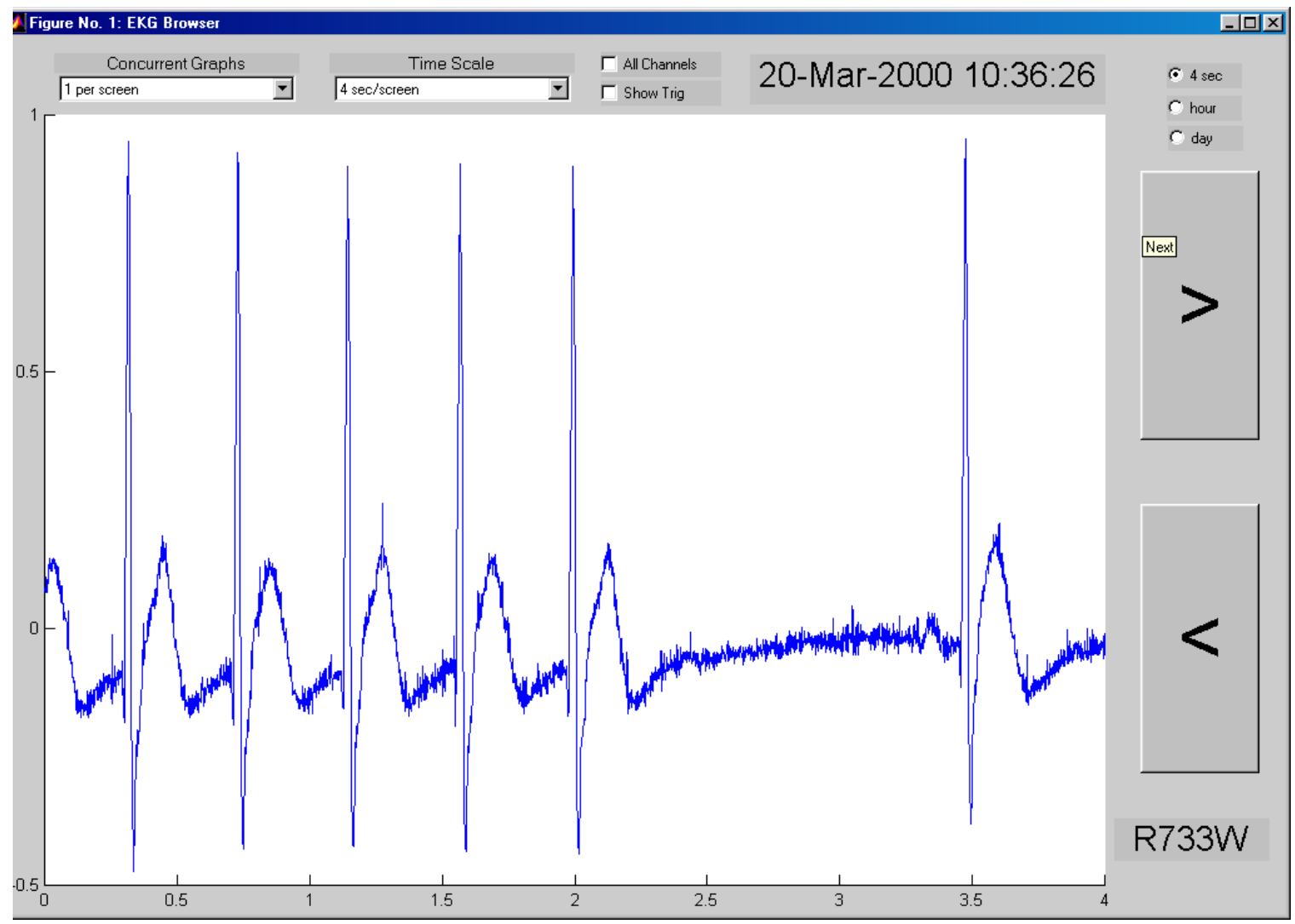

Figure 5.7 ECG signal; the beginning of bradycardia, four seconds per screen

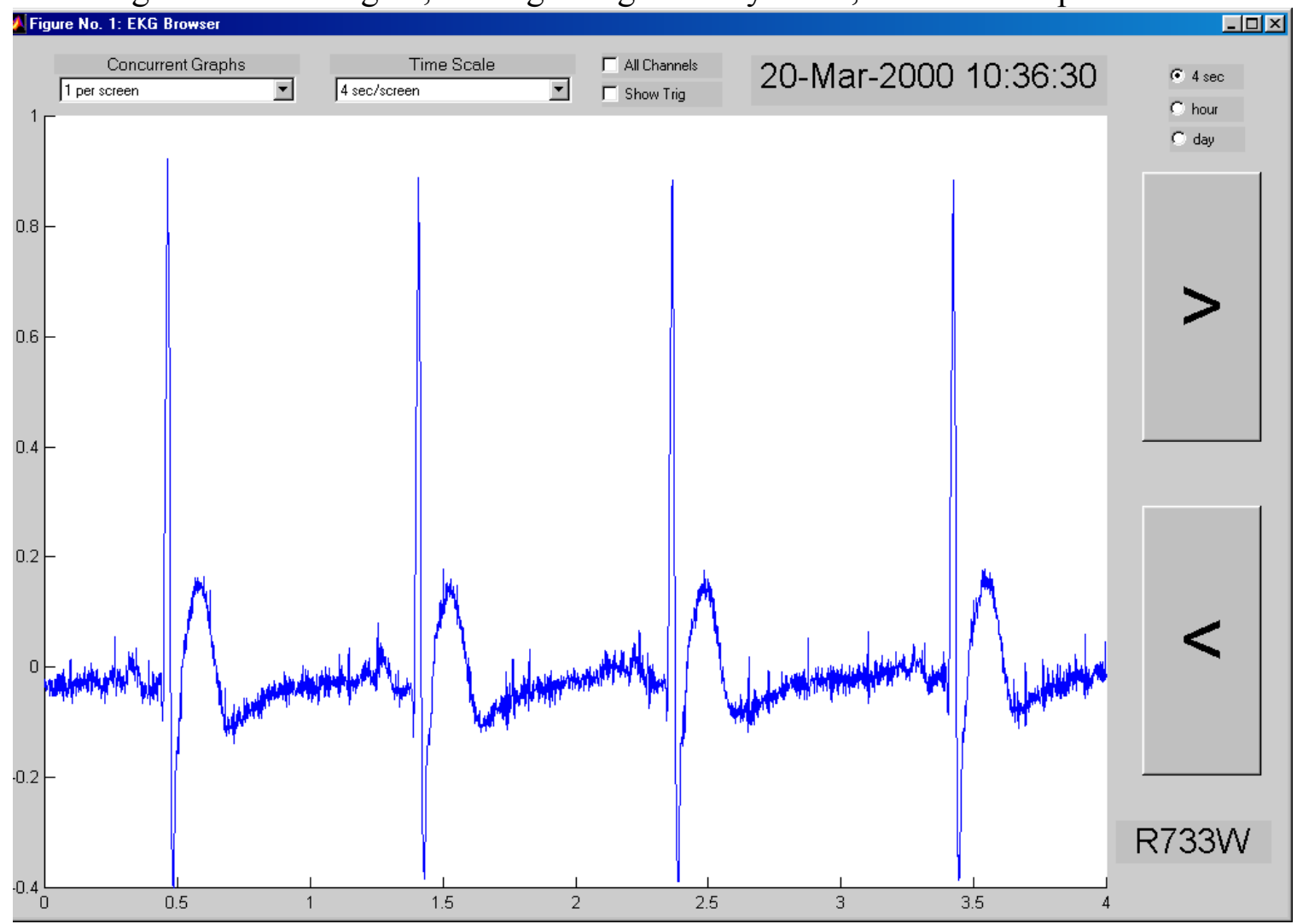

Figure 5.8 ECG signal, bradycardia, four seconds per screen 


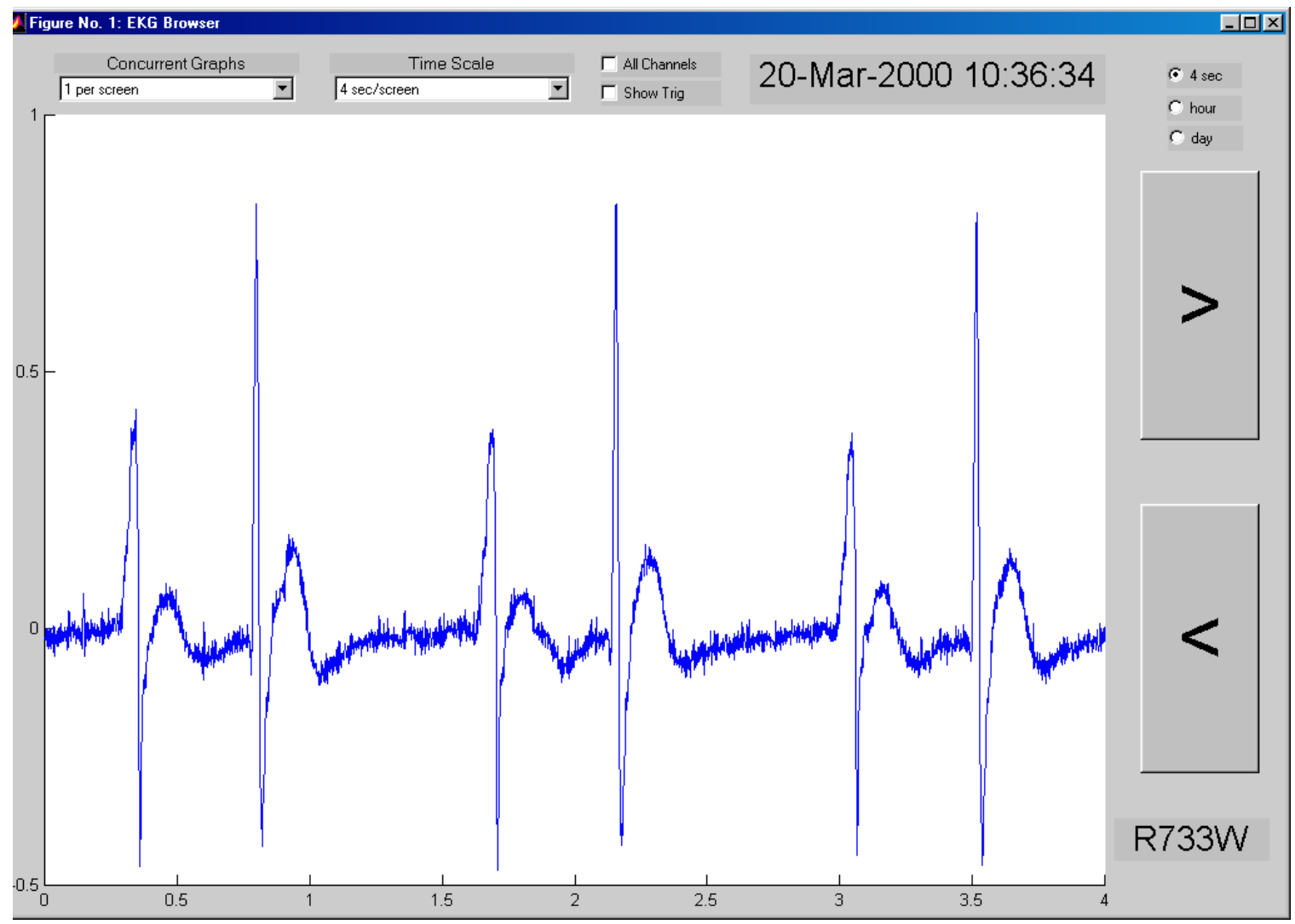

Figure 5.9 ECG signal, ventricular bigeminy, four seconds per screen

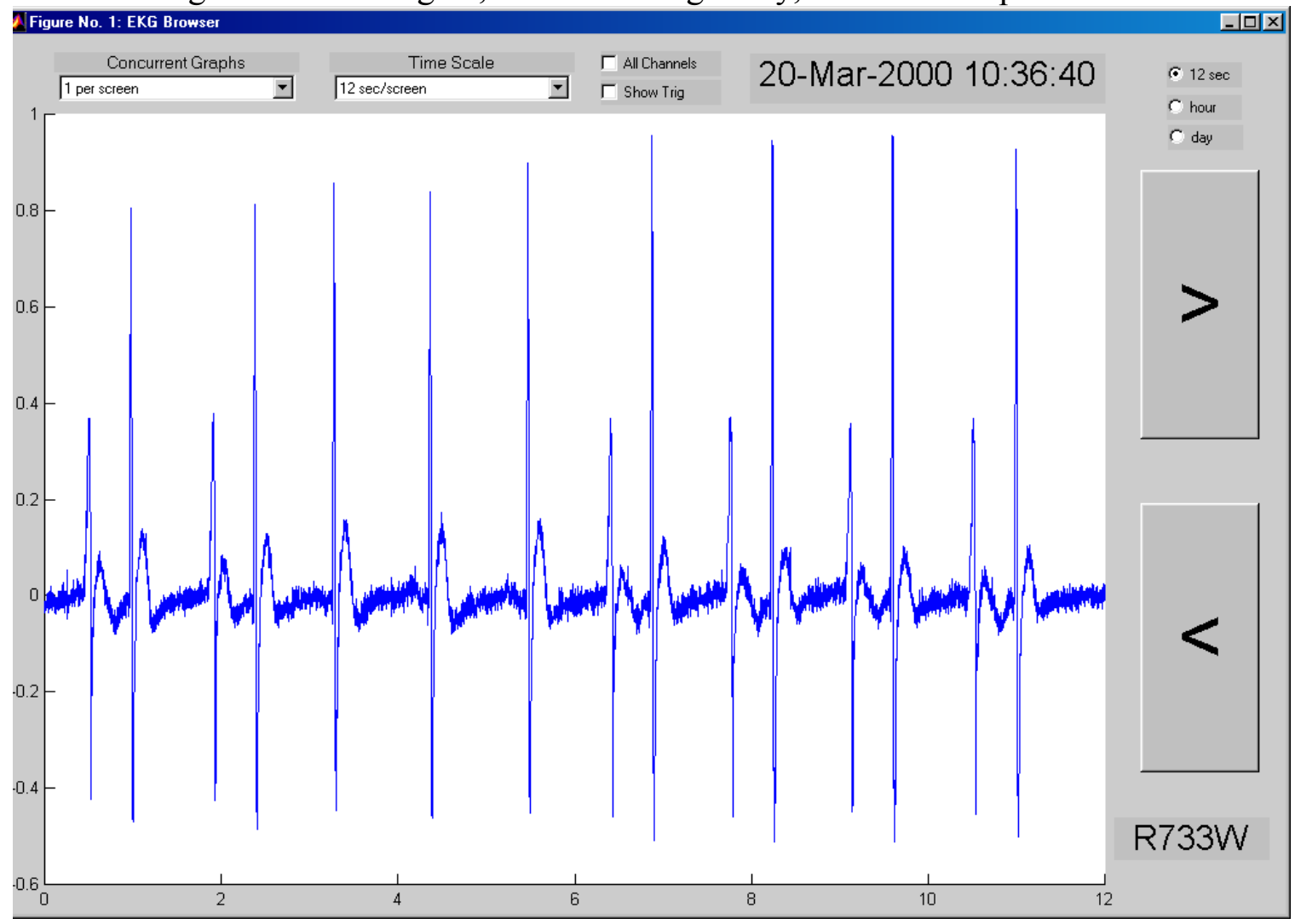

Figure 5.10 ECG signal, ventricular bigeminy, twelve seconds per screen 


\subsubsection{Rabbit R735W}

Rabbit R735W had two leads, an intracardiac lead and a surface lead. Data was processed and parameters relevant for HRV were computed based on the ECG signal from the intracardiac channel, which is clear and without noise. Figures with the evolution of ECG signal from the intracardiac channel are in appendix H. Figures with ECG signal from the surface lead are presented in this section for interpretation.

The ECG signal for rabbit R735W presents a different evolution compared with rabbit R733W. Figure 5.11 shows the ECG signal before the rabbit was injected with adriamycin. The $\mathrm{P}$ wave, the complex QRS and the T wave, are clear and visible. On eleventh of February the heart rate is faster (Figure 5.12). An altered shape is noticeable in figure 5.13 at one second per screen and in figure 5.14. On March $6^{\text {th }}$, figure 5.15 captures the moment in which the rabbit develops a myocardial infaction as noticed by elevation of the ST segment. A myocardial infarction is complete occlusion of a coronary artery. The area of myocardium supplied by the occluded coronary artery becomes non-viable and neither depolarizes nor contracts [22]. Figure 5.16 shows the moment before myocardial infarction (MI). Figure 5.17 shows ECG signal exactly after the injury. After myocardial infarction, the heart rate decreases as bradycardia develops and the animal dies. 


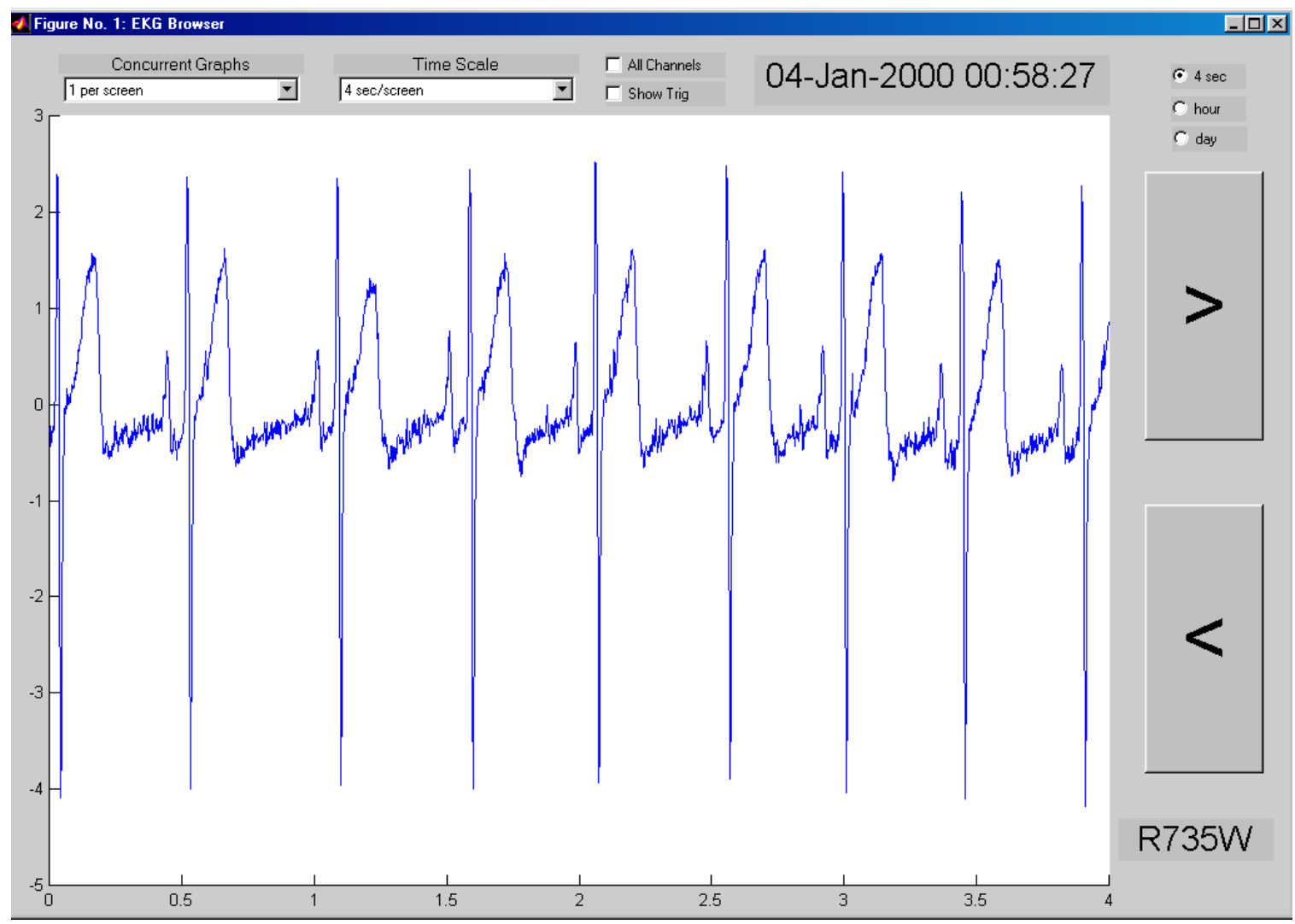

Figure 5.11 ECG signal before the first injection, four seconds per screen

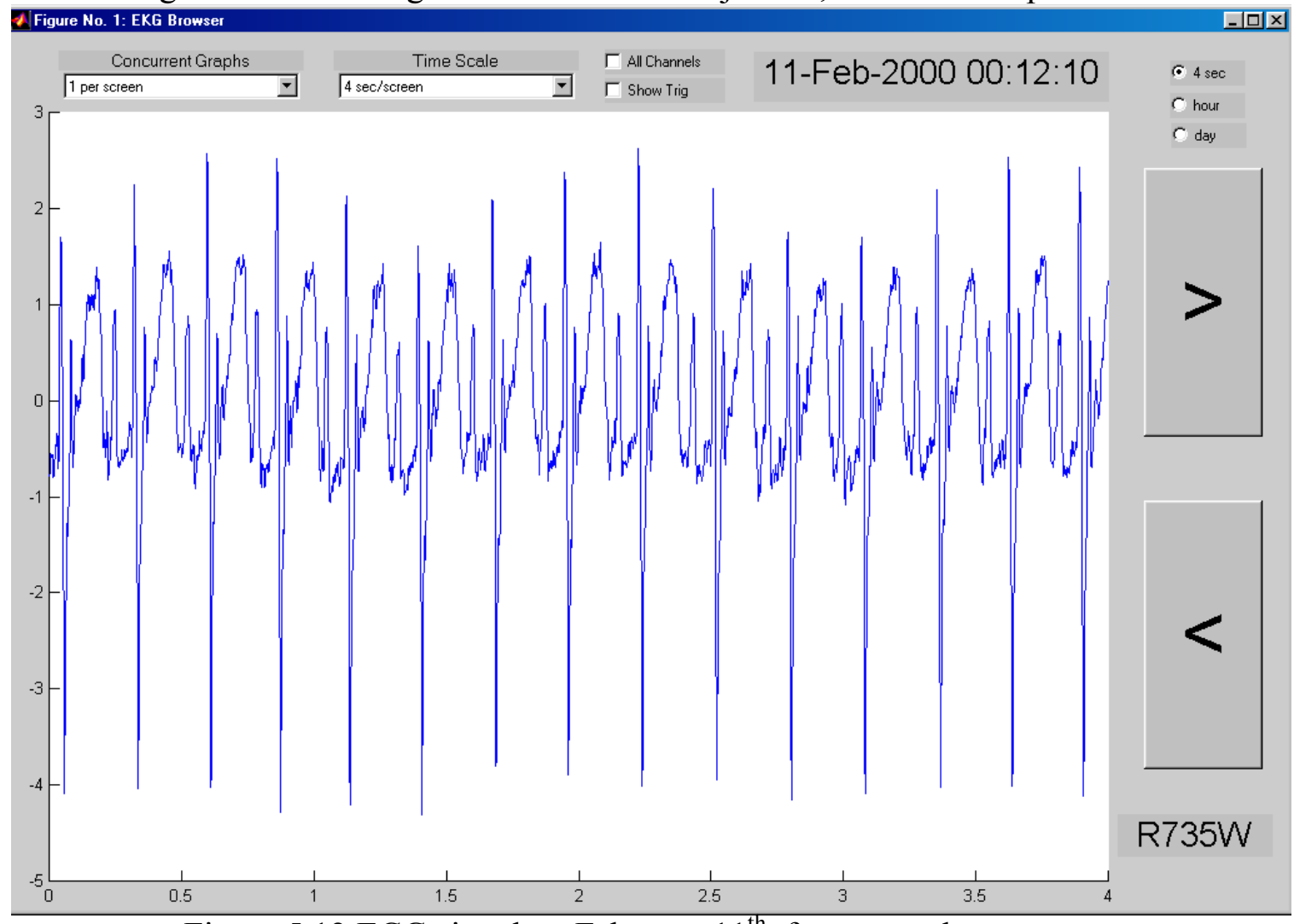

Figure 5.12 ECG signal on February $11^{\text {th }}$, four seconds per screen 


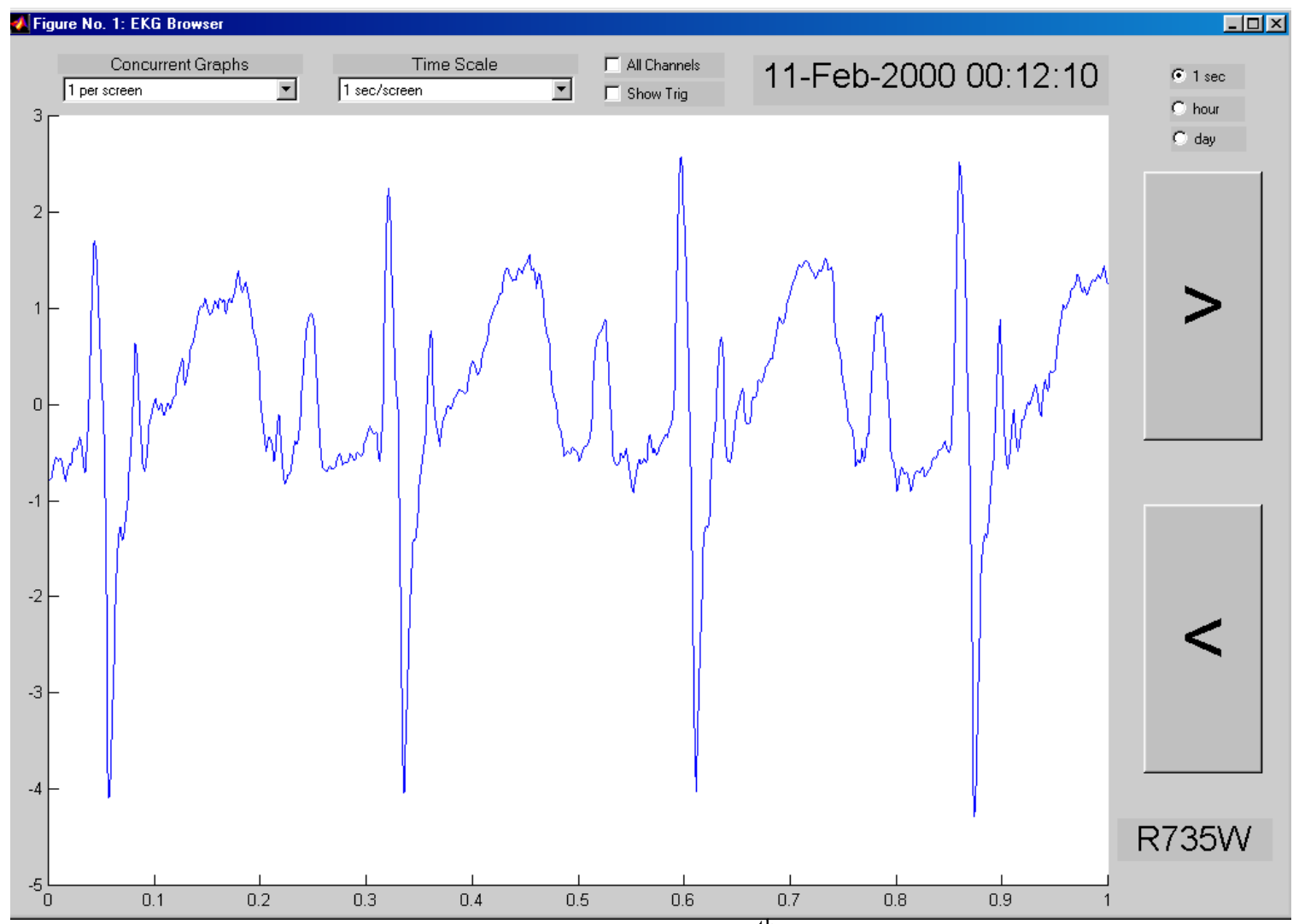

Figure 5.13 ECG signal on February $11^{\text {th }}$, one second per screen

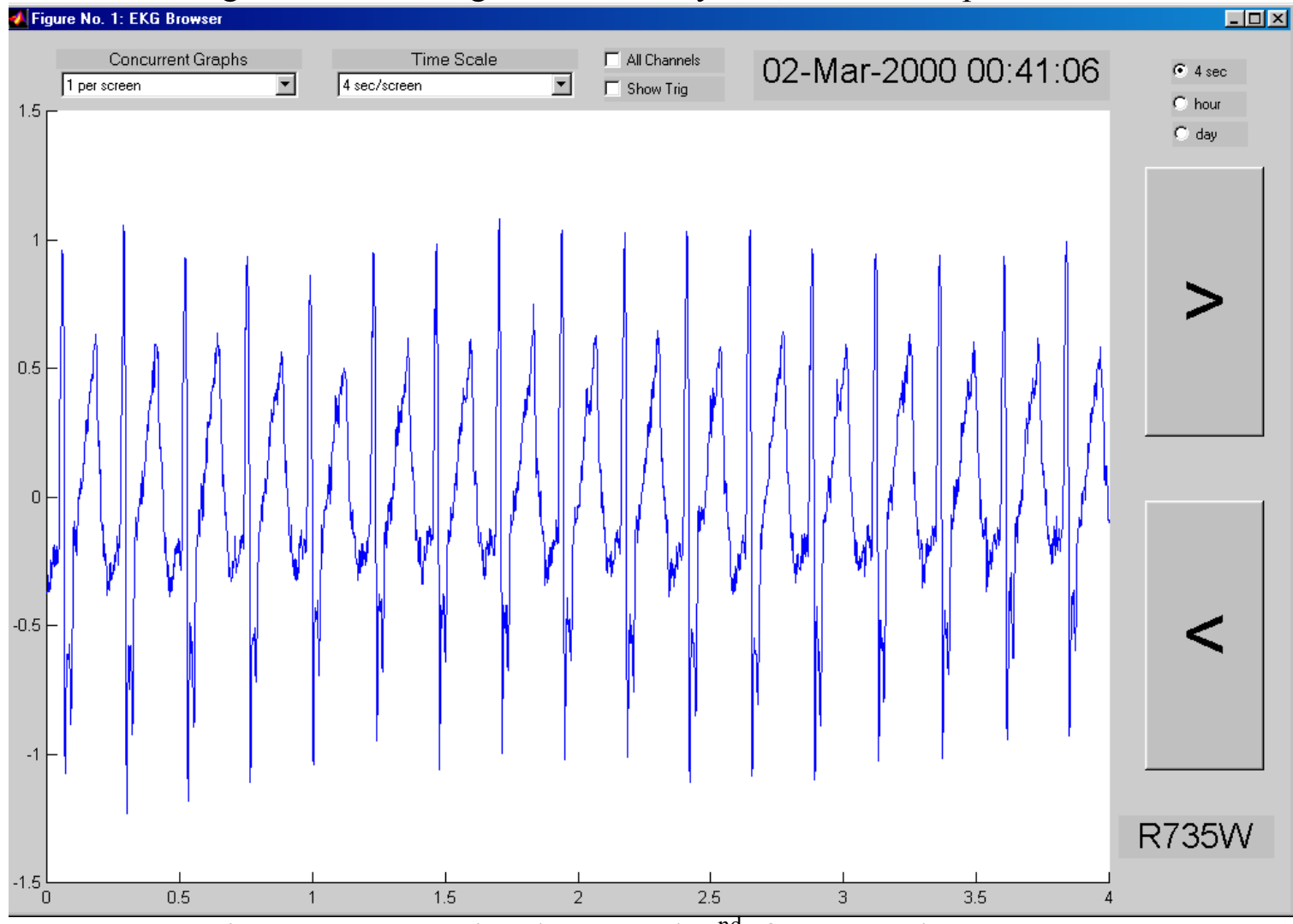

Figure 5.14 ECG signal on March $2^{\text {nd }}$, four seconds per screen 


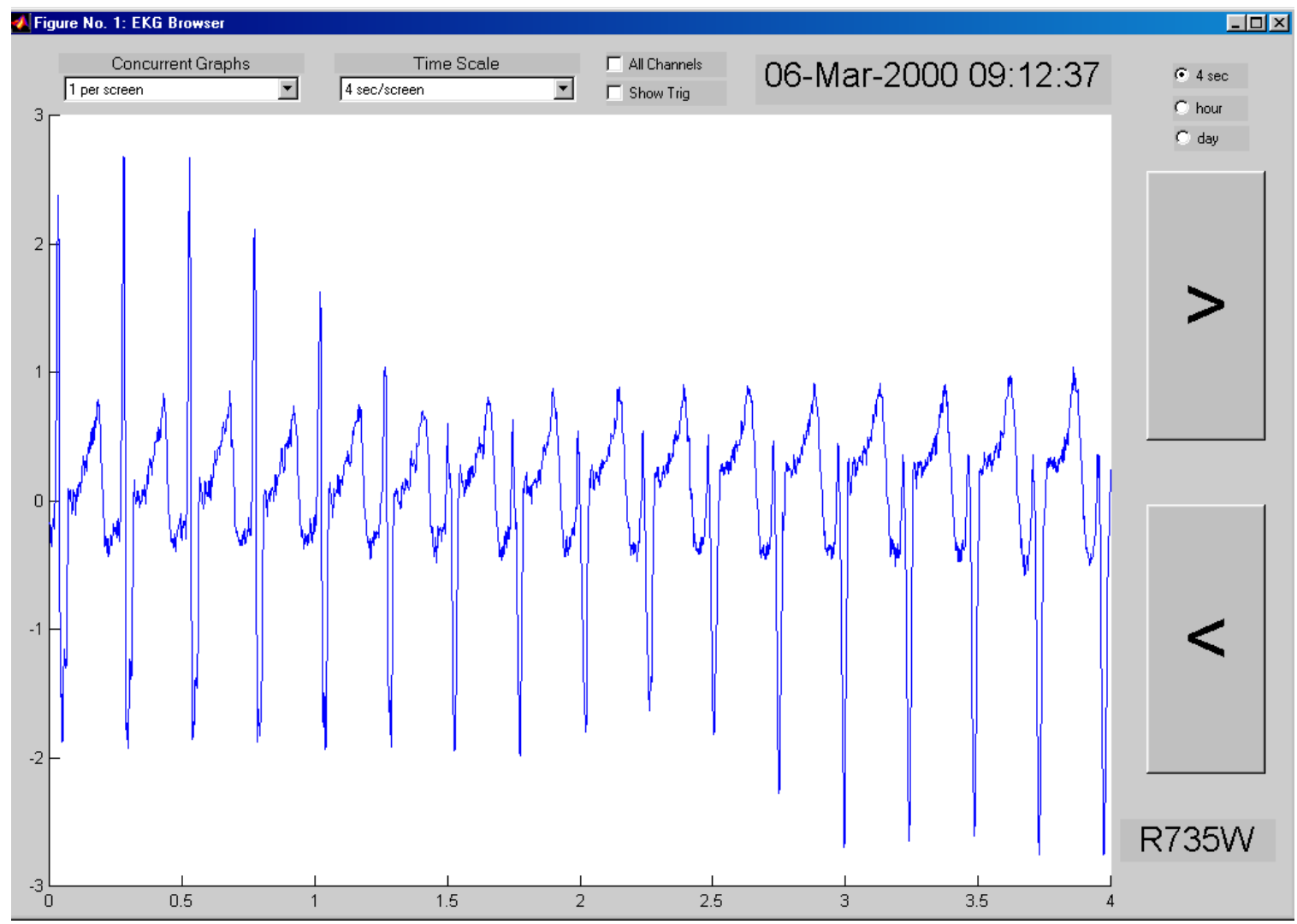

Figure 5.15 ECG signal, the moment of myocardial infarction, for seconds per screen

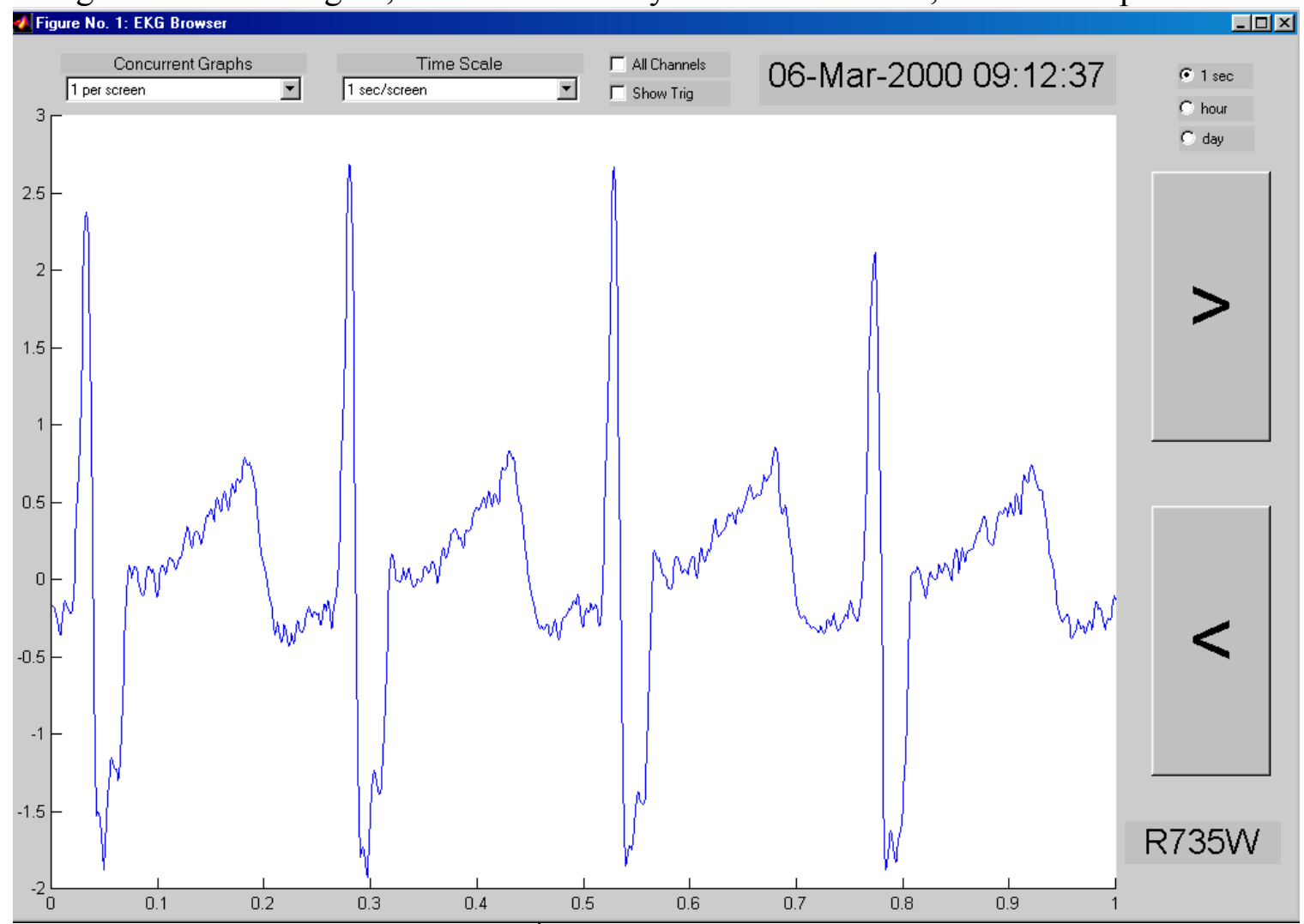

Figure 5.16 ECG signal on March $6^{\text {th }}$, before myocardial infaction, one second per screen 


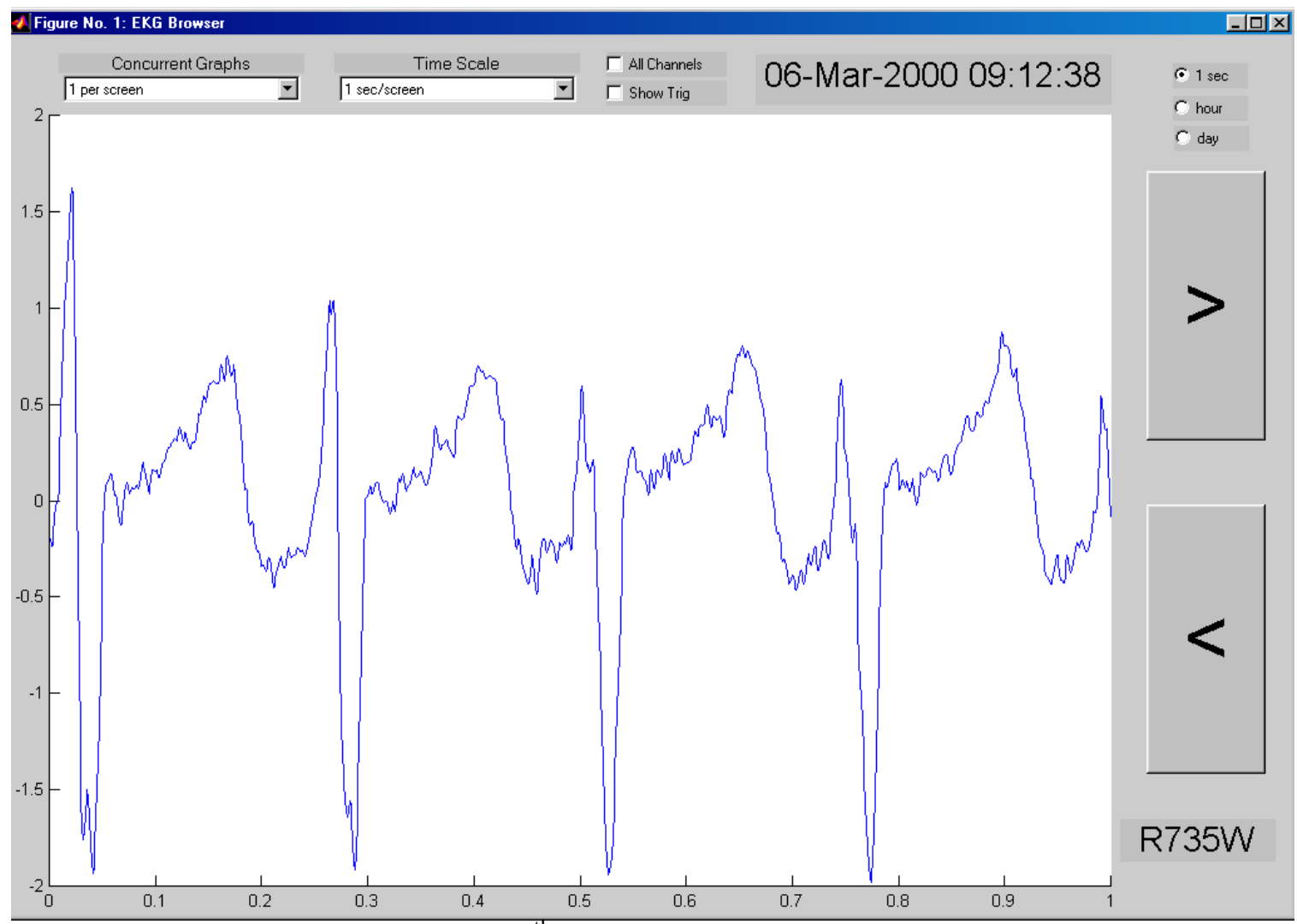

Figure 5.17 ECG signal on March $6^{\text {th }}$, after myocardial infarction, one second per screen

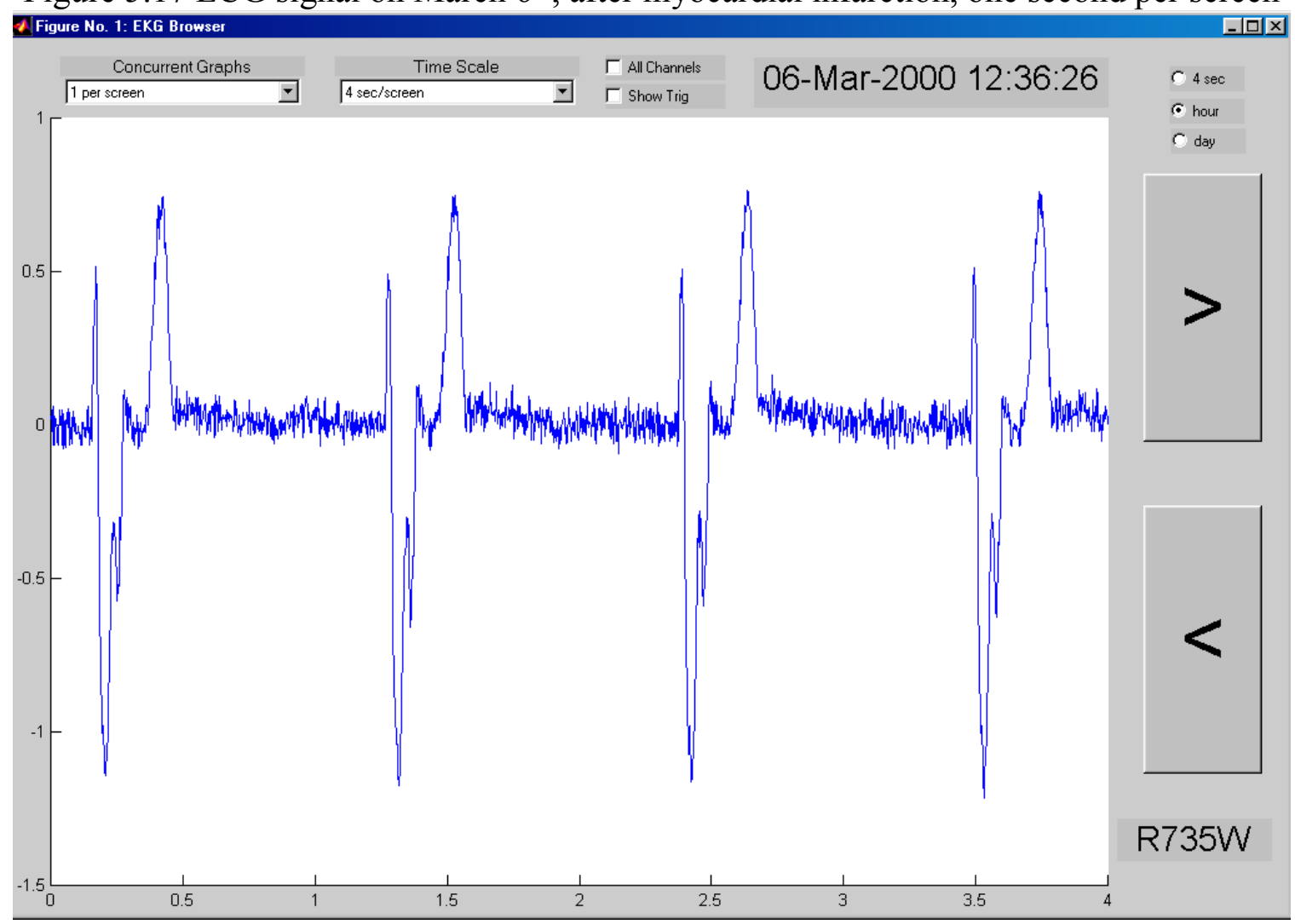

Figure 5.18 ECG signal, bradycardia, four seconds per screen 


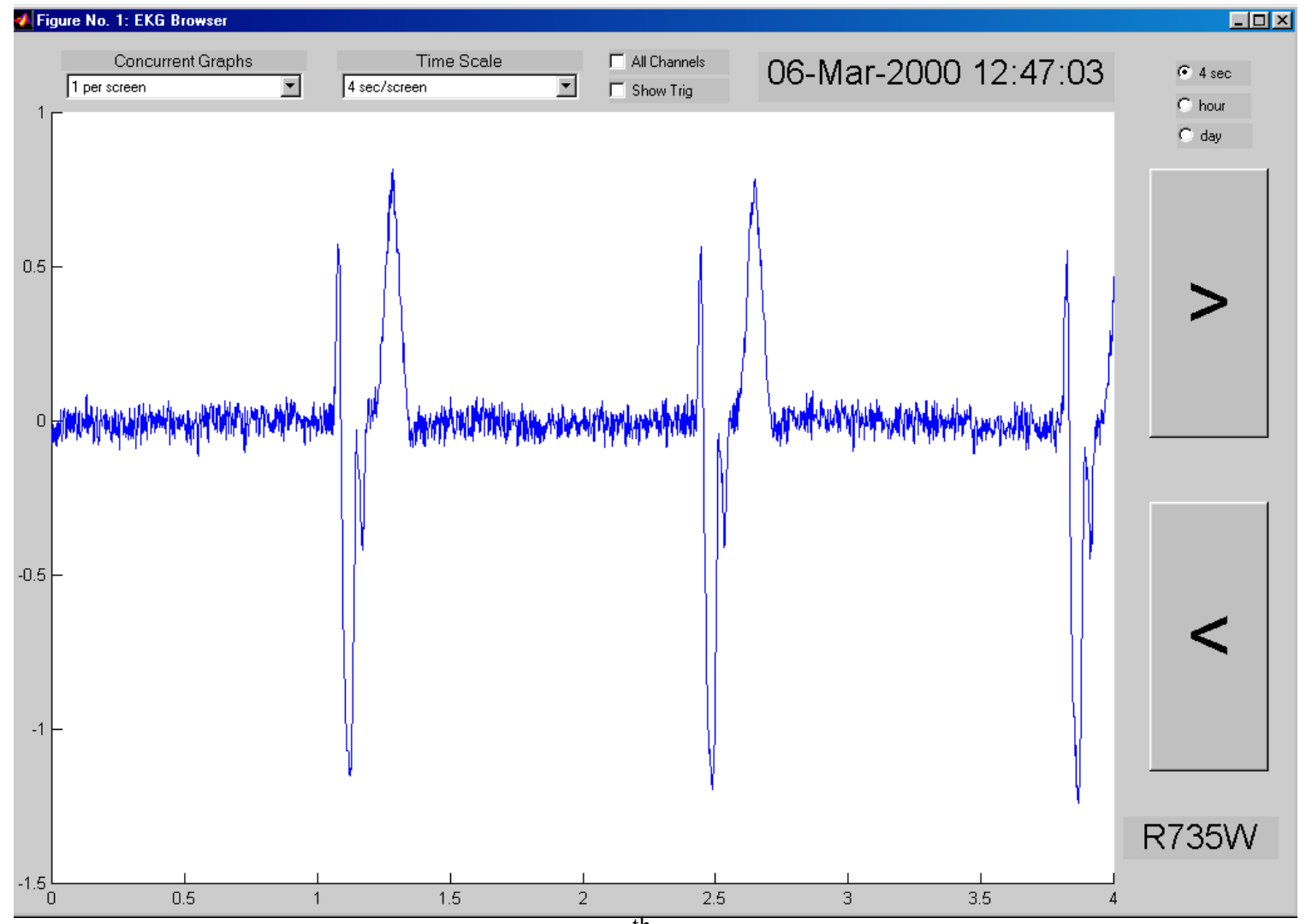

Figure 5.19 ECG signal on March $6^{\text {th }}$, bradycardia, four seconds per screen

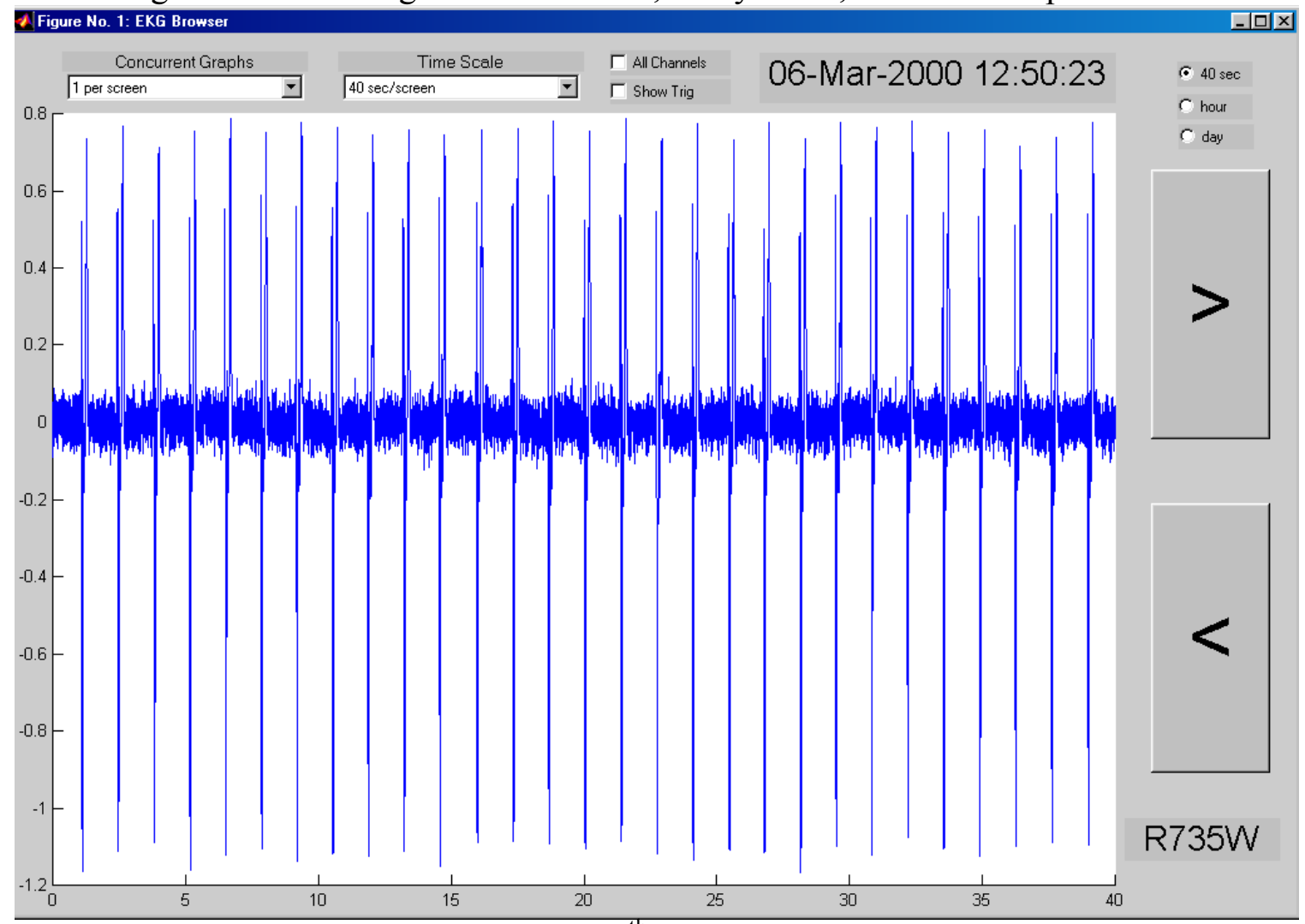

Figure 5.20 ECG signal on March $6^{\text {th }}$, bradycardia, forty seconds per screen 


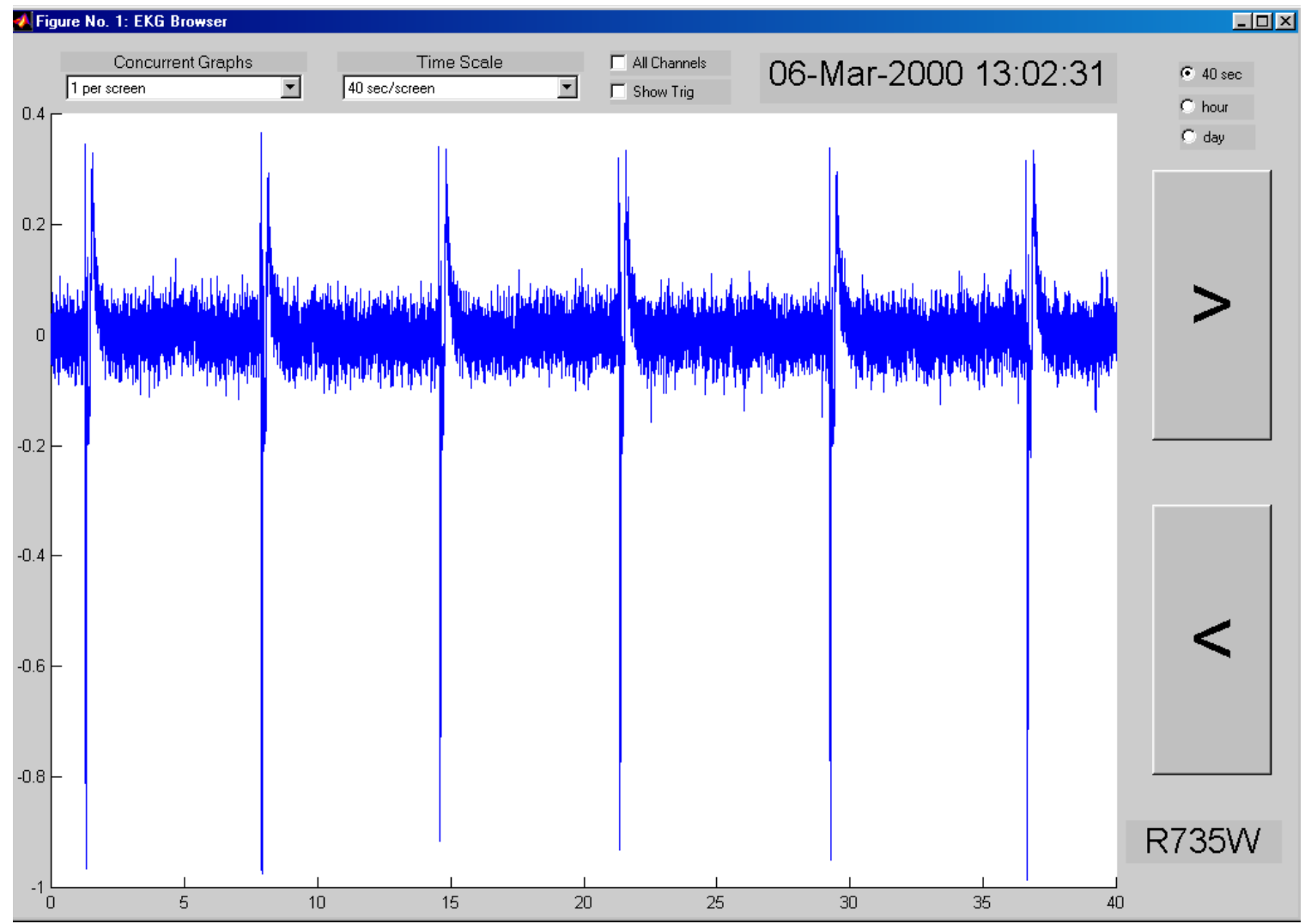

Figure 5.21 ECG signal, bradycardia, before the death, fourty seconds per screen

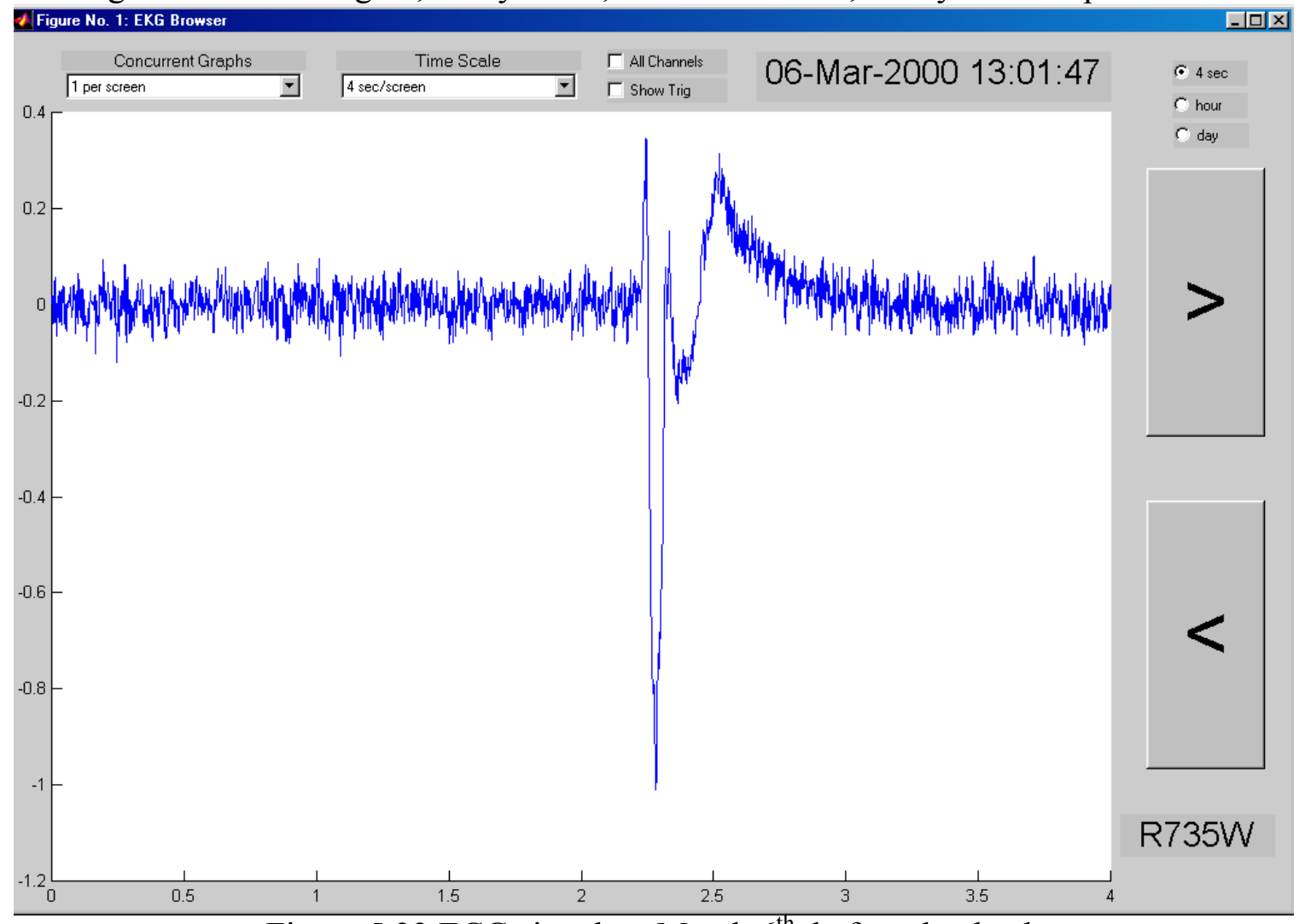

Figure 5.22 ECG signal on March $6^{\text {th }}$, before the death 


\subsection{Ways to capture and present the results.}

The research we performed in this research assumes knowledge from at least three points of view: signal processing knowledge, computer science knowledge and heart physiology knowledge. Due to the sheer mass of data, efficient and fast programs must be developed. One of the challenges, besides the efficient collection, storage and automated processing of the signals, was the development of methods to visualize the results. The quantity of data is overwhelming and is difficult for designing, coding and implementation, but also for verifying accurate results. The challenge is to find the most significant and suggestive ways to visualize the results. To handle also the massive amount of results, programs were developed with Matlab to organize data on:

- Individual plots (within a rabbit)

- $\quad$ Mixed plots (across rabbits)

Results are written to txt files, which can opened with Microsoft Excel. Final plots were designed in Excel.

\subsubsection{Individual plots, from averaged data, hourly and weekly}

Parameters relevant to HRV are computed for every 5-minute segment ECG and stored each in a vector of length 288 (one day has 288 5-minute segments). Data is averaged hourly, and vectors of length 24 are obtained for each day. These vectors form a matrix with 24 rows (24 hours) and 7 columns ( 7 days in a week). Data is again averaged for each hour, over the week and a single vector of length 24 (hours) is obtained for one week. These vectors were obtained for each rabbit, for all eleven parameters, and 5 weeks were plotted: week -1 , the first week prior to the injection, weeks 3, 5, 7 after the first injection, and week 9 for rabbits R710V and R733W and week 8 for rabbit R735W, since this rabbit died before the end of week 9. All the plots, for all eleven parameters are in the Appendix A.

The plots show the evolution of every parameter, over the weeks. In case of the control rabbit, R710V, which was injected with saline, the variation of all eleven parameters is the same, within a very small range of values. There are not noticeable differences from week to week. 
Figures 5.19 and 5.20 reveal the evolution of mean (MEAN) and coefficient of variance (CV) for rabbit $\mathrm{R} 710 \mathrm{~V}$.

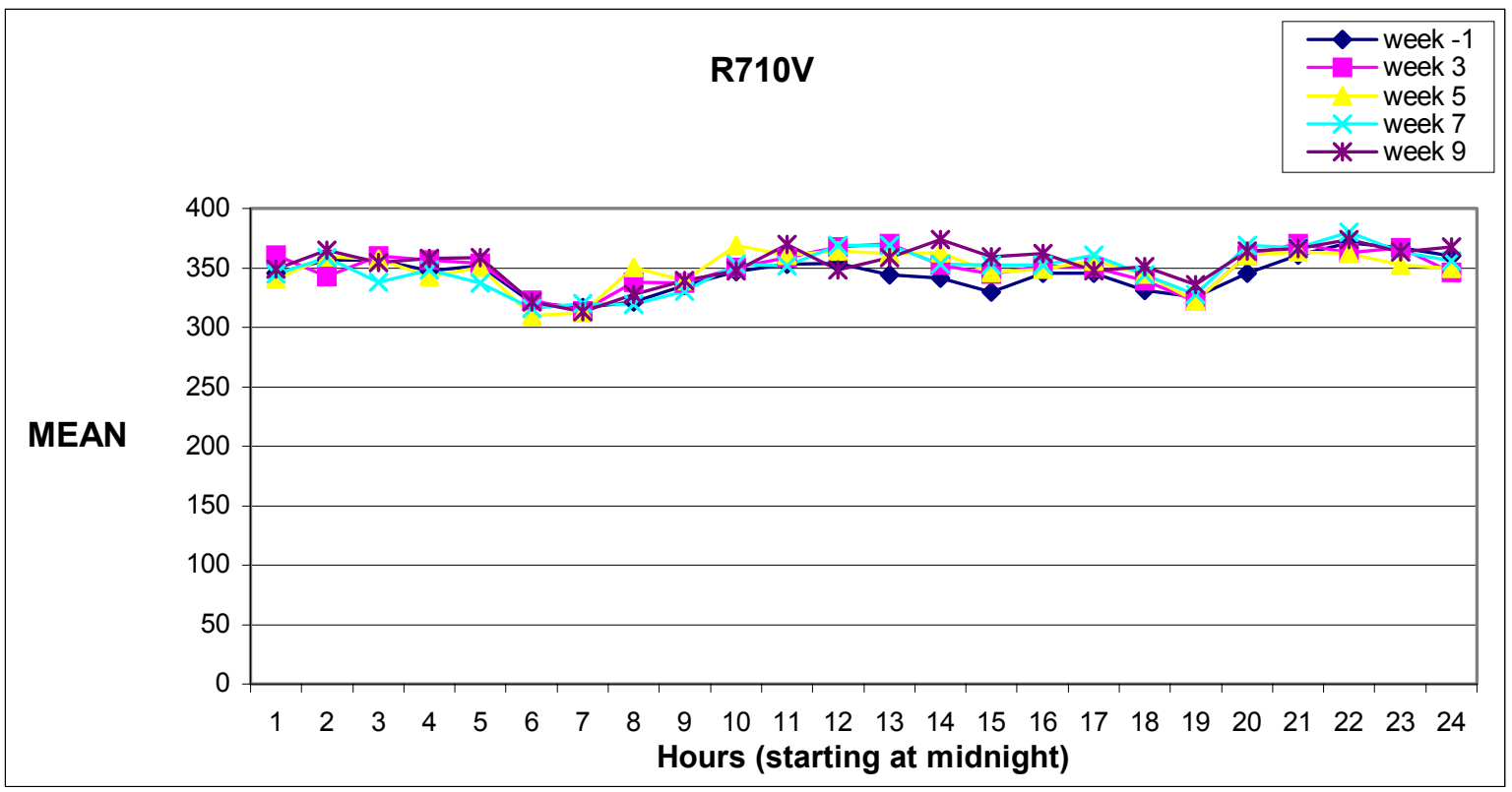

Figure 5.23 Mean, rabbit R710V

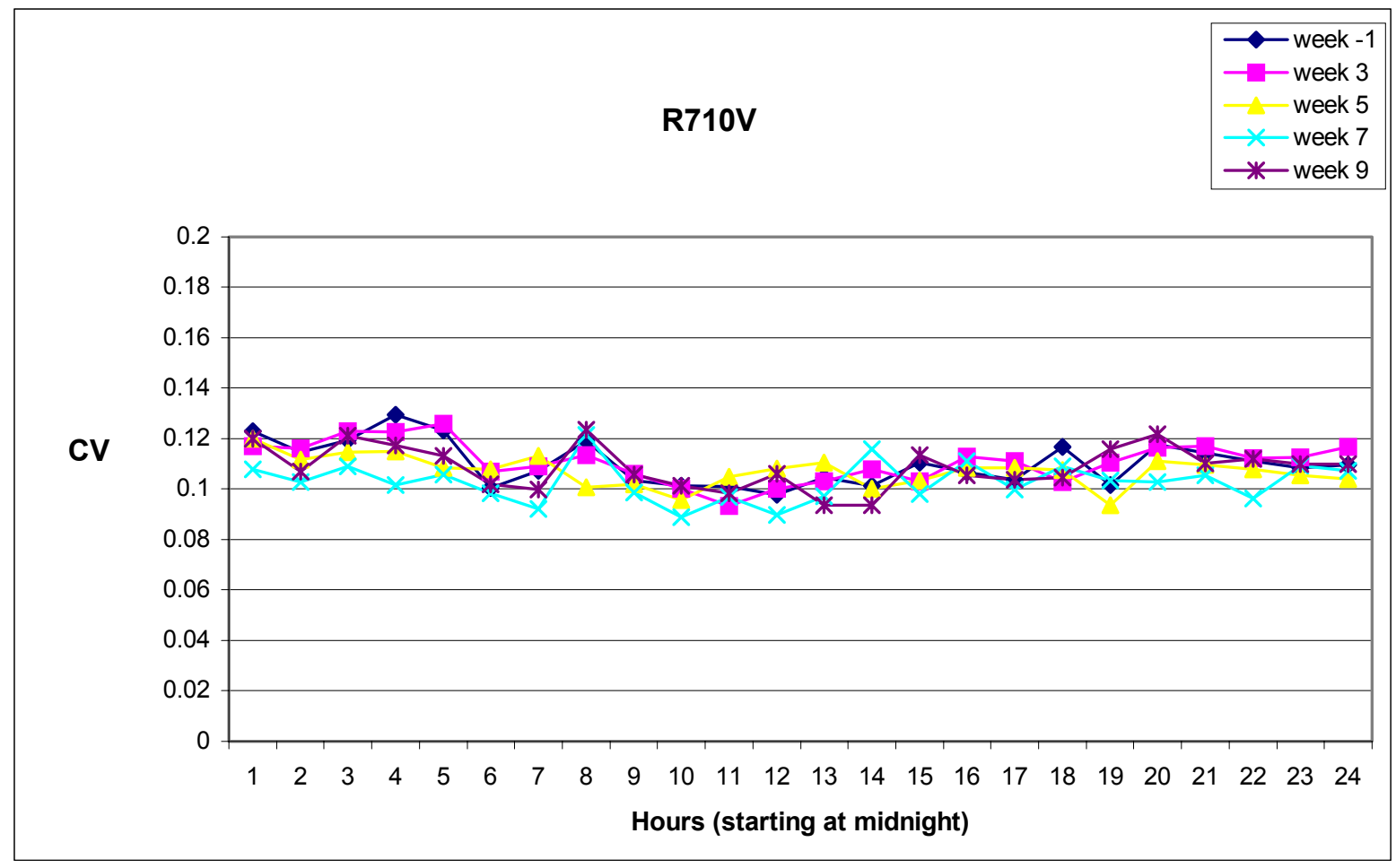

Figure 5.24 Coefficient of covariance, rabbit R710V 
High frequency (HF), which is a measure of parasympathetic activity, is higher during the night and lower during the day hours, and is in accordance with the variation in the healthy subjects (Figure 5.21).

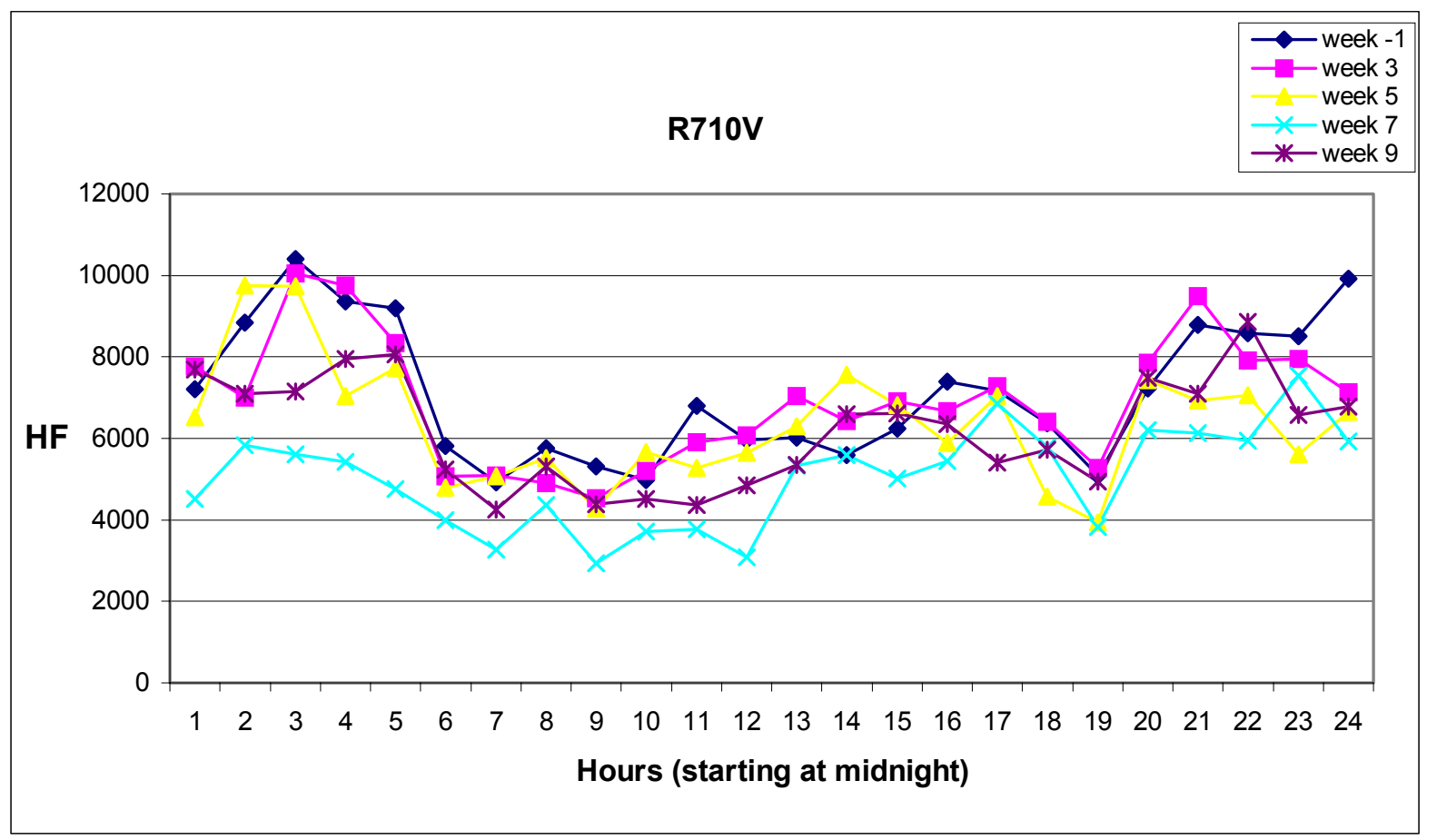

Figure 5.25 High frequency, rabbit R710V

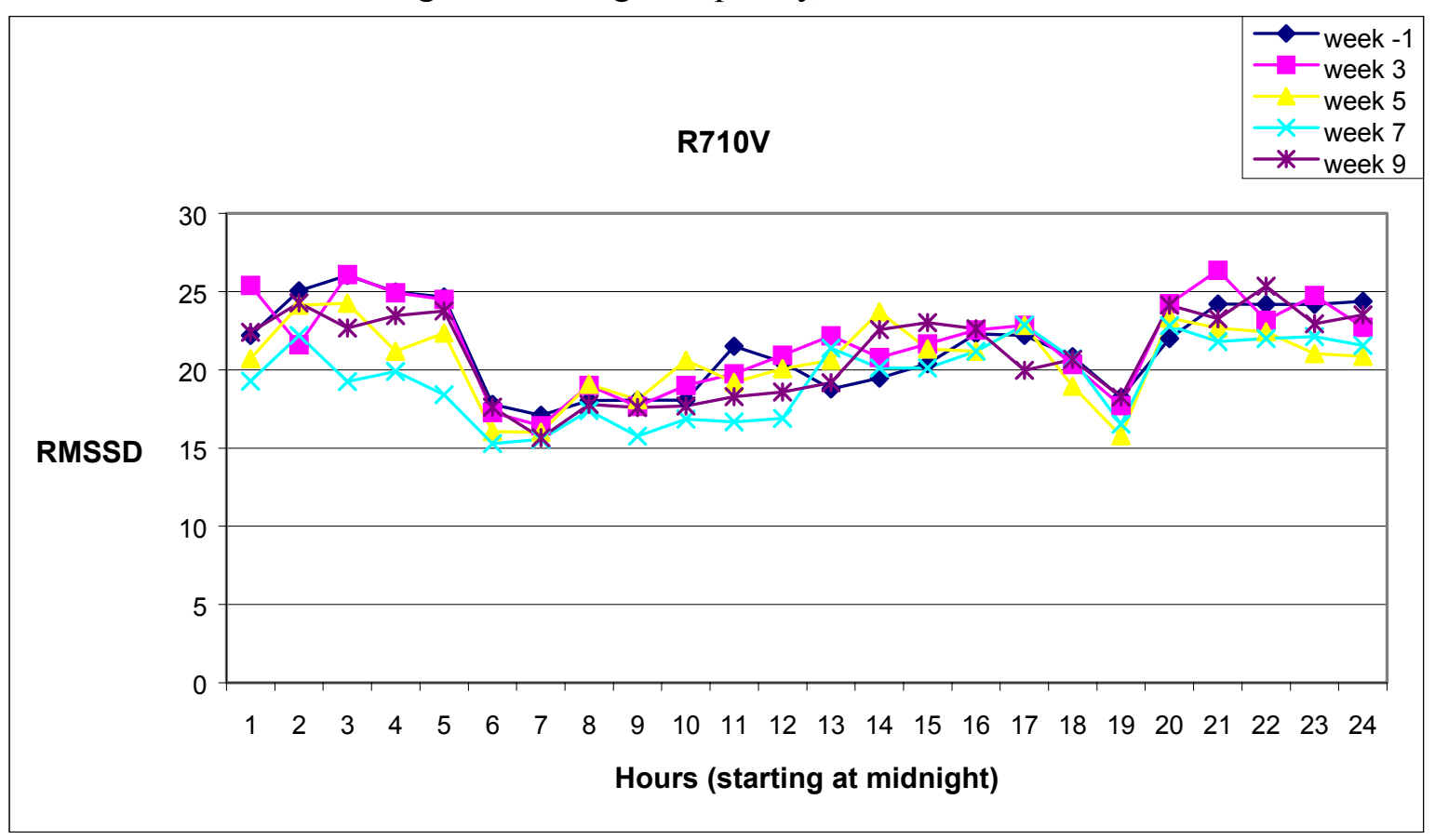

Figure 5.26 Root mean square successive differences, rabbit R710V 
RMSSD is another parameter relevant for parasympathetic activity, and in the case of control rabbit has the same pattern each week.

For rabbits $\mathrm{R} 733 \mathrm{~W}, \mathrm{R} 735 \mathrm{~W}$ the value of mean and median increase in week 3, and gradually decrease to a value lower than that of week- 1 (Figures 5.27 and 5.30). In addition, the mean becomes smoother, revealing a decline in variability over the day.

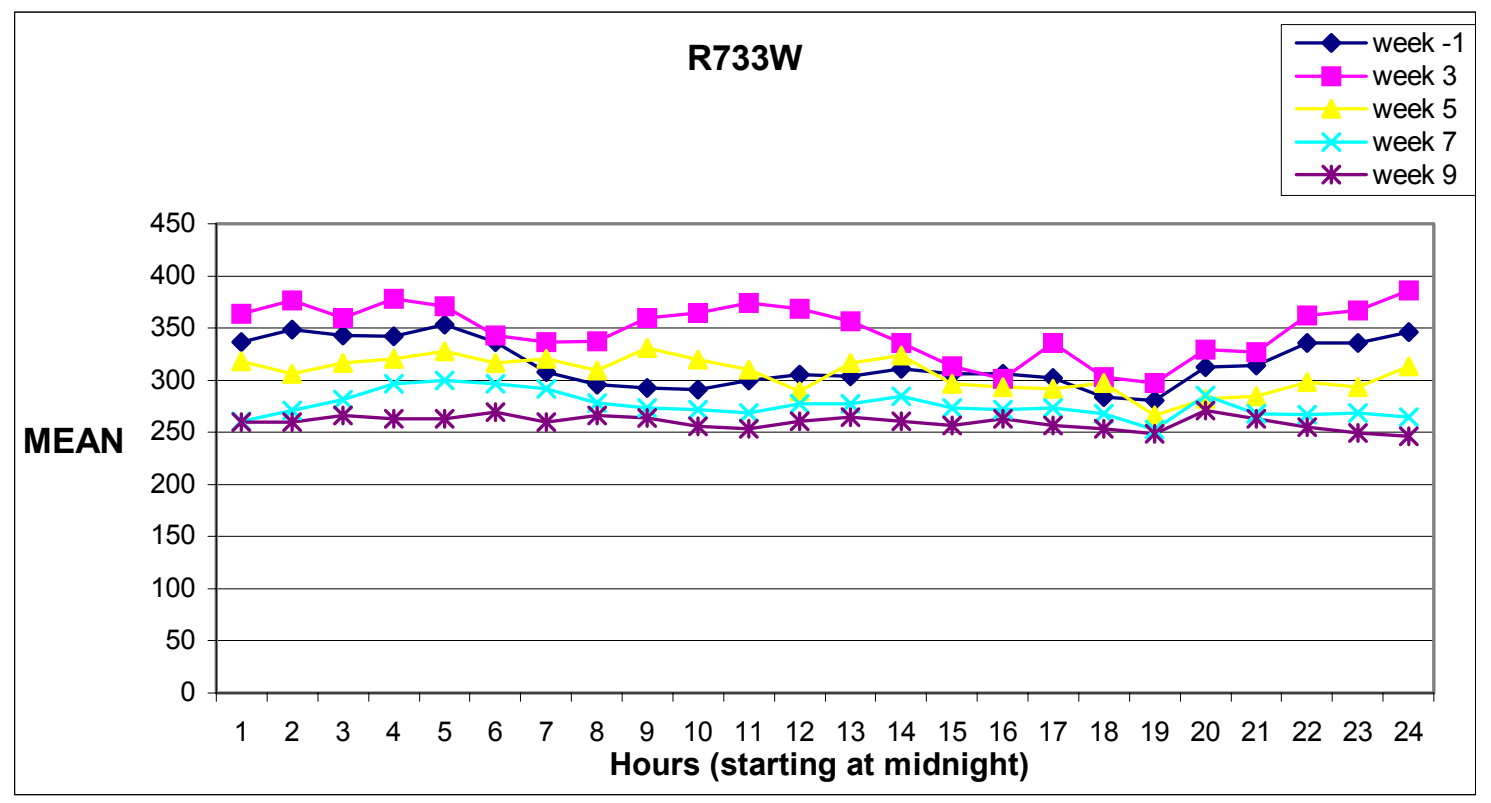

Figure 5.27 Mean, rabbit R733W

In the case of rabbit R733W all HRV parameters present an increase in week 3, with a higher variation during the day compared with week-1, and then a decrease with almost no variations by week 9 (Appendix A).

Special attention should be paid to the frequency domain measurements, high frequency (HF) for parasympathetic activity, low frequency (LF) for both parasympathetic and sympathetic activity, and the ratio LF/HF, which elicits the balance between the two (Figures 5.28 and 5.29). Before the injection, HF has larger values during the night compared with day hours and there is little variation during the night or day. After injections, values increase and there is a more variation during the day. The behavior of a healthy subject is lost. The ratio LF/HF (LHF) reveals higher values during the daytime in week-1, which is normal and corresponds to higher values of LF compared with HF. As the weeks pass, the pattern is lost; the ratio decreases to almost a line, with little variation. 


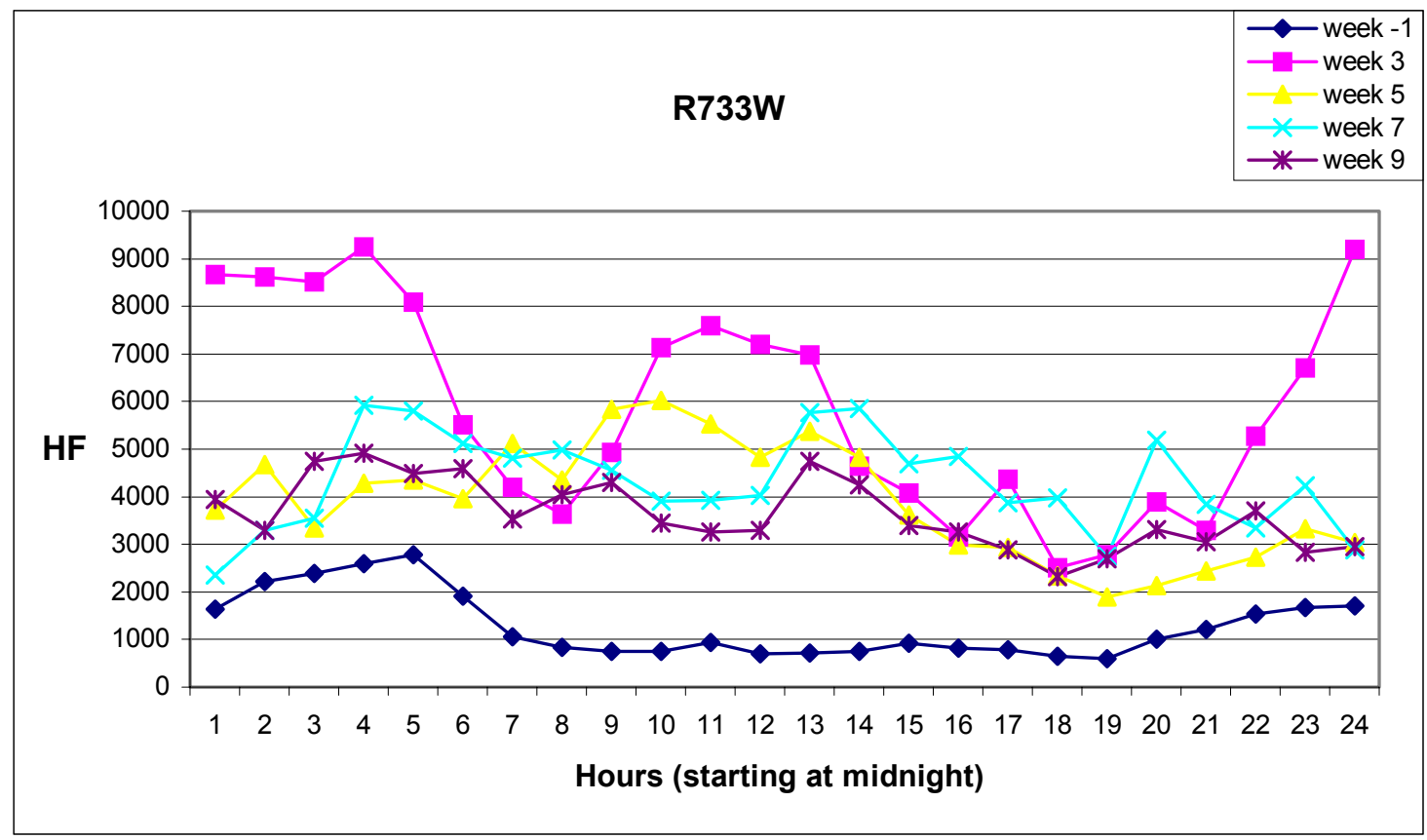

Figure 5.28 High frequency, rabbit R733W

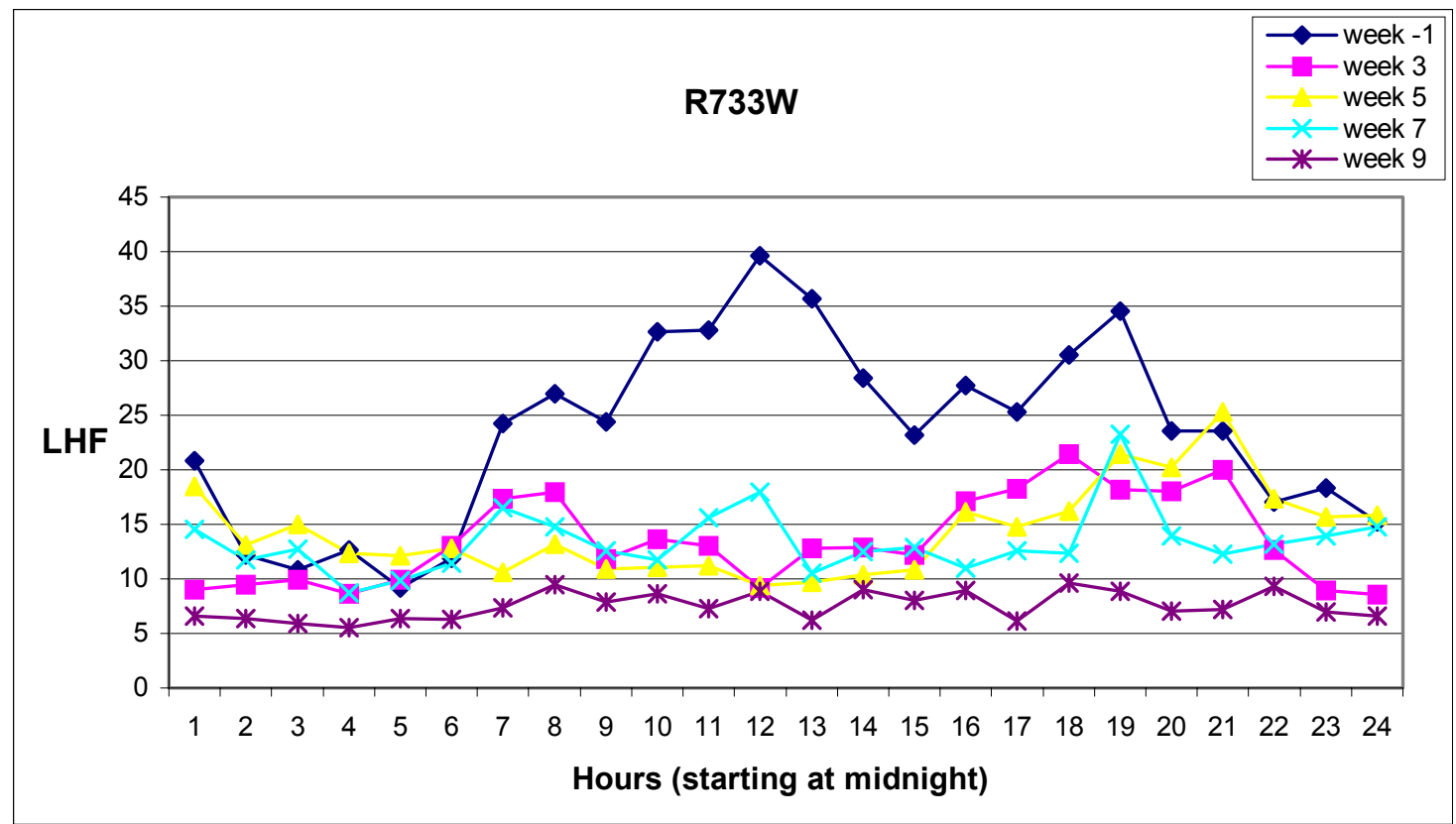

Figure 5.29 Ratio low frequency/high frequency, rabbit R733W

In case of rabbit R735W, all parameters IQR, RMSSD, DDIQR, SDSD, SDNN, CV present higher values in week 3 , compared with week -1 , and then become smoother and decrease to lower values than week- 1 by week 9 (Figure 5.31, Appendix A). 


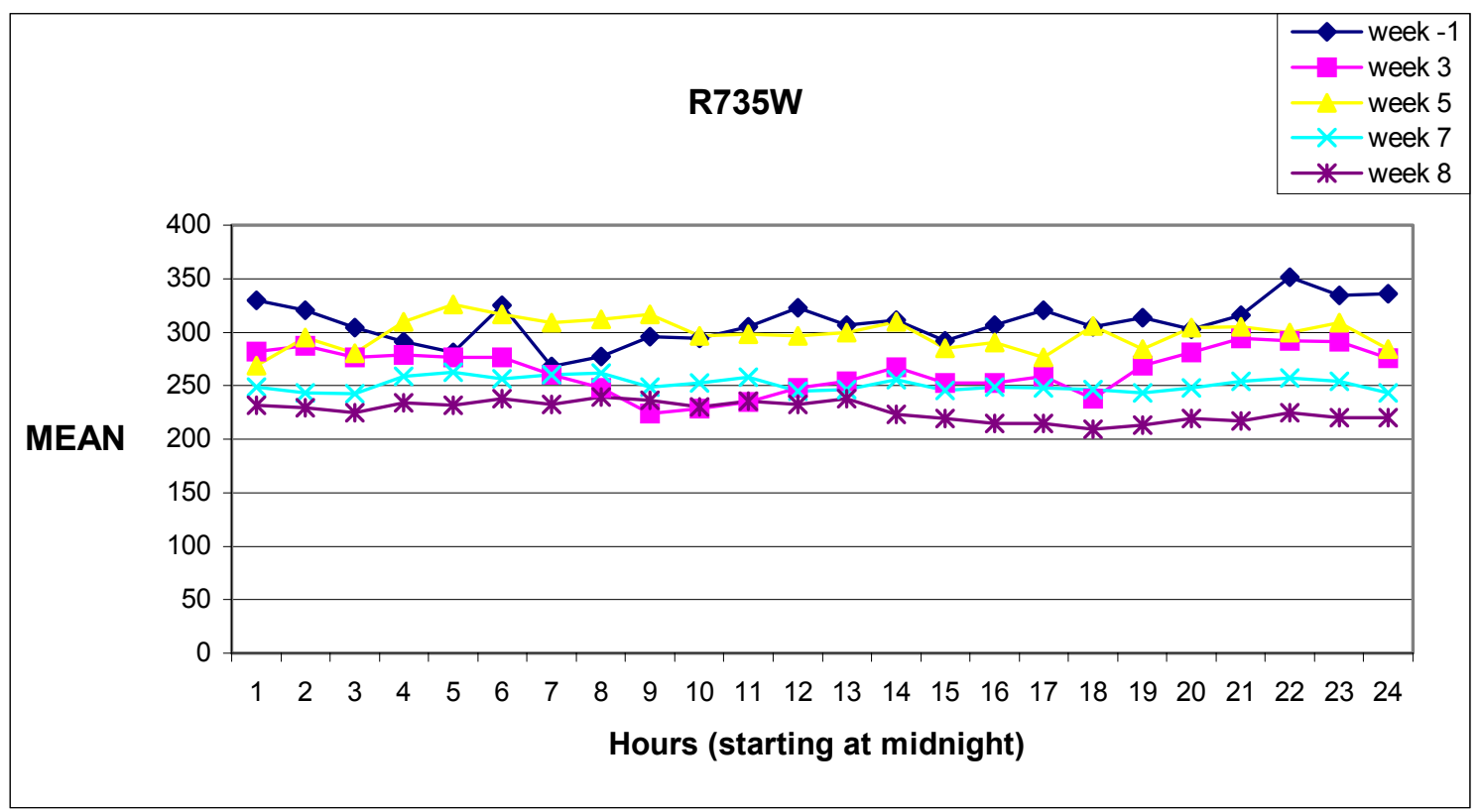

Figure 5.30 Mean, rabbit R735W

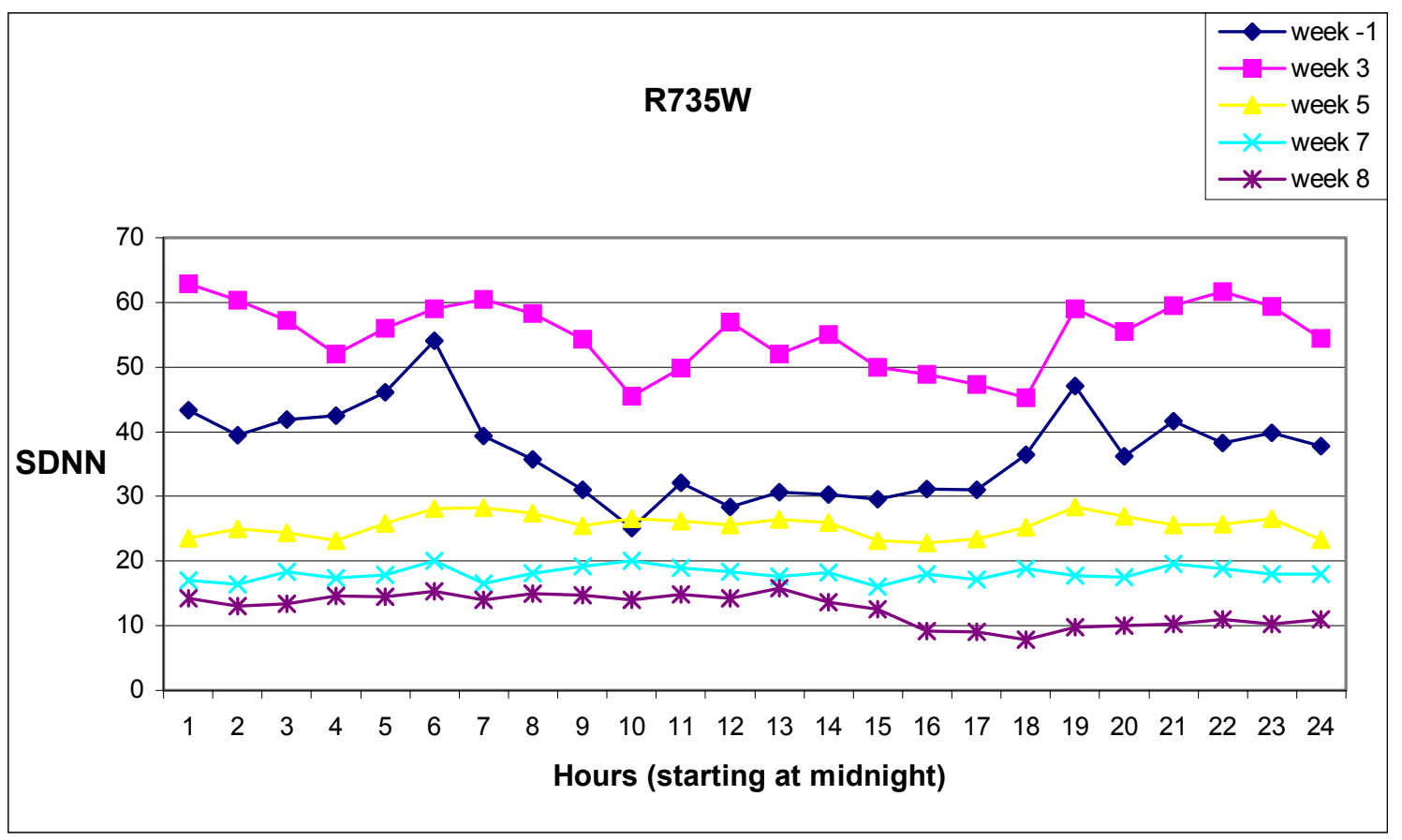

Figure 5.31 Standard deviation of RR intervals, rabbit R735W 
High frequency and the rest of parameters reveal an increase and then a drastically decrease of parasympathetic activity, fact relevant for heart failure and the adverse reaction of adriamycin (Figure 3.32)

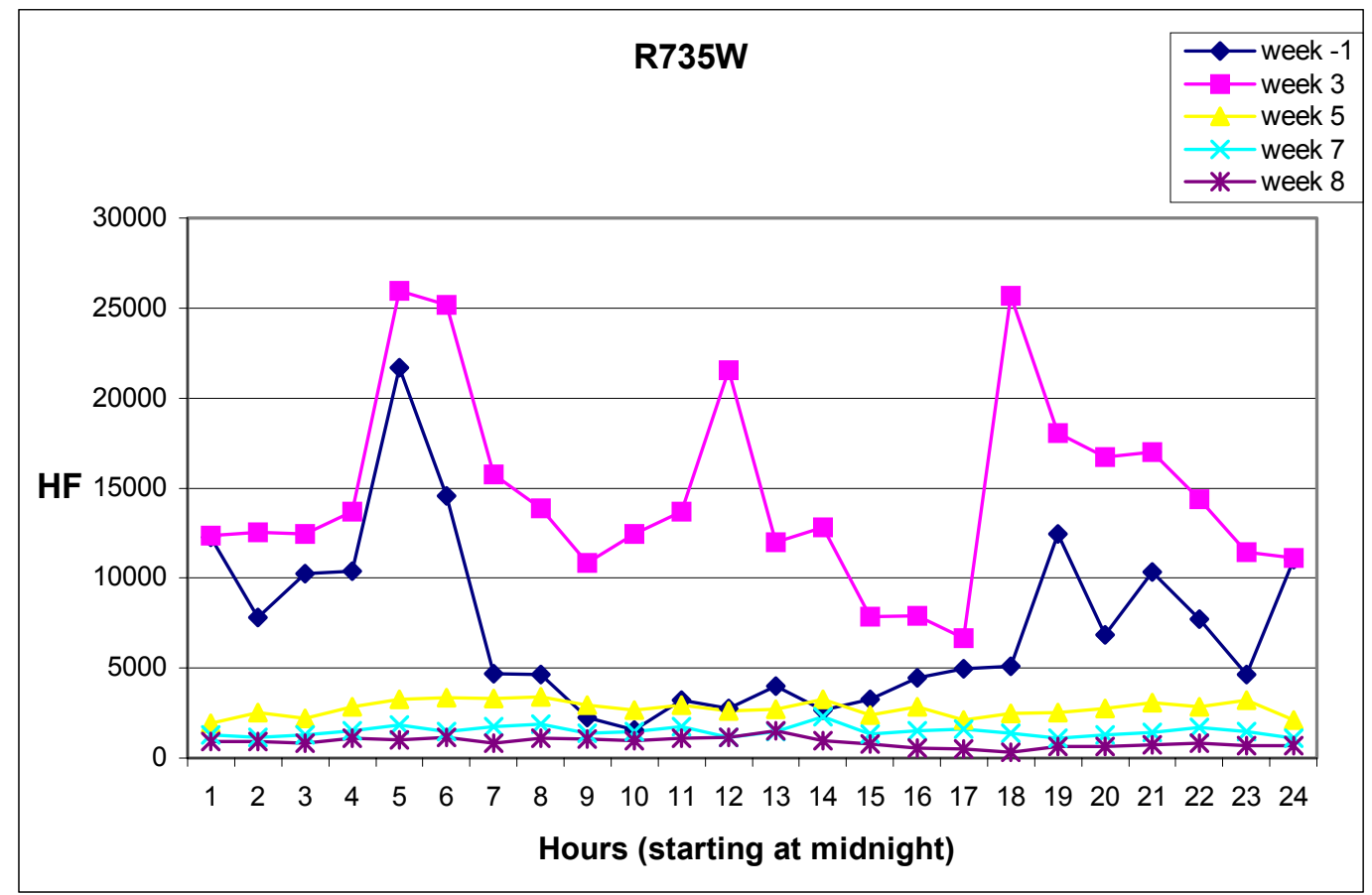

Figure 5.32 High frequency, rabbit R735W

\subsubsection{Individual plots where each 5 minute segment is averaged weekly}

Parameters relevant to HRV are computed for every 5-minute segment ECG and stored in a vector of length 288 for each day (one day has 2885 -minute segments). These vectors form a matrix with 288 rows and 7 columns, for the week. Values are averaged for each of 5-minute segments across the week, and a vector of length 288 is obtained for every week. Because values are not averaged across the hour as in the previous section, peaks and valleys are much more visible (Figures 5.33, 5.34, and 5.35). This type of plot is useful to give an overview and general understanding of the evolution of the parameters. If plotted with the appropriate software, it can be elongated and segments of interest can be then highlighted, zoomed or plotted. As in the previous kind of plots, these plots show a decrease of HRV for rabbit R733W and R735W. All plots, for all rabbits, and for all 5 weeks are in the Appendix B. 


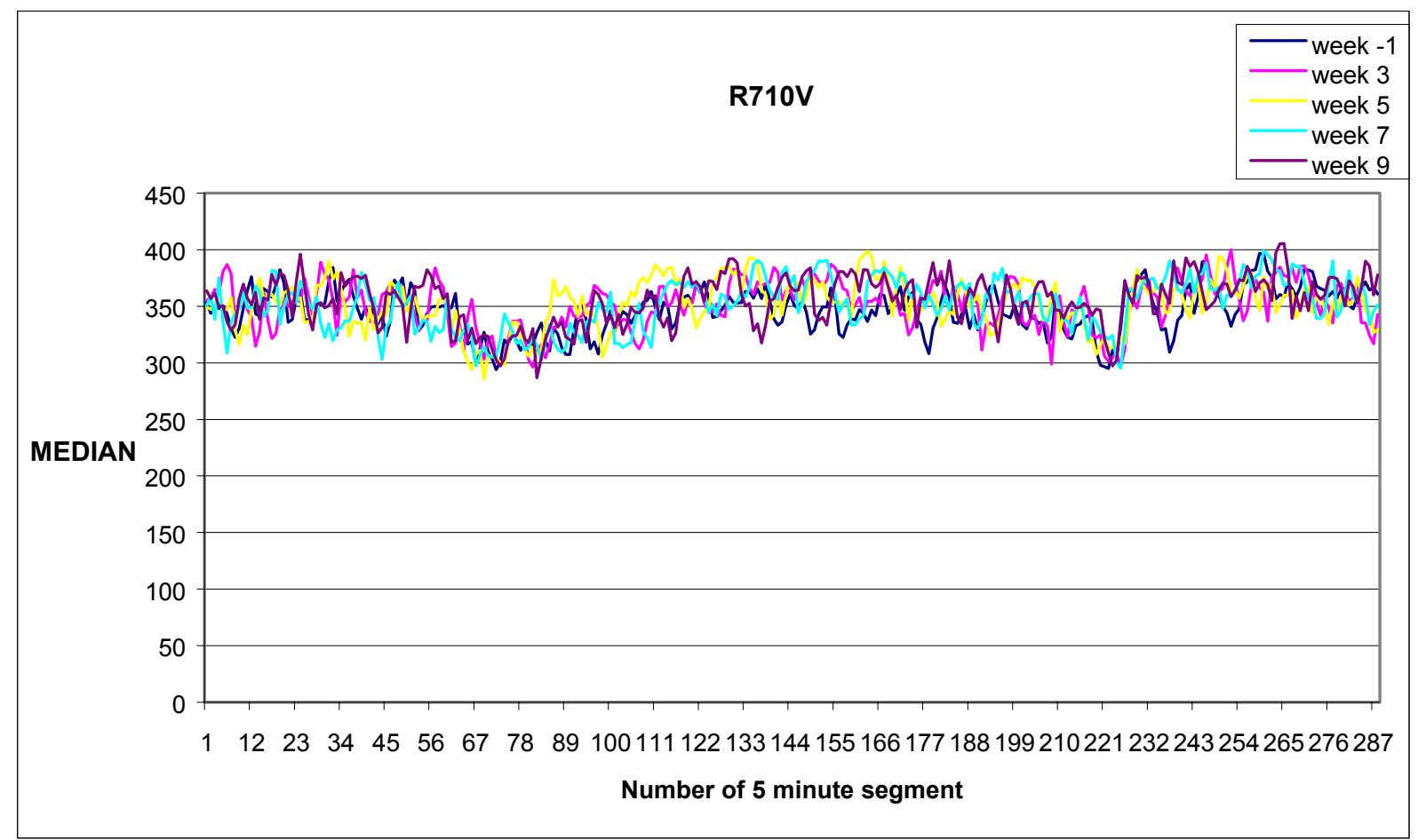

Figure 5.33 Median, rabbit R710V

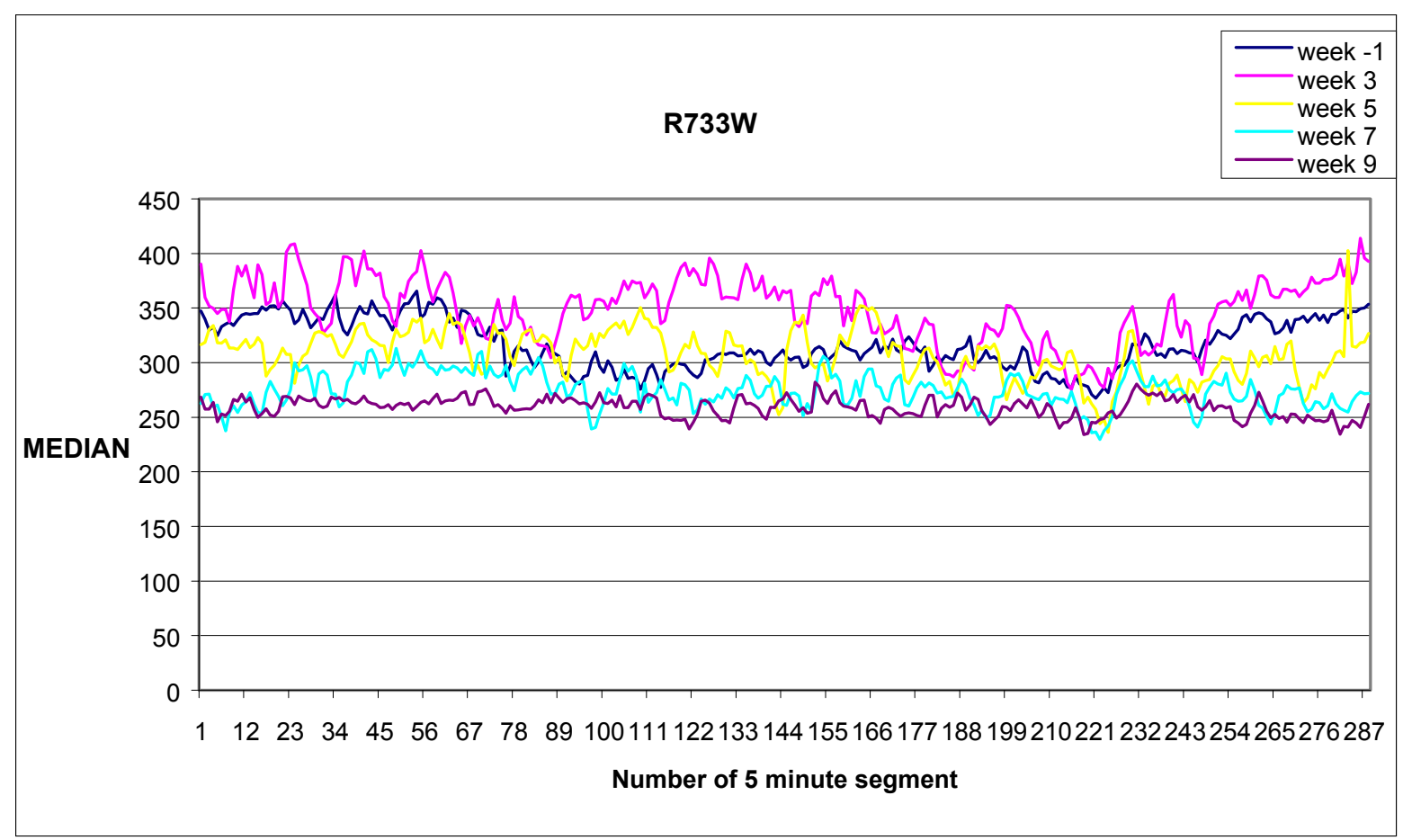

Figure 5.34 Median, rabbit R733W 


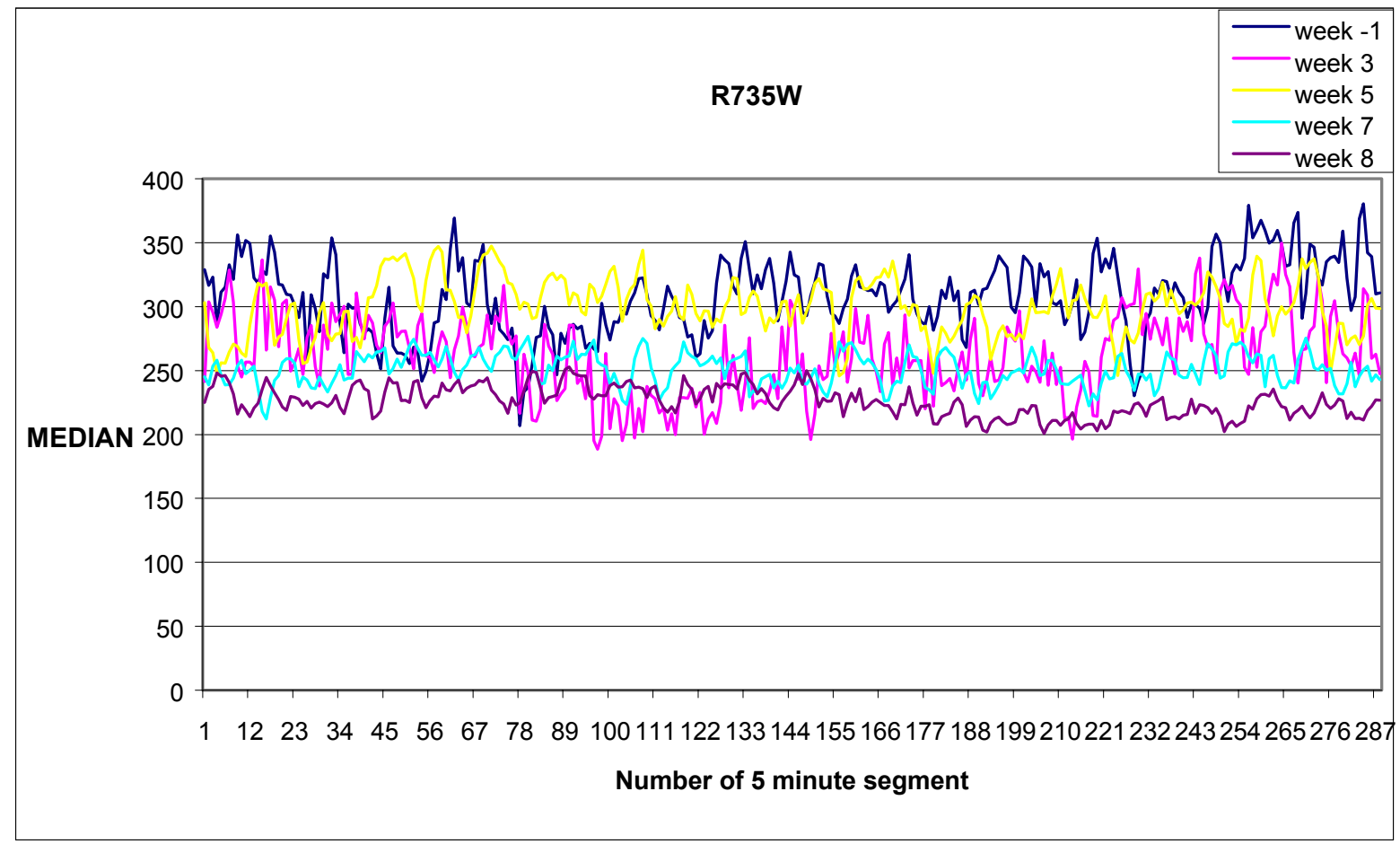

Figure 5.35 Median, rabbit R735W

\subsubsection{Mixed plots per week, averaged hourly and weekly}

Using the averaged weekly and hourly data described in section 4.2.1, the plots are now " mixed"; that is they contain the same parameter for all three rabbits, for one week. This type of plot is helpful to assess the difference between parameters of a healthy subject and a diseased one (Figures 5.36 and 5.37). In week- 1 and 3 no differences between groups can be seen. Starting with week 5 the differences are apparent and obvious. Standard deviation across the averaged data was also plotted for each hour and represented by the bars. 


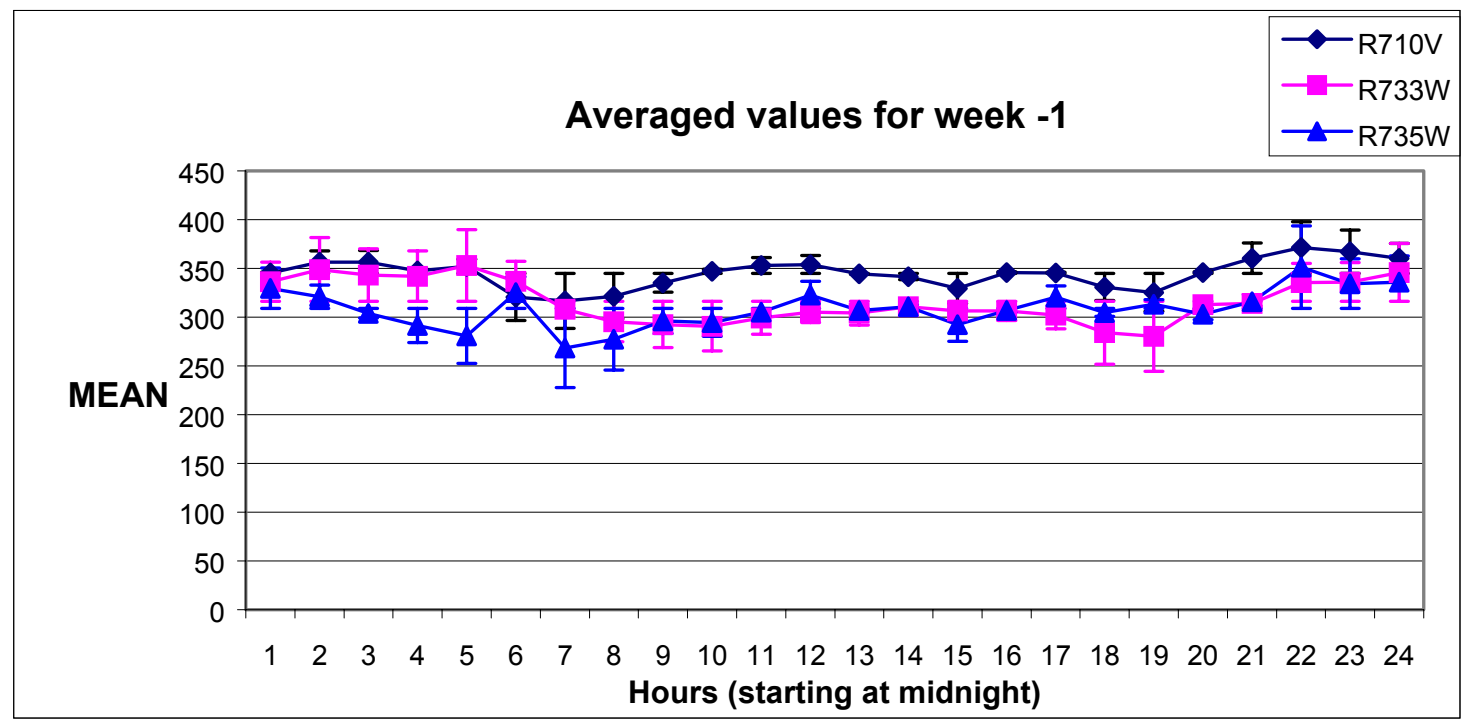

Figure 5.36 Mean, week -1

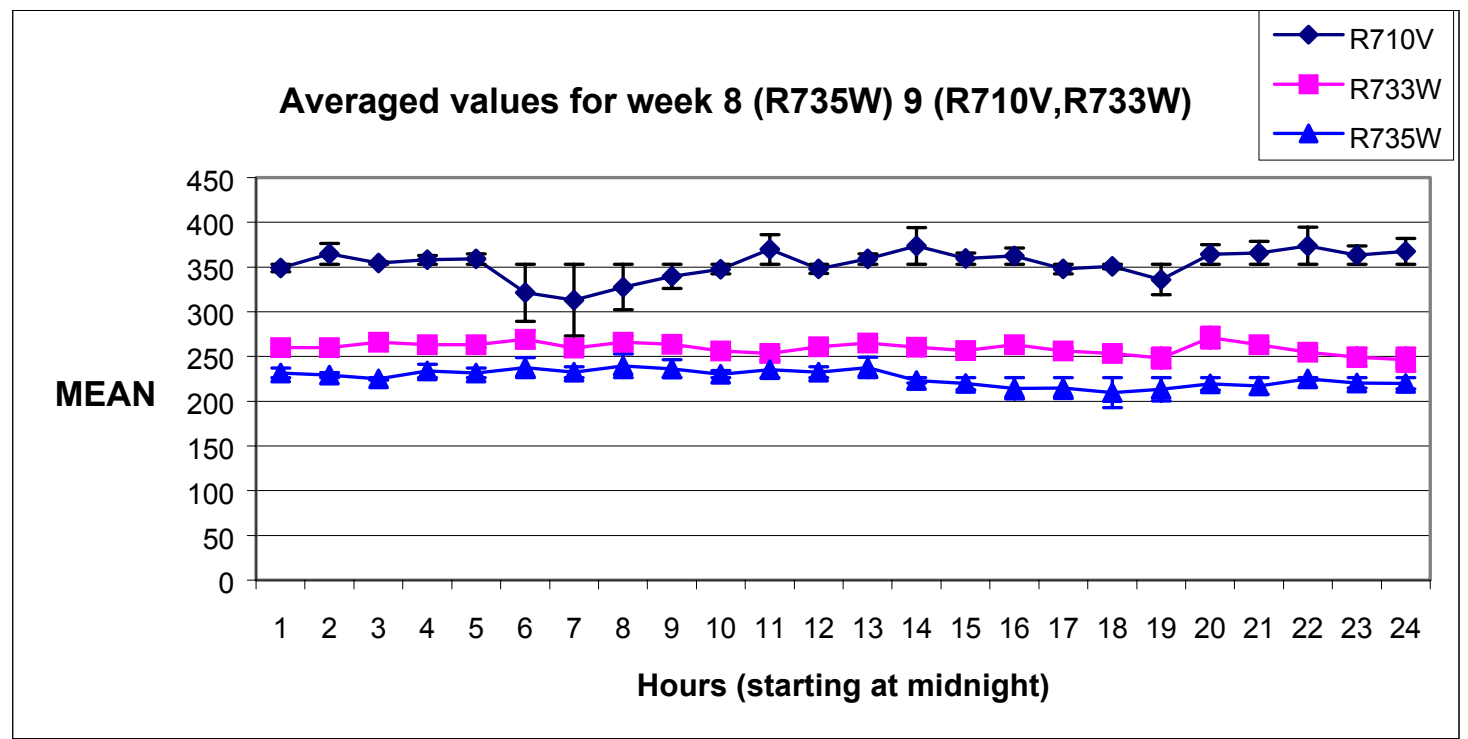

Figure 5.37 Mean, week 8 and 9

Compared with the control rabbit, the doxorubicin rabbit values decrease for all parameters computed in time domain, and frequency domain (Figures 5.38 and 5.39, Appendix C). 


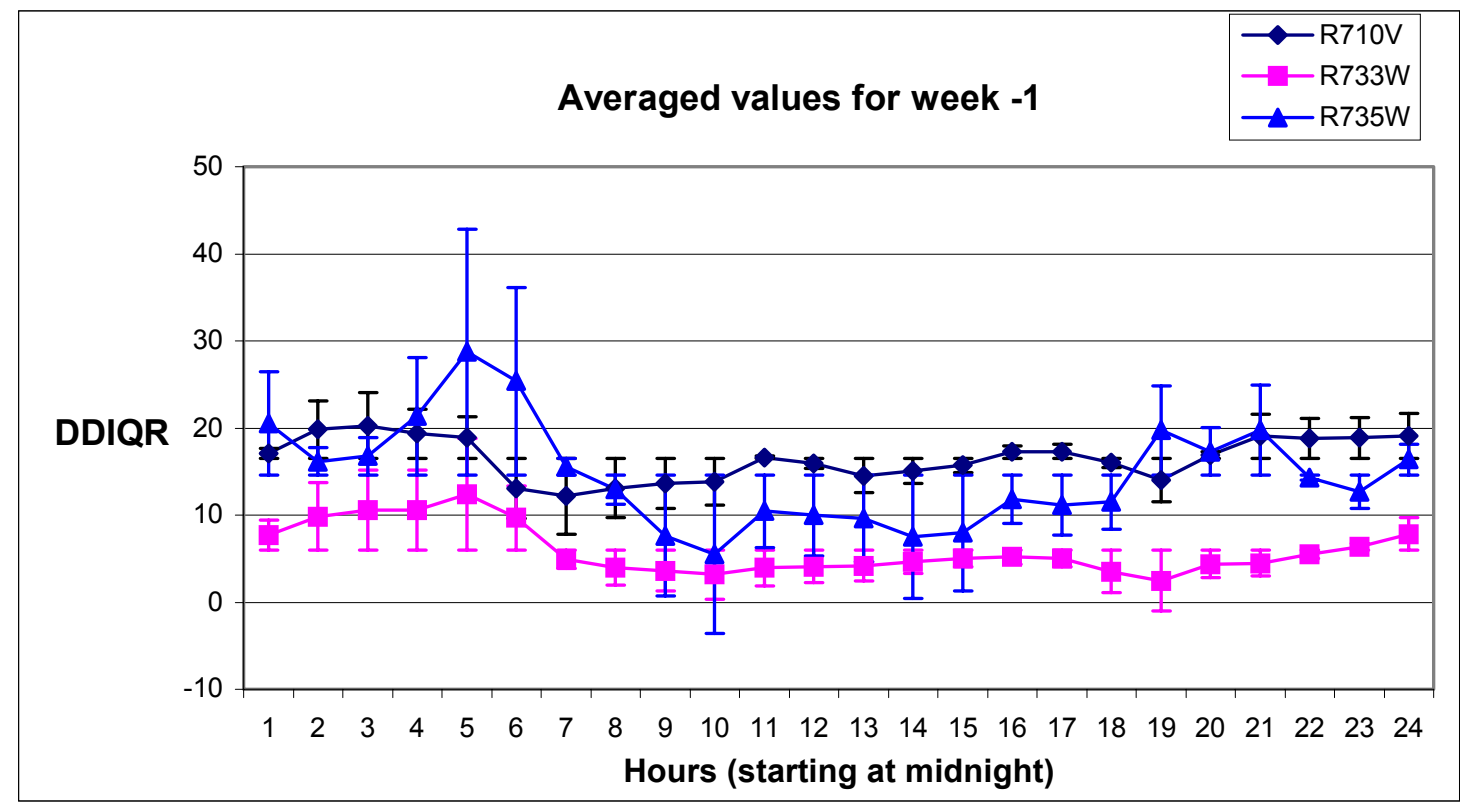

Figure 5.38 Variation of interquartile range of variation of RR intervals, week -1

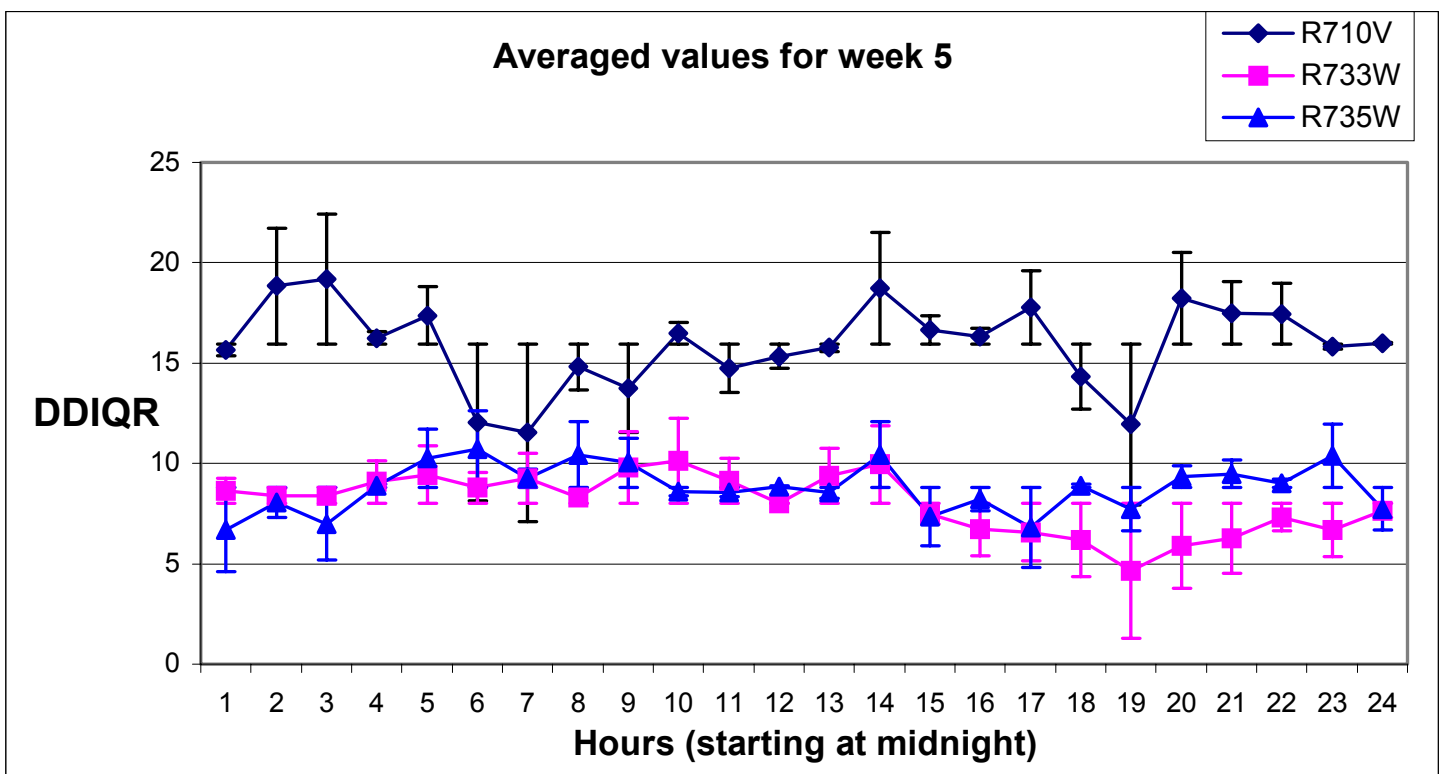

Figure 5.39 Variation of interquartile range of variation of RR intervals, week 5

The plots for all rabbits, all five weeks, and all parameters are in Appendix C.

\subsubsection{Individual and mixed plots, from averaged data per day and night}

It is well known that HRV exhibits a circadian rhythm, where heart rate variability varies across the day and night. This was apparent in the graphs in the previous sections. This section looks simply at day and night differences. 
In the rabbit room, light is automatically turned on at 6:00 AM and turned off at 6:00 PM. Taking this in account, all data for all five weeks was separated for day and night, and values were averaged for every rabbit, and each week. The results (Figure 5.40, 5.41 and 5.42, Appendix D) present two particular characteristics:

- Values for HRV among rabbits are close for week - 1; in week 3 values for rabbit $\mathrm{R} 735 \mathrm{~W}$ increase from week -1 , except for mean and median, while values for rabbit R733W in week 3 increase from week -1 but are still close to those of the control rabbit. Starting with week 5, values for injected rabbits are lower than the control rabbit.

- The difference between day and night, for the same rabbit and the same parameter is obvious in the first weeks. After injection, the points on the graphs for day and night become superimposed, so that along with a decrease of heart rate variability, there are also no differences between day and night. These results are expected, taking in account that all parameters present a smoother across the day as the injections progress.

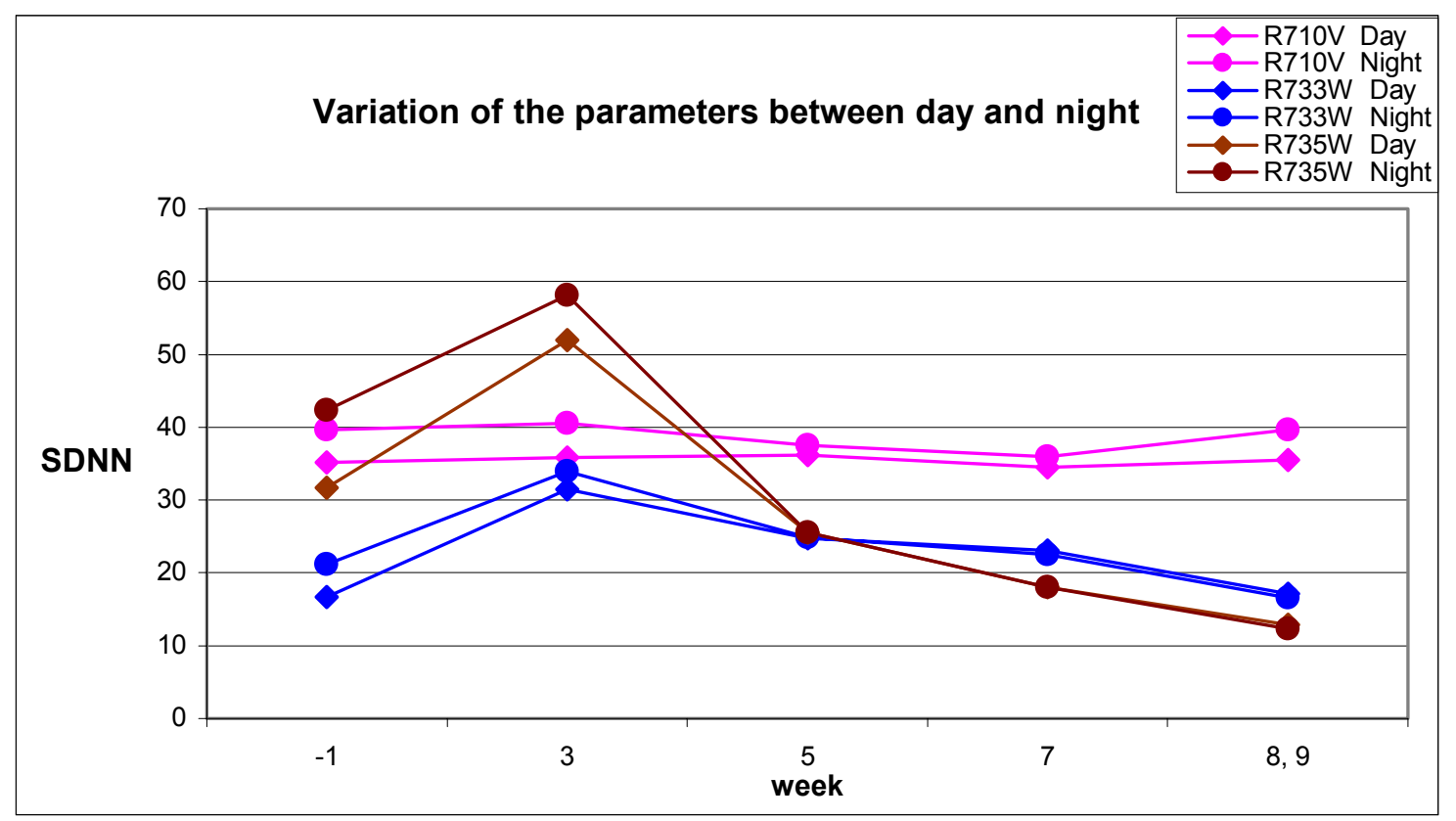

Figure 5.40 Variation of standard deviation between day and night 


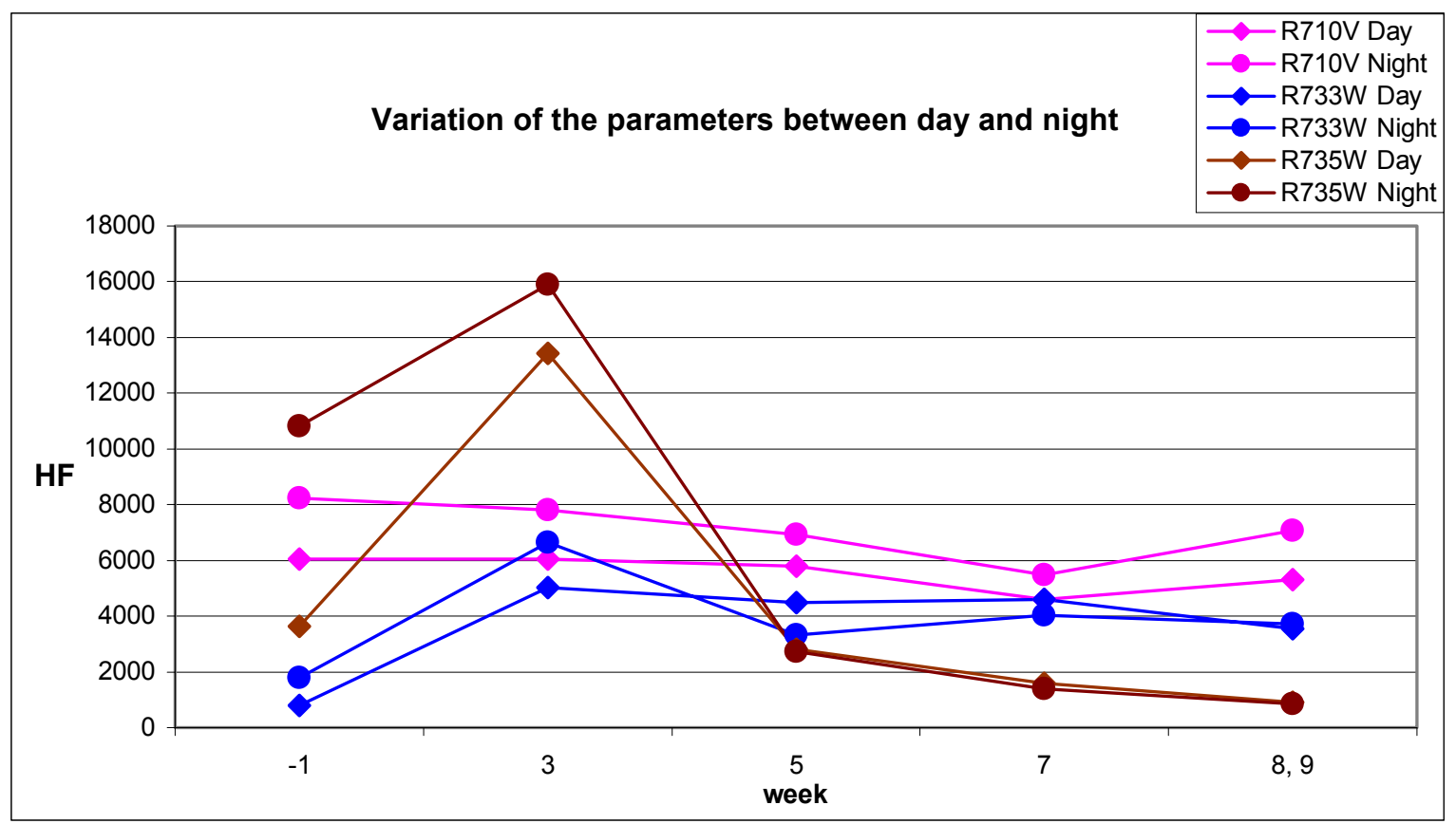

Figure 5.41 Variation of high frequency between day and night

The case of low frequency does not exhibit the common pattern. Instead, in week 7 values for rabbit $\mathrm{R} 733 \mathrm{~W}$ increase over the values of control rabbit.

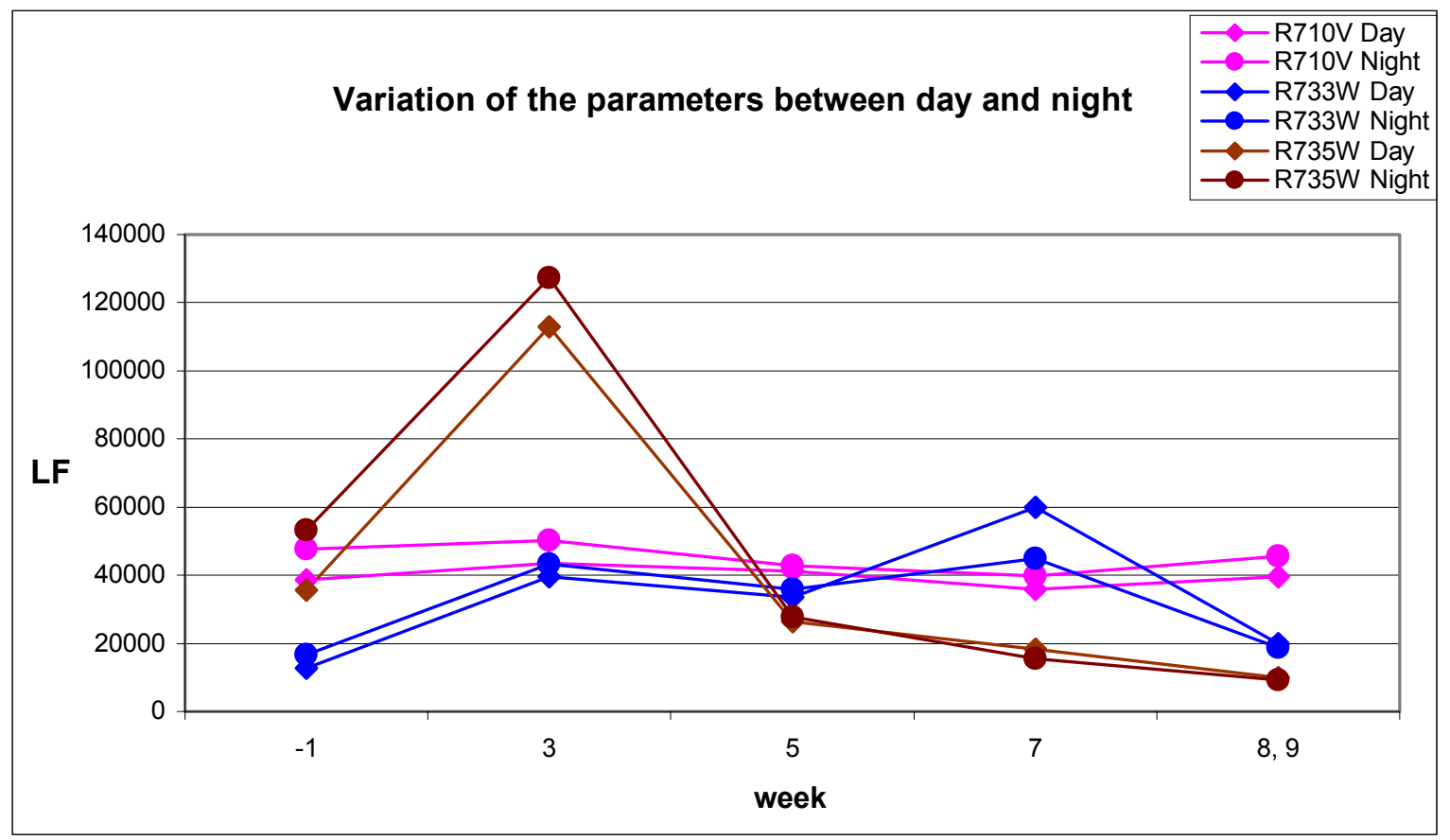

Figure 5.42 Variation of low frequency between day and night 


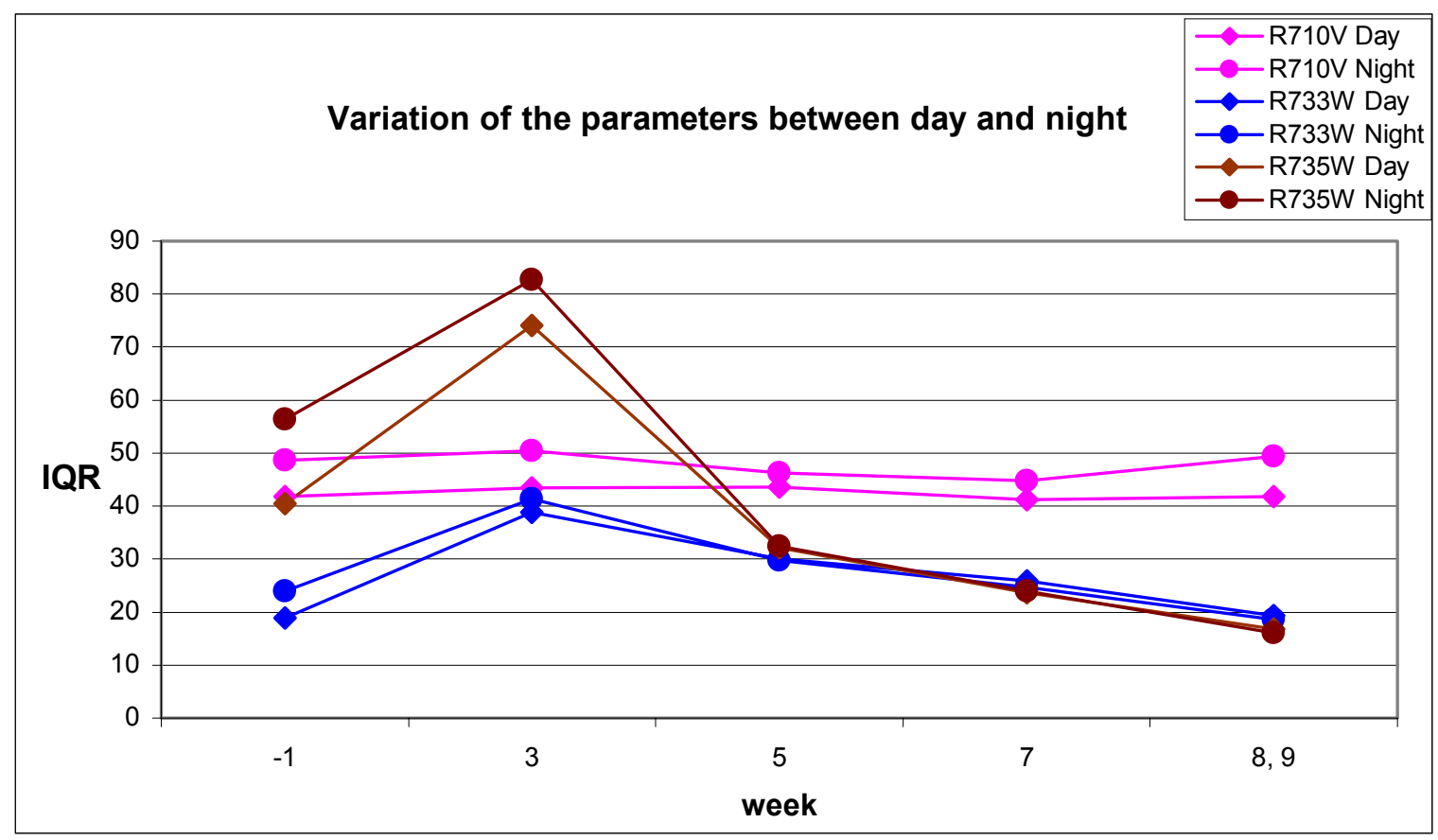

Figure 5.43 Variation of interquartile range of RR intervals between day and night

\subsubsection{Individual plots, one parameter versus another parameter}

Much information can be extracted if one parameter is plotted versus another one. Parameters relevant to HRV are computed for every 5-minute segment ECG and stored in a vector of length 288. One day has 288 5-minute segments. A new vector of length 2016 (288 length of the vector * 7 days) is obtained by appending the values of one parameter, for each day, across one week, for the same rabbit. The parameters are plotted one versus another for the same week and the same rabbit.

This format allows plotting values of every single five-minute segment (as opposed to averaged values of the previous sections). If compared, the plots of median versus mean reveals a different distribution of the points for injected rabbits and for the control rabbit (Figures 5.44, 5.45, and 5.46). For the control rabbit, points for all five weeks are distributed in the same manner. For injected rabbits, the area decreases over the weeks. It should be noted that this plot is expected to be linear since mean and median are estimating similar features. 


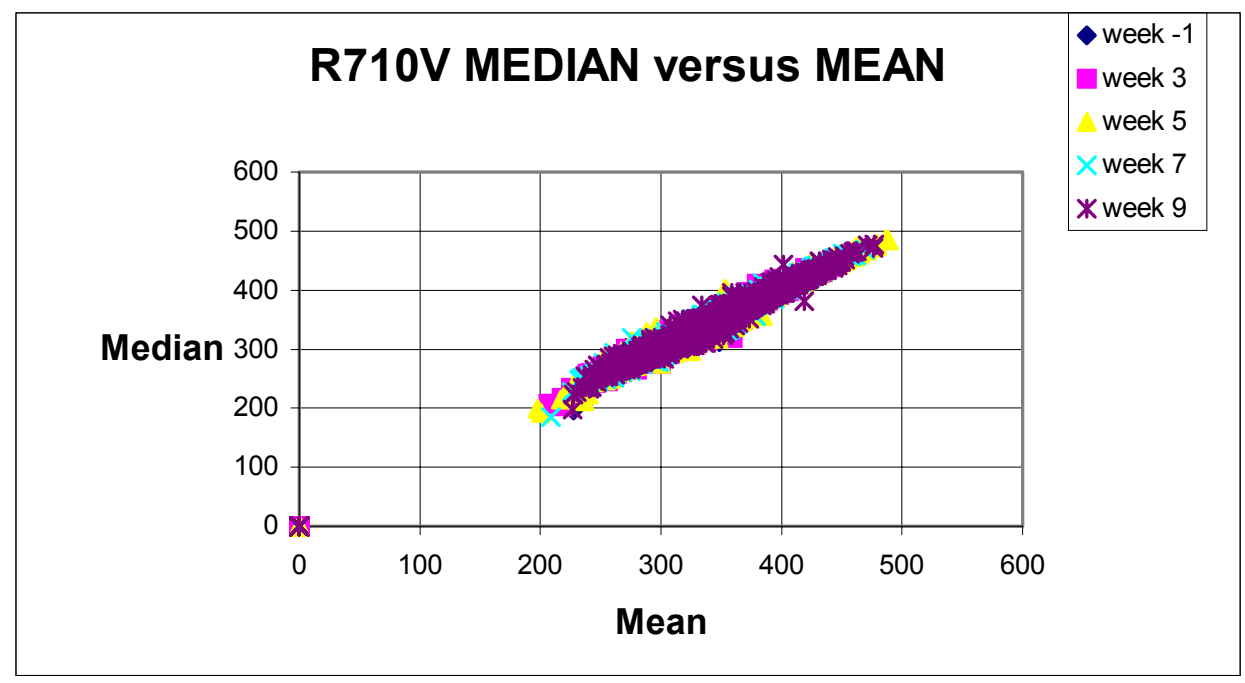

Figure 5.44 Median versus mean, rabbit R710V

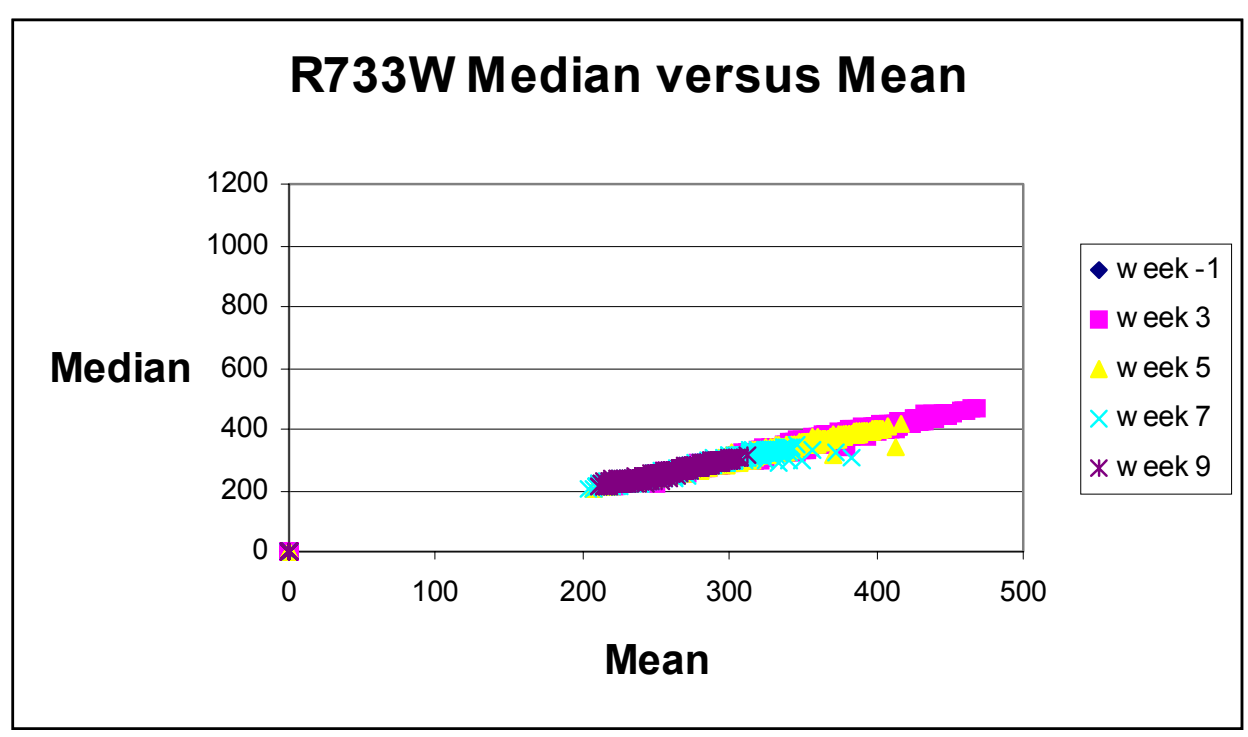

Figure 5.45 Median versus mean, rabbit R733W 


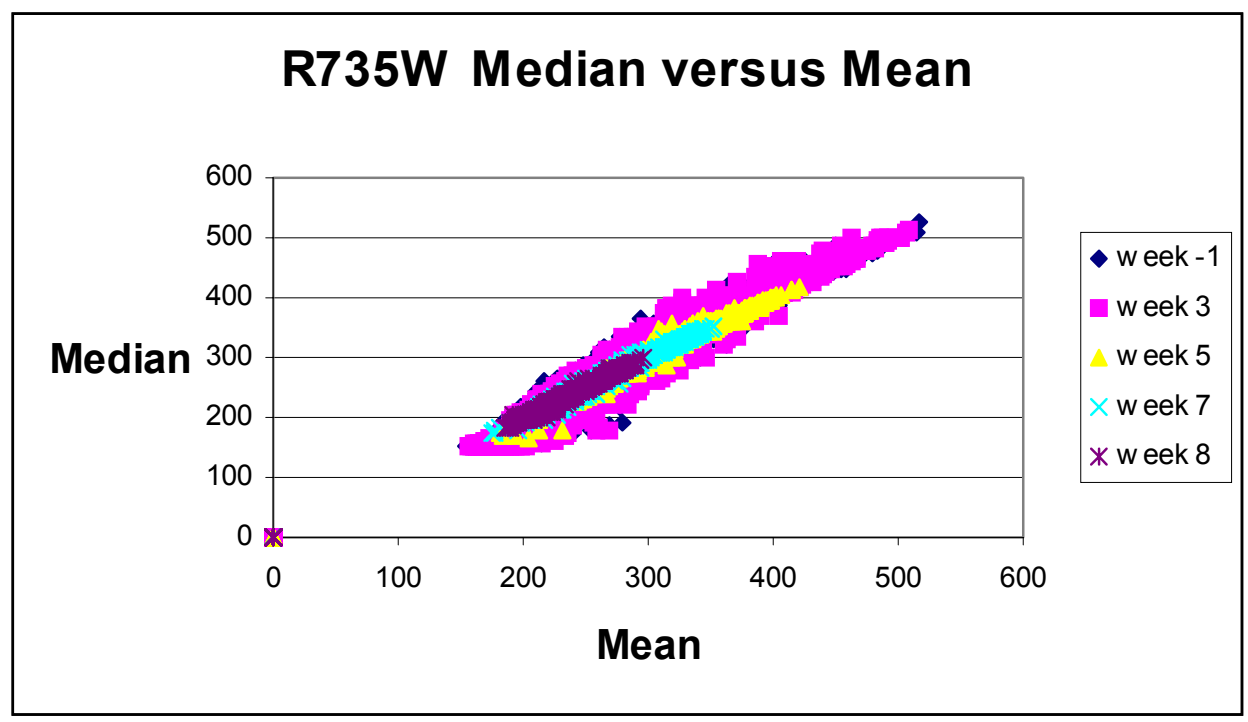

Figure 5.46 Median versus mean, rabbit R735W

The plot of median (MEDIAN) versus mean (MEAN) is relevant for the distribution of the points over the weeks. Other plots are more relevant since each axis contains different information. The following plots are included here (Figures 5.47 through 5.52) and in the Appendix E: interquartile range (IQR) versus mean (MEAN), interquartile range of variation of consecutive RR intervals (DDIQR) versus interquartile range of RR intervals (IQR), and root mean square successive difference (RMSSD) versus coefficient of variance (CV). IQR versus mean and DDIQR versus IQR shows a similar pattern as MEAN / MEDIAN plots when comparing the control rabbit with the injected rabbits.

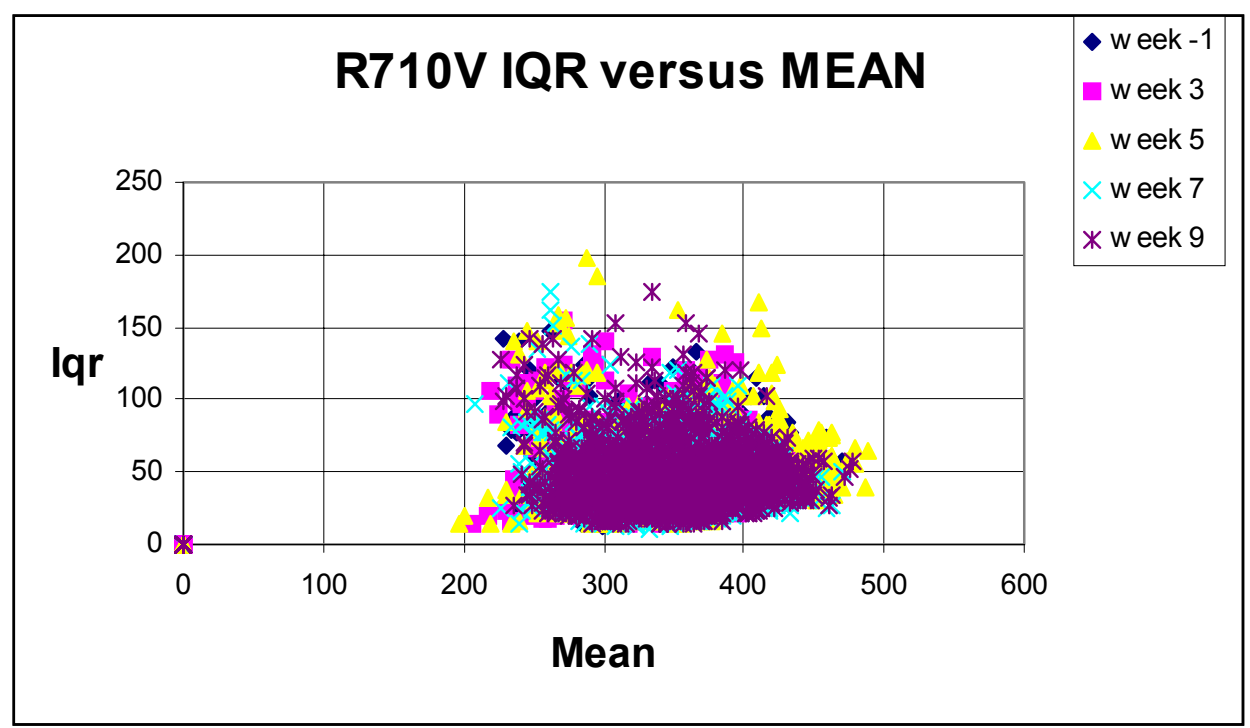

Figure 5.47 Interquartile range of RR intervals versus mean, rabbit R710V 


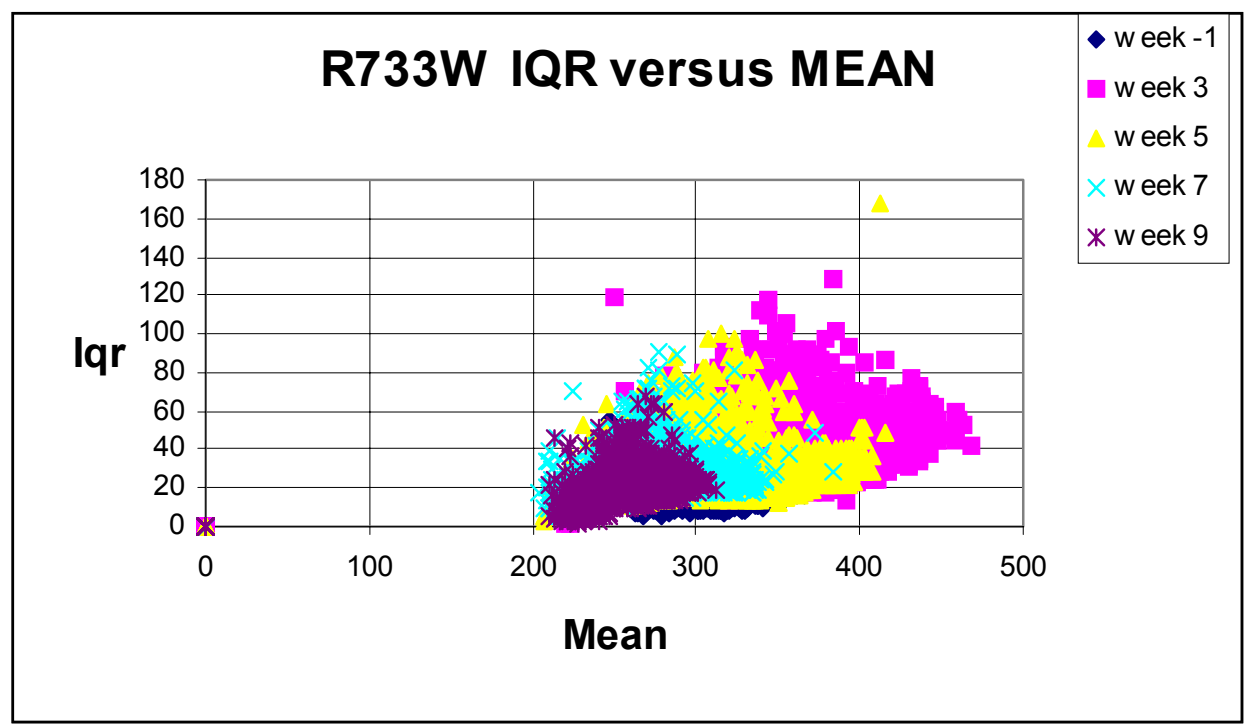

Figure 5.48 Interquartile range of RR intervals versus mean, rabbit R733W

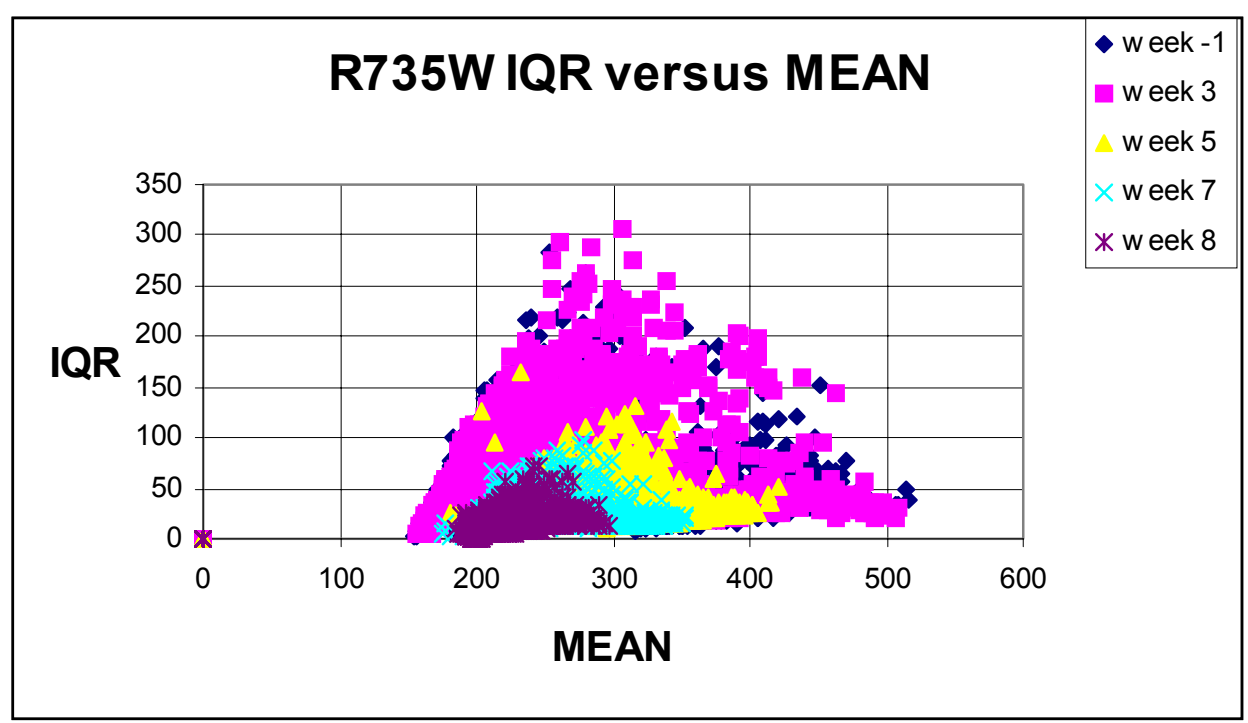

Figure 5.49 Interquartile range of RR intervals versus mean, rabbit R735W

Since this study only had three subjects, it is not possible to quantify these results. With more subjects not only the distribution can be studied, but also the shape and the size of the area, and the position on the graph. 


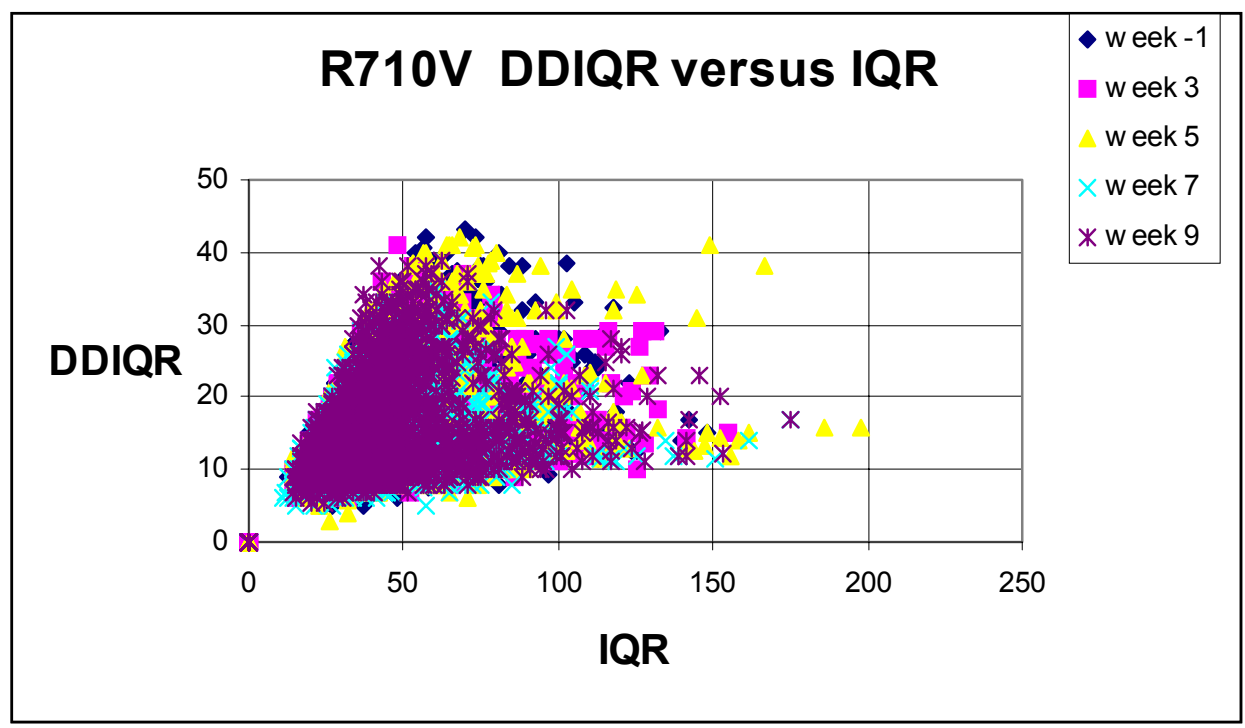

Figure 5.50 Interquartile range of variation of $R R$ intervals versus interquartile range of $R R$ intervals, rabbit $\mathrm{R} 710 \mathrm{~V}$

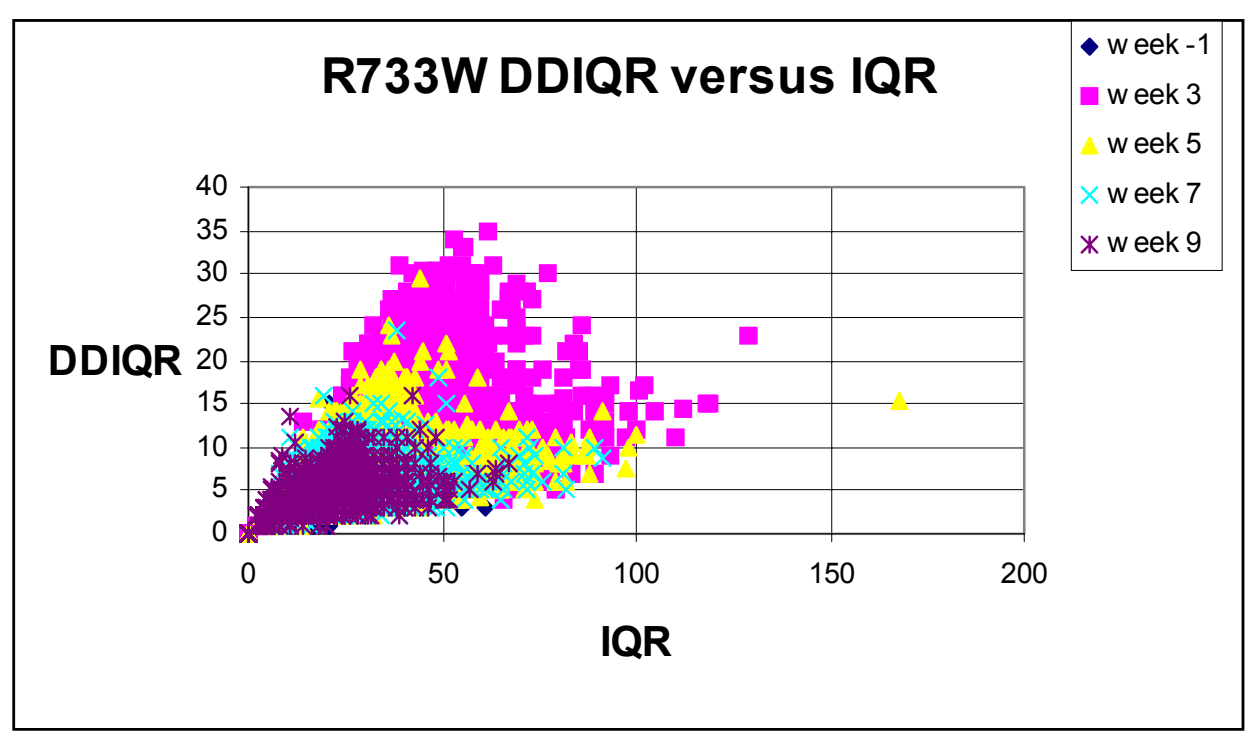

Figure 5.51 Interquartile range of variation of RR intervals versus interquartile range of RR intervals, rabbit R733W 


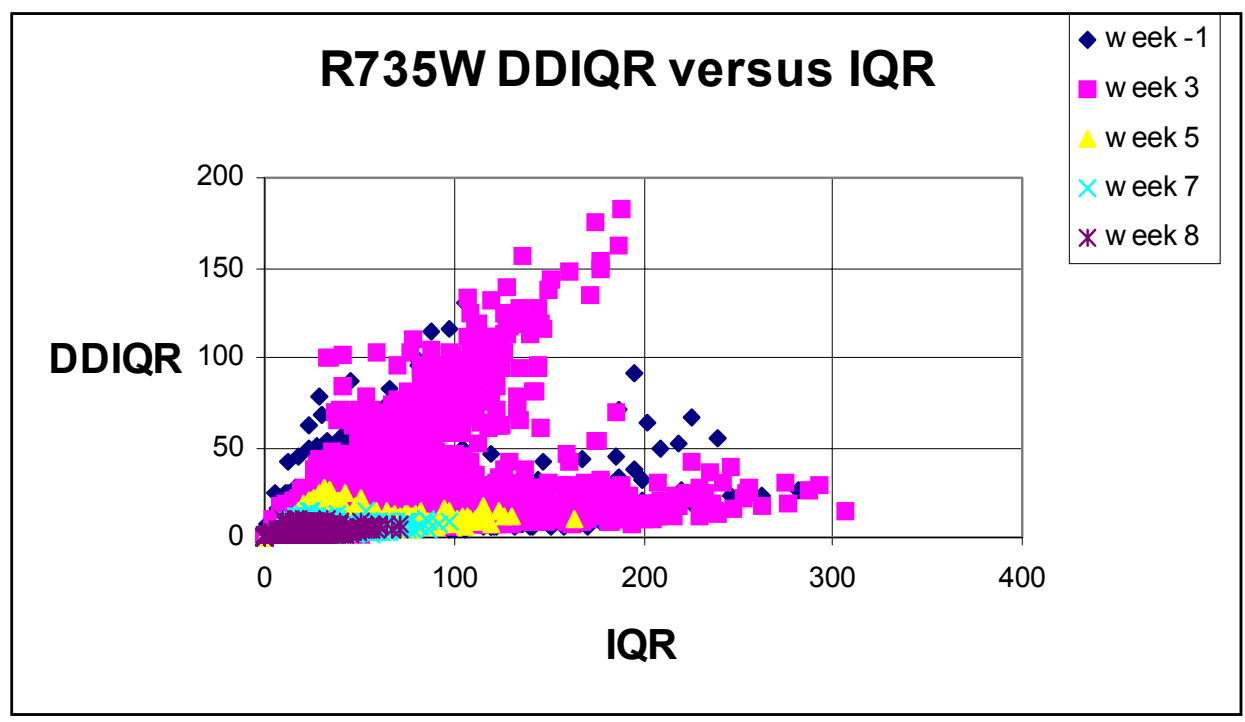

Figure 5.52 Interquartile range of variation of $R R$ intervals versus interquartile range of $R R$ intervals, rabbit $\mathrm{R} 735 \mathrm{~W}$

\subsubsection{Individual plots, all data from five weeks plotted versus hour}

The circadian rhythm of heart rate variability is reflected in the periodicity of the parameters computed. Another way to plot the data, in order to see the periodicity, is to plot parameters versus hour. Parameters relevant to HRV are computed for every five-minute segment ECG and stored each in a vector of length 288. Data is averaged hourly, and vectors of length 24 are obtained for each day. These vectors form a matrix with 24 rows ( 24 hours/day) and 35 columns ( 5 weeks $* 7$ days/week). The first row is plotted versus hour 1 , row 2 is plotted versus hour 2 and a plot with 24 bars is obtained. The plots show peaks and valleys, and the periodicity for the control rabbit is obvious (Figures 5.53 and 5.56). In case of injected rabbits, the data is much more spread on vertical, and periodicity is lost to some extent (Figures 5.54, 5.55, 5.57, and 5.58). Since the data before and after injections are included on the same plot some periodicity can still seen. The plots for all rabbits and all parameters are in Appendix F. 


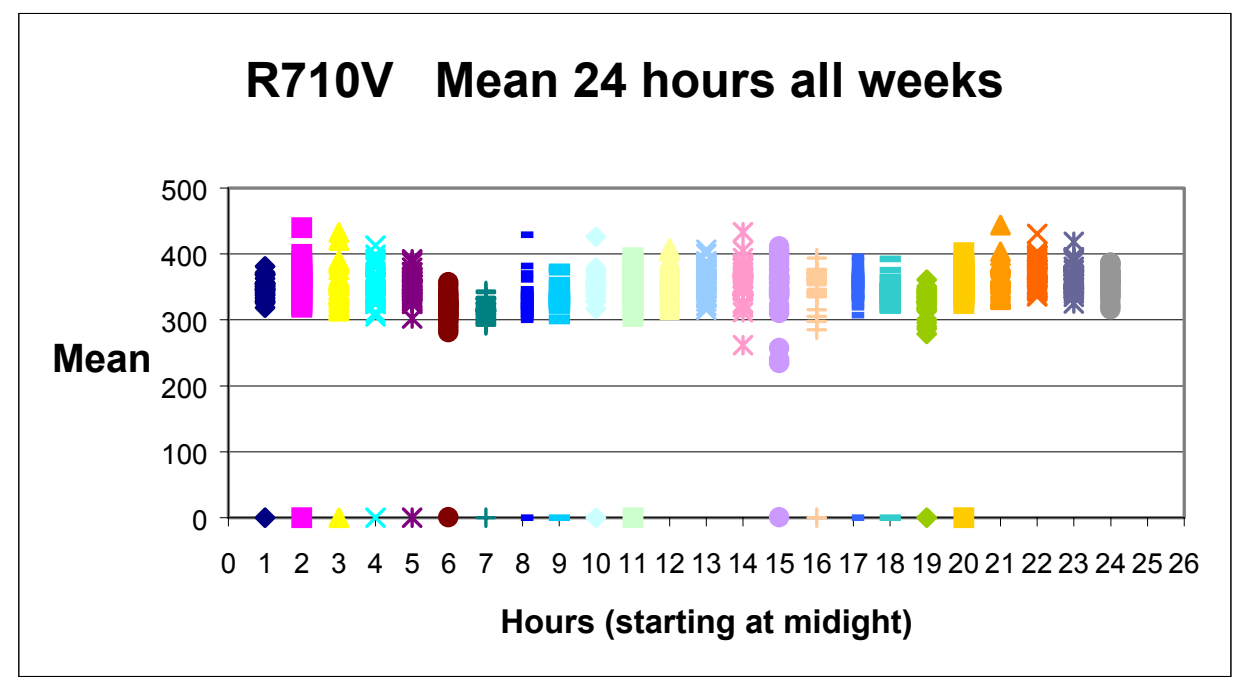

Figure 5.53 Mean, 24 hours all weeks, rabbit R710V

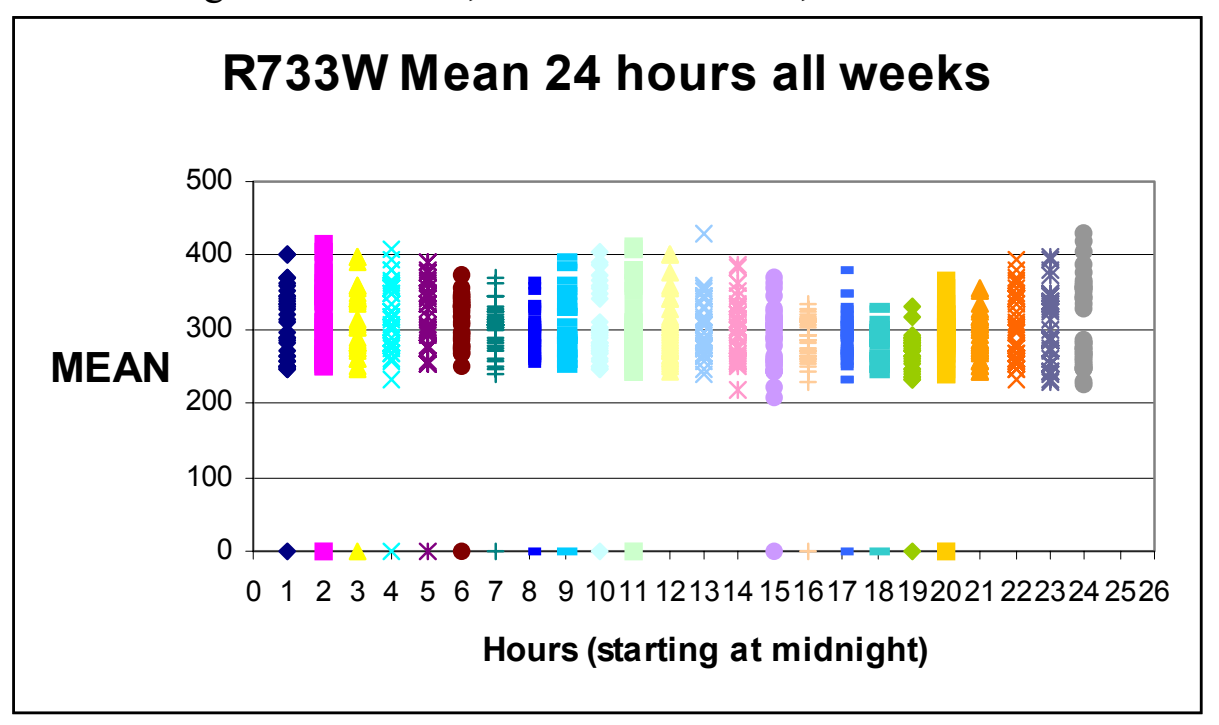

Figure 5.54 Mean, 24 hours all weeks, rabbit R733W 


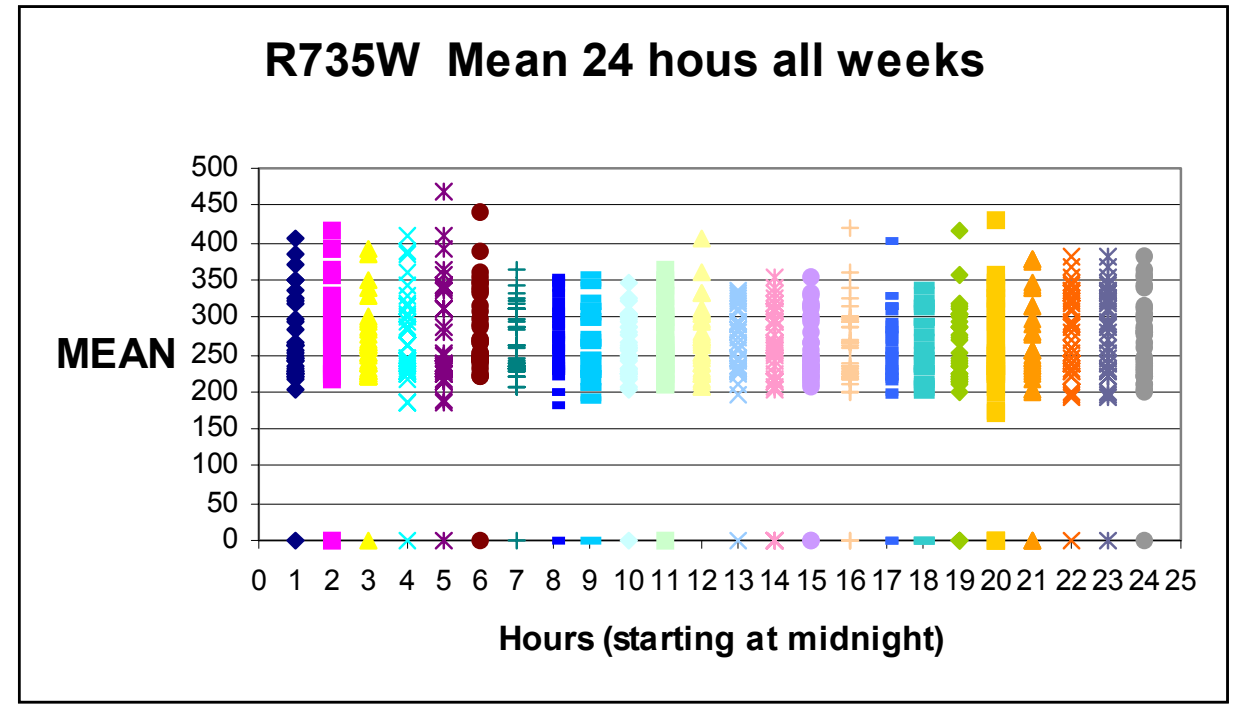

Figure 5.55 Mean, 24 hours all weeks, rabbit R735W

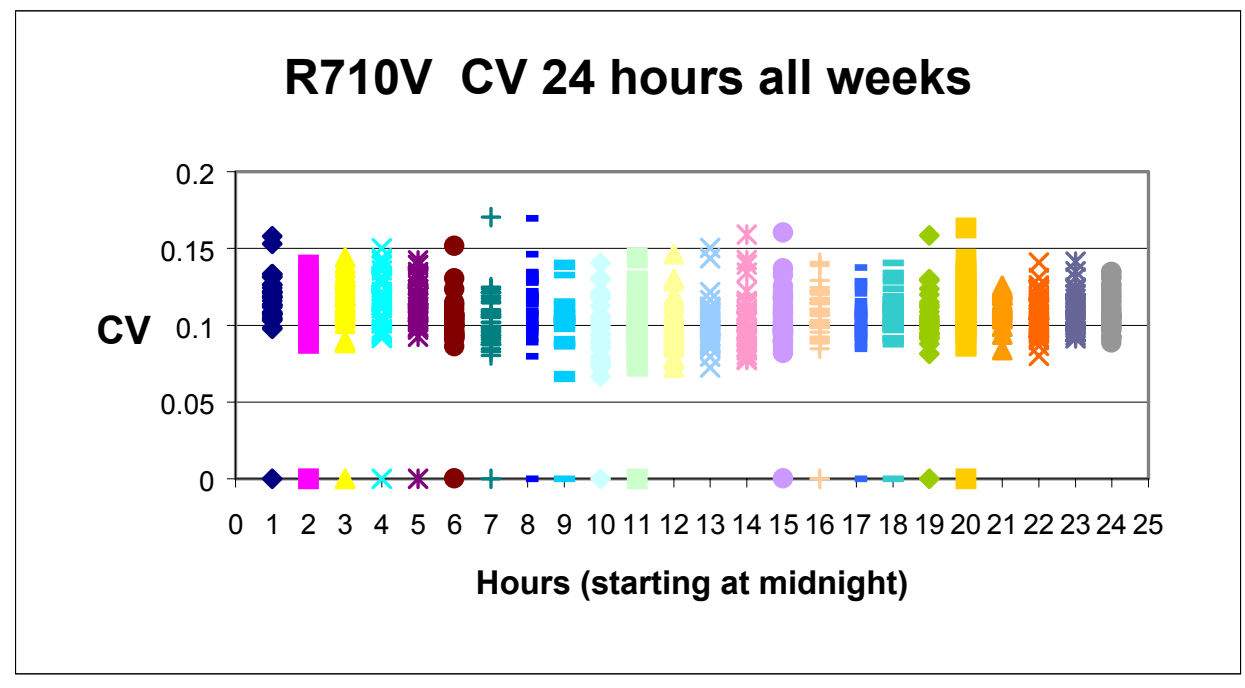

Figure 5.56 Coefficient of variance, 24 hours all weeks, rabbit R710V 


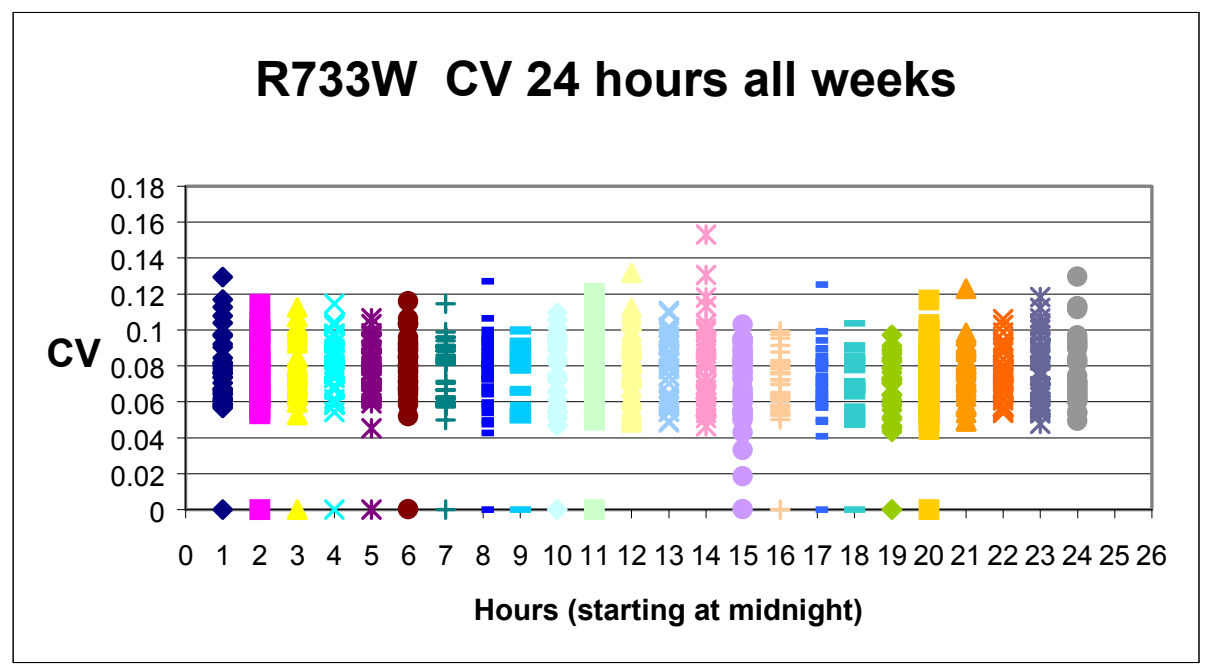

Figure 5.57 Coefficient of variance, 24 hours all weeks, rabbit R733W

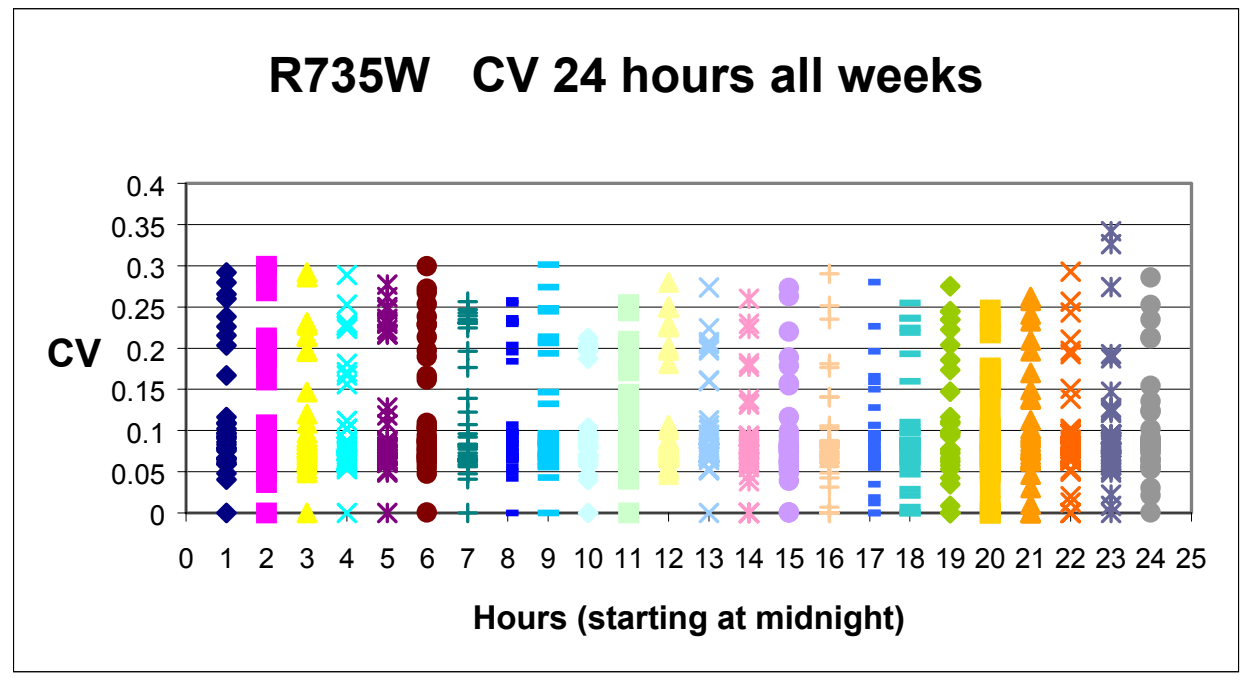

Figure 5.58 Coefficient of variance, 24 hours all weeks, rabbit R735W 


\section{Chapter 6 CONCLUSIONS}

The rapid progress in the computing area allows today a new type of biomedical research based on modern, accurate tools and methods. The significant relationship between the autonomic nervous system and cardiovascular mortality is recognized, and efforts are done to find new ways to break unknown barriers in heart diseases. Heart rate variability is a tool for assessing the integrity of the autonomic nervous system, for describing the interaction between physiological states and autonomic control and the pathophysiology of the heart diseases. This research demonstrates that electrocardiogram can be recorded and changes of ECG features including HRV can be captured, processed, analyzed, and stored for month, even years. Parameters such as mean, median, standard deviation, coefficient of variance and others, which are relevant for heart rate variability are computed and presented in unique ways such that conclusions regarding the variation of heart rate variability, and the progression of heart disease in the experiment can be made. Chapter number five shows the results of the laboratory tests, which demonstrate the heart muscle damage, describes and interprets the electrocardiogram for the injected rabbits. Six types of plots help us to understand the progression of ECG signal from normal state to a diseased state, and shows that heart rate variability is lower in case of heart diseases like myocardial infarction, bradycardia and ventricular bigeminy. The massive amount of data and results can be handled with appropriate methods.

More work must be done for optimal collection, processing, and analysis of the data. In particular, compression schemes need to be explored to minimize the amount of storage space needed. New ways must be found to organize the massive amount of data from continuously recordings and to visualize it in the most significant and suggestive way. Particular suggestions include developing methods using multimedia databases, datamining techniques and sophisticated interfaces. Statistical methods must be applied to quantify the changes of heart rate variability in heart diseases. 


\section{REFERENCES}

1. M. Roelke, J. N. Ruskin, "Dilated Cardiomyopathy: Ventricular Arrhythmias and Sudden Death", Cardiac Electrophysiology: From Cell to Bedside, D. P. Zipes, J. Jalife, eds., Philadelphia, PA:W. B. Saunders Company, 1995, pp. 774-753.

2. L. S. Gettes, W. E. Cascio, W. E. Sanders, "Mechanism of Sudden Cardiac Death", Cardiac Electrophysiology: From Cell to Bedside, D. P. Zipes, J. Jalife, eds., Philadelphia, PA:W. B. Saunders Company, 1995, pp. 527-538.

3. J. E. Poole, G. H. Bardy, "Sudden Cardiac Death", Cardiac Electrophysiology: From Cell to Bedside, D. P. Zipes, J. Jalife, eds., Philadelphia, PA:W. B. Saunders Company, 1995, pp. 812-832.

4. Hon EH, Lee ST. " Electronic evaluations of the fetal heart rate patterns preceding fetal death: further observations", Am J Obstet Gynecol., vol. 87, pp.814-826, 1965

5. Marek Malik, A. John Camm. "Heart rate variability" , Futura Publishing Company.Inc, pp. 449-462, 293-309, 1995

6. Gnag Y, Keeling PJ, Fei L, et al. “ An analysis of heart rate variability in patients with congestive heart failure” , Br Heart J , pp. 69-273, 1993

7. Dadoun M, Morand PG. " Heart rate variability in heart failure as a function of Etiology”, J Heart Failure, pp.1-197, 1993

8. Poole-Wilson PA. "Future perspectives in the management of congestive heart failure", Am J Cardiol., vol.66, pp.462-467, 1990

9. Casolo GC,Fazi A.,Boddi M. “ Twenty-four heart rate behavior in patients with impaired left ventricular function due to coronary artery disease”, Cardiology, vol.74, pp.116-123, 1987

10. Zsolt Ori, George Monir, Jerry Weiss, Xavier Sayhouni, Donald H. Singer. " Heart rate variability. Frequency Domain Analysis”, Cardiology Clinics, vol.10, pp.449-533, 1992

11. Martin GJ, Magid MN,Valentini V, et al. " Heart rate variability and the evaluation of risk of sudden cardiac death”, Clin Res, pp.35-302A, 1987 
12. Martin GJ, Magid NM, Myers G, et al. "Heart rate variability and sudden death secondary to coronary artery disease during ambulatory ECG monitoring", Am J Cardiol, vol.60, pp.86-89, 1987

13. Myers GA,Martin GJ, Magid NM, et al. "Power spectral analysis of heart rate variability in sudden cardiac death. Comparison to other methods", IEEE Trans Biomed Eng, vol.33, pp. 1149-1156, 1986

14. Kiowski W, Osswald S. "Circadian variation of ischemic cardiac events" , J Cardivasc Pharmacol, vol.21, pp.45-48, 1993

15. Mulcahy D., Cunningham D., Crean P., et al. " Circadian variation of the total ischemic buden and its alteration with anti- anginal agents" .

16. Caloso G, Balli E, Fazi A, et al. "Twenty- four hour spectral analysis of heart rate variability in congestive heart failure secondary to coronary artery disease" , Am J Cardiol, vol.67, pp.1154-1158, 1991

17. Gang Yi, Xiao-Hua Guo, Michael Reardon, et al. " Circadian variation of the QT interval in patients with sudden cardiac death after myocardial infarction" , Am J Cardiol, vol.81, pp.950-955, 1998

18. "Heart Rate Variability. Standards of Measurement, Physiological Interpretation, and Clinical Use. Special Report”, Circulation, vol.93, Nr.5, March 1, pp.1043-1065, 1996

19. David S. Jacobs, MD, Wayne R. De Mott, MD, Harold J. Grady, PhD, et al." Laboratory Test Handbook, CONCISE with disease index", Lexi-Comp' s Clinical Reference Library.

20. Lauralee Sherwood. "Fundamentals of physiology. A human perspective", second edition. Chapter 7, pp. 204-236.

21. Joel G.Hardman, Lee E.Limbird, et al. "The pharmacological Basis of Therapeutics", ninth edition. Goodman \& Gilman’ s, chapter 51, pp1265-1266.

22. Dale Dubin, MD. " Rapid Interpretation of EKG' s", COVER Publishing Company, Edition V, pp. 63-92, 245-258.

23. S. Yakubo, Y. Ozawa, K. Komaki, “ Intra-QRS High-frequency ECG Changes with Ischemia: Is It Possible to Evaluate These Changes Using the Signal-averaged Holter ECG in Dogs?” J of Electrocardiology, vol. 28 (suppl), pp. 234-238, 1995.

24. A. Nogami, K. Taniguchi, J. Takeuchi, "Serial Analysis of Spontaneous and Induced Ventricular Arrhythmias in a Canine Model of Myocardial Infarction," Japan Heart J, vol. 29, pp. 437-453, 1988. 
25. C. F. Opitz, G. F. Mitchell, M. A. Pfeffer, J. M. Pfeffer, “Arrhythmias and Death After Coronary Artery Occlusion in the Rat: Continuous Telemetric ECG Monitoring in Conscious, Untethered Rats," Circulation, vol. 92, pp. 253-261, 1995.

26. F. Carre, Y. Lessard, P. Coumel, L. Ollivier, S. Besse, Y. Lecarpentier, B. Swynghedauw, "Spontaneous Arrhythmias in Various Models of Cardiac Hypertrophy and Senescence of Rats. A Holter Monitoring Study," Cardiovascular Research, vol. 26, pp. 698-705, 1992.

27. John D. Doherty, Stuart M.Cobbe. "Electrophysiological changes in animal model of chronic cardiac failure”, Cardiovascular Research, vol.24, pp.309-316,1990.

28. A. Strashun, "Adriamycin, Congestive Cardiomyoathy, and Metaiodobenylguanidine," $J$ Nuclear Medicine, vol. 33, pp. 215-222, 1992.

29. A. Dindogru, M. Barcos, E. S. Henderson, H. J. Wallace, "Electrocardiographic Changes following Adriamycin Treatment," Medical Pediatric Oncology, vol. 5, pp. 65-71, 1978.

30. D. D. Von Hoff, M. Rozencweig, M. Layard, M. Slavik, F. M. Muggia, "DaunomycinInduced Cardiotoxicity in Children and Adults," Am J Medicine, vol. 62, pp. 200-208, 1977.

31. R. D. Couch, K. K. Loh, J. Sugino, " Sudden Cardiac Death Following Adriamycin Therapy," Cancer, vol. 48, pp. 38-39, 1981.

32. D. Langton, B. Jover, B.P. McGrath, J. Ludbrook. “ Cardiovascular responses to graded treadmill exercise, serially during the development of congestive heart failure", Cardiovascular Research, vol. 24, pp.959-968, 1990.

33. S. Wakasugi, A. J. Fischman, J. W. Babich, R. J. Callahan, D. R. Elmaleh, R. Wilkinson, H. W. Strauss, "Myocardial Substrate Utilization and Left Ventricular Function in Adriamycin Cardiomyopathy," J Nucl Med, vol. 34, pp. 1529-1535, 1993.

34. A. Calderone, J. de Champlain, J. L. Rouleau, "Adriamycin-Induced Changes to the Myocardial Beta-Adrenergic system in the Rabbit," J Mol Cell Cardiol, vol. 23, pp. 333$342,1991$.

35. H. Shenasa, A. Calderone, M. Vermeulen, P. Paradis, H. Stephens, R. Cardinal, J. de Champlain, J. L. rouleau, "Chronic Doxorubicin Induced Cardiomyopathy in Rabbits: Mechanical, Intracellular Action Potential, and Beta-Adrenergic Characteristics of the Failing Myocardium," Cardiovascular Research, vol. 24, pp. 591-604, 1990.

36. W. Cheng, A. G. Justicz, M. S. Soberman, N. P. alazraki, W. P. Santamore, J. D. Sink, "Effects of Dynamic Cardiomyoplasty on Indices of Left Ventricular Systolic and Diastolic Function in a Canine Model of Chronic Heart Failure," J Thorac Cardiovasc Surg, vol. 103, pp. 1207-13, 1992. 
37. E.J. Kelso, r. F. Geraghty, B. J. McDermott, C. H.S. Cameron, D. P. Nicholls, B. Silke, "Characterization of A Cellular Model of Cardiomyopathy, in the Rabbit, Produced by Chronic Administration of the Anthracycline, Epirubicin," J Mol Cell Cardiol, vol.29, pp. 3385-3397, 1997.

38. Heikki V. Huikuri, MD, Kenneth M.Kessler, MD, et al. " Reproducibility and circadian rhythm of heart rate variability in healthy subjects" , Am. Journal of Cardiology, vol. 65, pp.391-393, feb. 1990

39. Joao Freitas, MD, Pedro Lago, $\mathrm{PhD}$, et al. "Circadian heart rate variability rhythm in shift workers", Journal of electrocardiology, vol.30, nr.1, pp.39-44, 1997.

40. Jumichiro Hayano, MD, Wei Jiang, MD, Robert Waugh, MD, et al. " Stability over time of circadian rhythm of variability of heart rate in patients with stable coronary artery disease" , American Heart Journal, vol.134, Nr.3, pp.411-418.

41. Robert W.Peters, MD, Steve McQuillan, BS, et al. "Interaction of septadian and circadian rhythm in life-threatening ventricular arrhythmias in patients with implantable cardioverter defibrillators", American Journal of Cardiology, vol.84, pp.555-557, sept.1999.

42. H.R.Arntz, MD, S.N.Willich, MD, et al. "Circadian Variation of sudden cardiac death reflects age-related variability in ventricular fibrillation", Circulation, vol.88, Nr.5, pp.2284-2289, nov.1993.

43. Maarten van den Buuse and Simon C. Malpas. " 24-hour recordings of blood pressure, heart rate and behavioral activity in rabbits by radio-telemetry: effects of feeding and hypertension" , Physiology and behavior, vol.62, Nr.1, pp.83-89, 1997.

44. Keiichiro Sato, Fumio Chatani, Shuzo Sato. "Circadian and short-term variabilities in blood pressure and heart rate measured by telemetry in rabbits and rats", Journal of autonomic nervous system, Nr.54, pp.235-246, 1995.

45. V.Eijzenbach, J.H.Sneek and C.Borst. "Arterial pressure and heart period in the conscious rabbit: diurnal rhythm and influence of activity" , Clinical and experimental Pharmacology and Physiology, vol.13, pp.585-592, 1986.

46. E.M. Vaughan Williams, P.D.Dennis, C. Garnham. " Circadian rhythm of heart rate in the rabbit: Prolongation of action potential duration by sustained beta adrenoceptor blockade is not due to associated bradycardia", Cardiovascular research, vol.20, pp.528-535, 1986. 
47. Viatcheslav A. Moguilevski, Louise Shiel, Judith Oliver, Barry P.McGrath. " Power spectral analysis of heart rate variability reflects the level of cardiac autonomic activity in rabbits", Journal of autonomic nervous system, Nr.58, pp.18-24, 1996.

48. Robert E. Kleiger, MD, FACC, Phyllis K.Stein, PhD, et al. " Time domain measurements of heart rate variability” , Cardiology Clinics, vol.10, Nr.3, pp. 487-498 
Appendix A Individual plots, from averaged data, hourly and weekly 


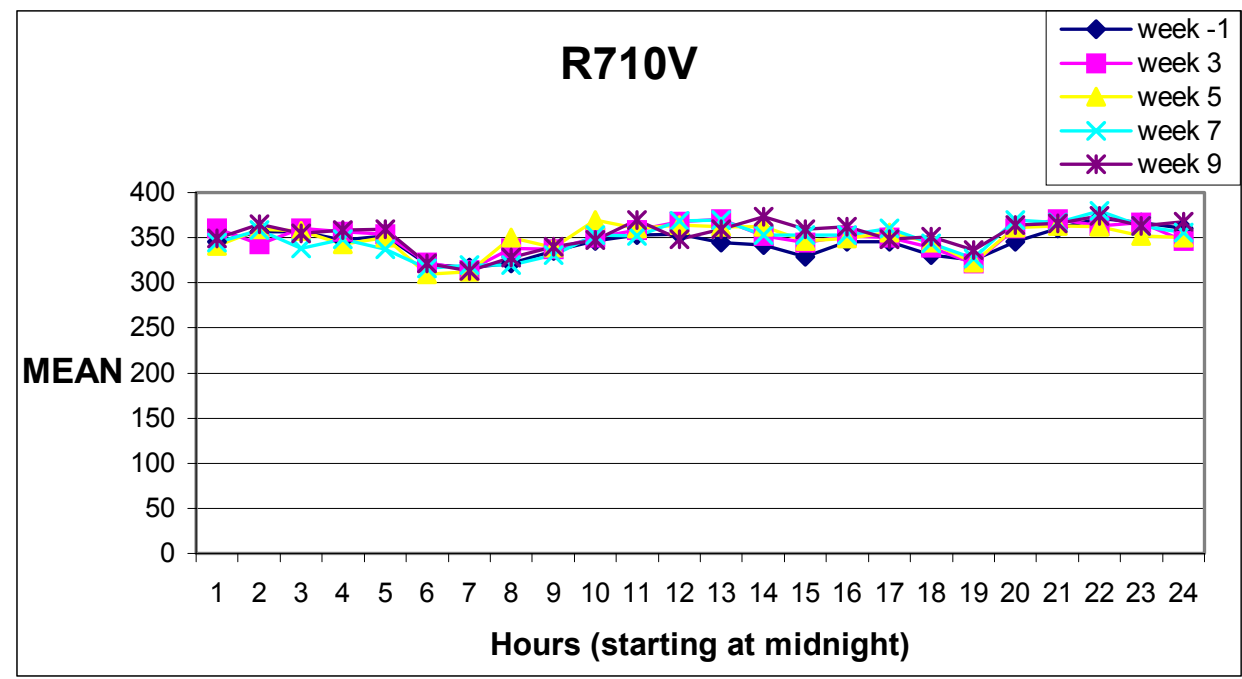

Figure A.1 MEAN R710V

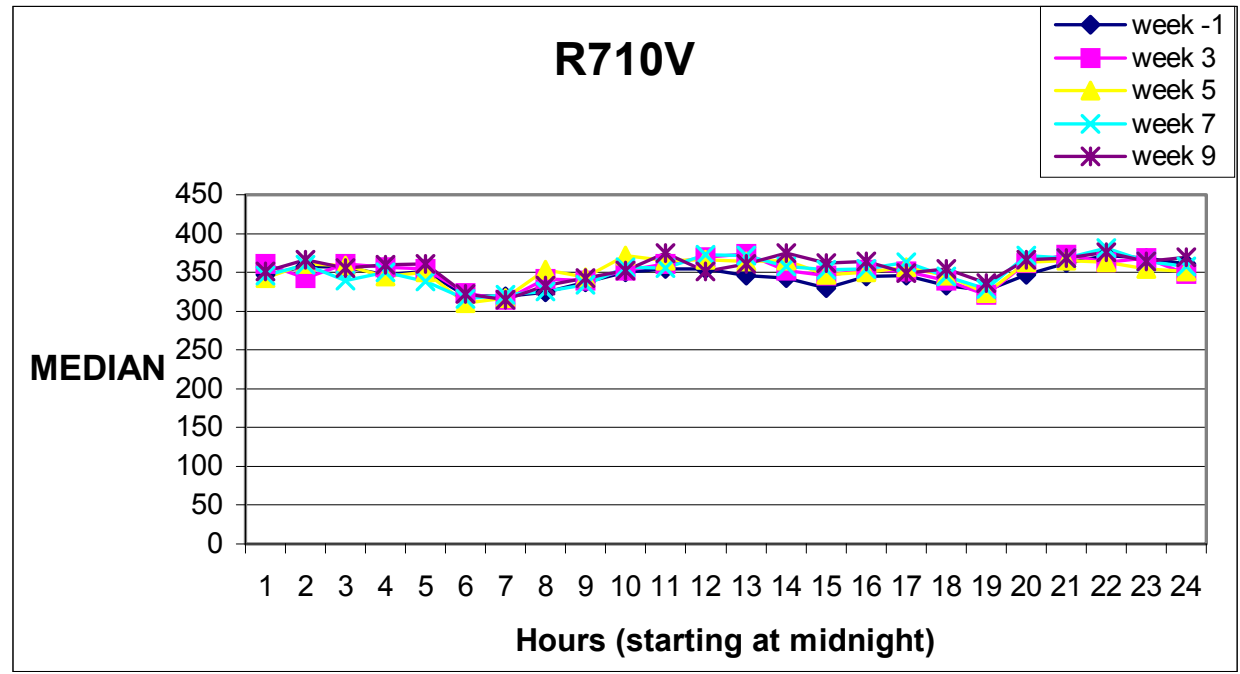

Figure A.2 MEDIAN R710V

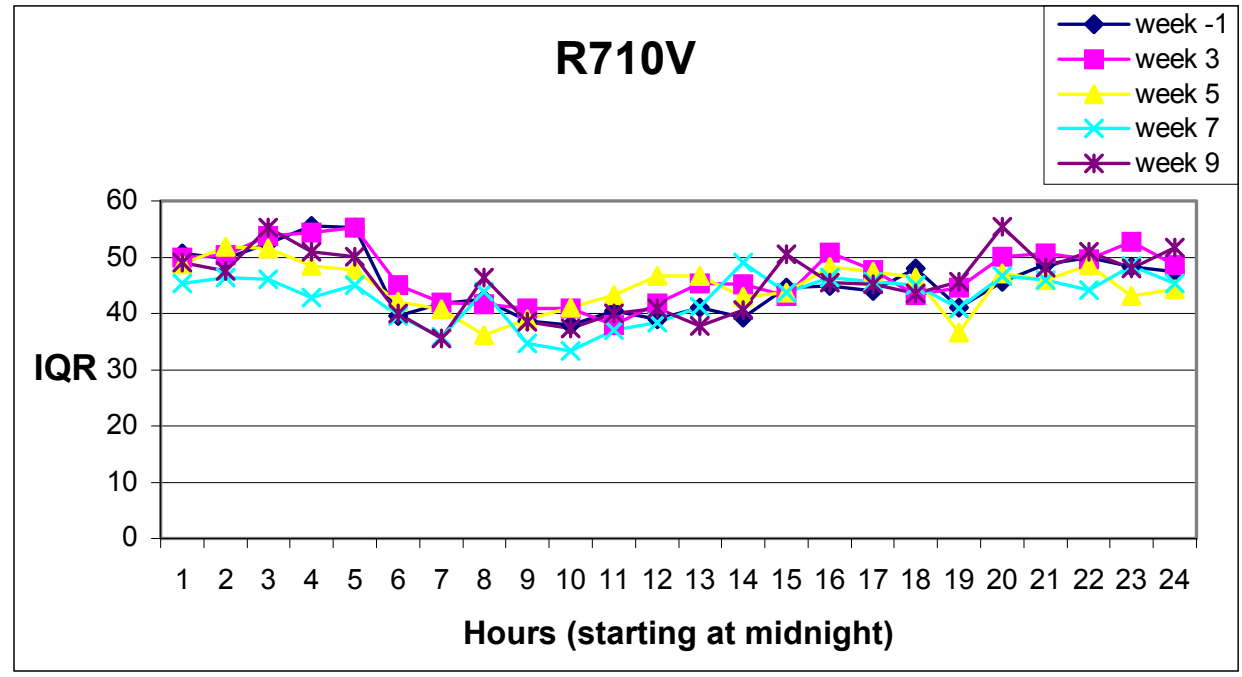

Figure A.3 IQR R710V 


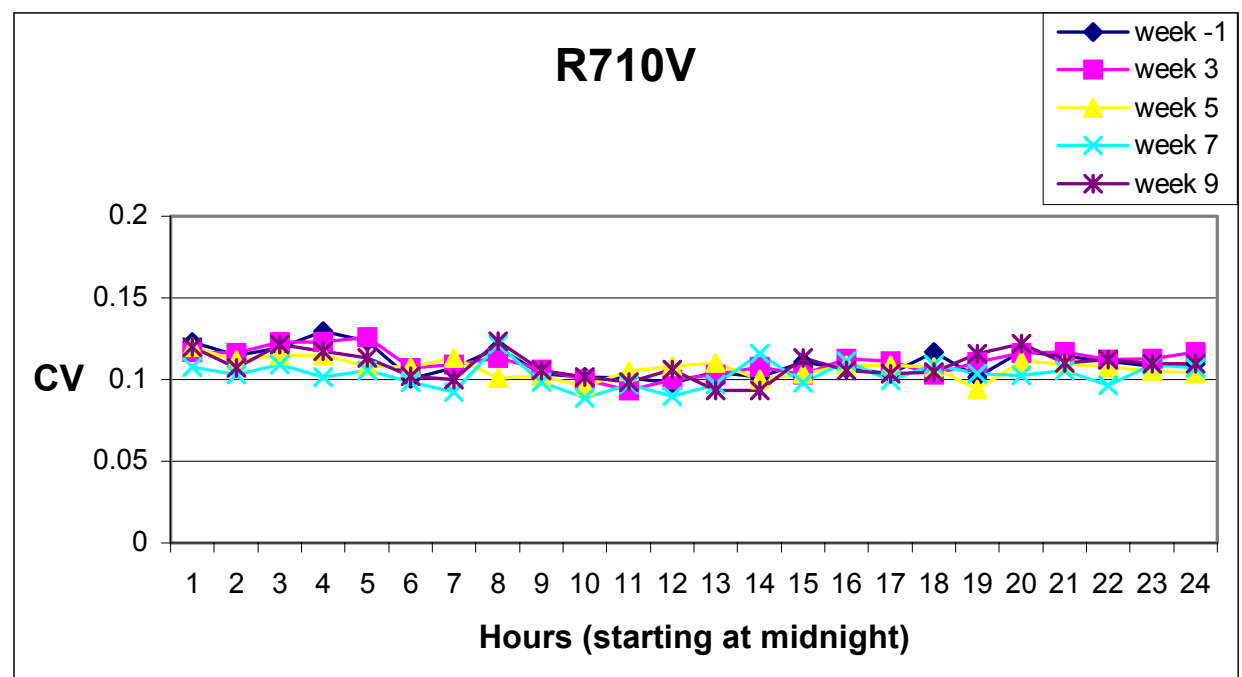

Figure A.4 CV R710V

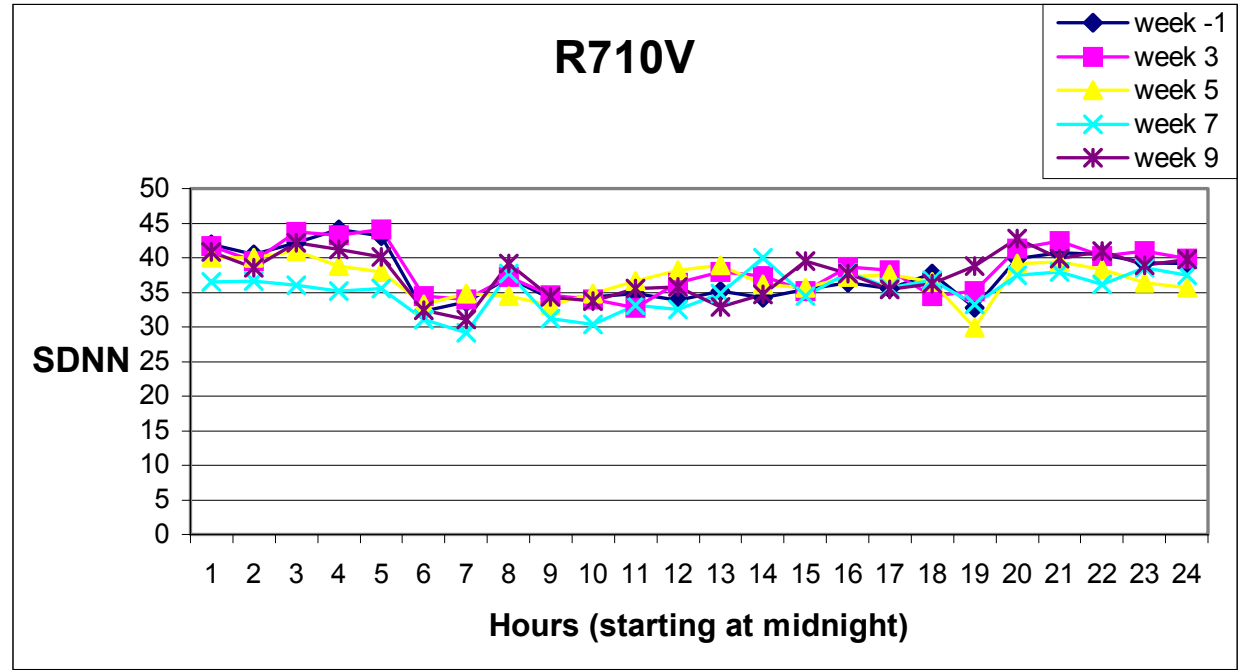

Figure A.5 SDNN R710V

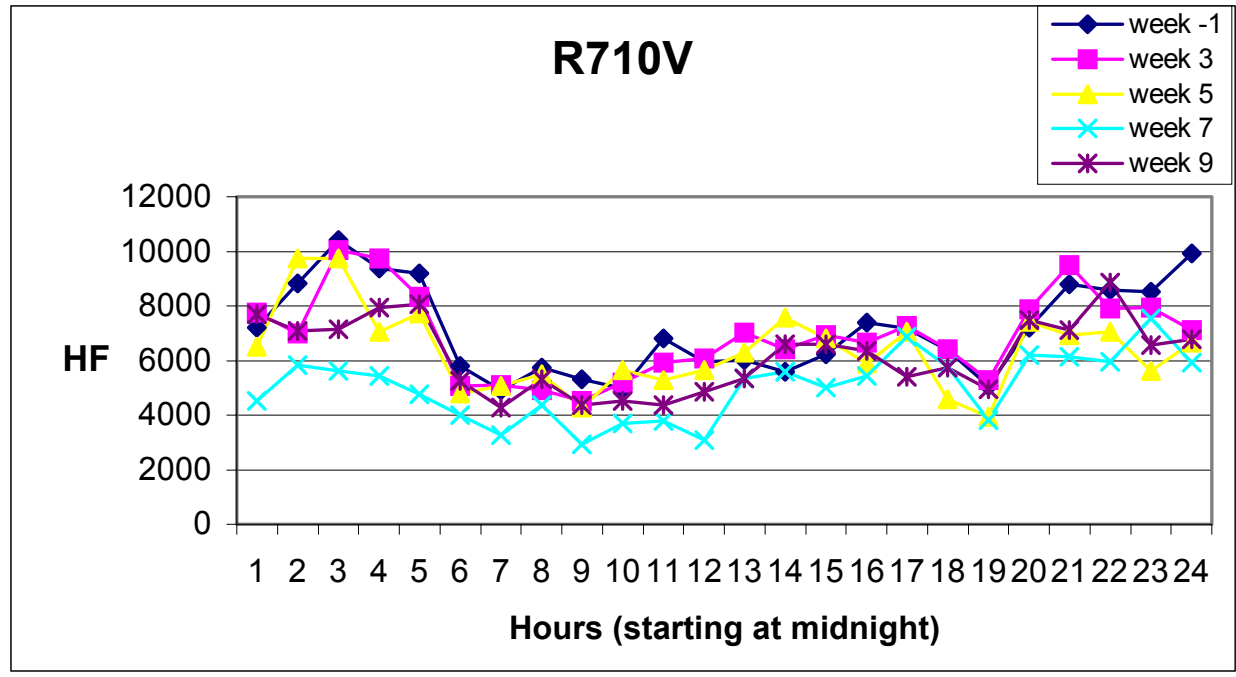

Figure A.6 HF R710V 


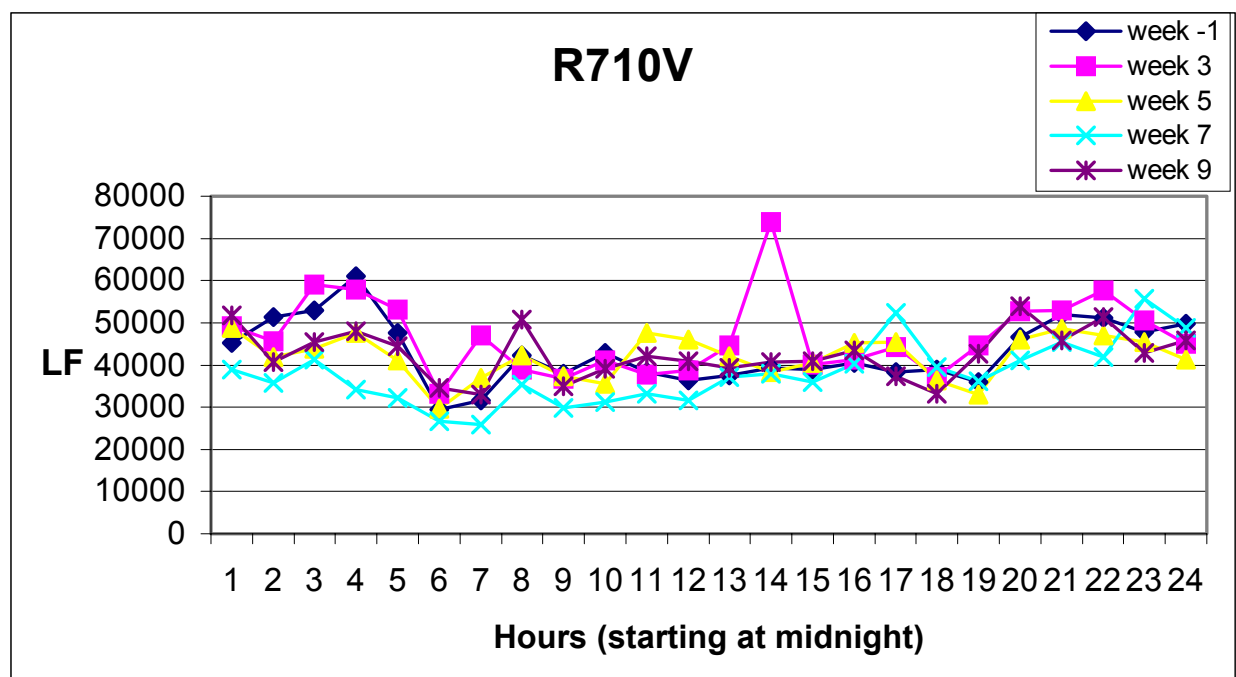

Figure A.7 LF R710V

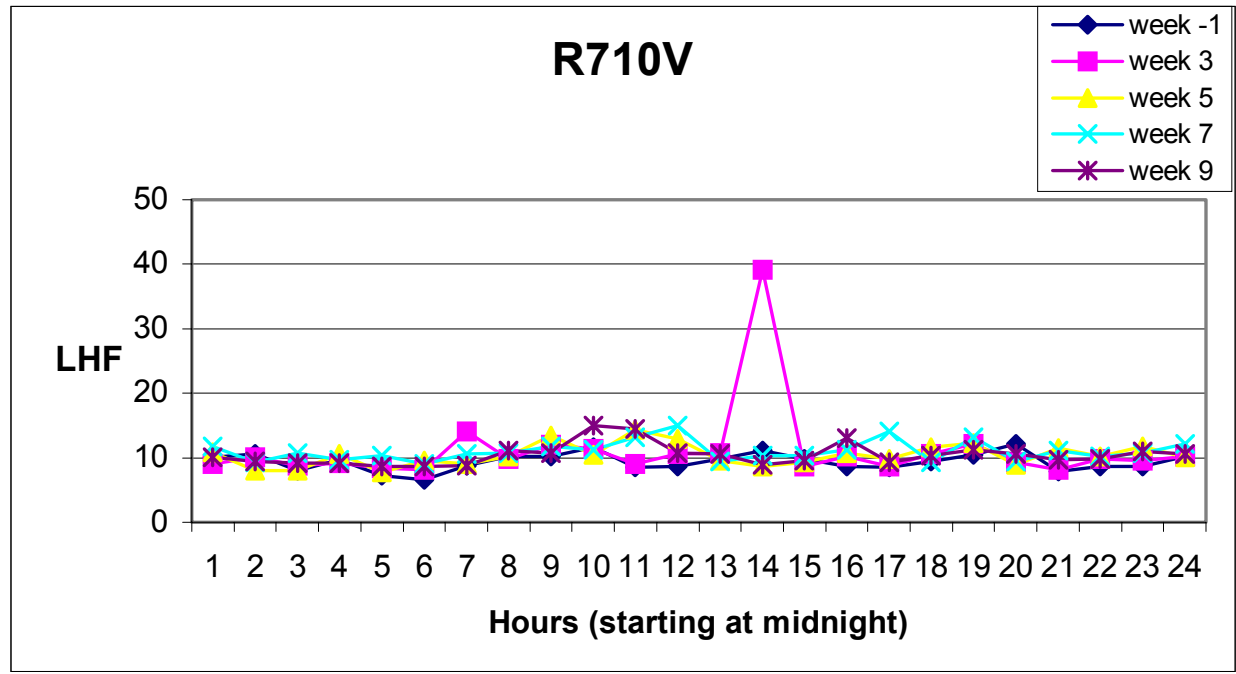

Figure A.8 LHF R710V

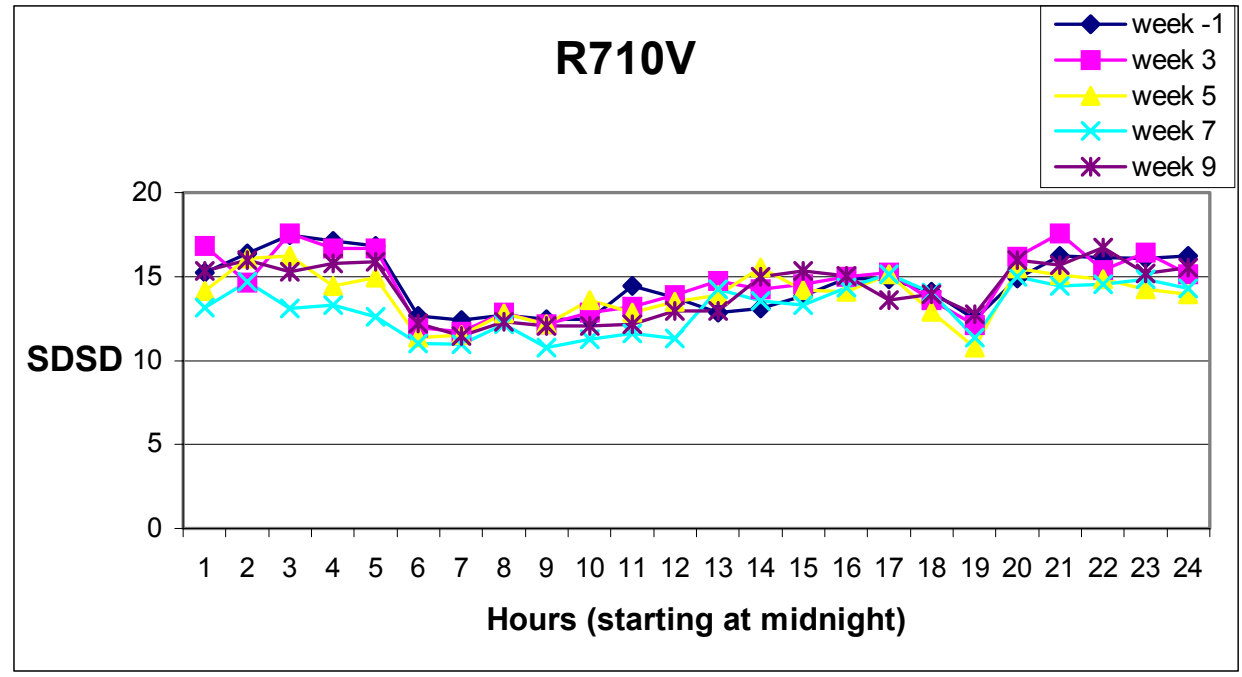

Figure A.9 SDSD R710V 


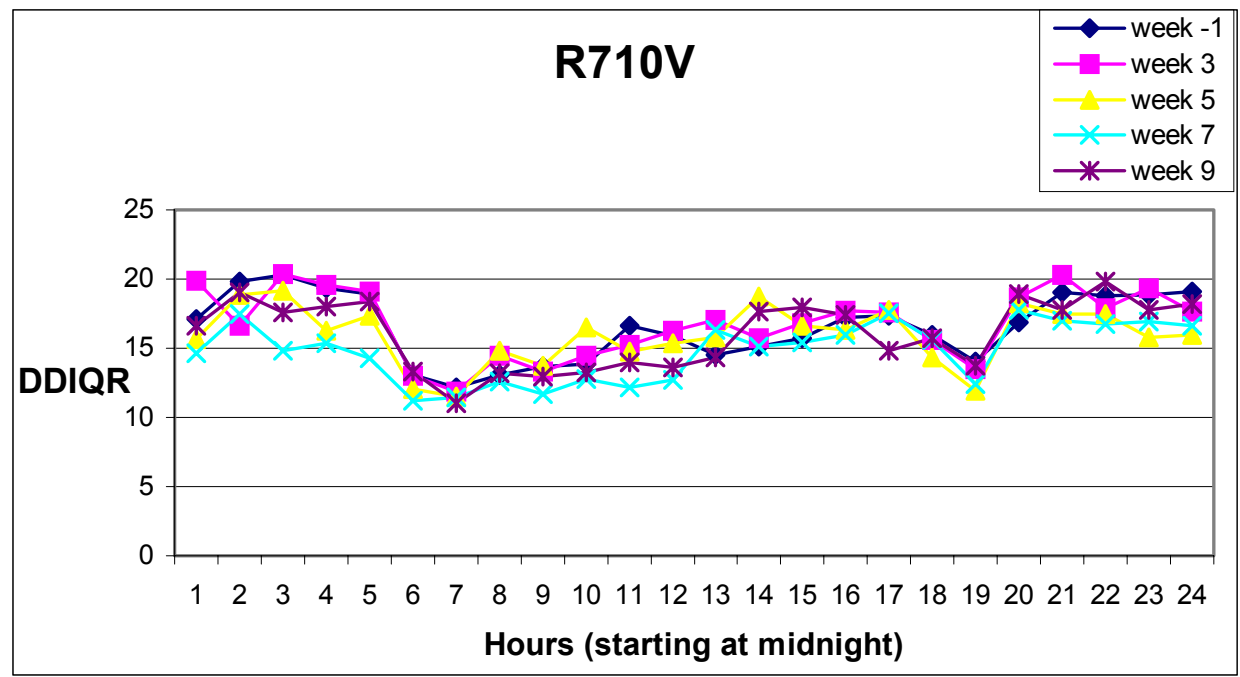

Figure A.10 DDIQR R710V

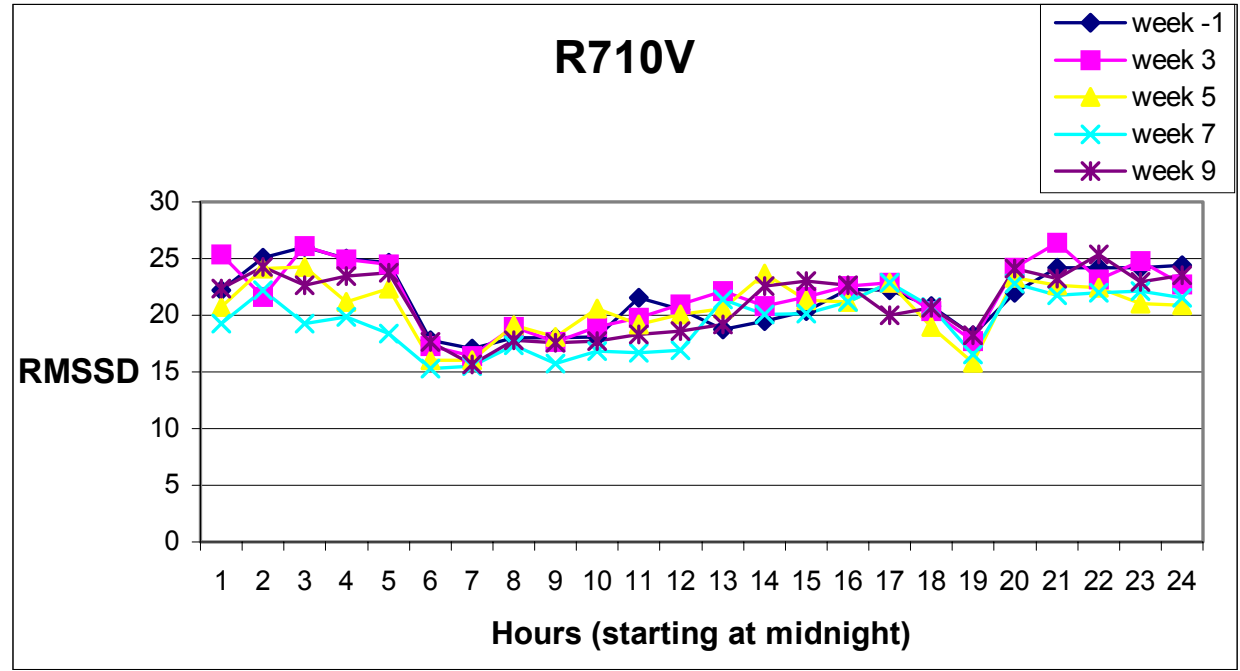

Figure A.11 RMSSD R710V

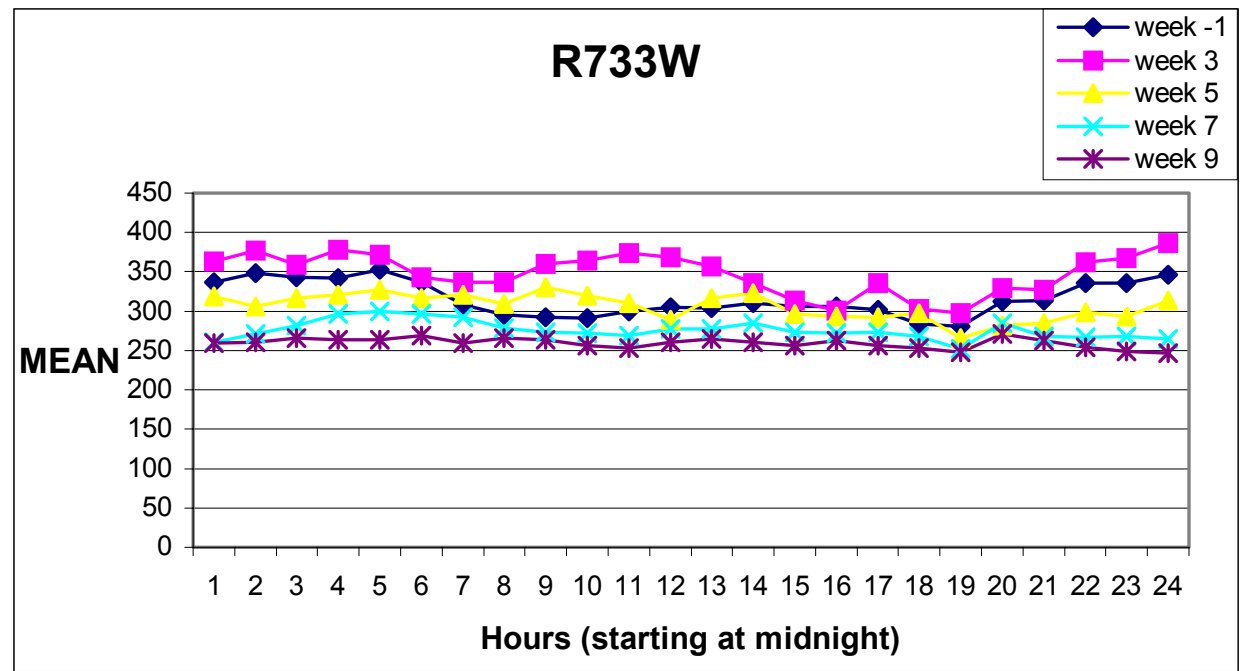

Figure A.12 MEAN R733W 


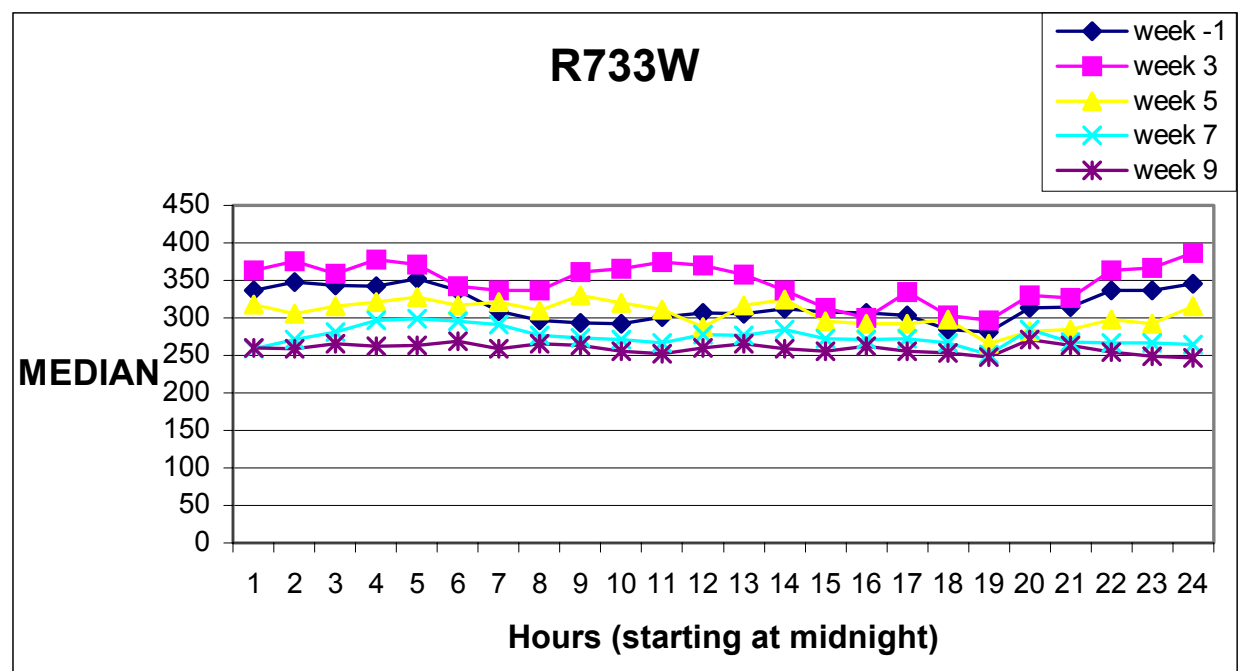

Figure A.13 MEDIAN R733W

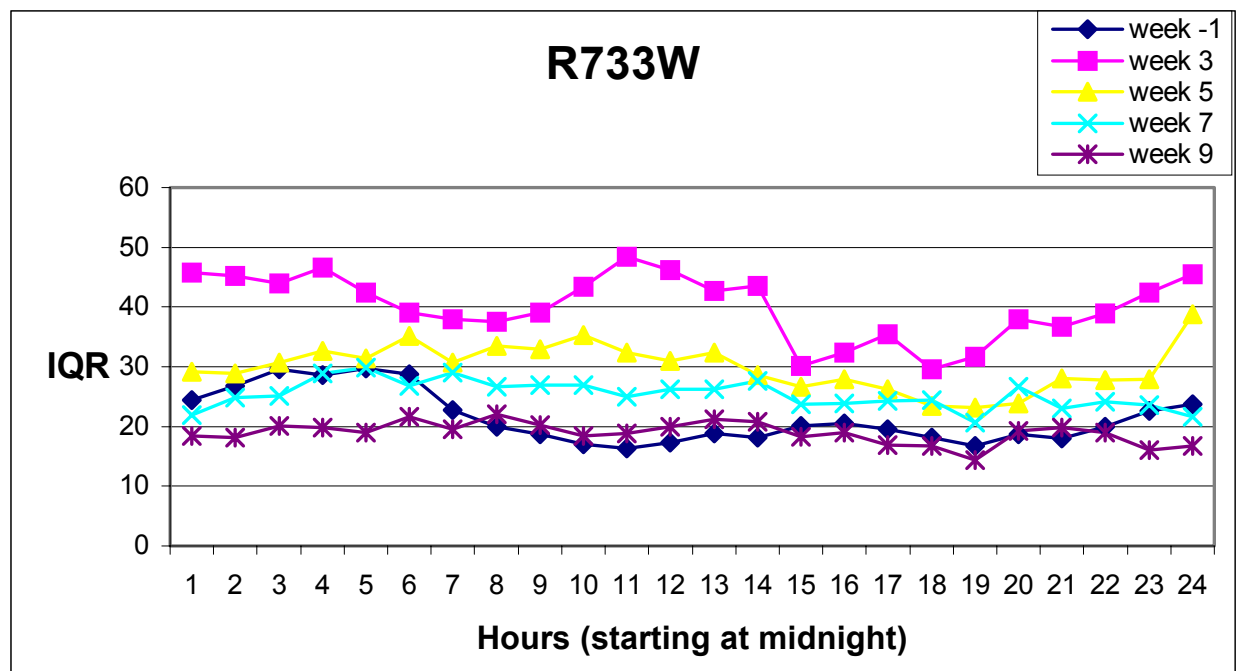

Figure A.14 IQR R733W

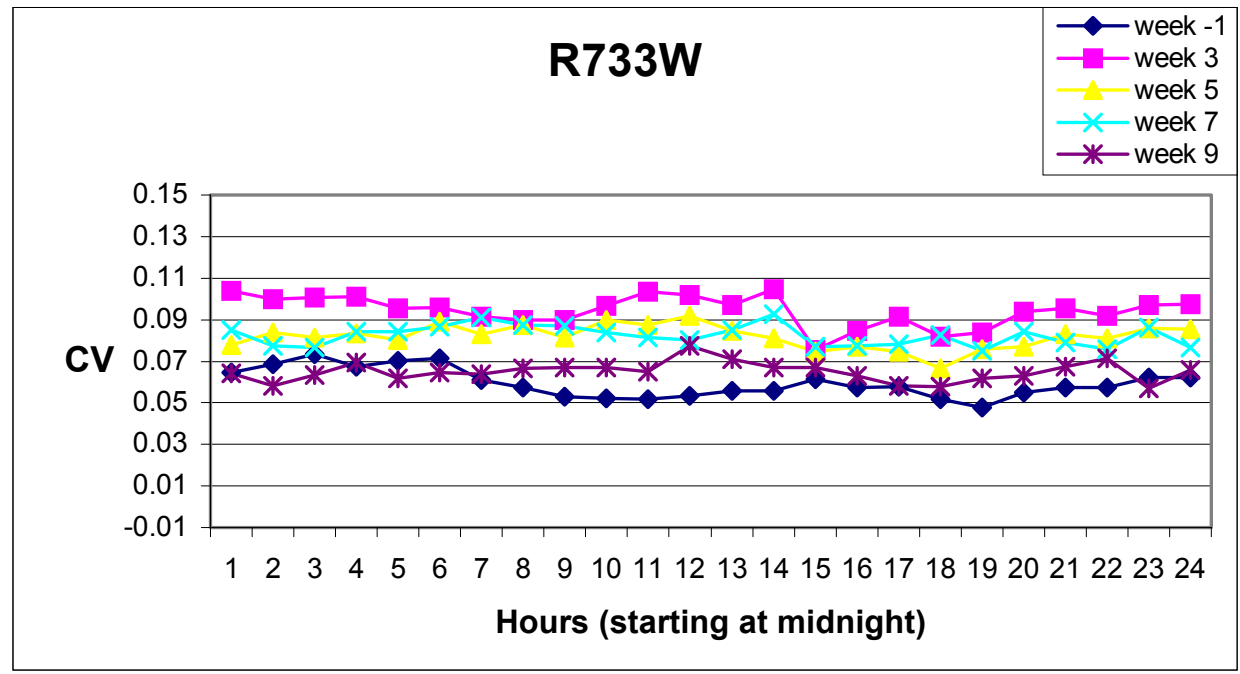

Figure A.15 CV R733W 


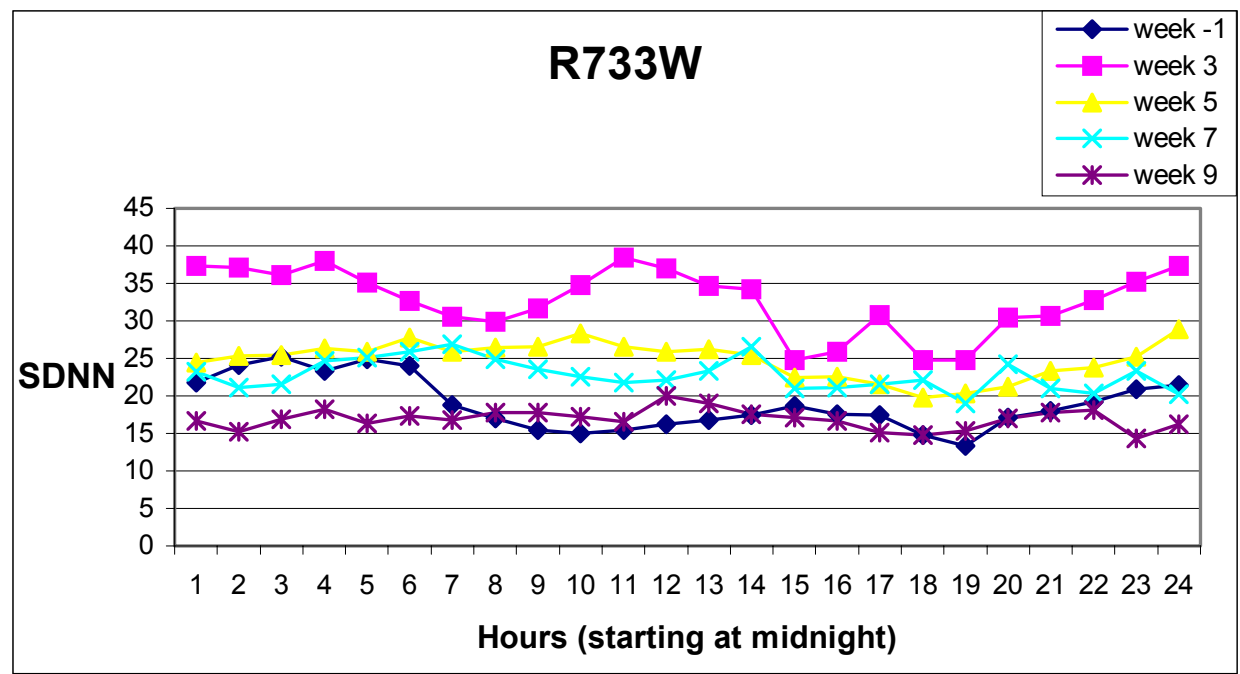

Figure A.16 SDNN R733W

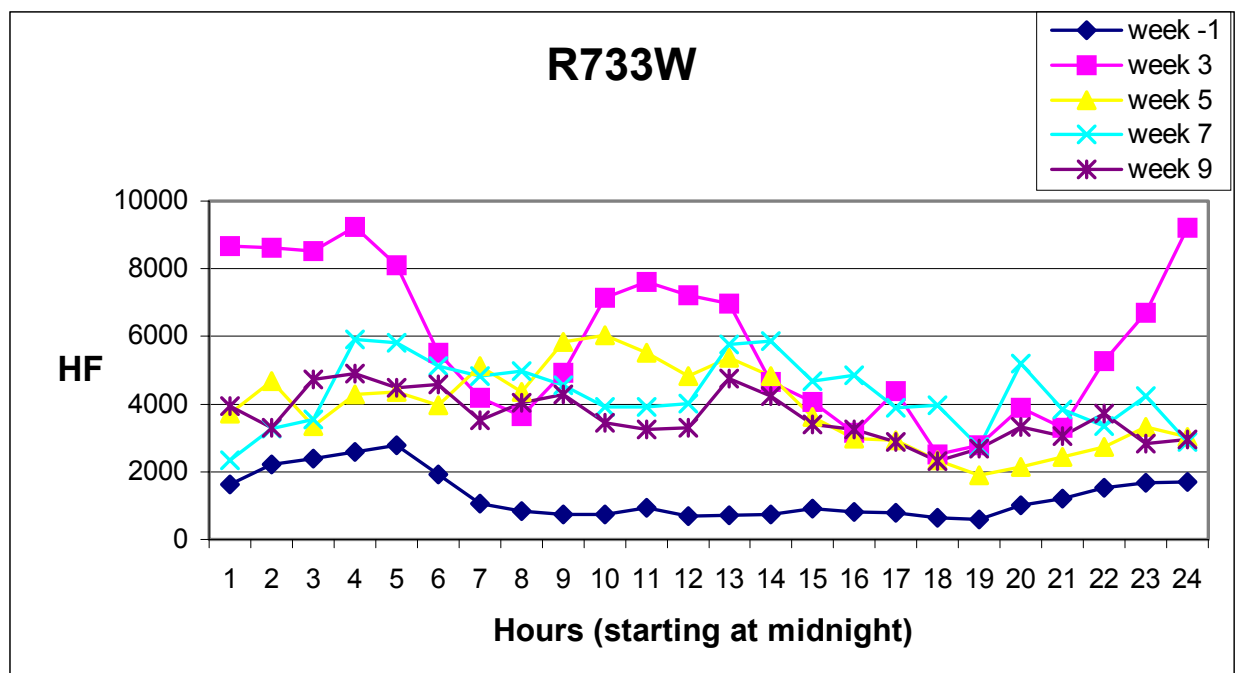

Figure A.17 HF R733W

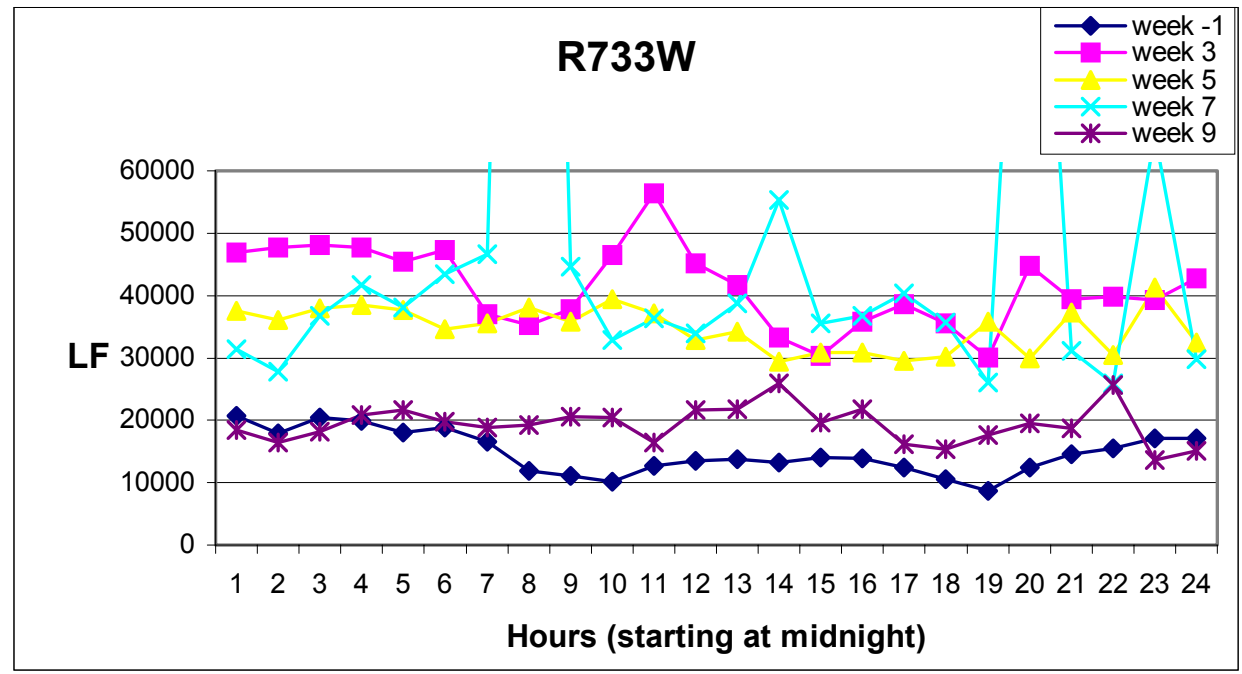

Figure A.18 LF R733W 


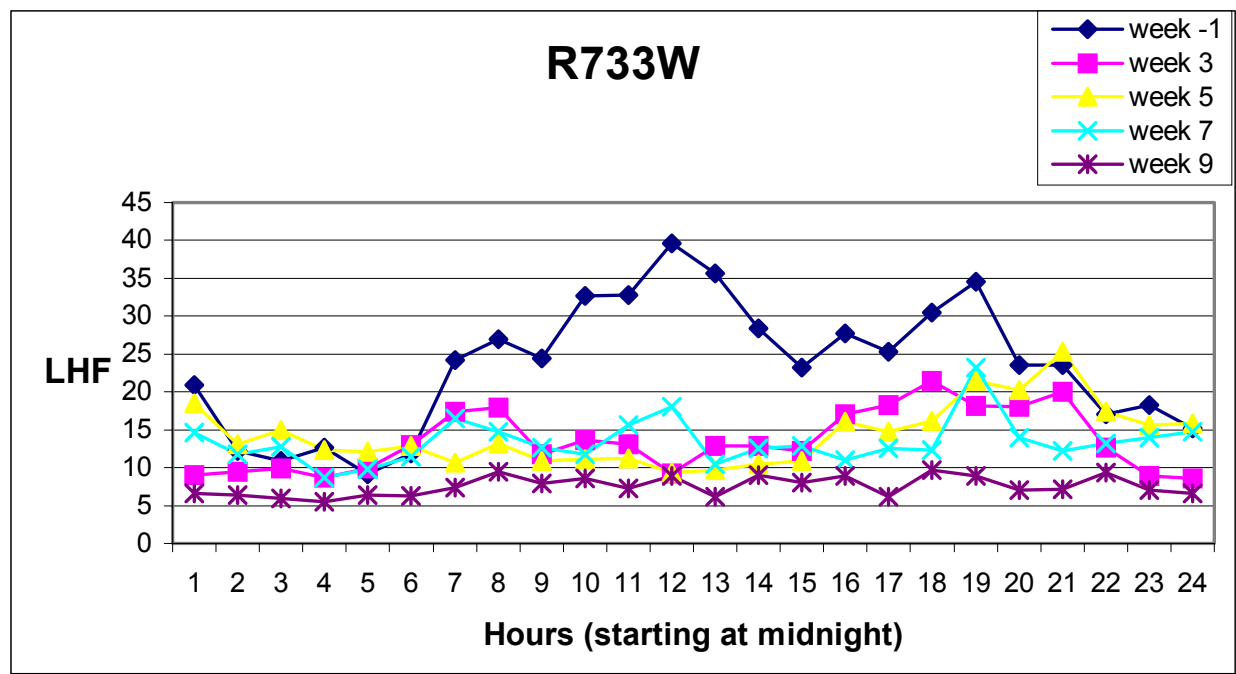

Figure A.19 LHF R733W

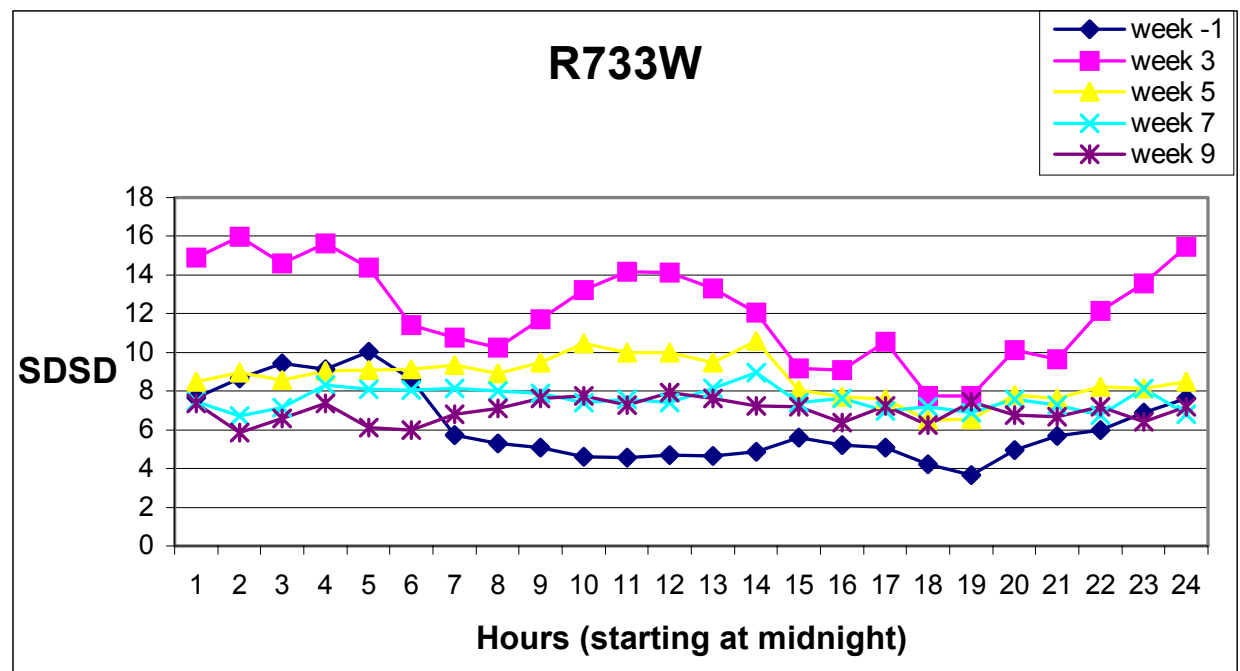

Figure A.20 SDSD R733W

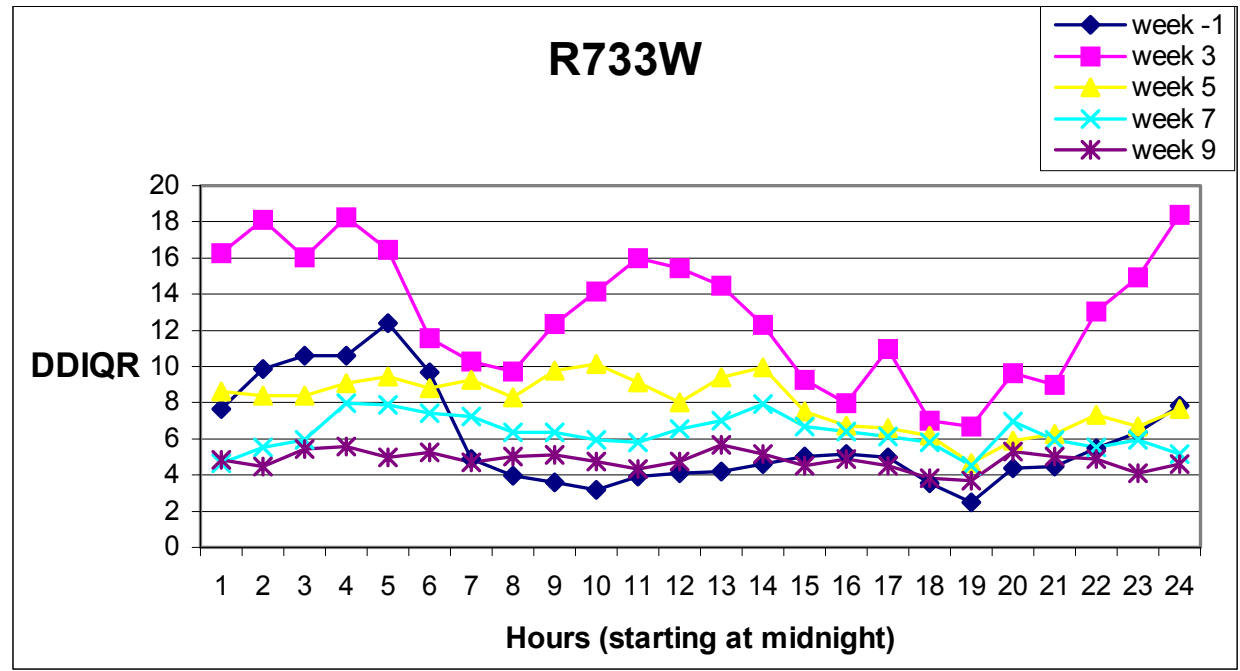

Figure A.21 DDIQR R733W 


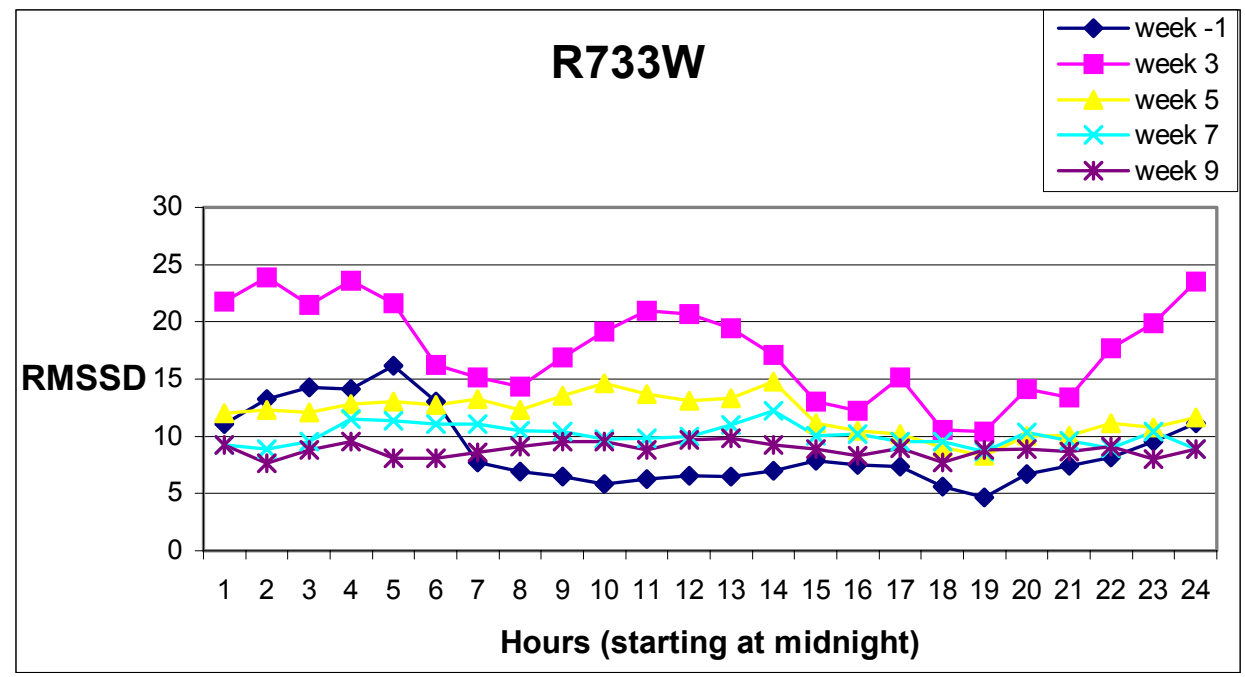

Figure A.22 RMSSD R733W

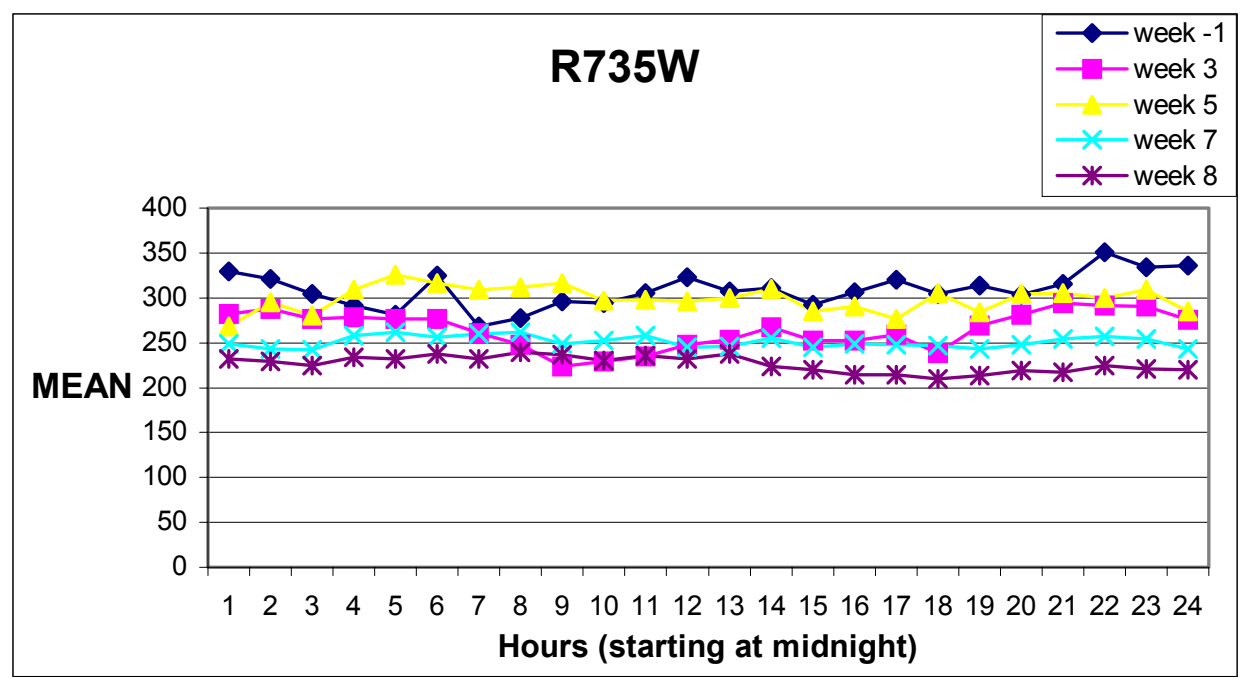

Figure A.23 MEAN R735W

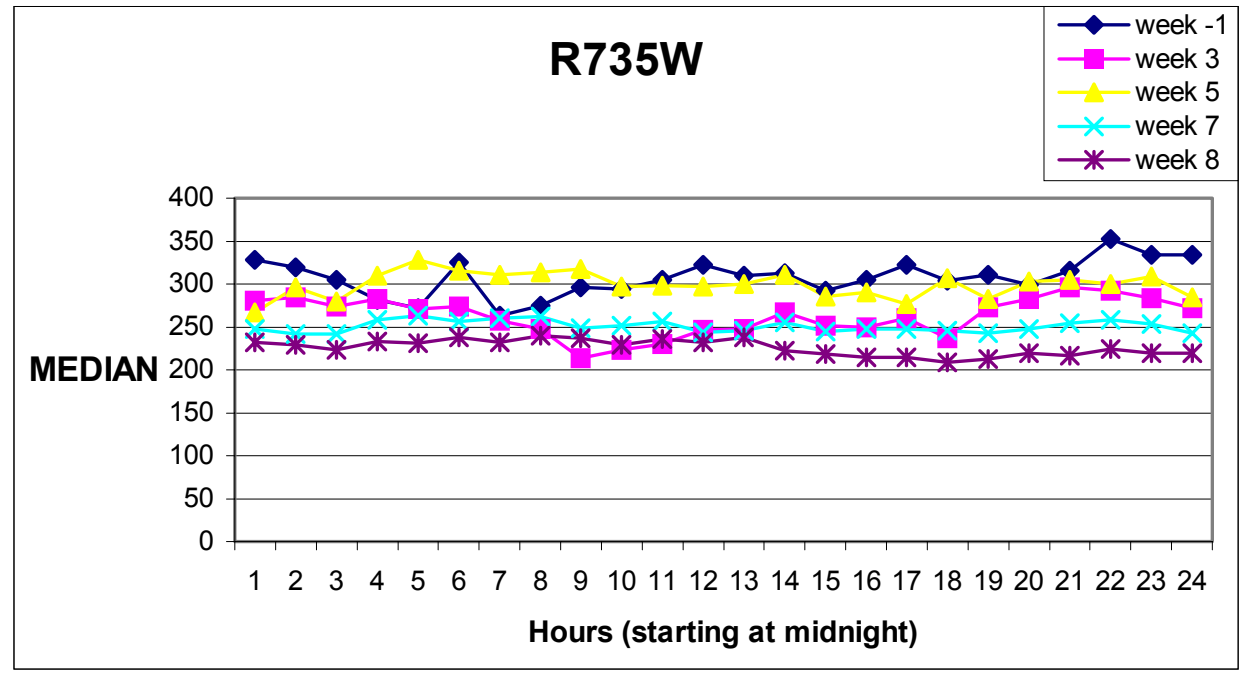

Figure A.24 MEDIAN R735W 


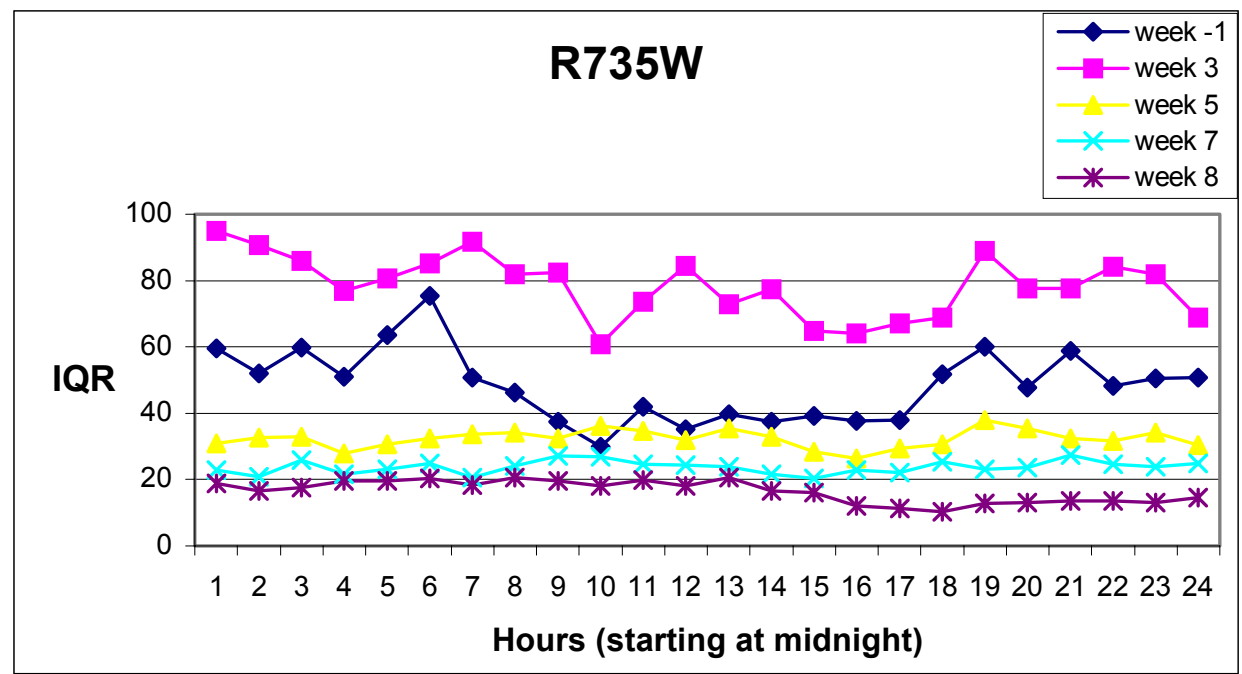

Figure A.25 IQR R735W

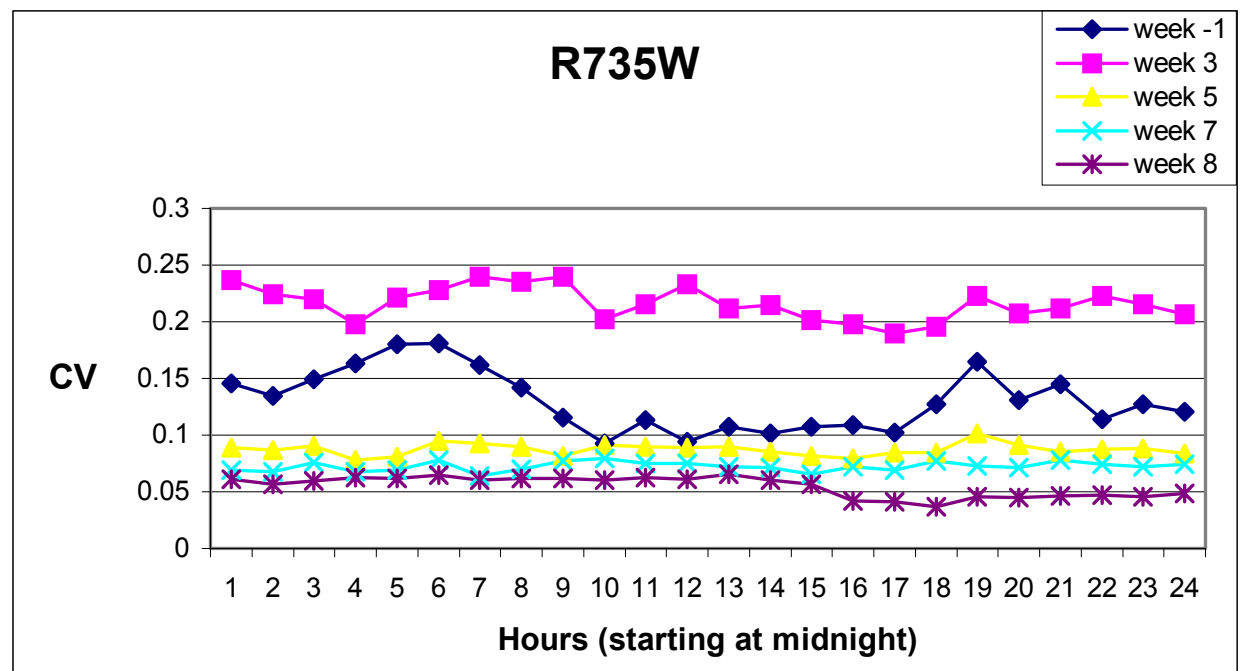

Figure A.26 CV R735W

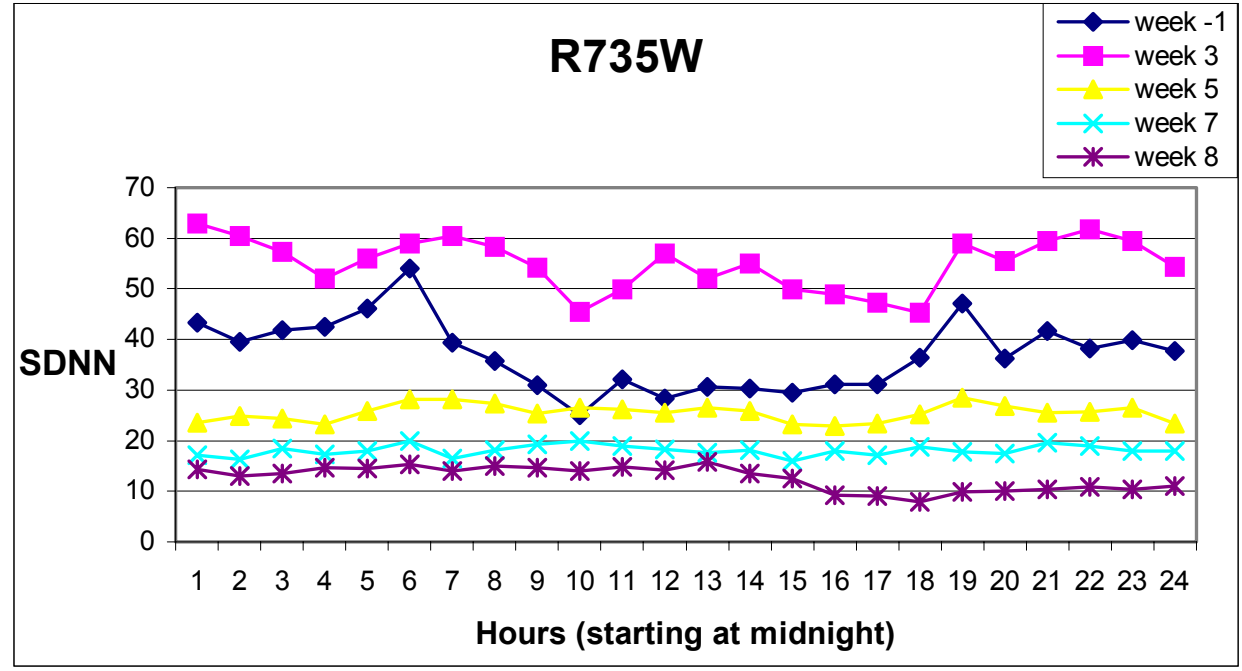

Figure A.27 SDNN R735W 


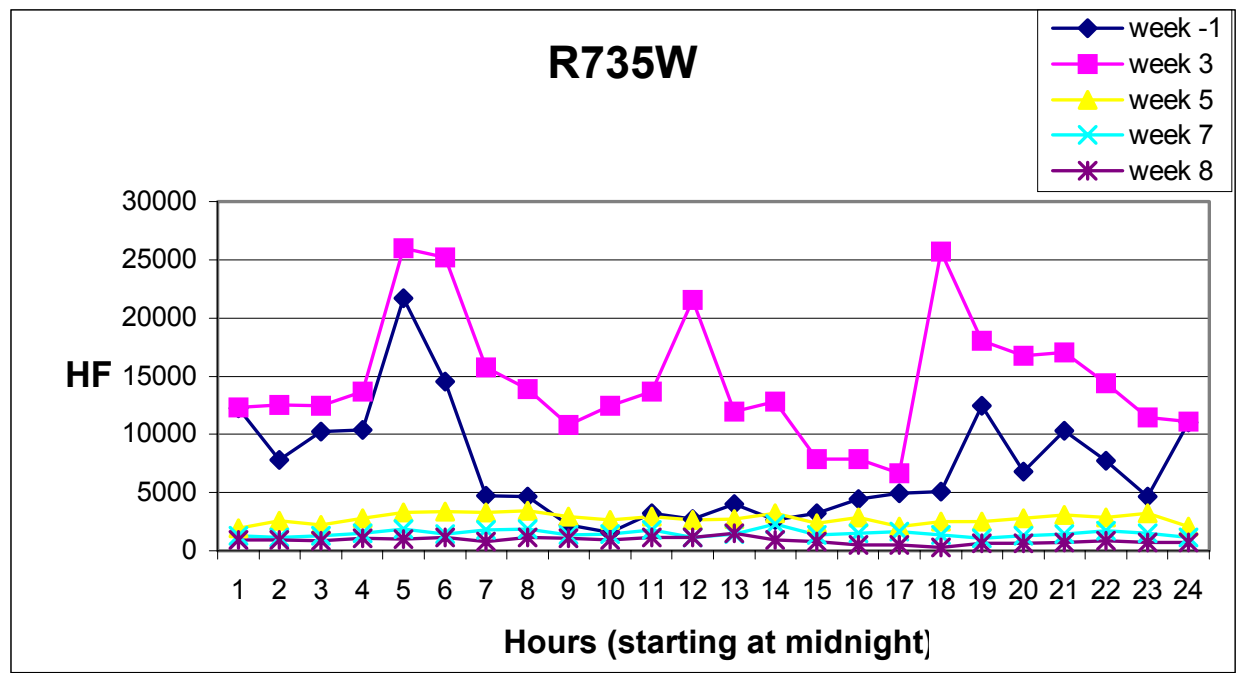

Figure A.28 HF R735W

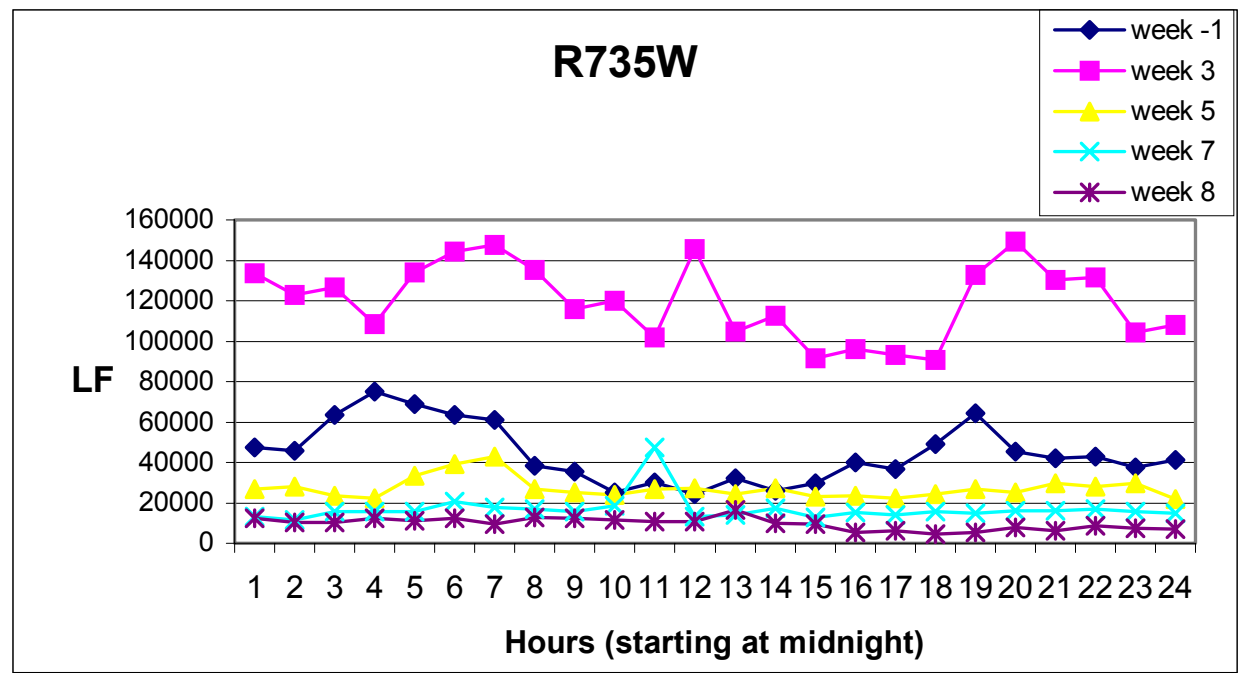

Figure A.29 LF R735W

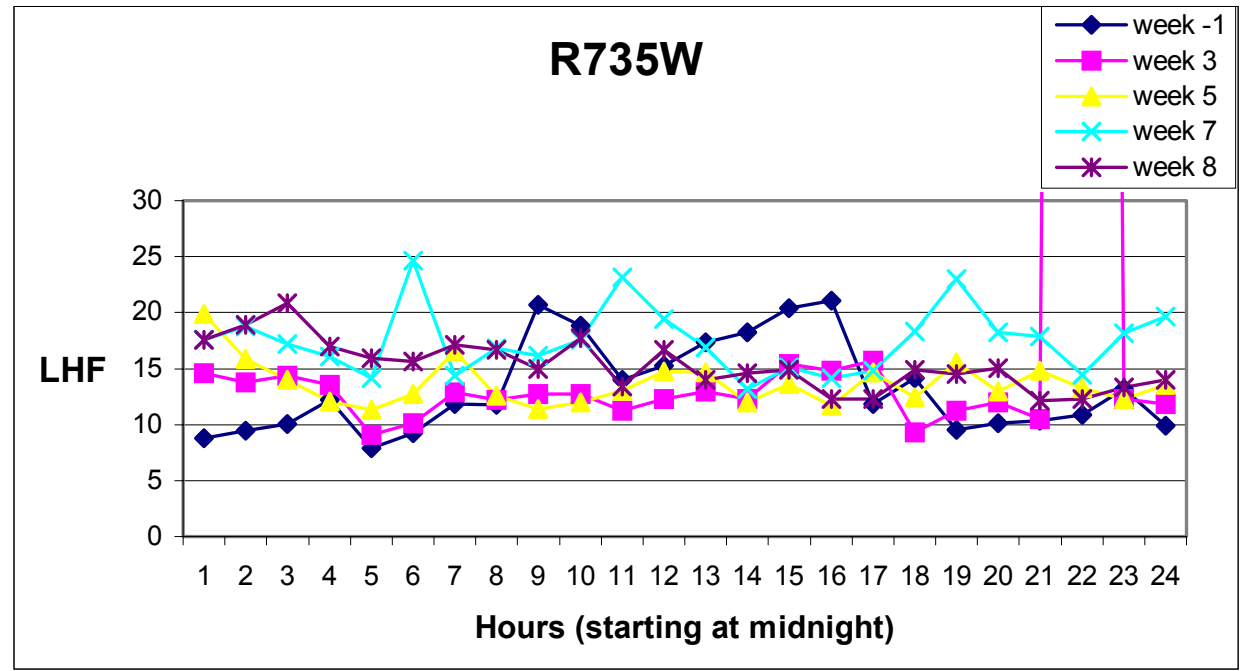

Figure A.30 LHF R735W 


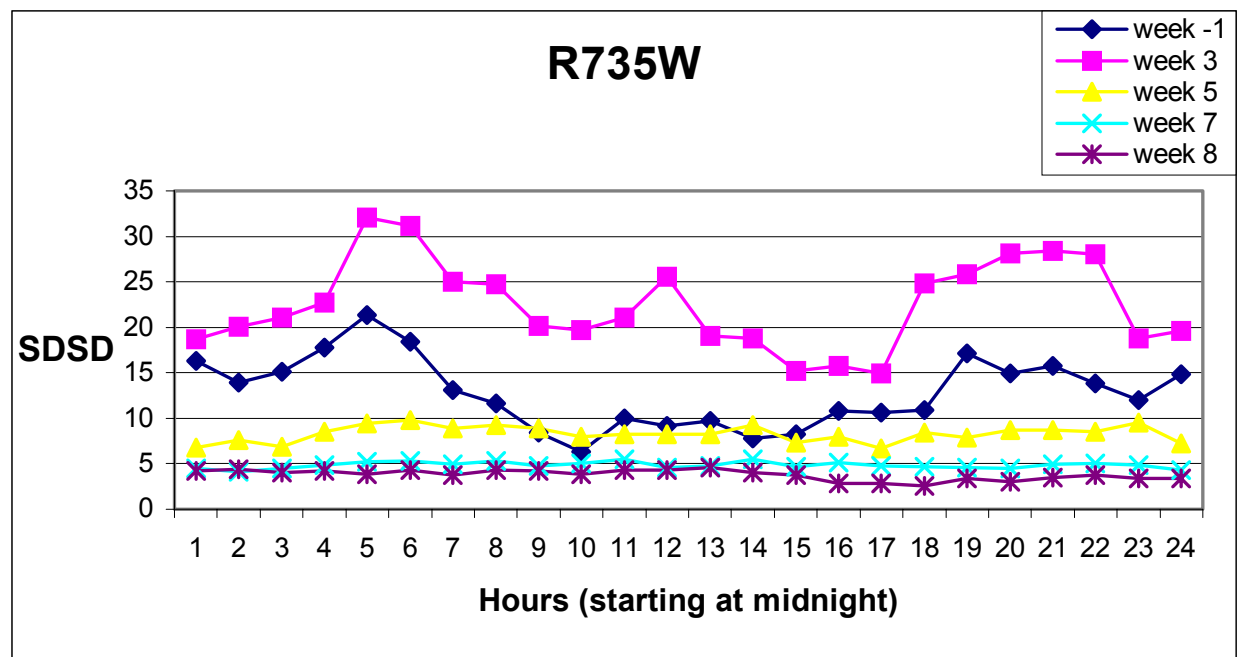

Figure A.31 SDSD R735W

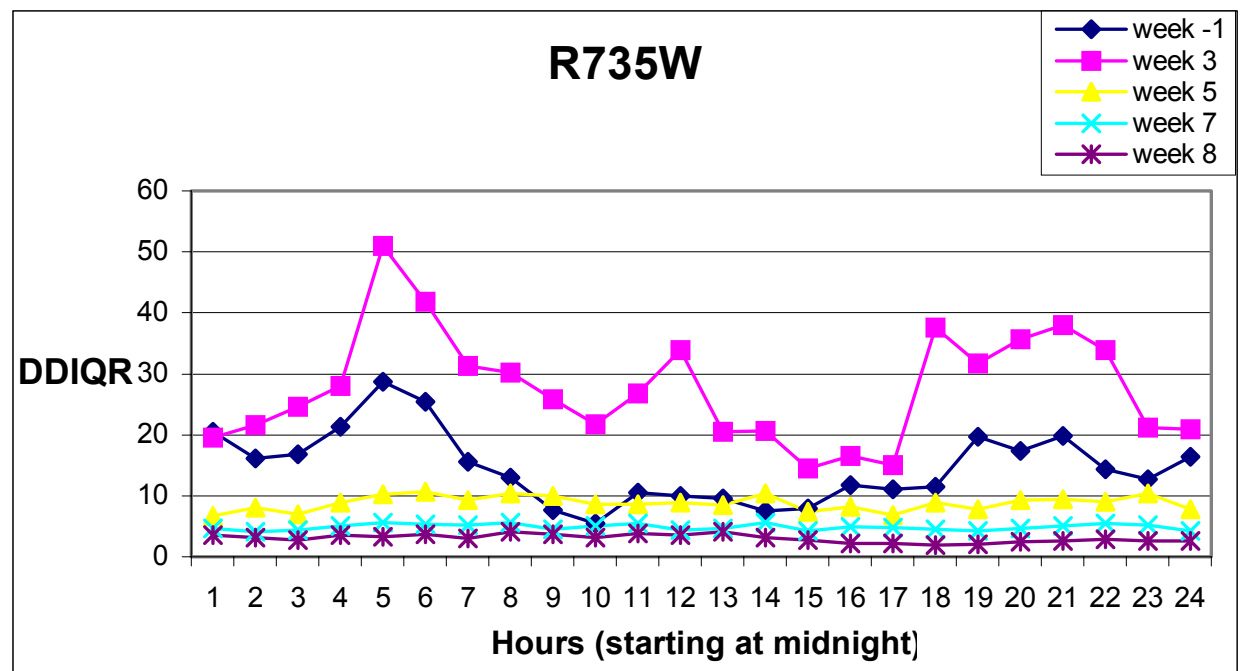

Figure A.32 DDIQR R735W

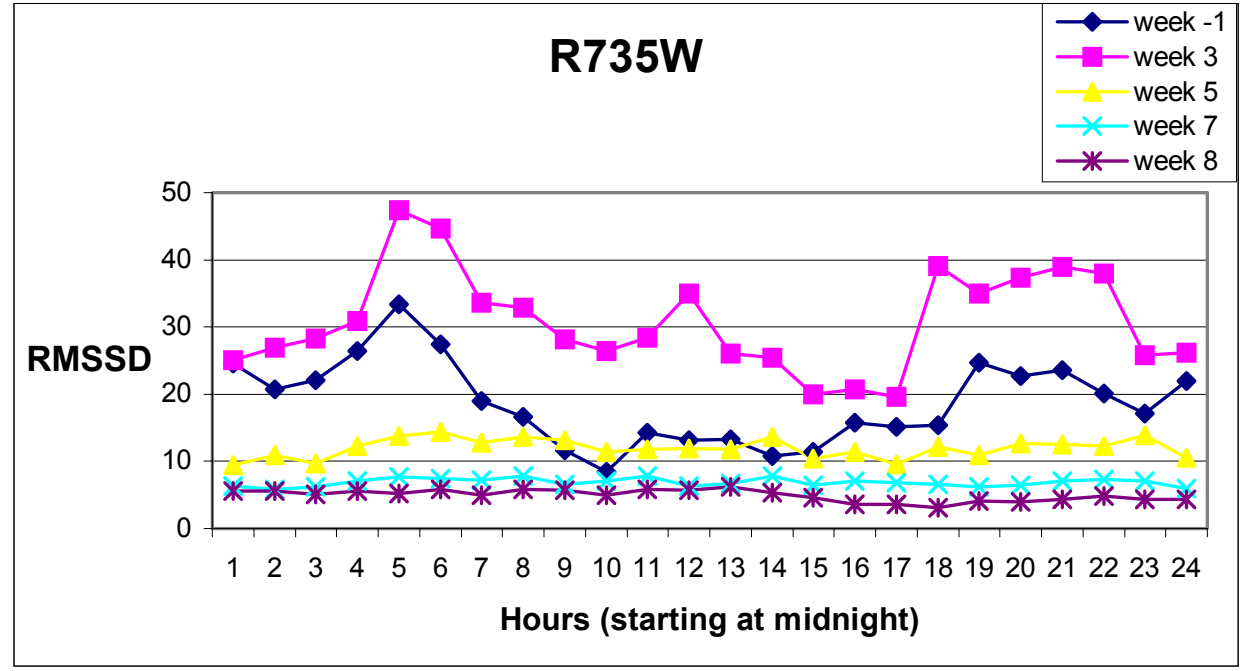

Figure A.33 RMSSD R735W 
Appendix B Individual plots where each 5 minute segment is averaged weekly 


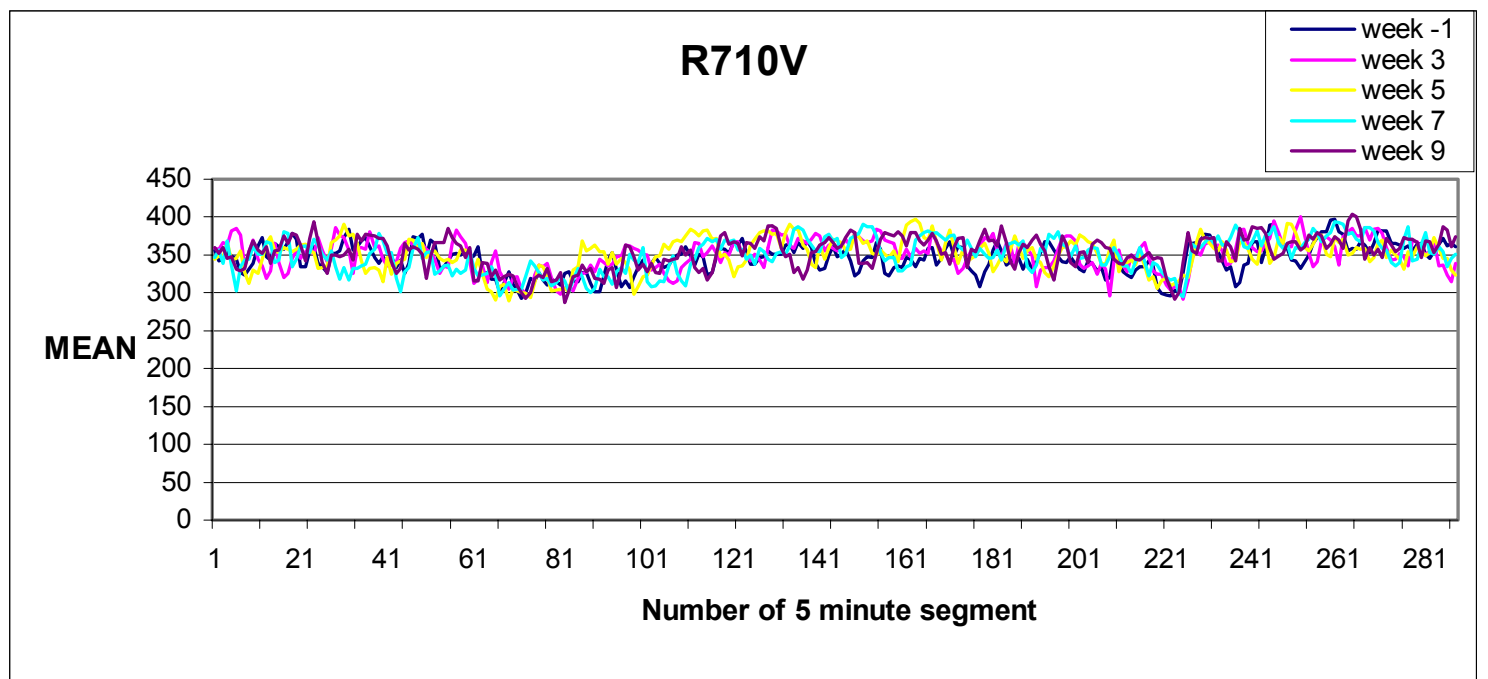

Figure B.1 MEAN R710V

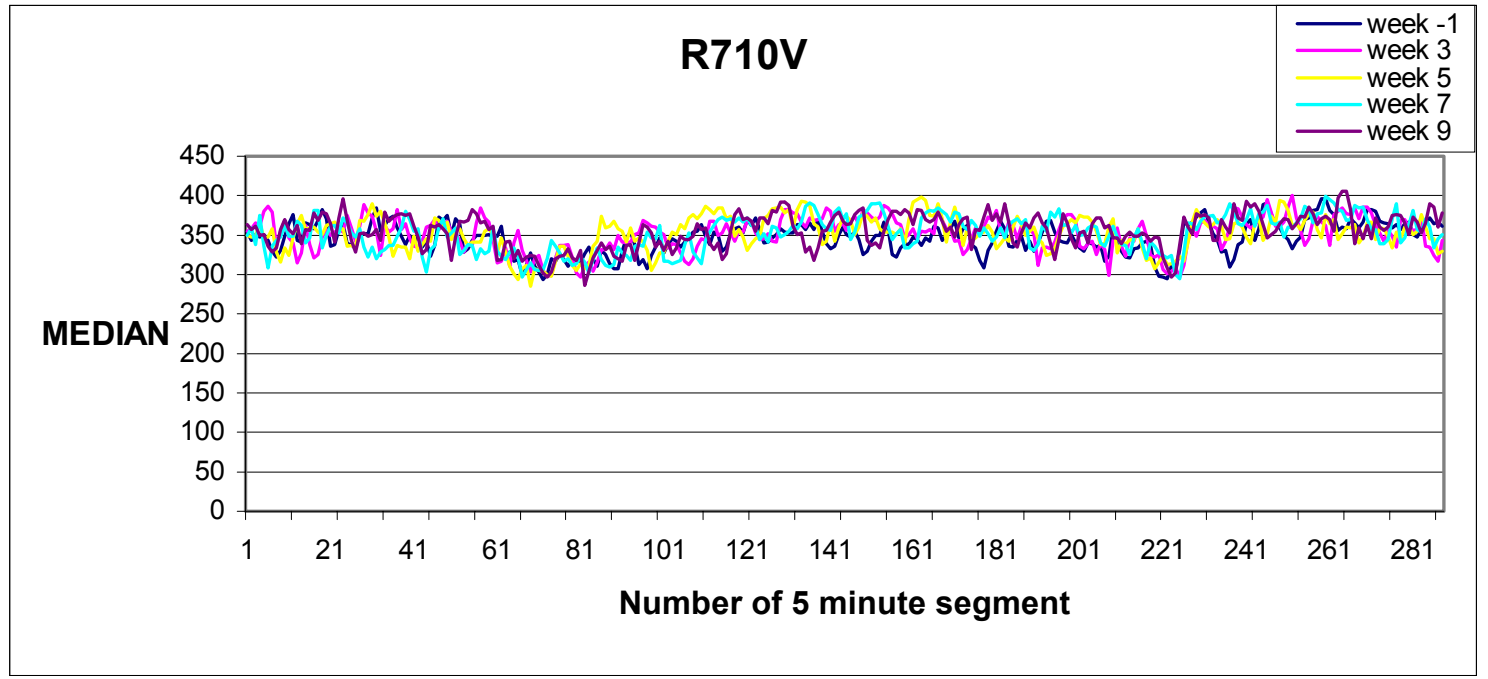

Figure B.2 MEDIAN R710V

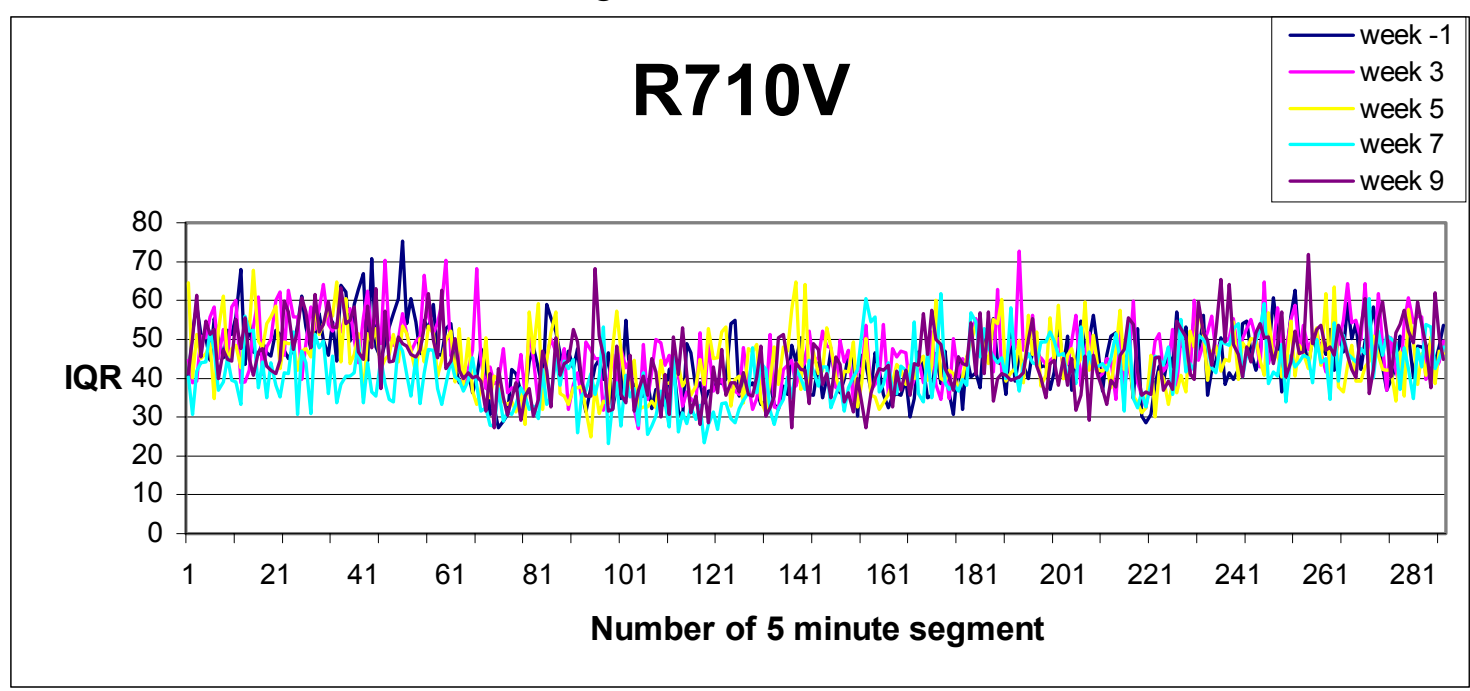

Figure B.3 IQR R710V 


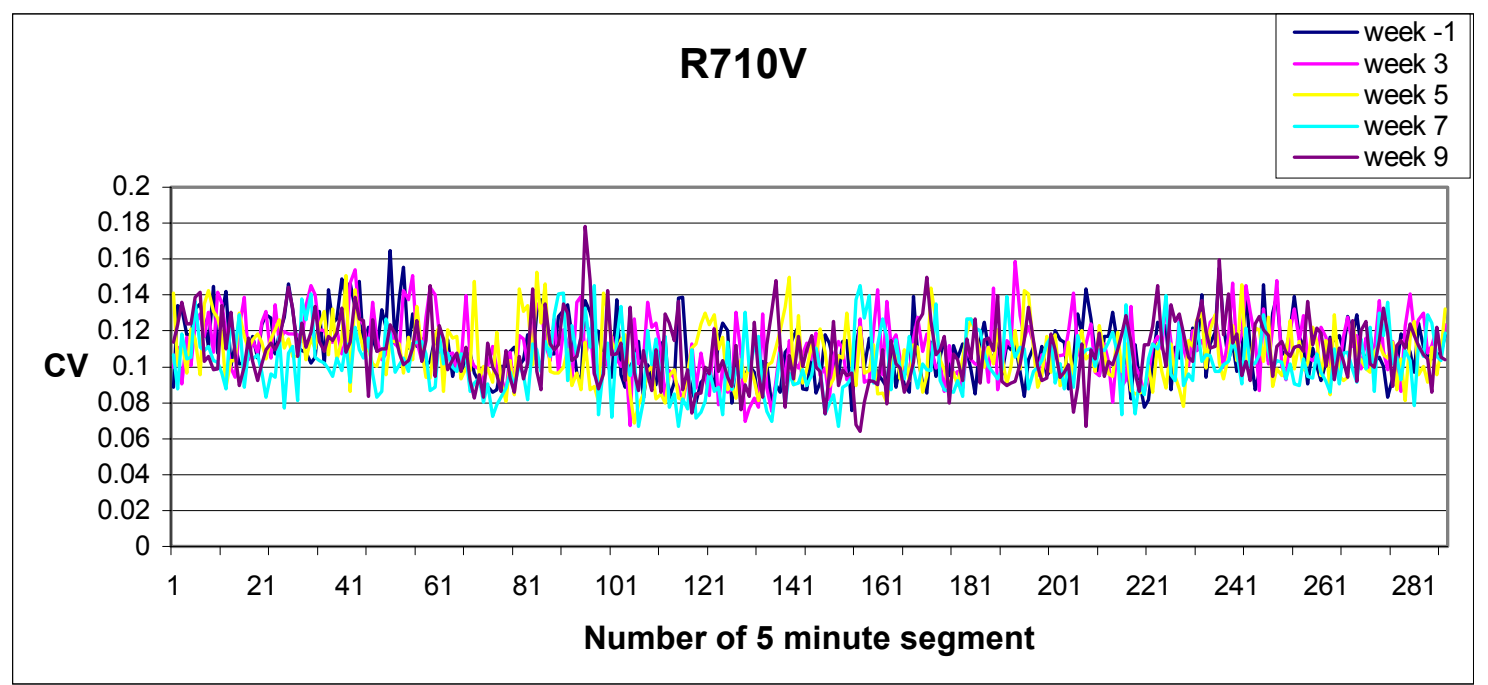

Figure B.4 CV R710V

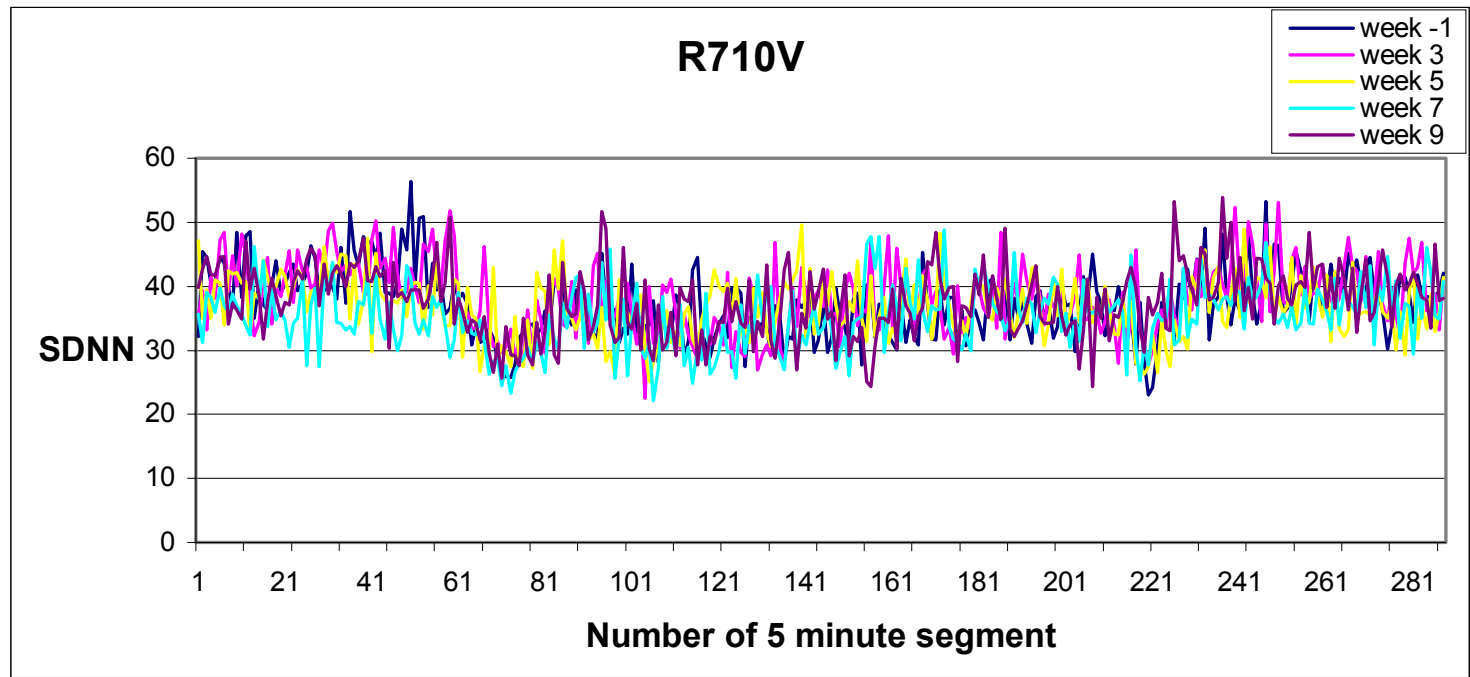

Figure B.5 SDNN R710V

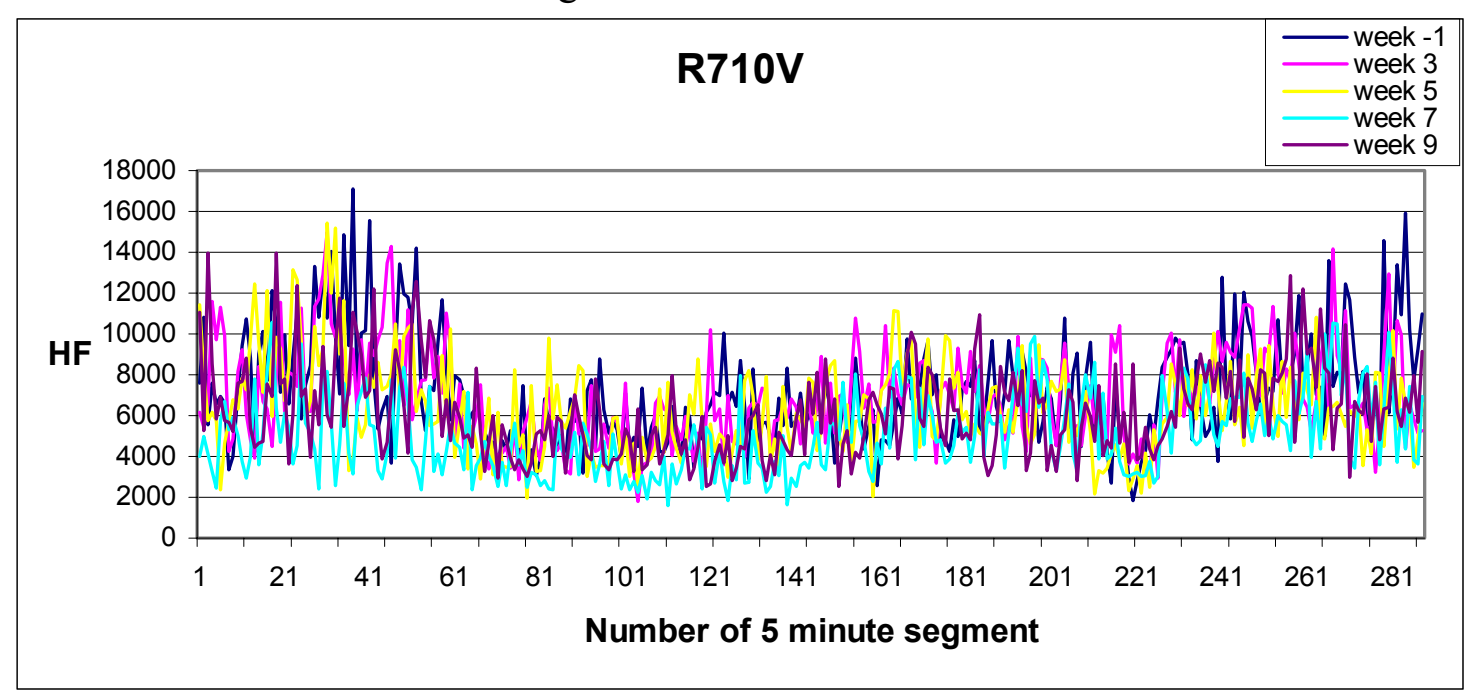

Figure B.6 HF R710V 


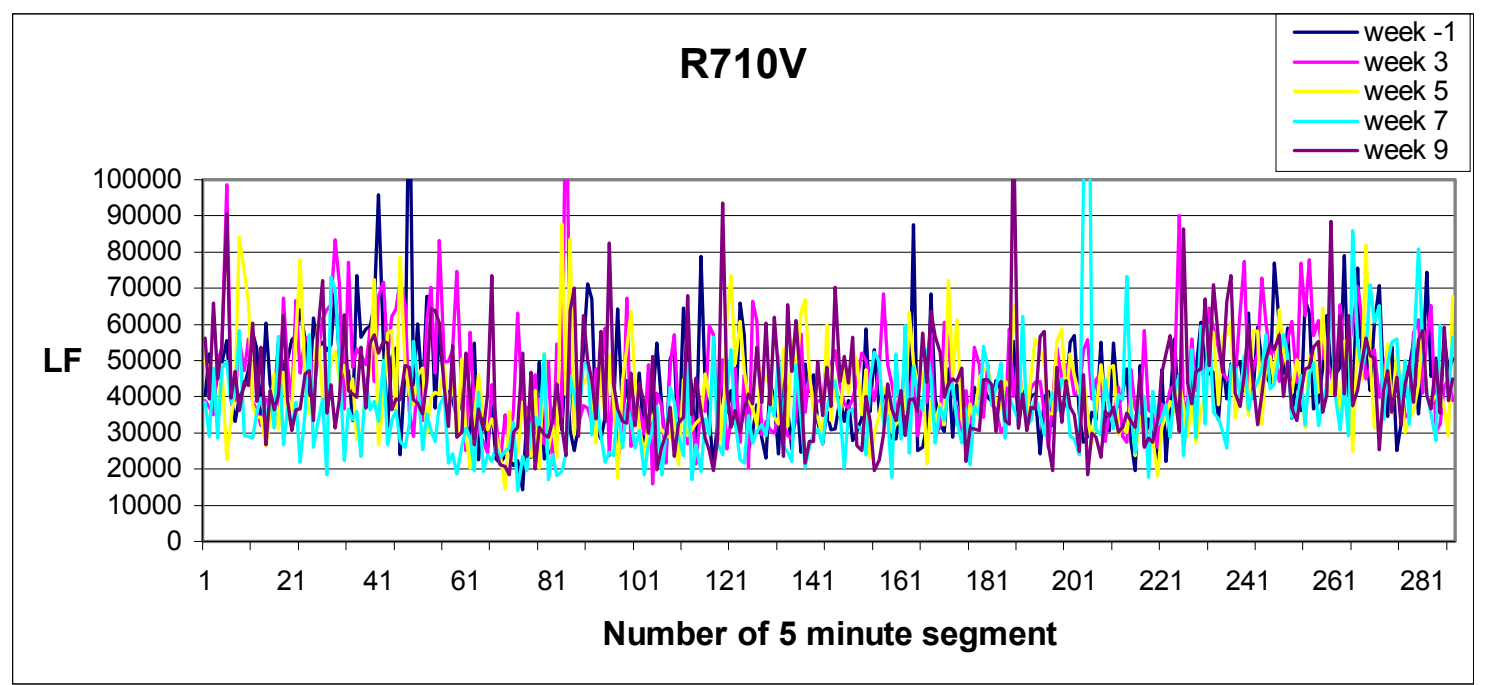

Figure B.7 LF R710V

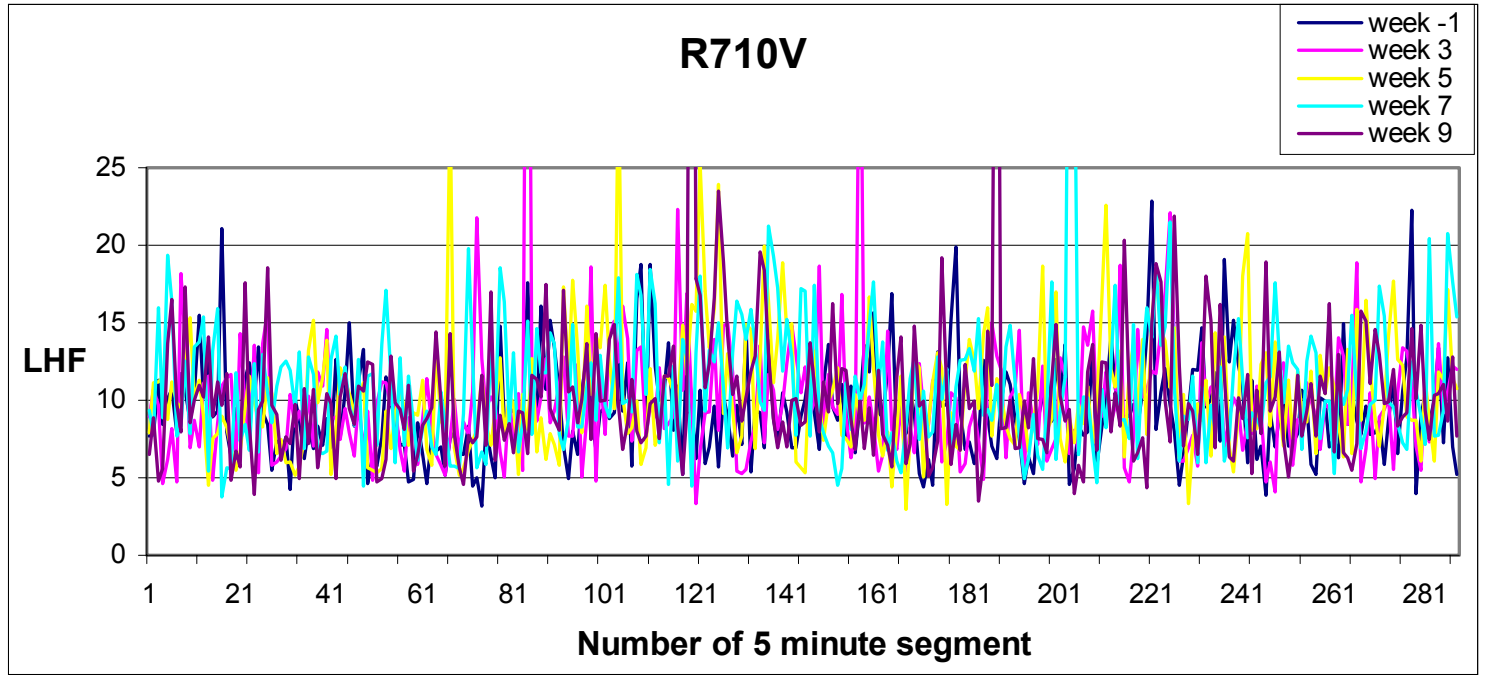

Figure B.8 LHF R710V

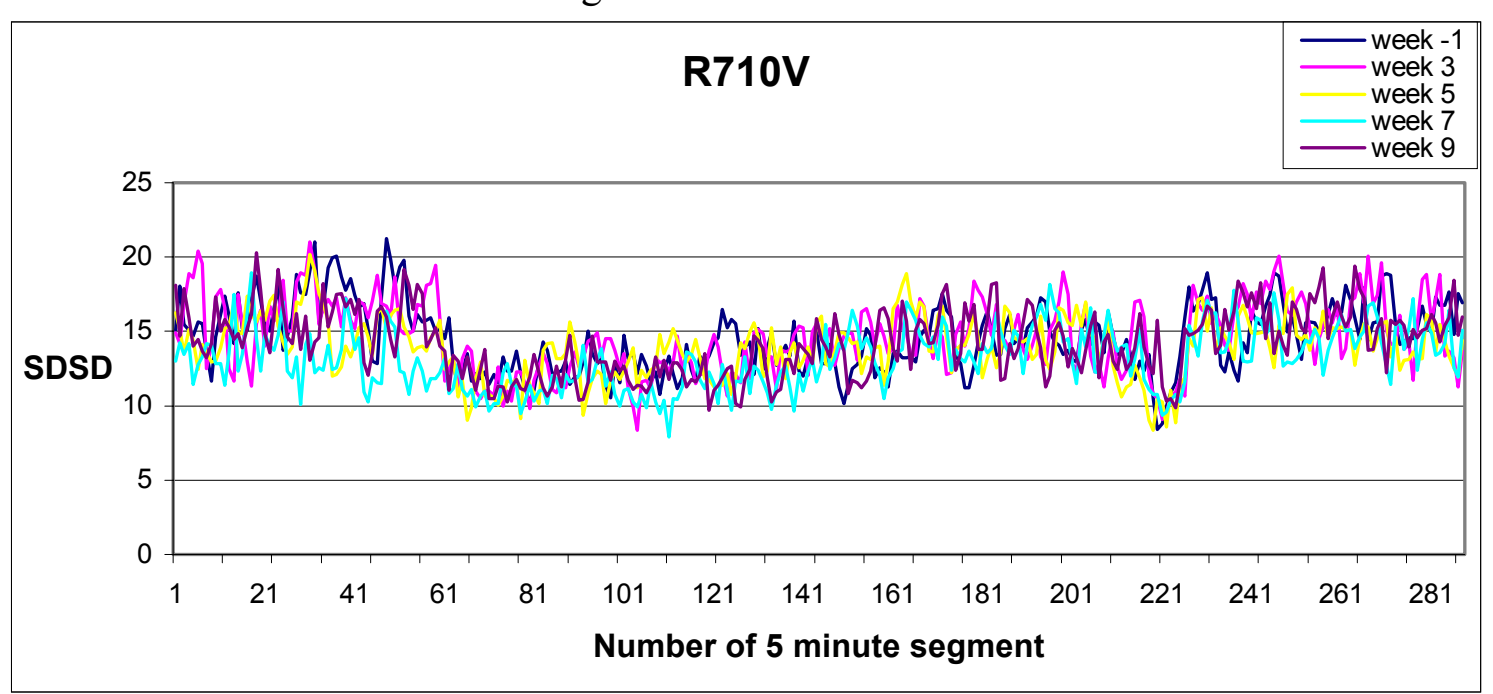

Figure B.9 SDSD R710V 


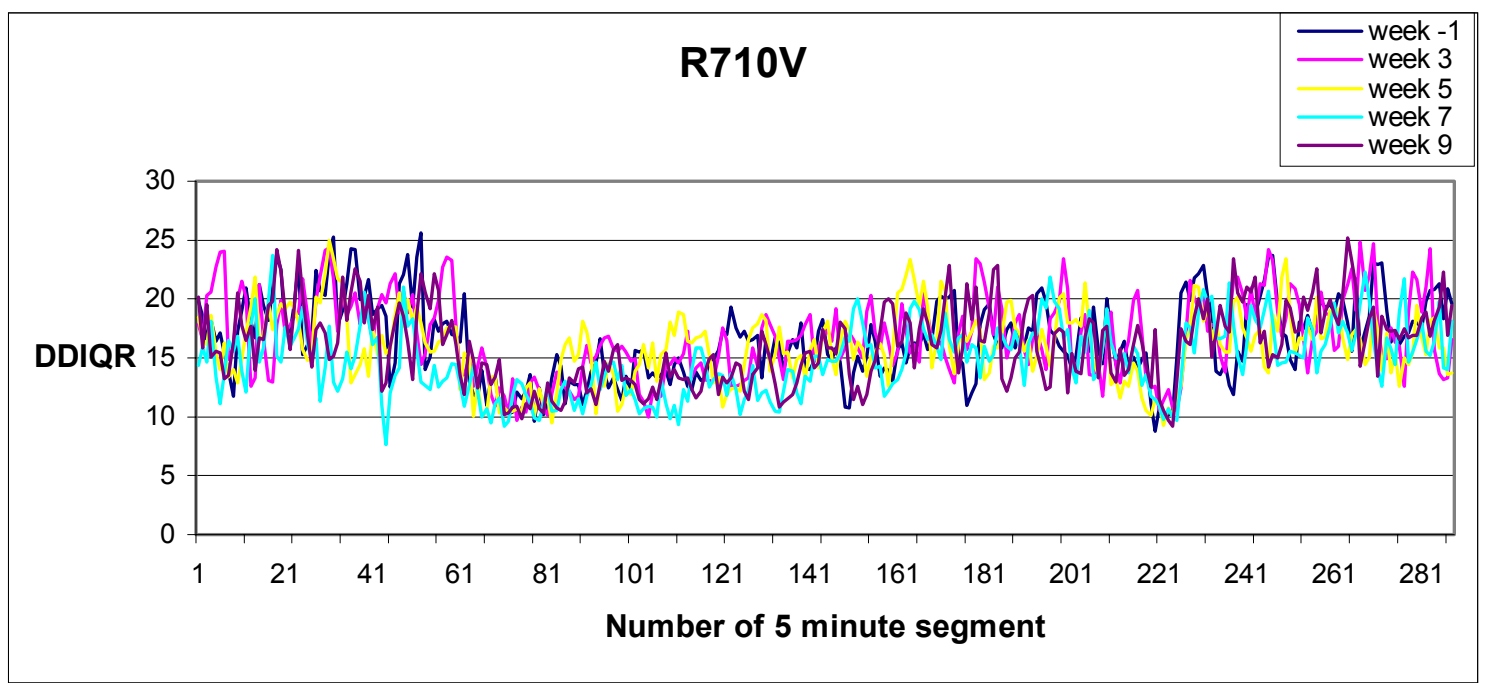

Figure B.10 DDIQR R710V

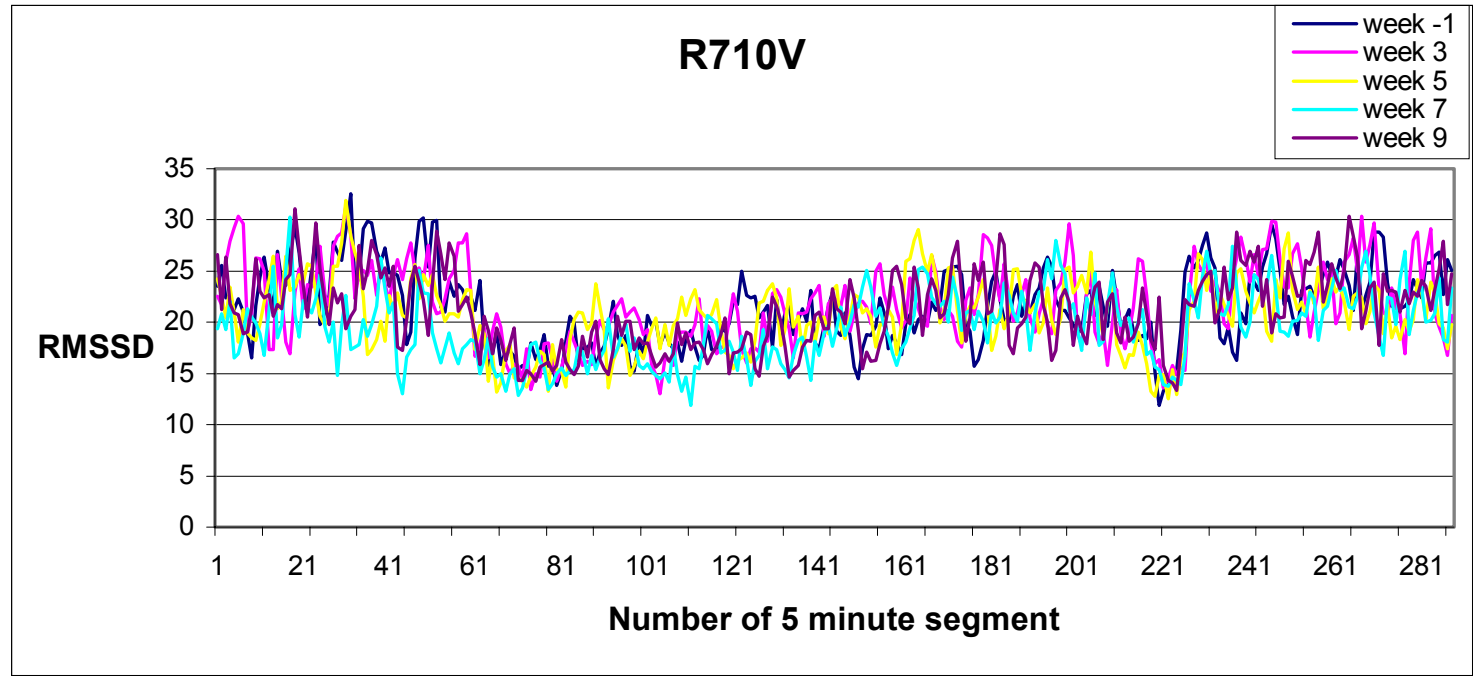

Figure B.11 RMSSD R710V

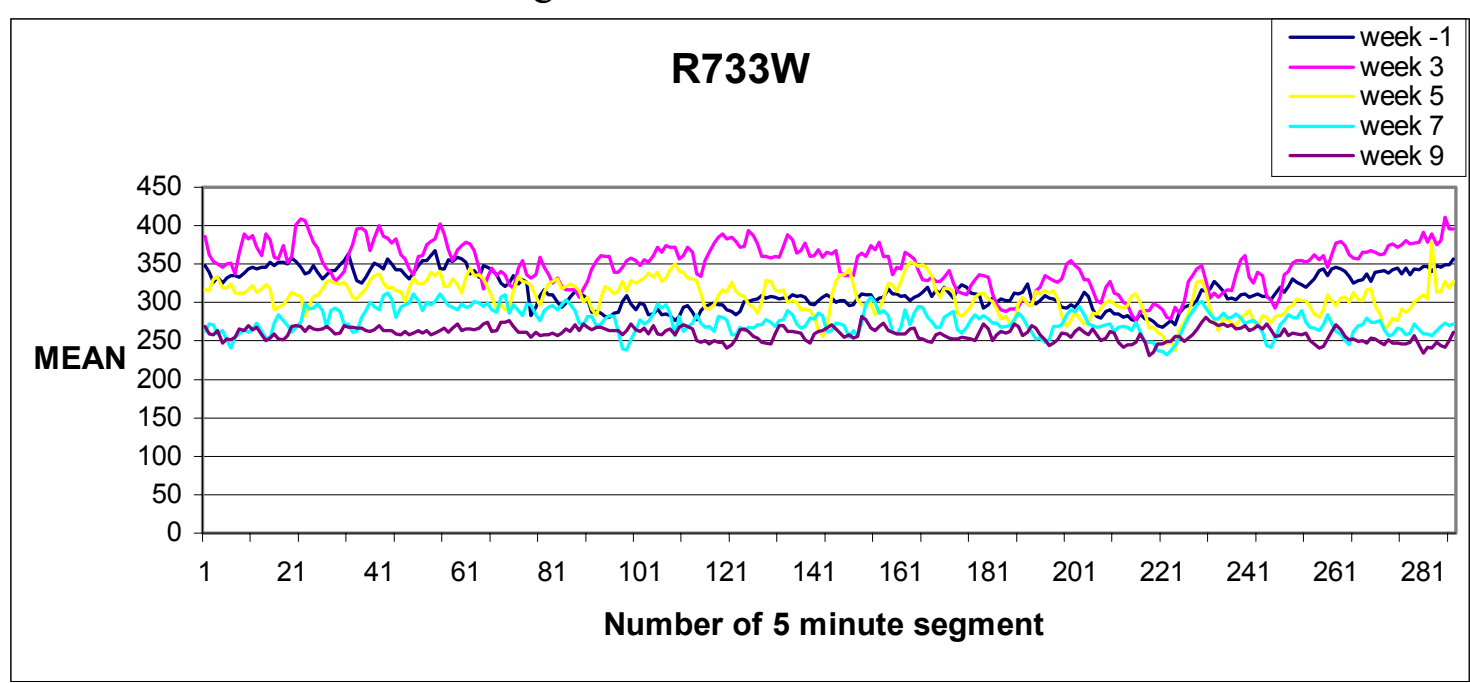

Figure B.12 MEAN R733W 


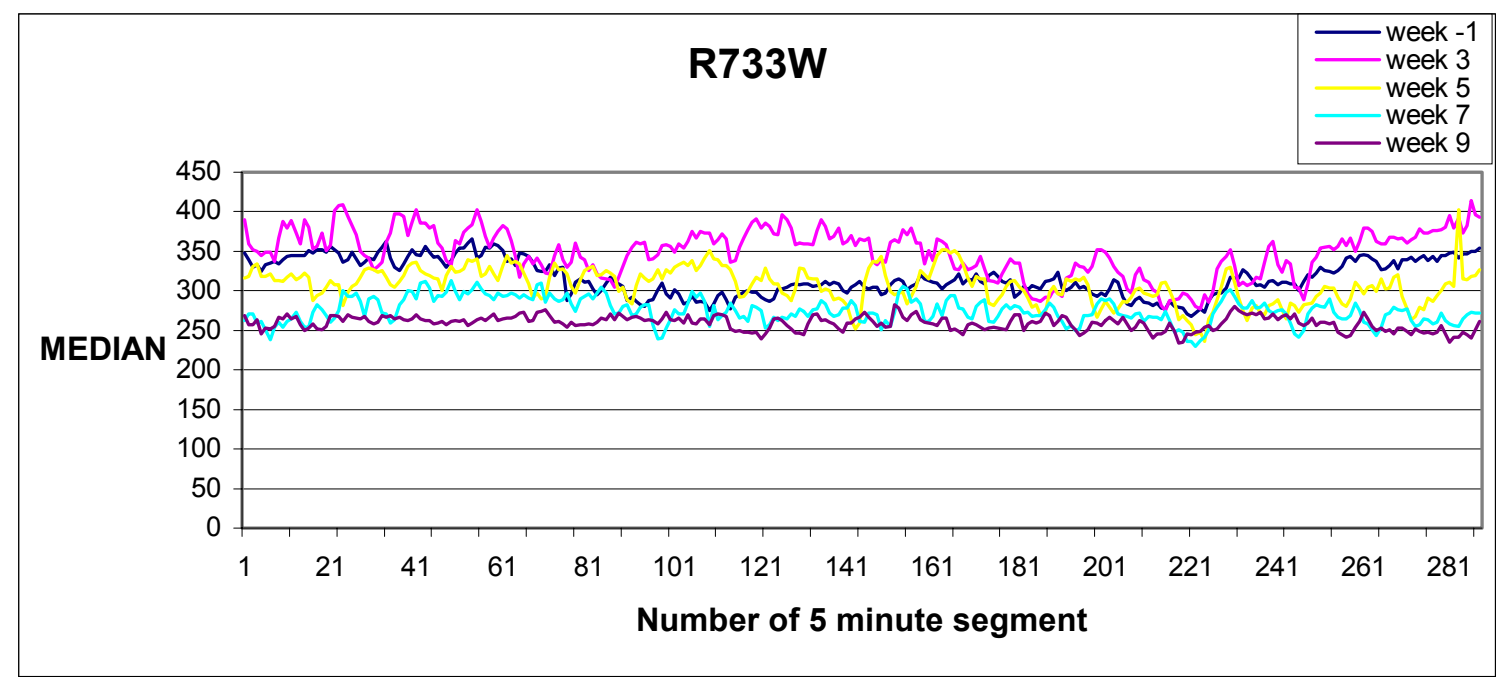

Figure B.13 MEDIAN R733W

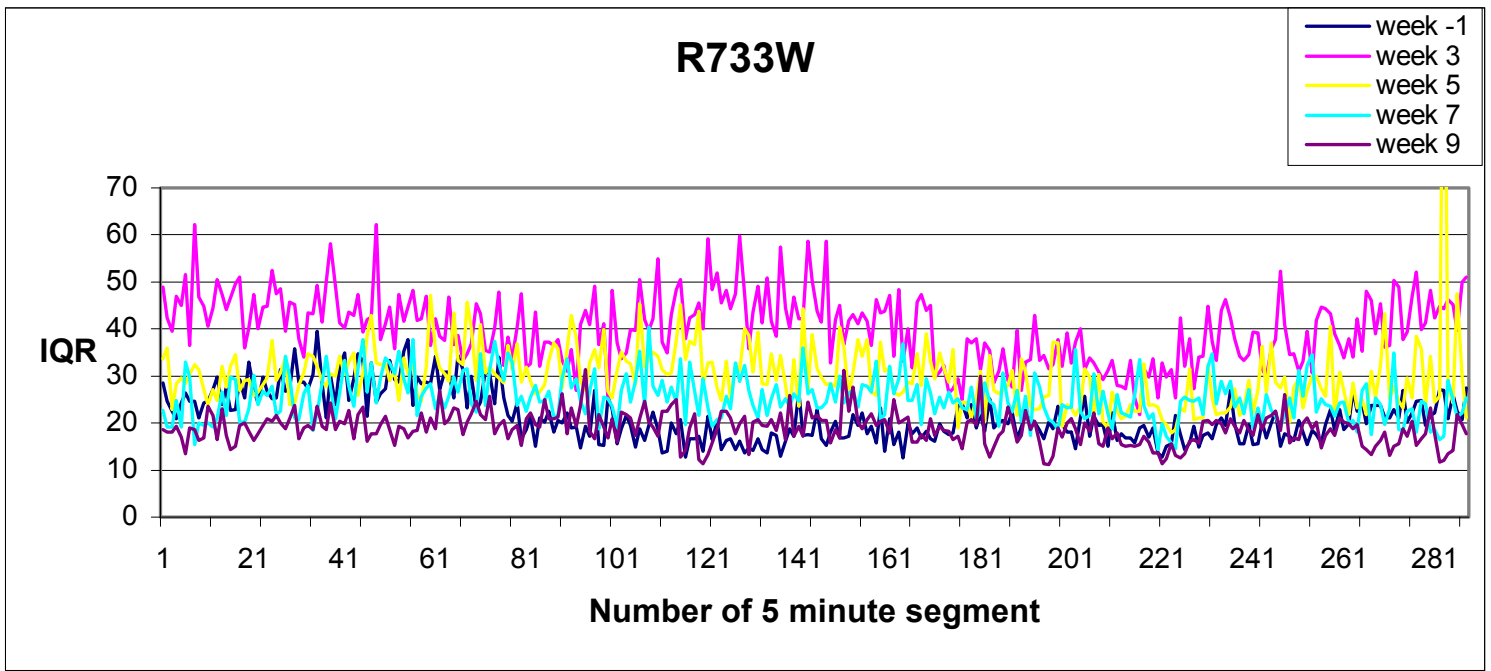

Figure B.14 IQR R733W

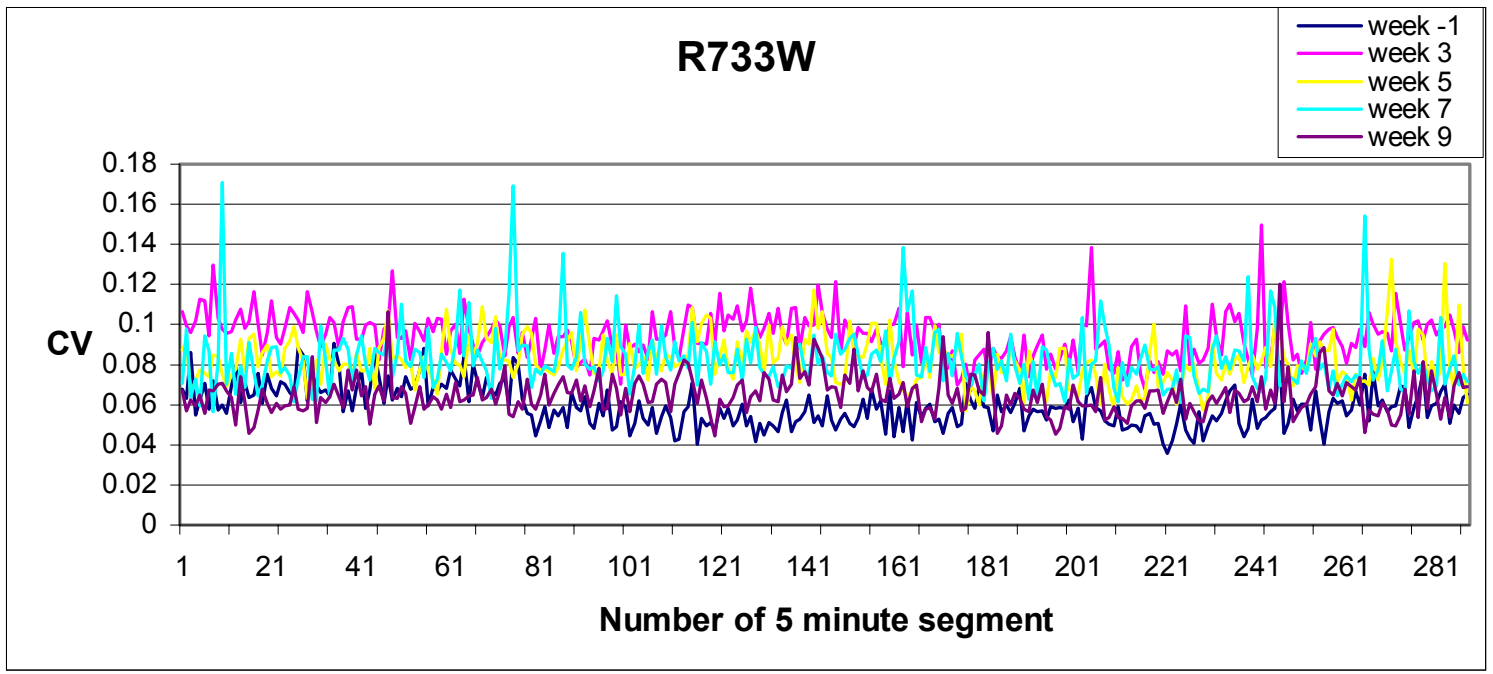

Figure B.15 CV R733W 


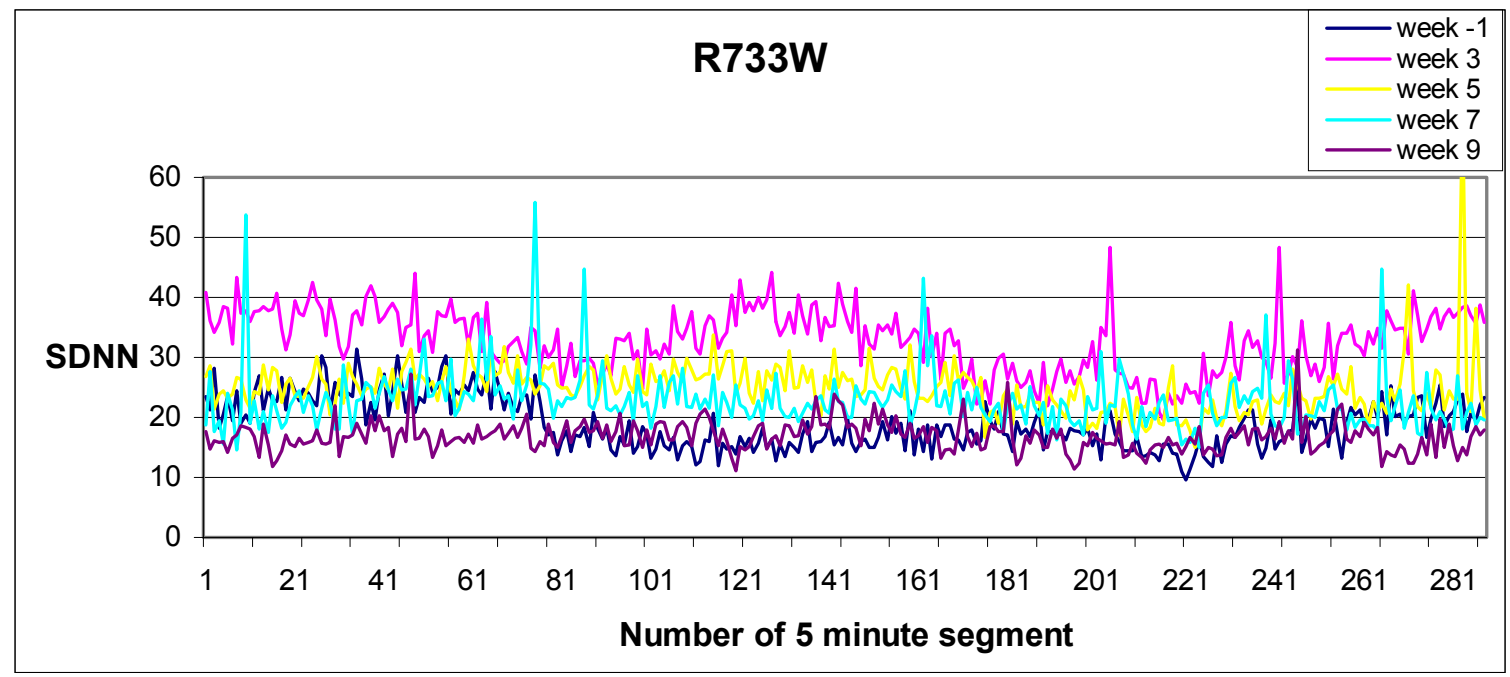

Figure B.16 SDNN R733W

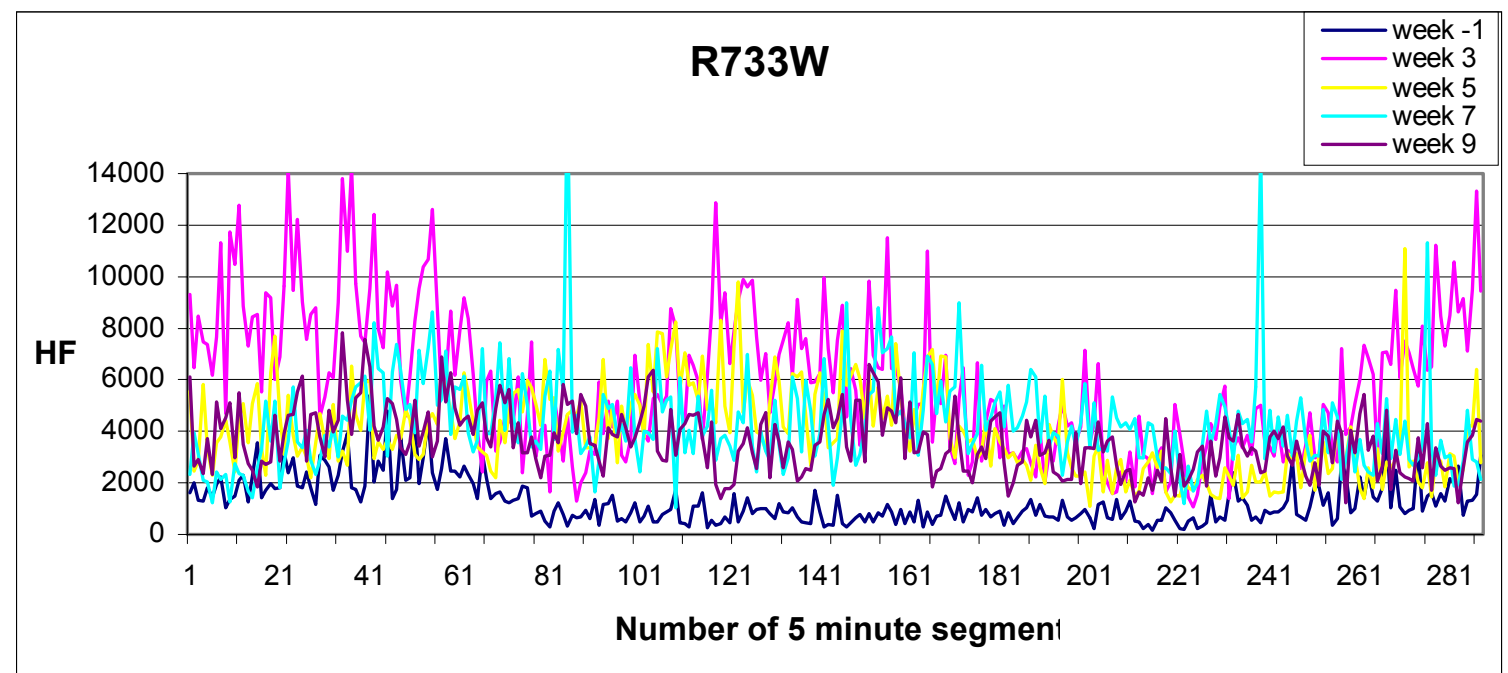

Figure B.17 HF R733W

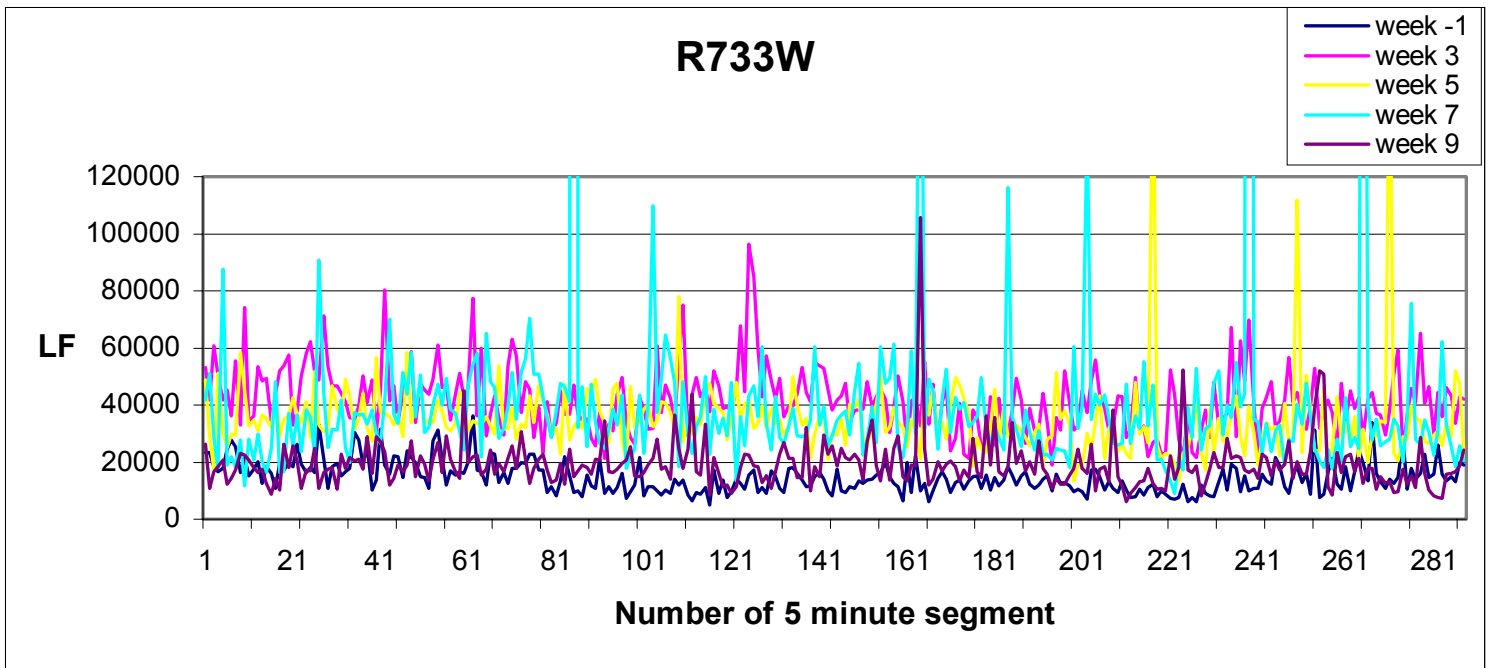

Figure B.18 LF R733W 


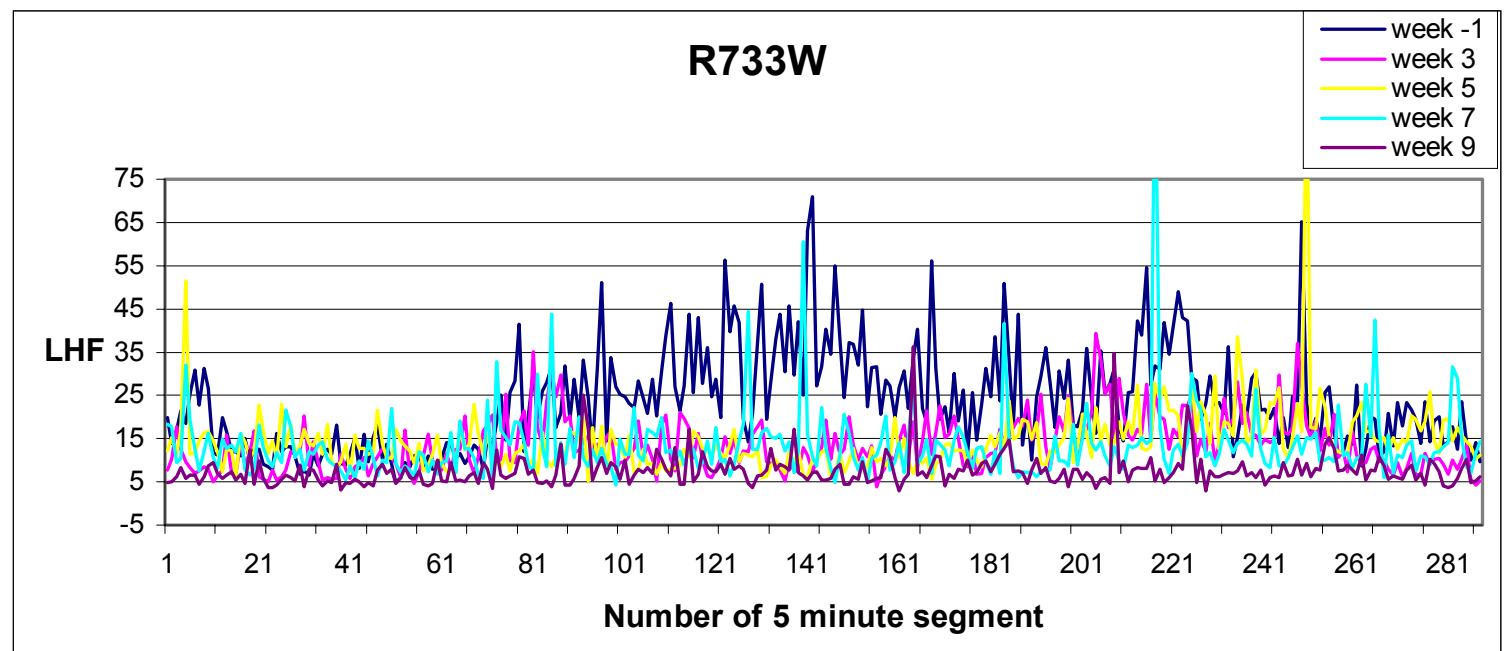

Figure B.19 LHF R733W

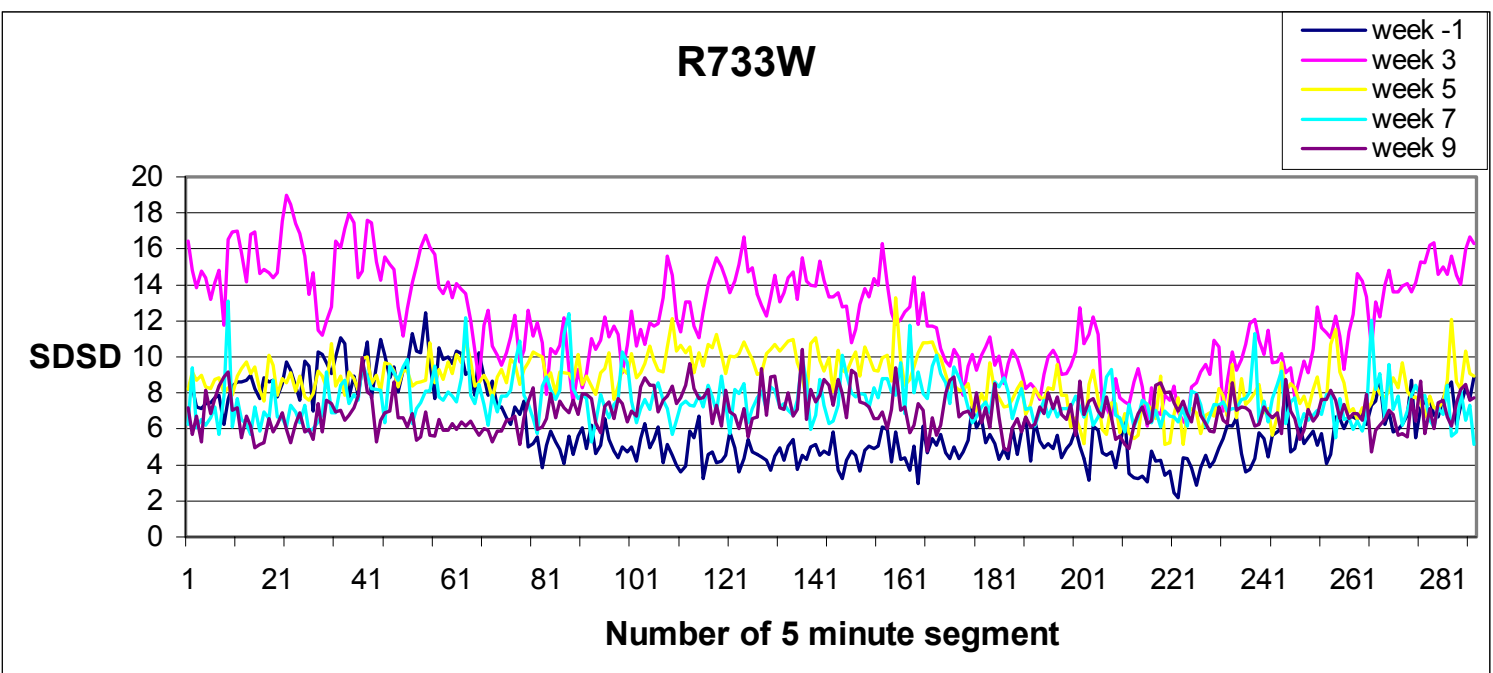

Figure B.20 SDSD R733W

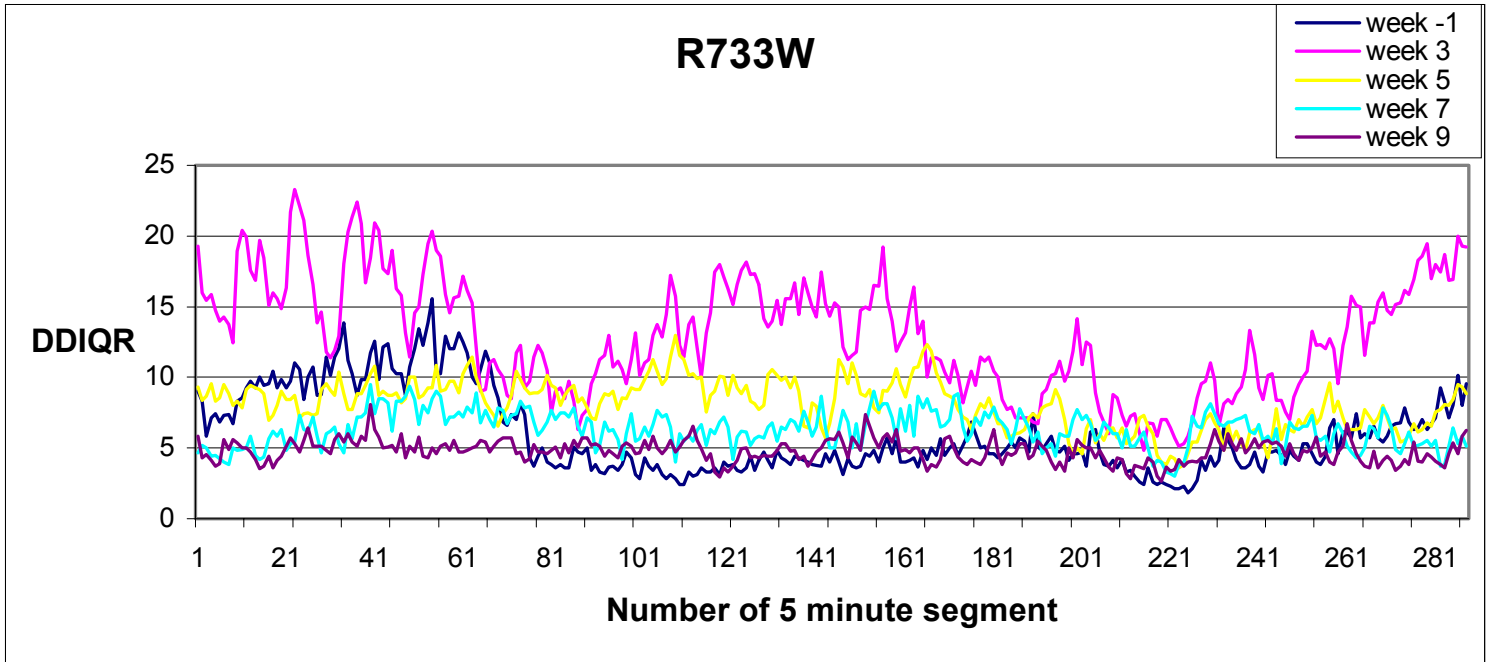

Figure B.21 DDIQR R733W 


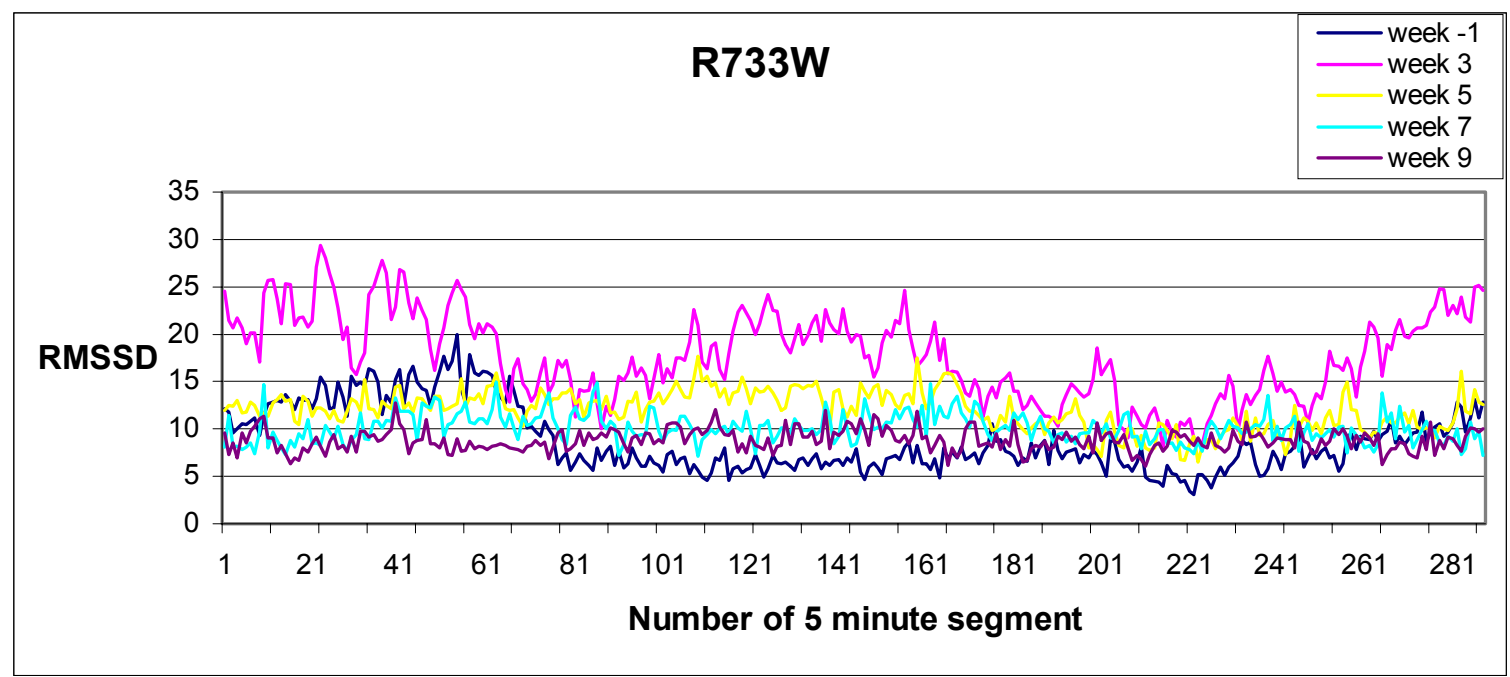

Figure B.22 RMSSD R733W

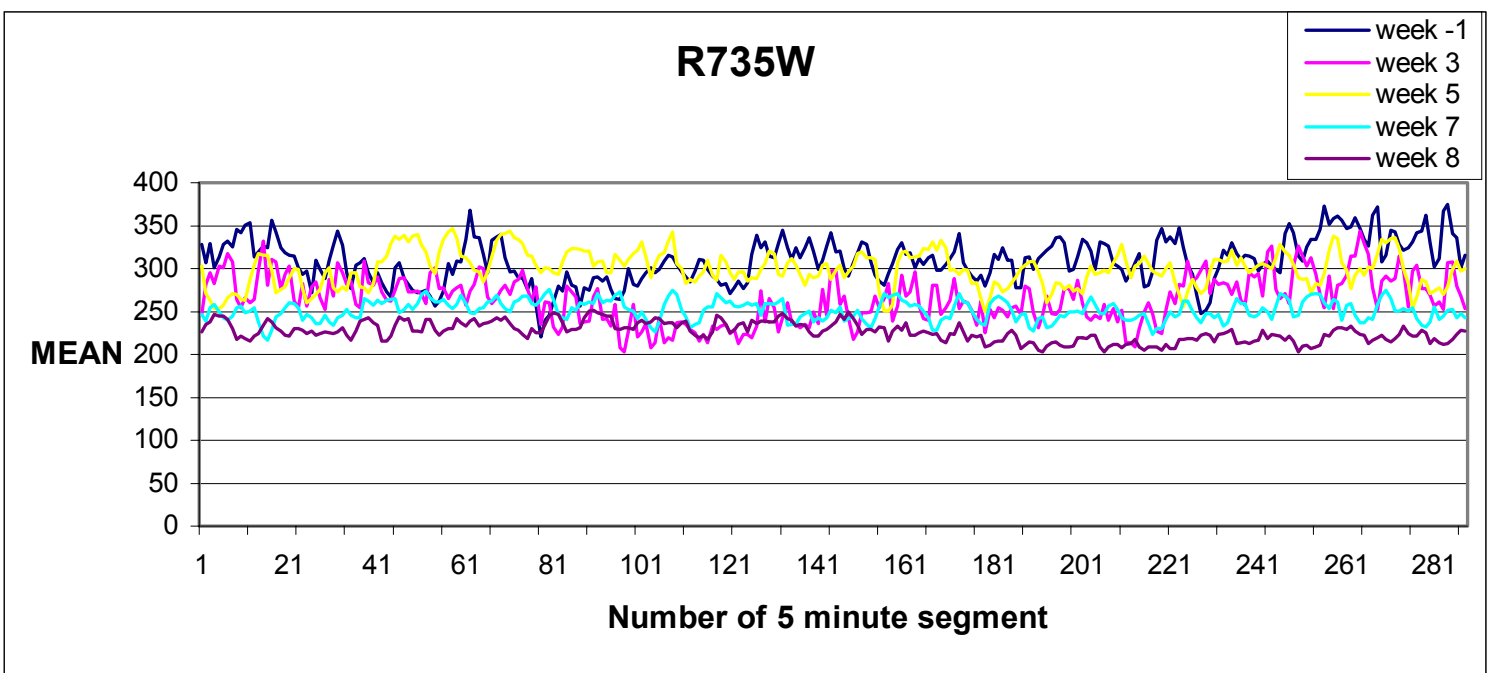

Figure B.23 MEAN R735W

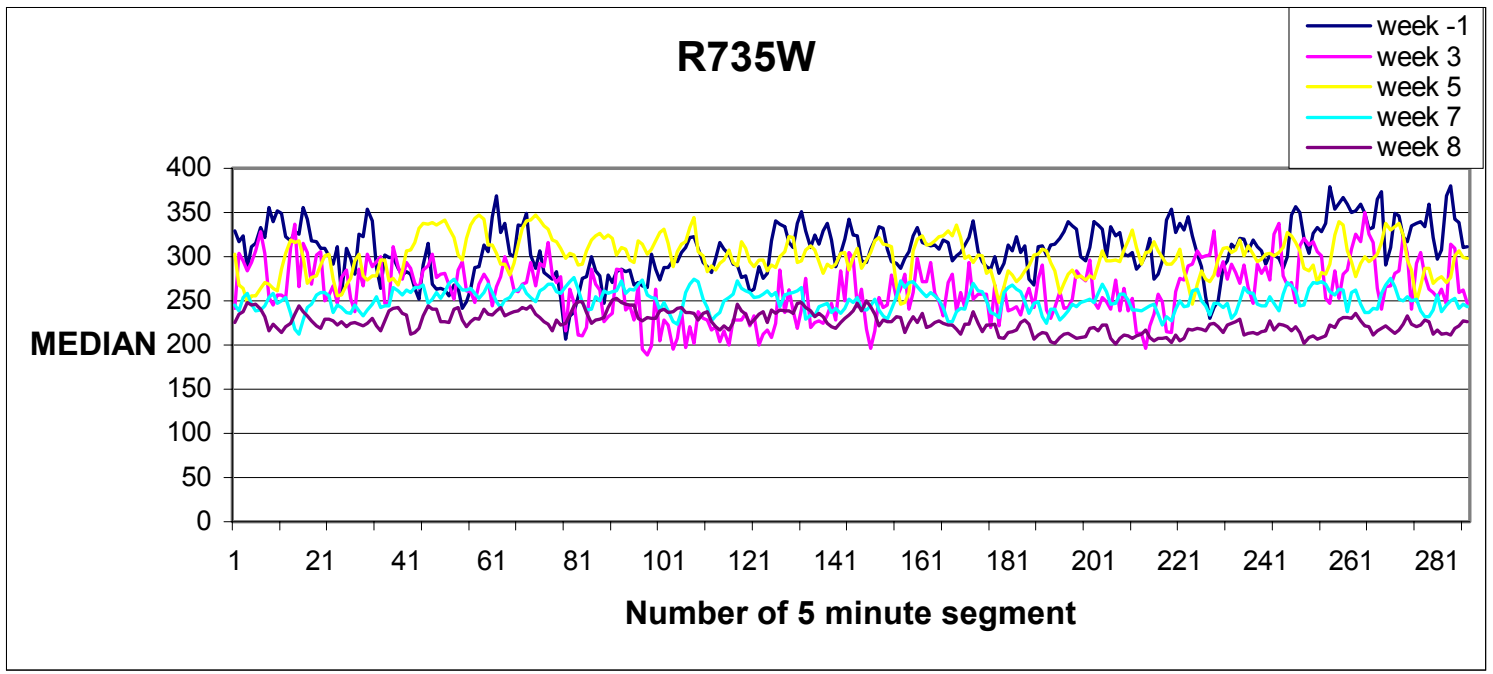

Figure B.24 MEDIAN R735W 


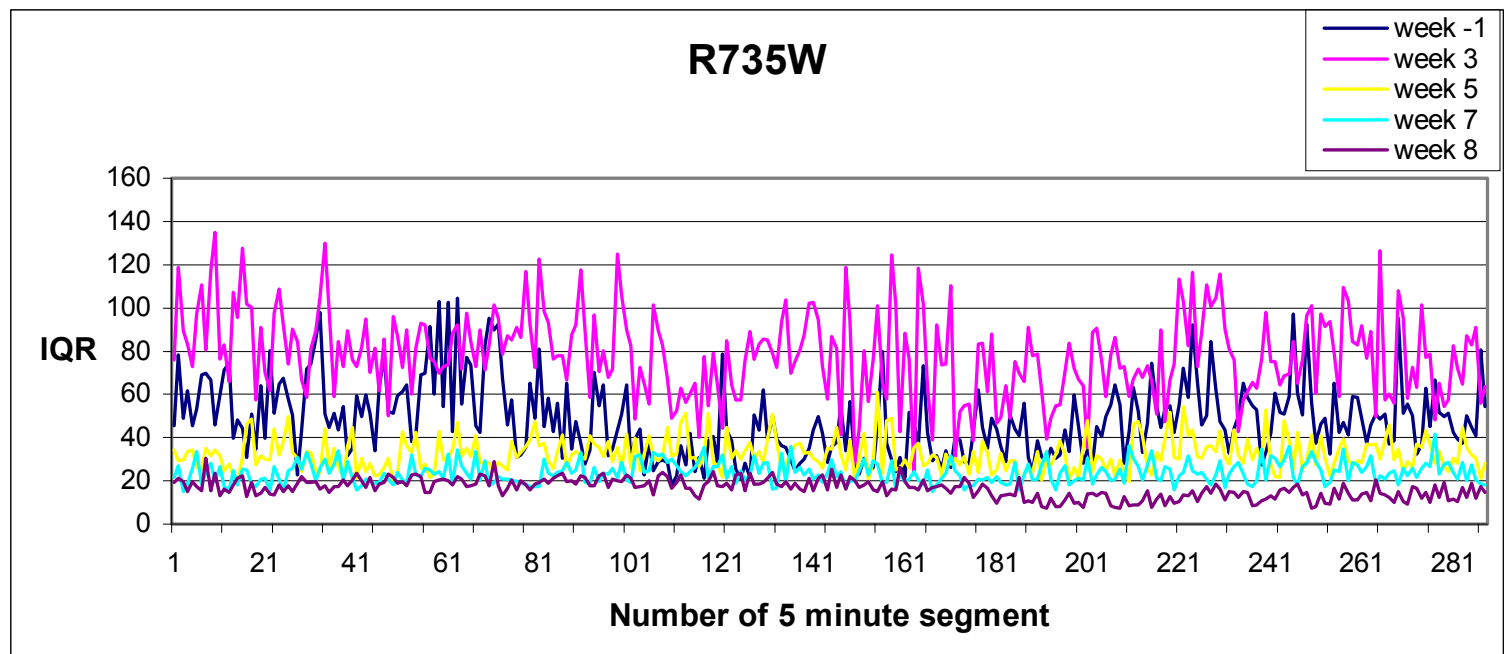

Figure B.25 IQR R735W

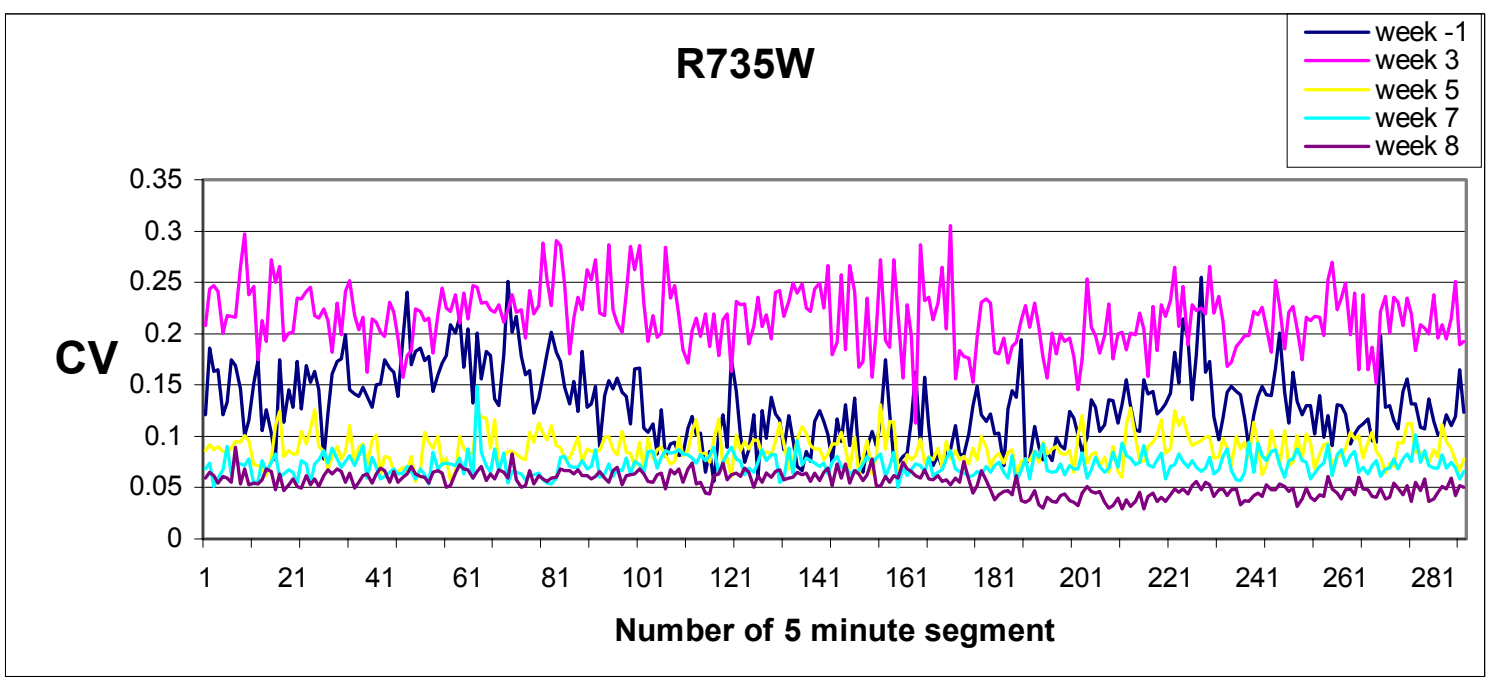

Figure B.26 CV R735W

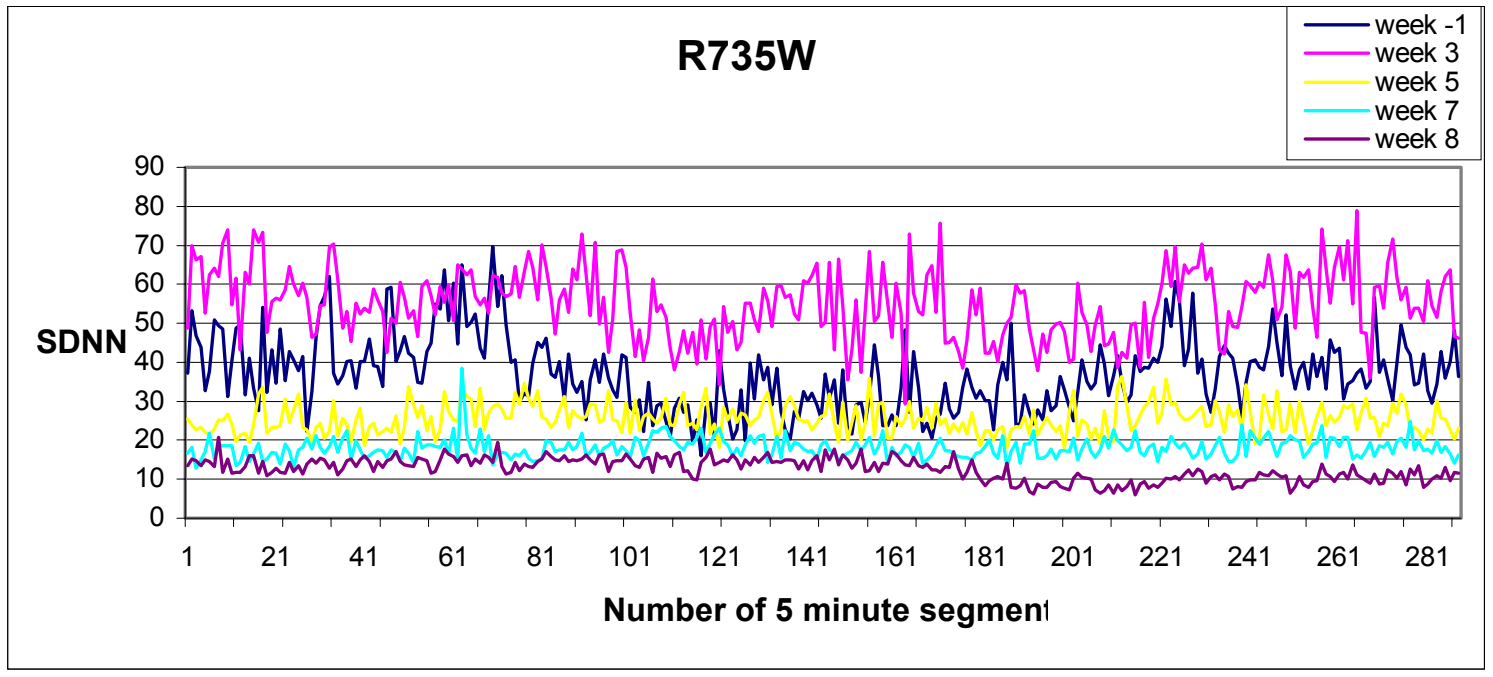

Figure B.27 SDNN R735W 


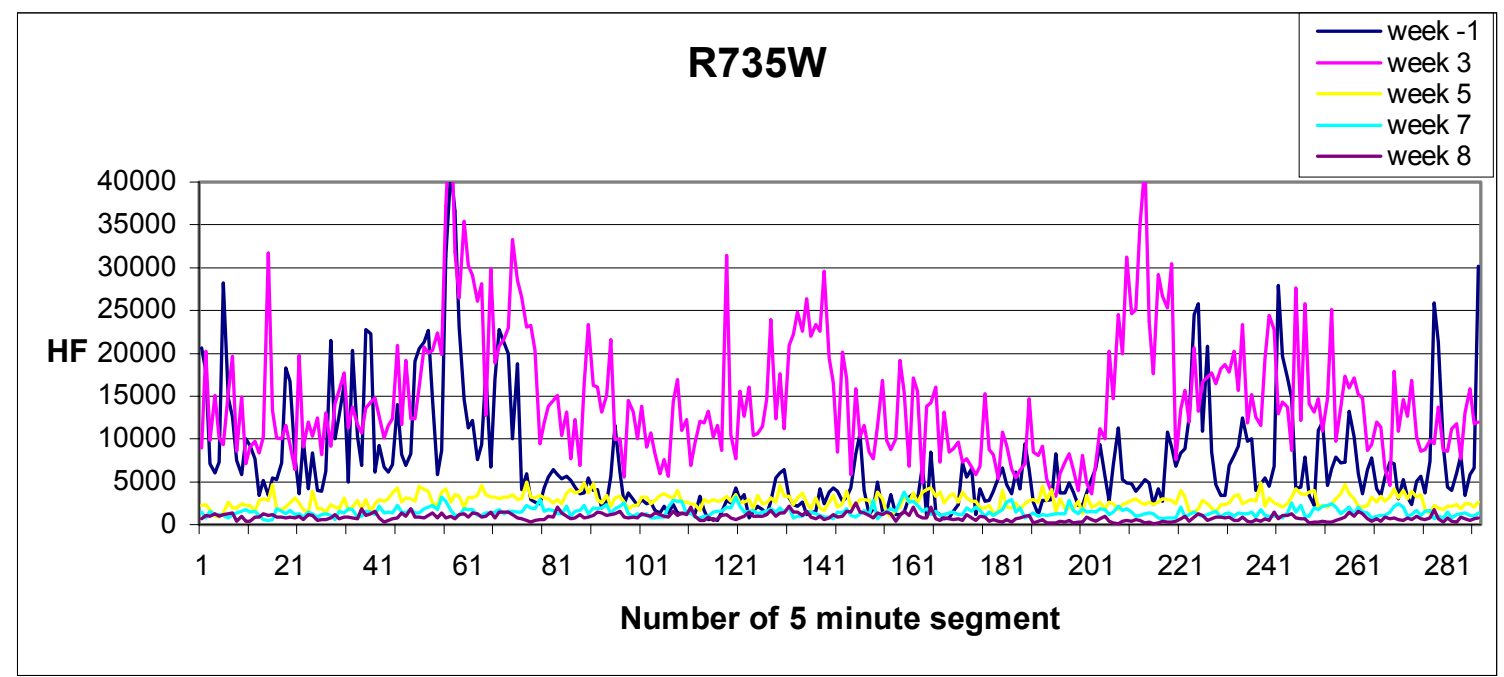

Figure B.28 HF R735W

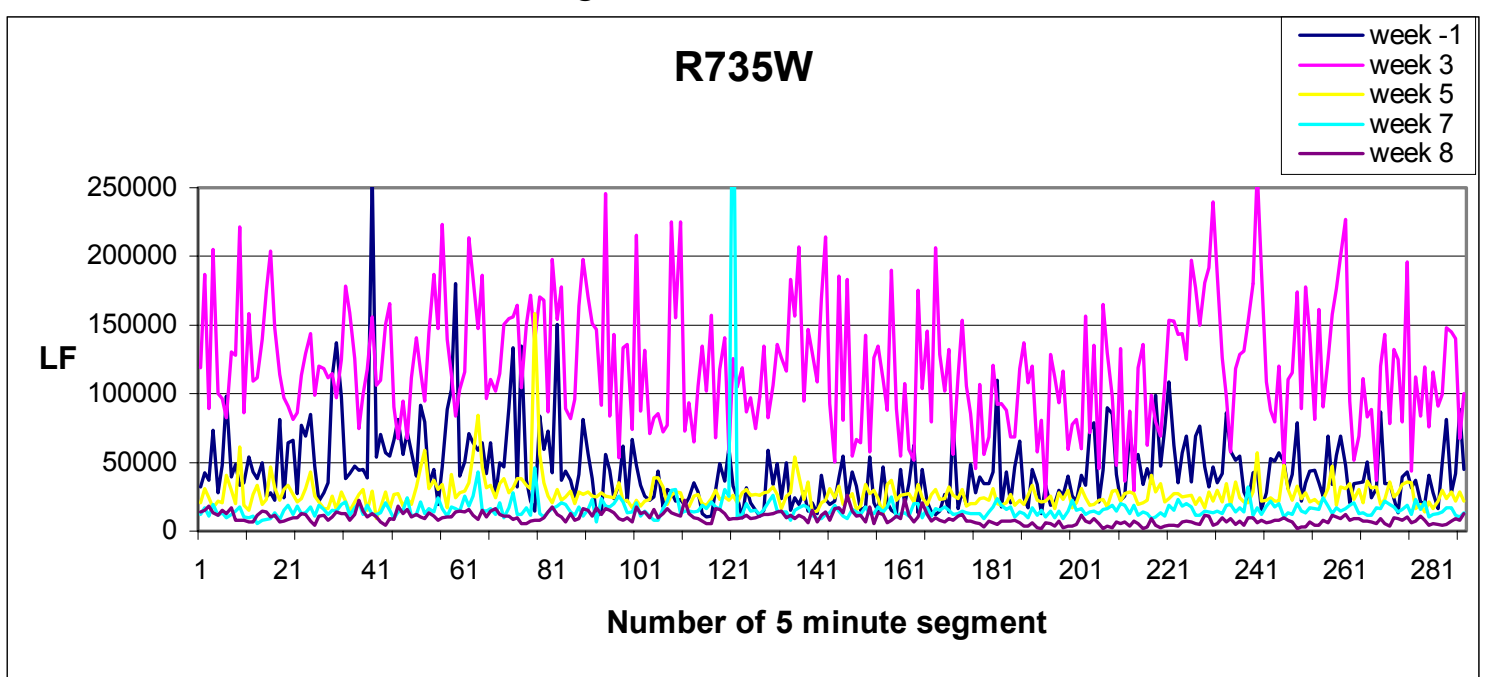

Figure B.29 LF R735W

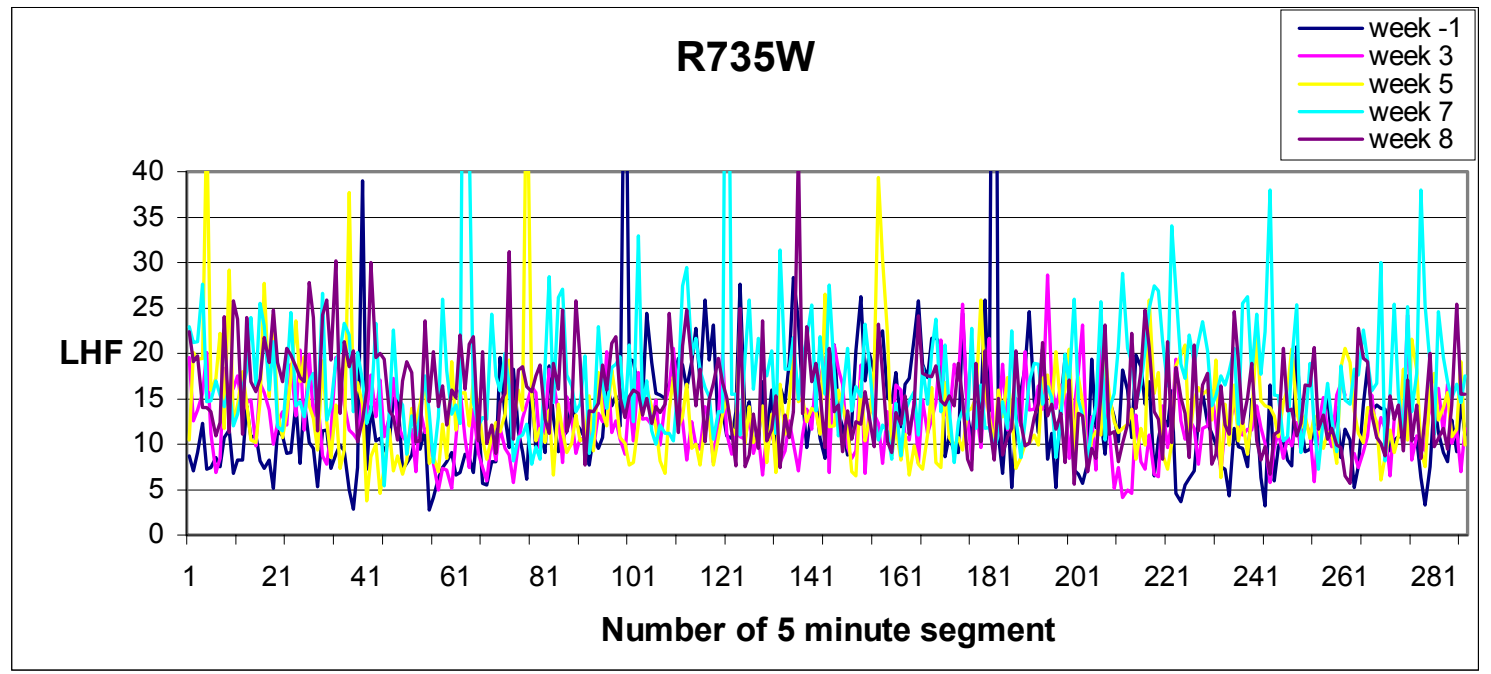

Figure B.30 LHF R735W 


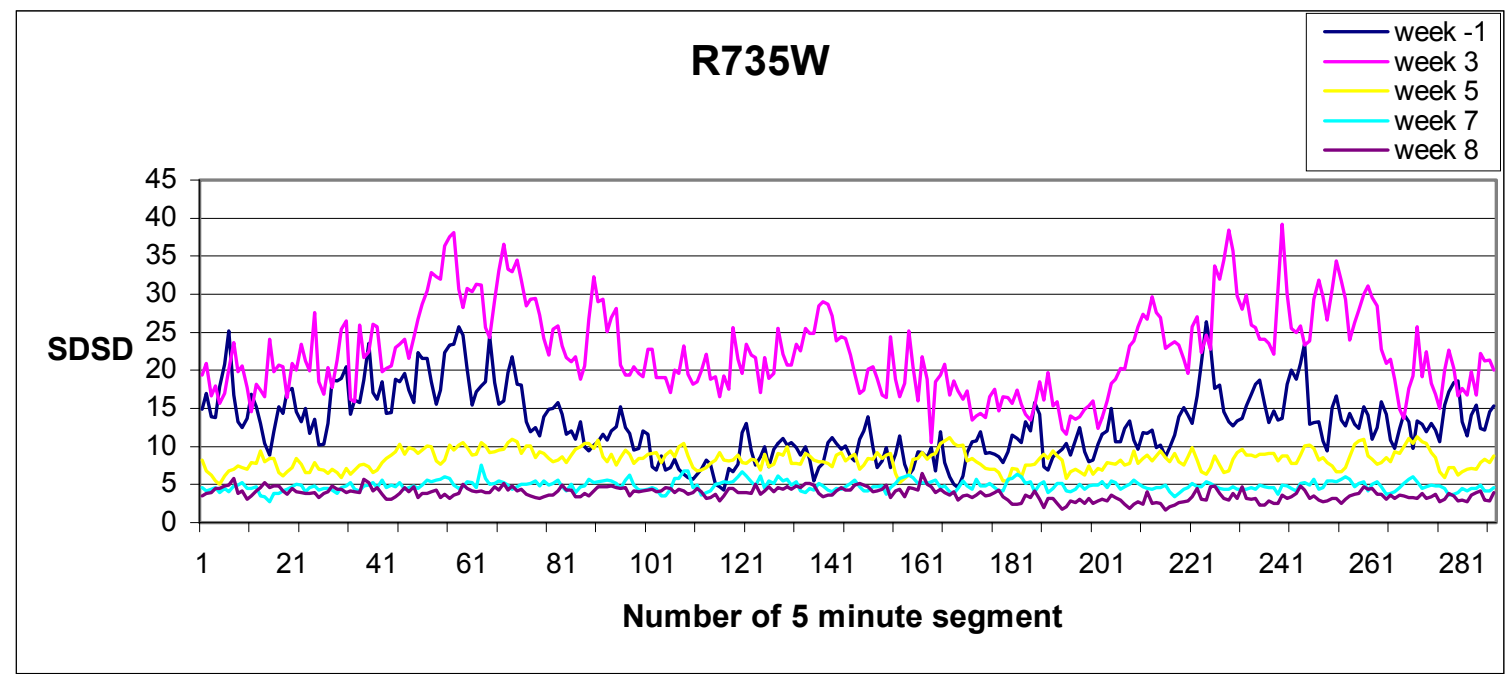

Figure B.31 SDSD R735W

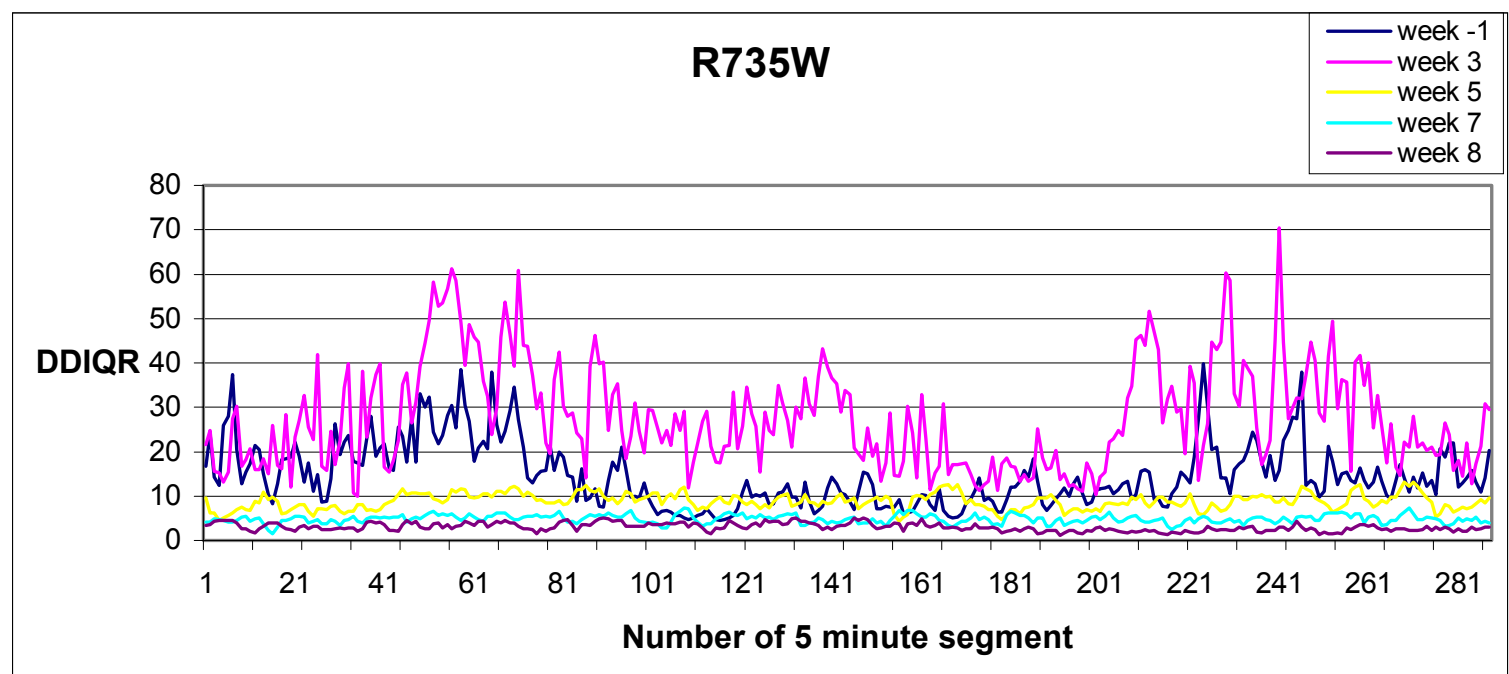

Figure B.32 DDIQR R735W

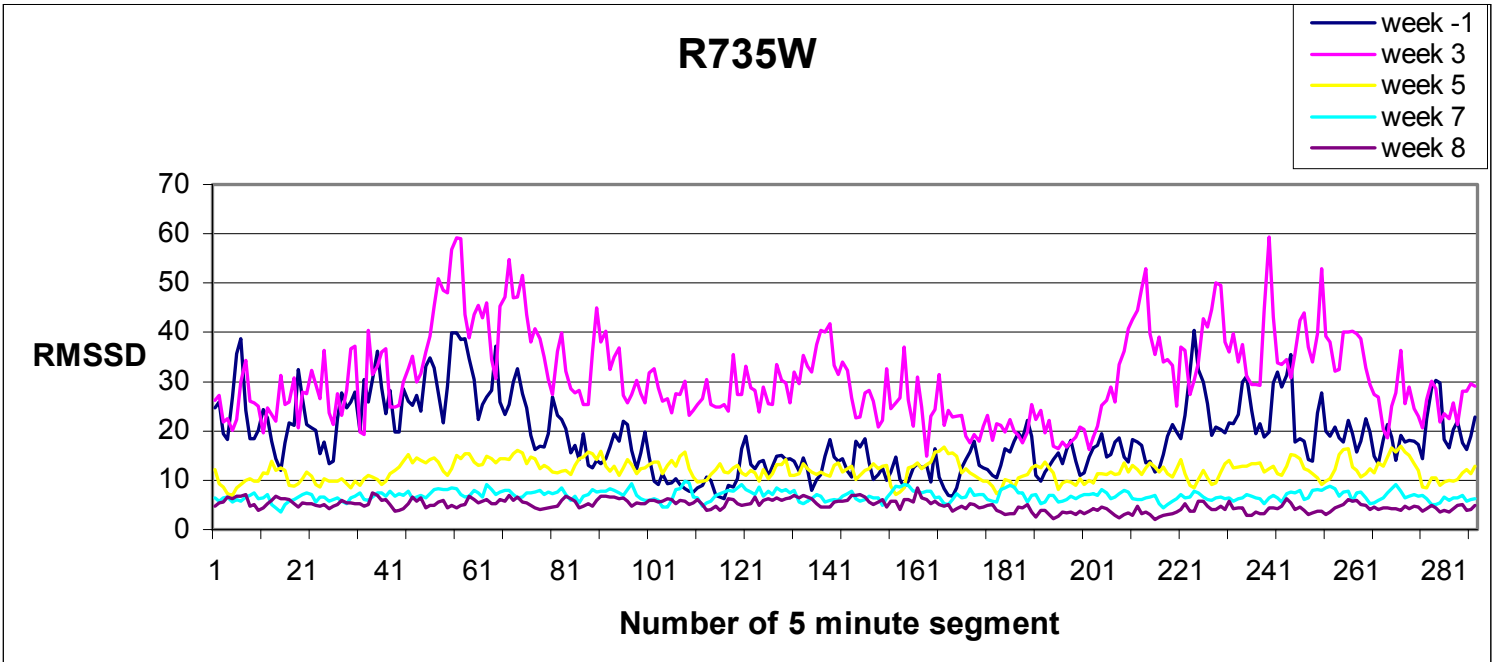

Figure B.33 RMSSD R735W 
Appendix C Mixed plots per week, averaged hourly and weekly 


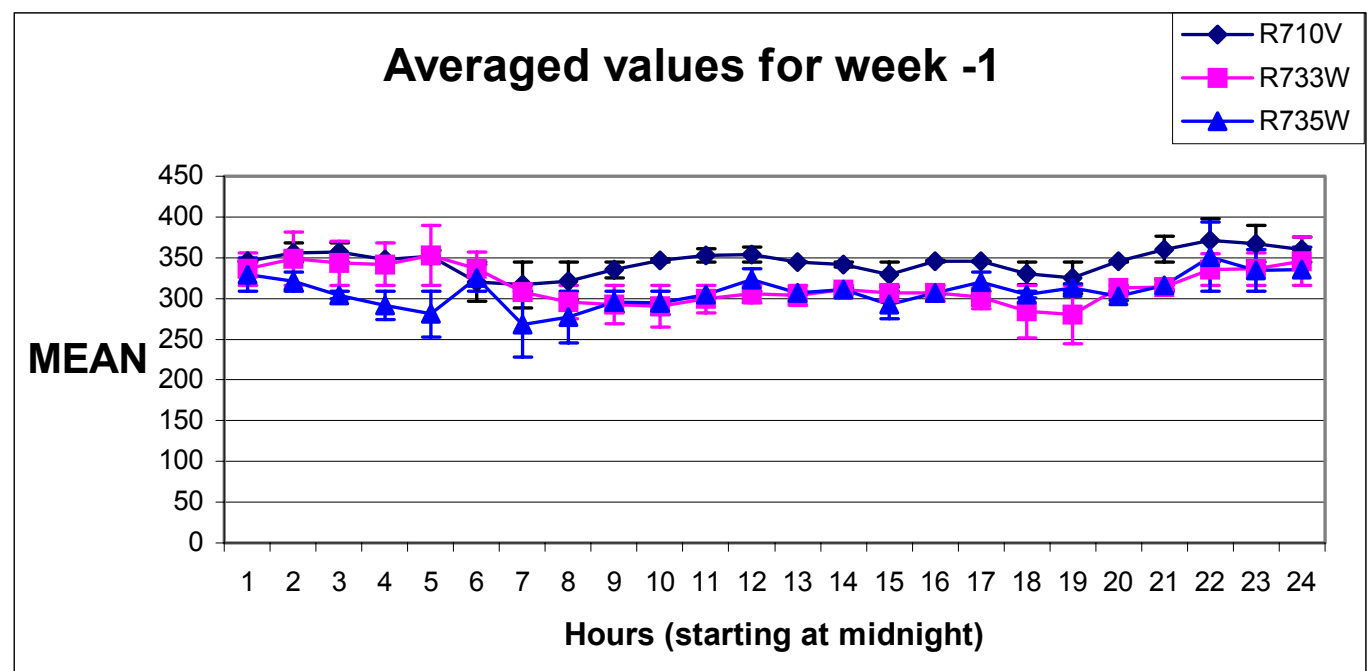

Figure C.1 MEAN week-1

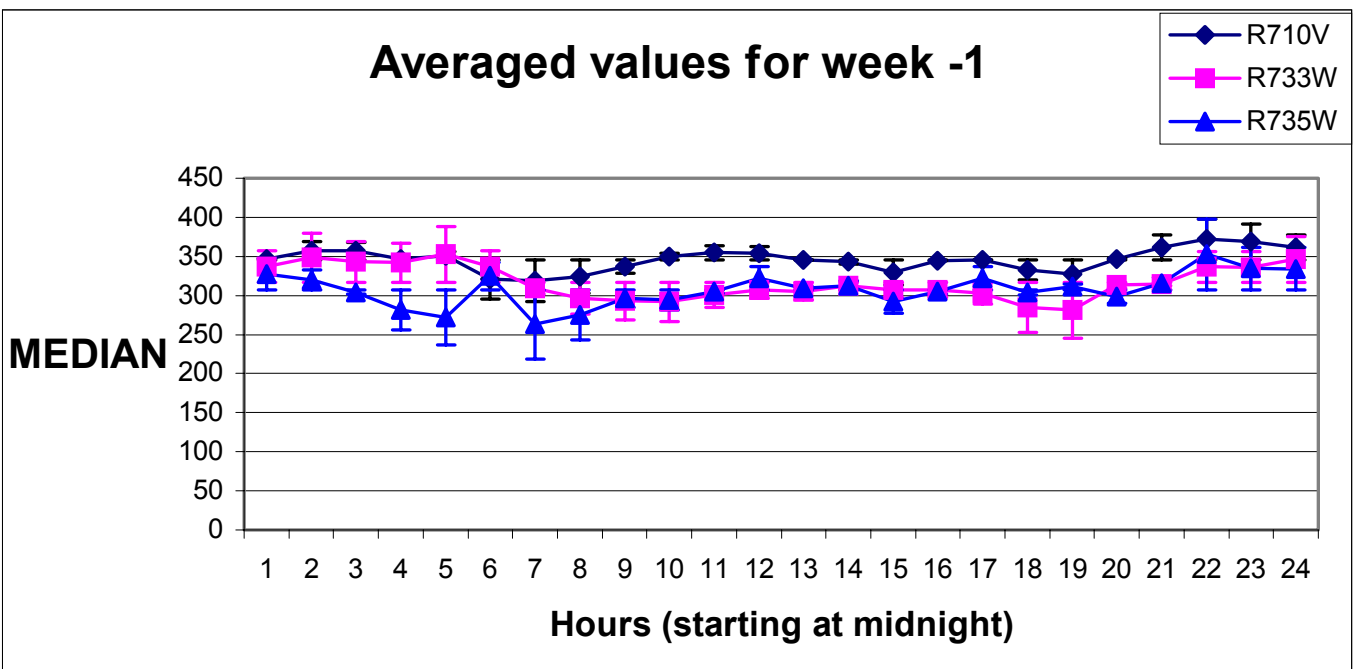

Figure C.2 MEDIAN week-1

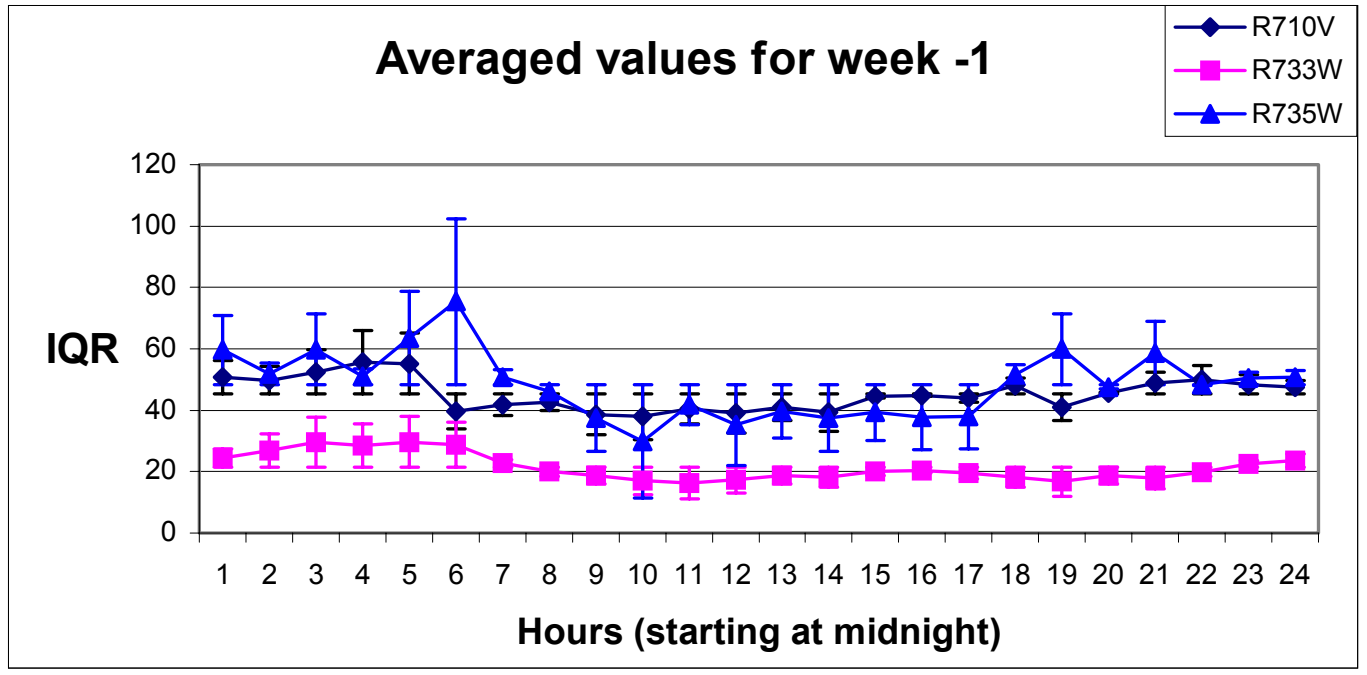

Figure C.3 IQR week-1 


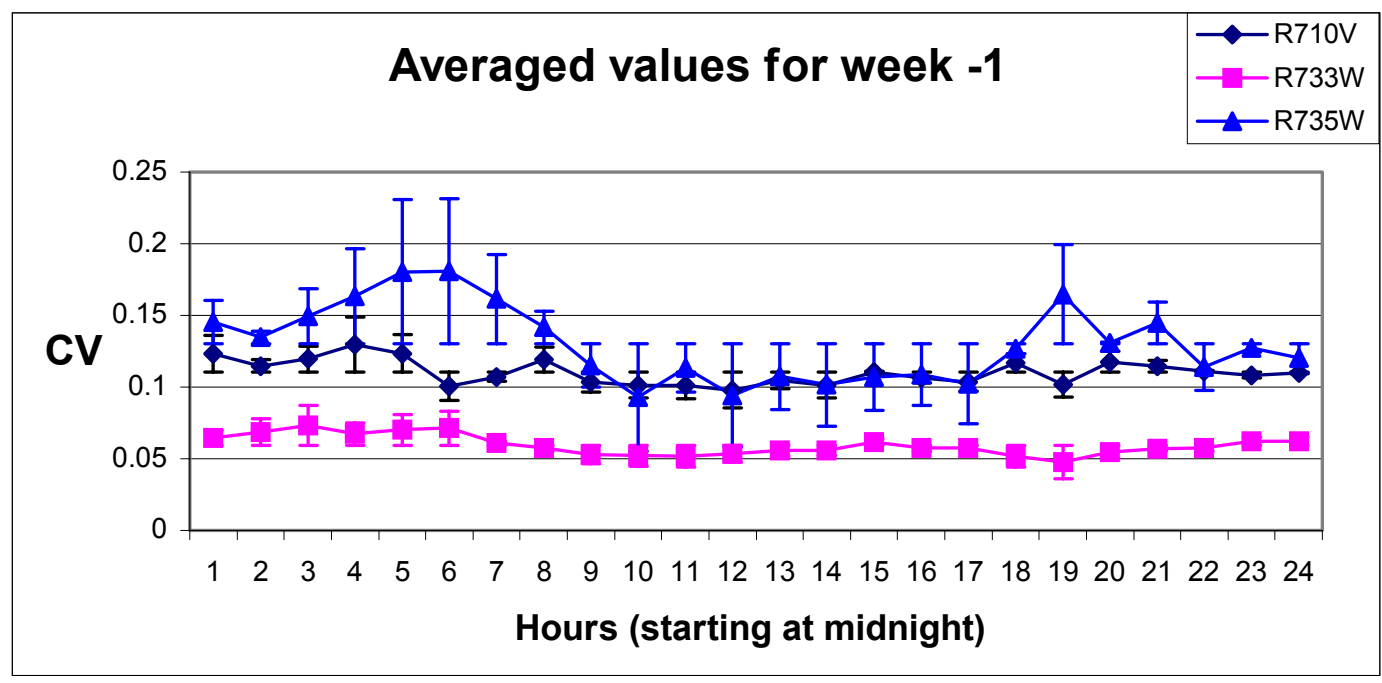

Figure C.4 CV week-1

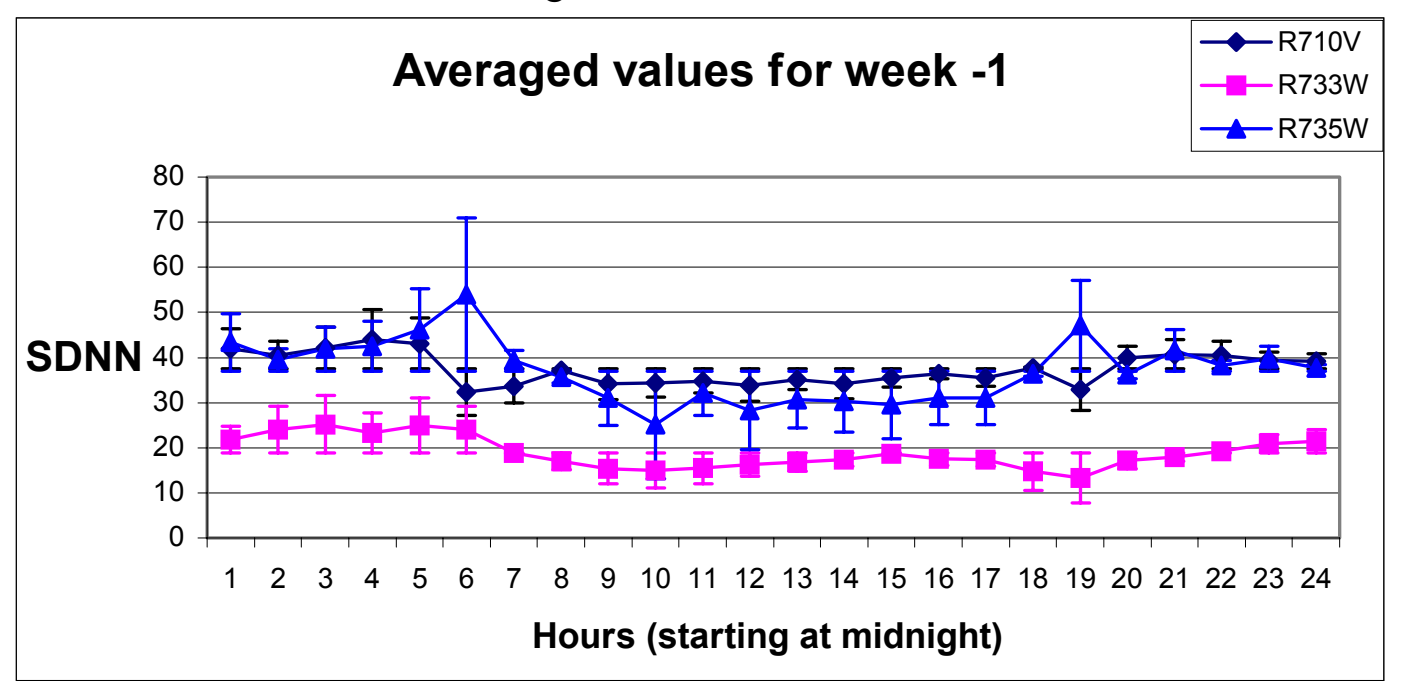

Figure C.5 SDNN week-1

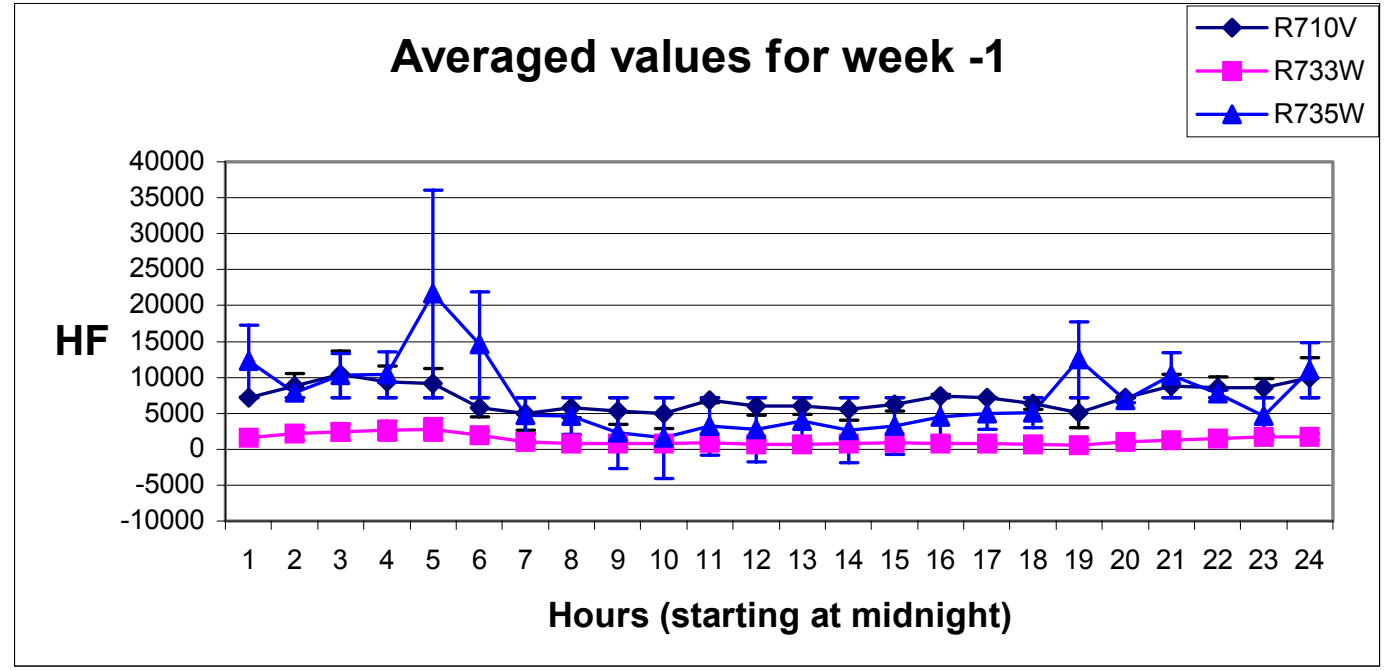

Figure C.6 HF week-1 


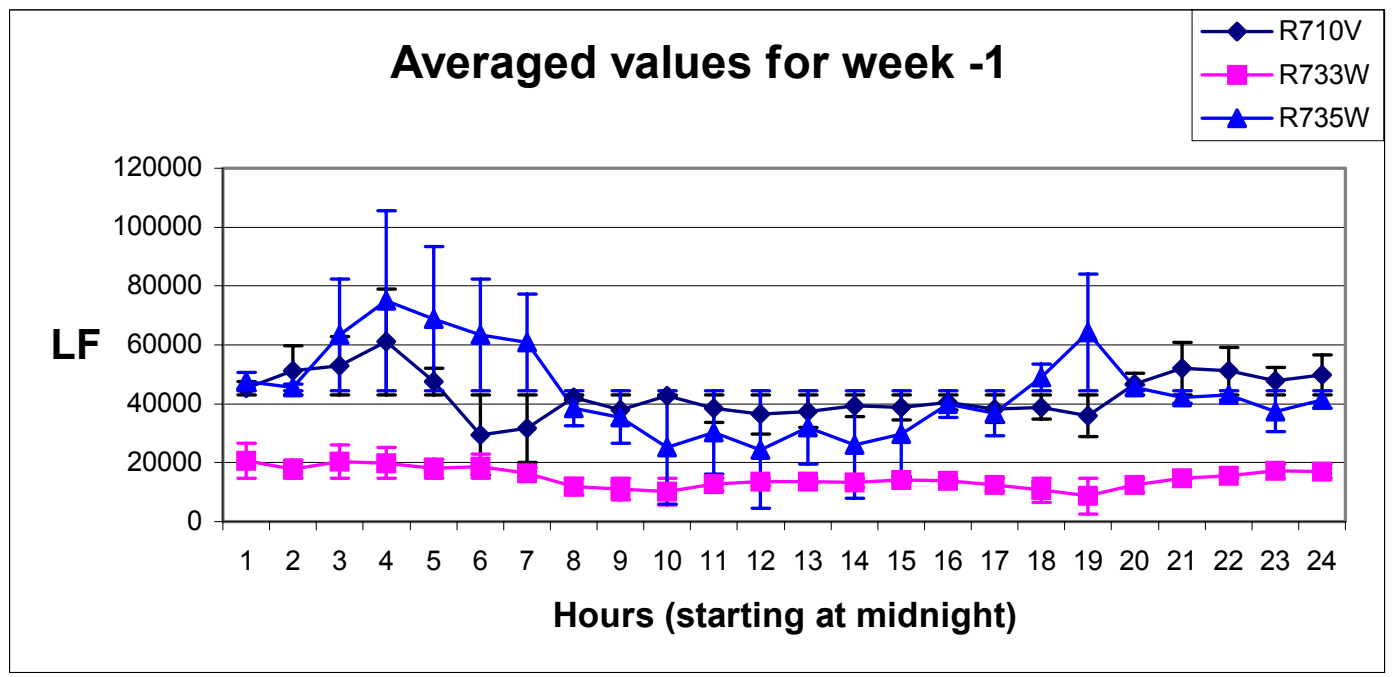

Figure C.7 LF week-1

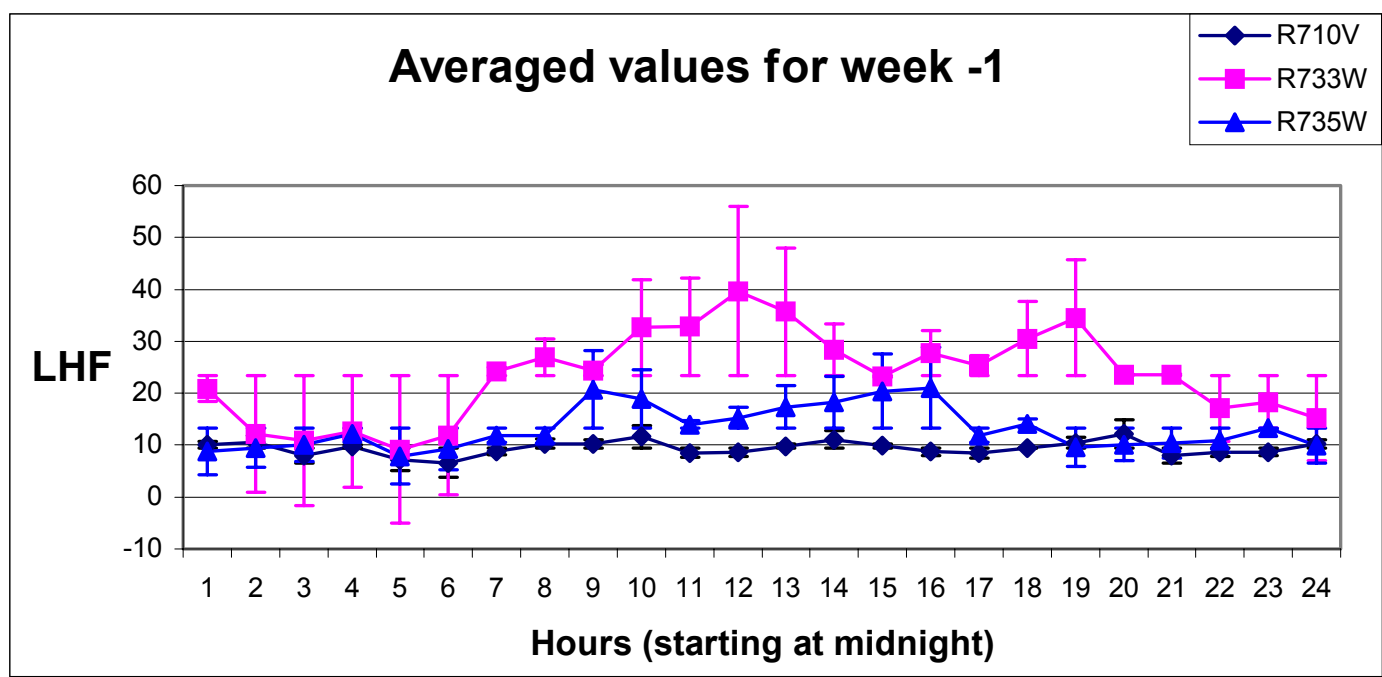

Figure C.8 LHF week-1

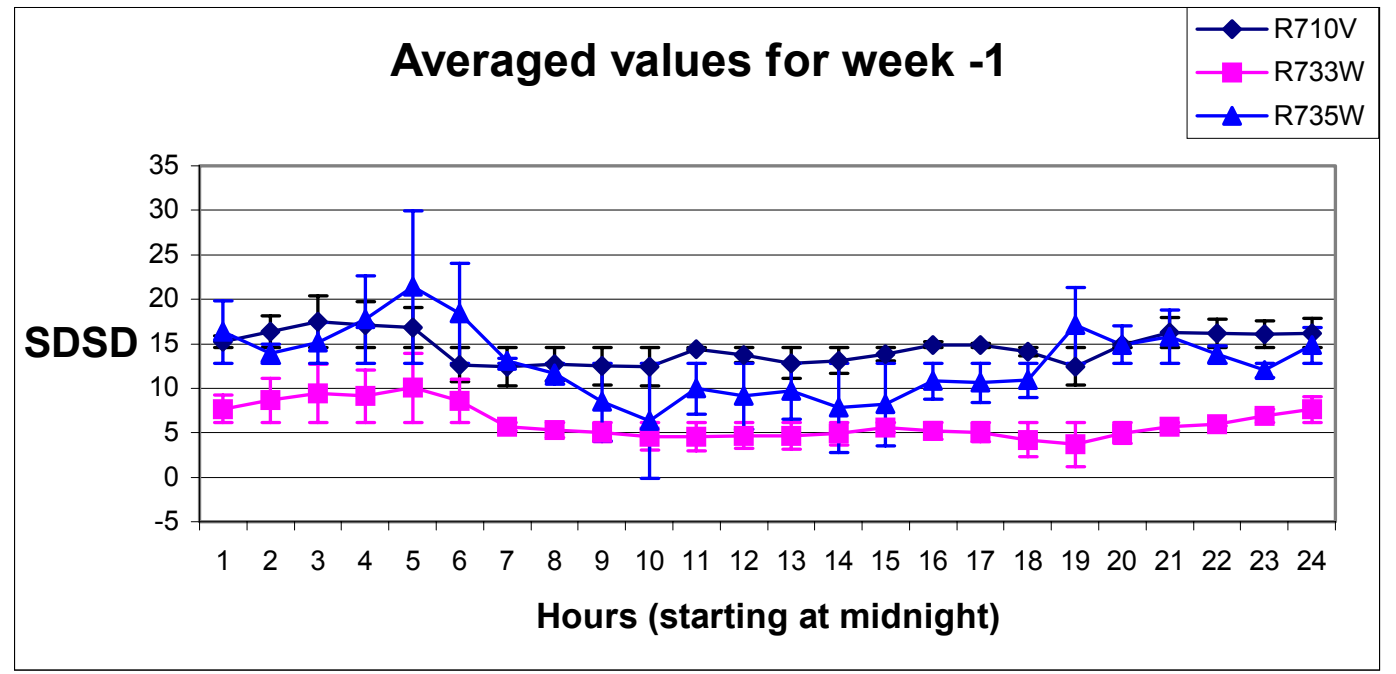

Figure C.9 SDSD week-1 


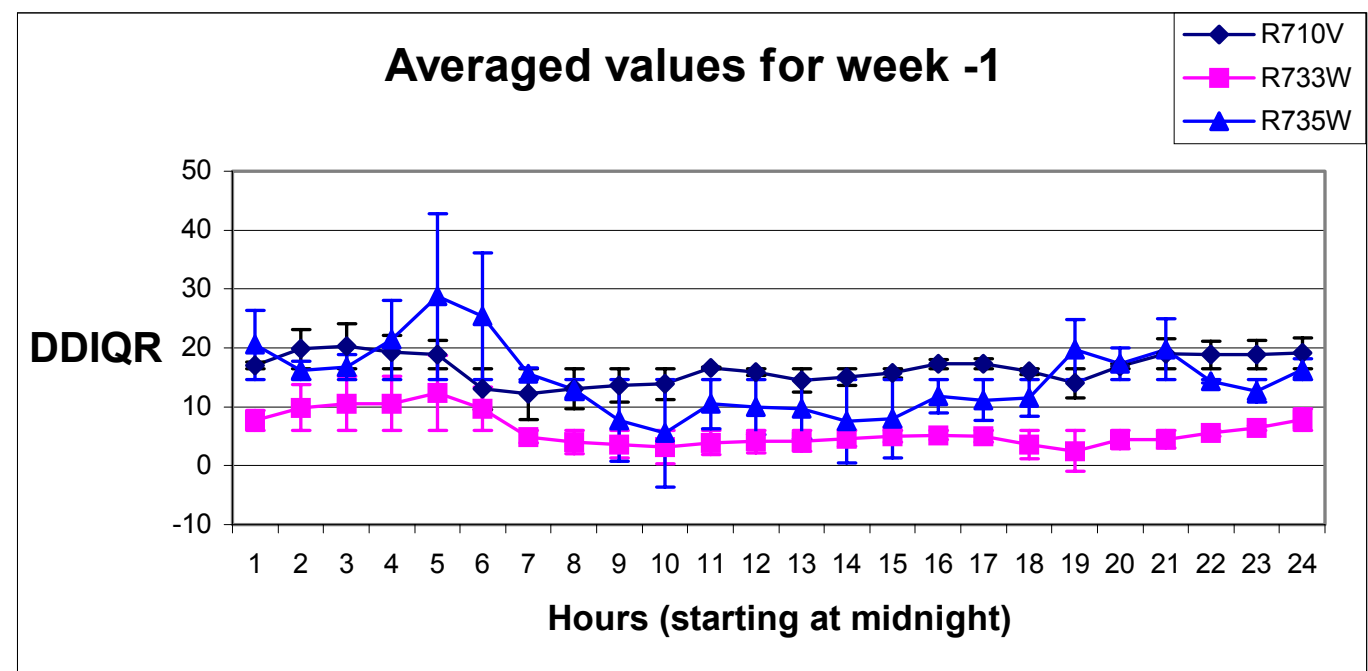

Figure C.10 DDIQR week-1

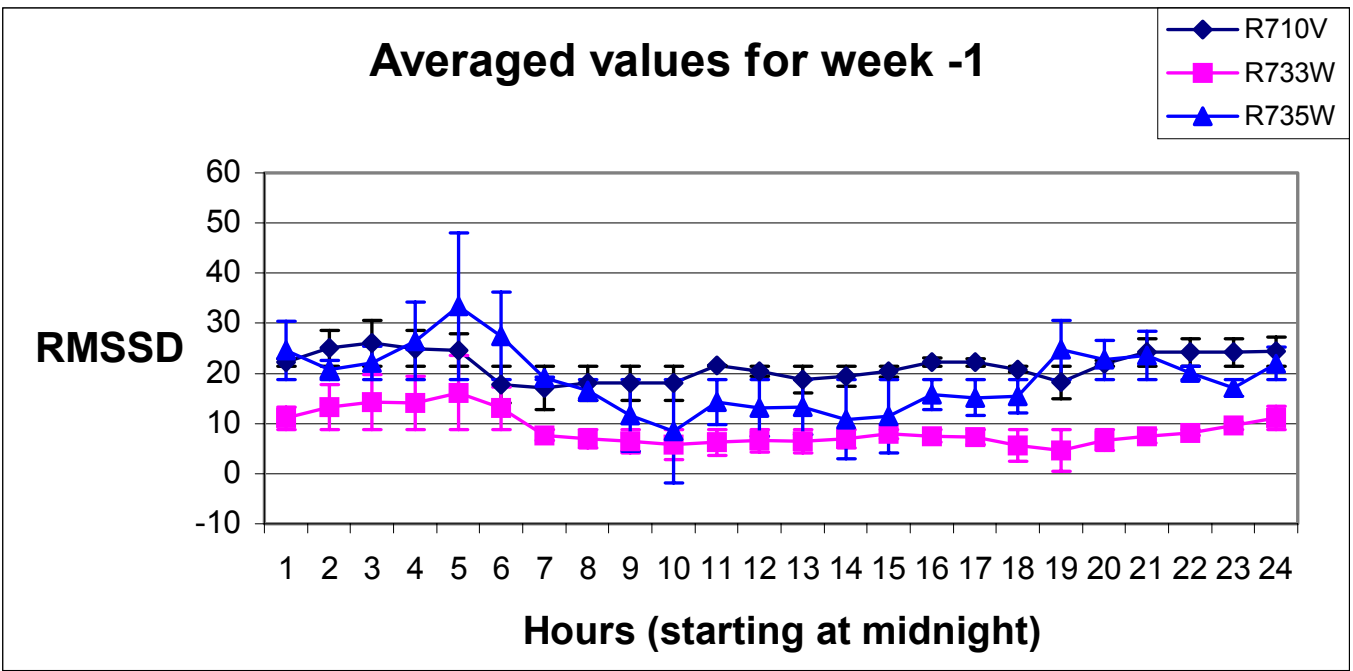

Figure C.11 RMSSD week-1

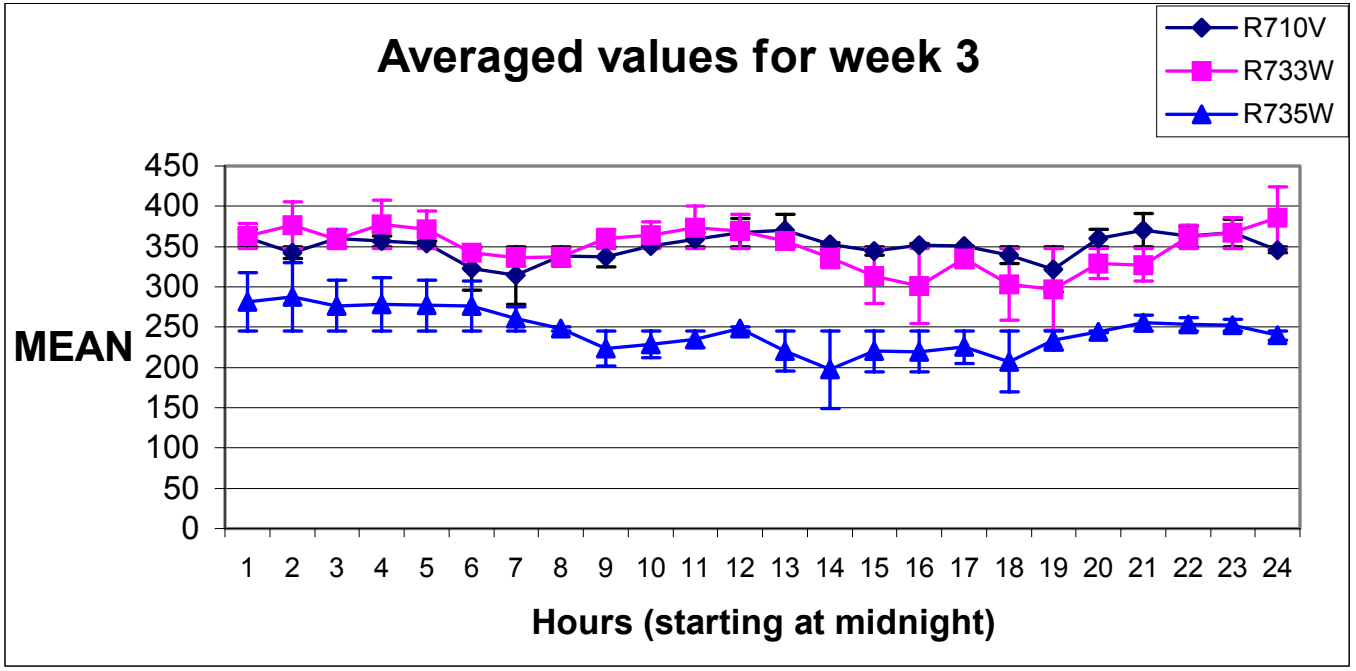

Figure C.12 MEAN week 3 


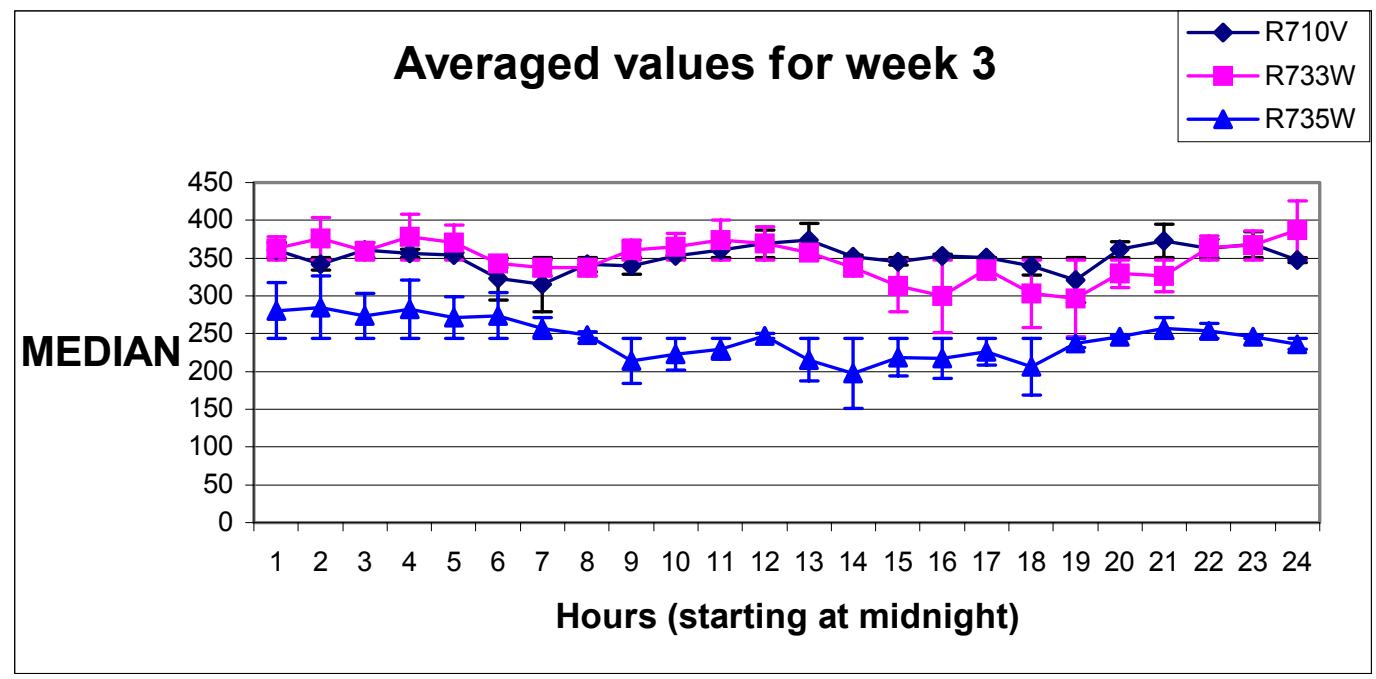

Figure C.13 MEDIAN week 3

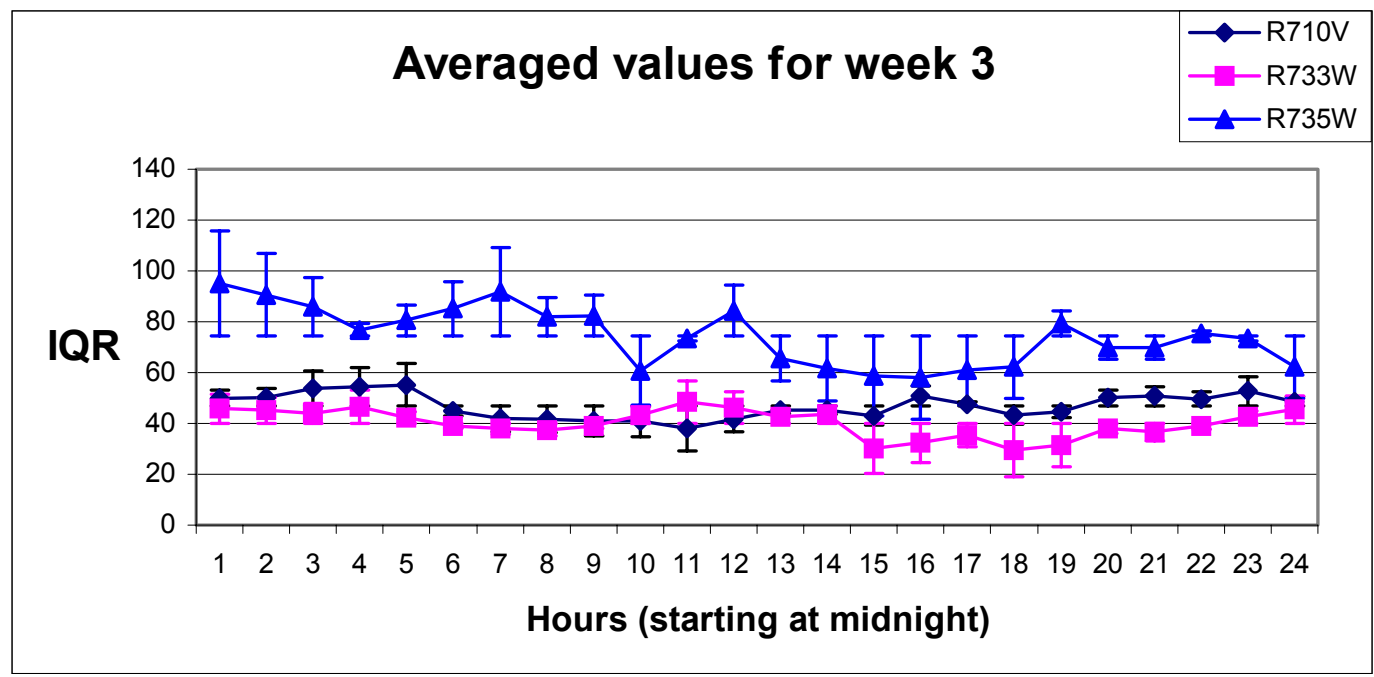

Figure C.14 IQR week 3

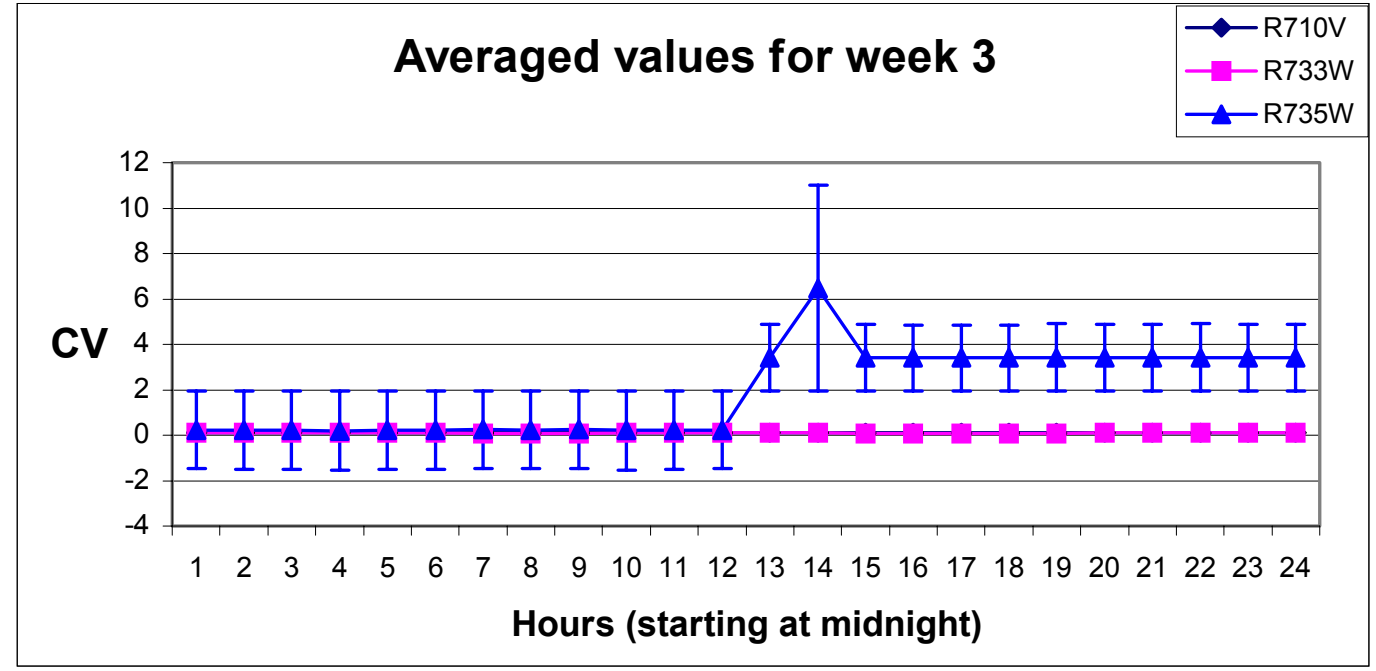

Figure C.15 CV week 3 


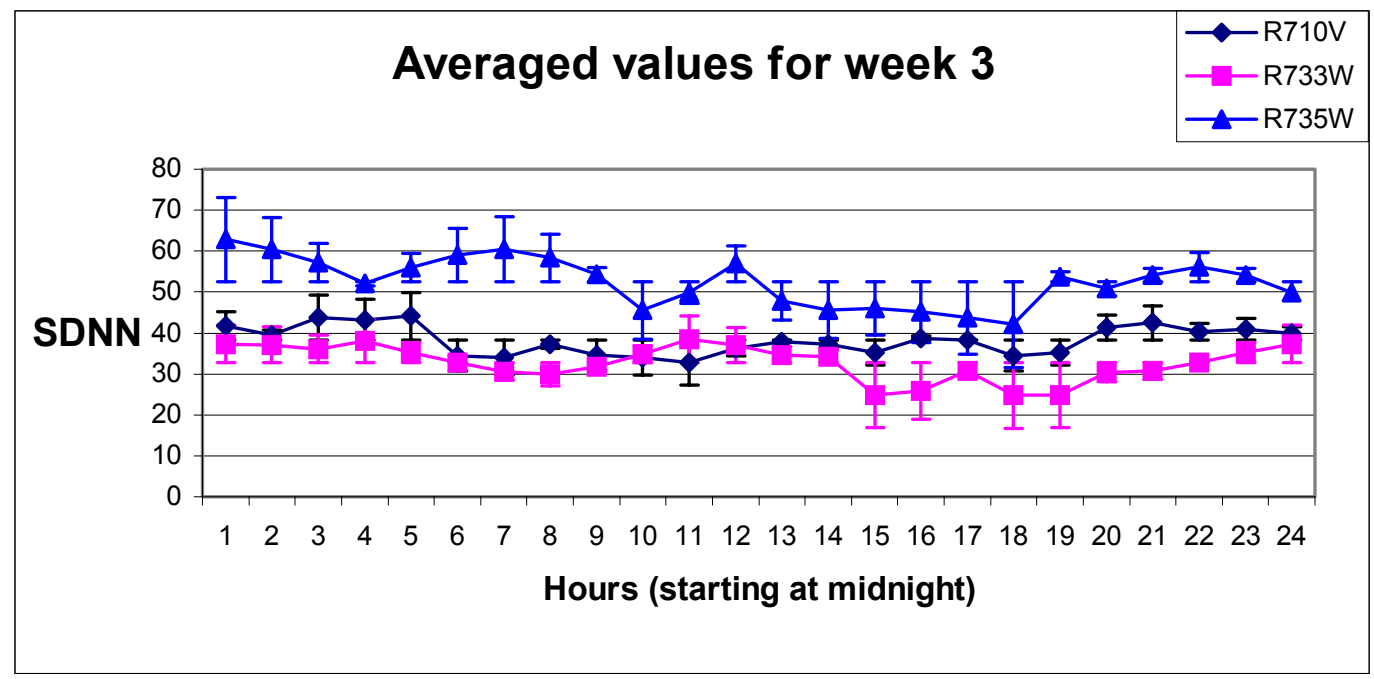

Figure C.16 SDNN week 3

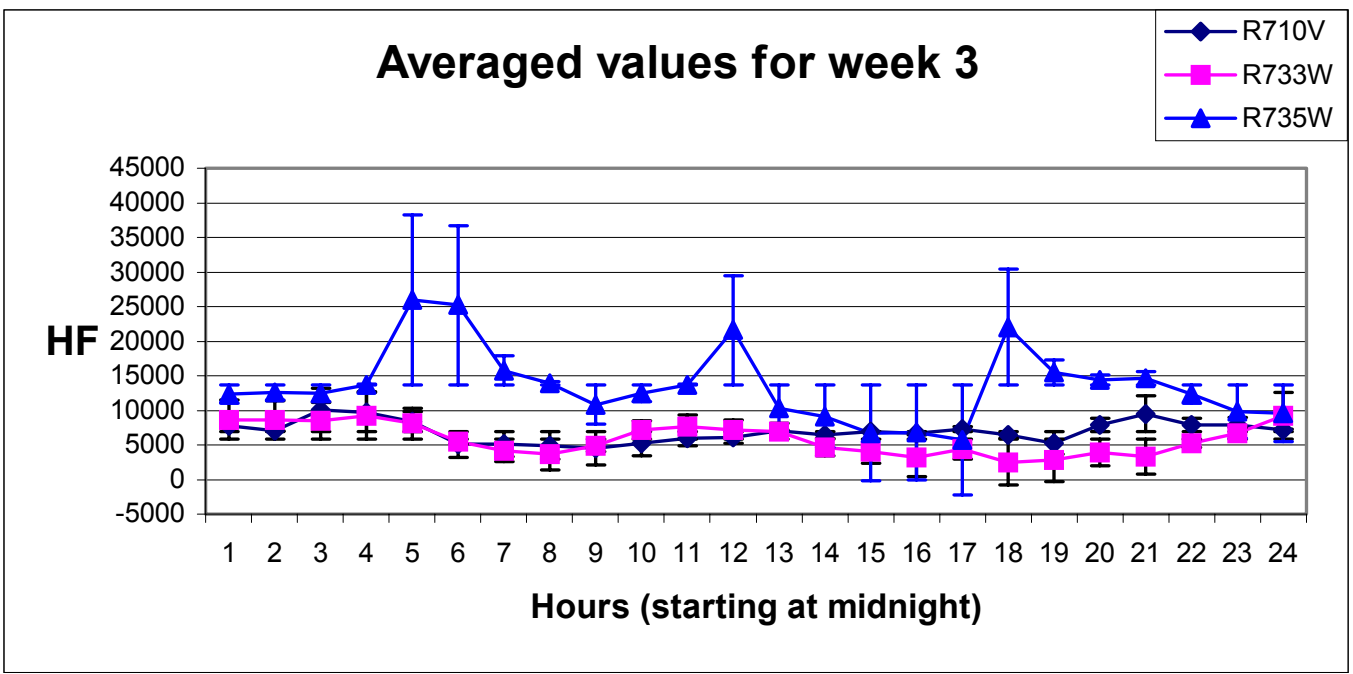

Figure C.17 HF week 3

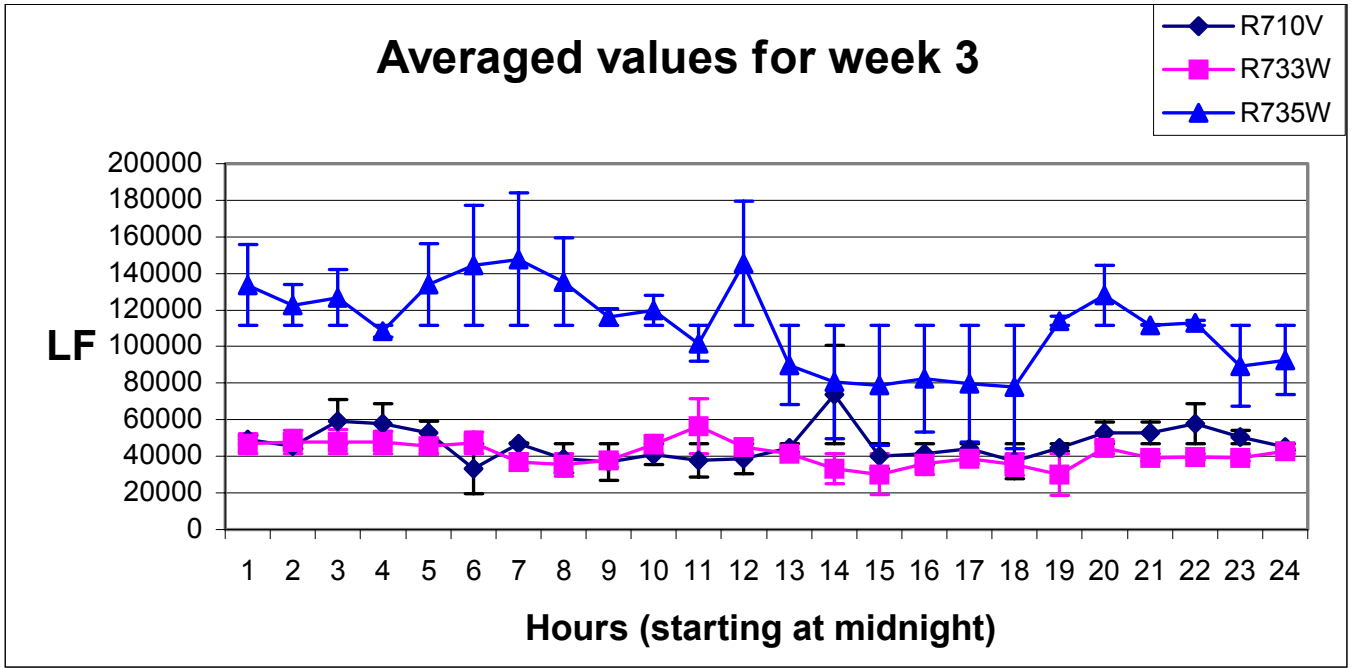

Figure C.18 LF week 3 


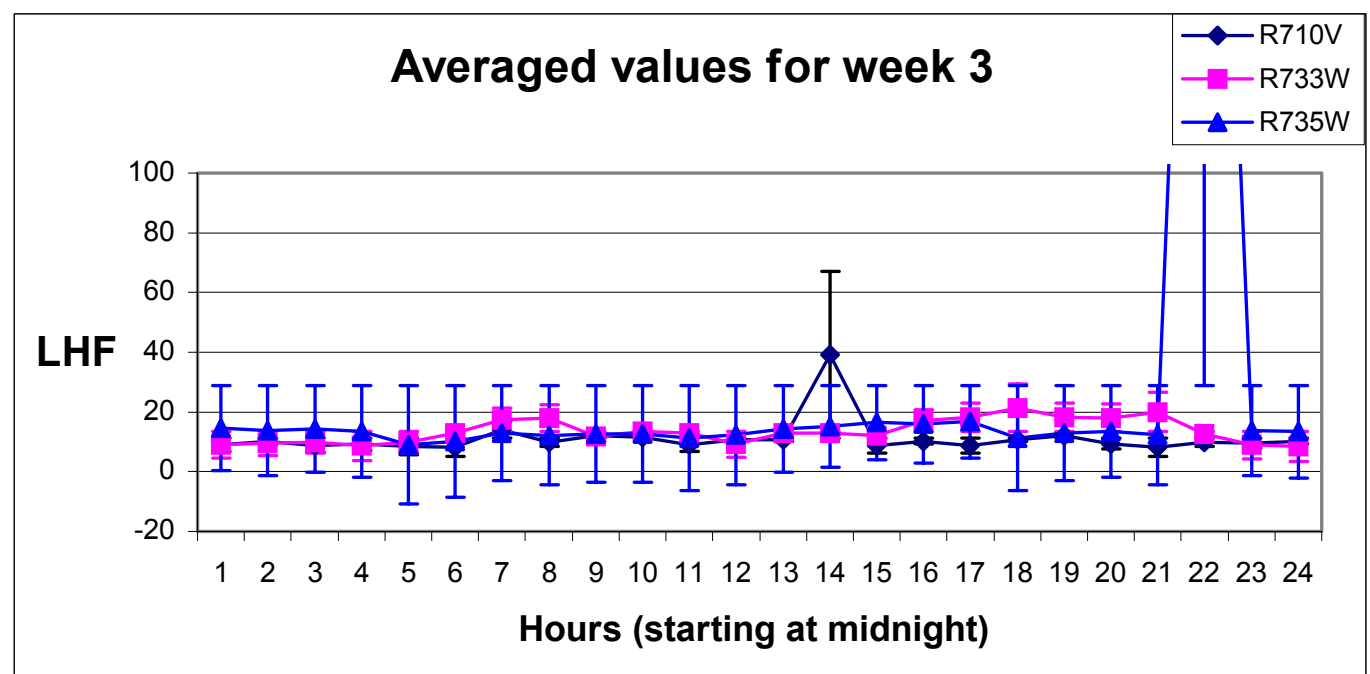

Figure C.19 LHF week 3

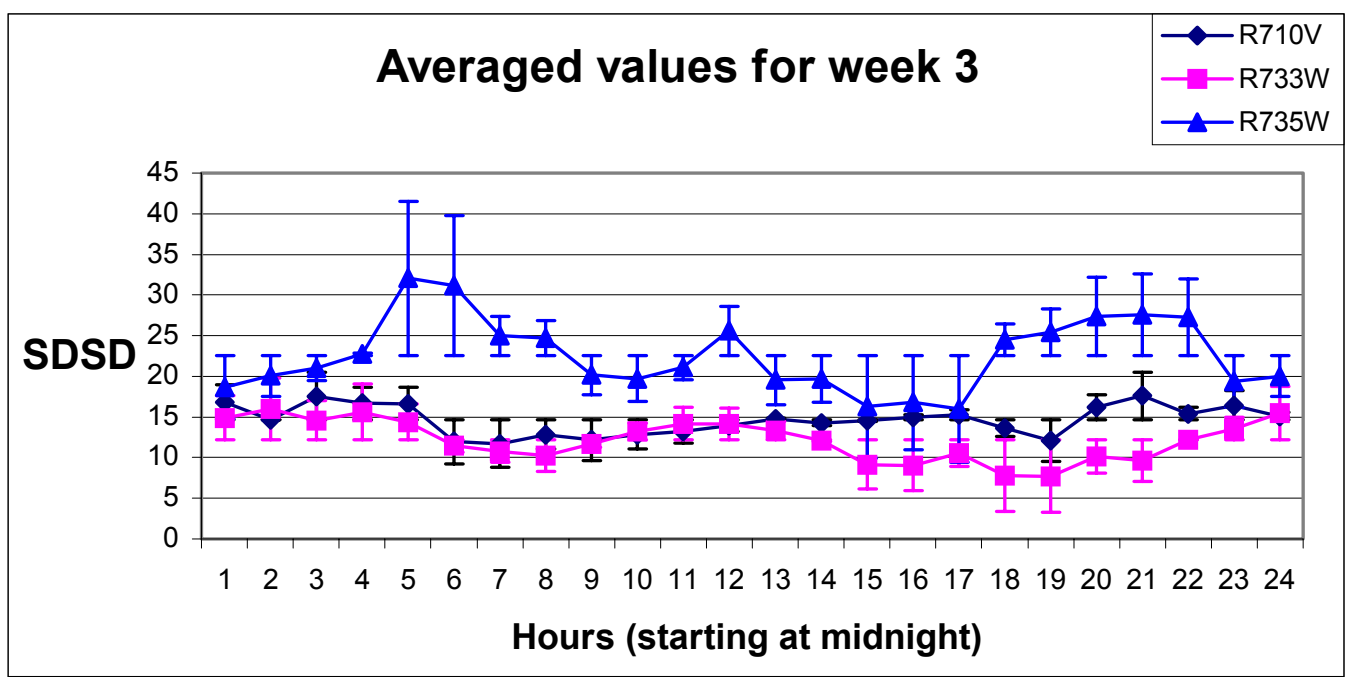

Figure C.20 SDSD week 3

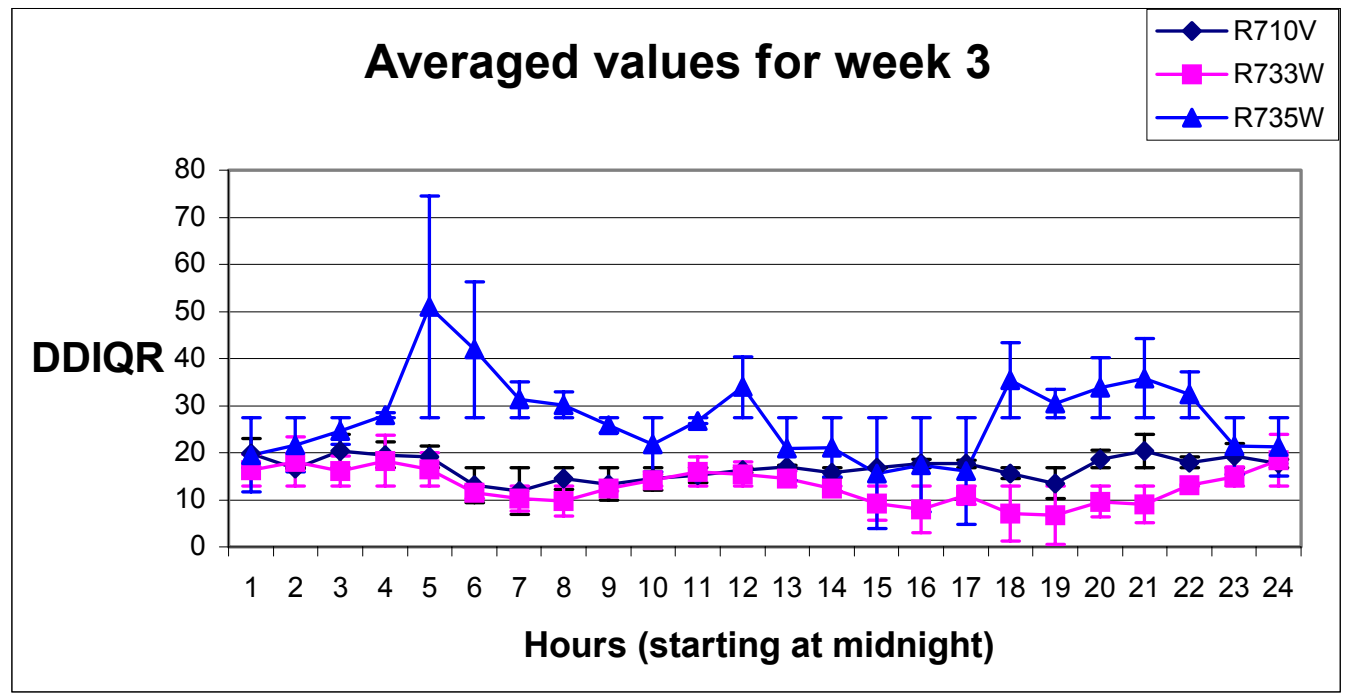

Figure C.21 DDIQR week 3 


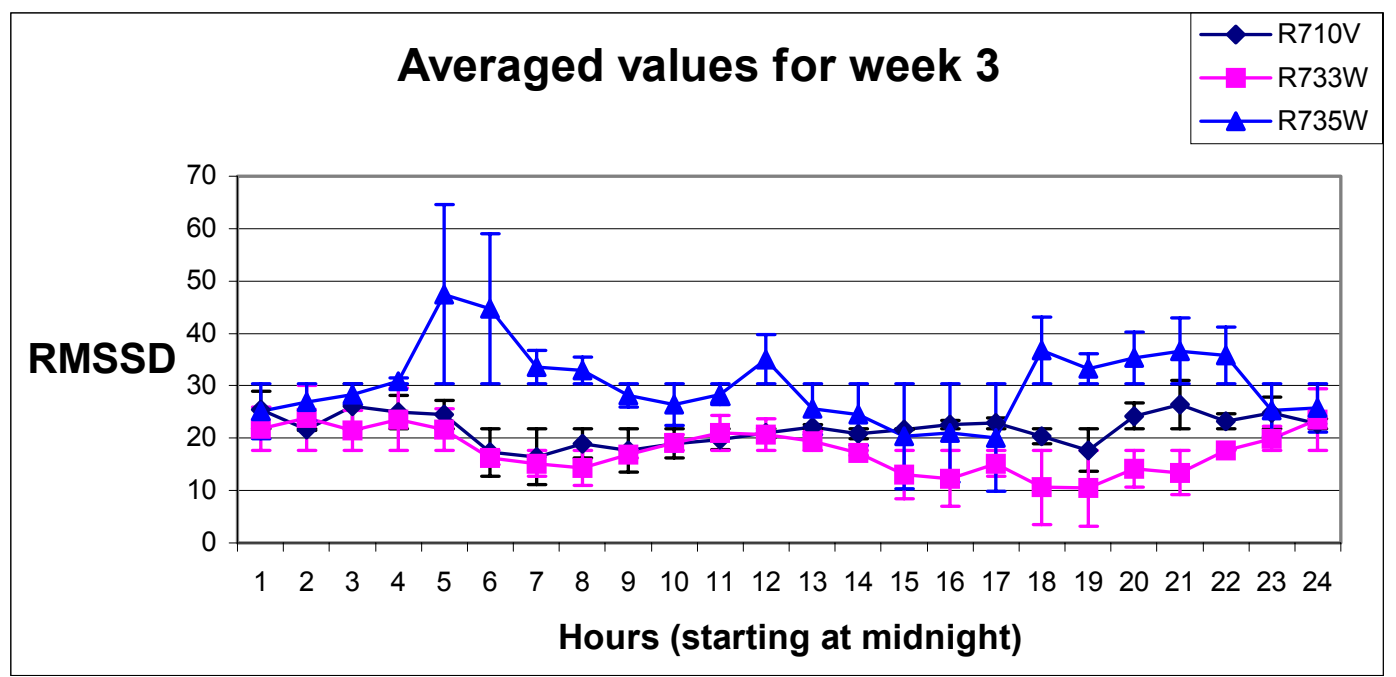

Figure C.22 RMSSD week 3

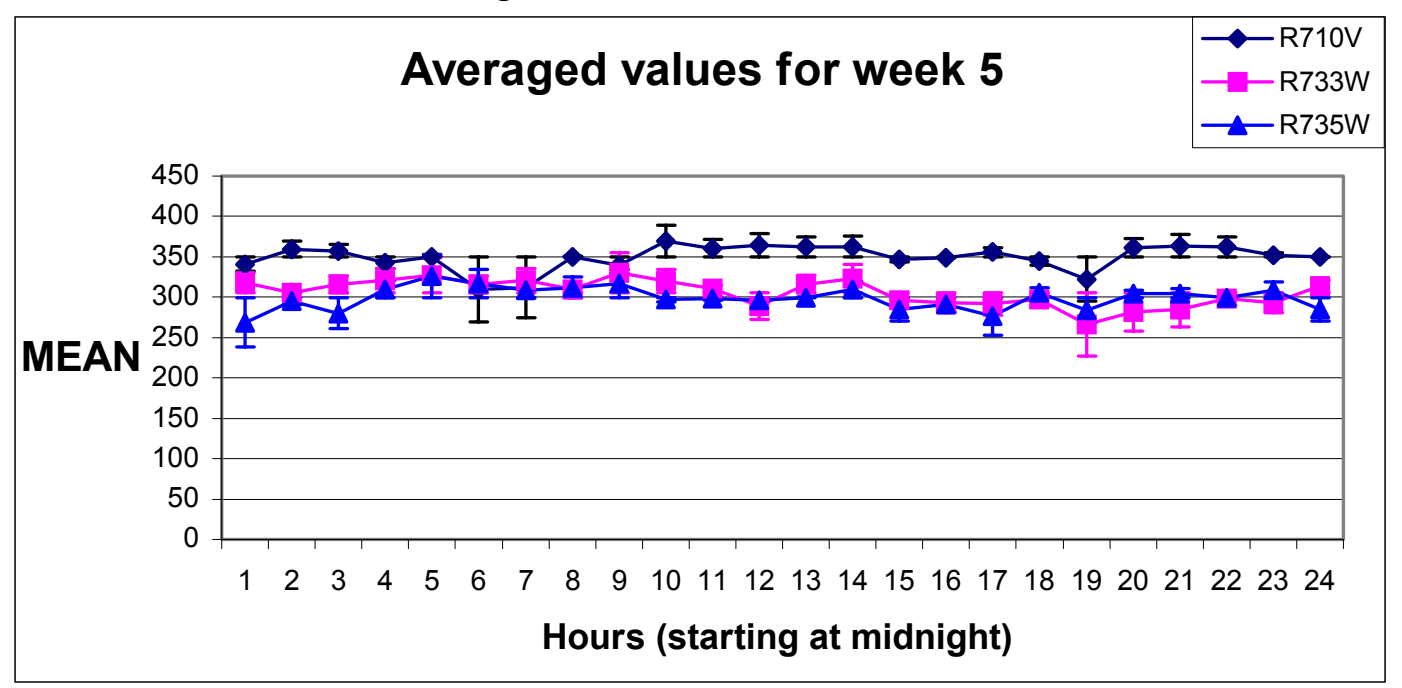

Figure C.23 MEAN week 5

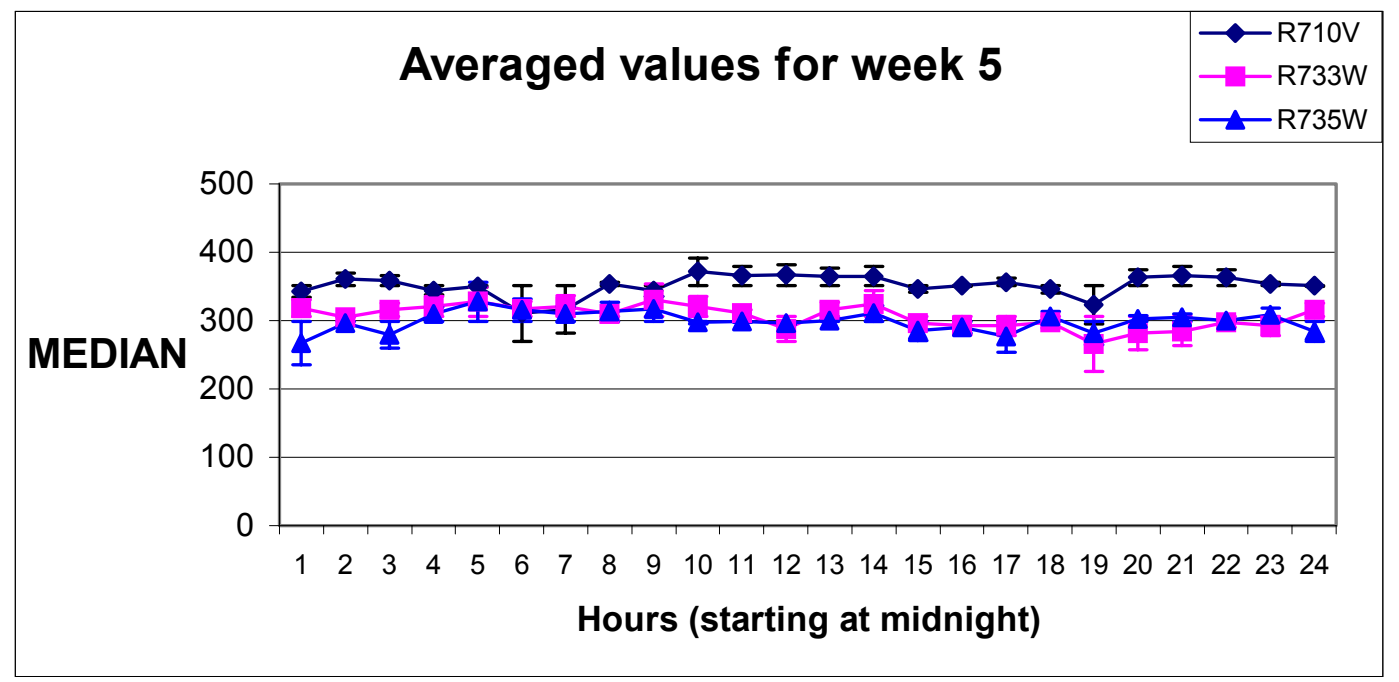

Figure C.24 MEDIAN week 5 


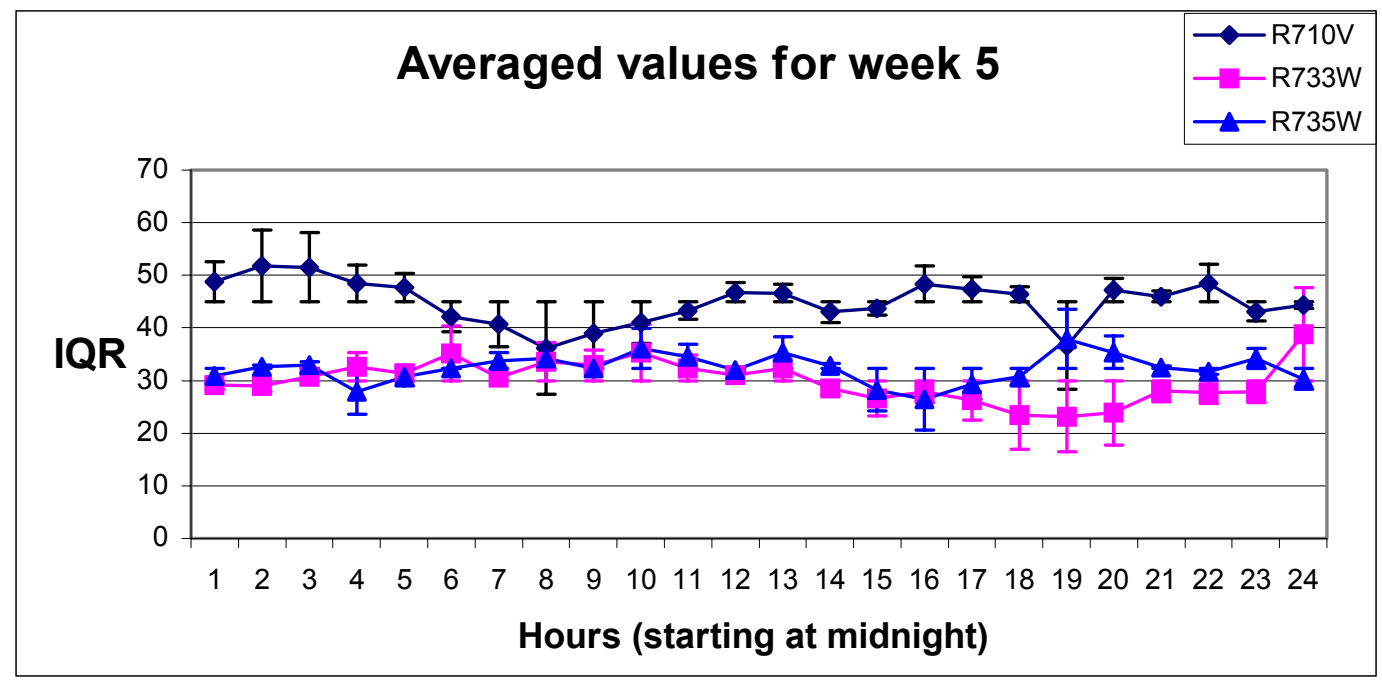

Figure C.25 IQR week 5

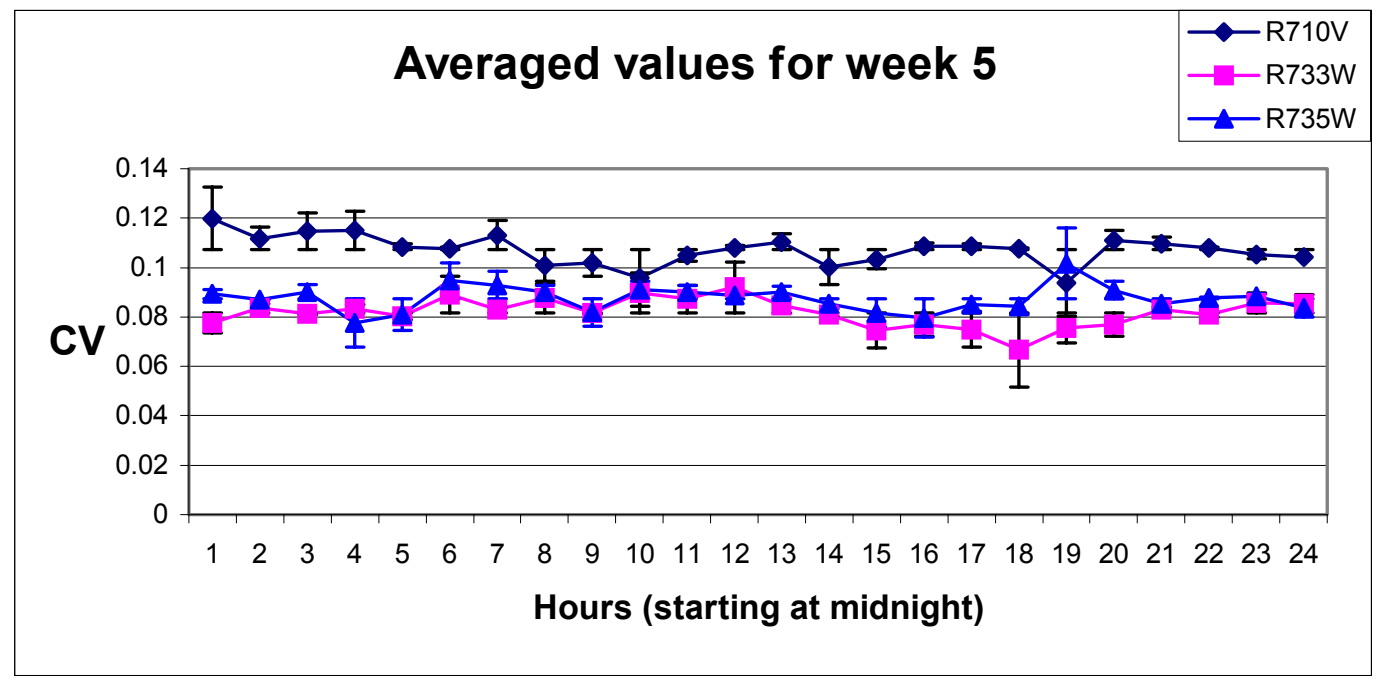

Figure C.26 CV week 5

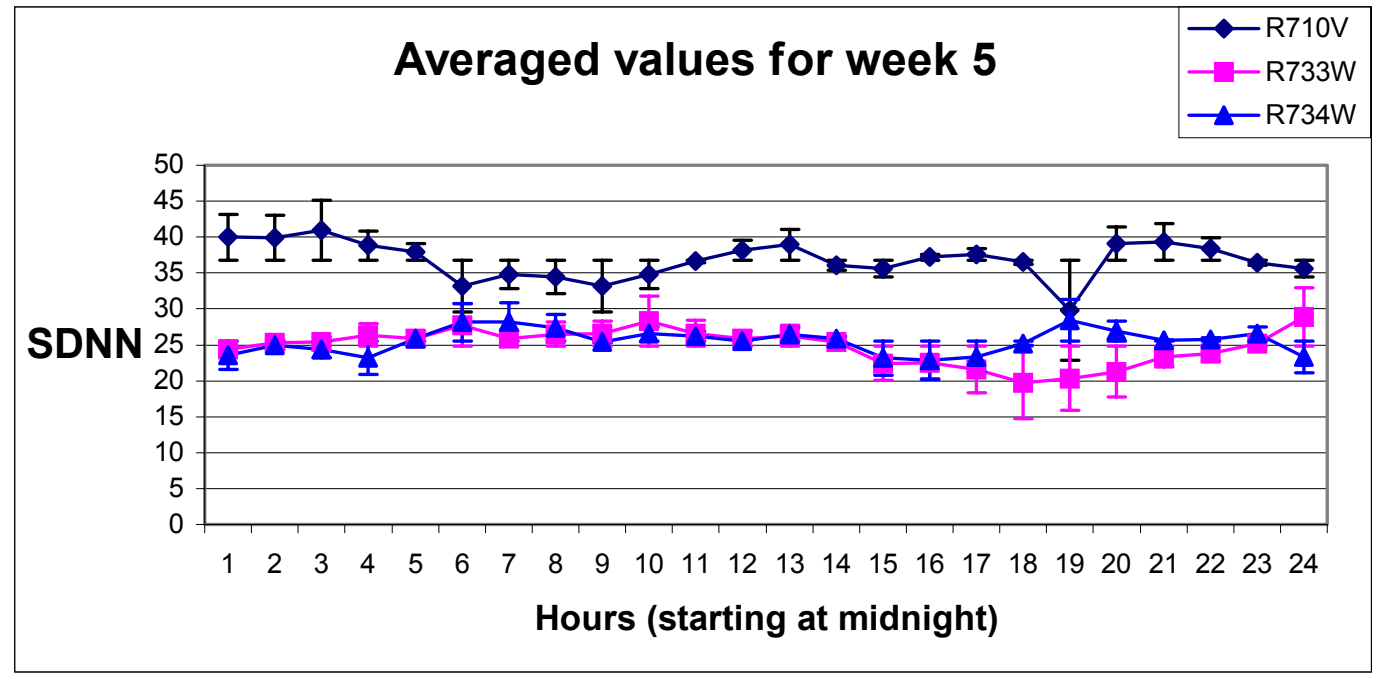

Figure C.27 SDNN week 5 


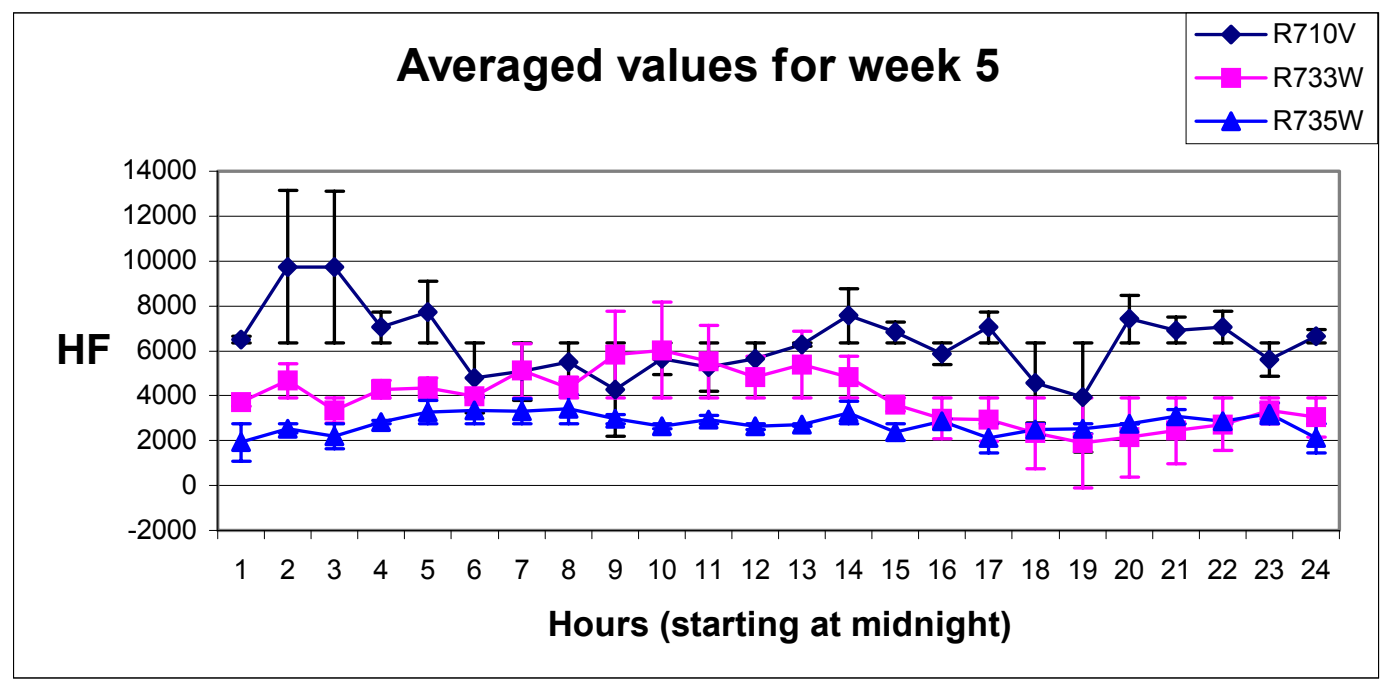

Figure C.28 HF week 5

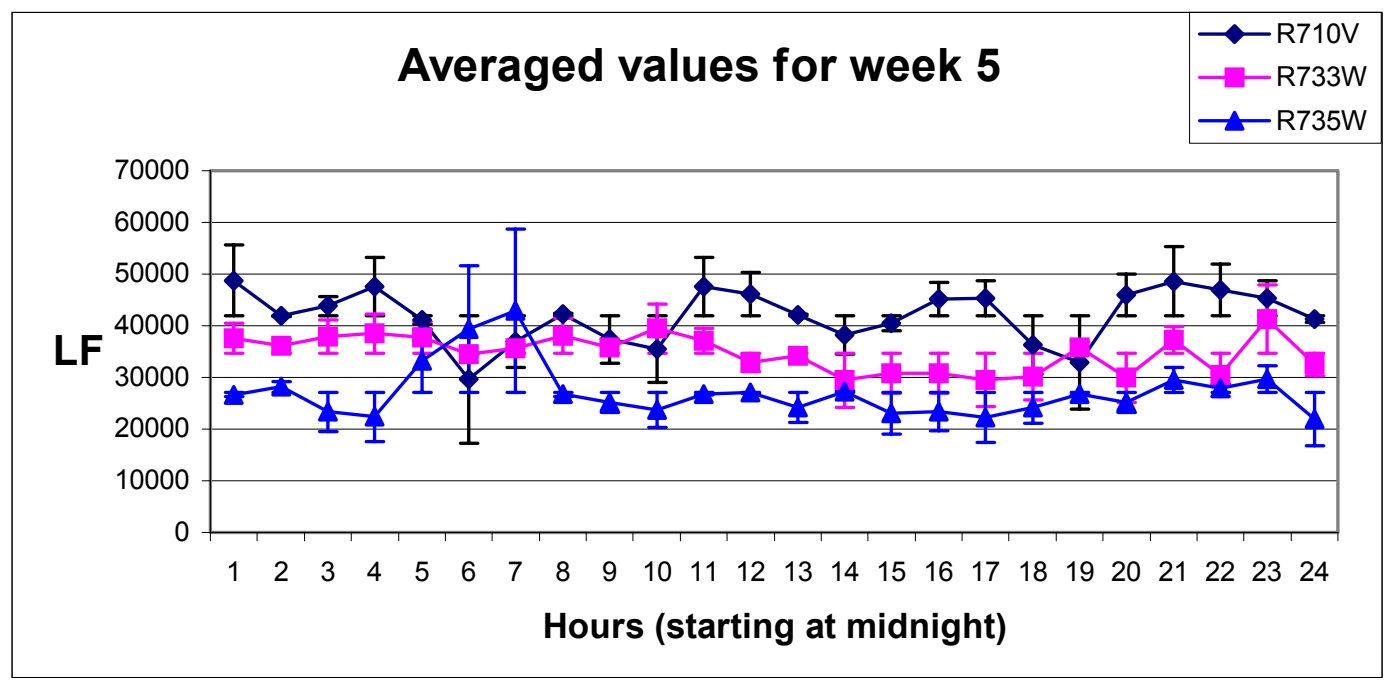

Figure C.29 LF week 5

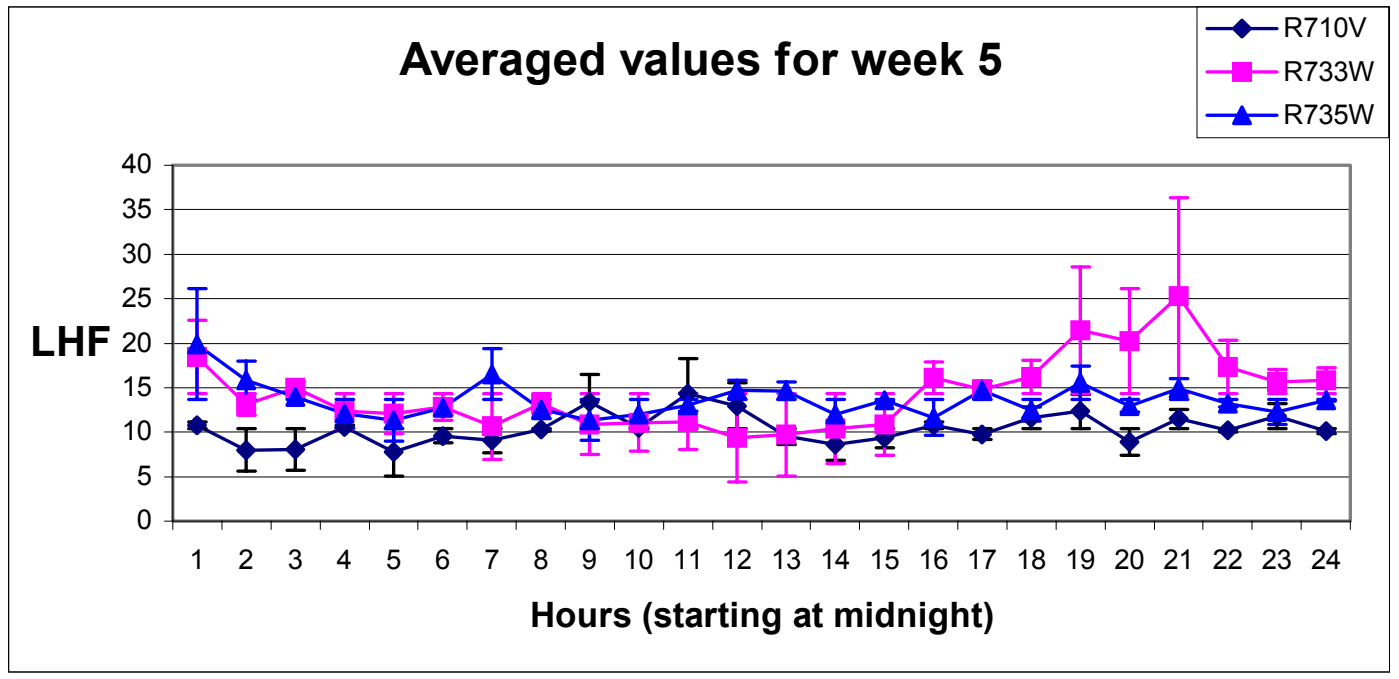

Figure C.30 LHF week 5 


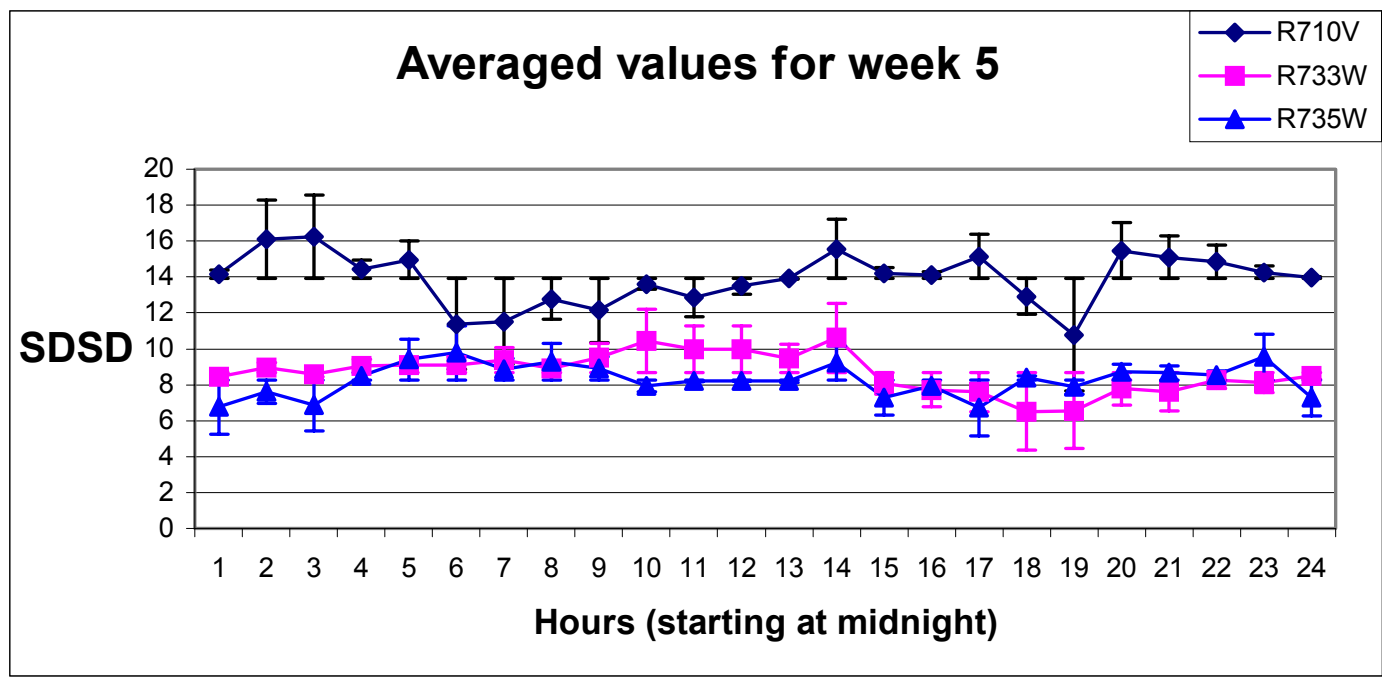

Figure C.31 SDSD week 5

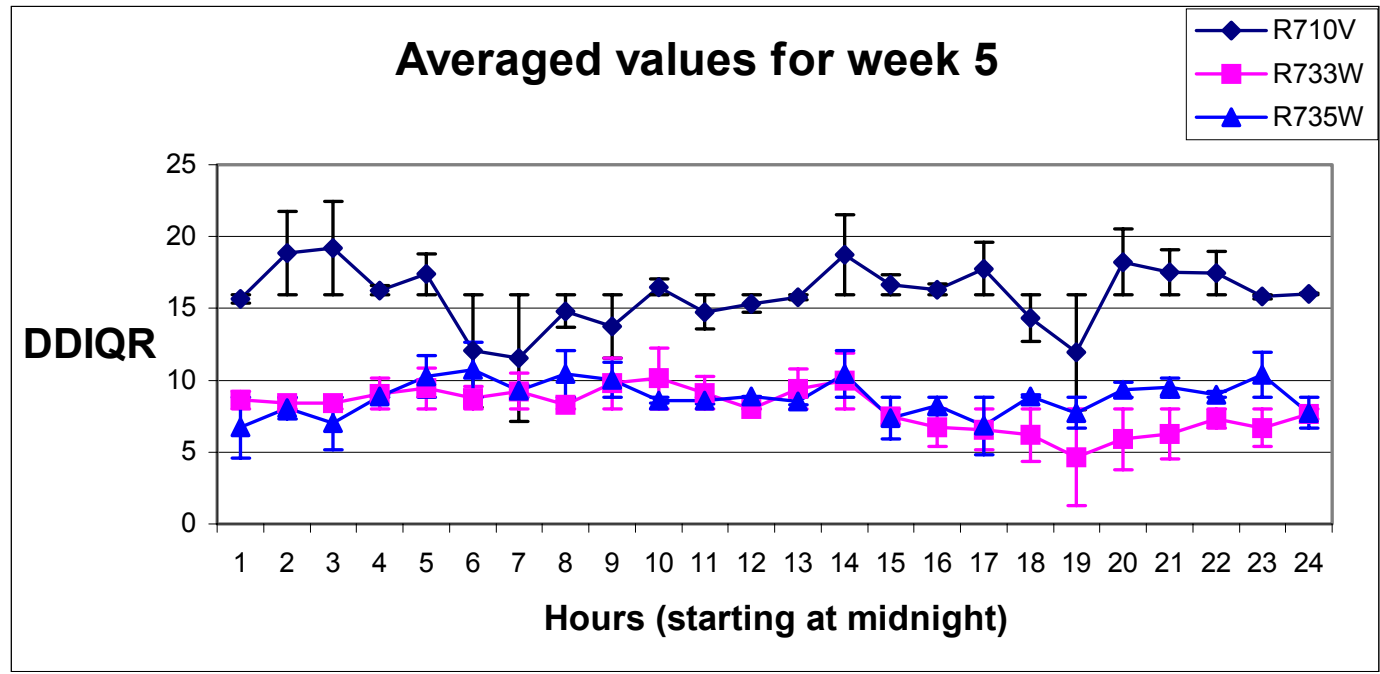

Figure C.32 DDIQR week 5

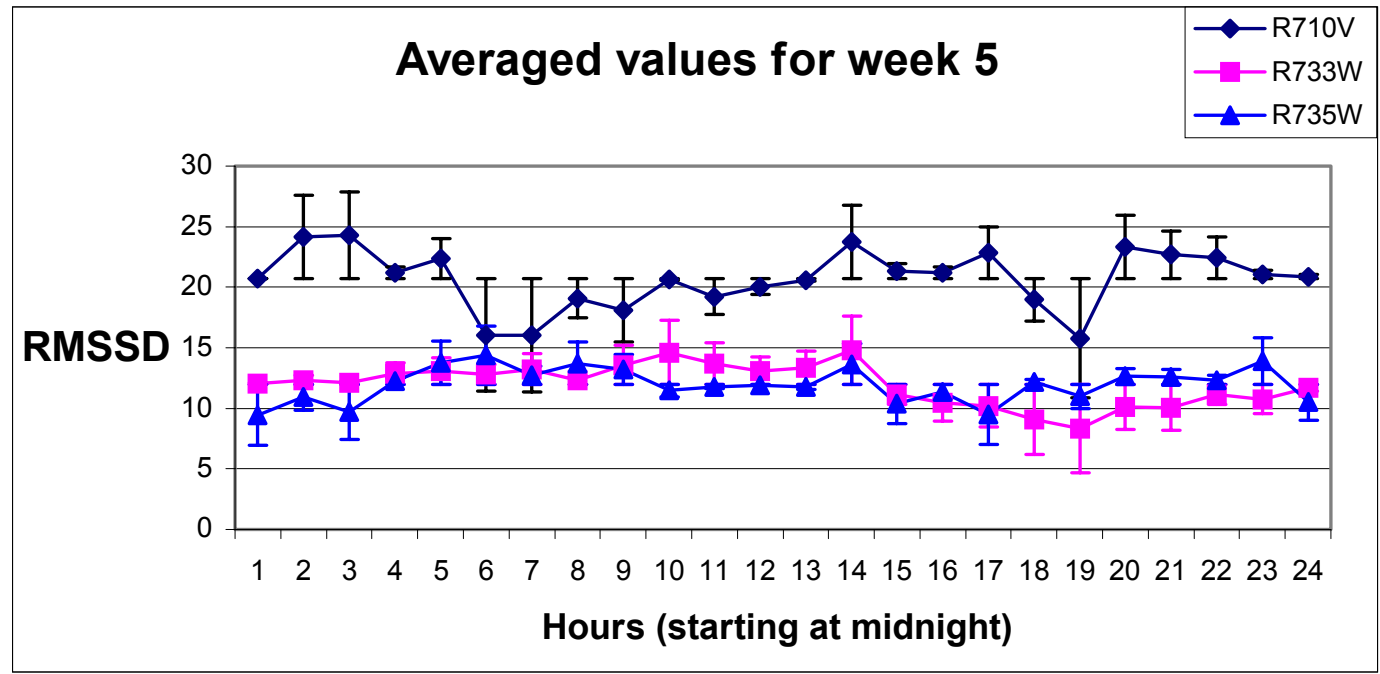

Figure C.33 RMSSD week 5 


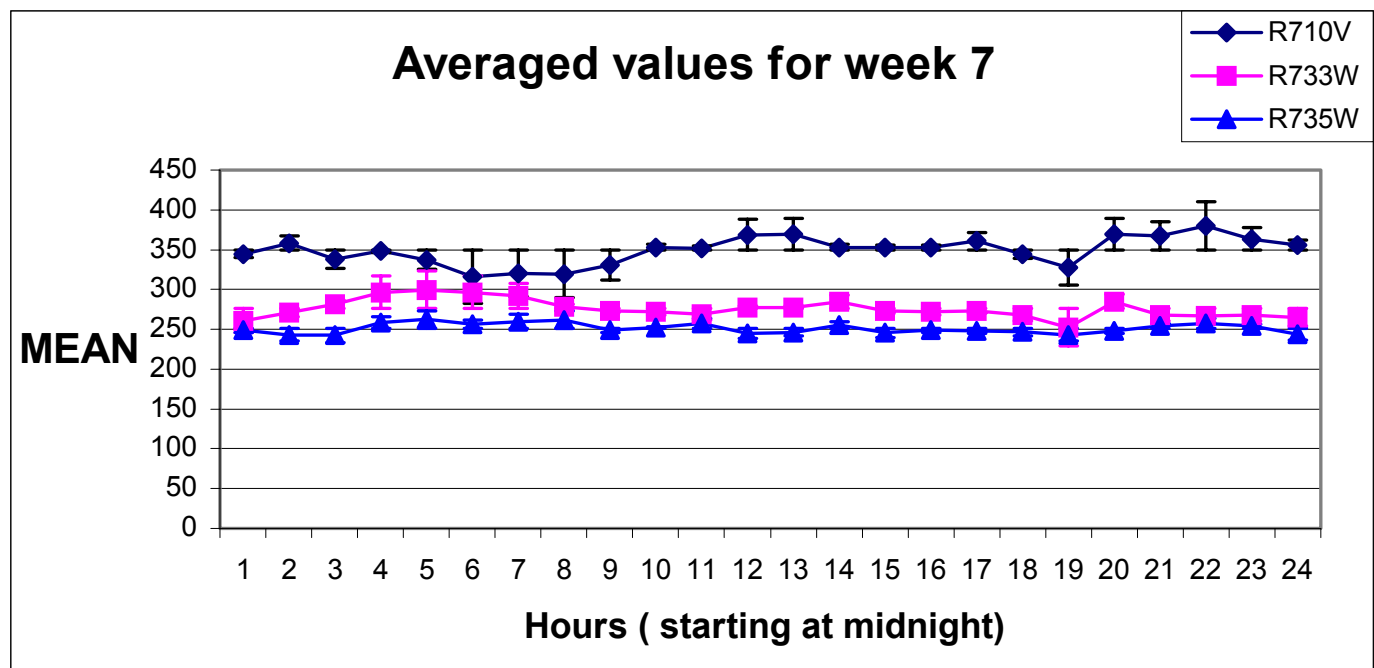

Figure C.34 MEAN week 7

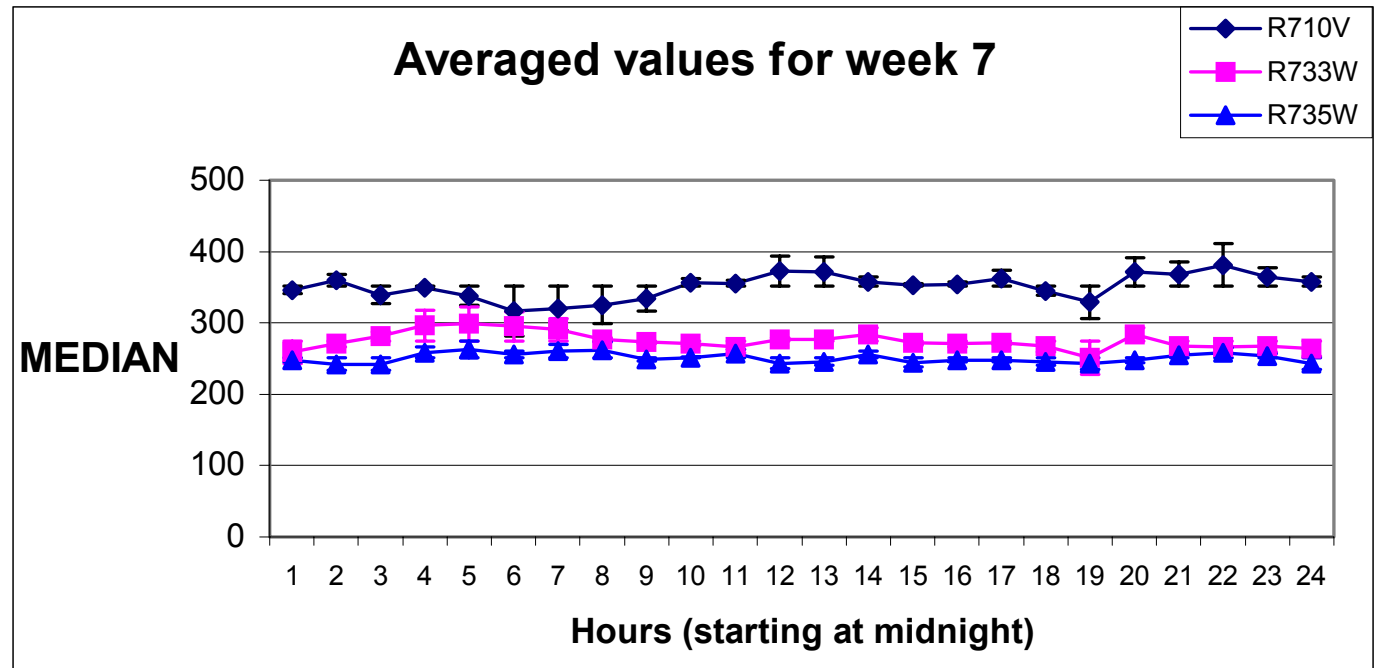

Figure C.35 MEDIAN week 7

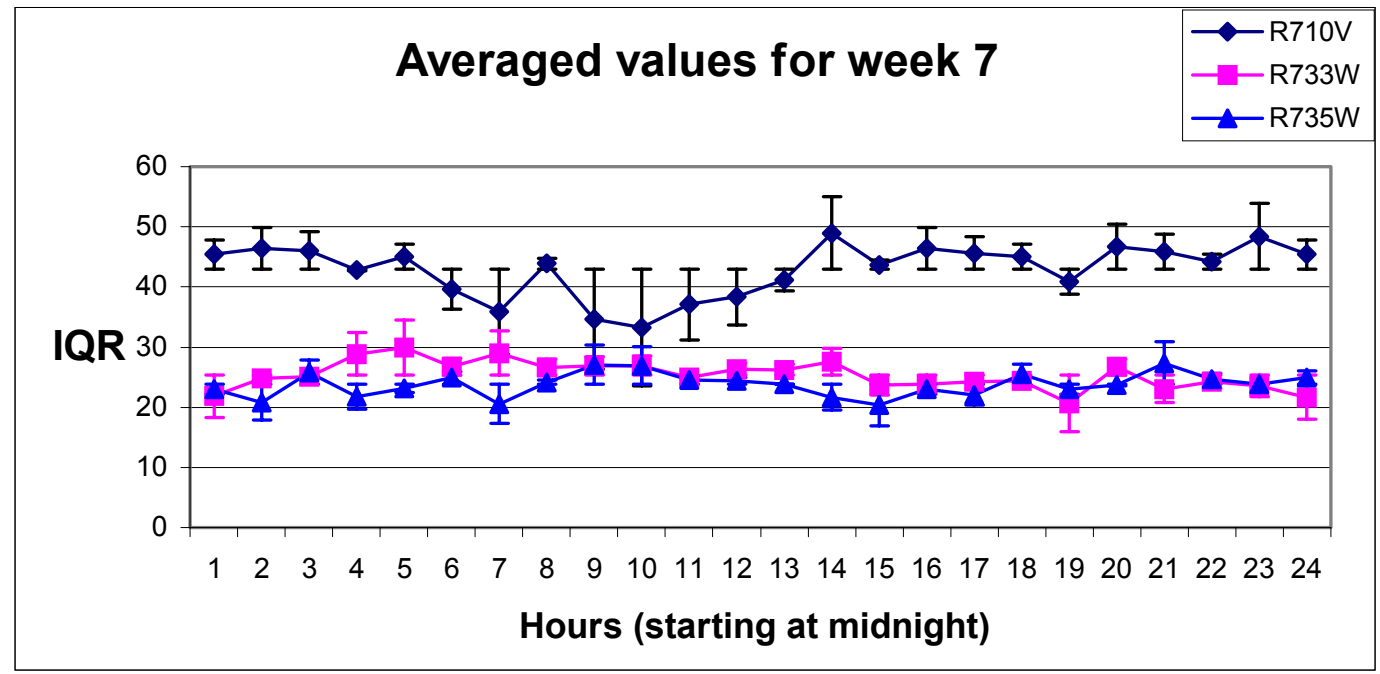

Figure C.36 IQR week 7 


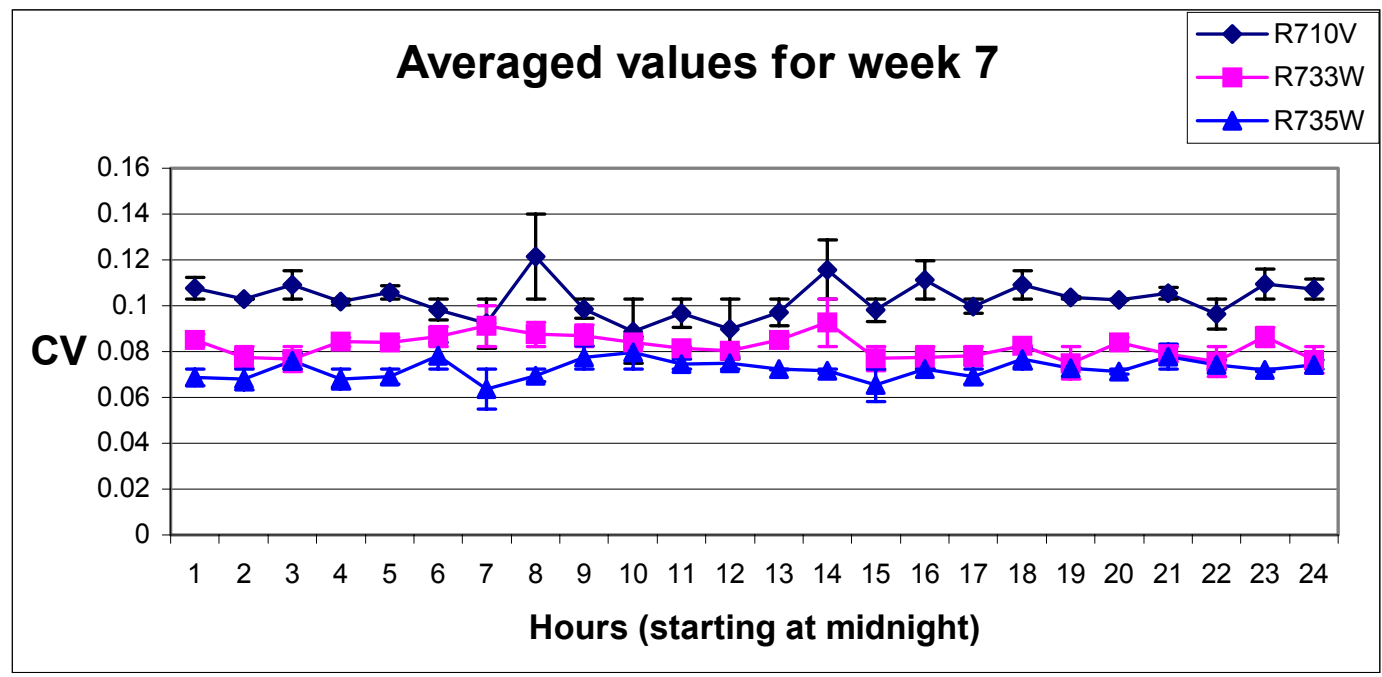

Figure C.37 CV week 7

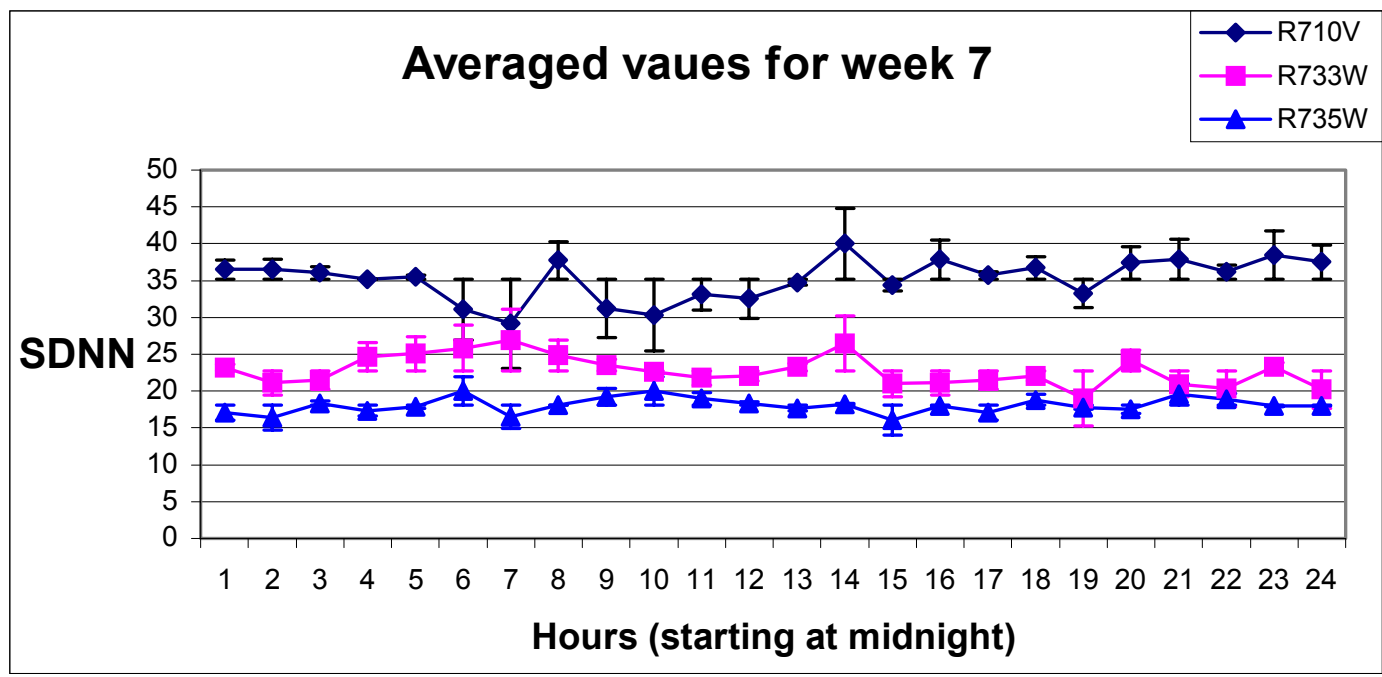

Figure C.38 SDNN week 7

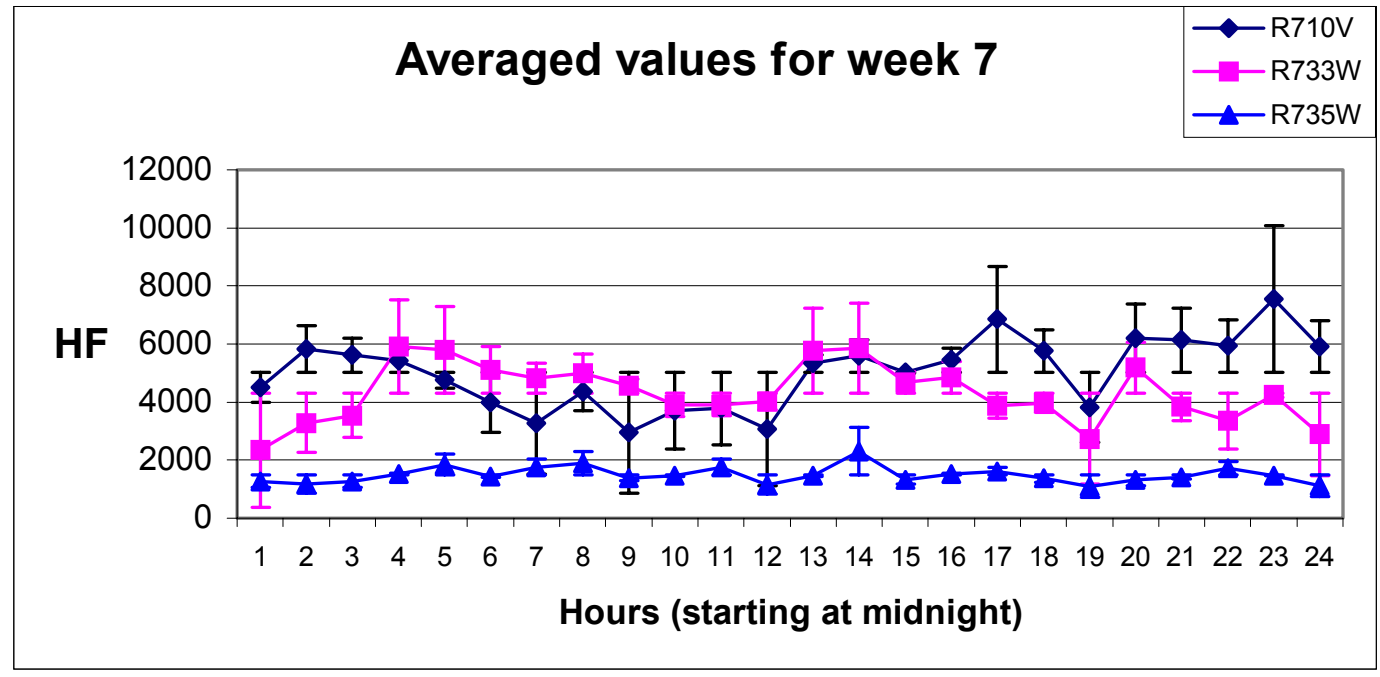

Figure C.39 HF week 7 


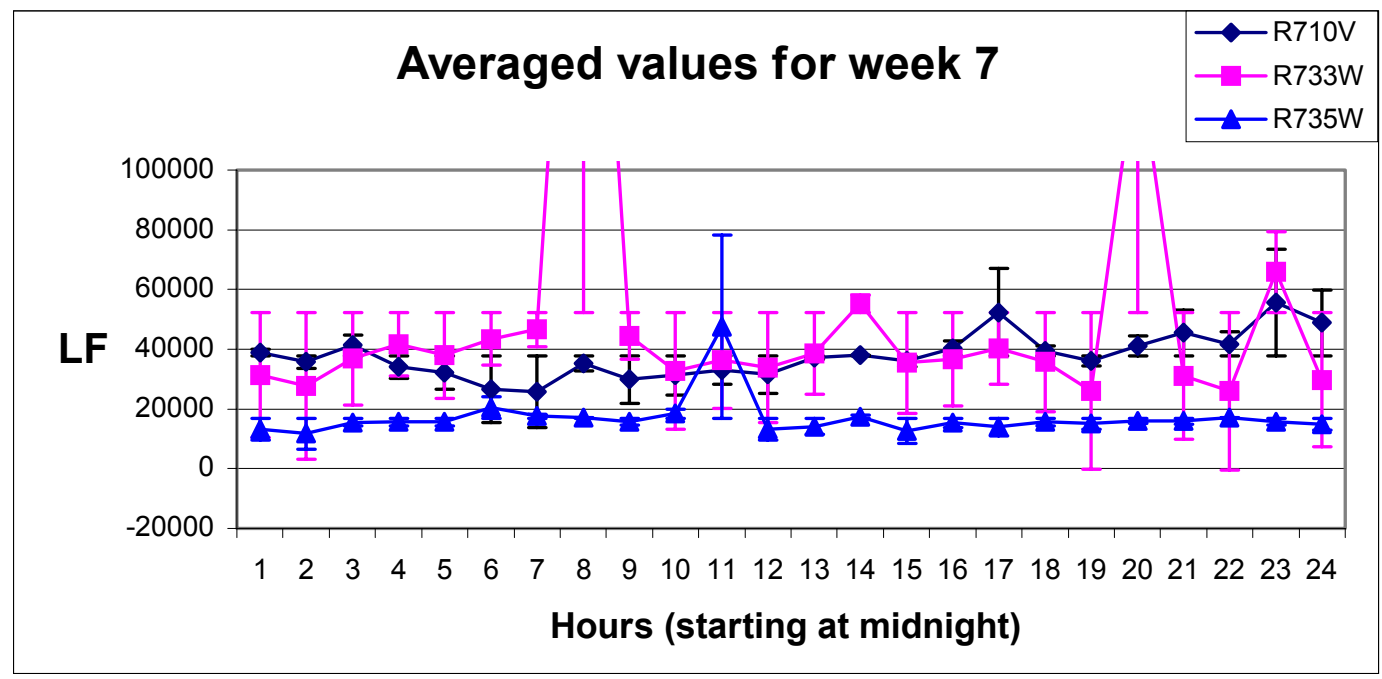

Figure C.40 LF week 7

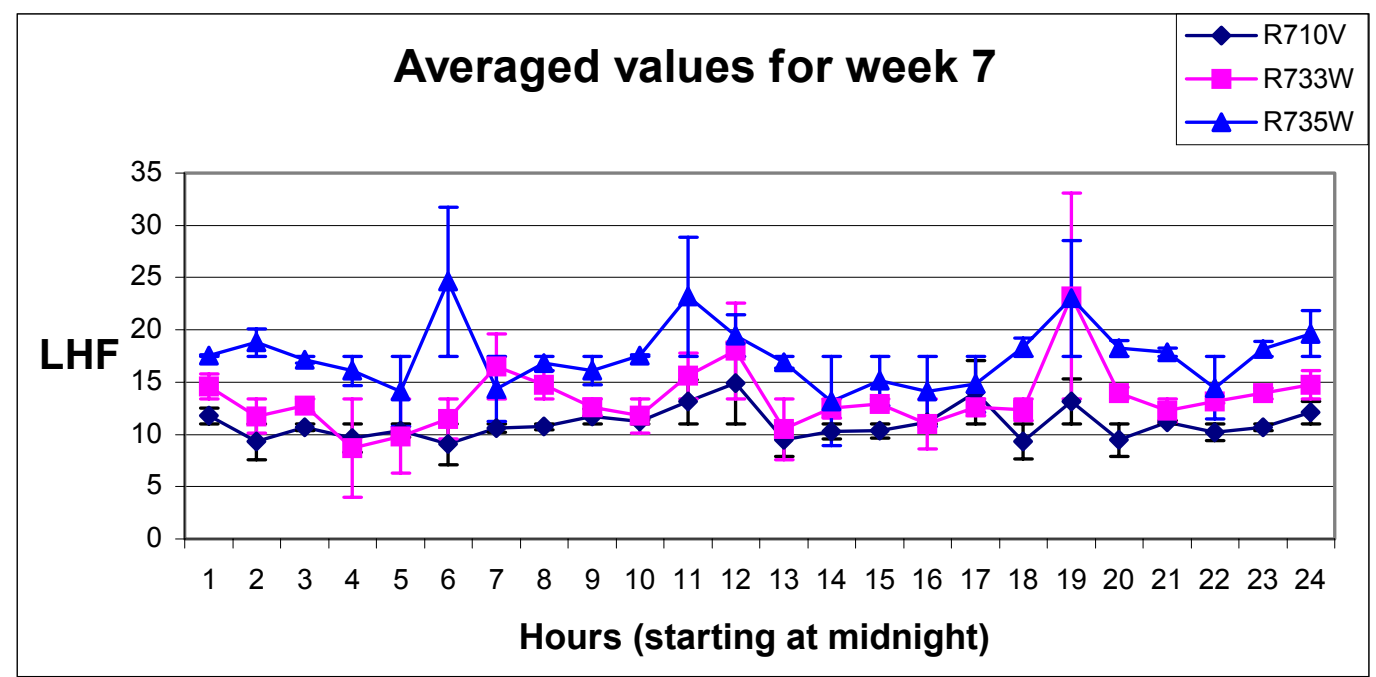

Figure C.41 LHF week 7

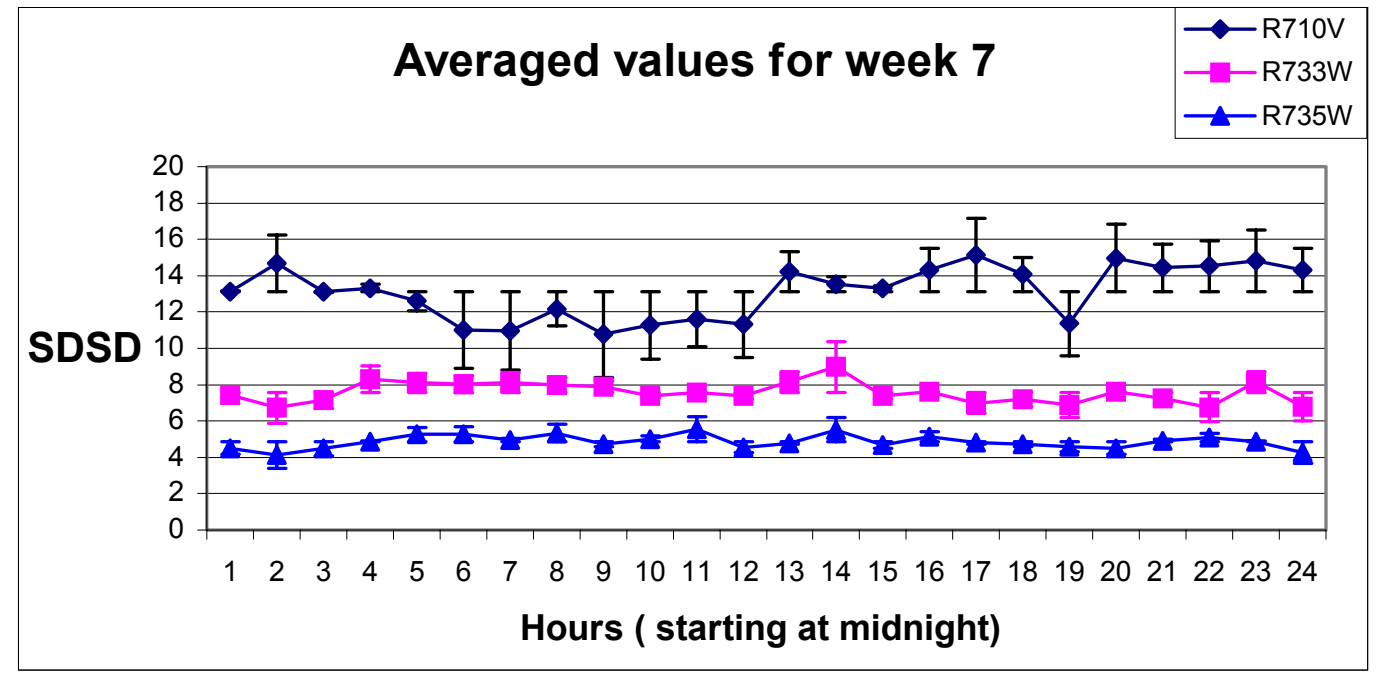

Figure C.42 SDSD week 7 


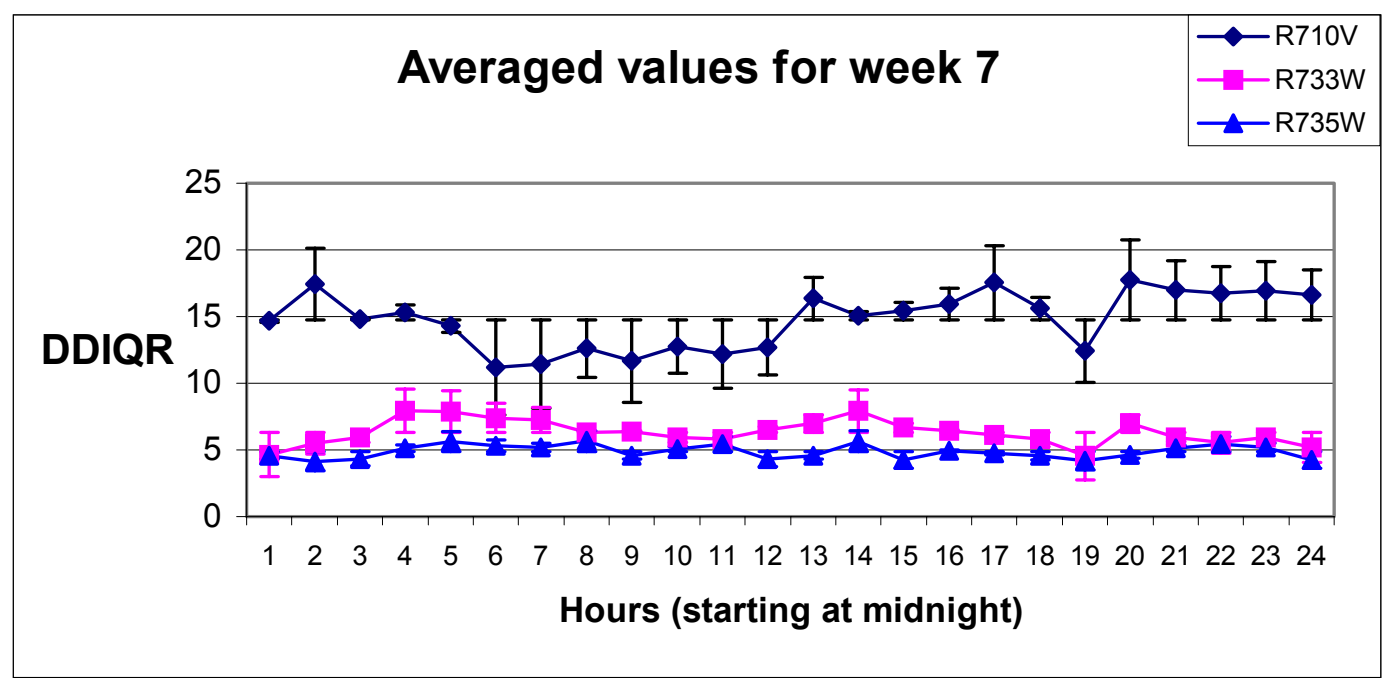

Figure C.43 DDIQR week 7

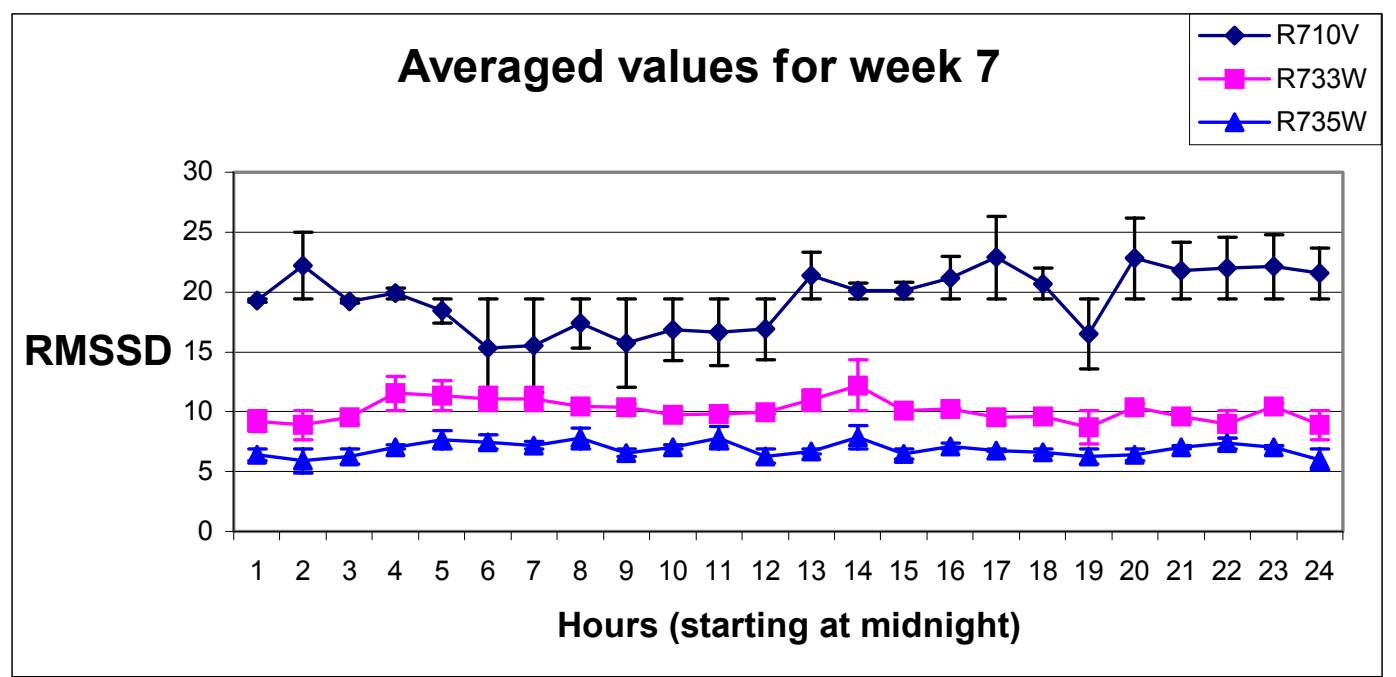

Figure C.44 RMSSD week 7

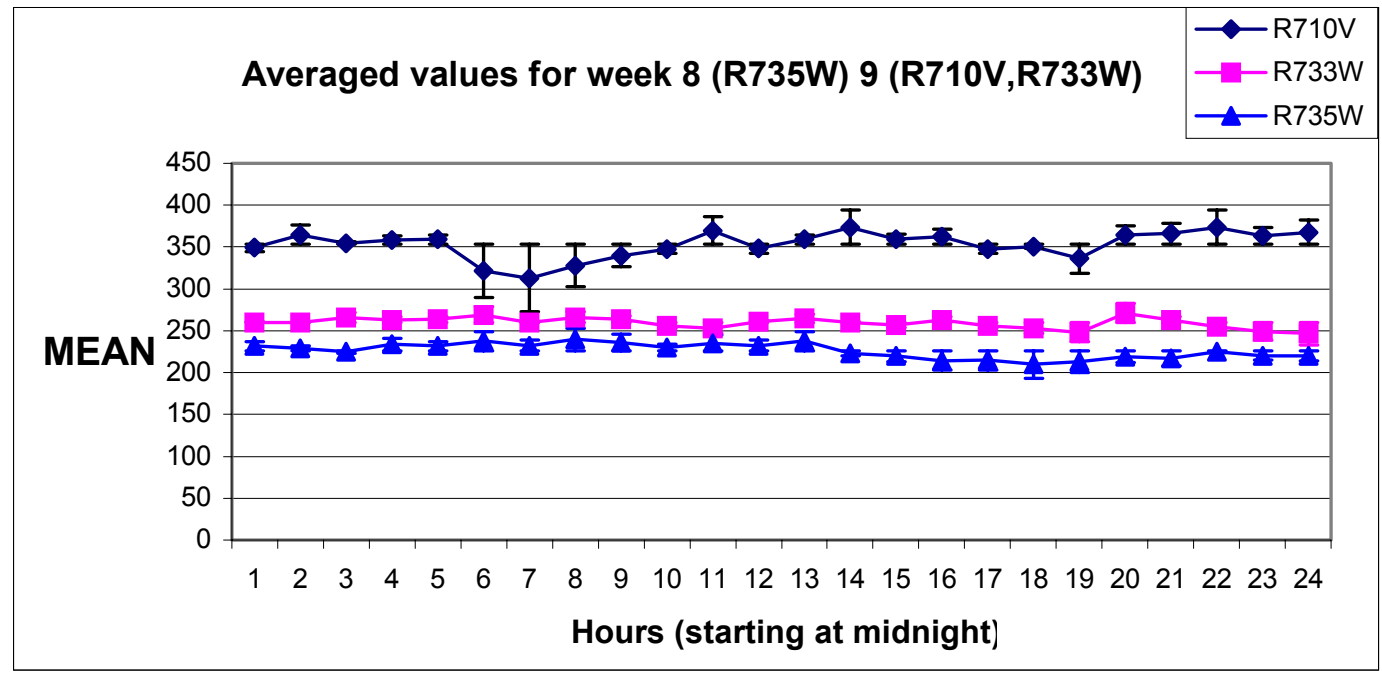

Figure C.45 MEAN week 8, 9 


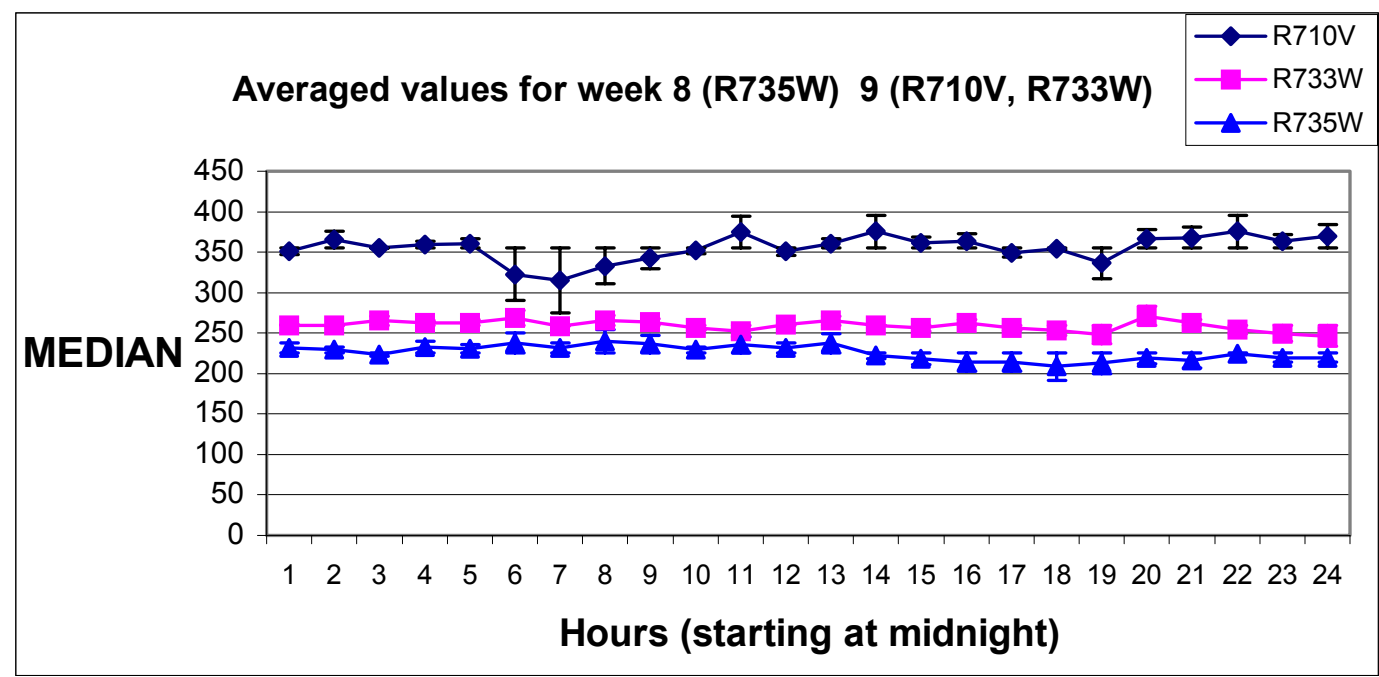

Figure C.46 MEDIAN week 8, 9

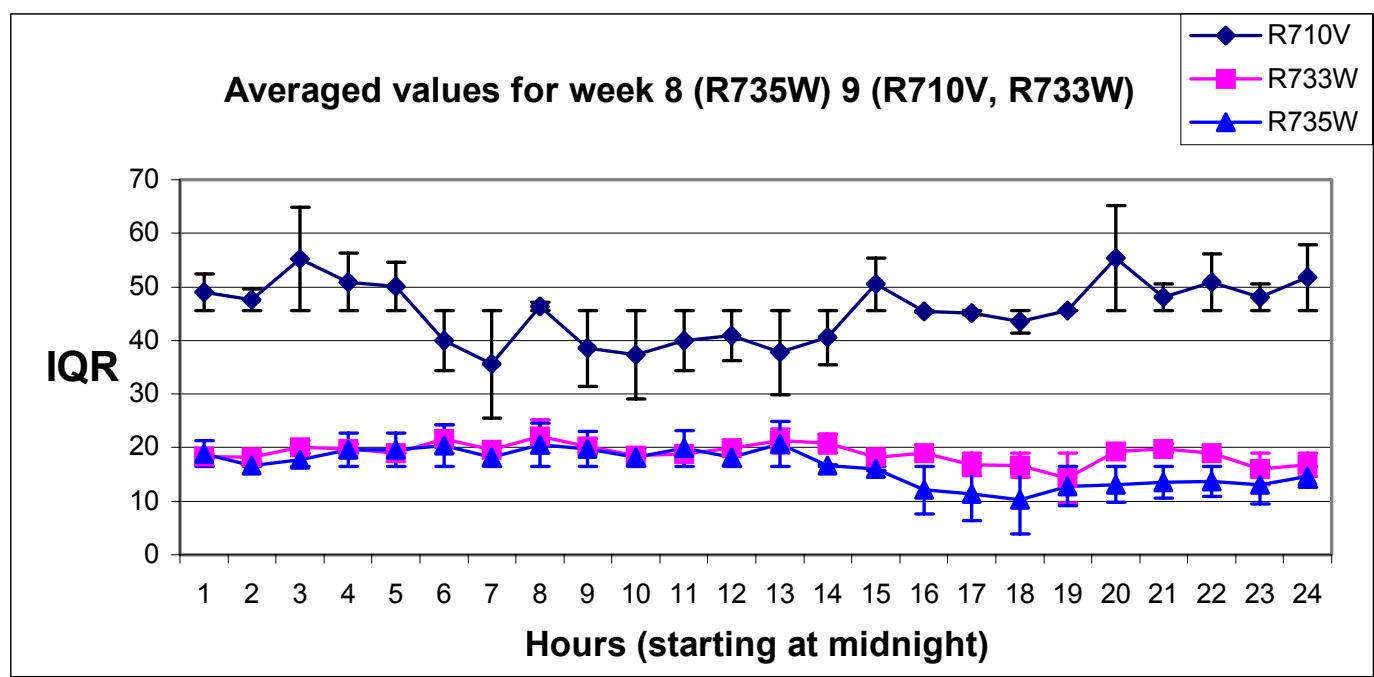

Figure C.47 IQR week 8, 9

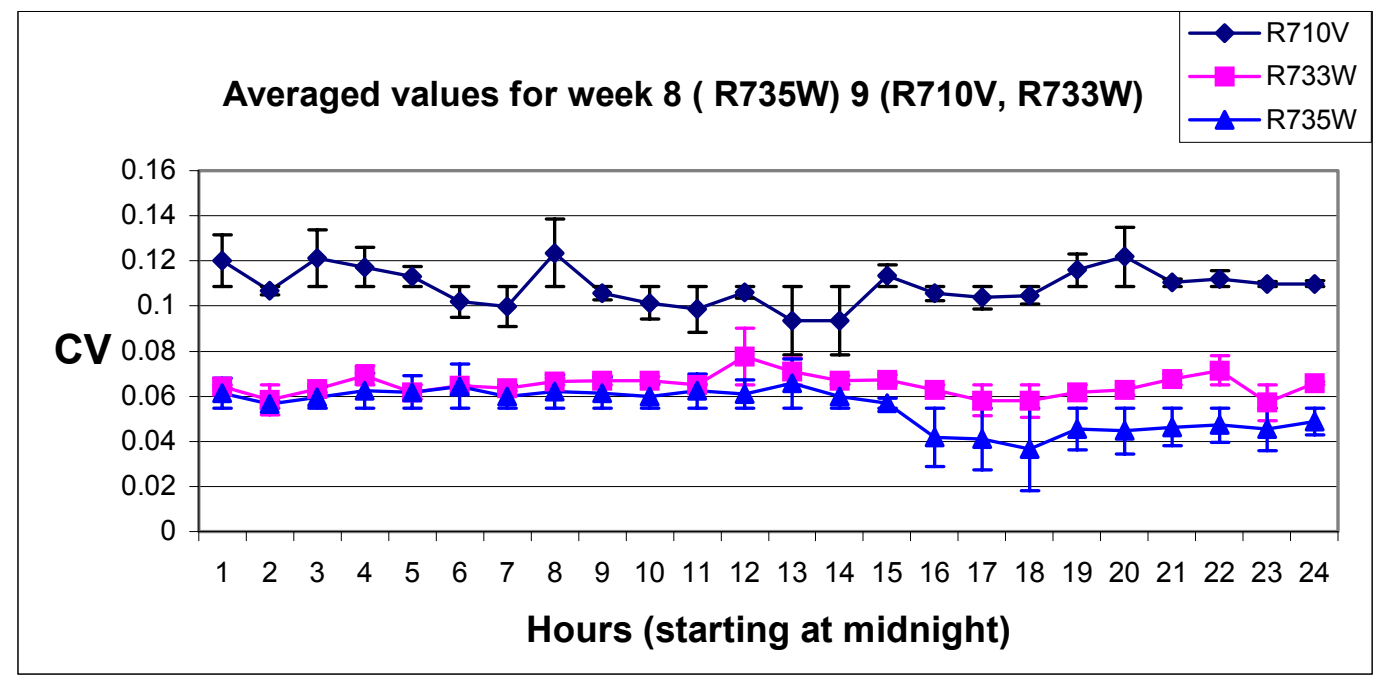

Figure C.48 CV week 8, 9 


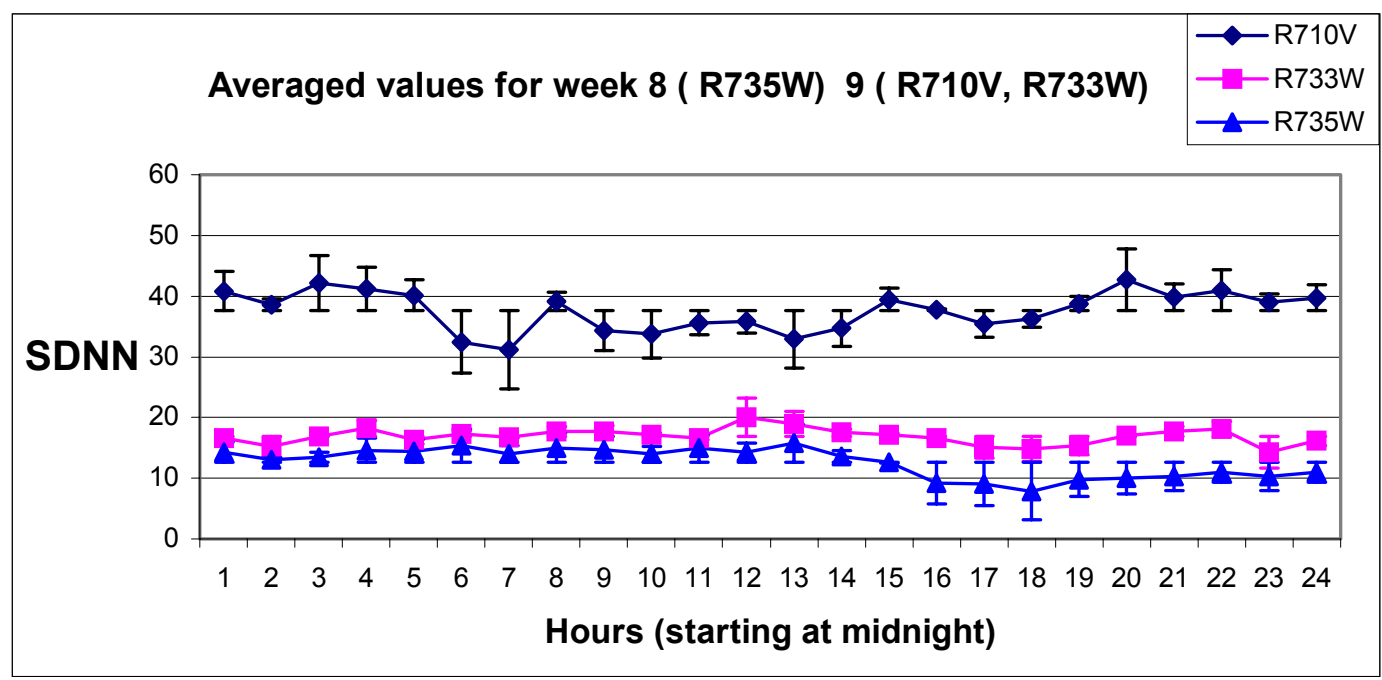

Figure C.49 SDNN week 8, 9

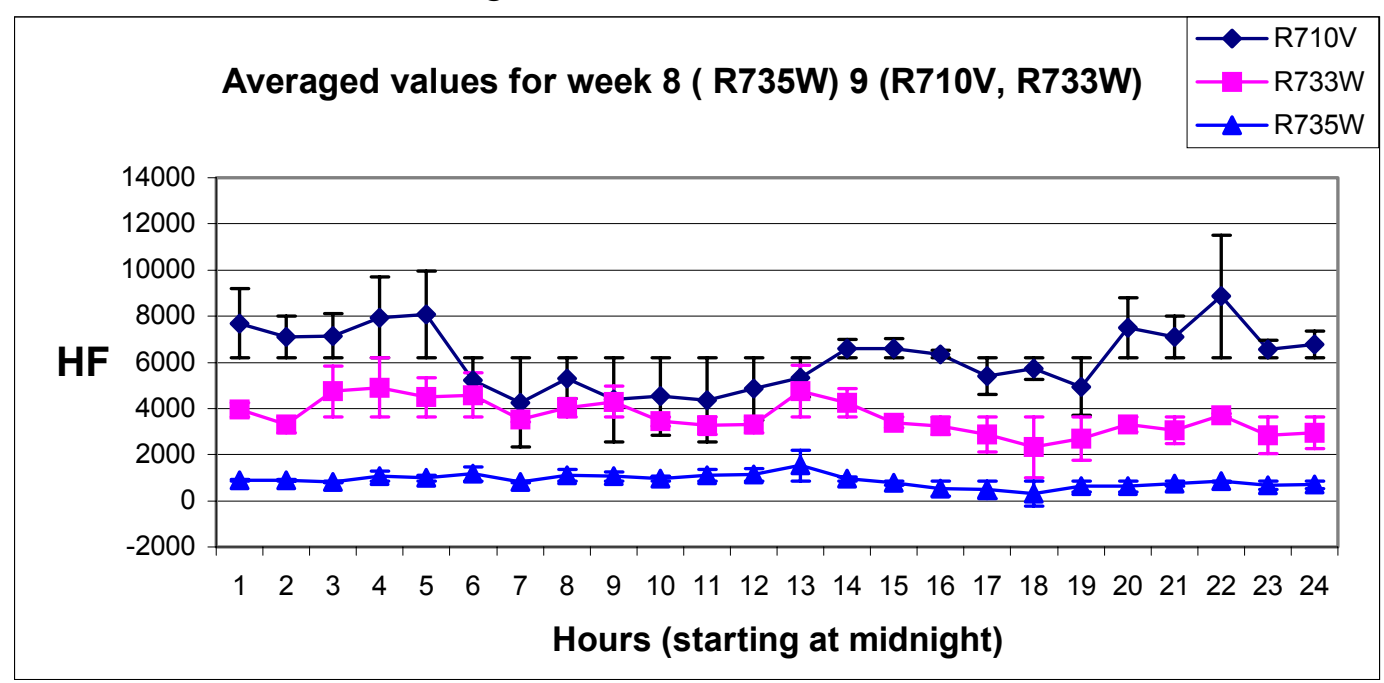

Figure C.50 HF week 8, 9

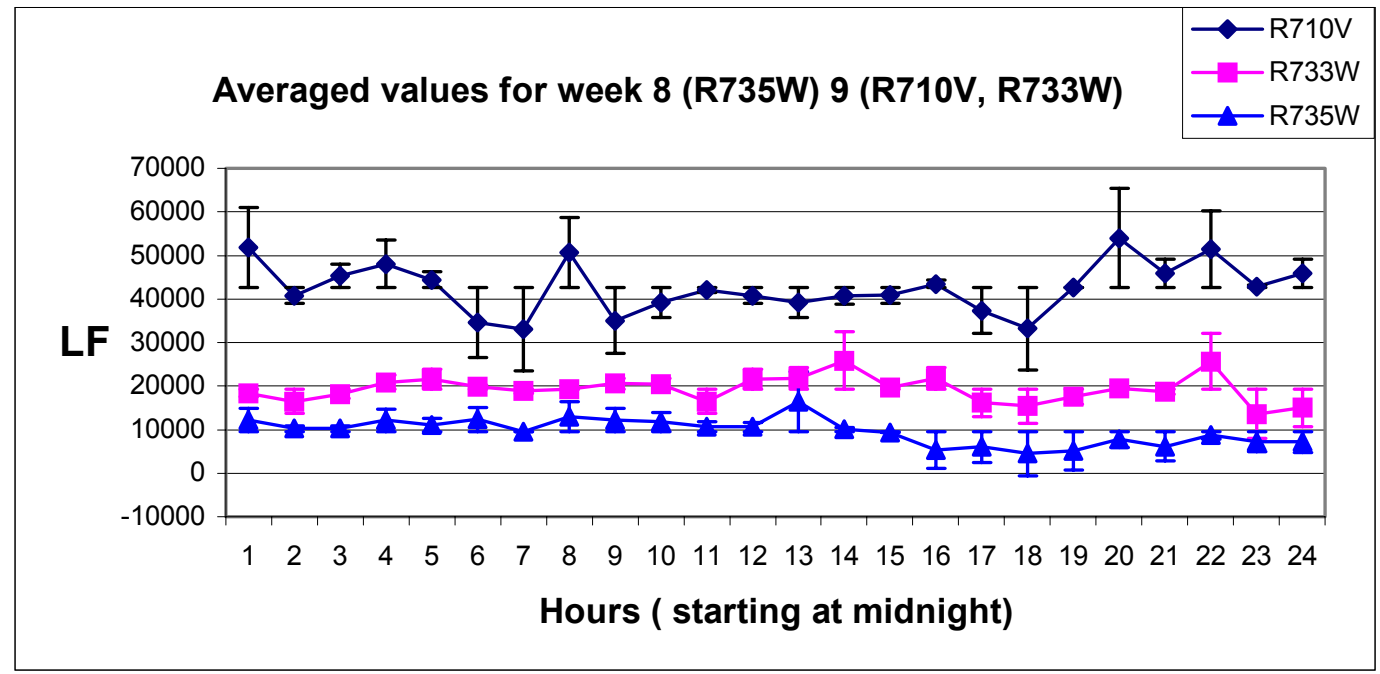

Figure C.51 LF week 8, 9 


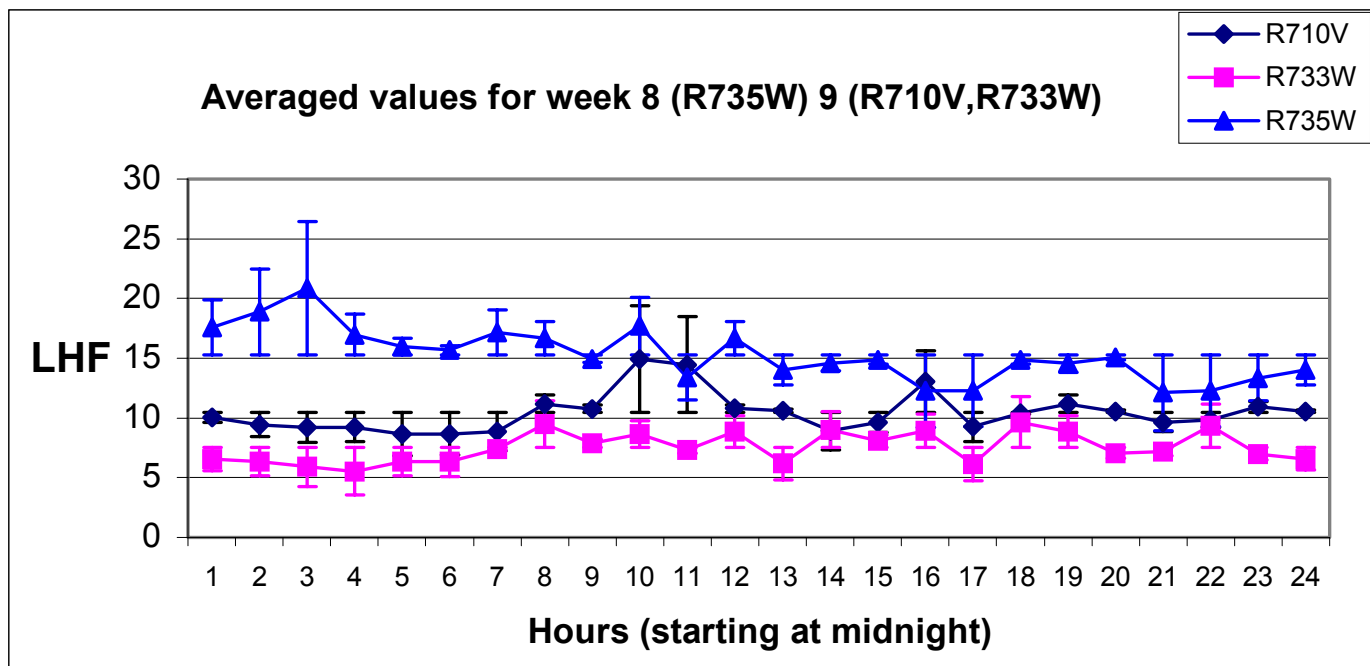

Figure C.52 LHF week 8, 9

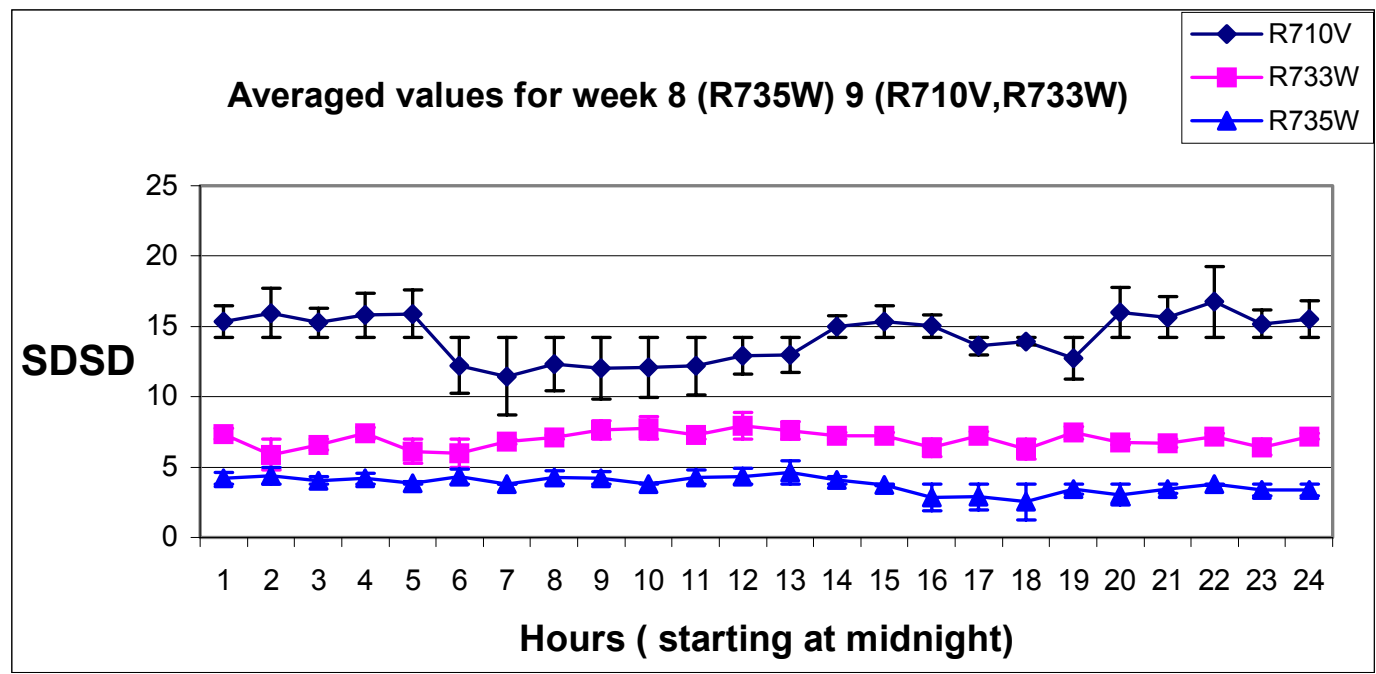

Figure C.53 SDSD week 8, 9

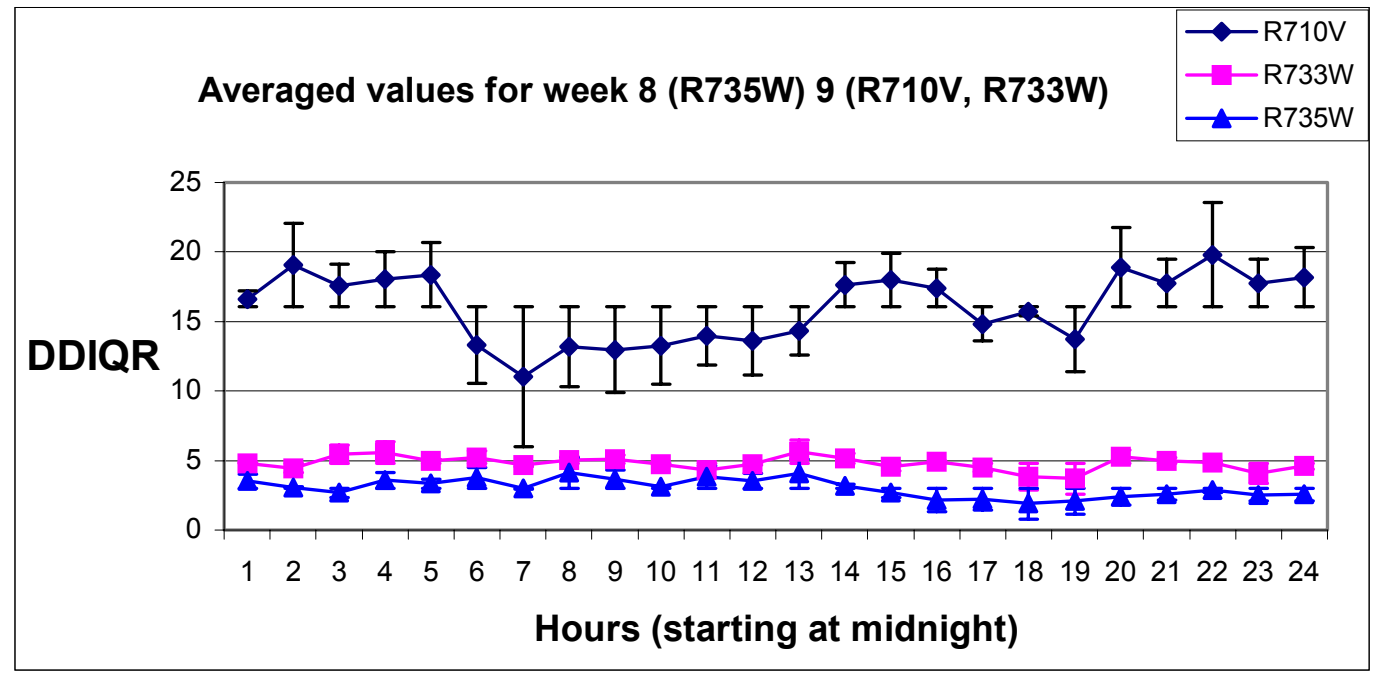

Figure C.54 DDIQR week 8, 9 


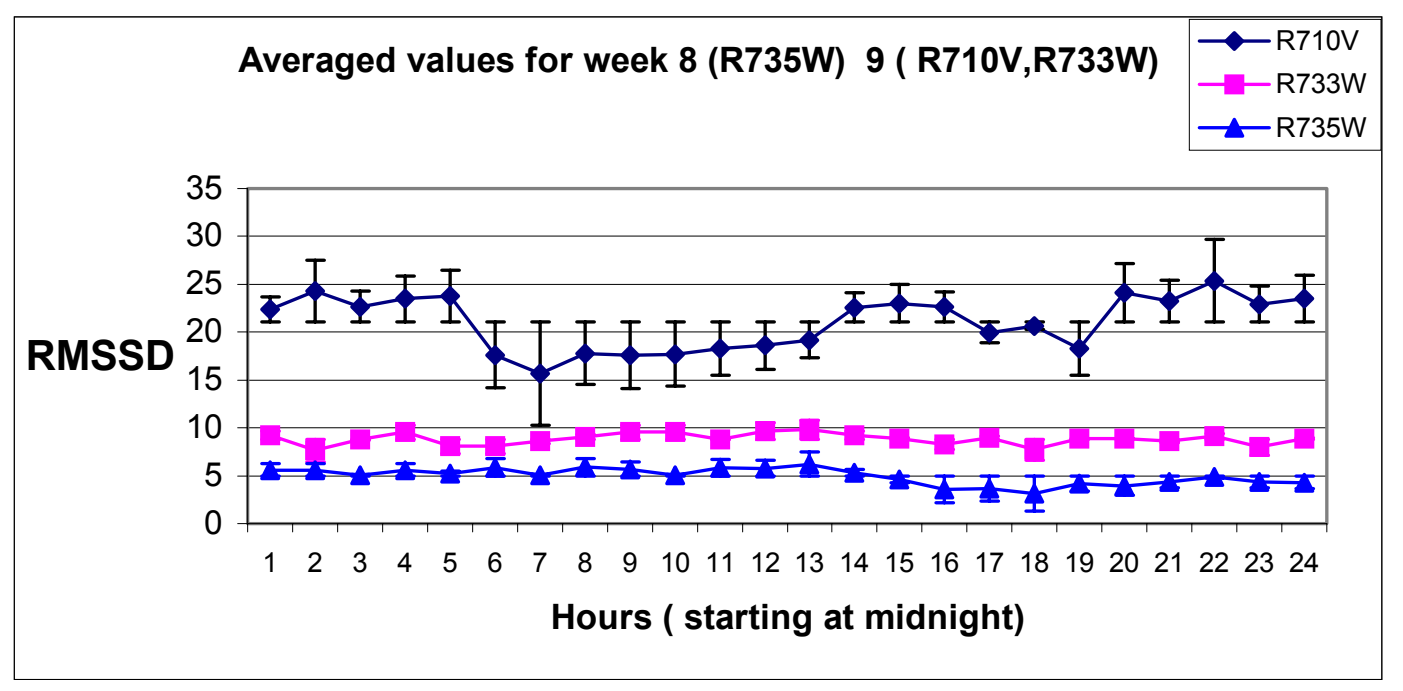

Figure C.55 RMSSD week 8, 9 
Appendix D Individual and mixed plots from averaged data per day and night 


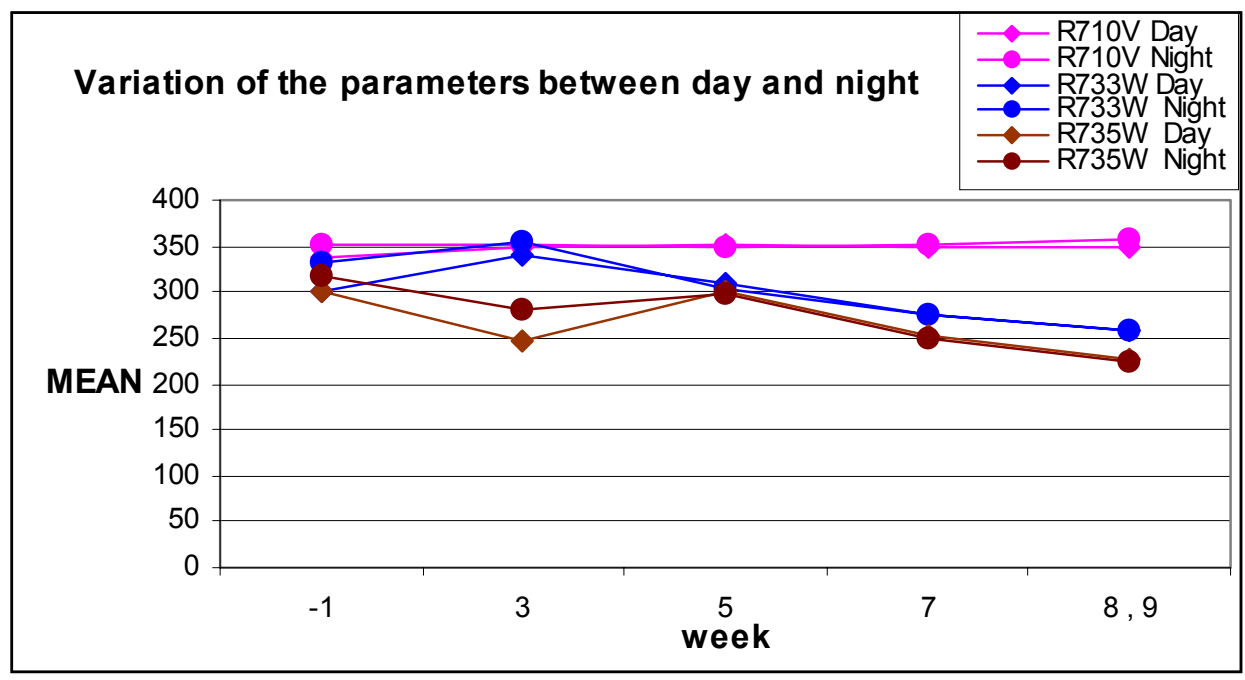

Figure D.1 MEAN

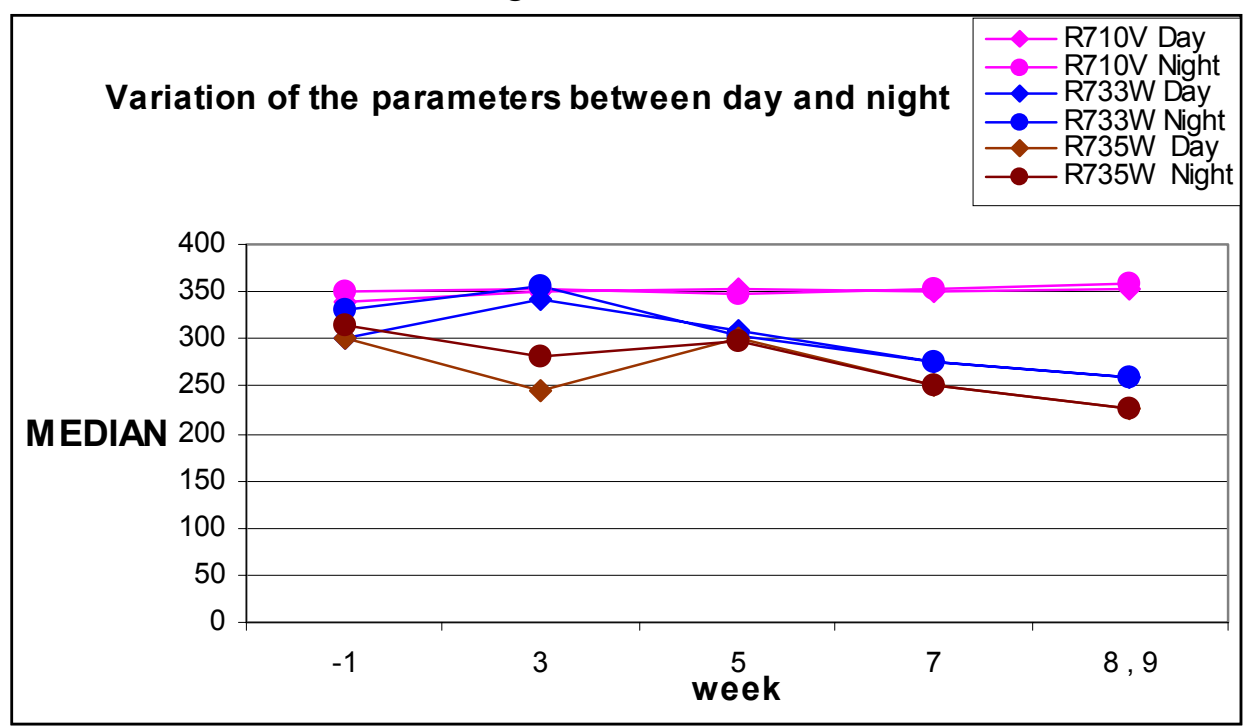

Figure D.2 MEDIAN 


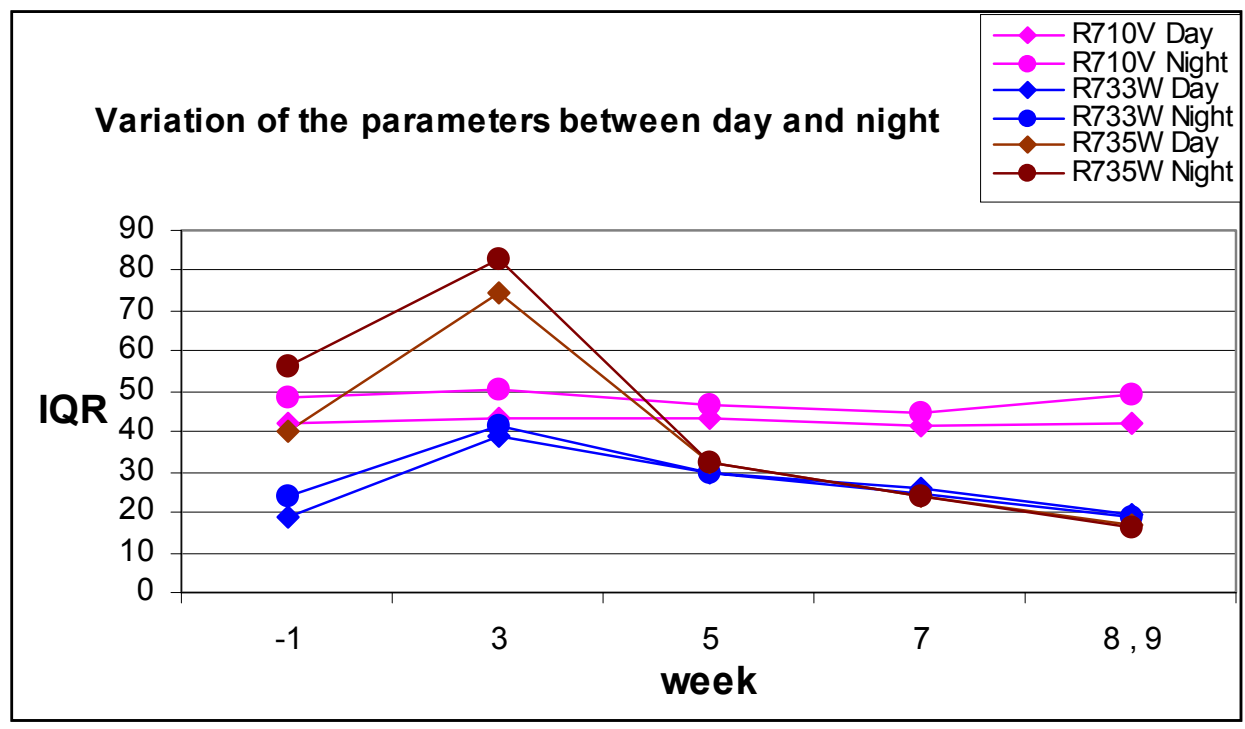

Figure D.3 IQR

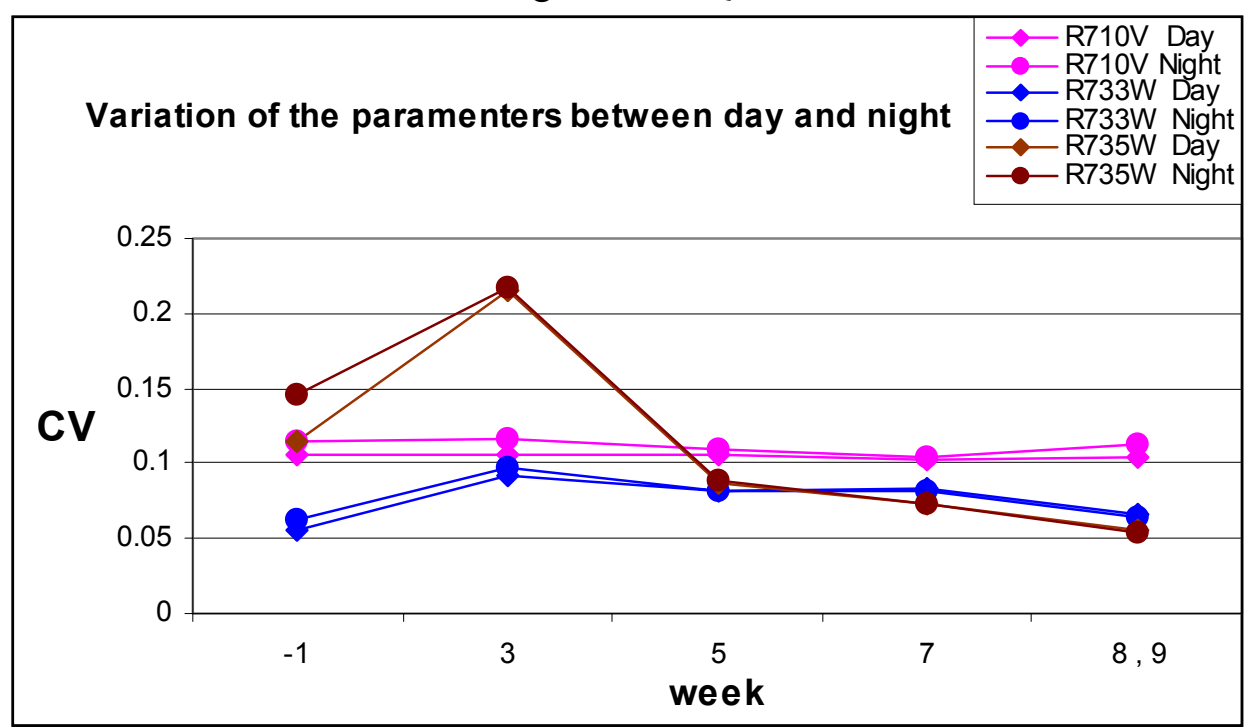

Figure D. $4 \mathrm{CV}$ 


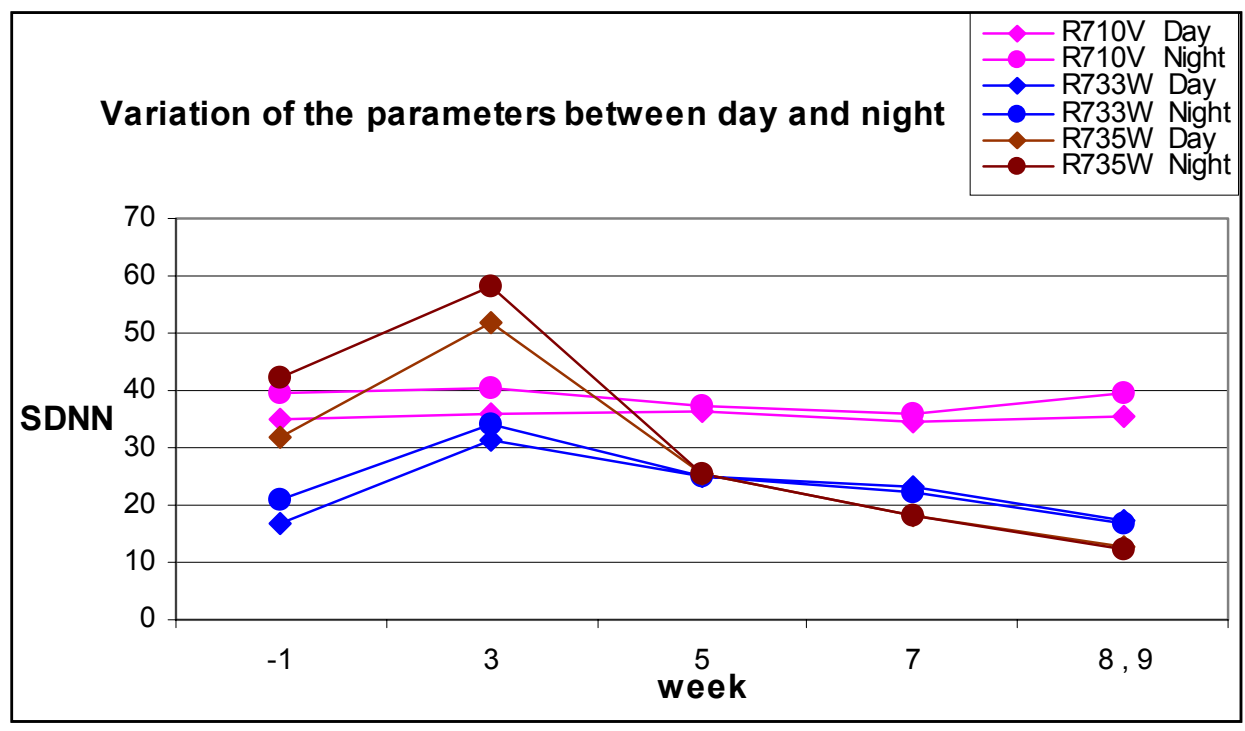

Figure D.5 SDNN

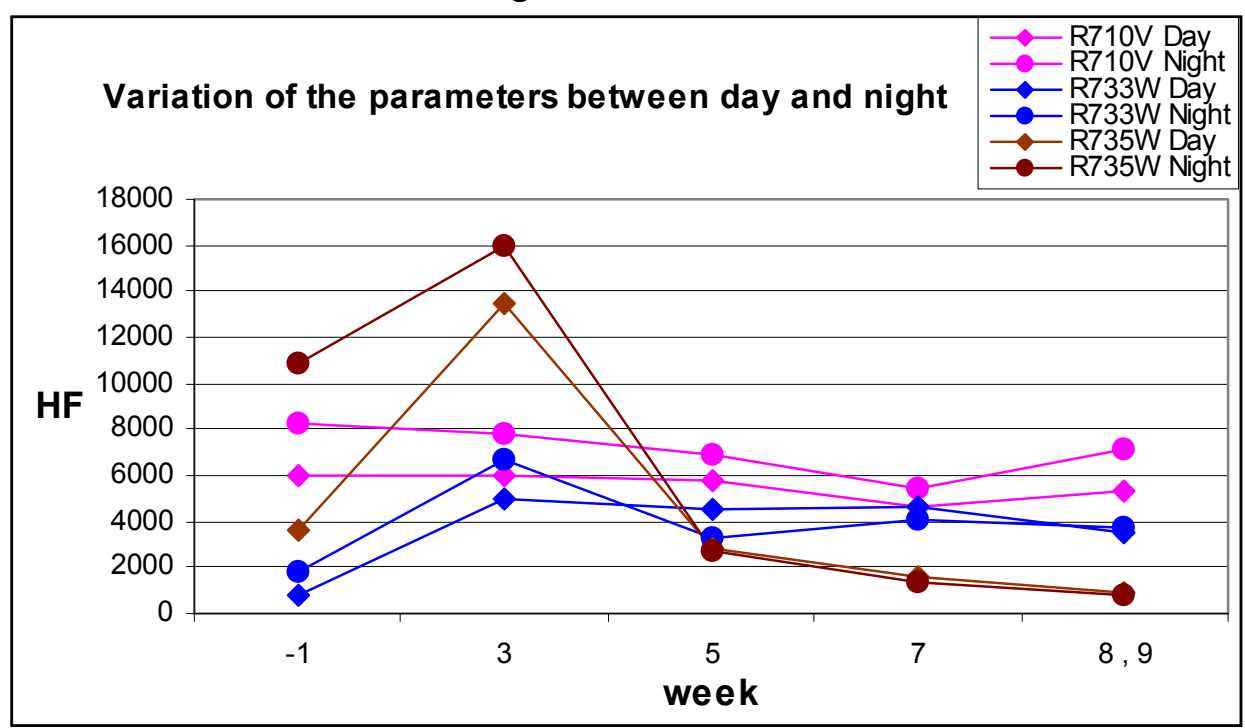

Figure D.6 HF 


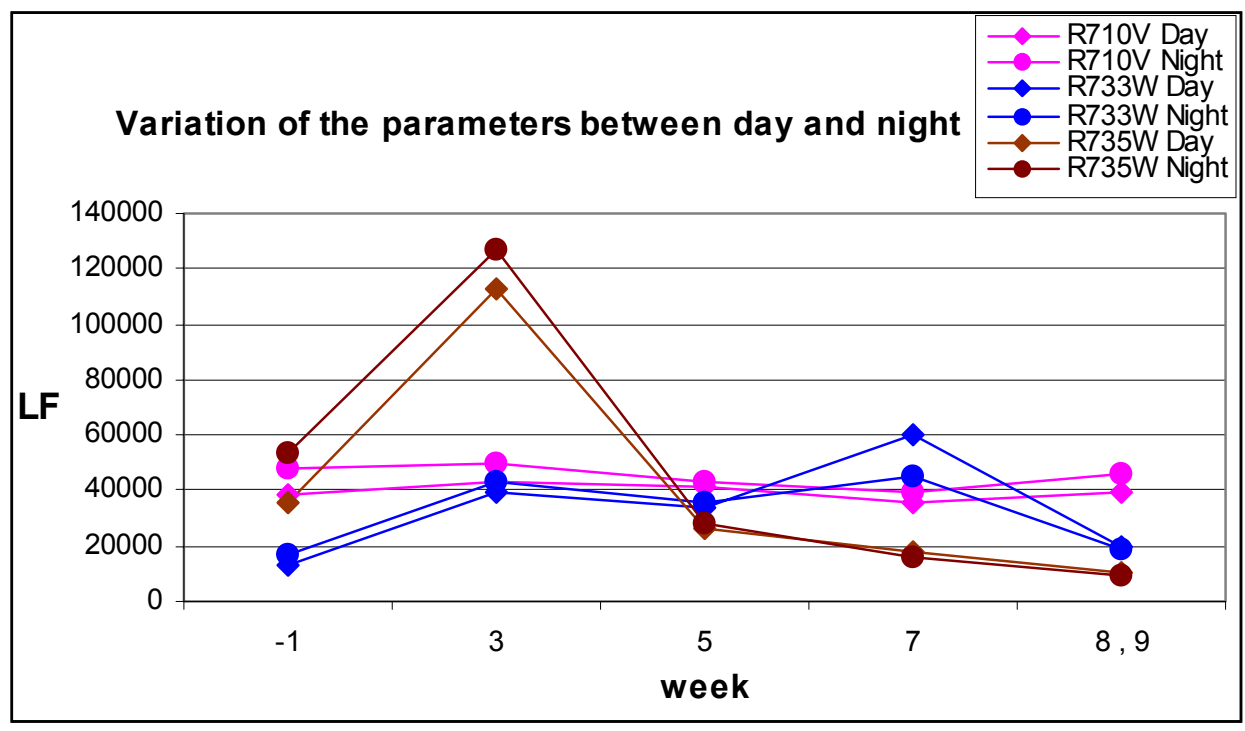

Figure D.7 LF

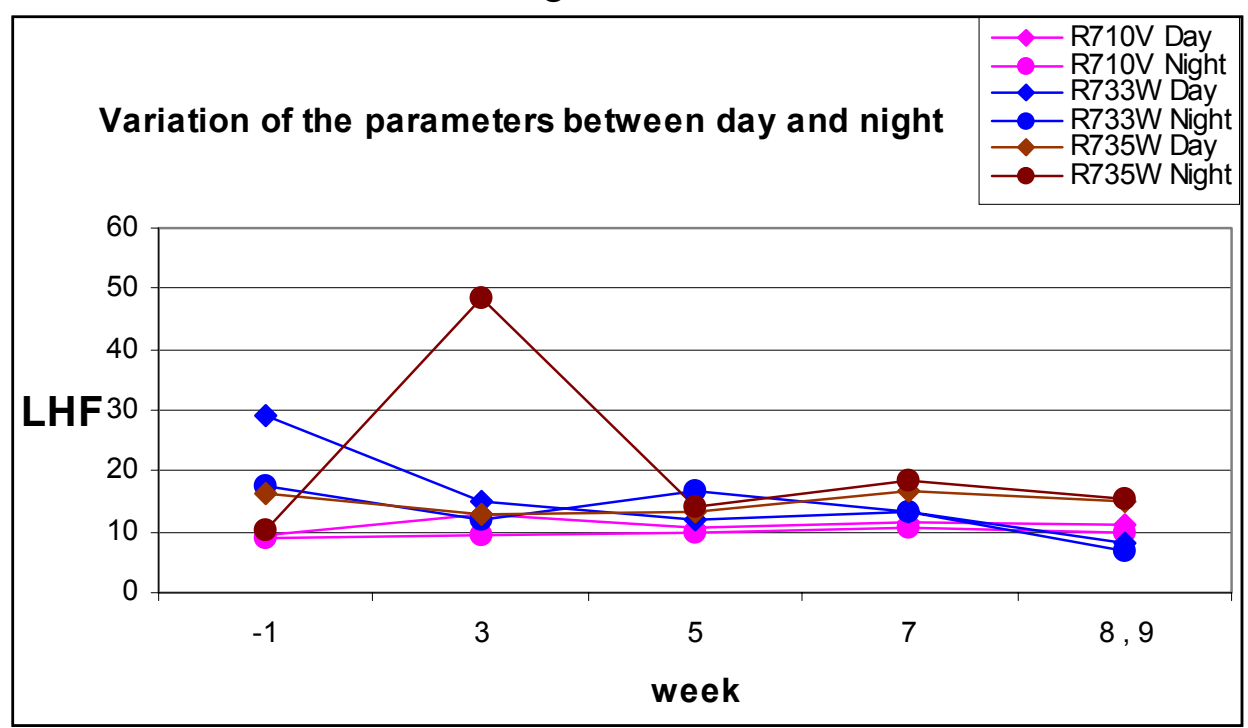

Figure D.8 LHF 


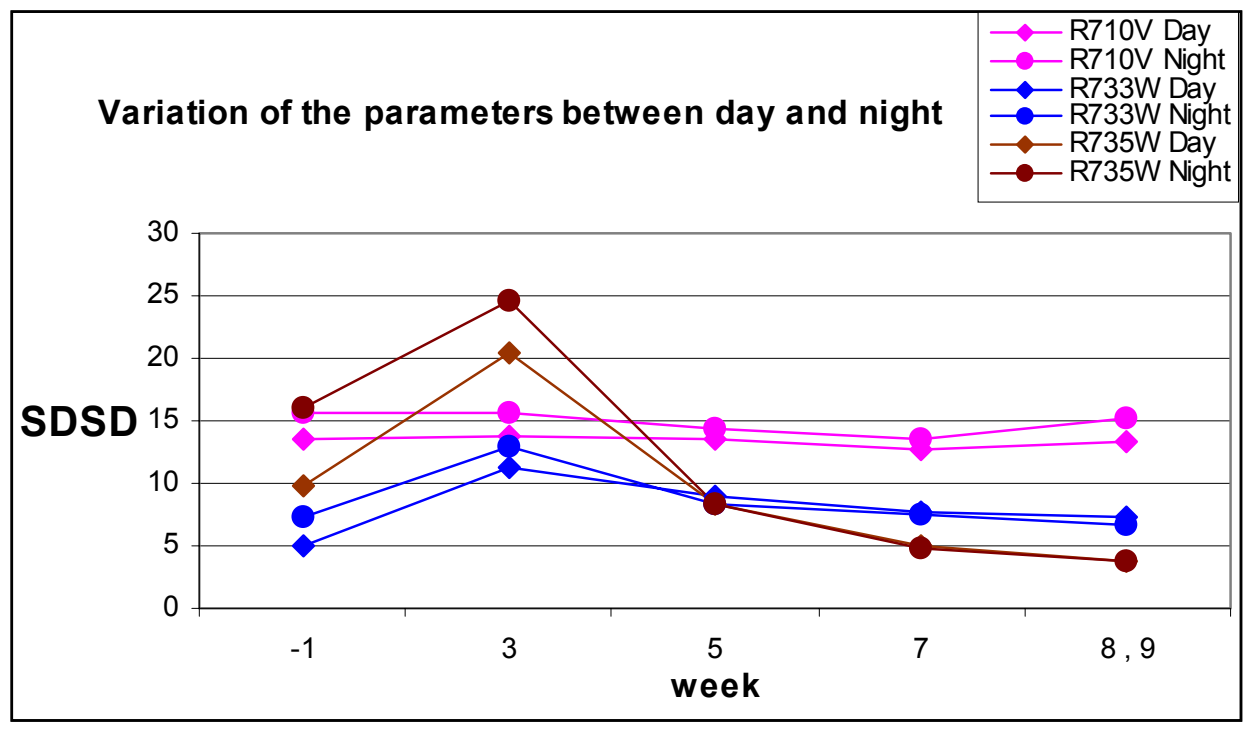

Figure D.9 SDSD

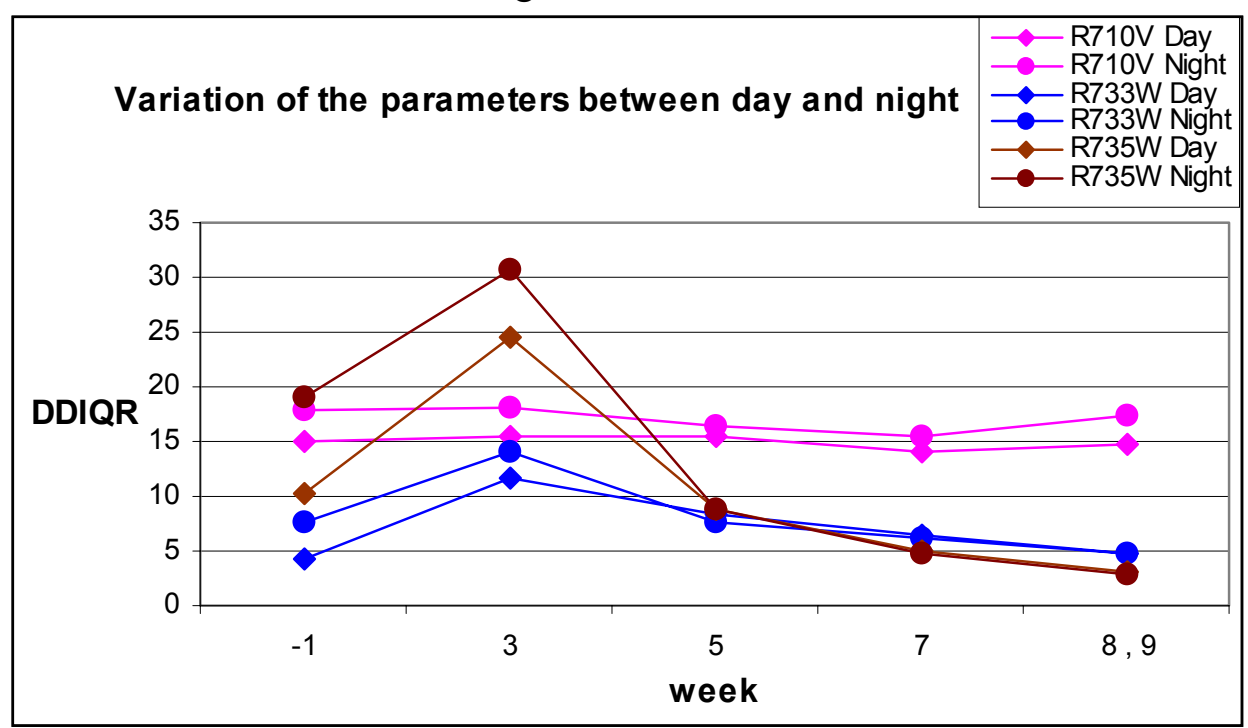

Figure D.10 DDIQR 


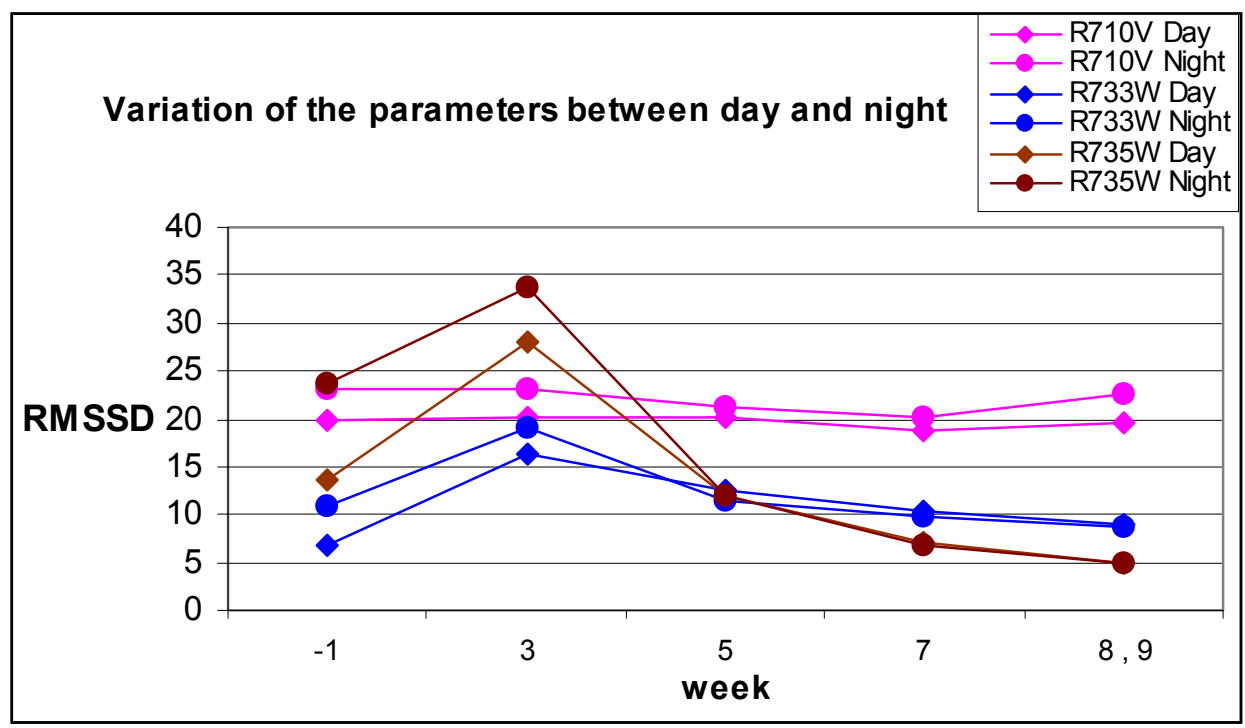

Figure D.11 RMSSD 
Appendix E Individual plots, one parameter versus another parameter 


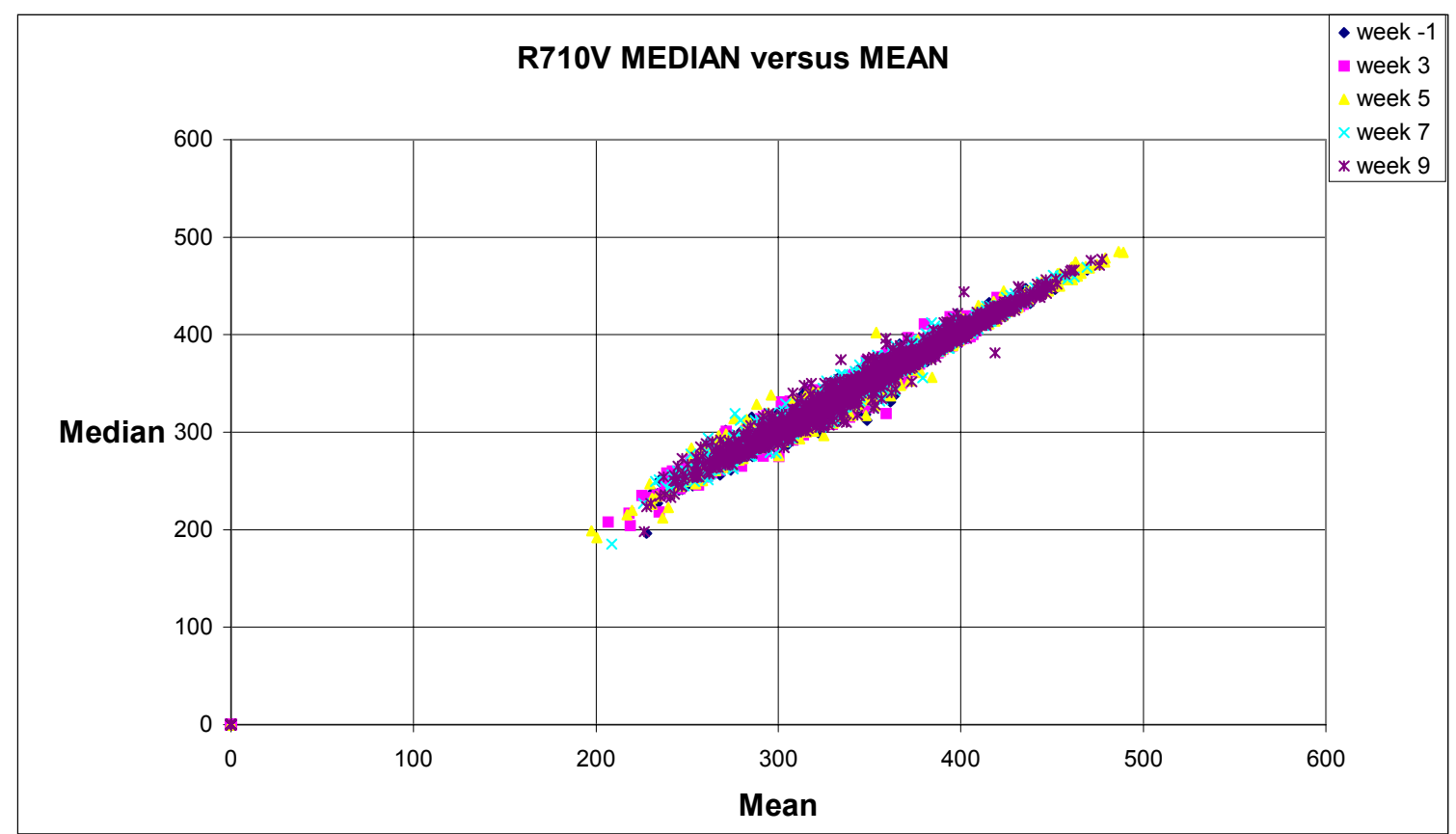

Figure E.1 MEDIAN versus MEAN R710V

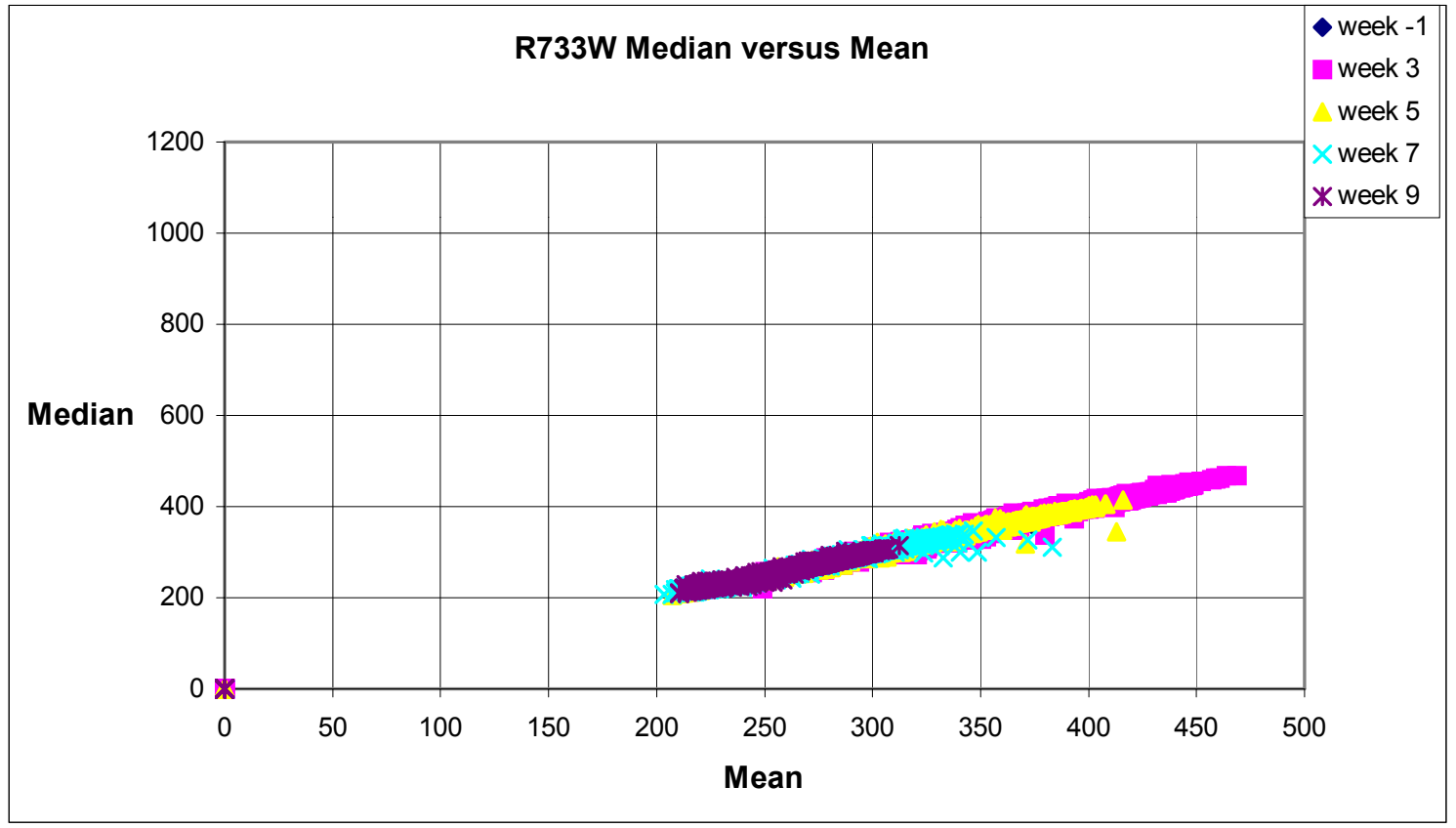

Figure E.2 MEDIAN versus MEAN R733W 


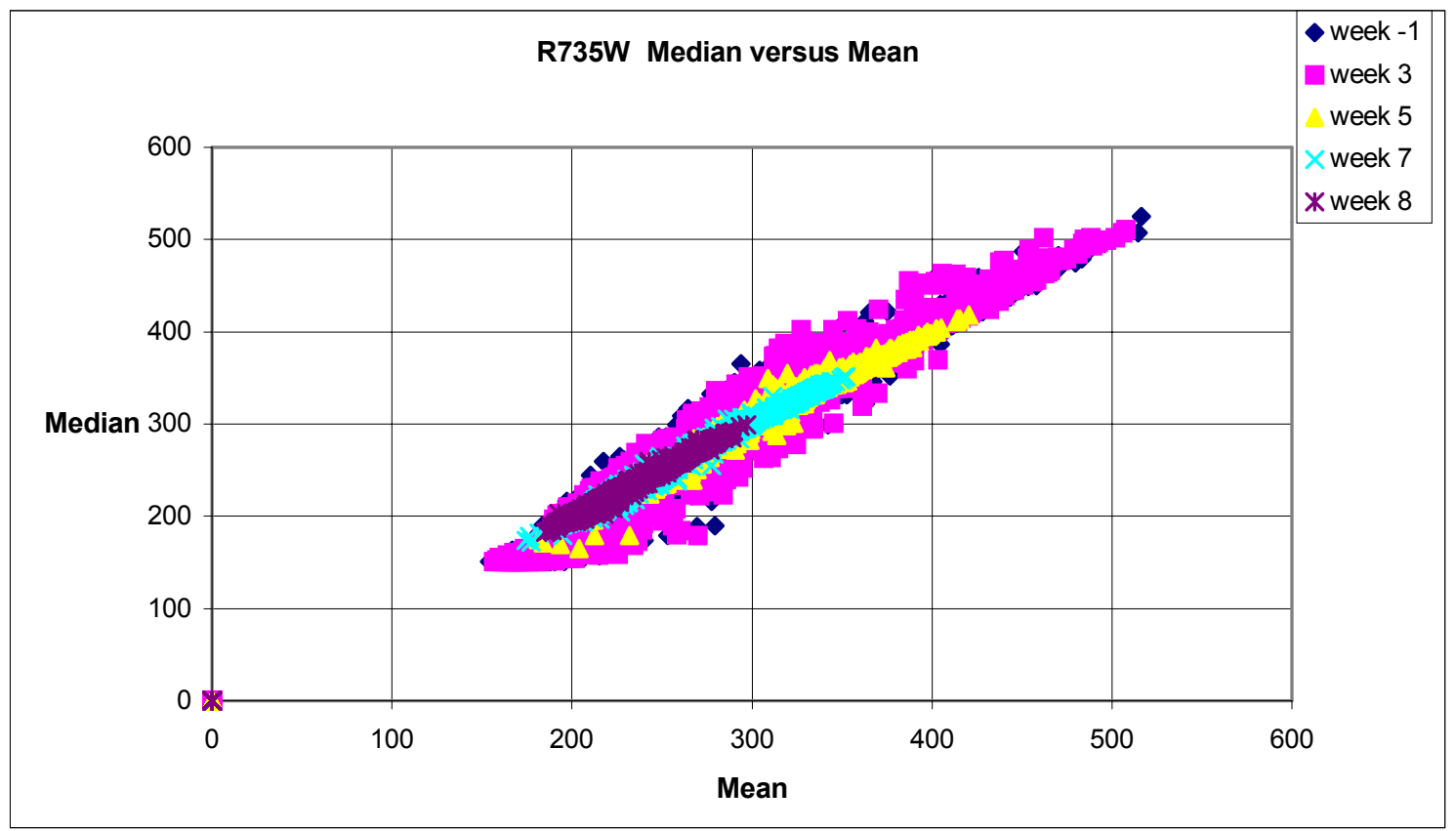

Figure E.3 MEDIAN versus MEAN R735W

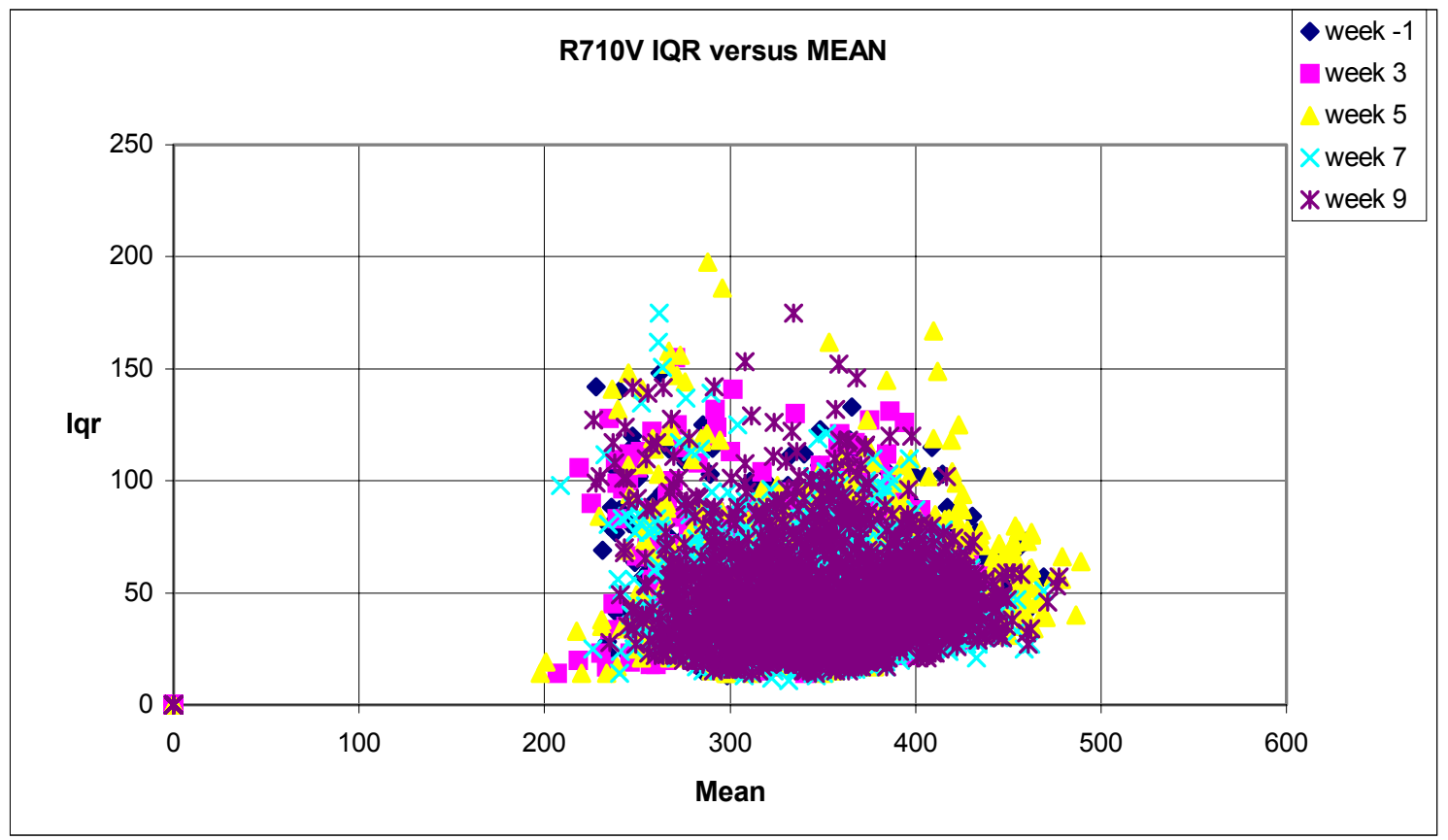

Figure E.4 IQR versus MEAN R710V 


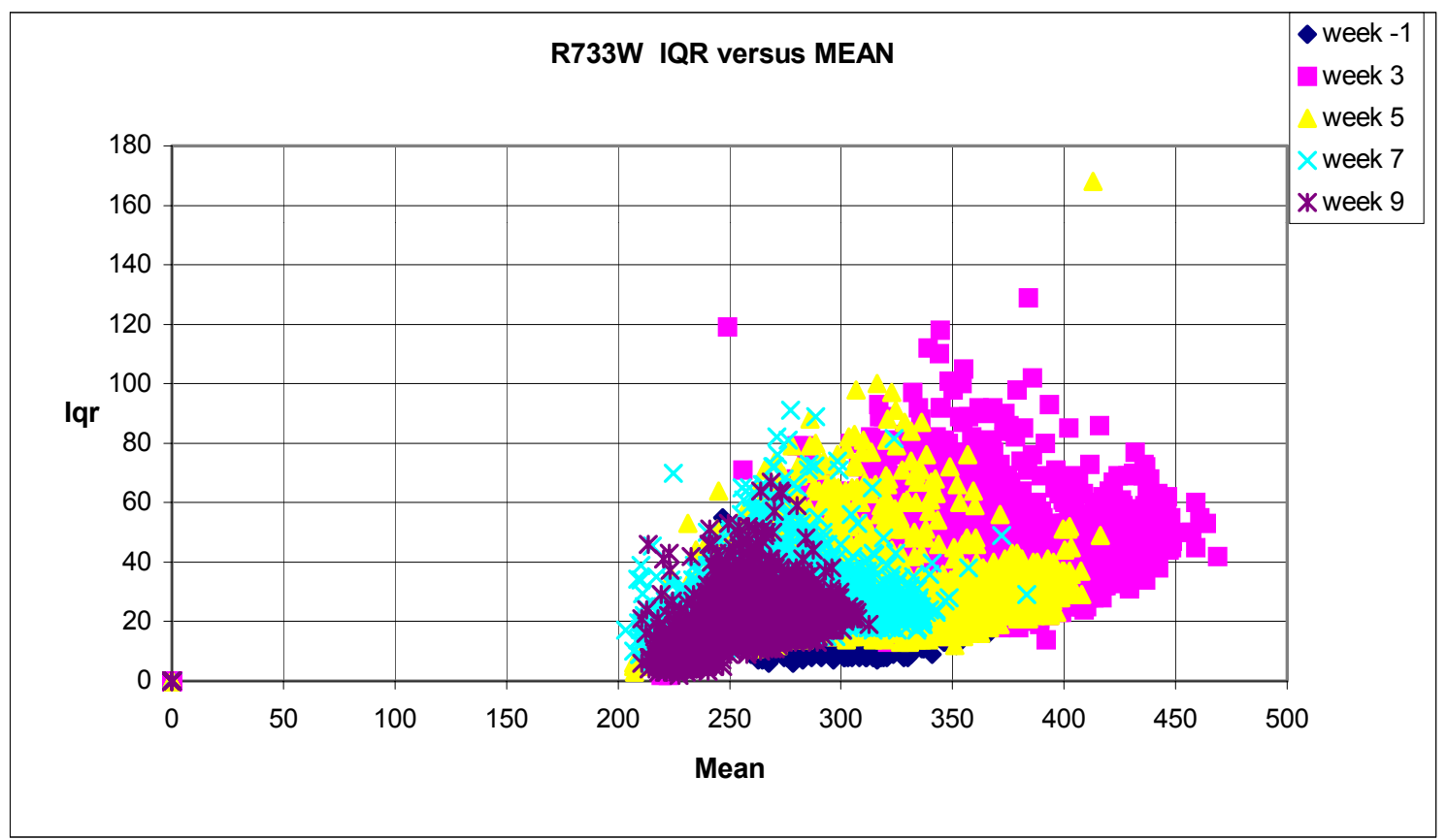

Figure E.5 IQR versus MEAN R733W

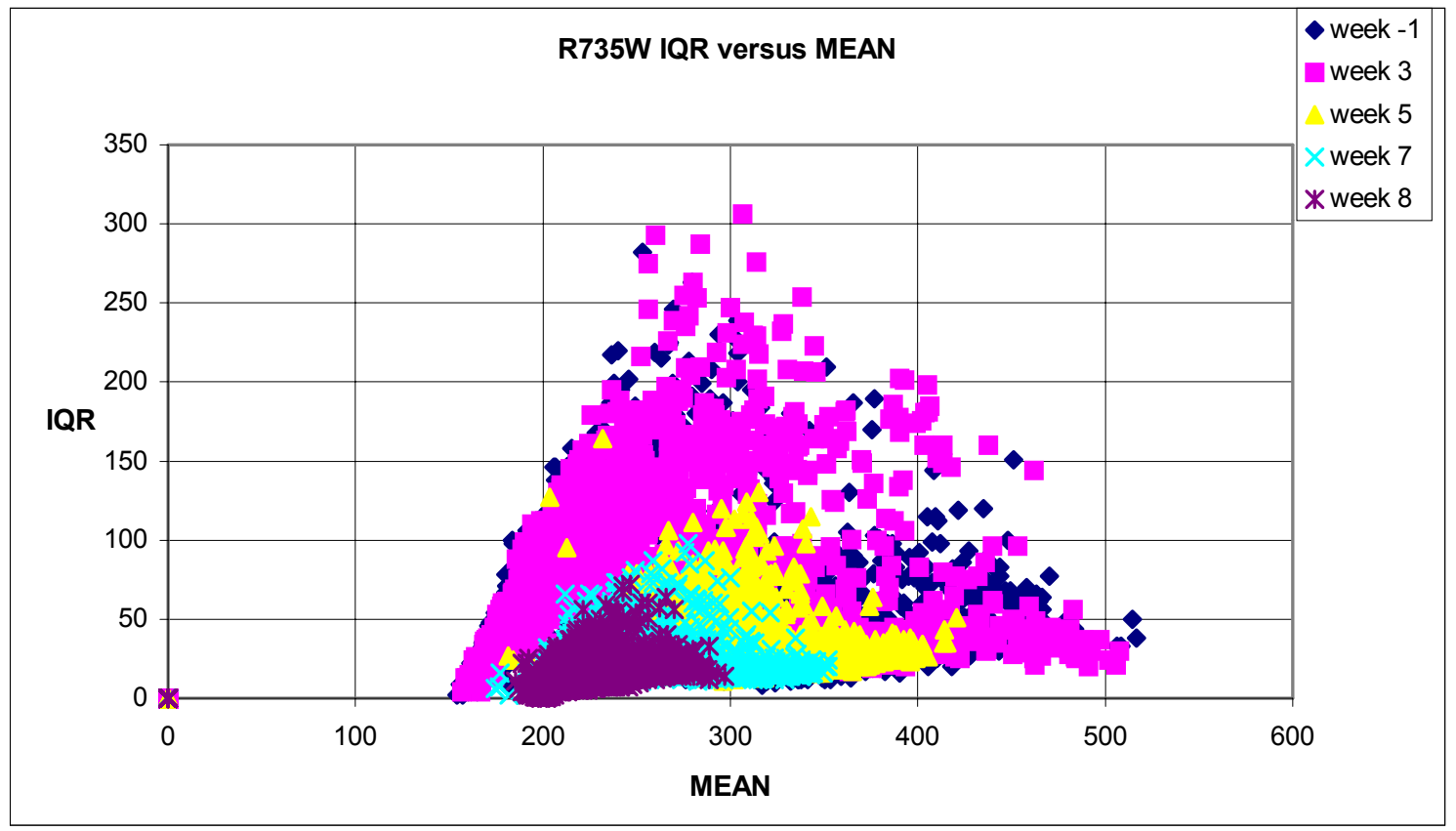

Figure E.6 IQR versus MEAN R735W 


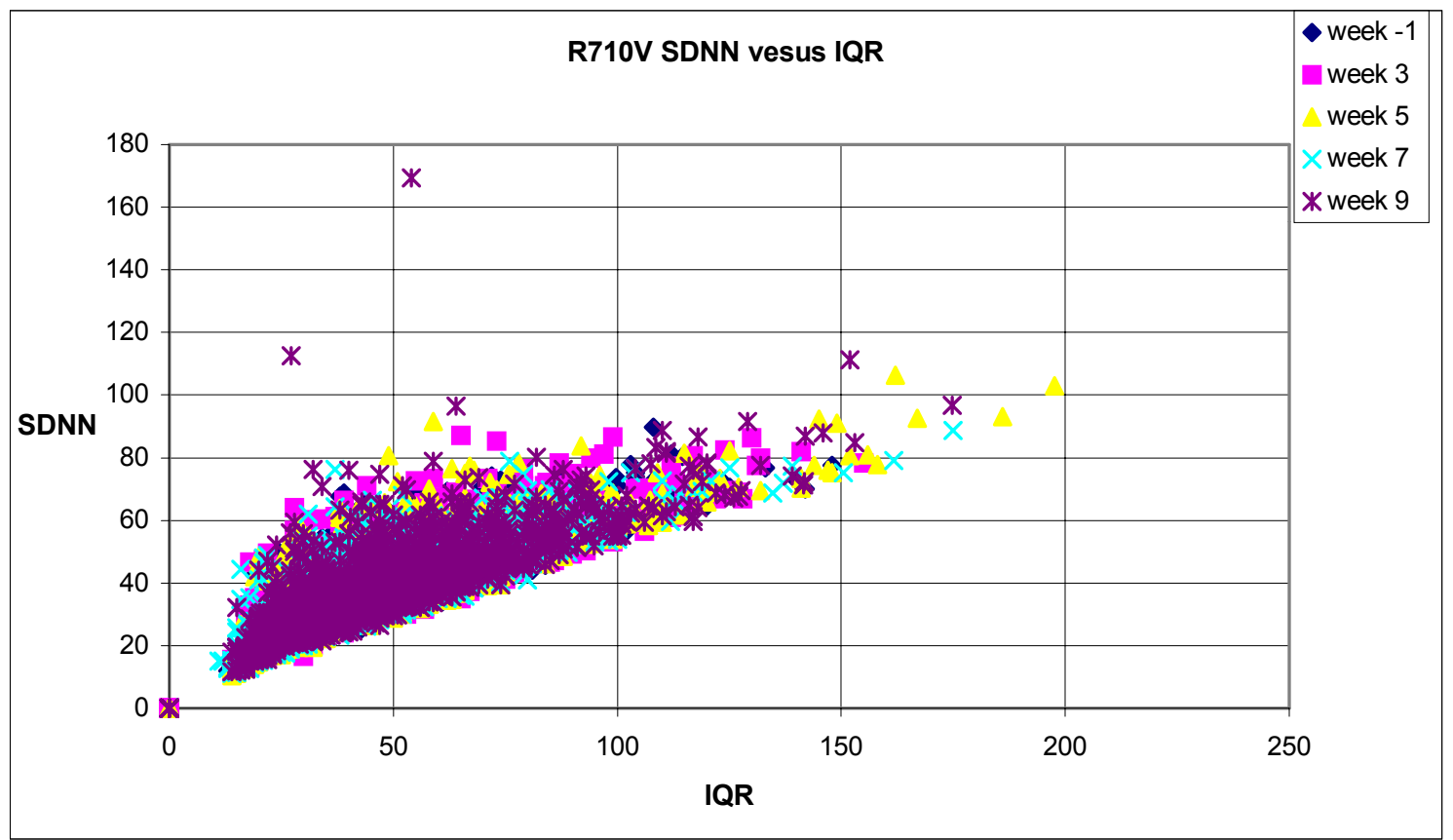

Figure E.7 SDNN versus IQR R710V

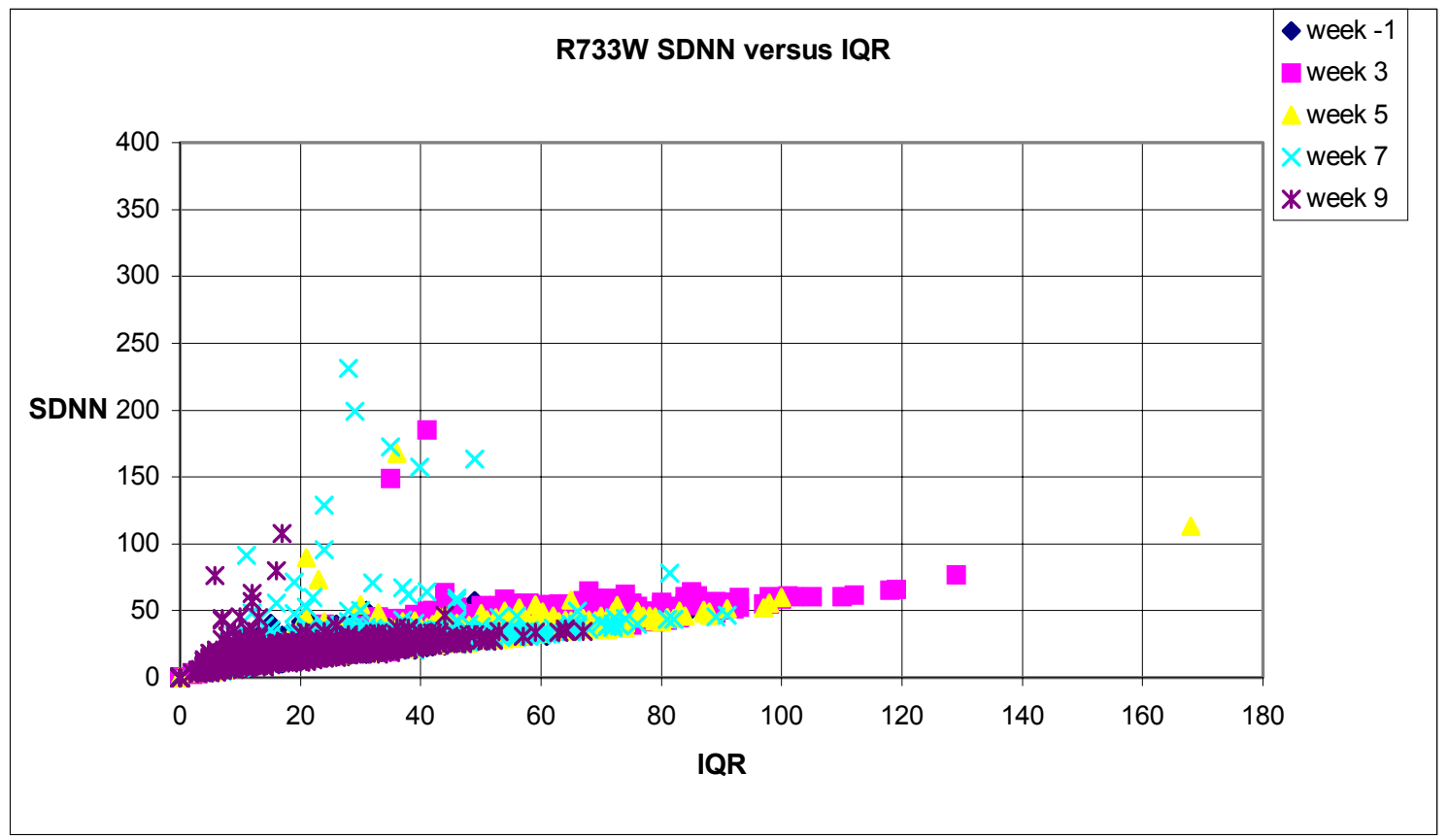

Figure E.8 SDNN versus IQR R733W 


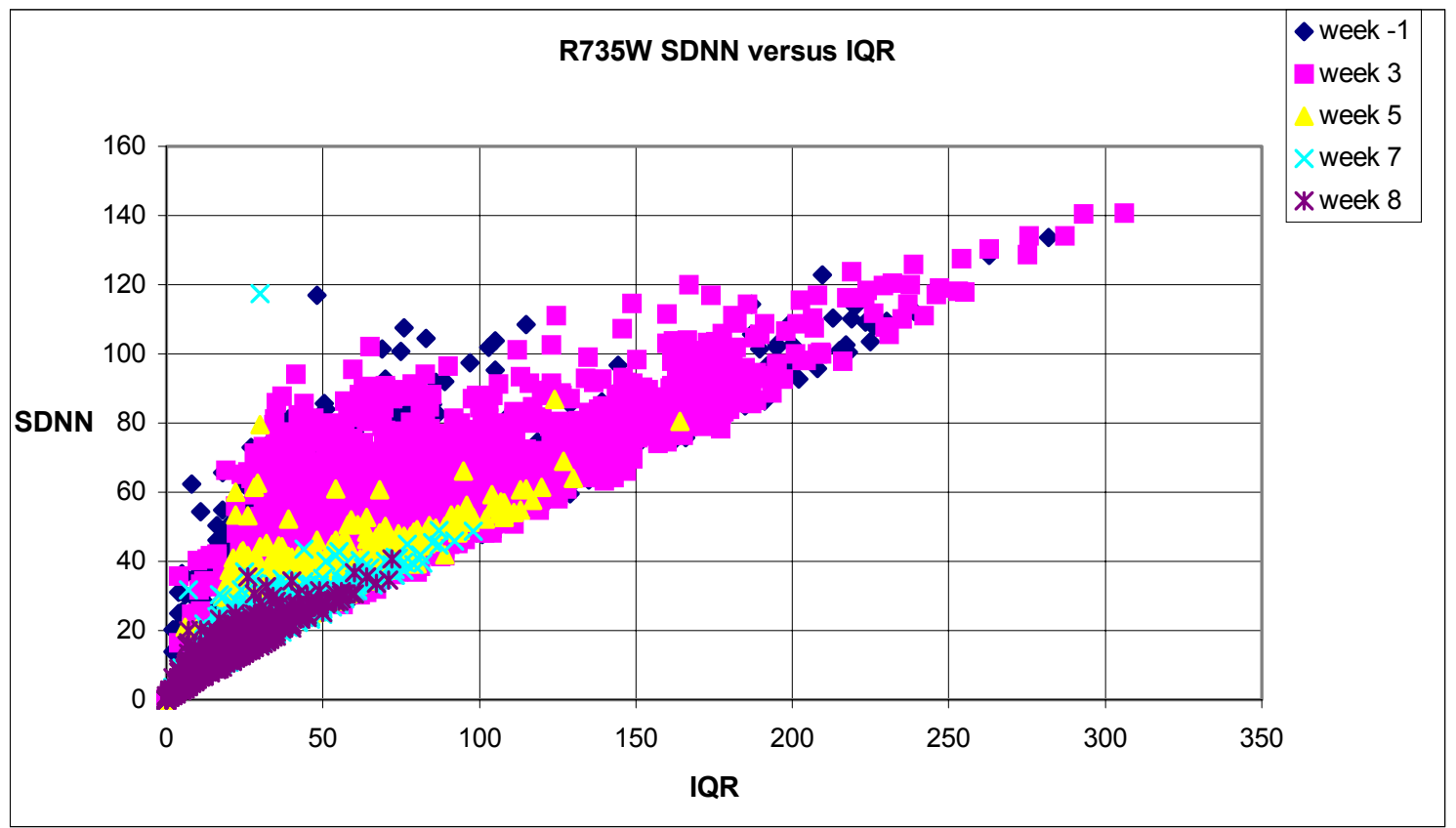

Figure E.9 SDNN versus IQR R735W

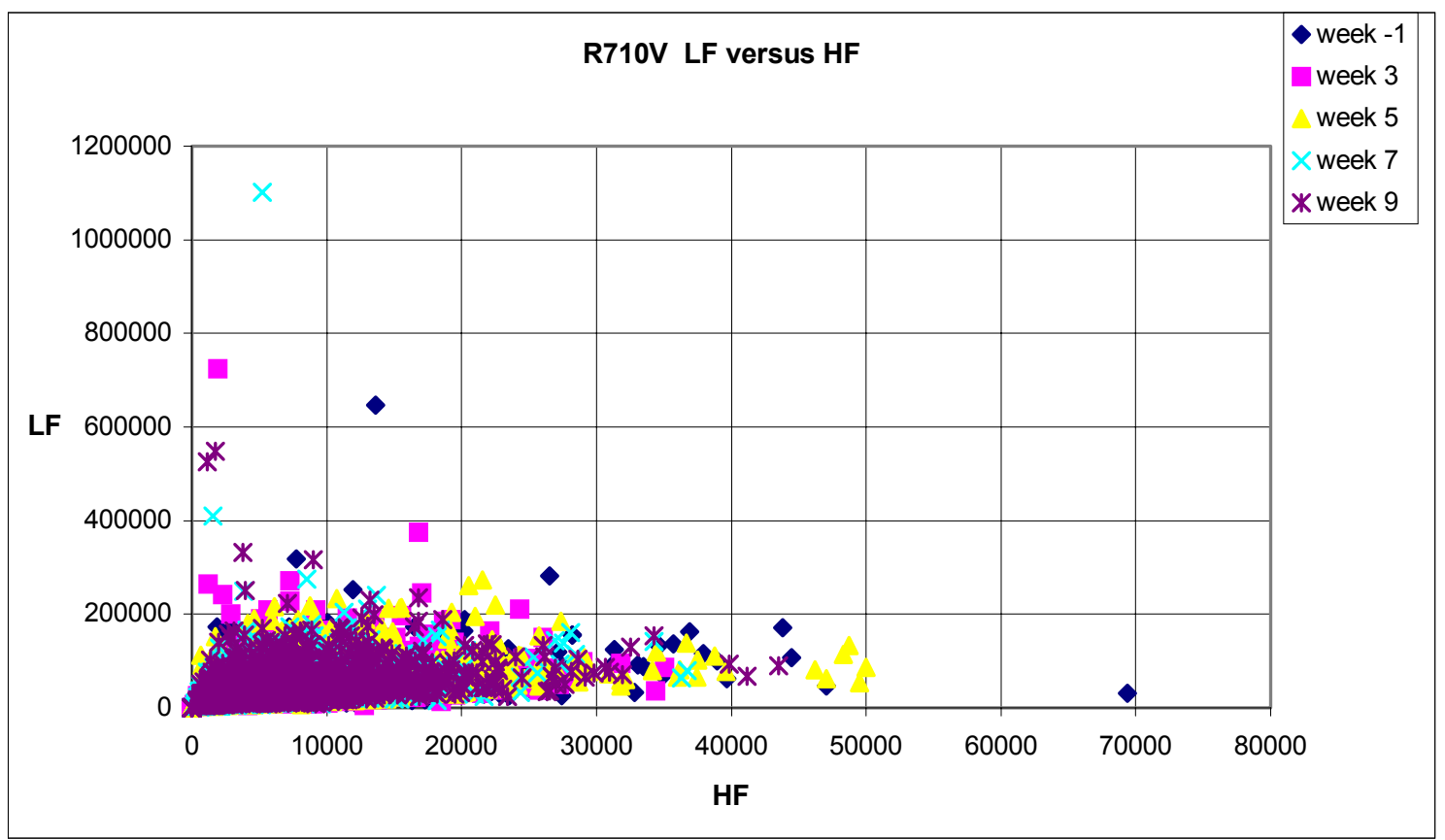

Figure E.10 LF versus HF R710V 


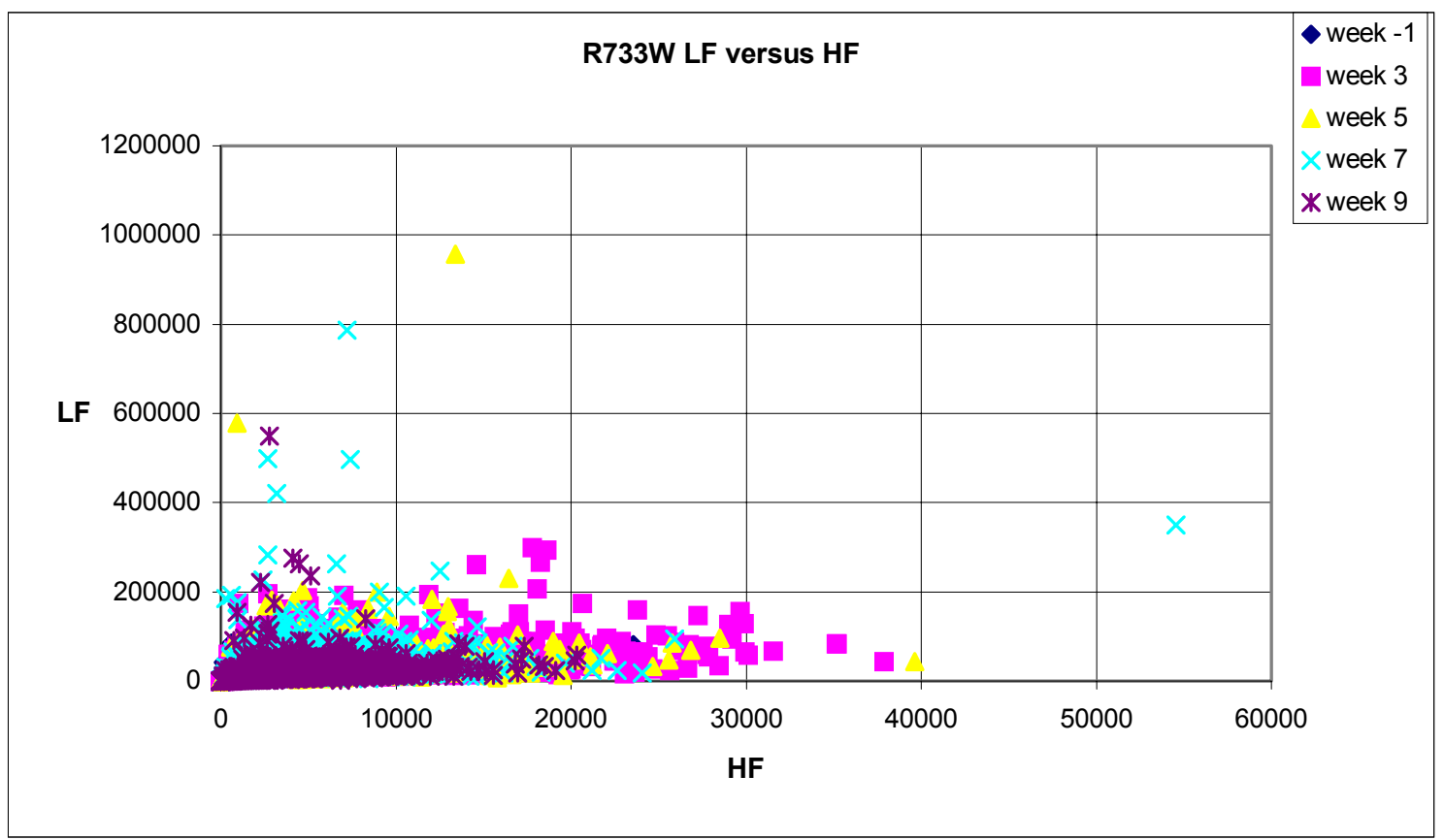

Figure E.11 LF versus HF R733W

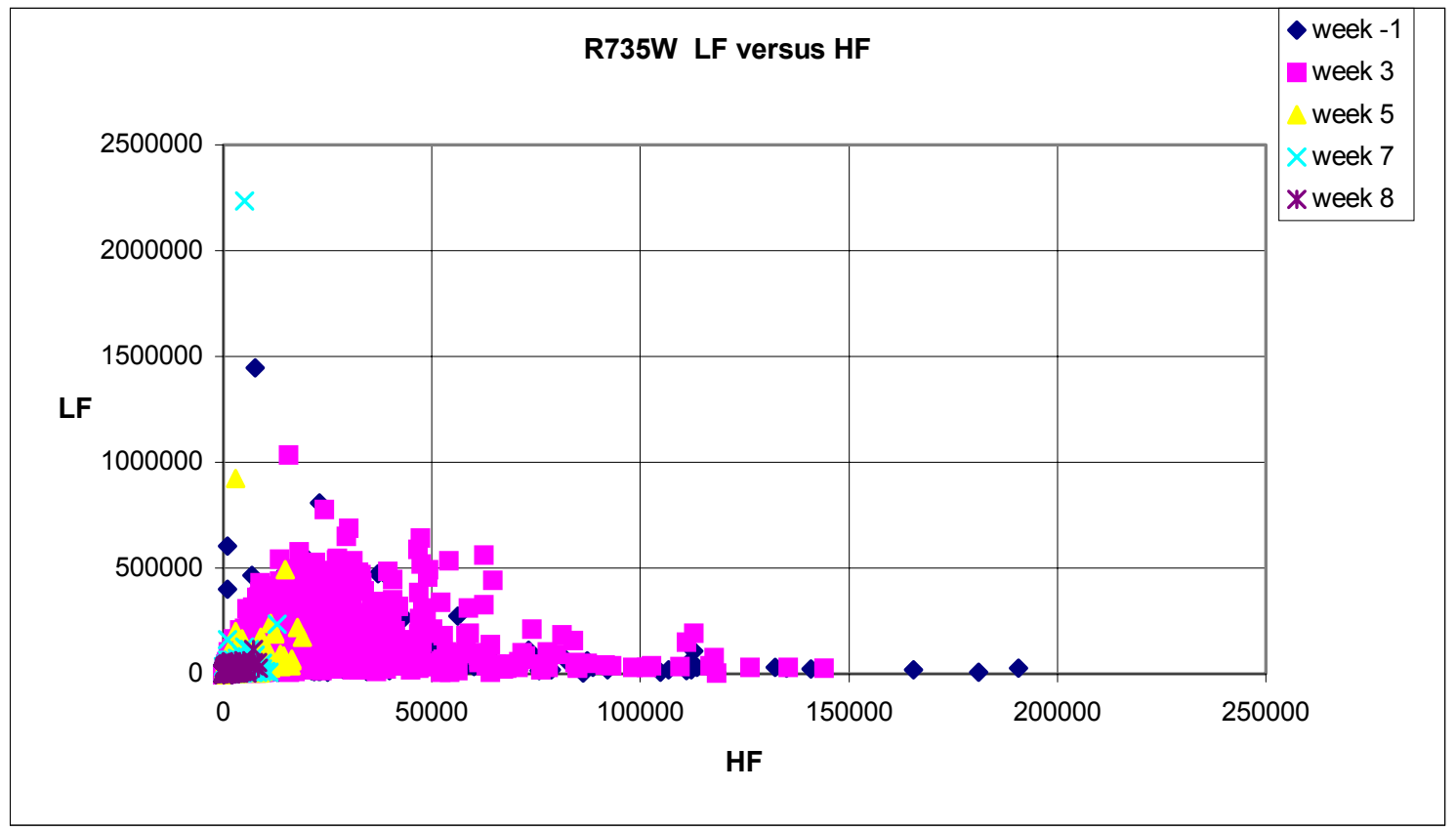

Figure E.12 LF versus HF R735W 


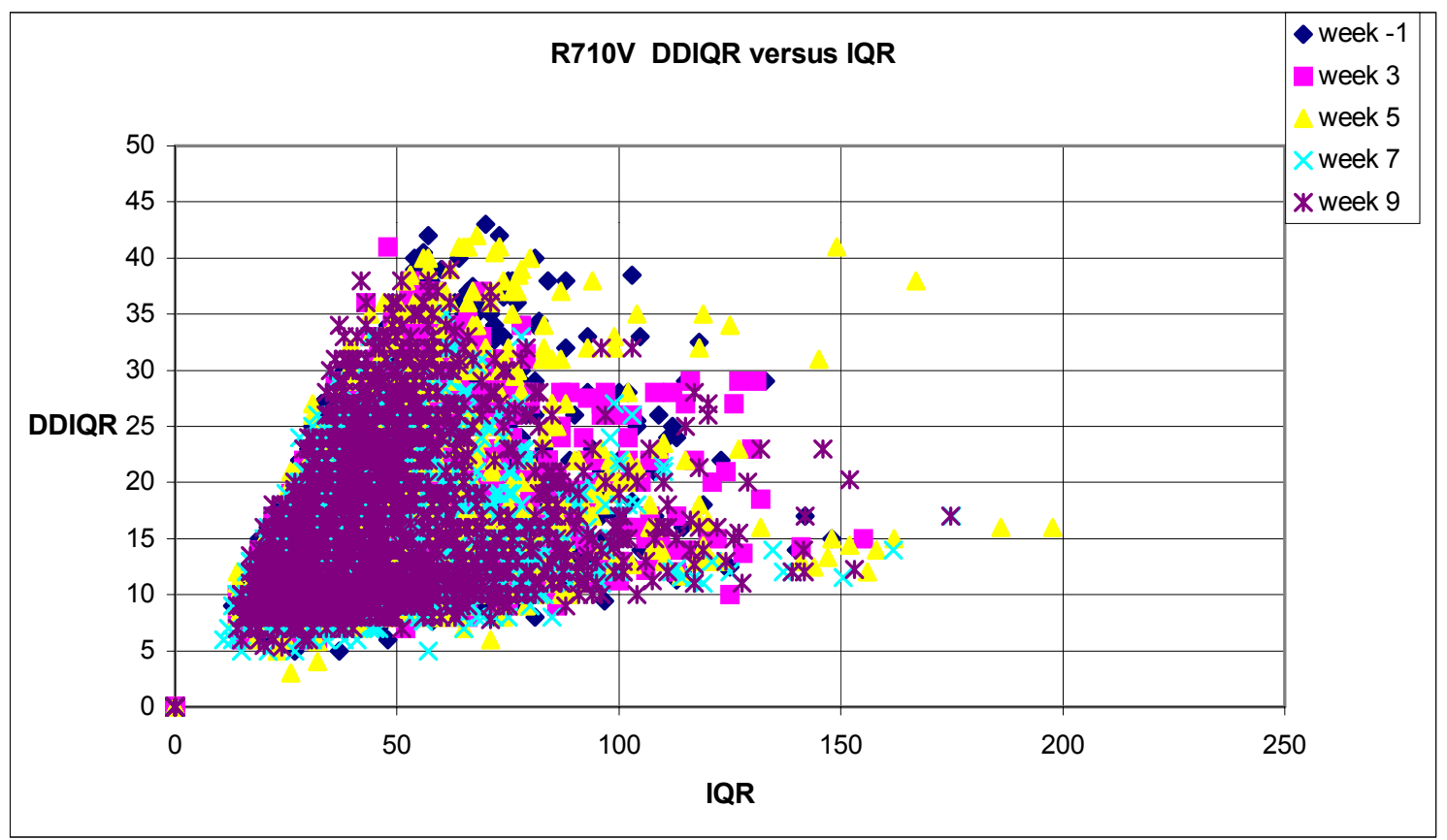

Figure E.13 DDIQR versus IQR R710V

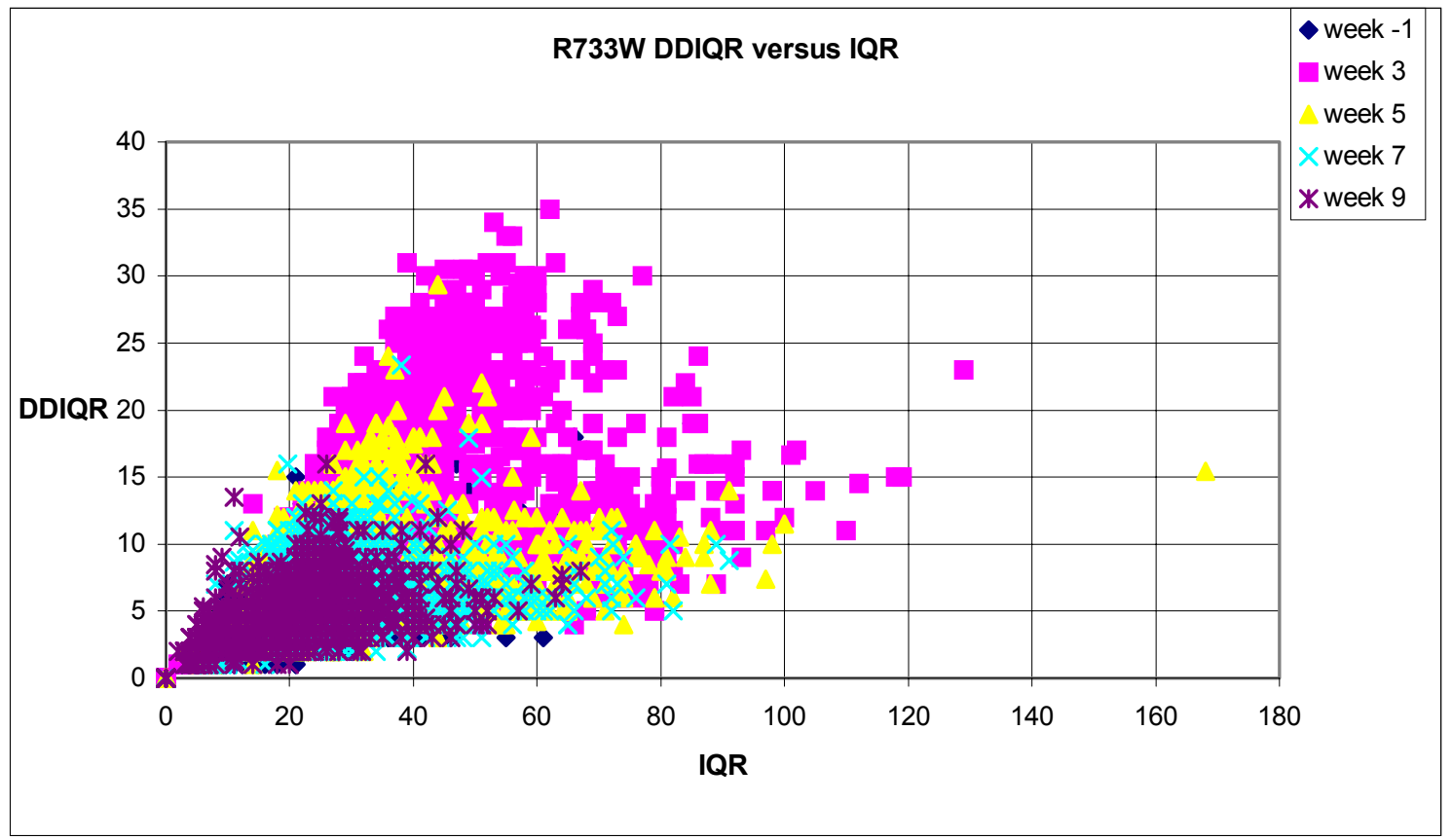

Figure E.14 DDIQR versus IQR R733W 


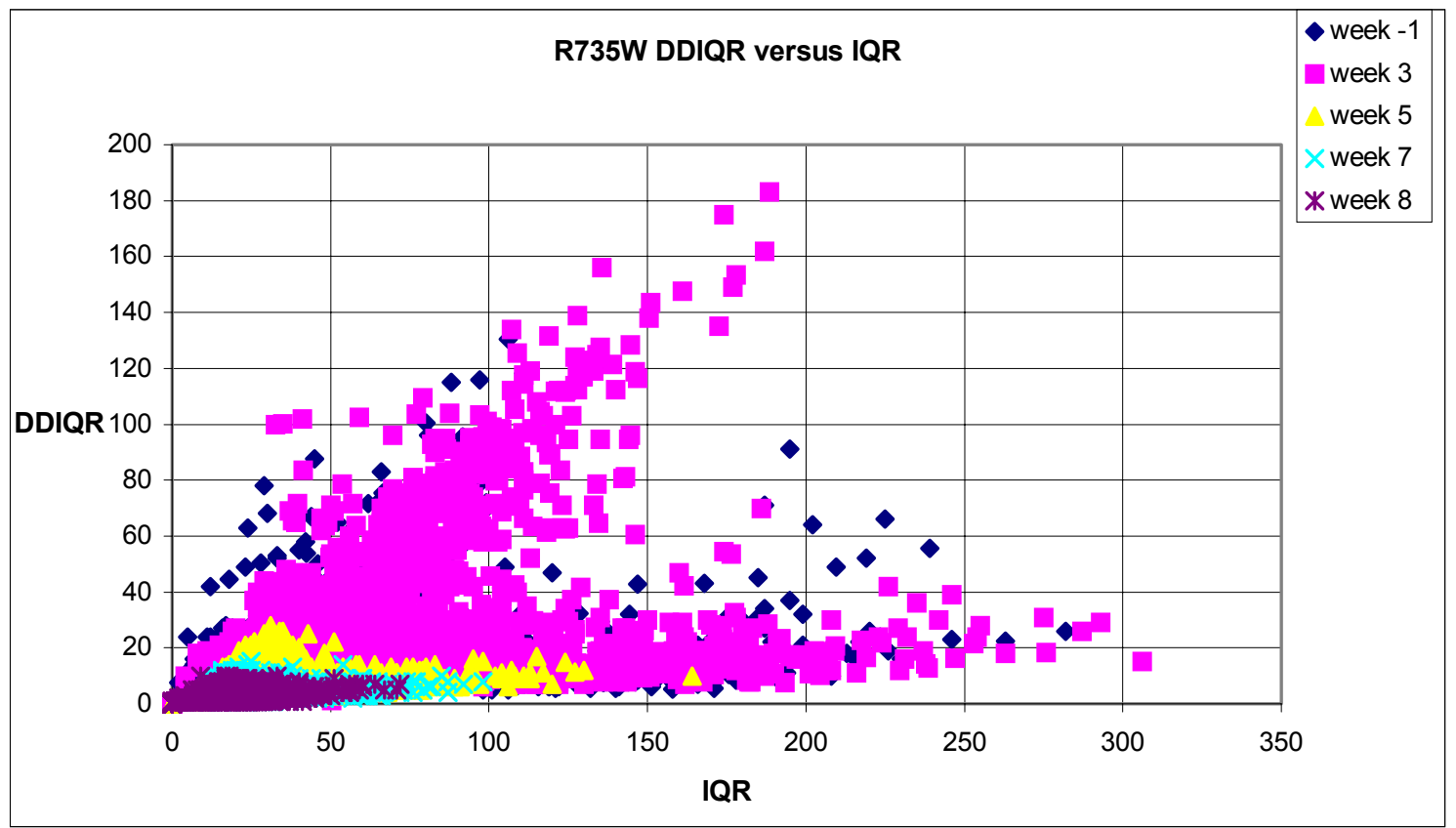

Figure E.15 DDIQR versus IQR R735W

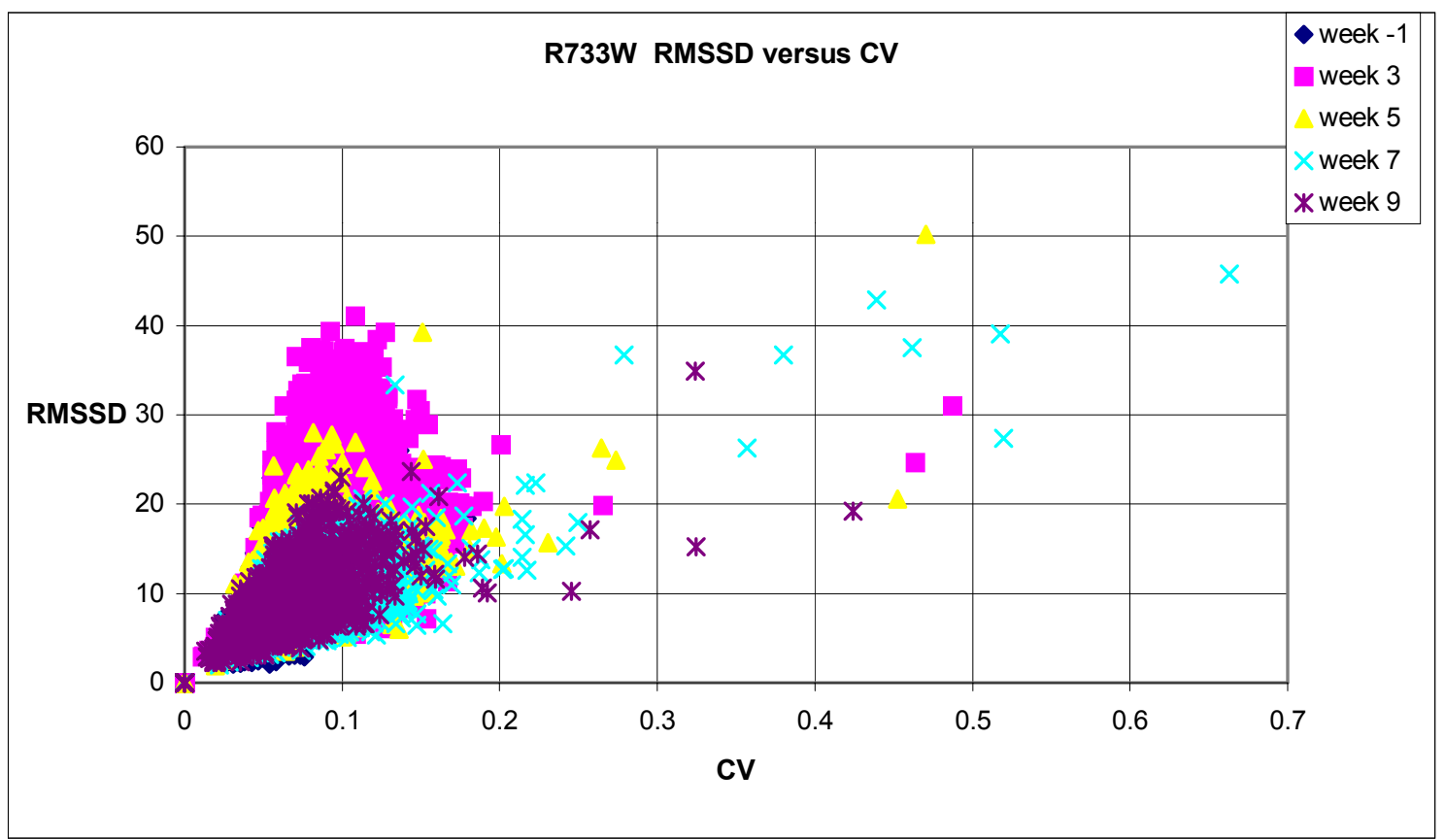

Figure E.16 RMSSD versus CV R733W 


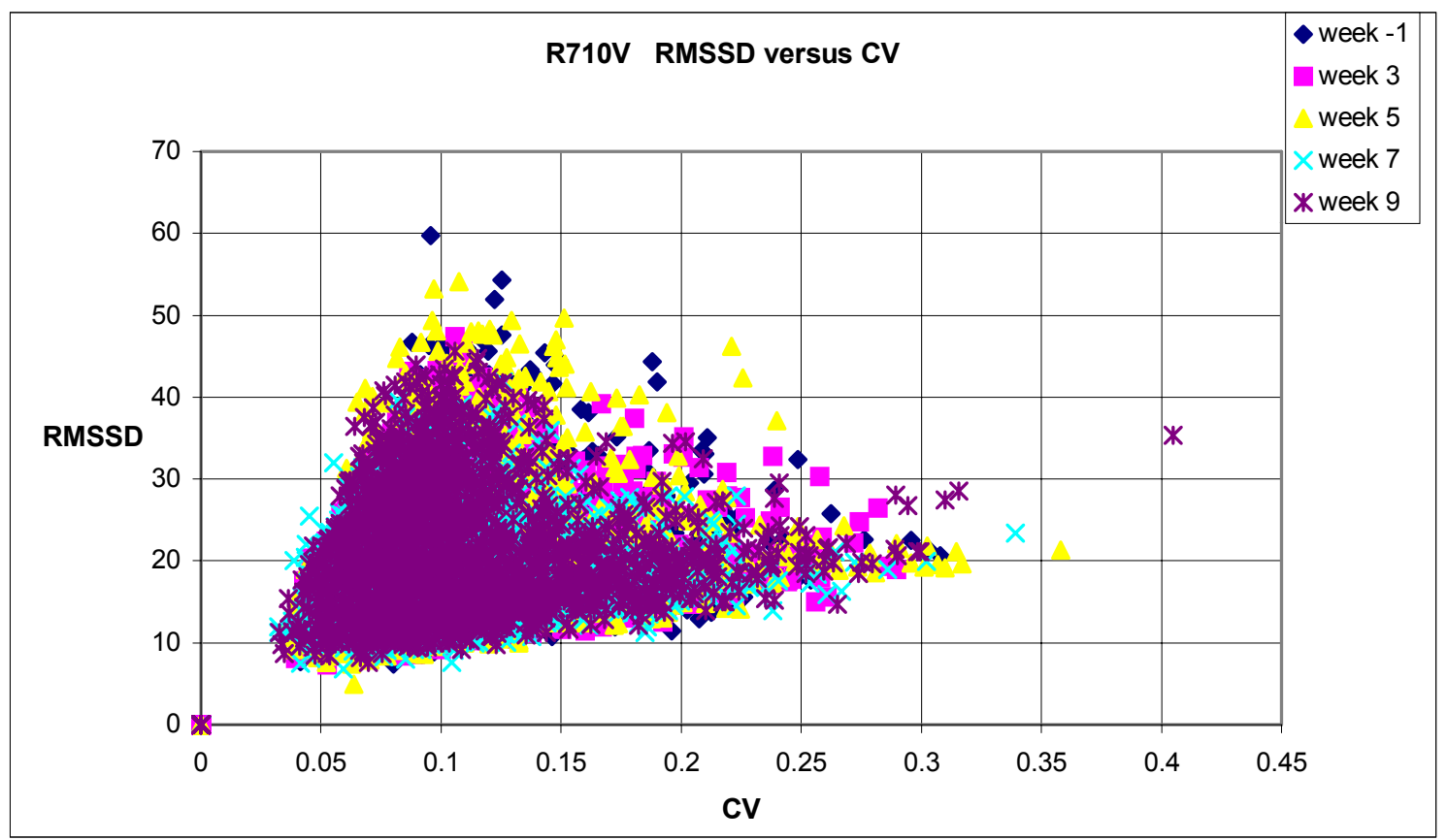

Figure E.17 RMSSD versus CV R710V

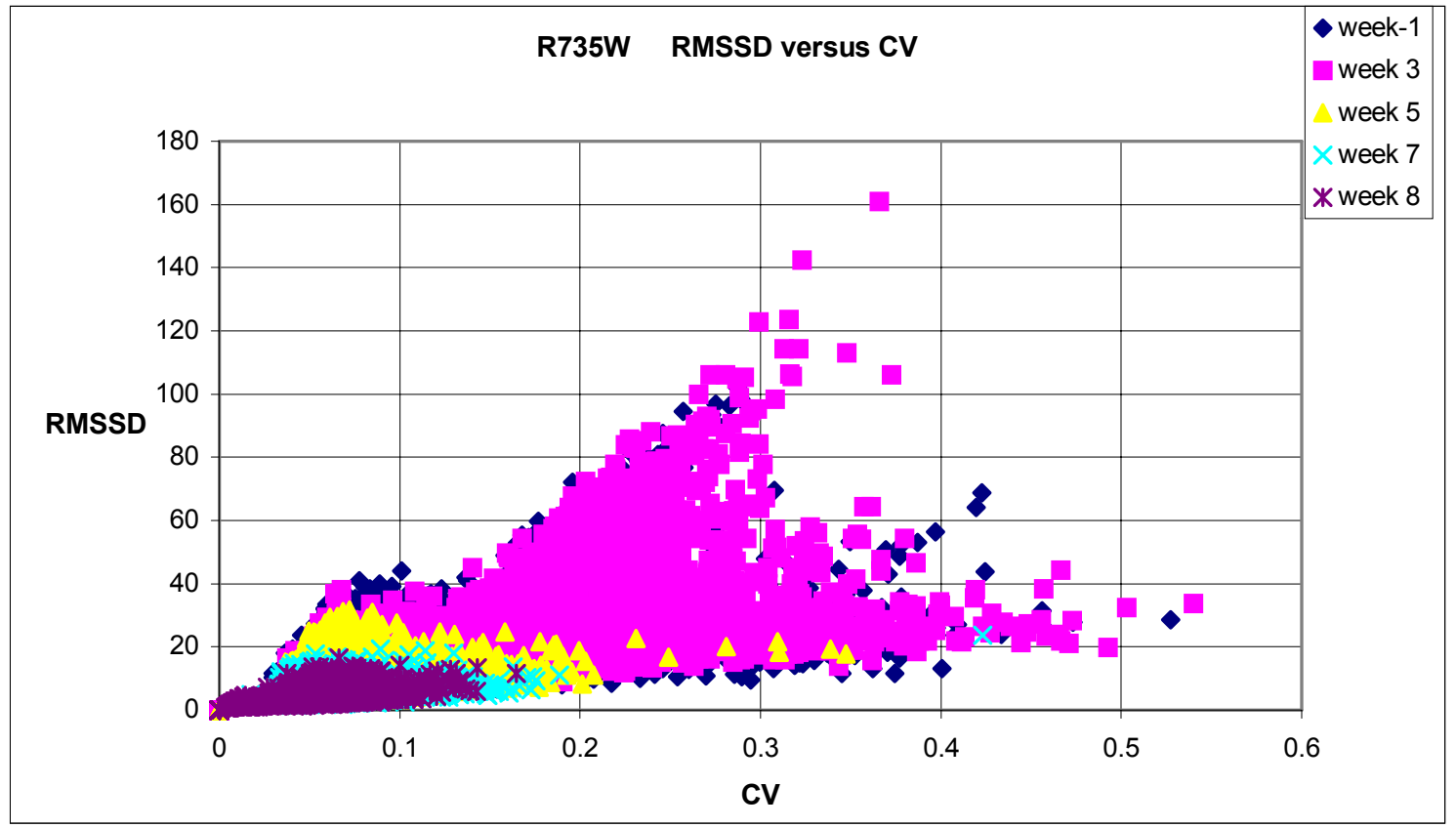

Figure E.18 RMSSD versus CV R735W 
Appendix F Individual plots, all data from five weeks plotted versus hour 


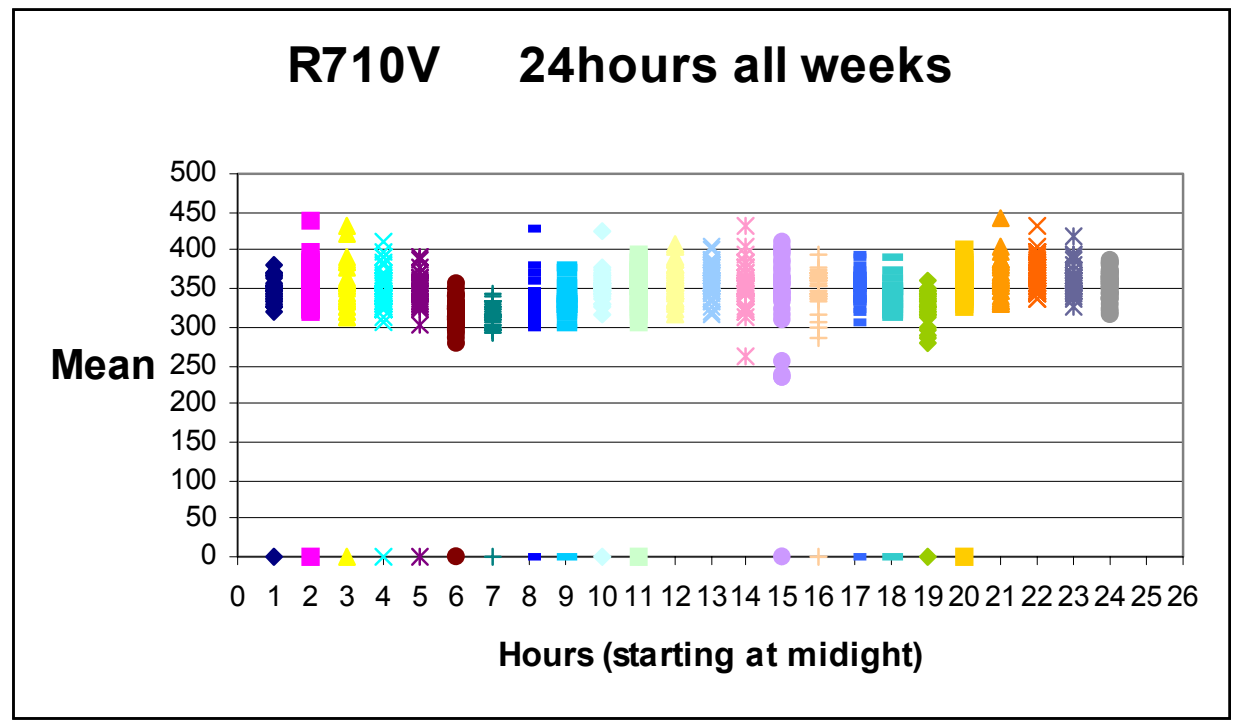

Figure F.1 MEAN R710V

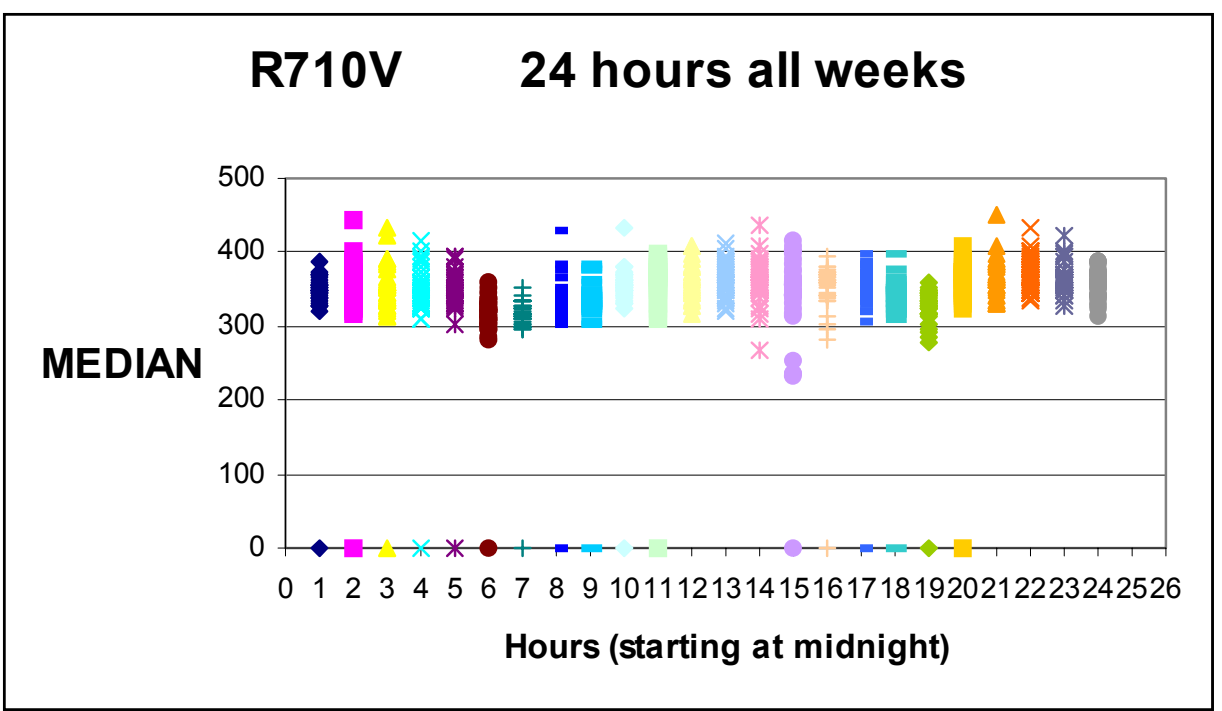

Figure F.2 MEDIAN R710V 


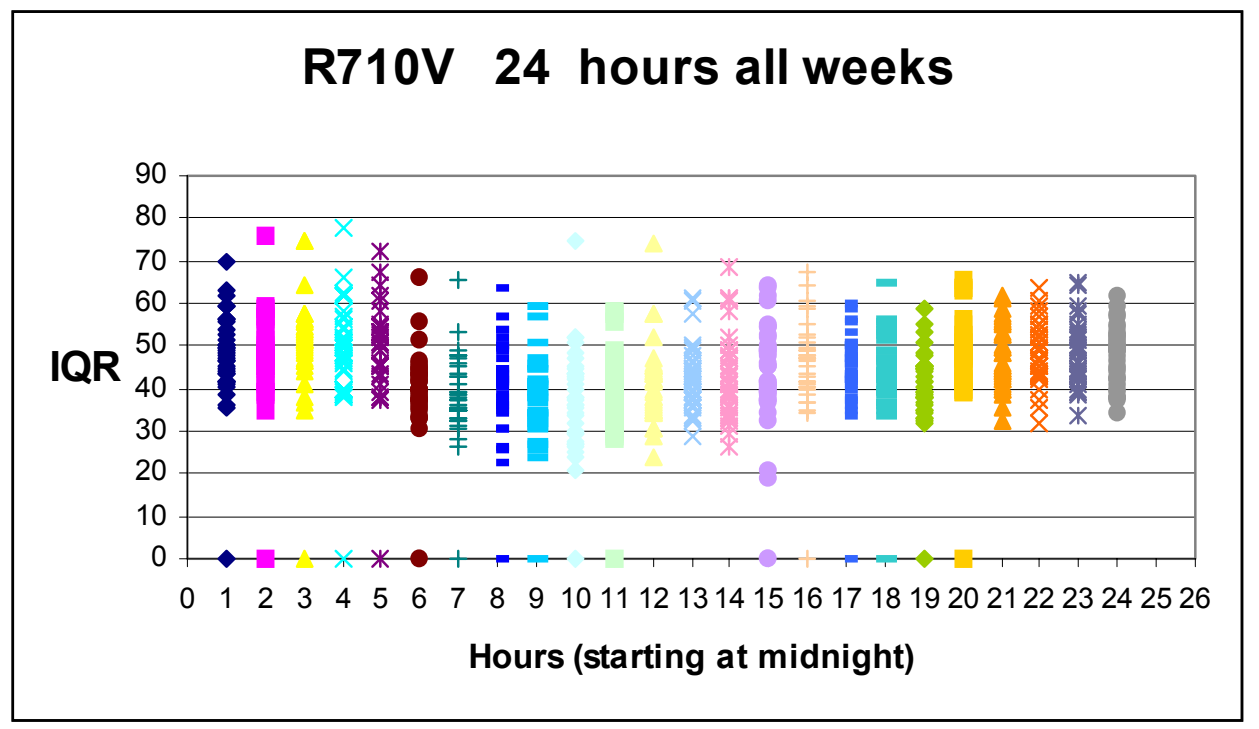

Figure F.3 IQR R710V

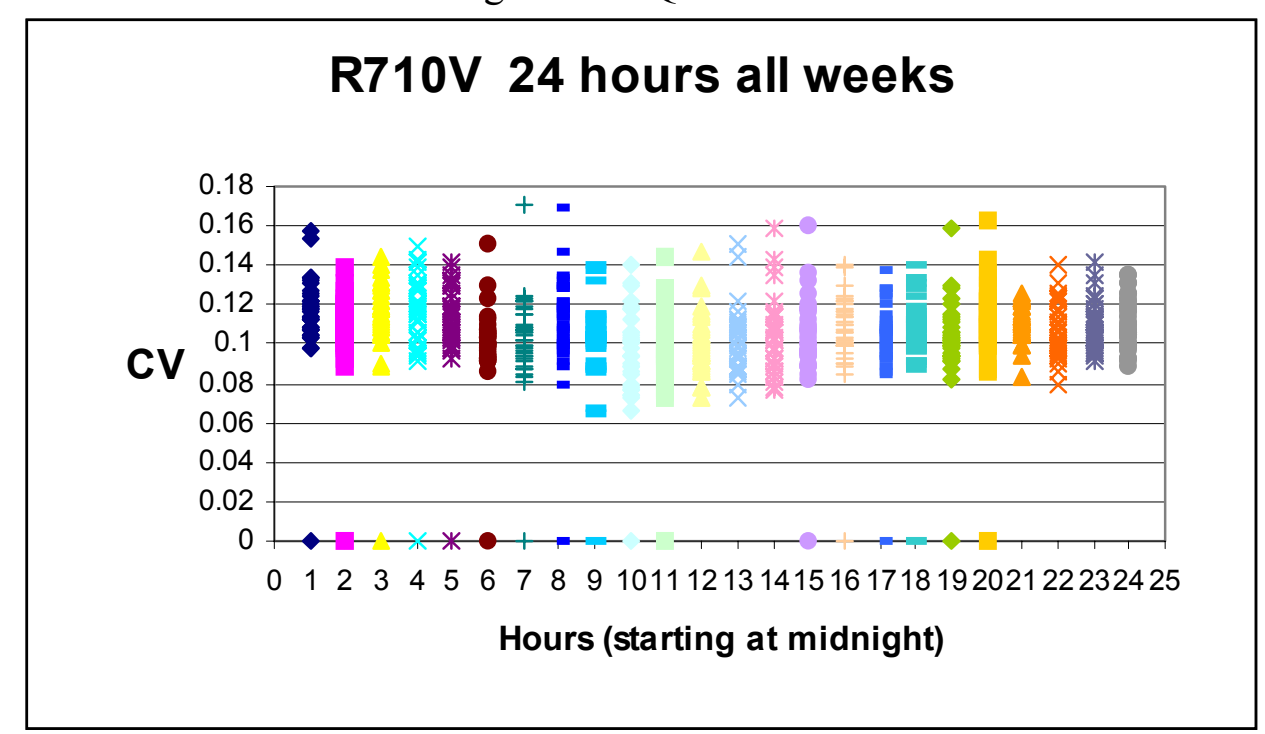

Figure F.4 CV R710V 


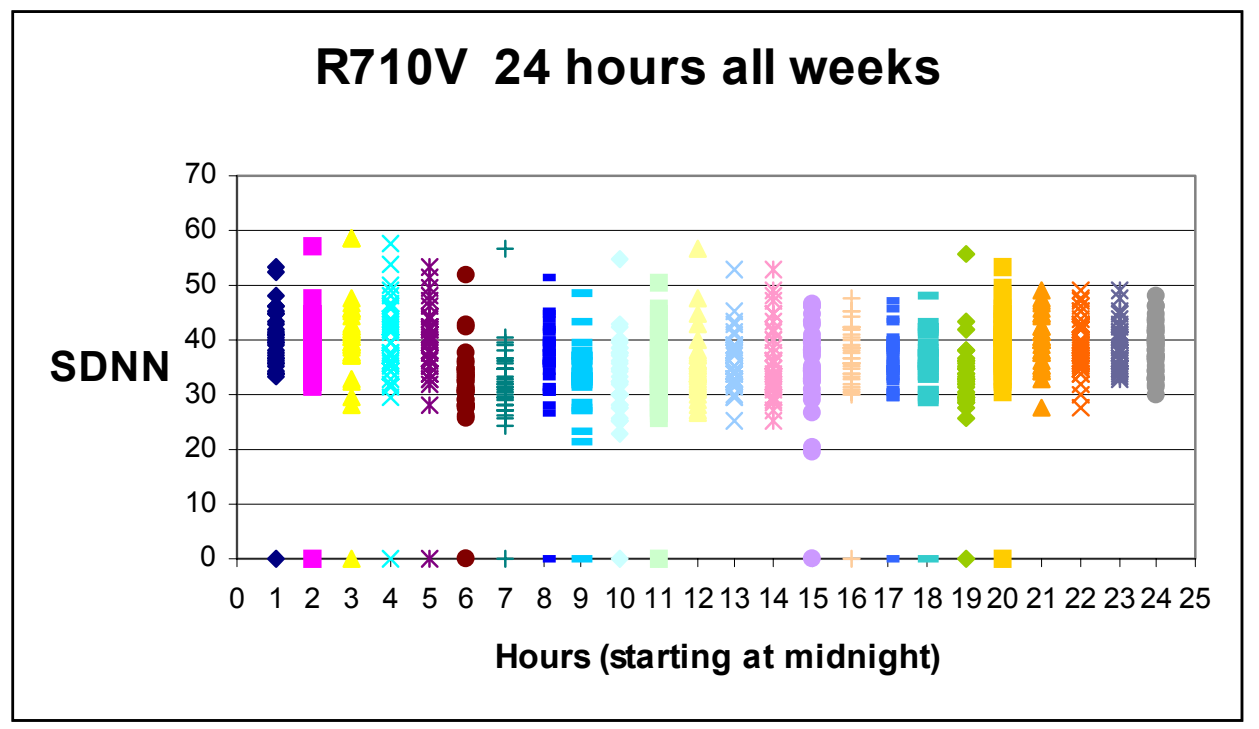

Figure F.5 SDNN R710V

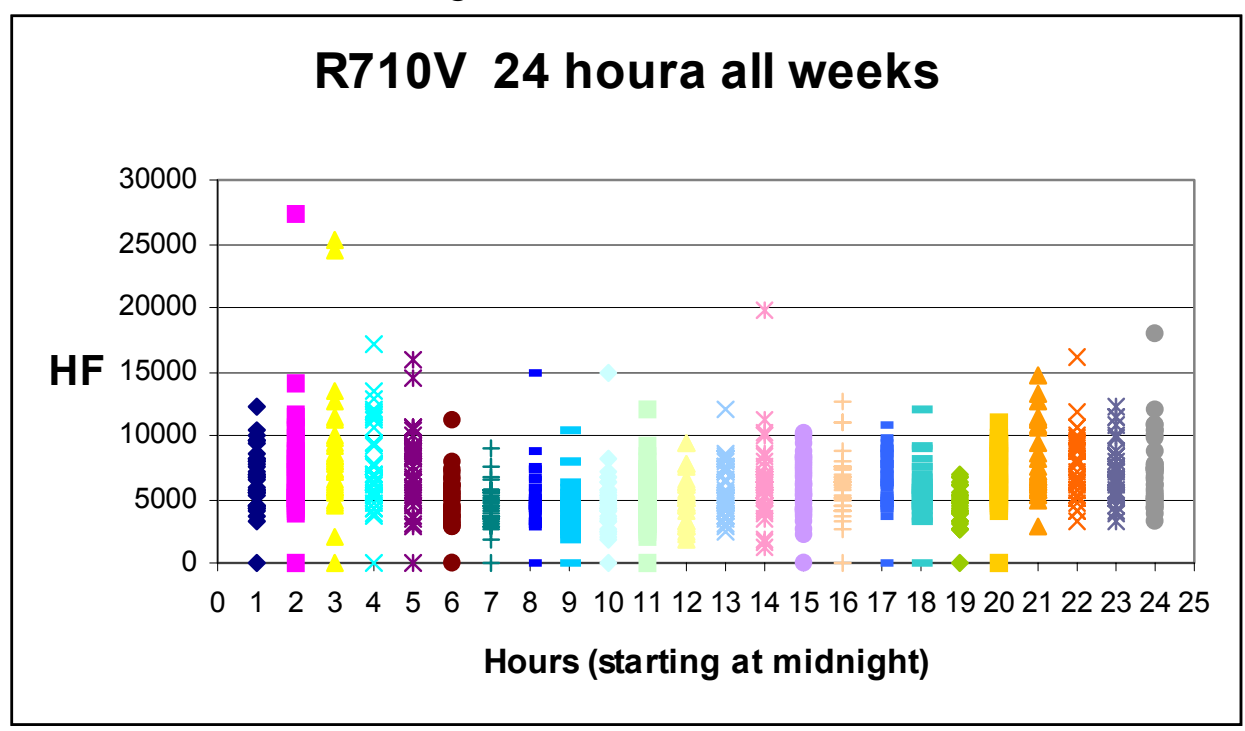

Figure F.6 HF R710V 


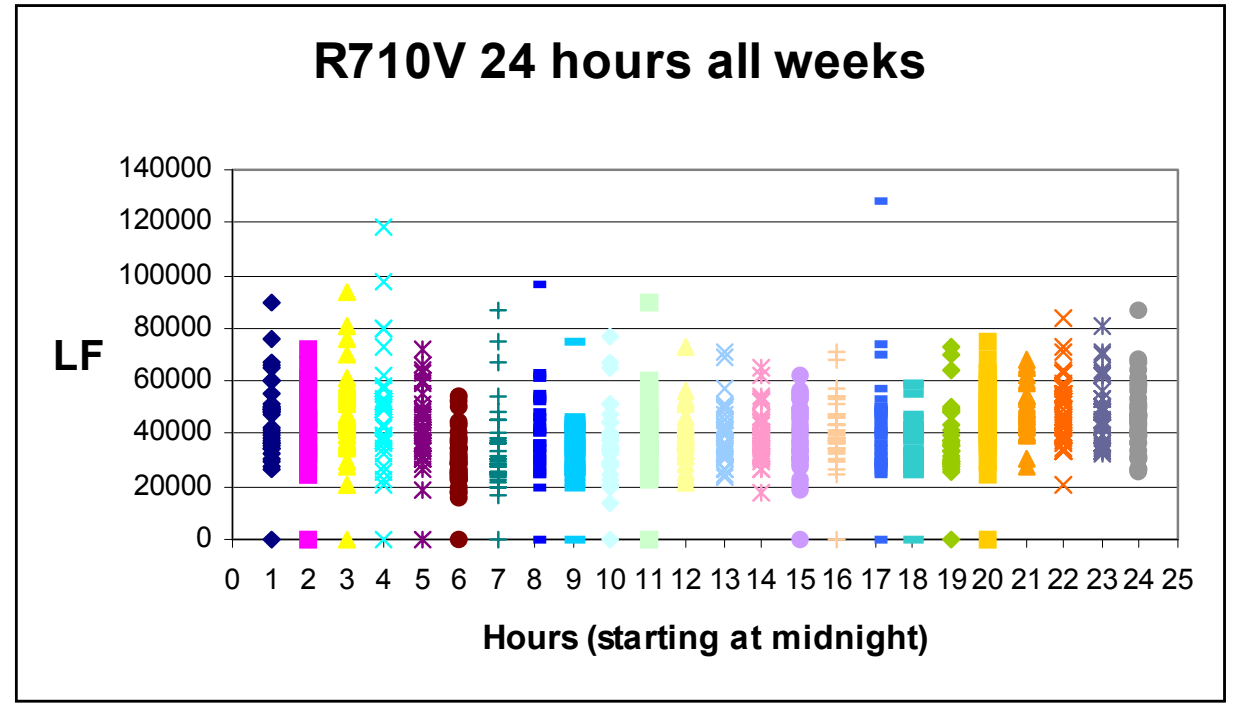

Figure F.7 LF R710V

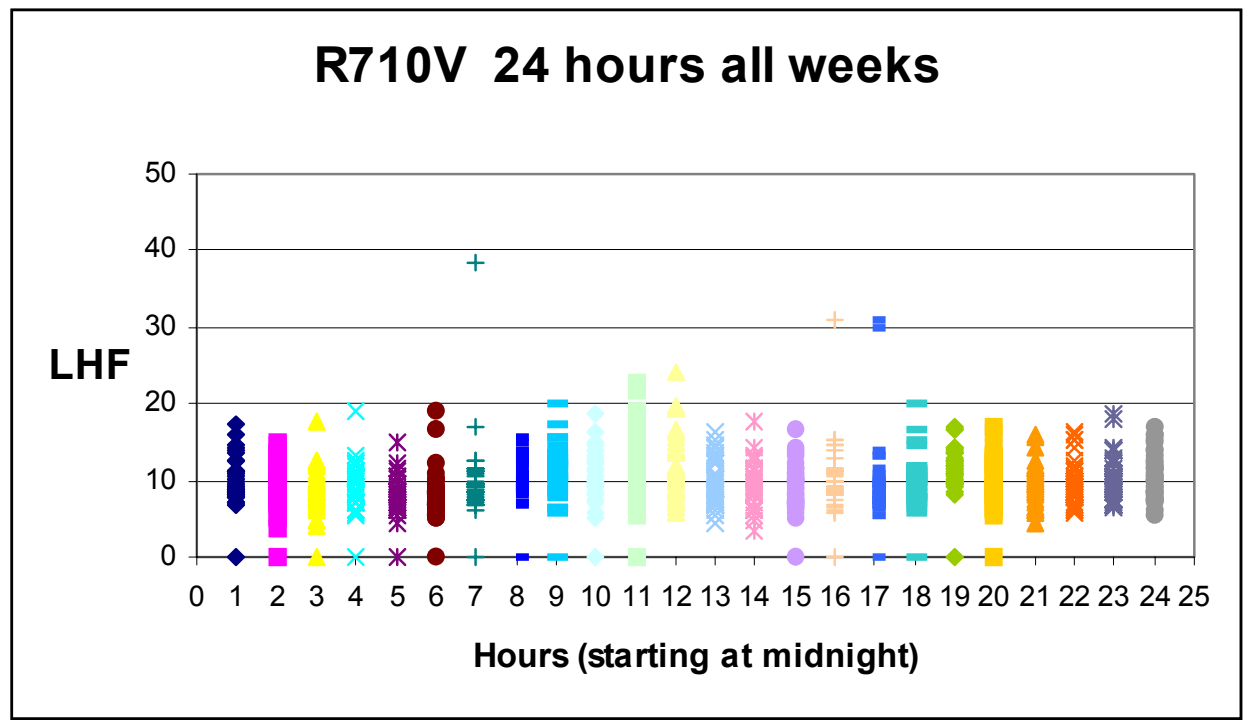

Figure F.8 LHF R710V 


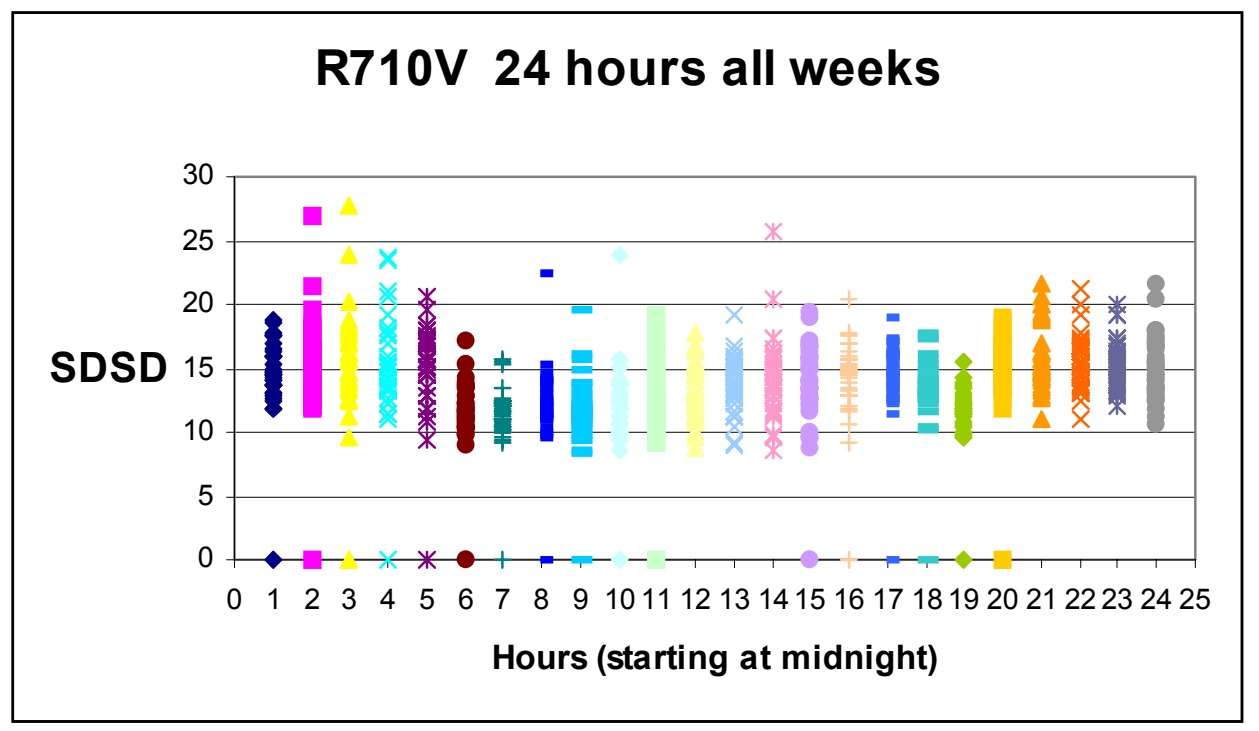

Figure F.9 SDSD R710V

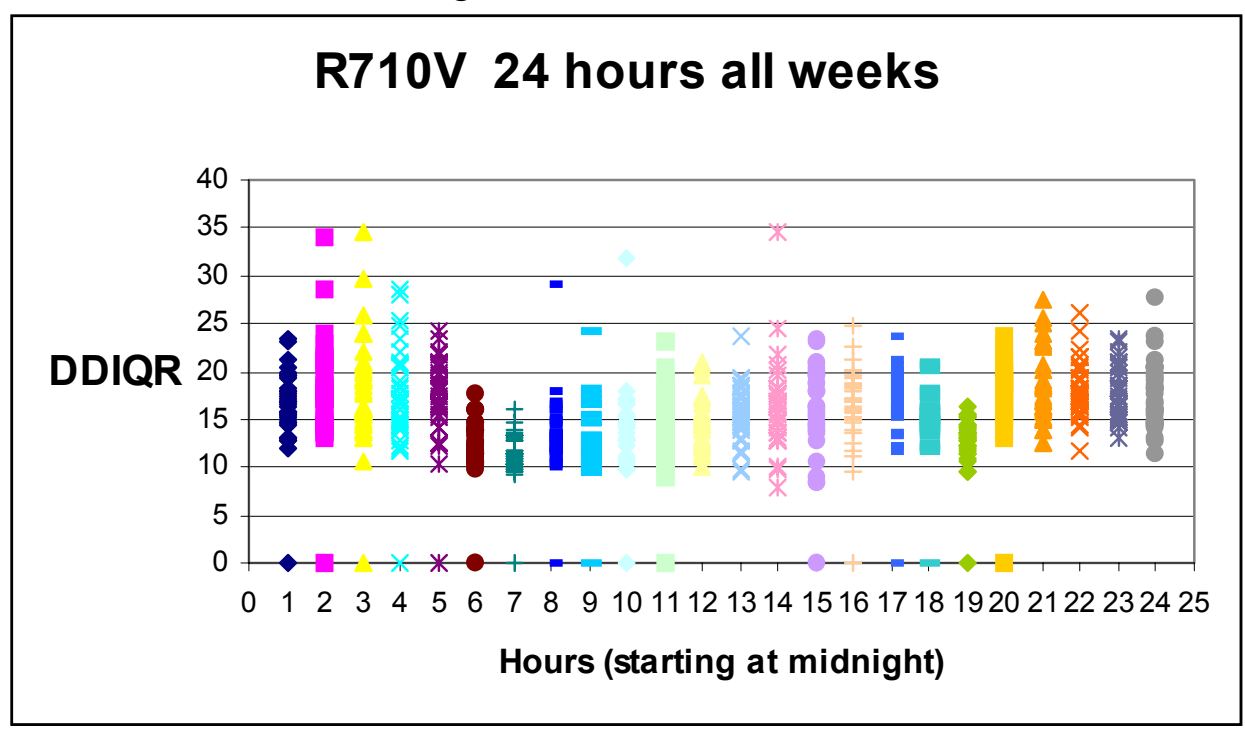

Figure F.10 DDIQR R710V 


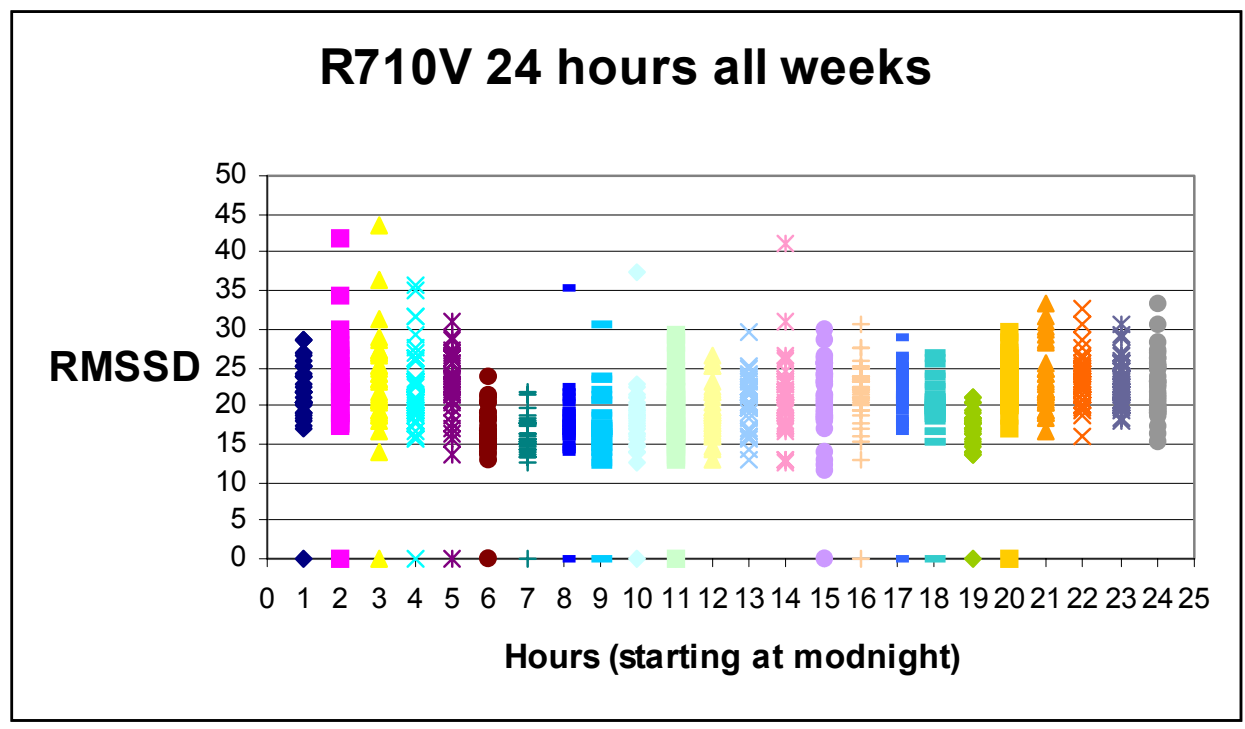

Figure F.11 RMSSD R710V

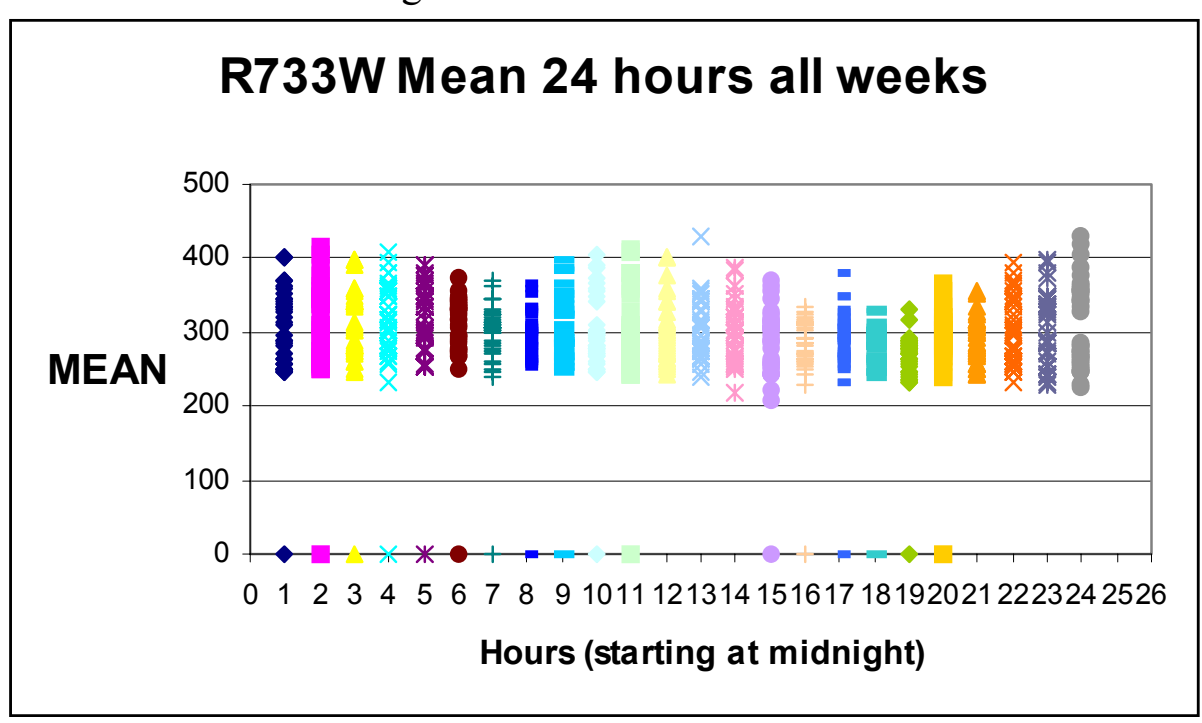

Figure F.12 MEAN R733W 


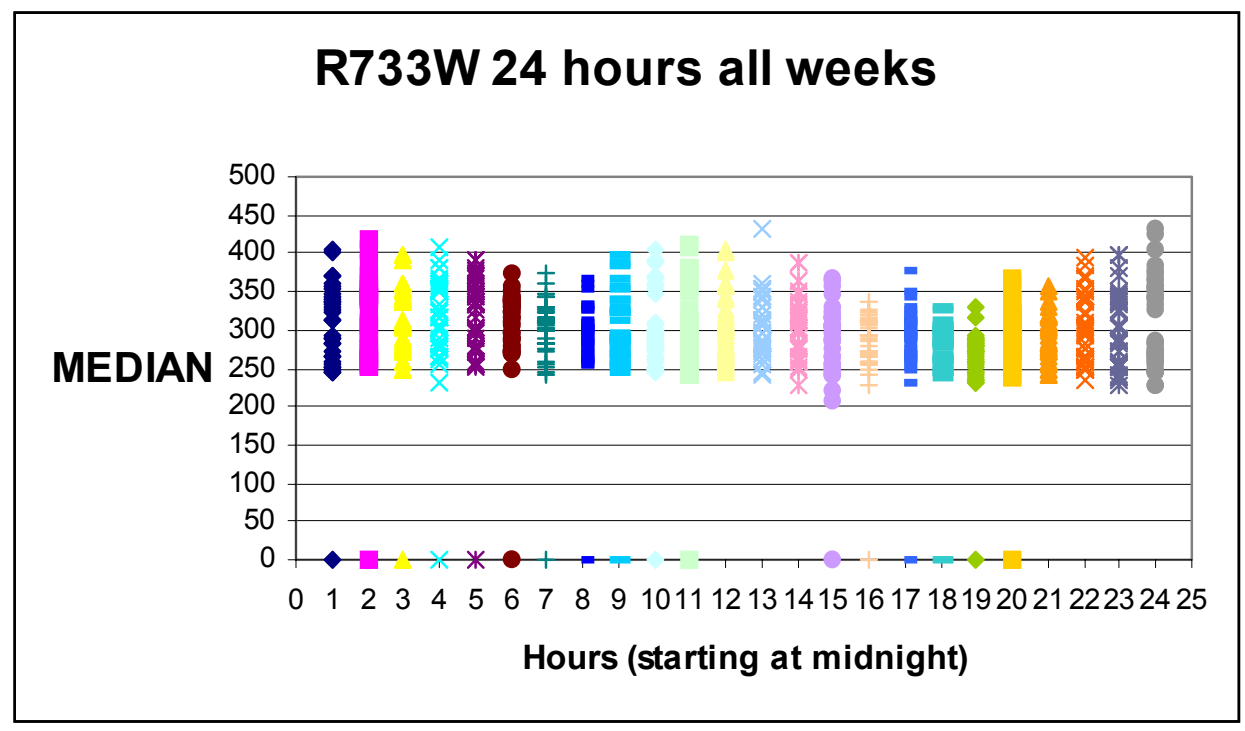

Figure F.13 MEDIAN R733W

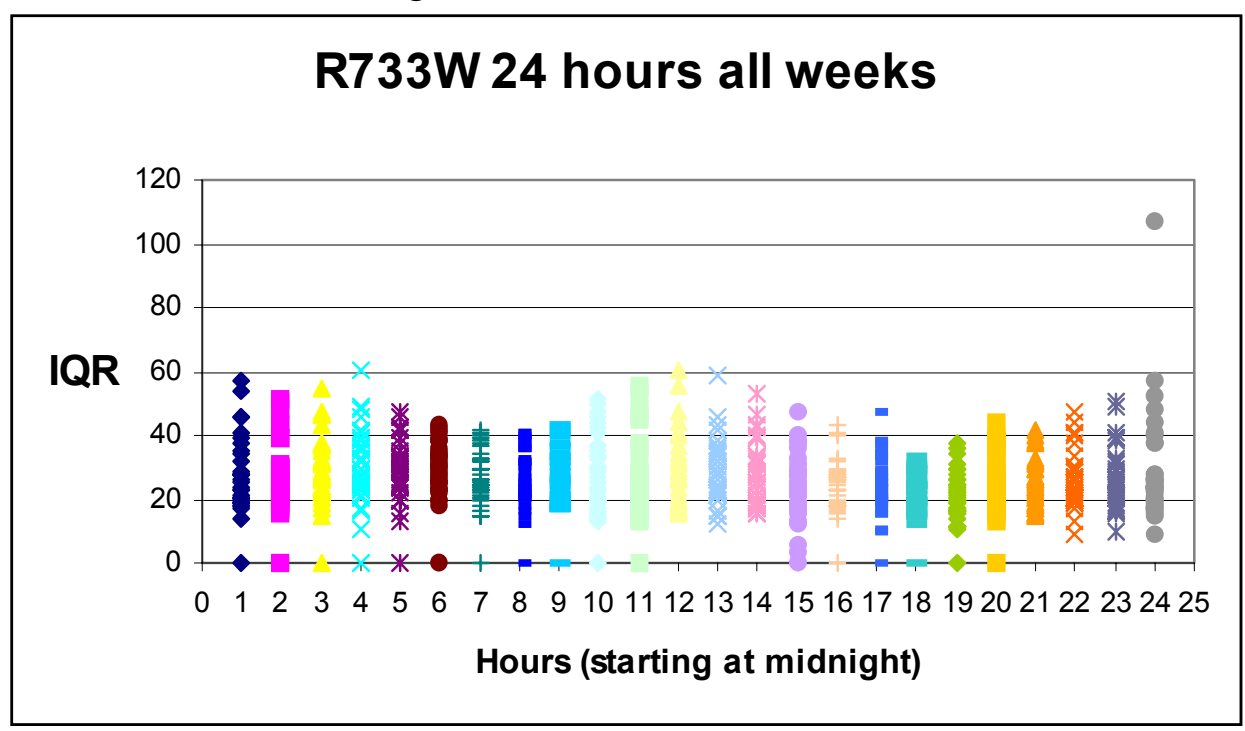

Figure F.14 IQR R733W 


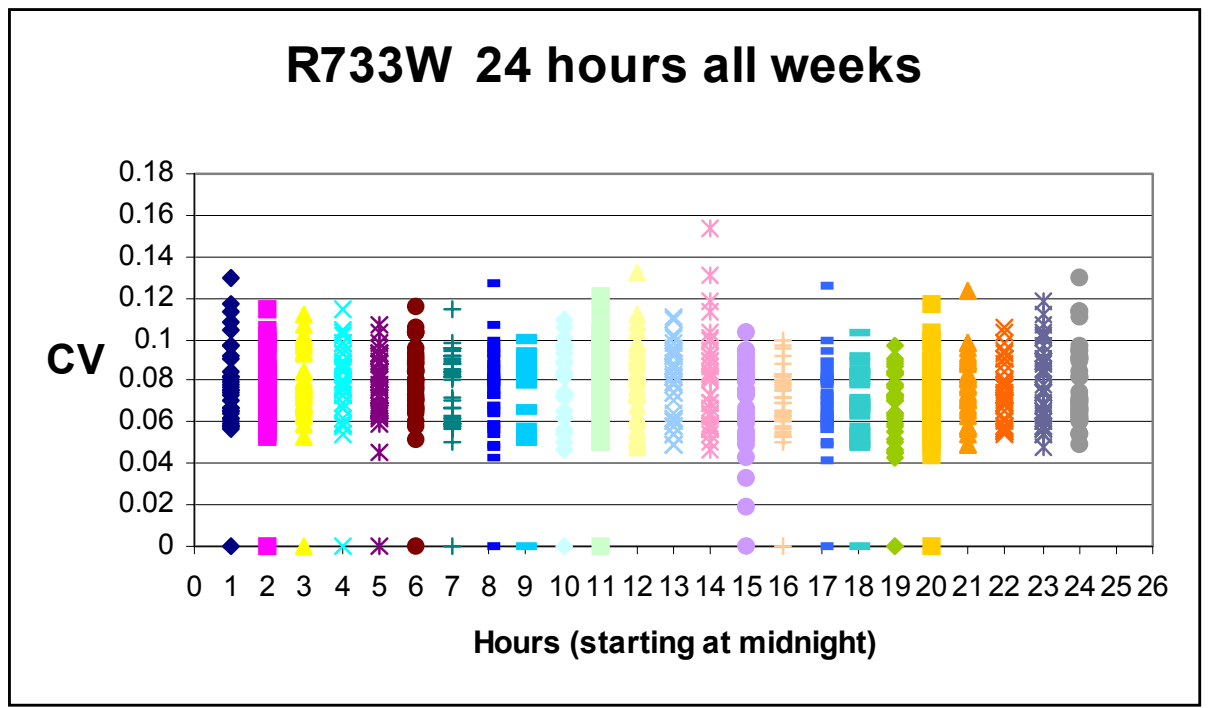

Figure F.15 CV R733W

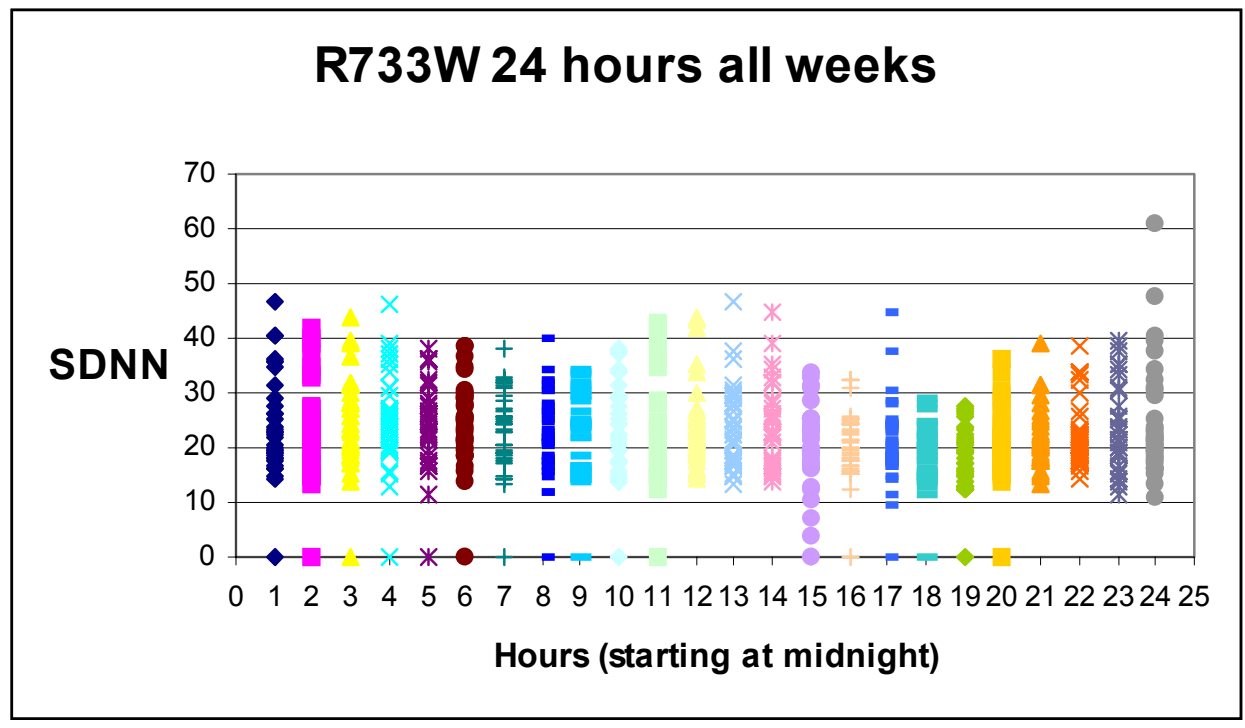

Figure F.16 SDNN R733W 


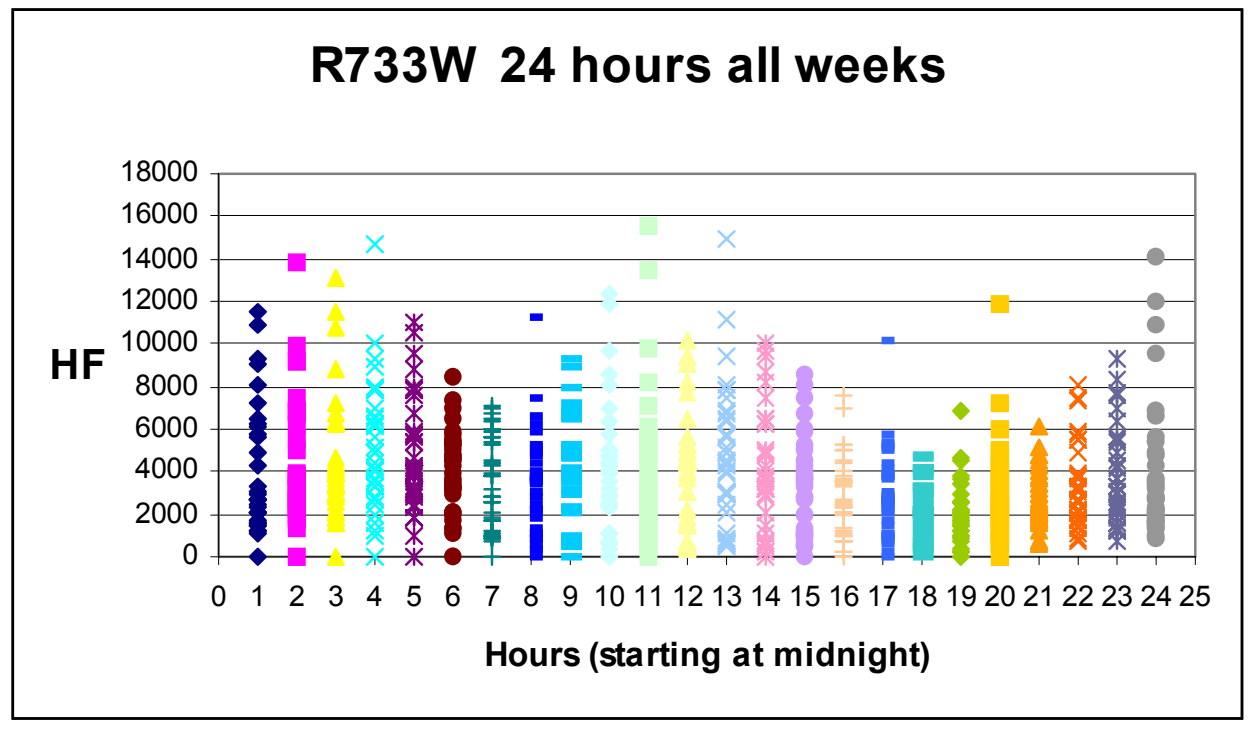

Figure F.17 HF R733W

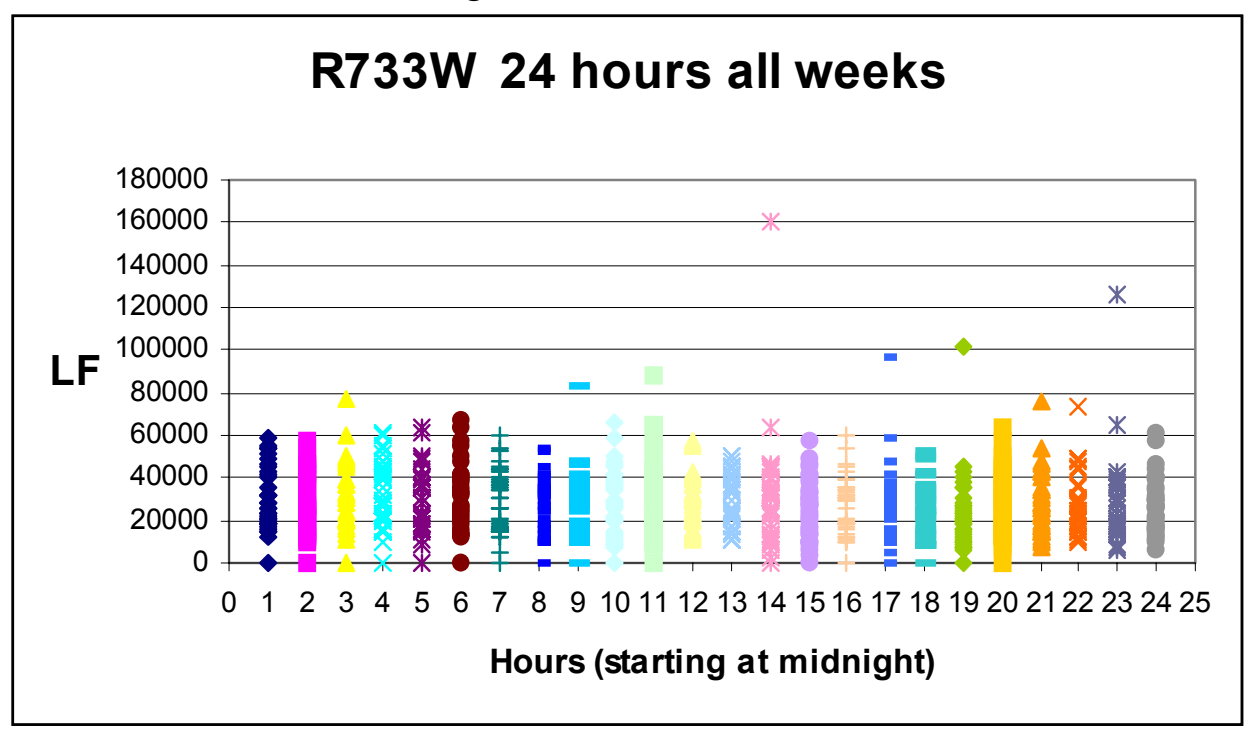

Figure F.18 LF R733W 


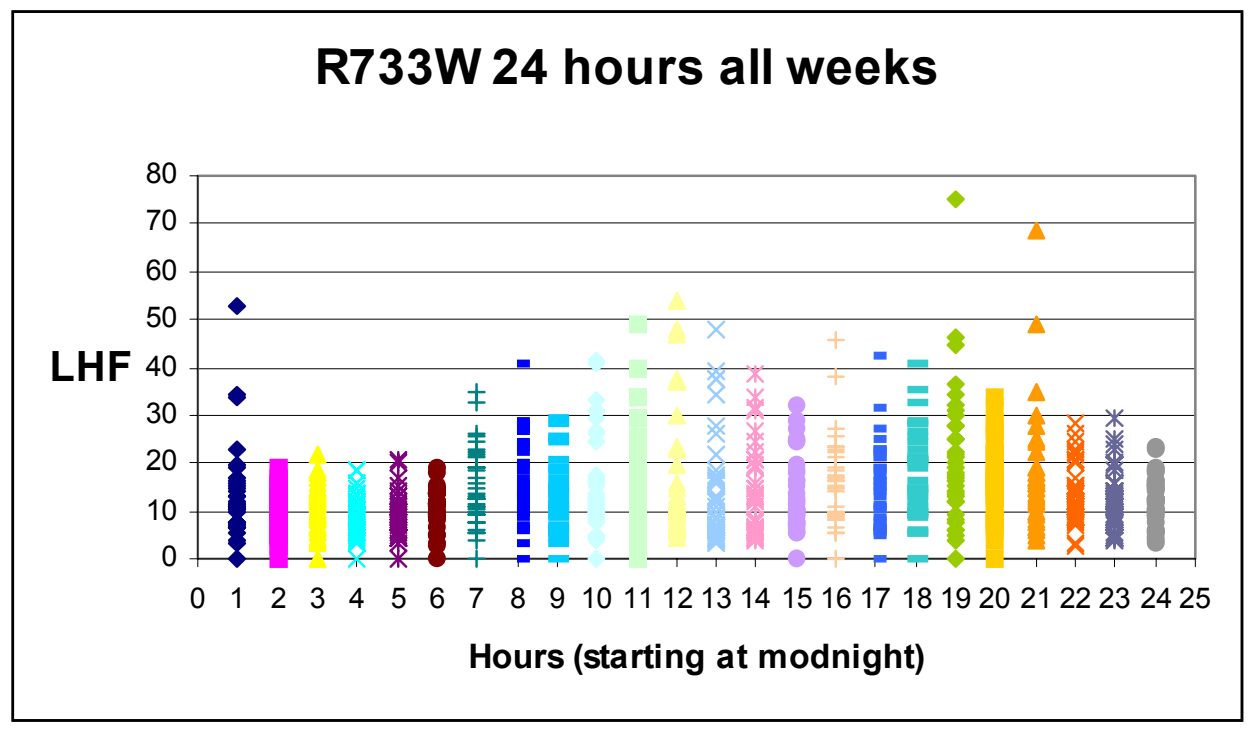

Figure F.19 LHF R733W

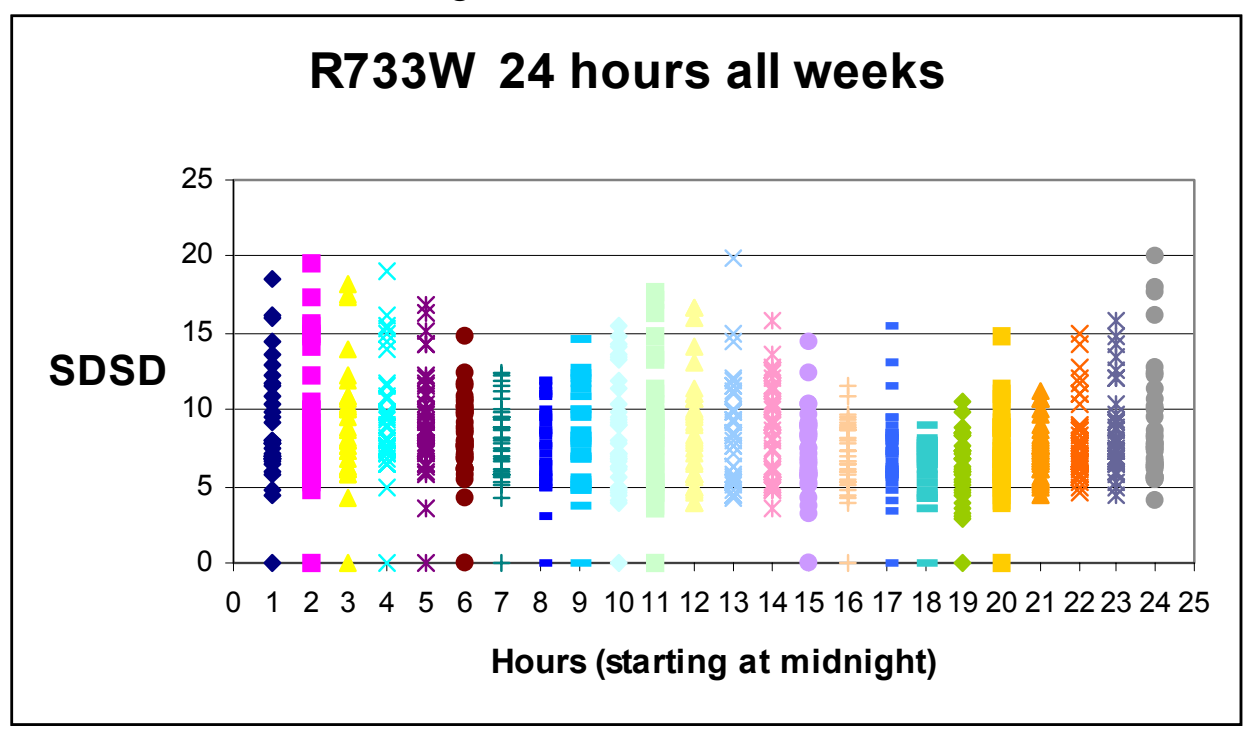

Figure F.20 SDSD R733W 


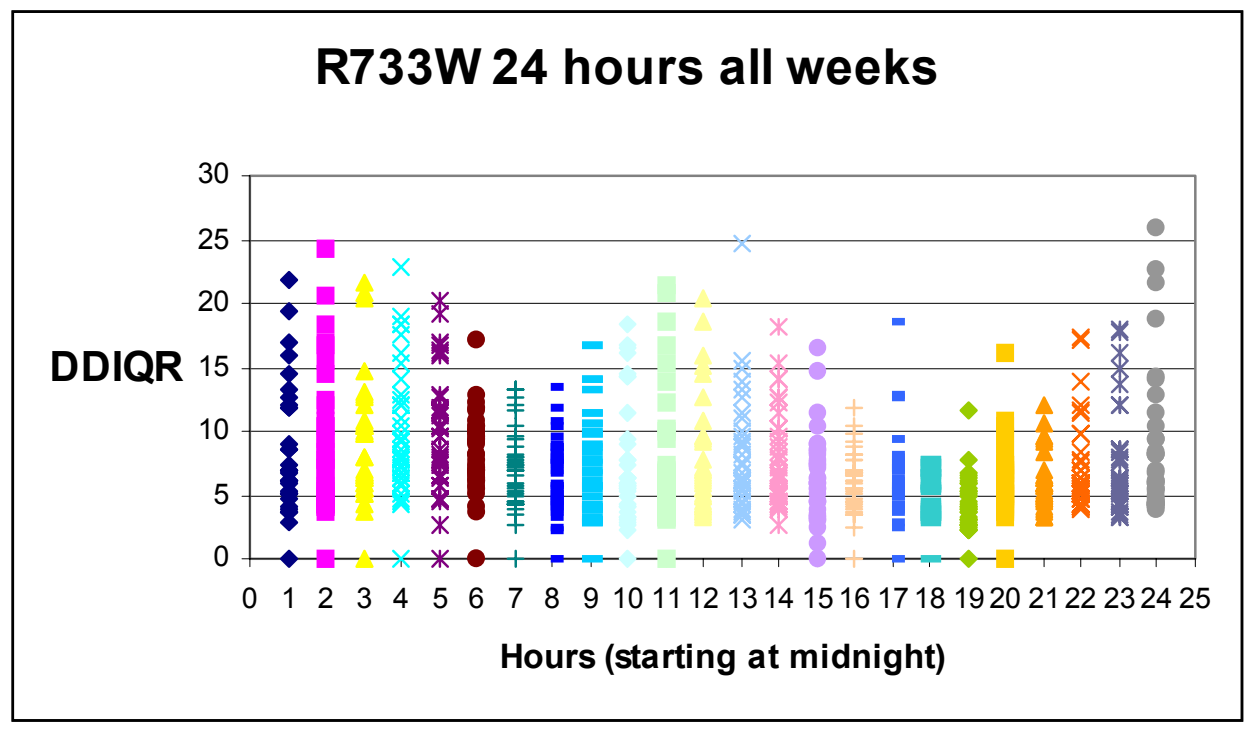

Figure F.21 DDIQR R733W

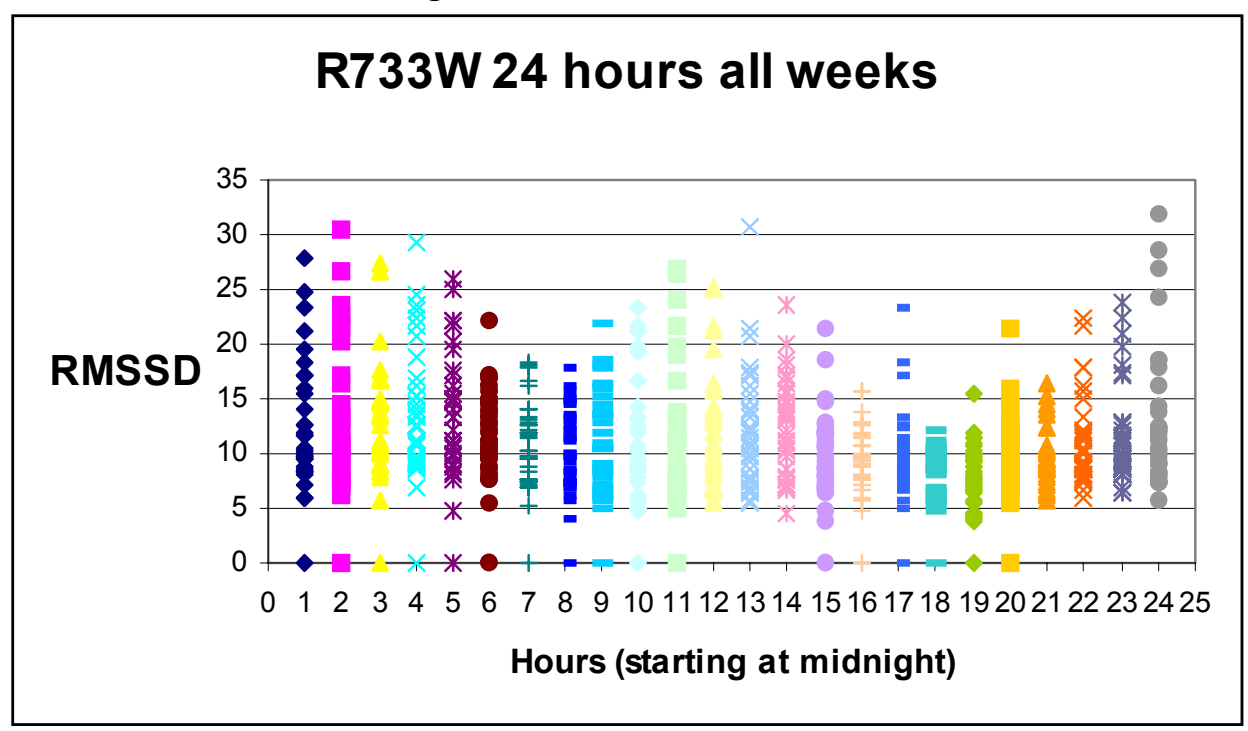

Figure F.22 RMSSD R733W 


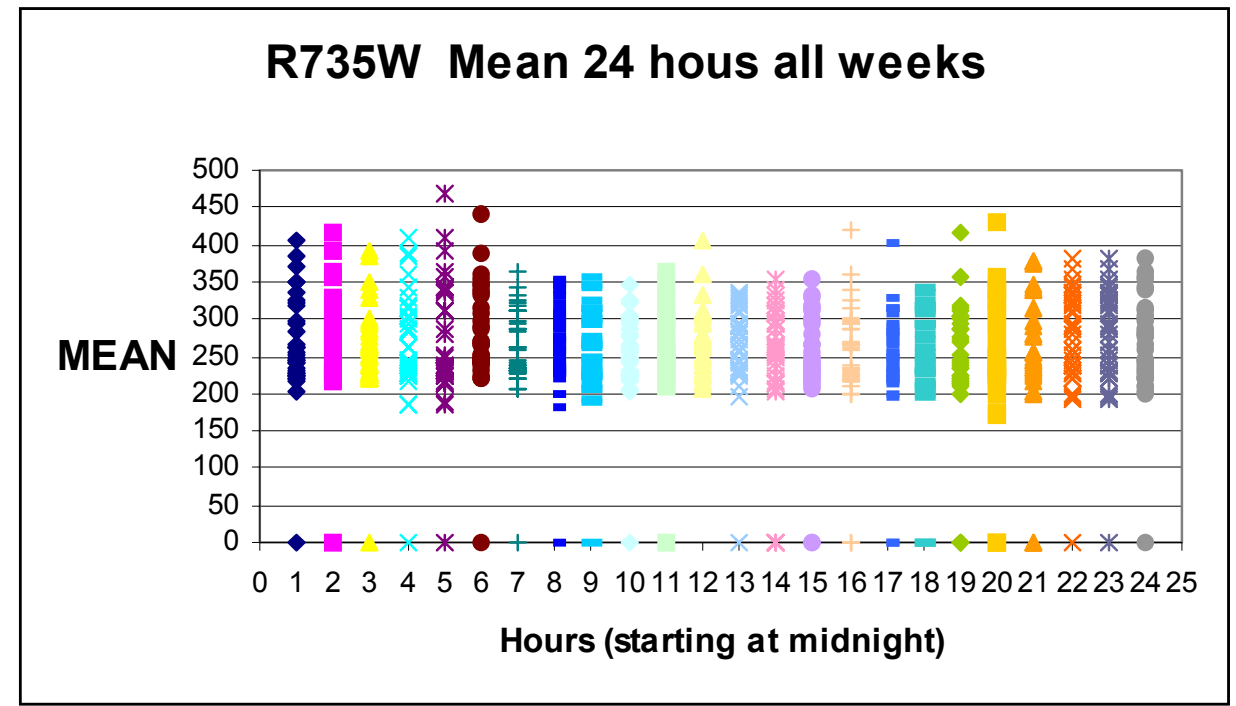

Figure F.23 MEAN R735W

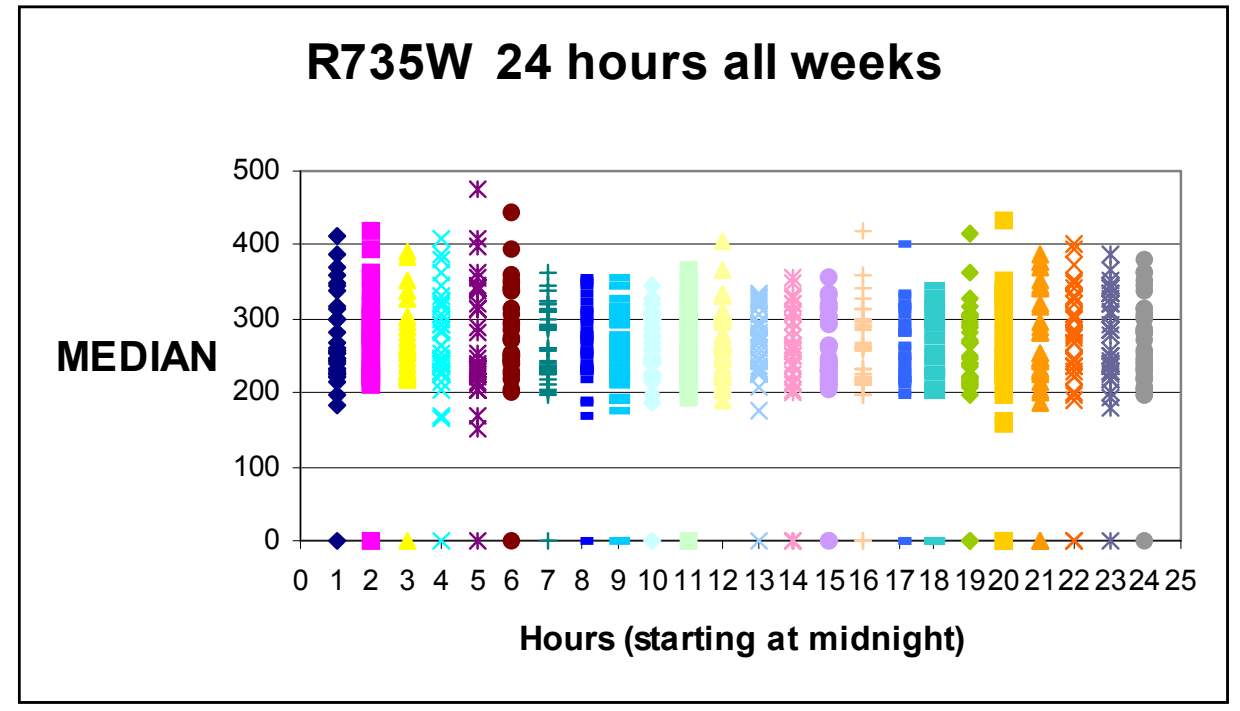

Figure F.24 MEDIAN R735W 


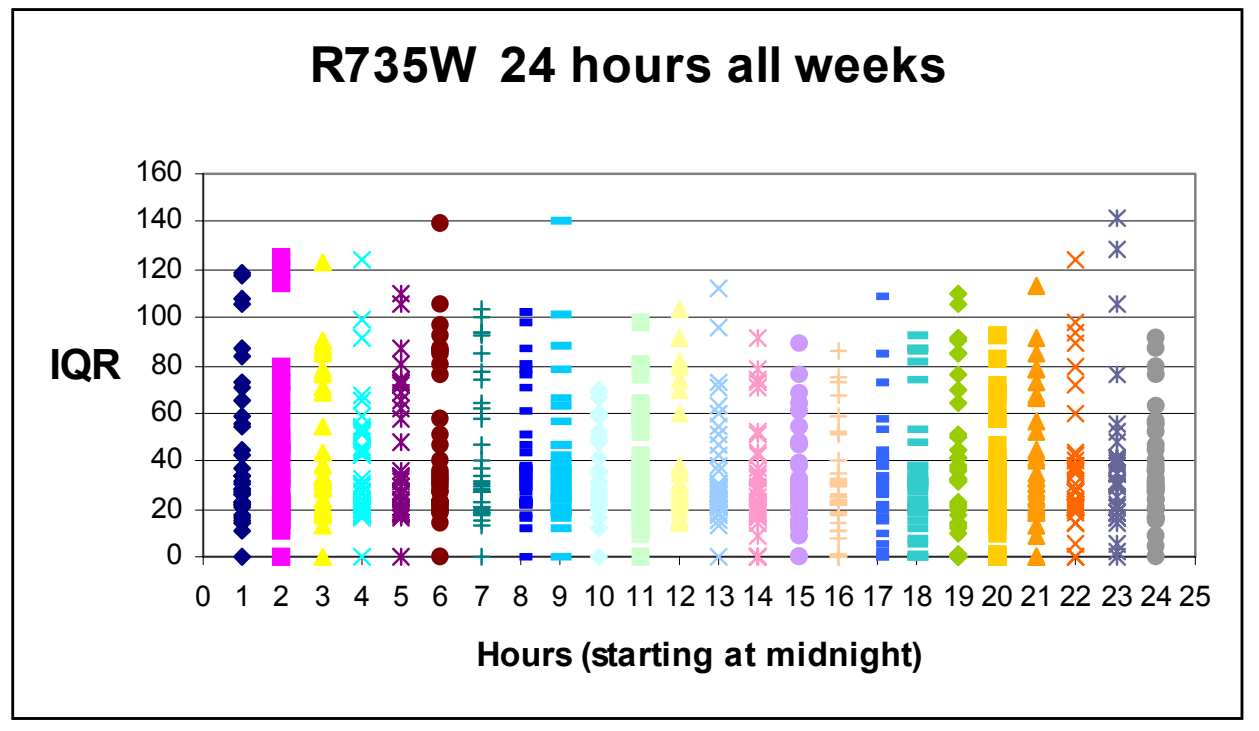

Figure F.25 IQR R735W

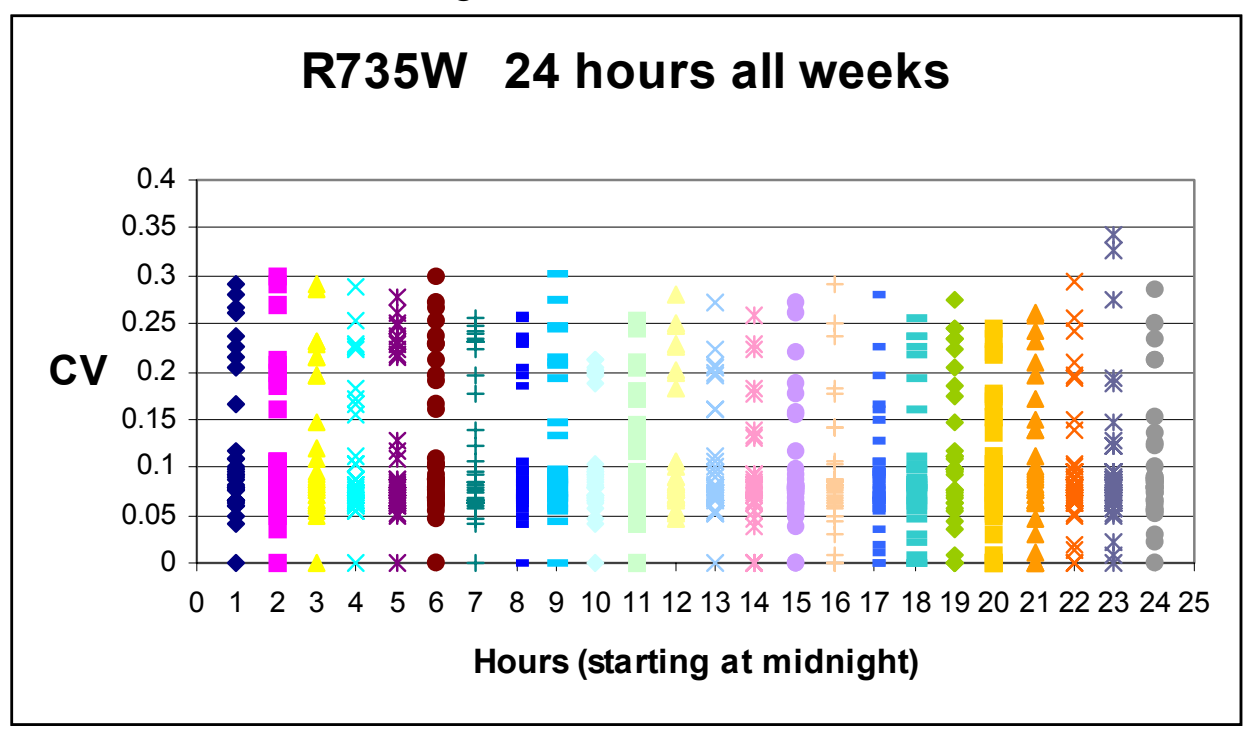

Figure F.26 CV R735W 


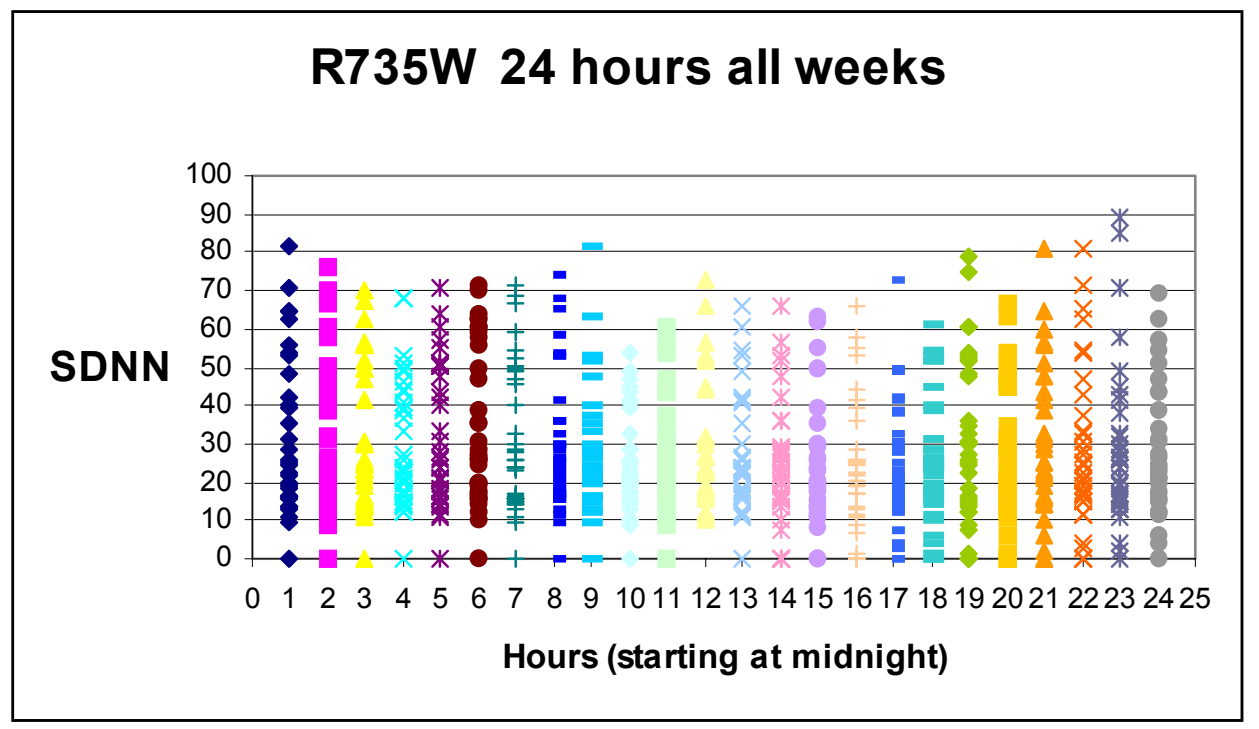

Figure F.27 SDNN R735W

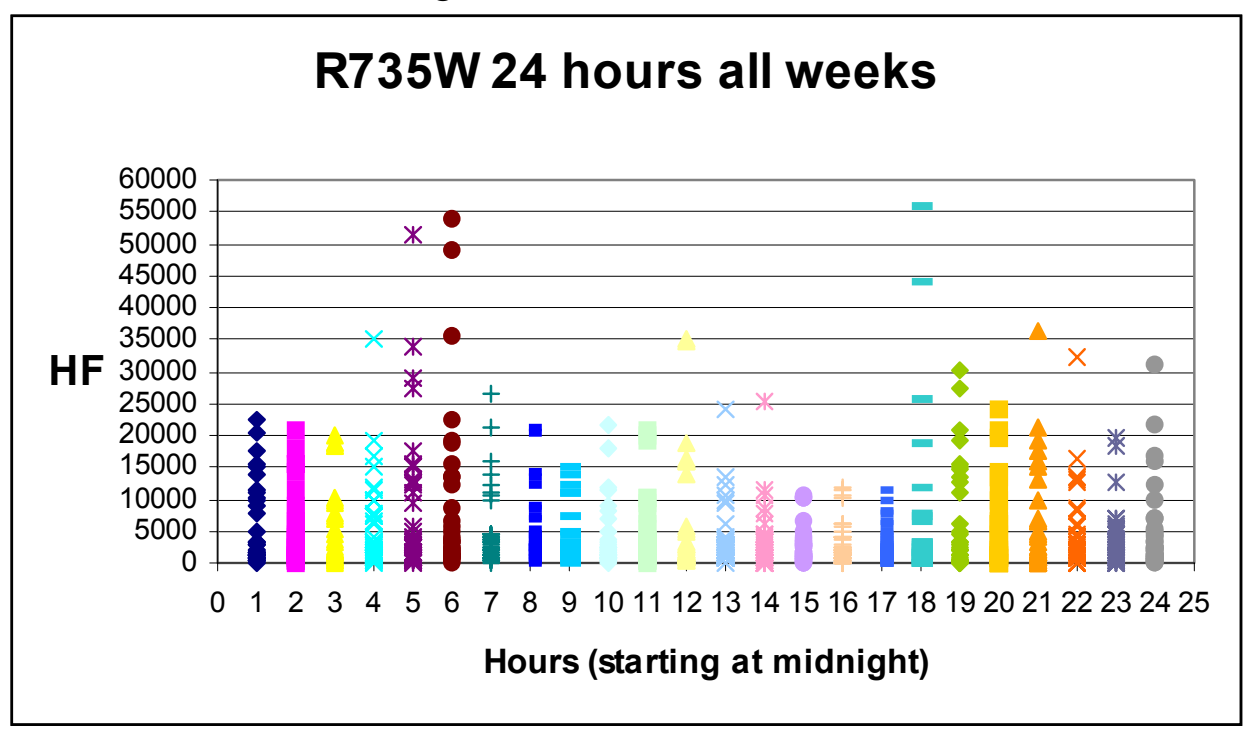

Figure F.28 HF R735W 


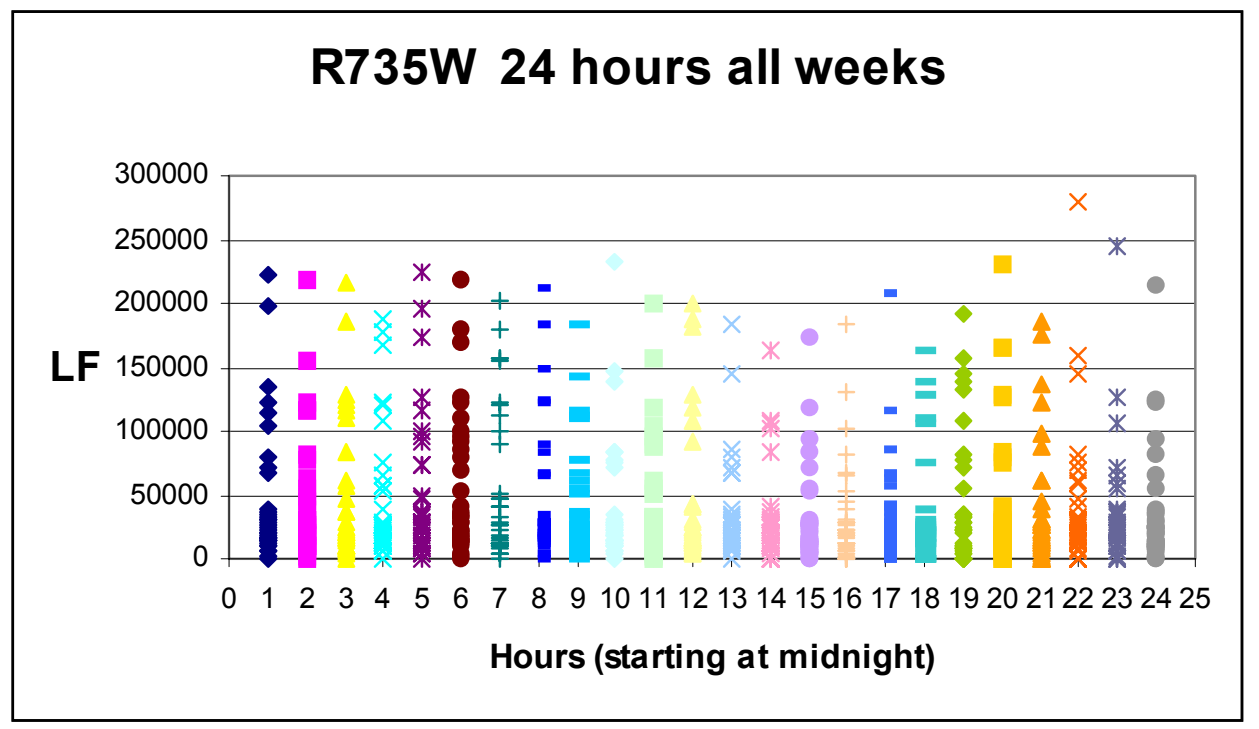

Figure F.29 LF R735W

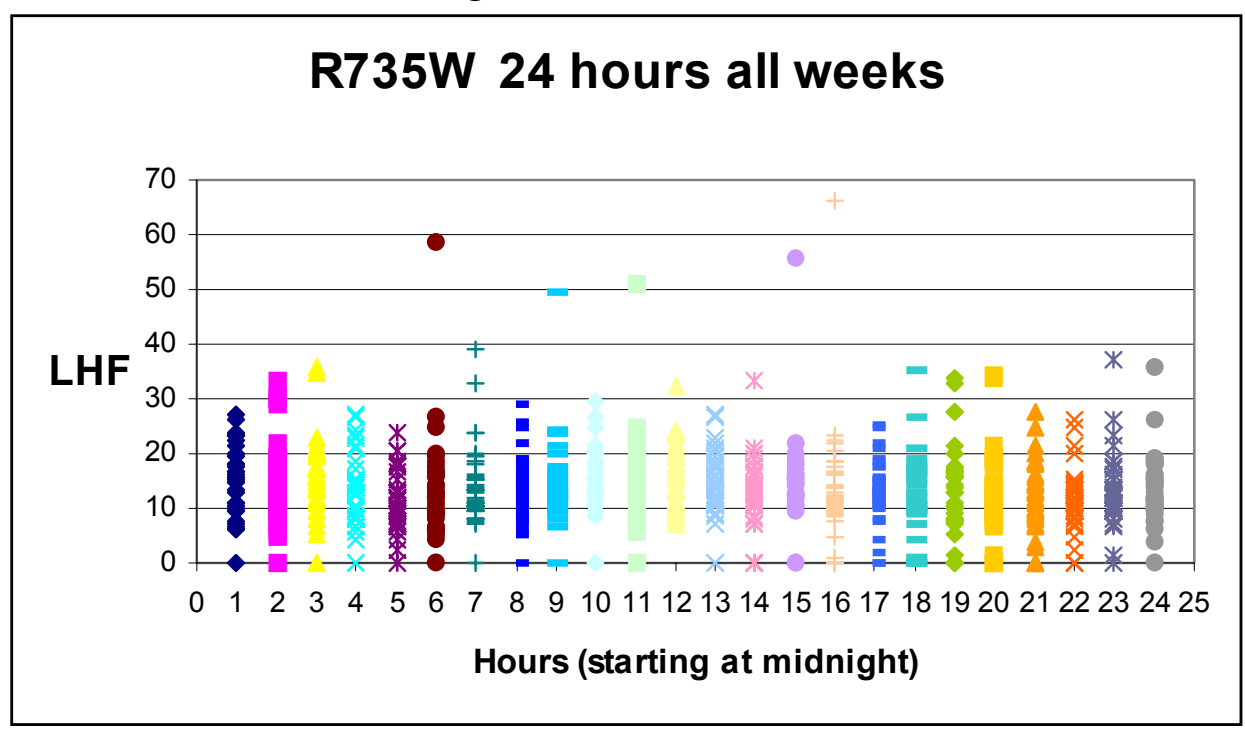

Figure F.30 LHF R735W 


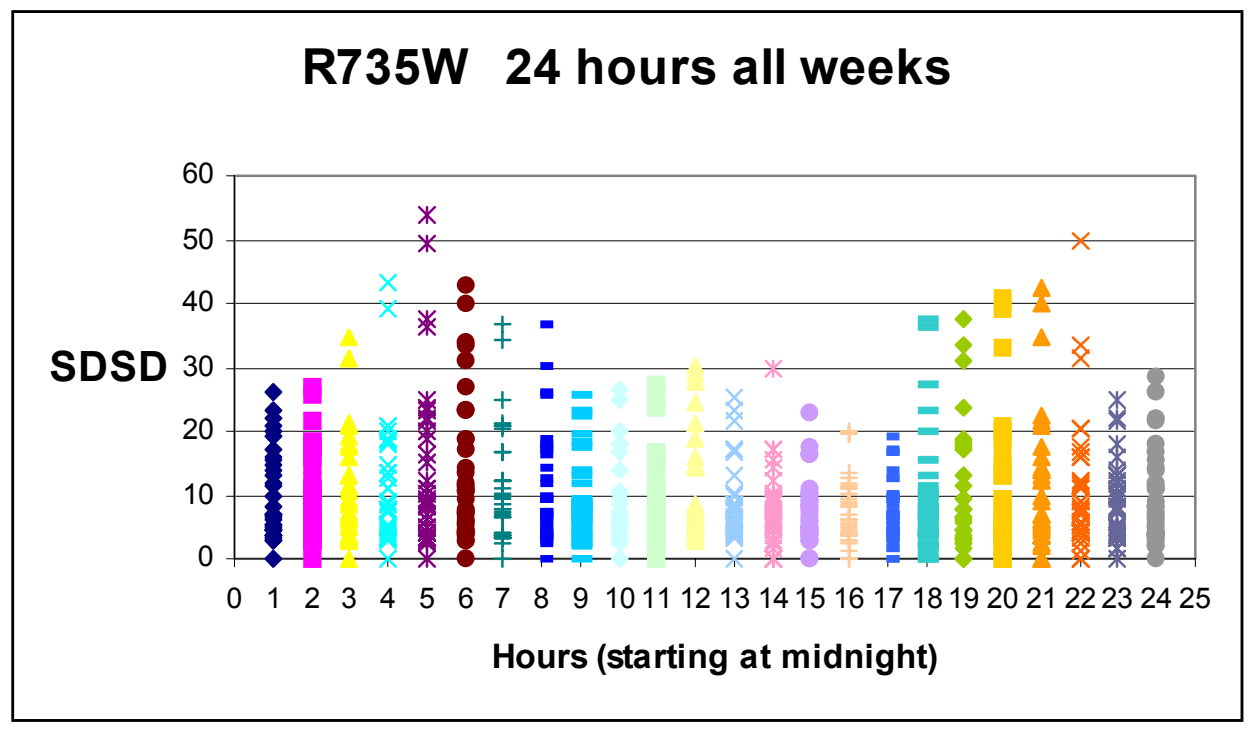

Figure F.31 SDSD R735W

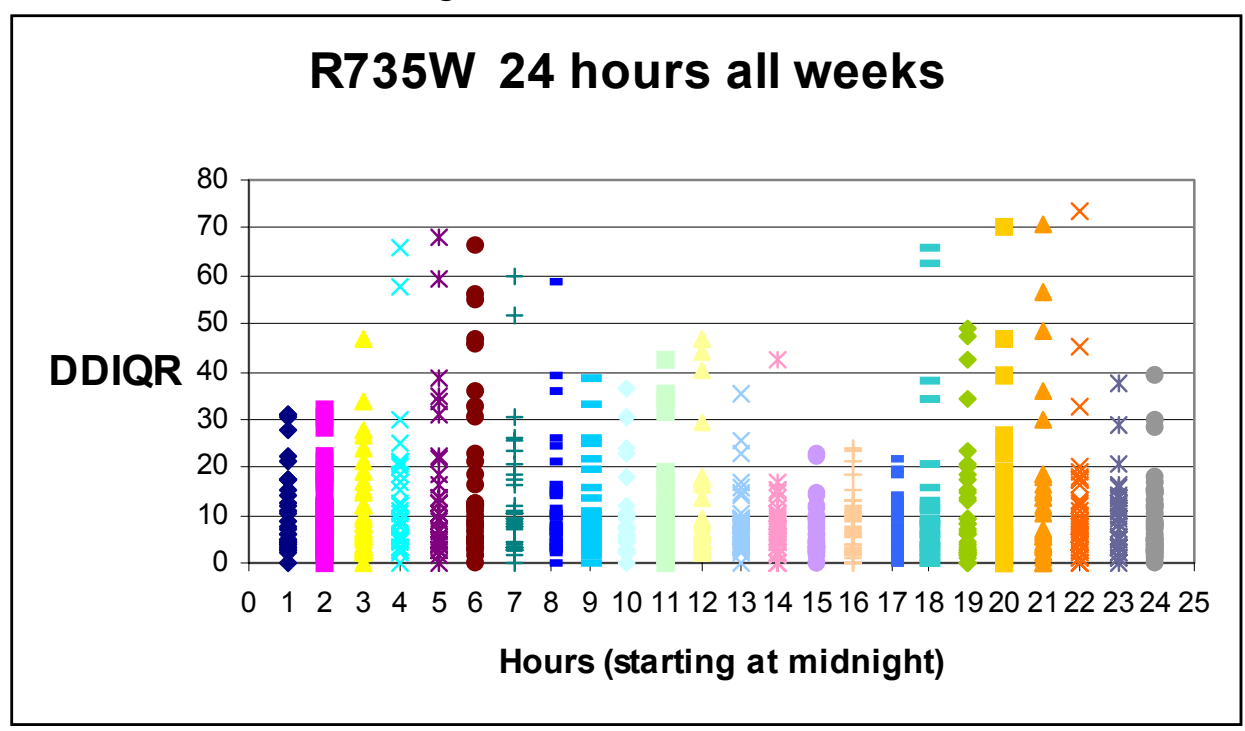

Figure F.32 DDIQR R735W 


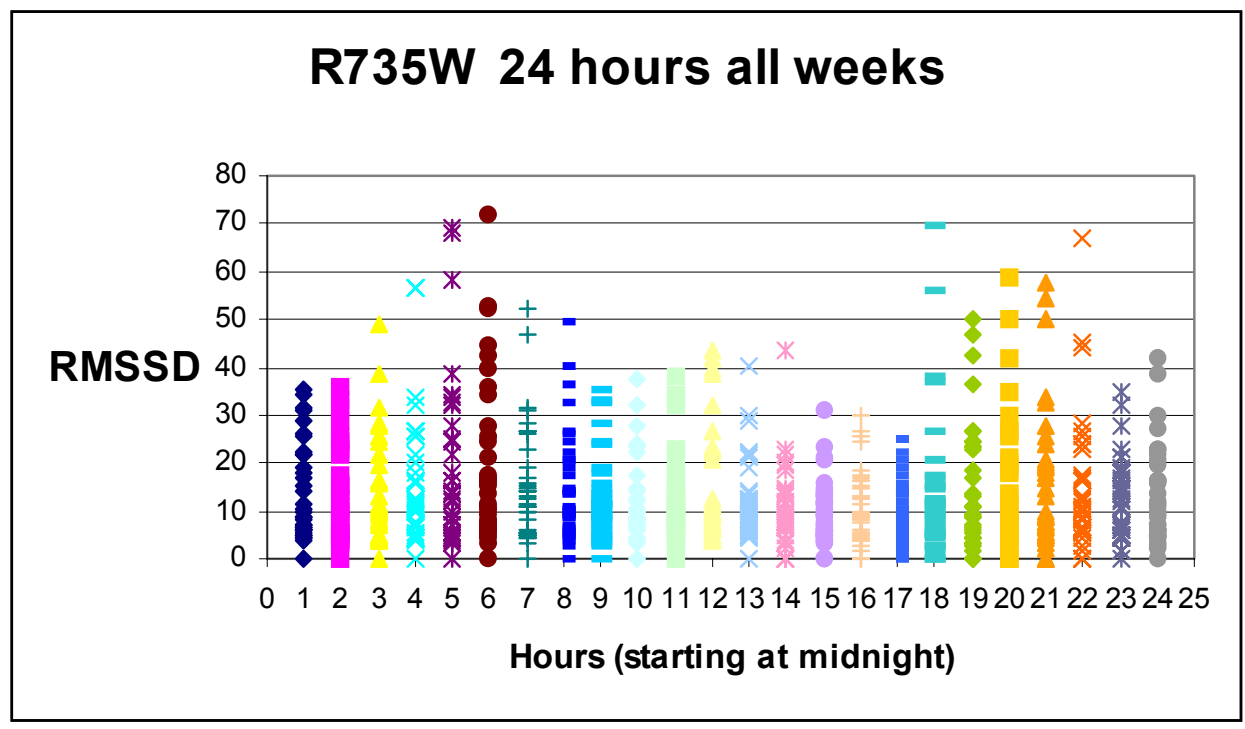

Figure F.33 RMSSD R735W 
Appendix G Laboratory analysis and tests 


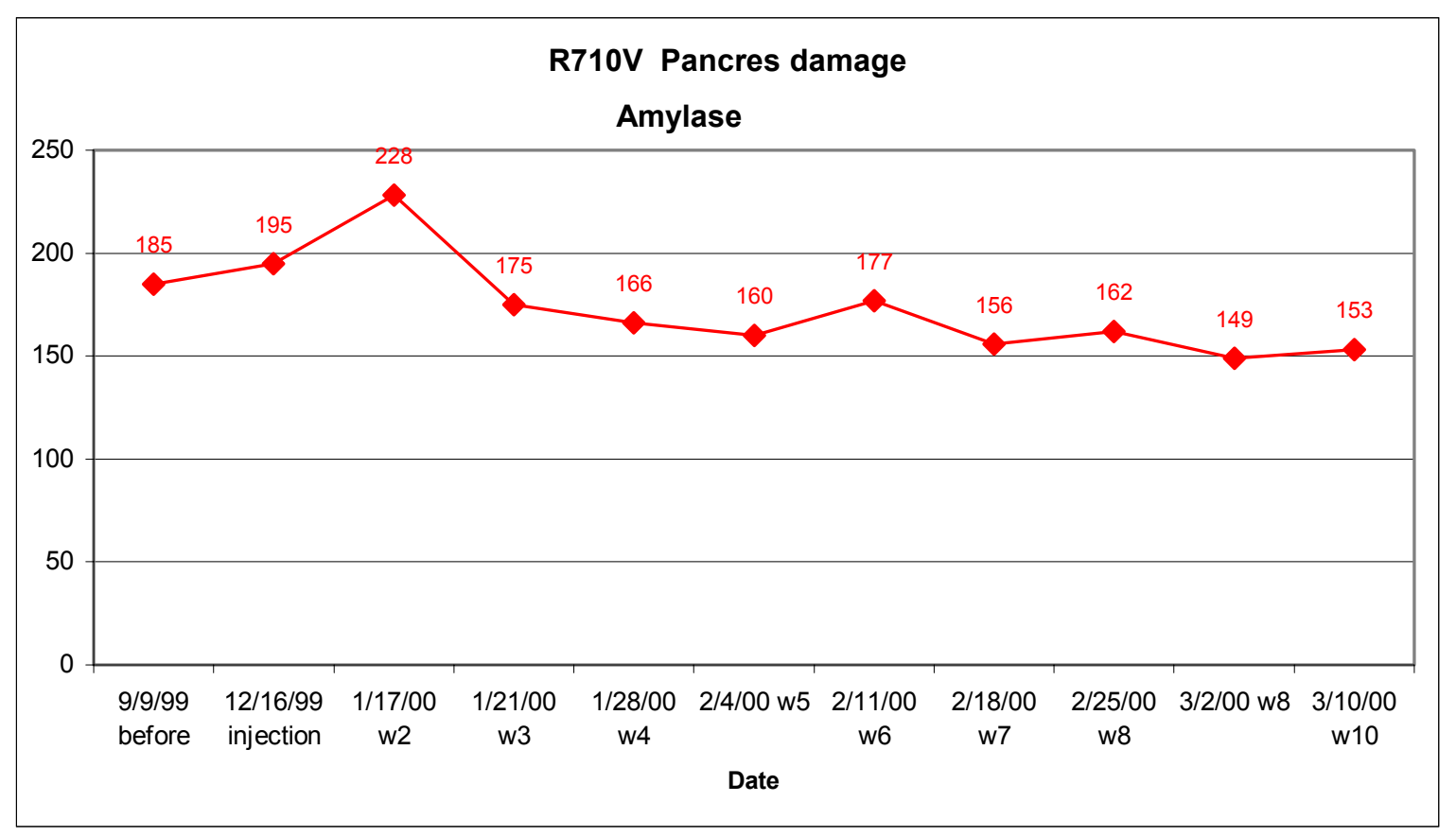

Figure G.1 Amylase R710V

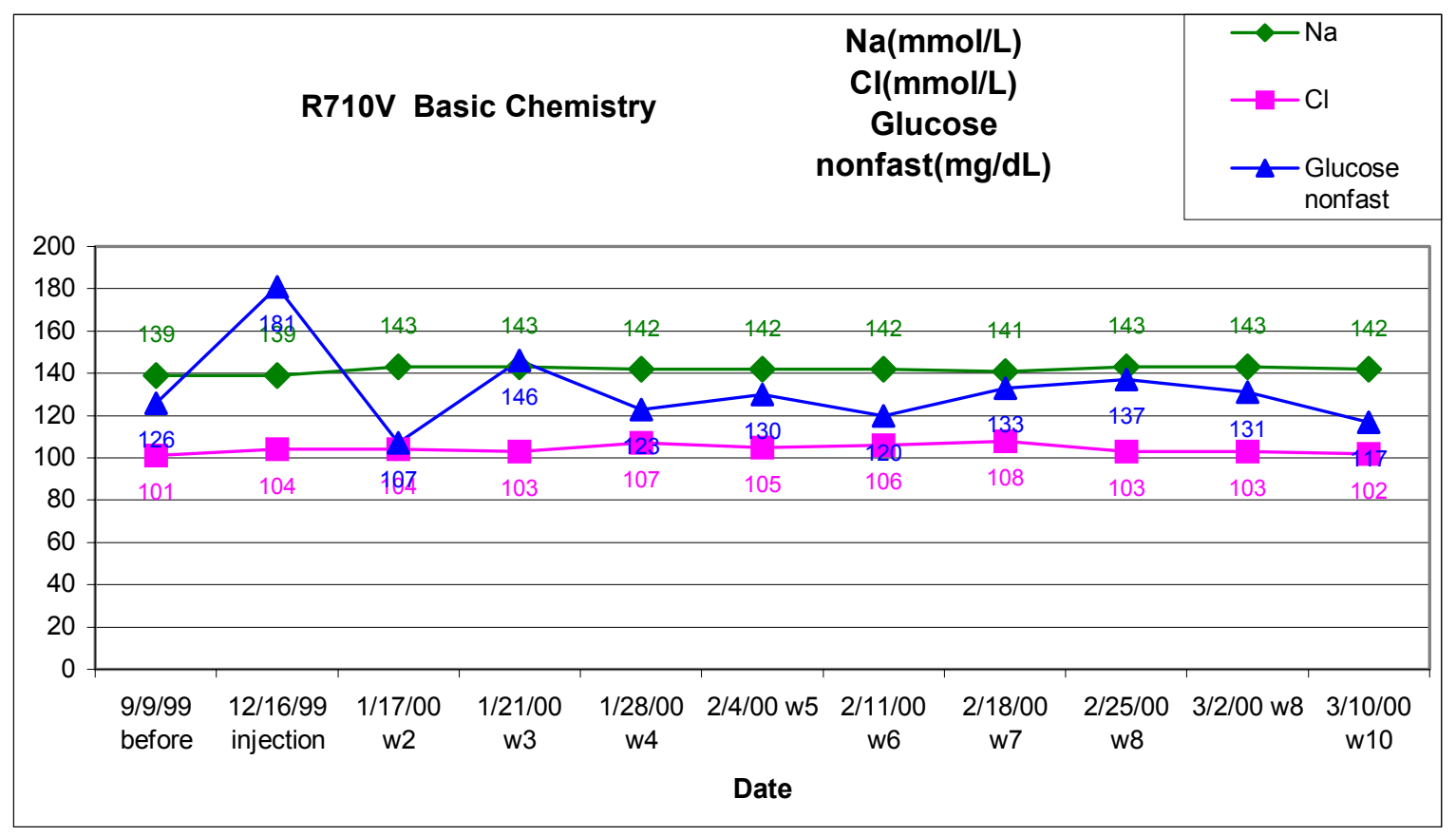

Figure G.2 Basic chemistry R710V 


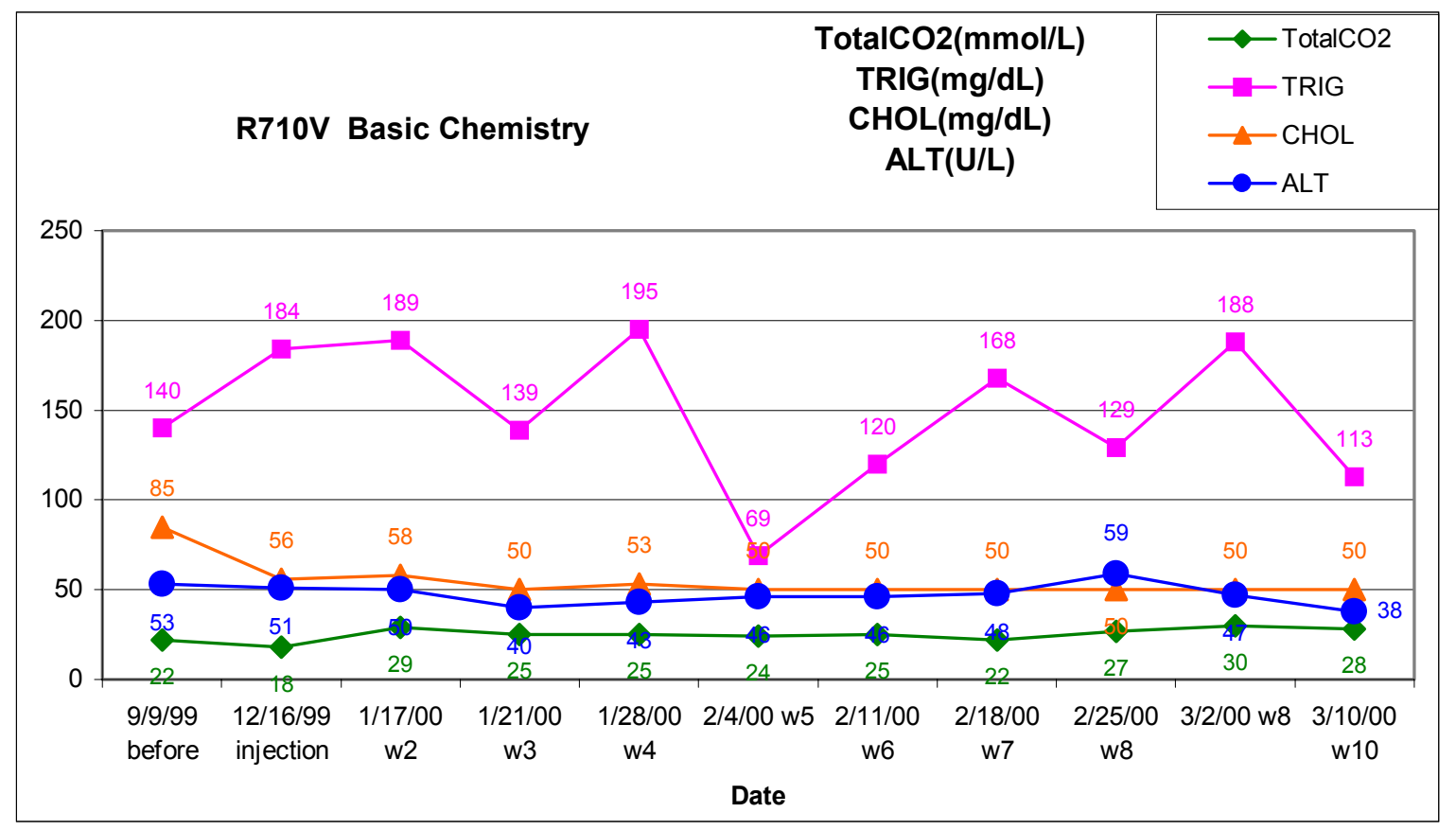

Figure G.3 Basic chemistry R710V

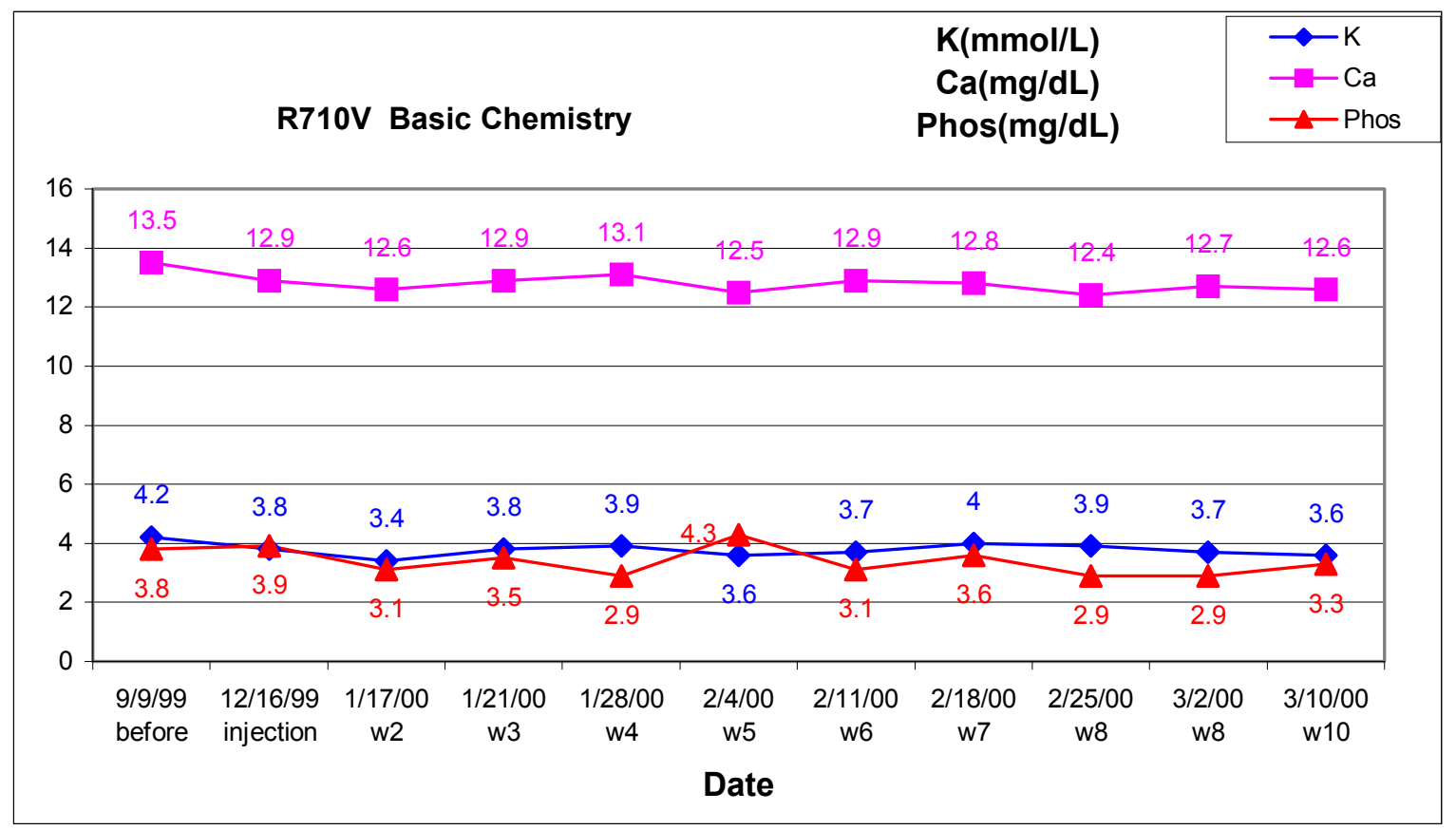

Figure G.4 Basic chemistry R710V 


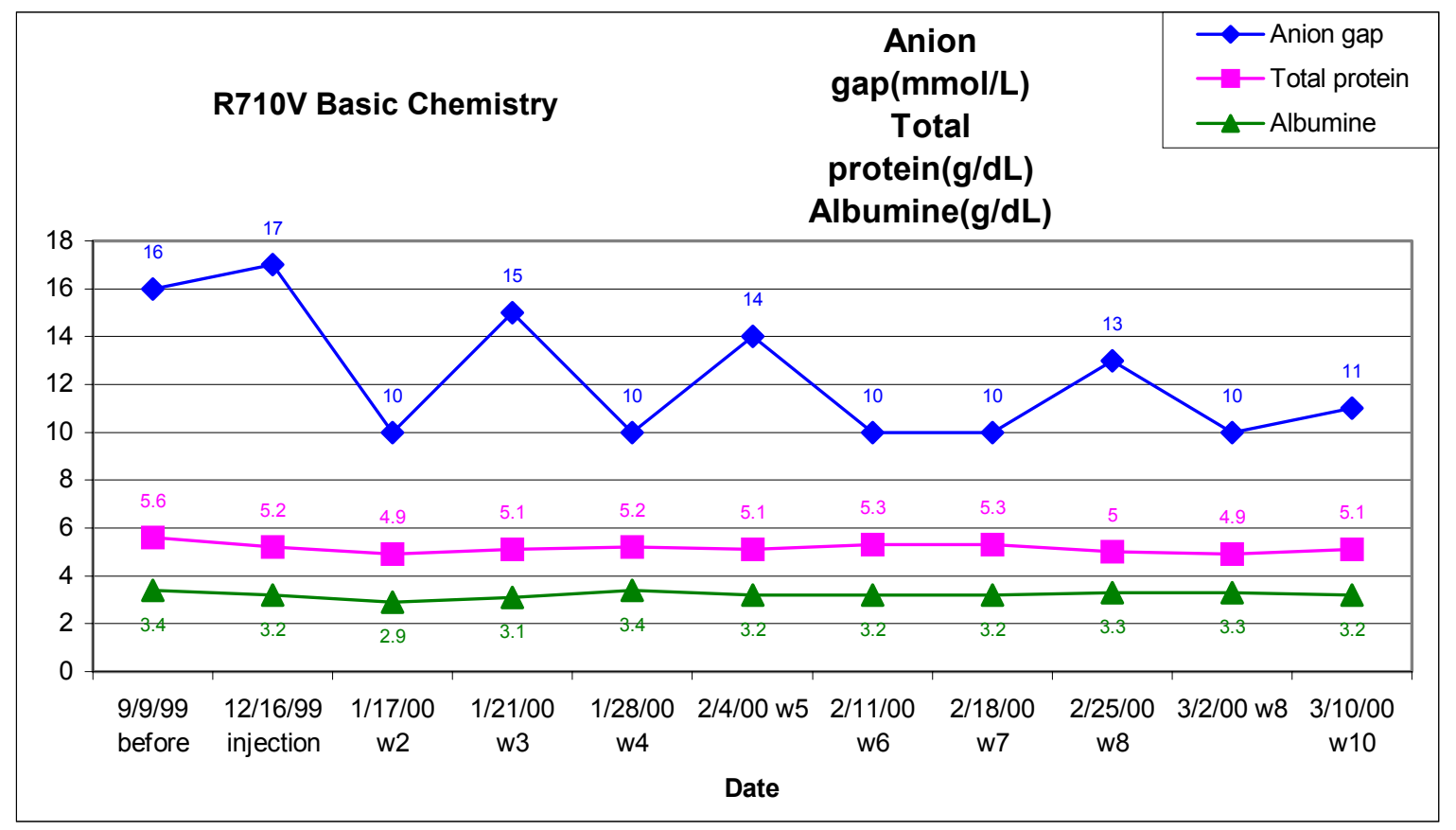

Figure G.5 Basic chemistry R710V

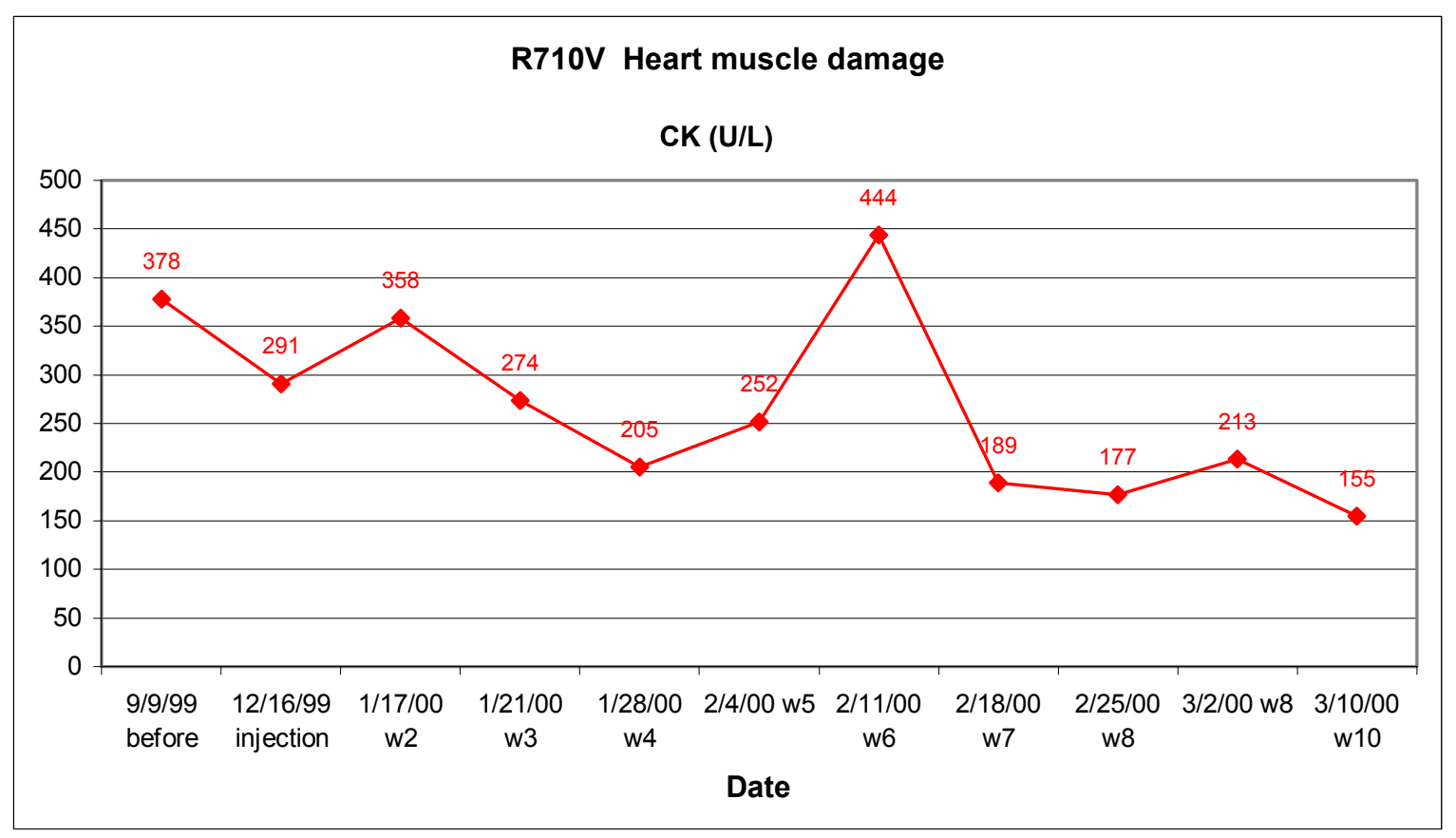

Figure G.6 Creatine kinase R710V 


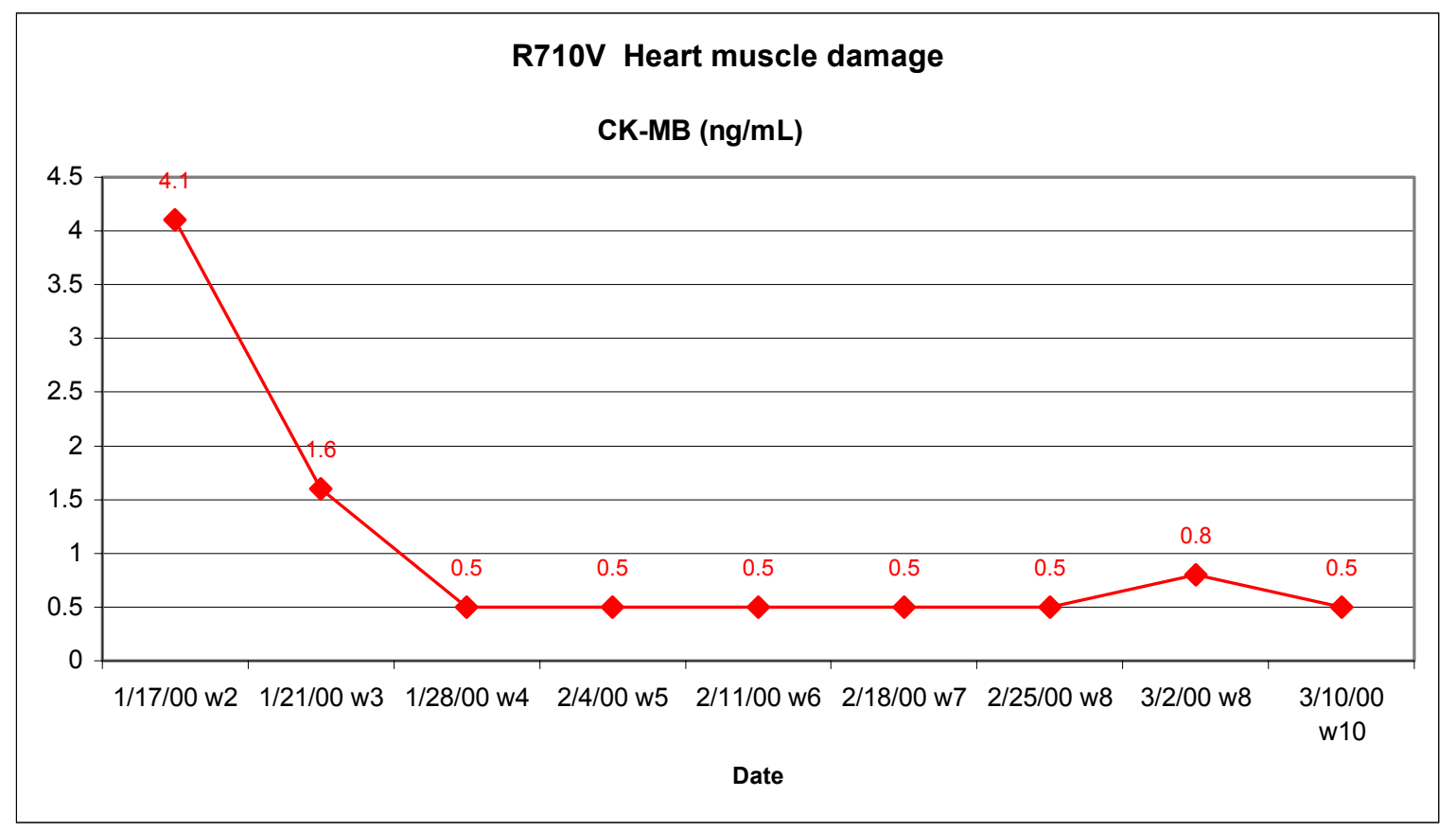

Figure G.7 Creatine kinase - MB R710V

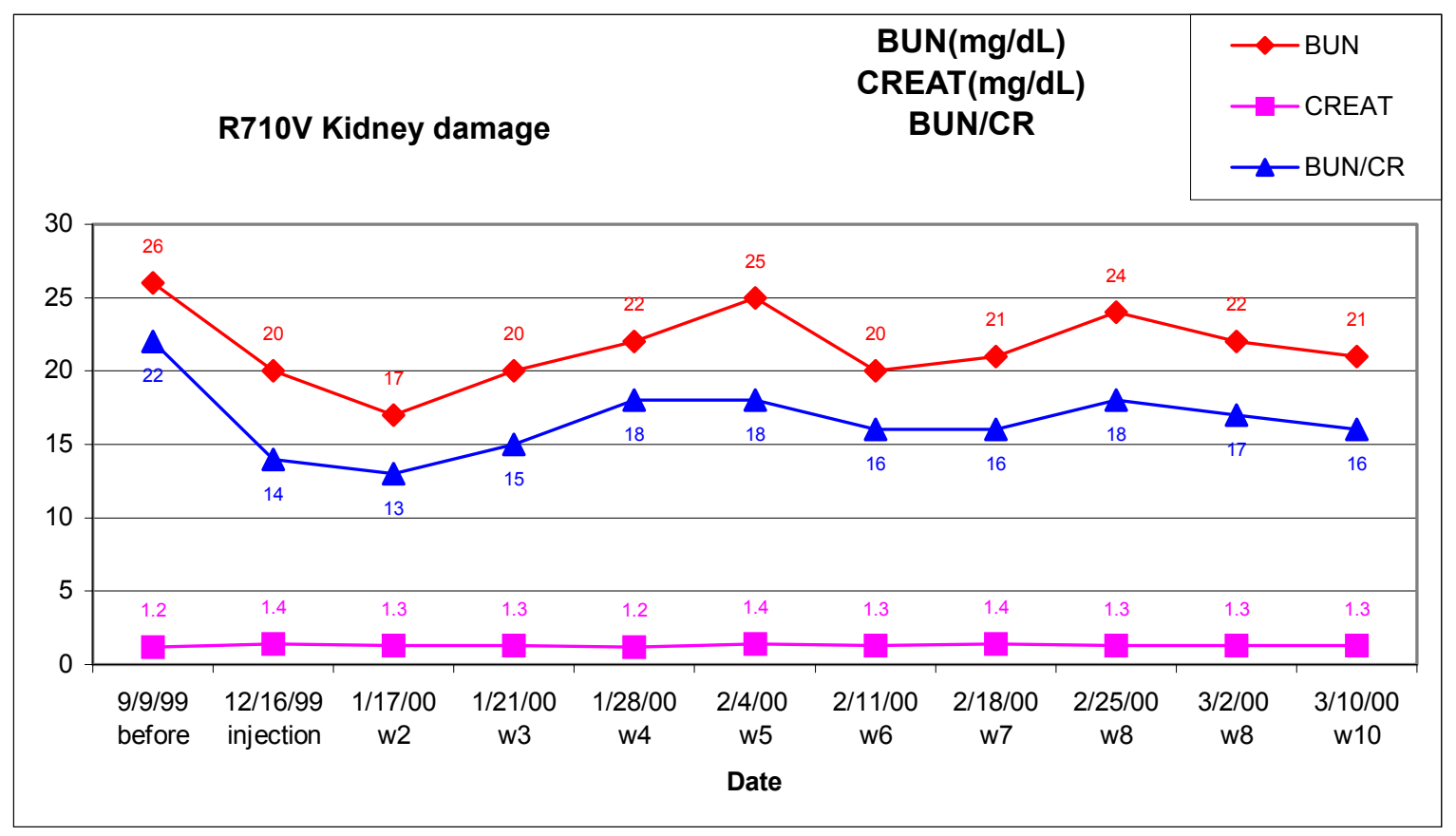

Figure G.8 Kidney damage R710V 


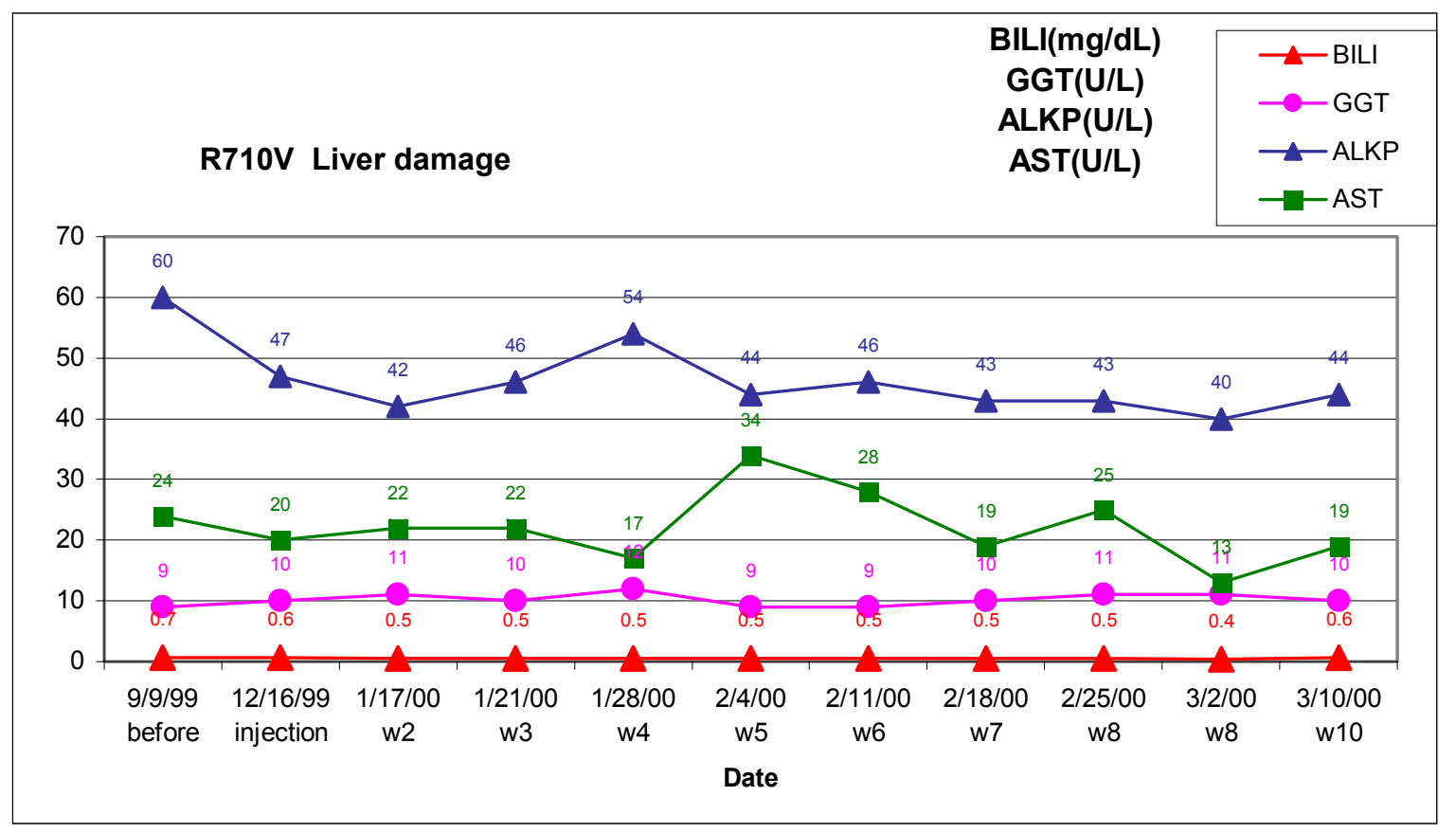

Figure G.9 Liver damage R710V

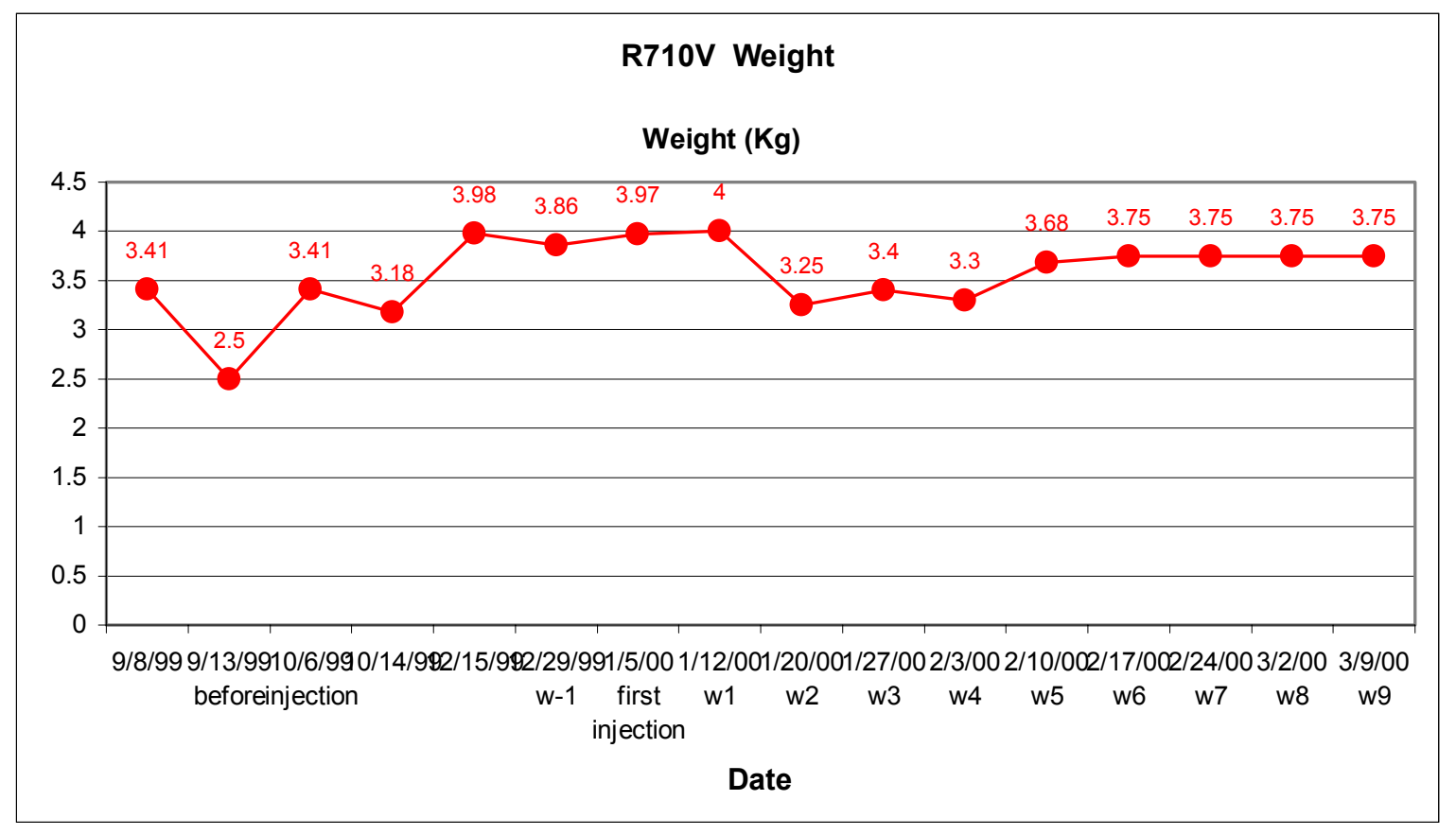

Figure G.10 Weight R710V 


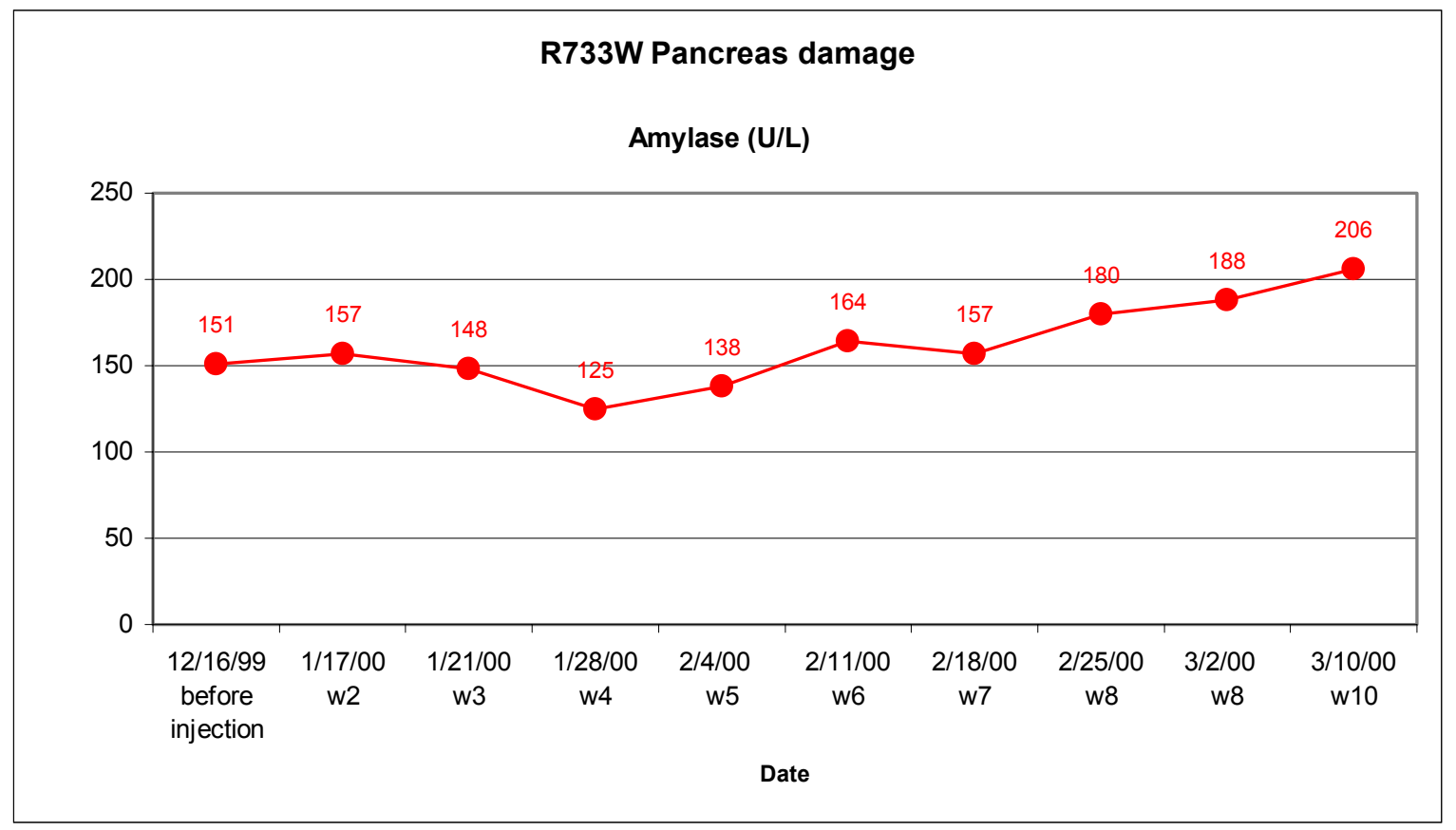

Figure G.11 Amylase R733W

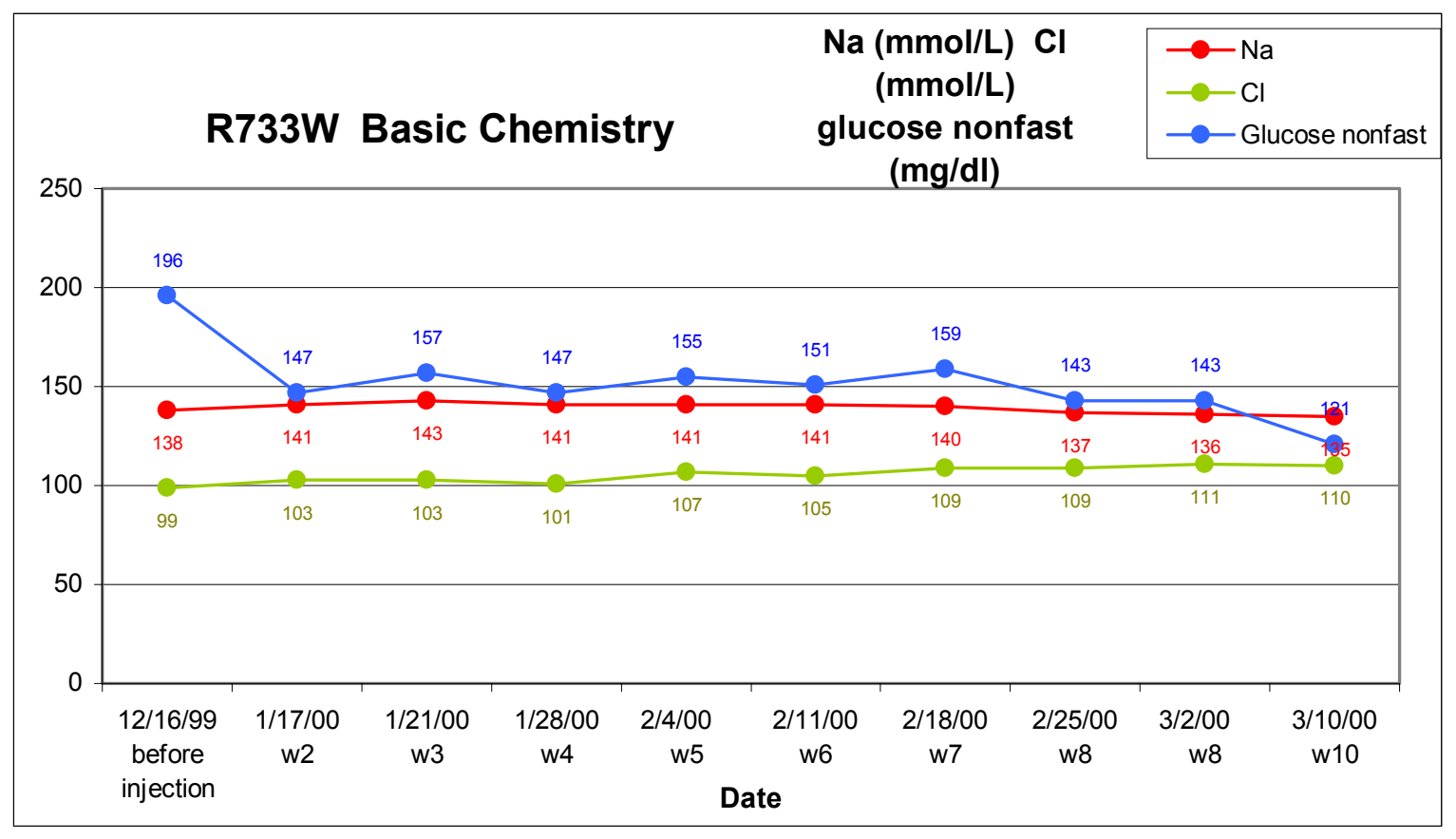

Figure G.12 Basic chemistry R733W 


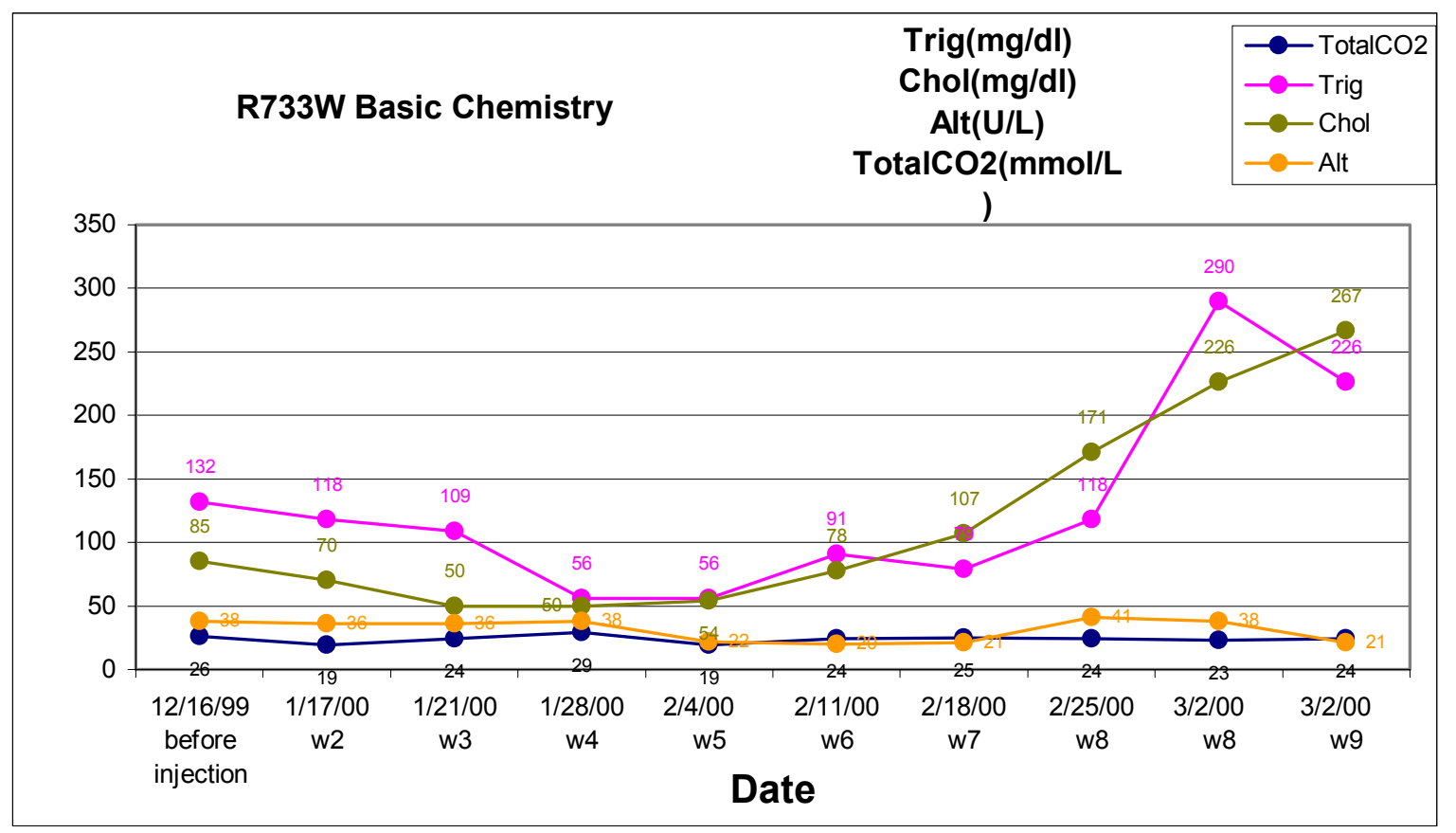

Figure G.13 Basic chemistry R733W

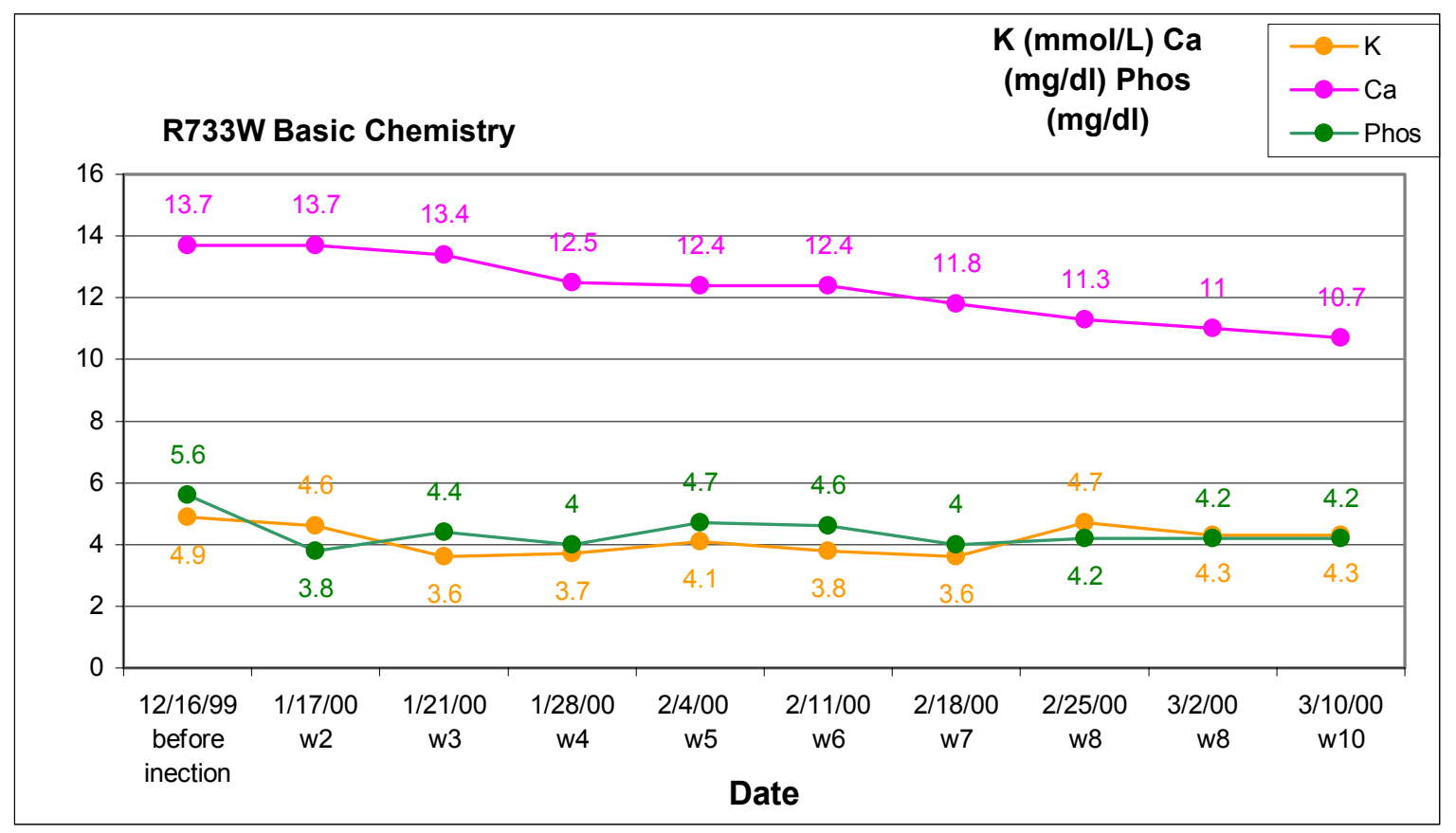

Figure G.14 Basic chemistry R733W 


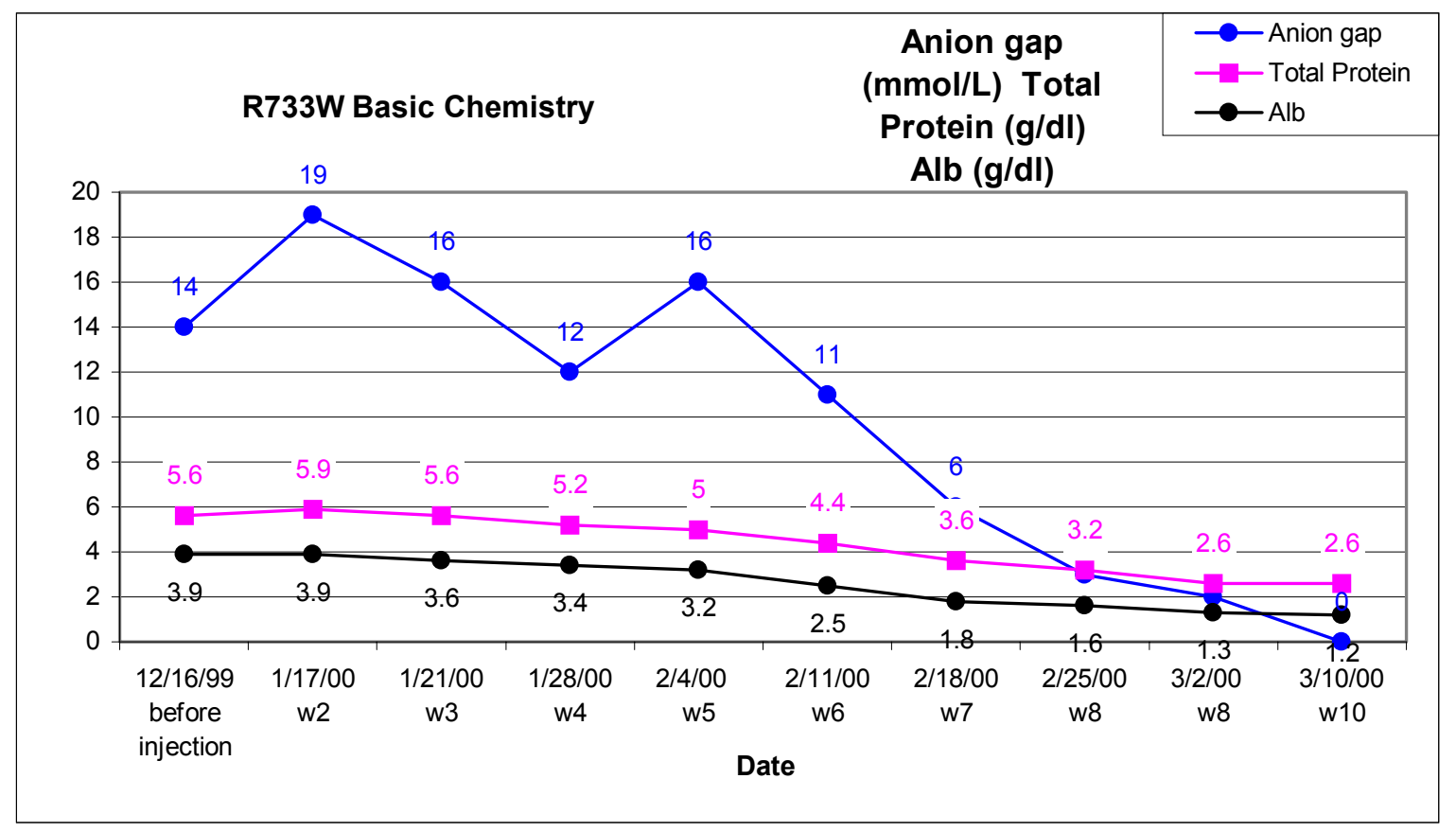

Figure G.15 Basic chemistry R733W

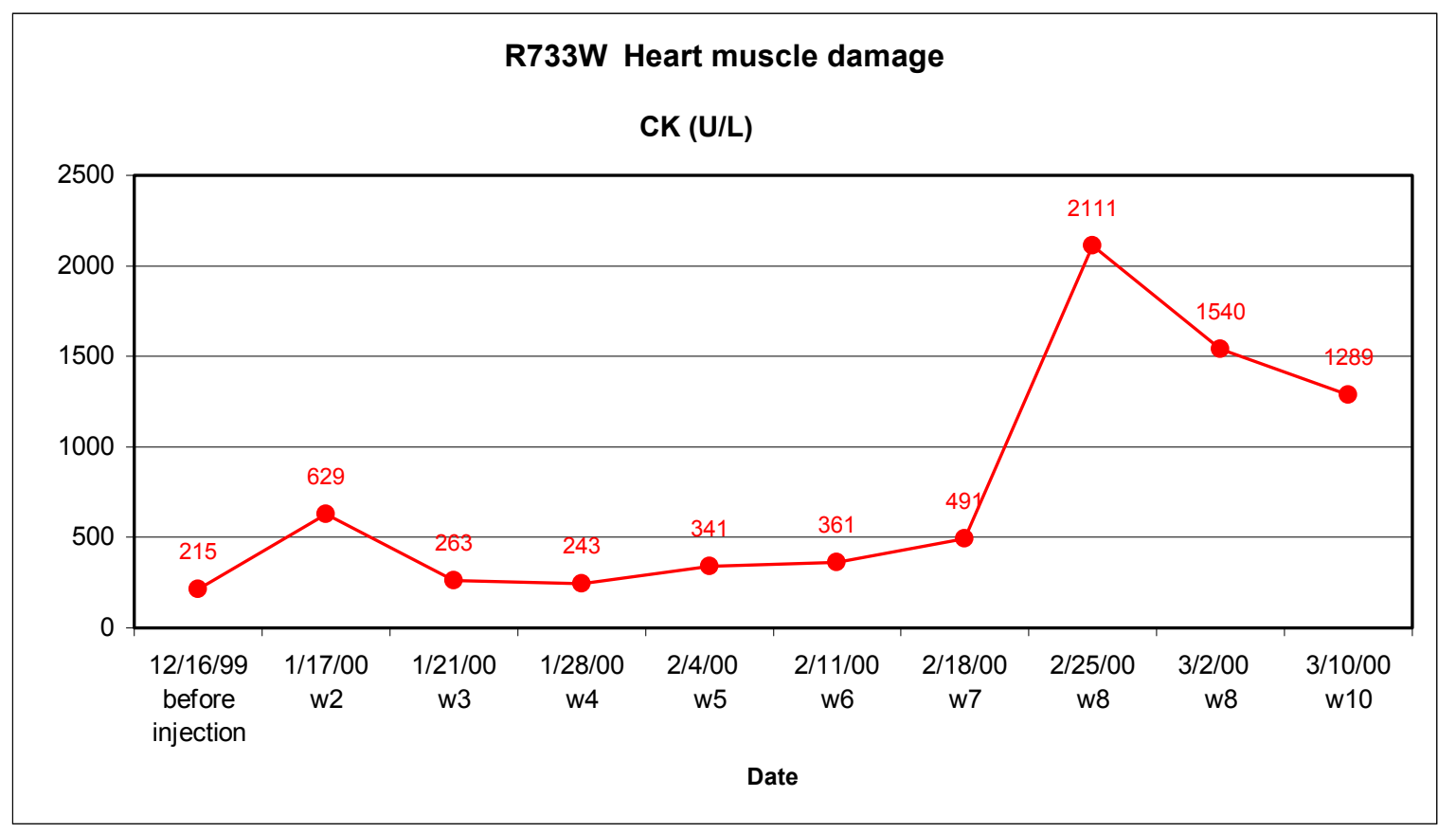

Figure G.16 Creatine kinase R733W 


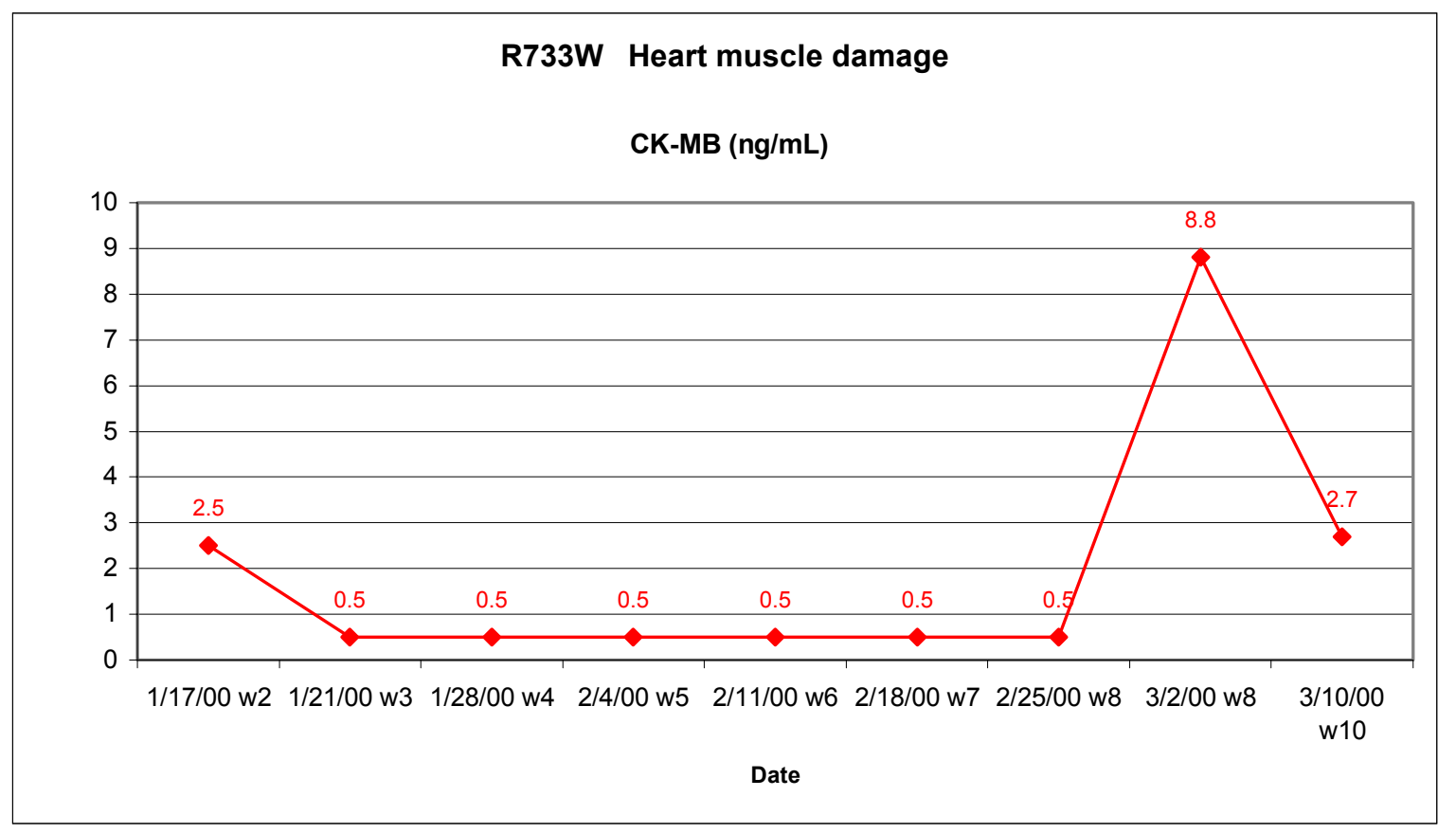

Figure G.17 Creatine kinase - MB R733W

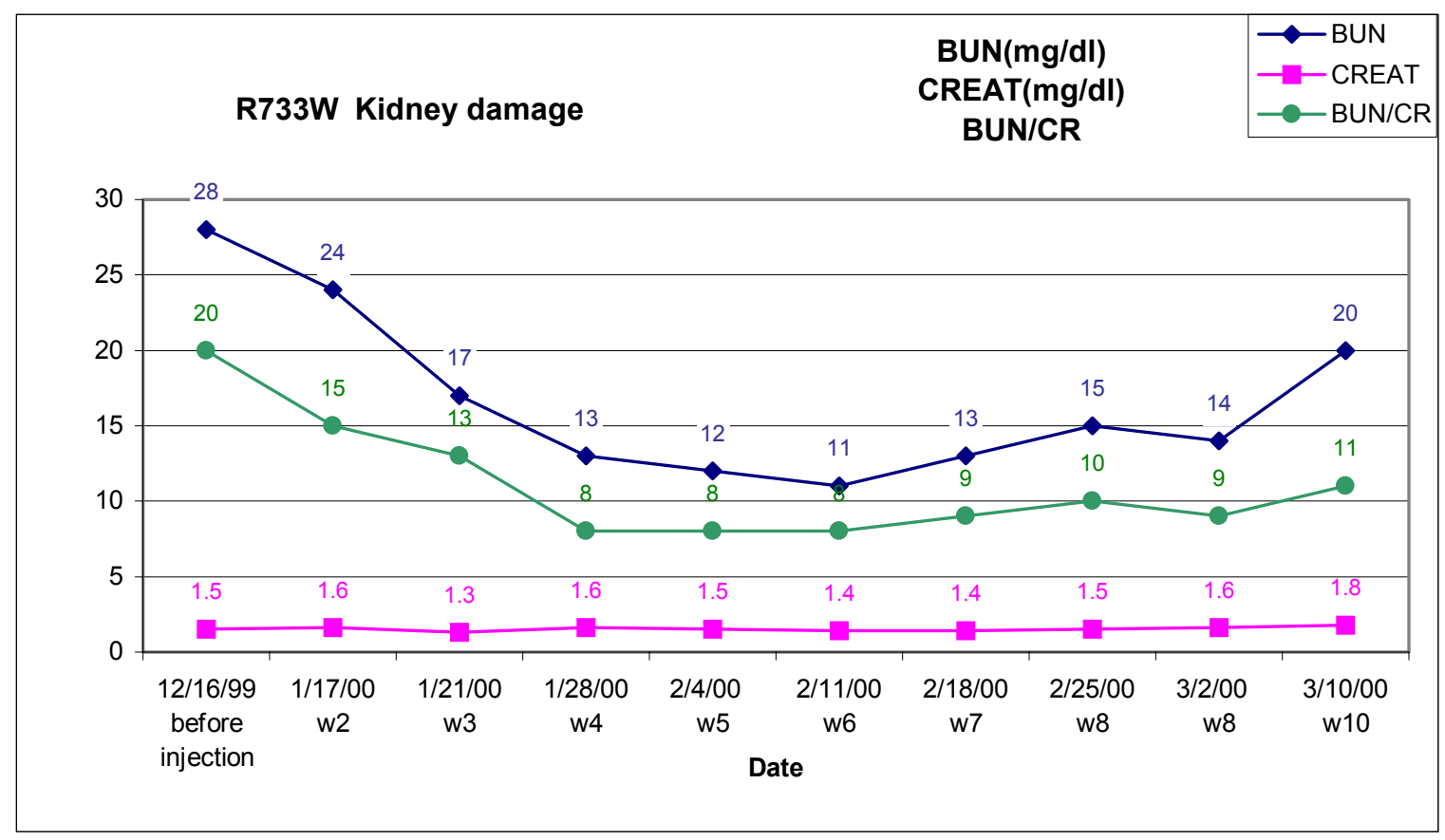

Figure G.18 Kidney damage R733W 


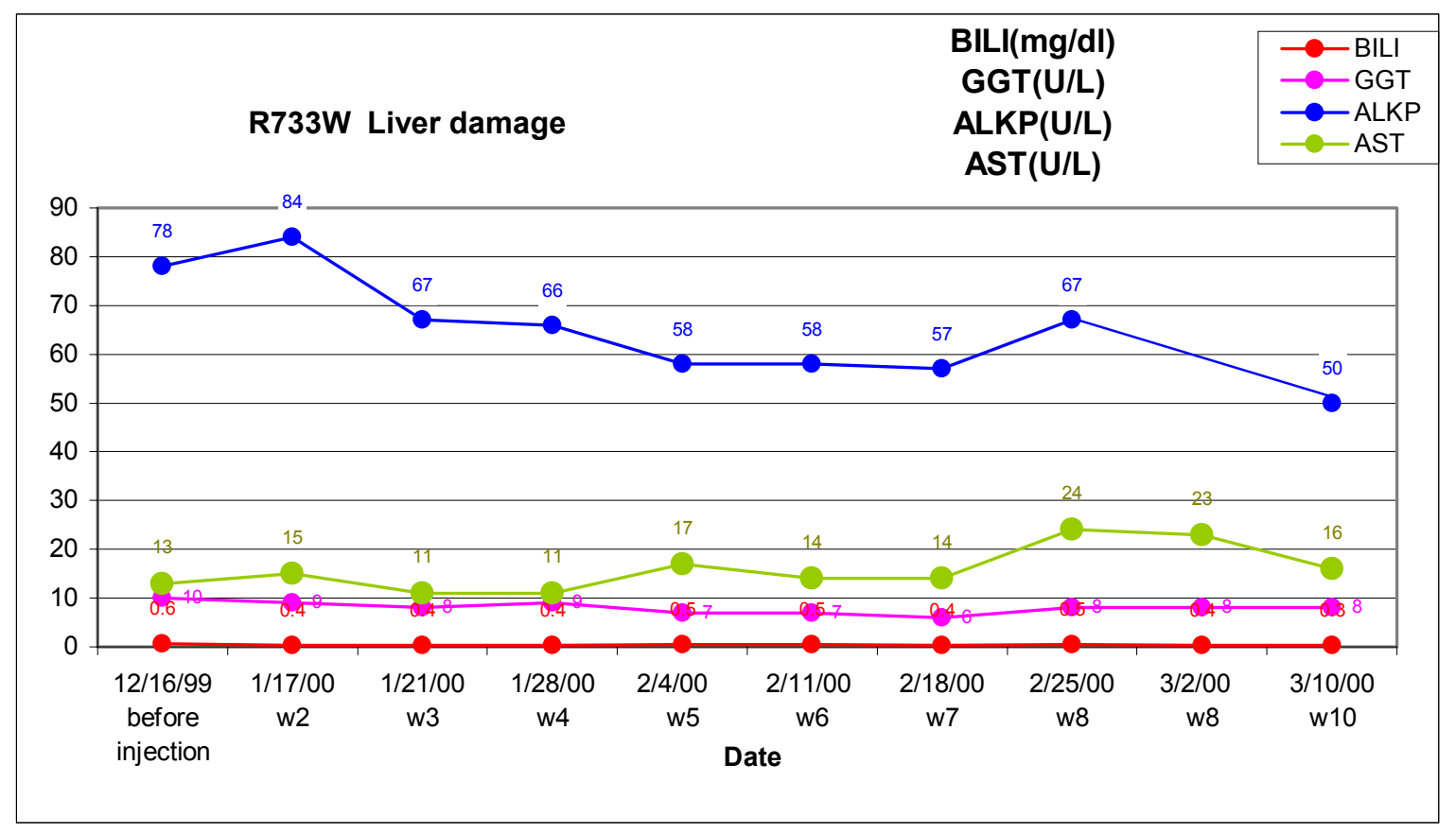

Figure G.19 Liver damage R733W

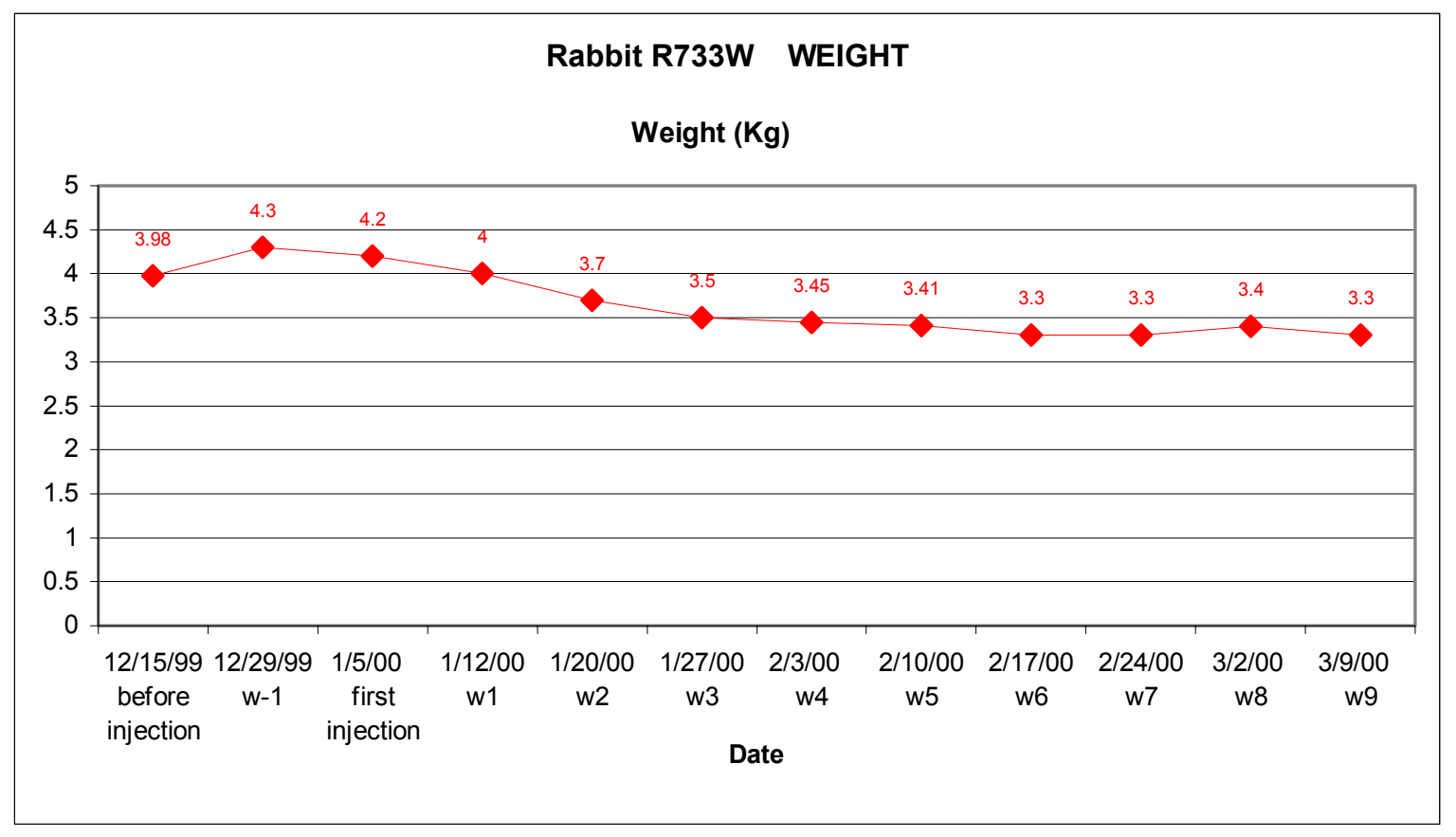

Figure G.20 Weight R733W 


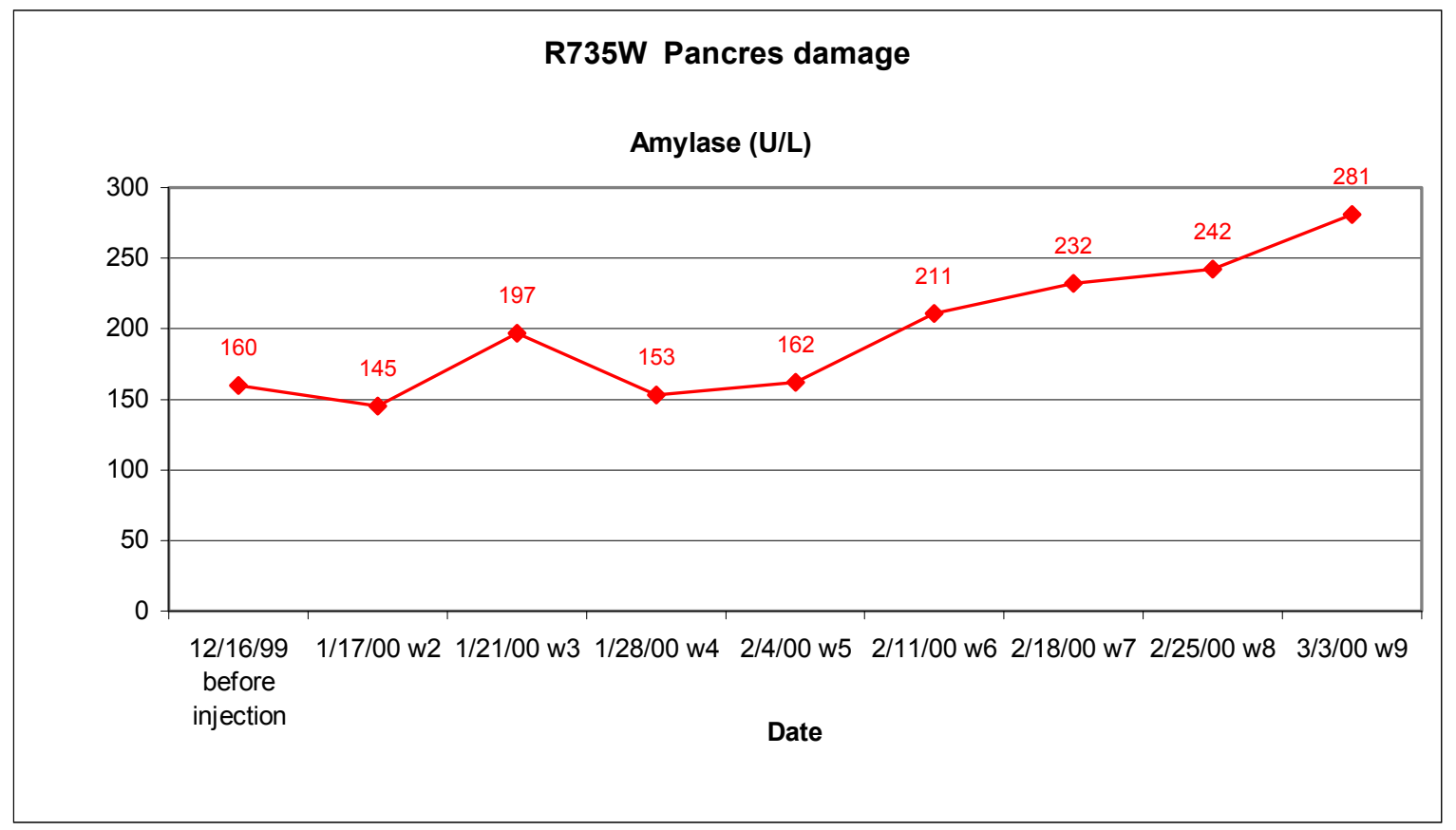

Figure G.21 Amylase R735W

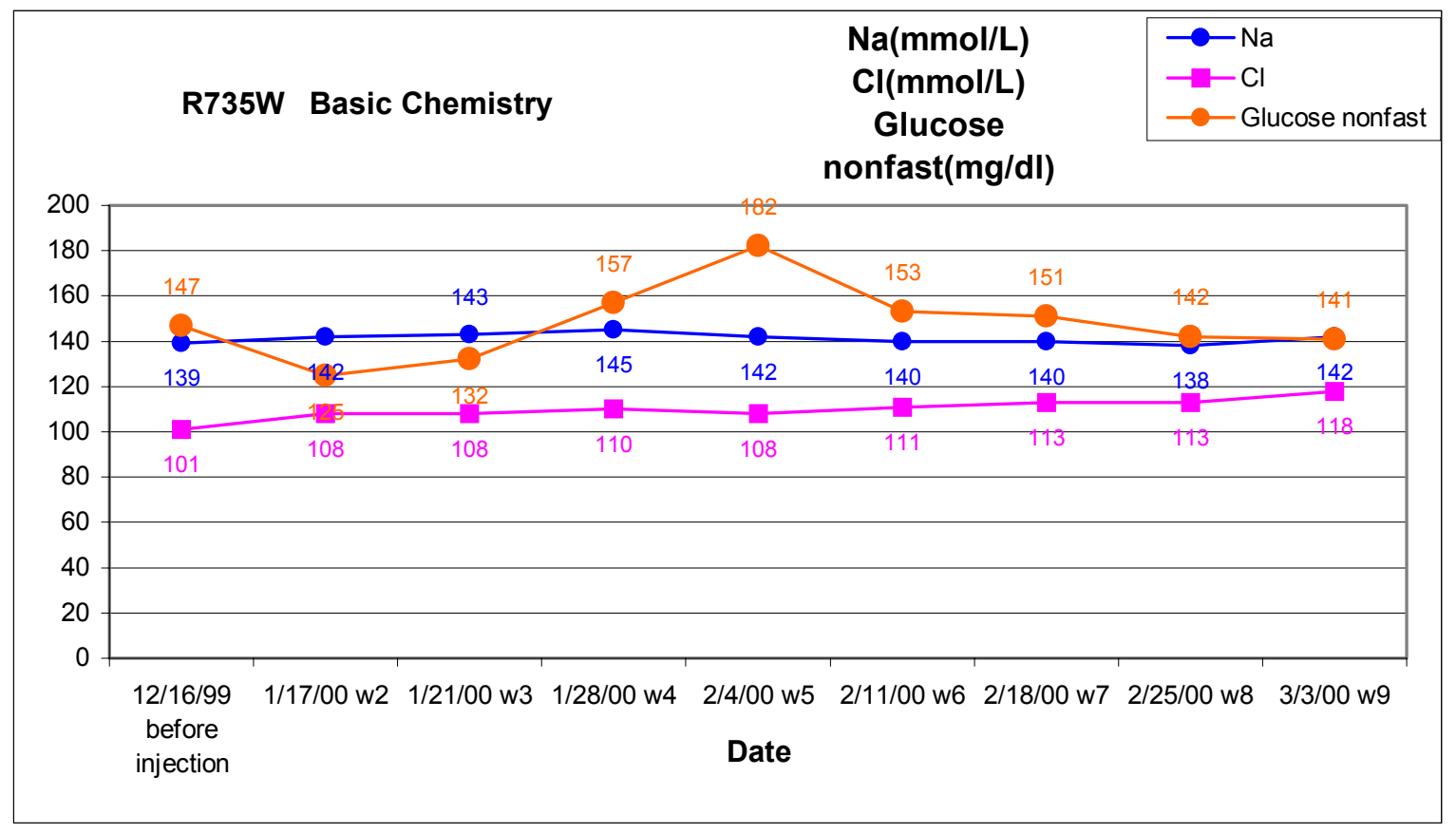

Figure G.22 Basic chemistry R735W 


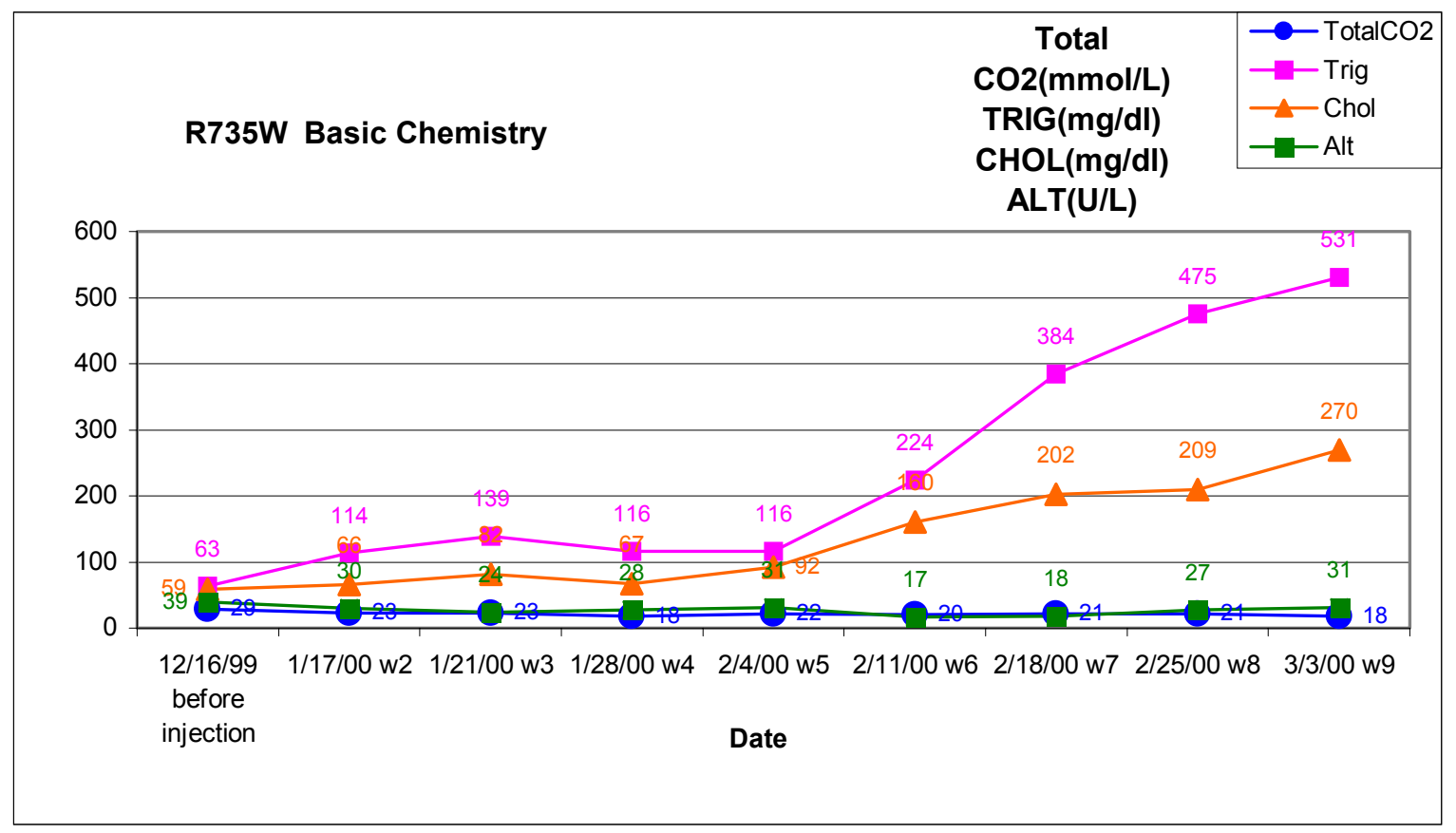

Figure G.23 Basic chemistry R735W

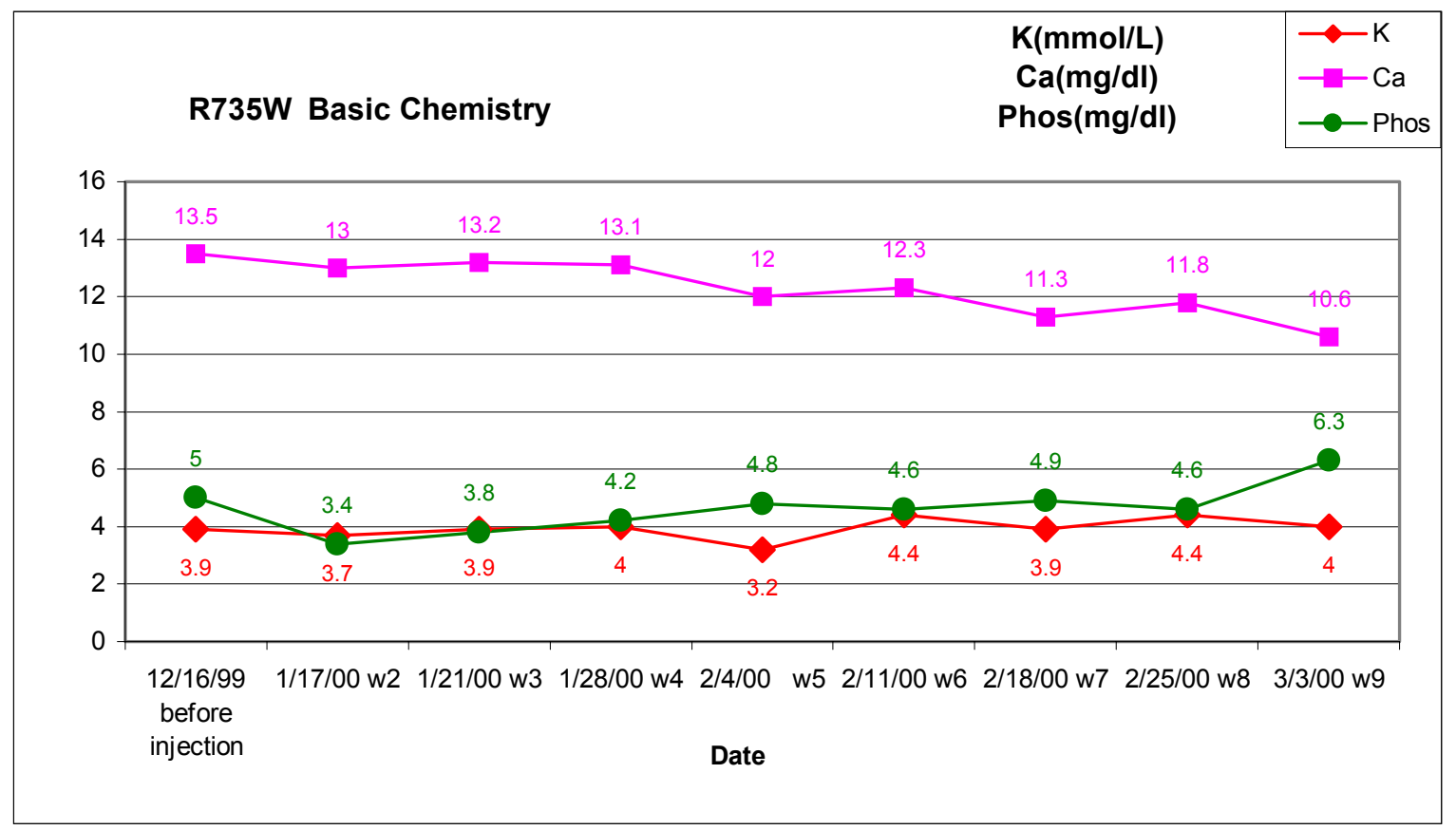

Figure G.24 Basic chemistry R735W 


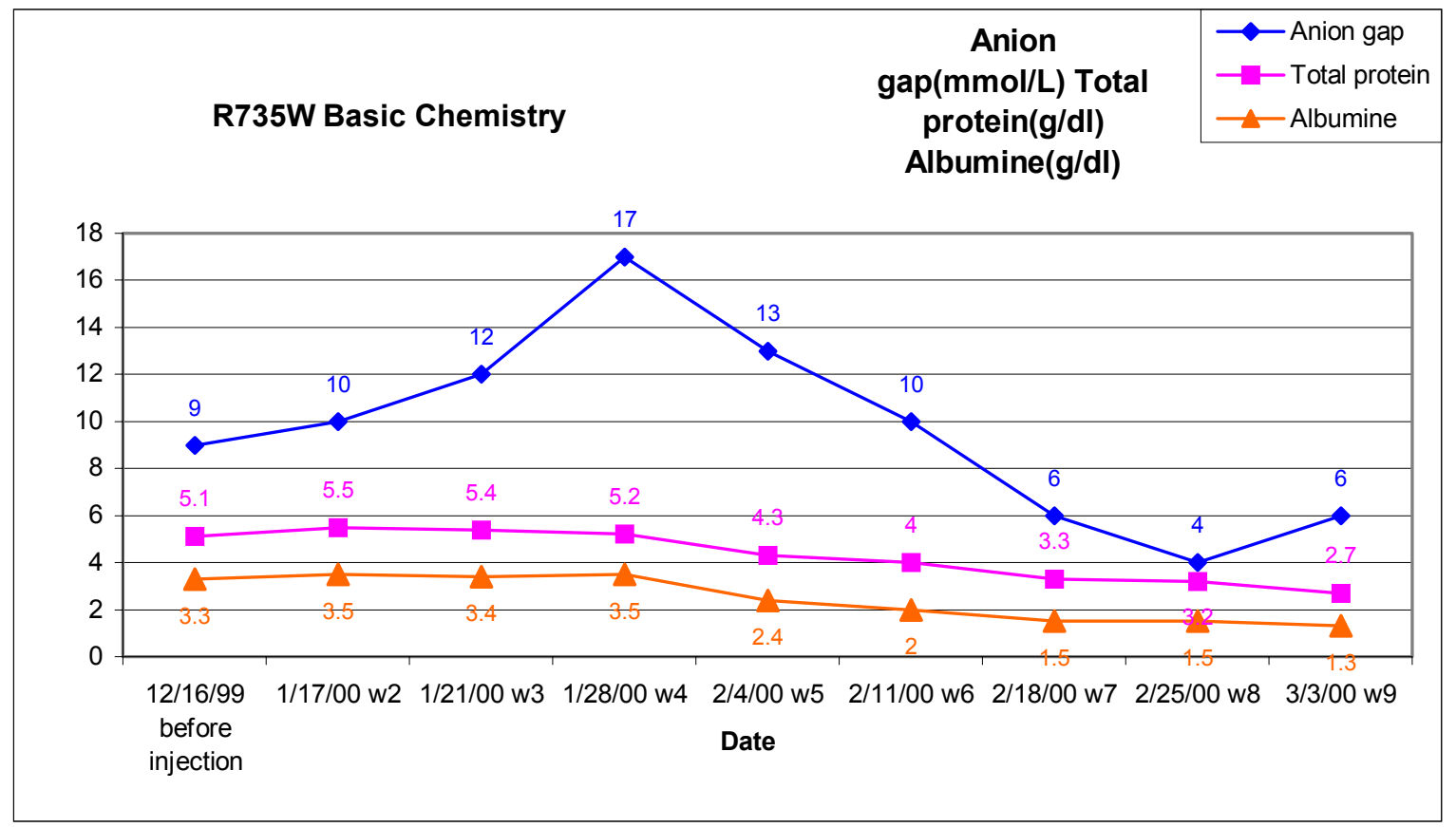

Figure G.25 Basic chemistry R735W

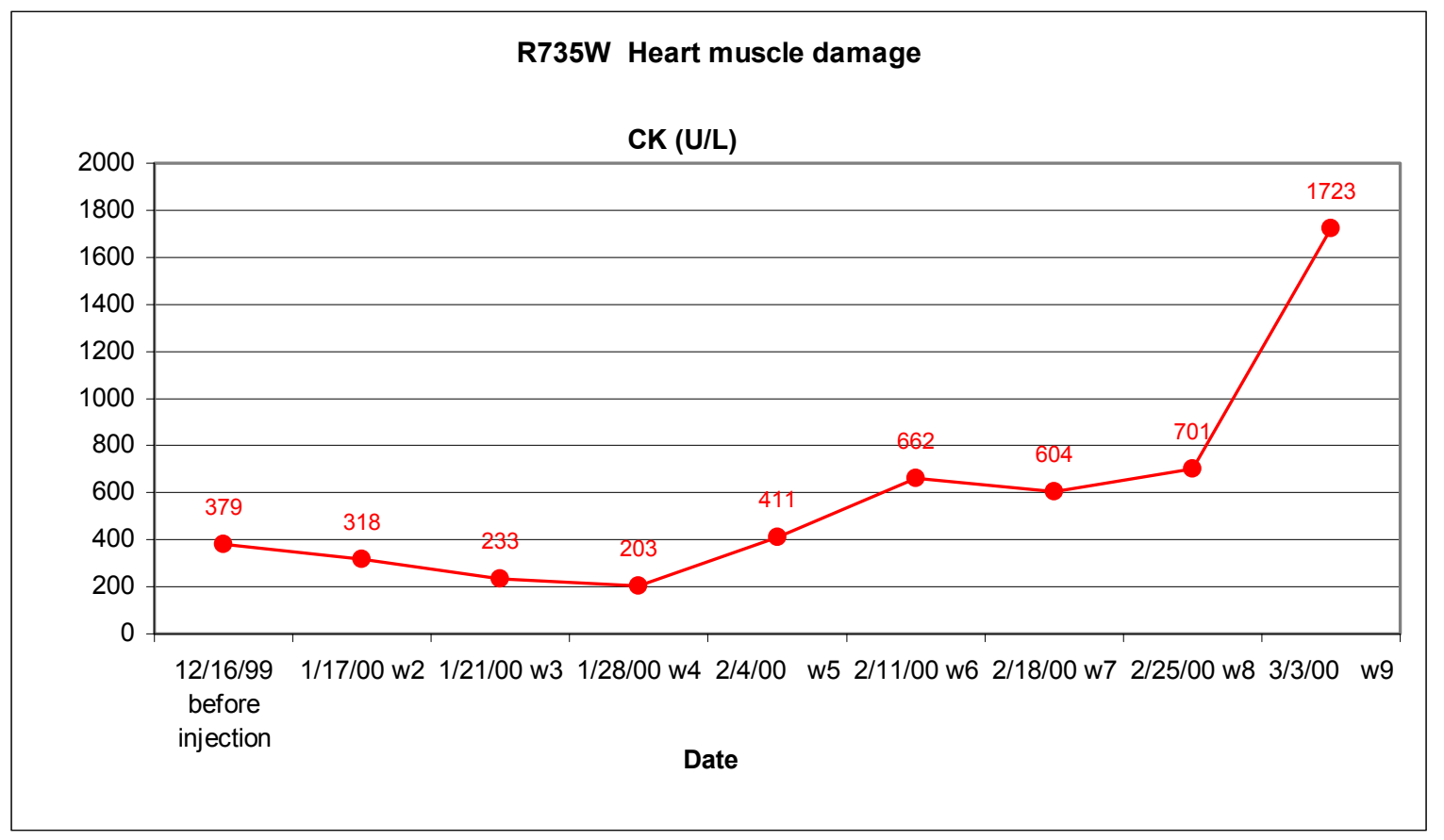

Figure G.26 Creatine kinase R735W 


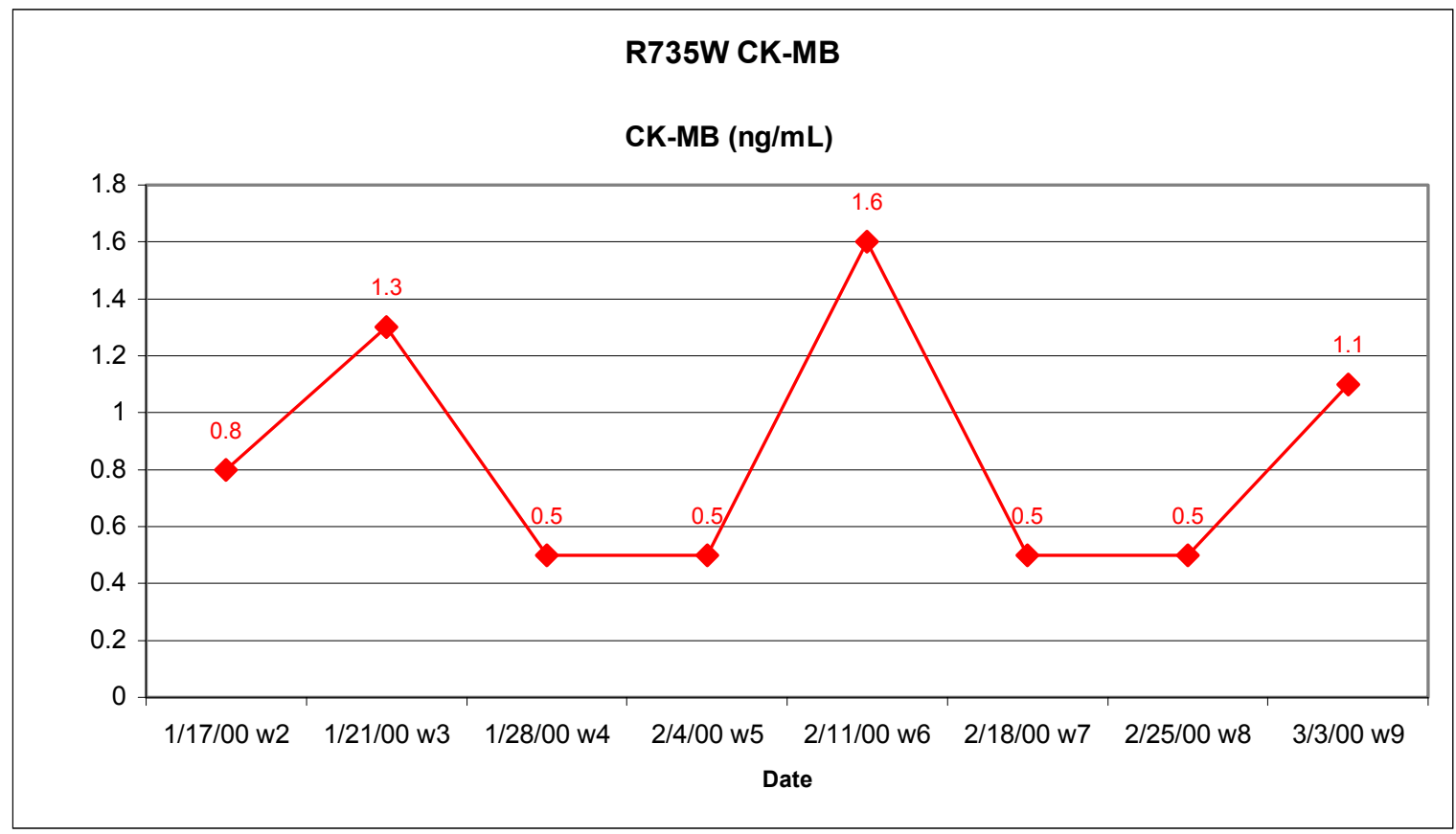

Figure G.27 Creatine kinase - MB R735W

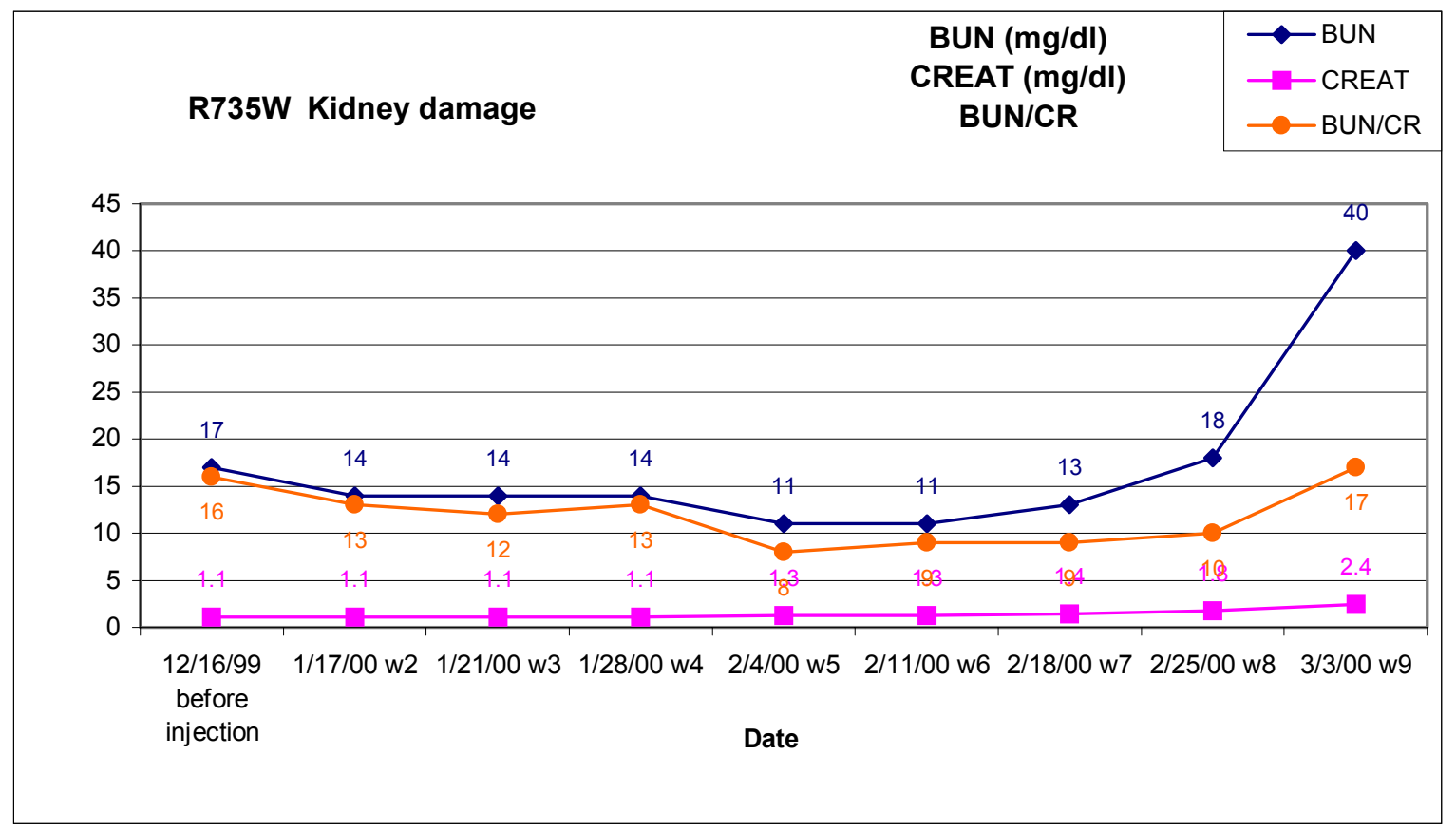

Figure G.28 Kidney damage R735W 


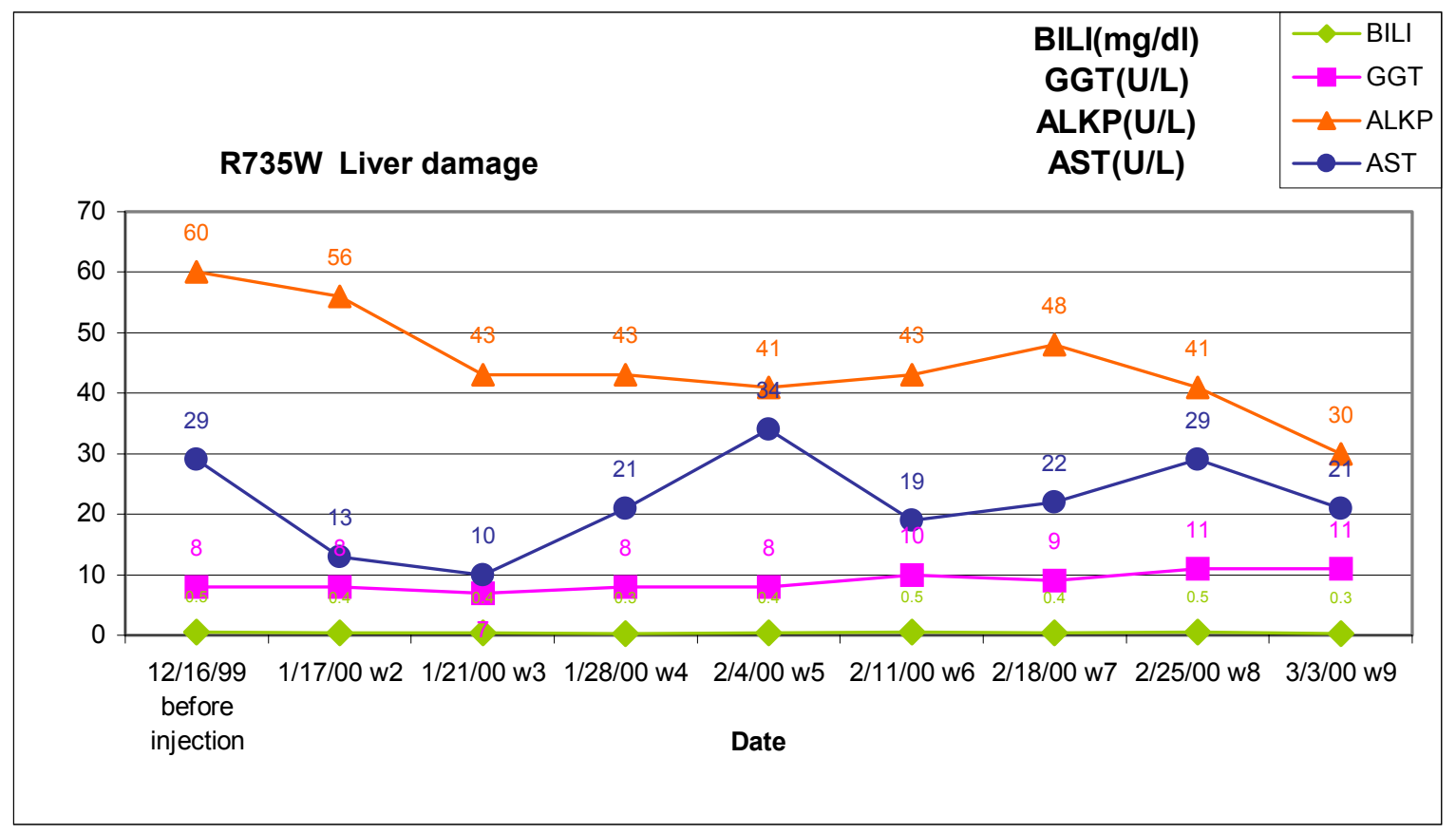

Figure G.29 Liver damage R735W

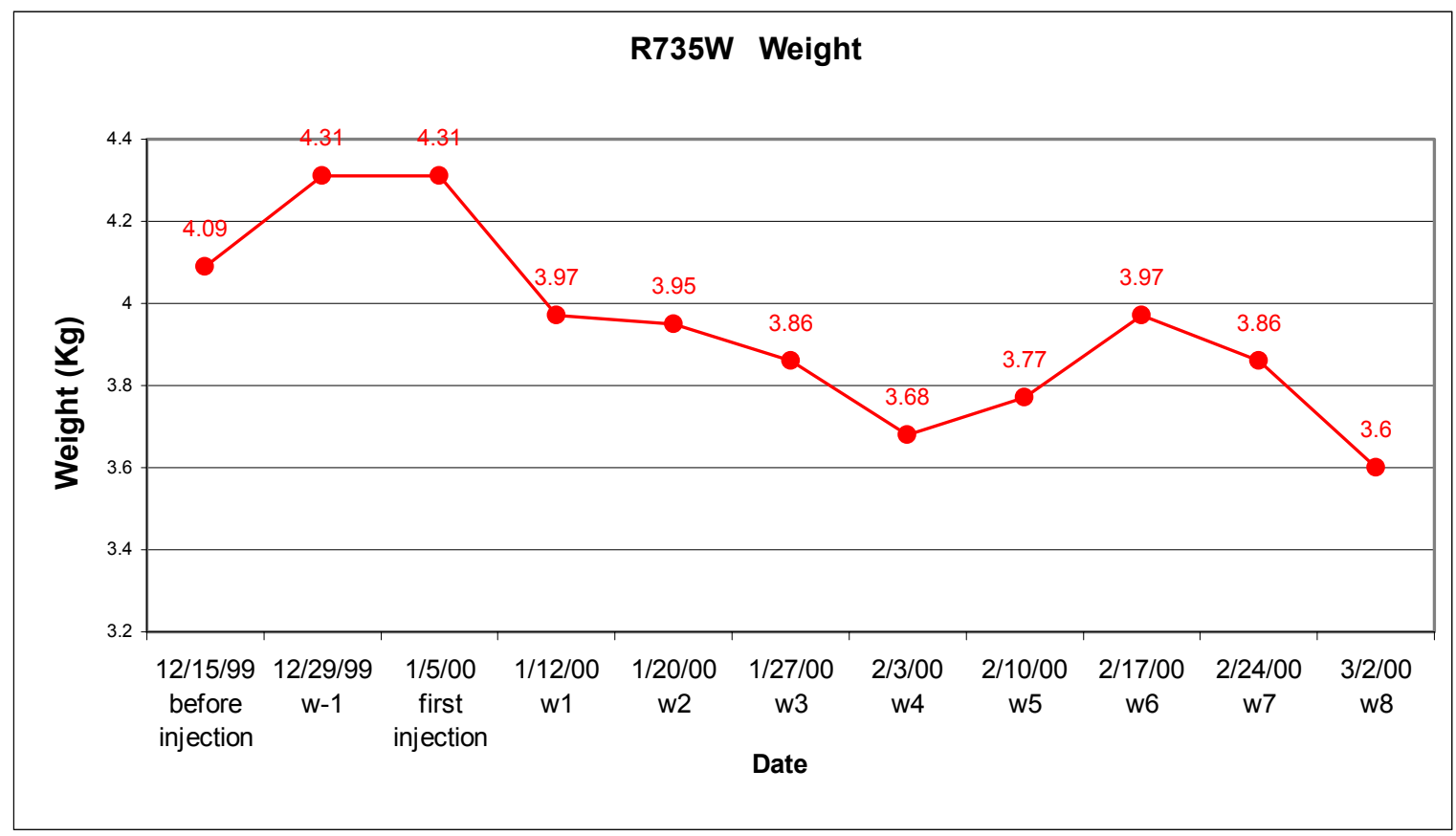

Figure G.30 Weight R735W 


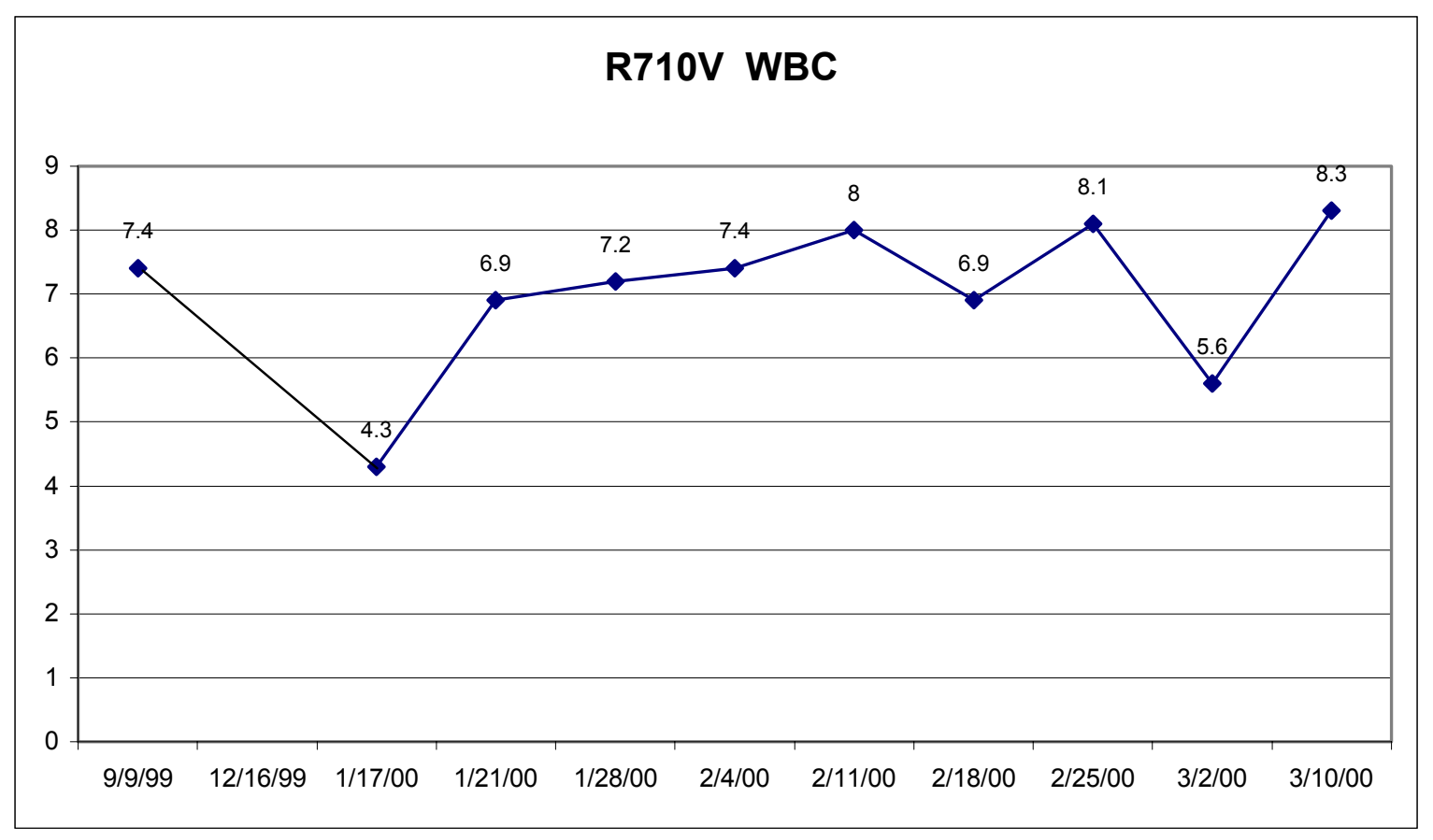

Figure G.31 WBC R710V

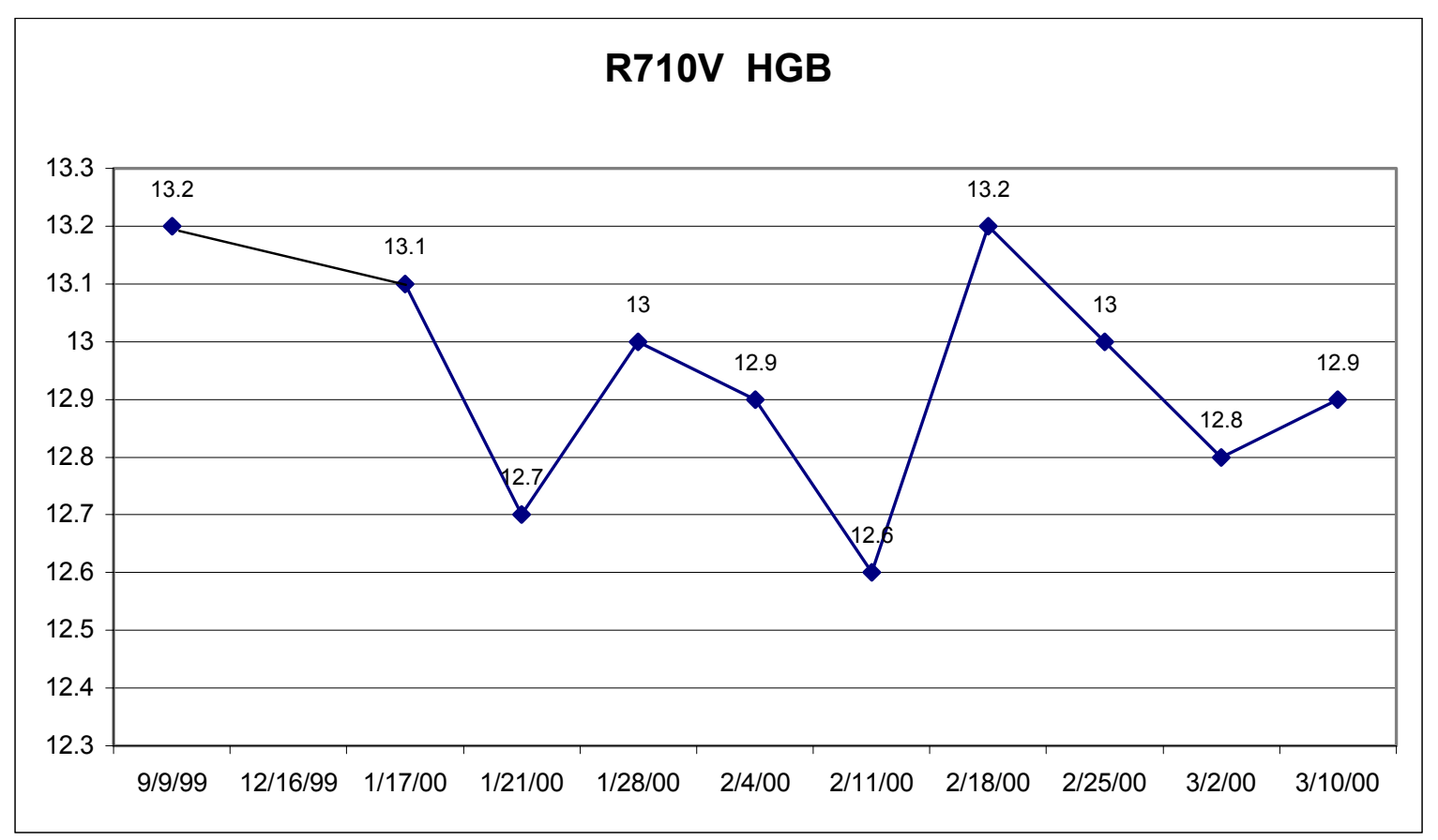

Figure G.32 HGB R710V 


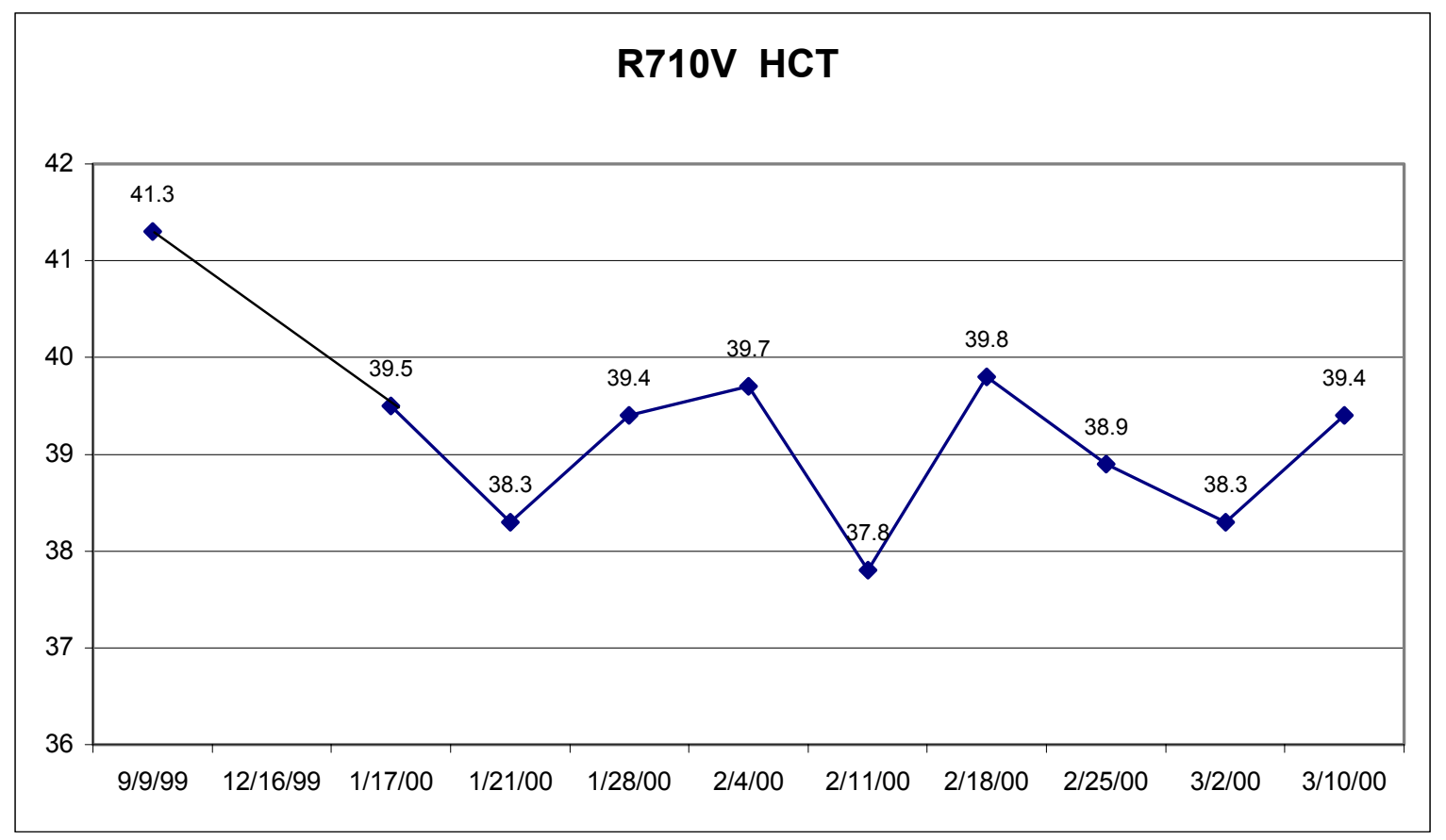

Figure G.33 HCT R710V

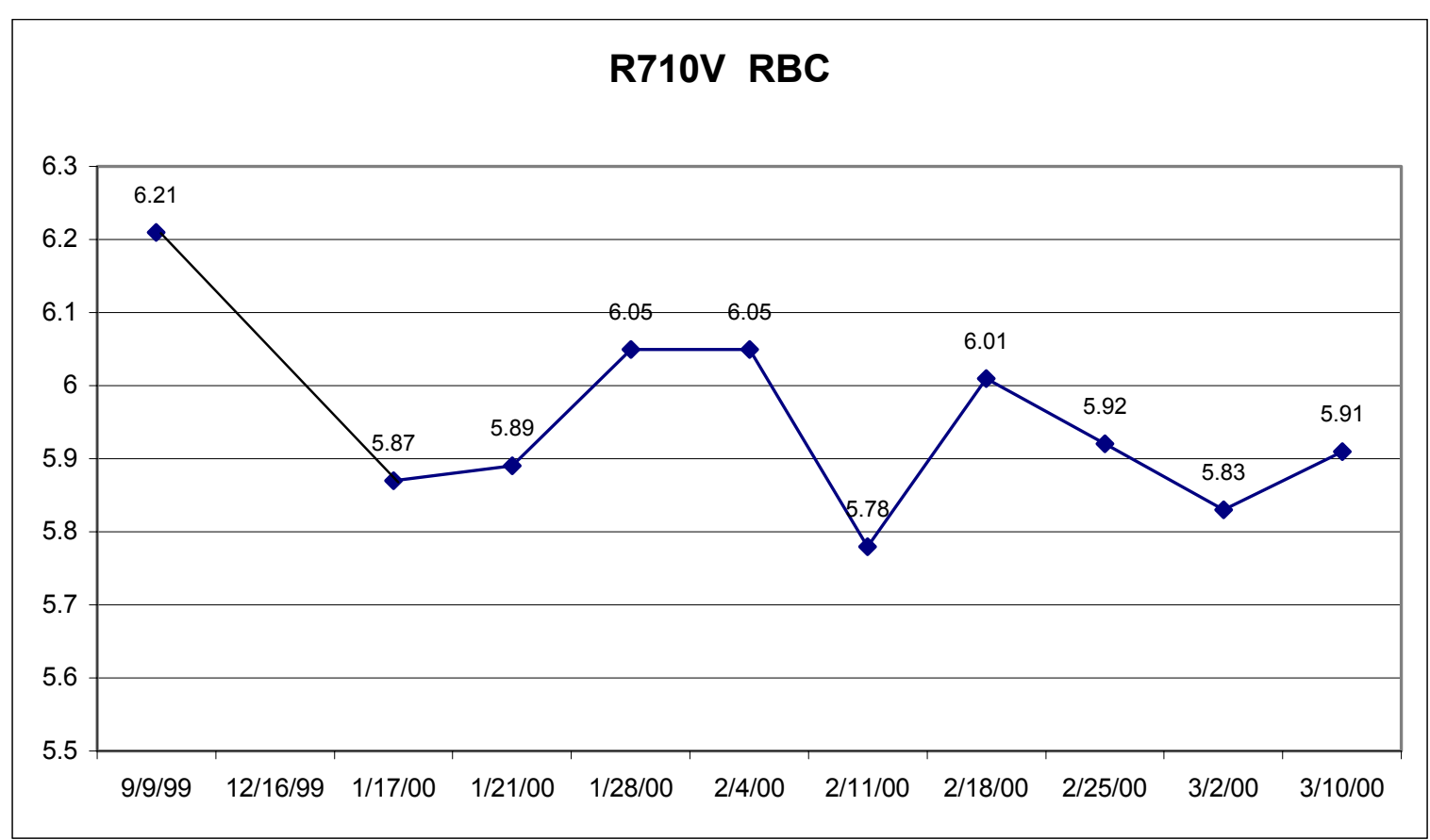

Figure G.34 RBC R710V 


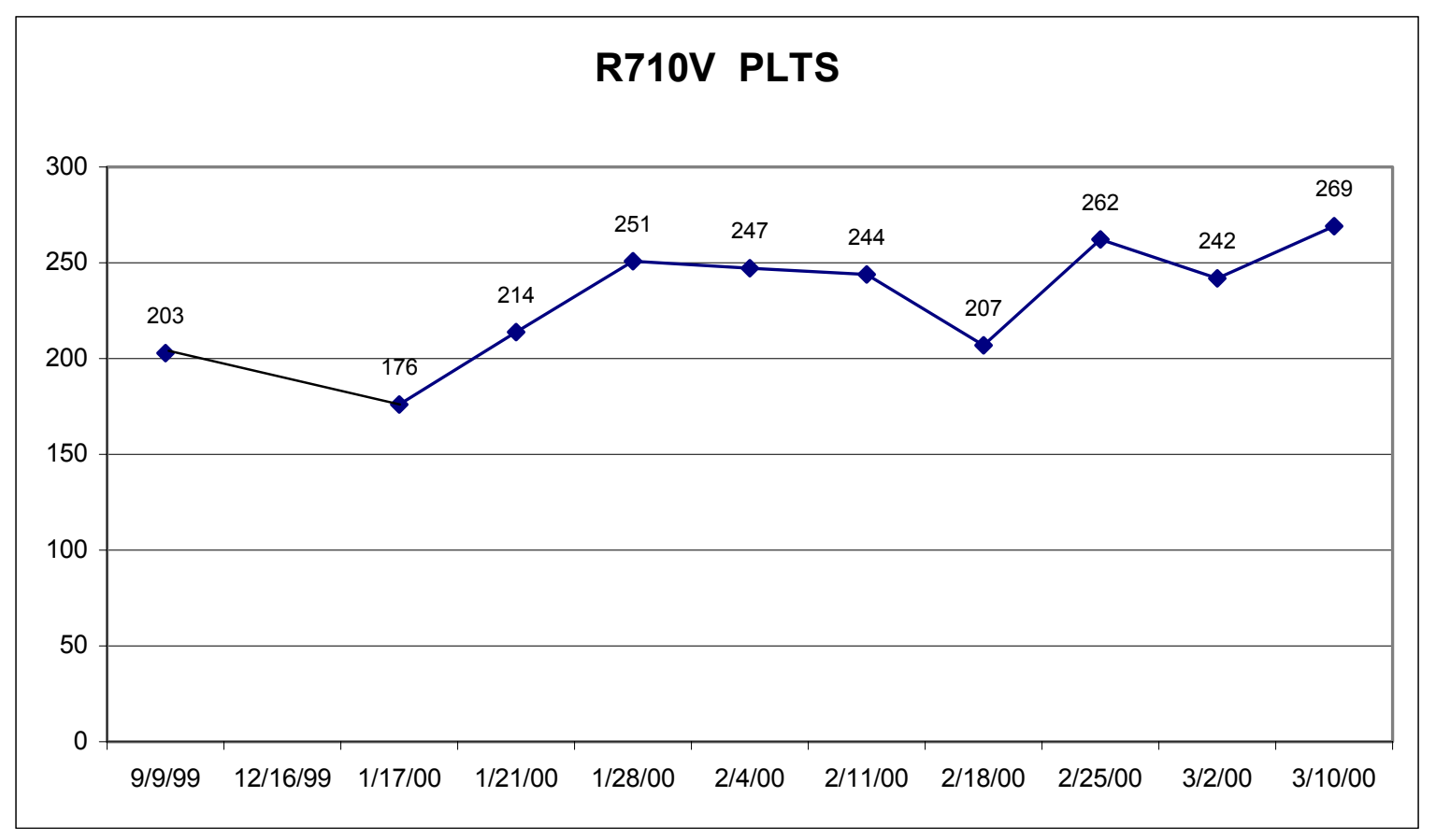

Figure G.35 Platelets R710V

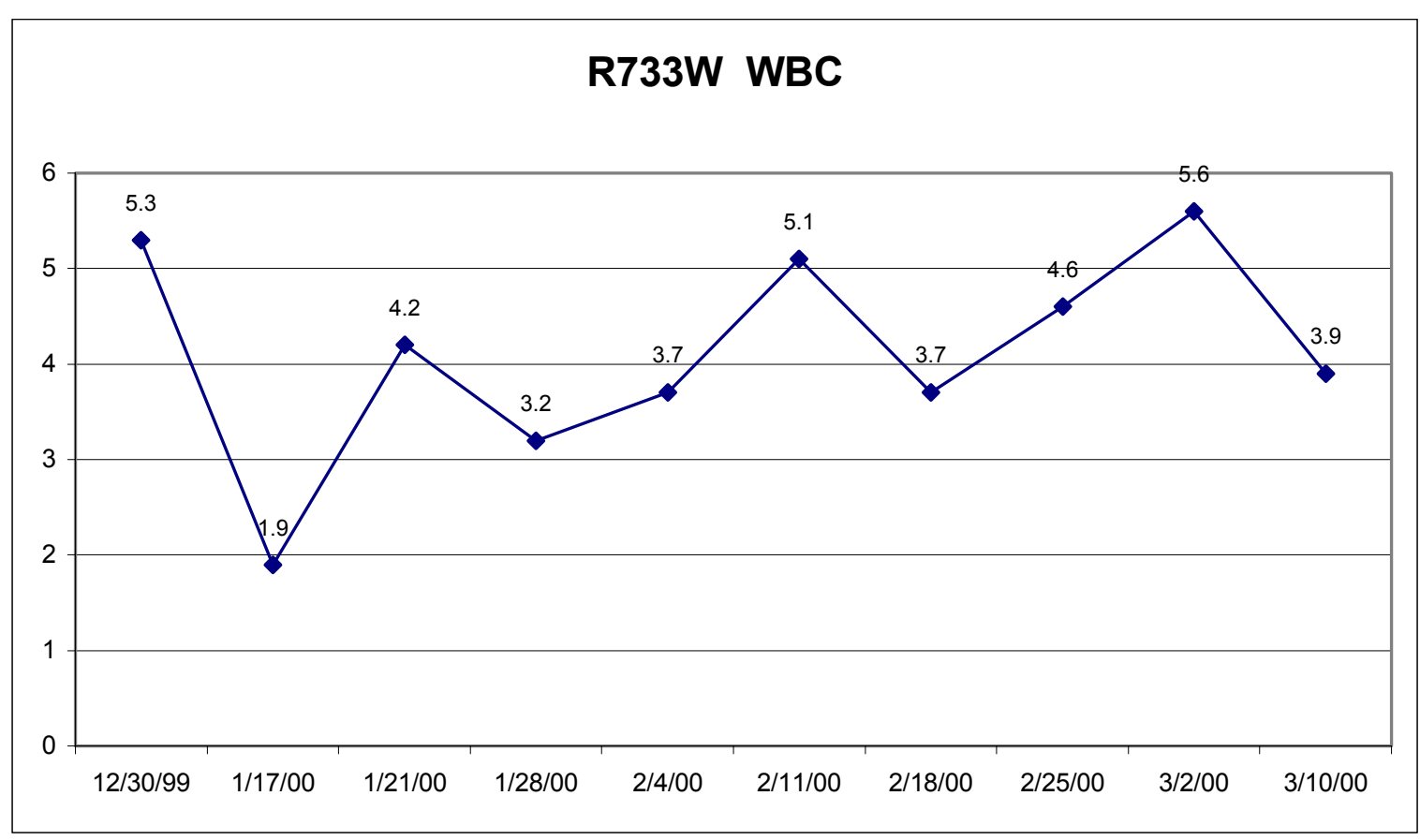

Figure G.36 WBC R733W 


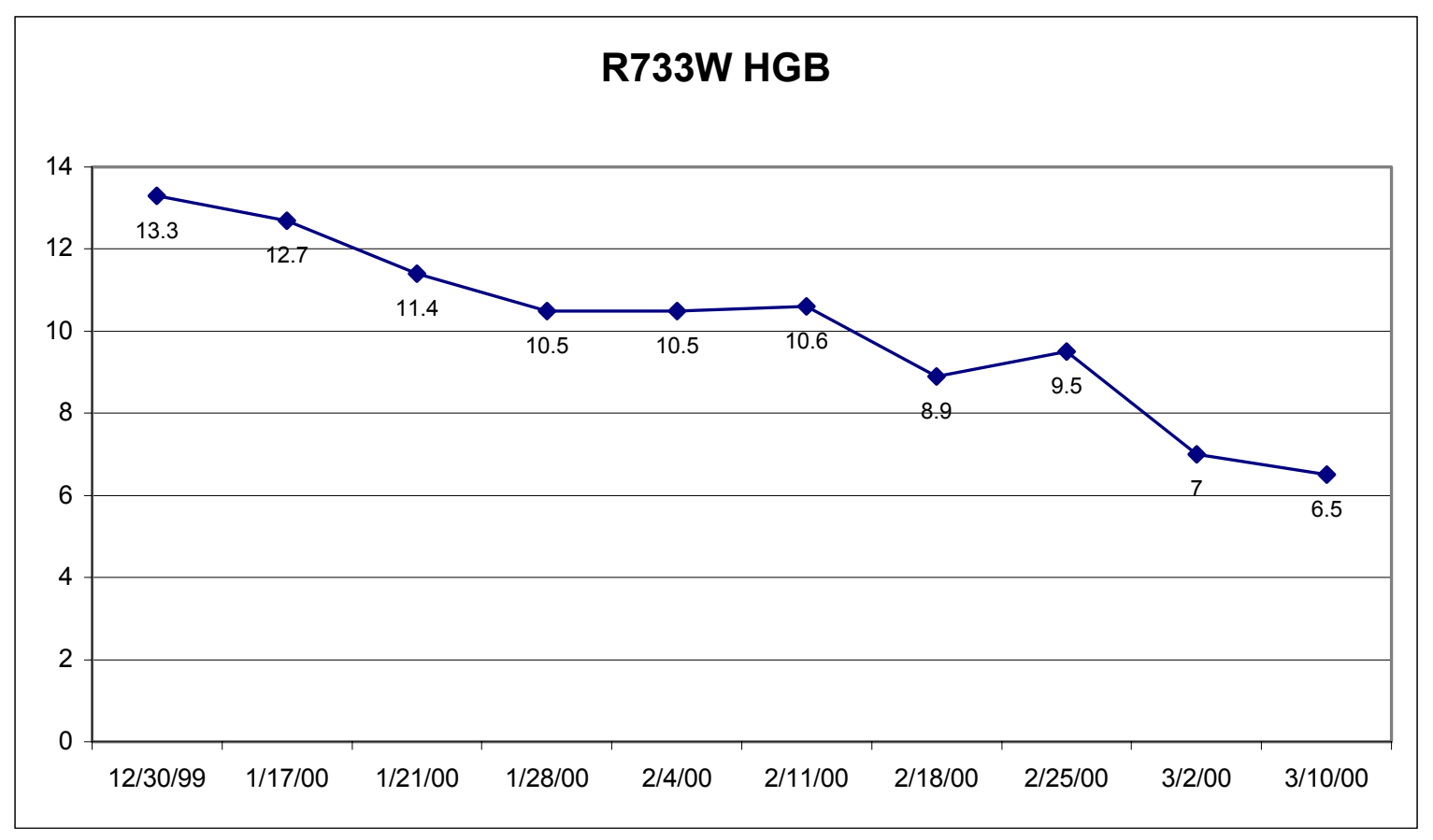

Figure G.37 HGB R733W

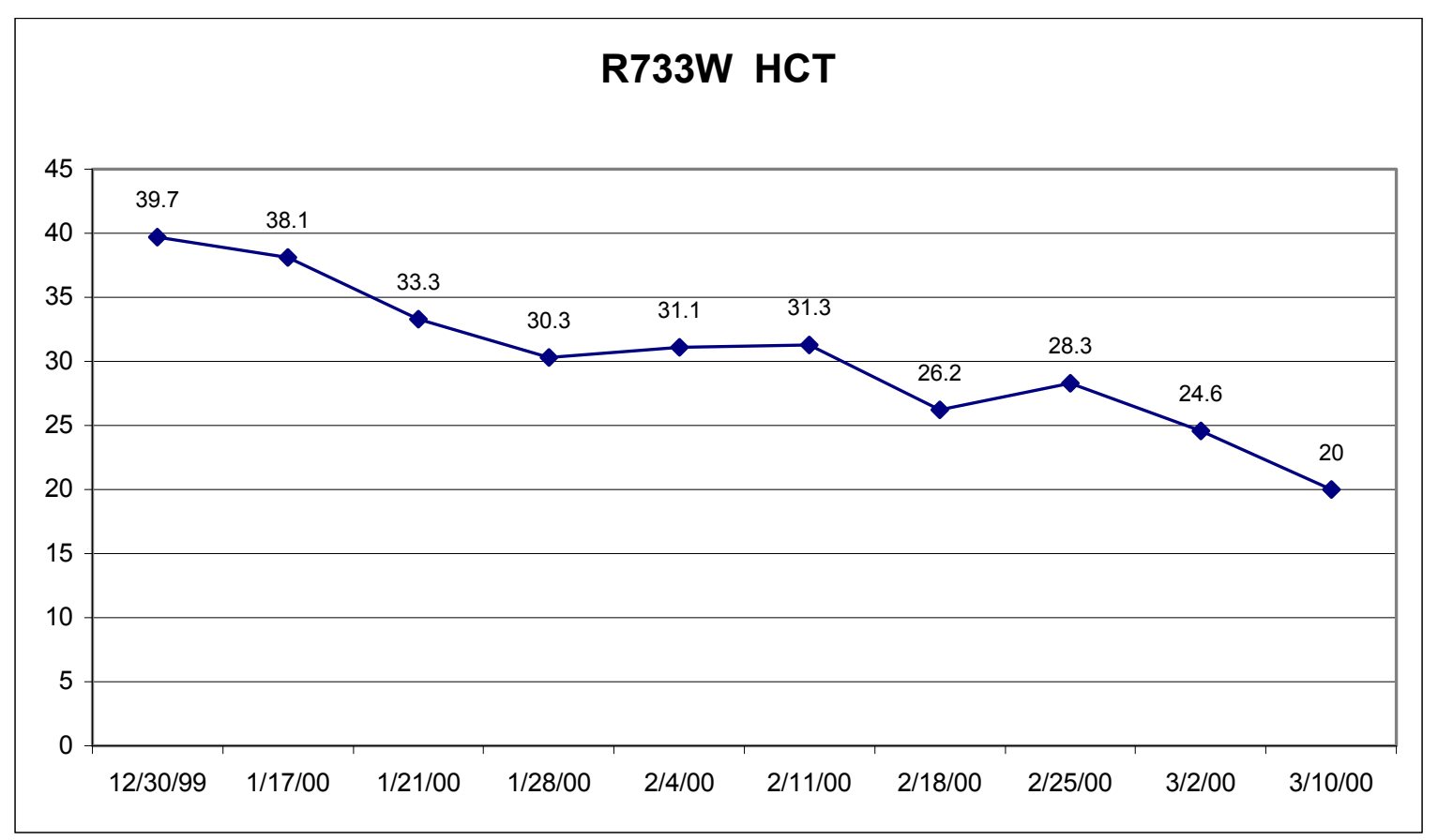

Figure G.38 HCT R733W 


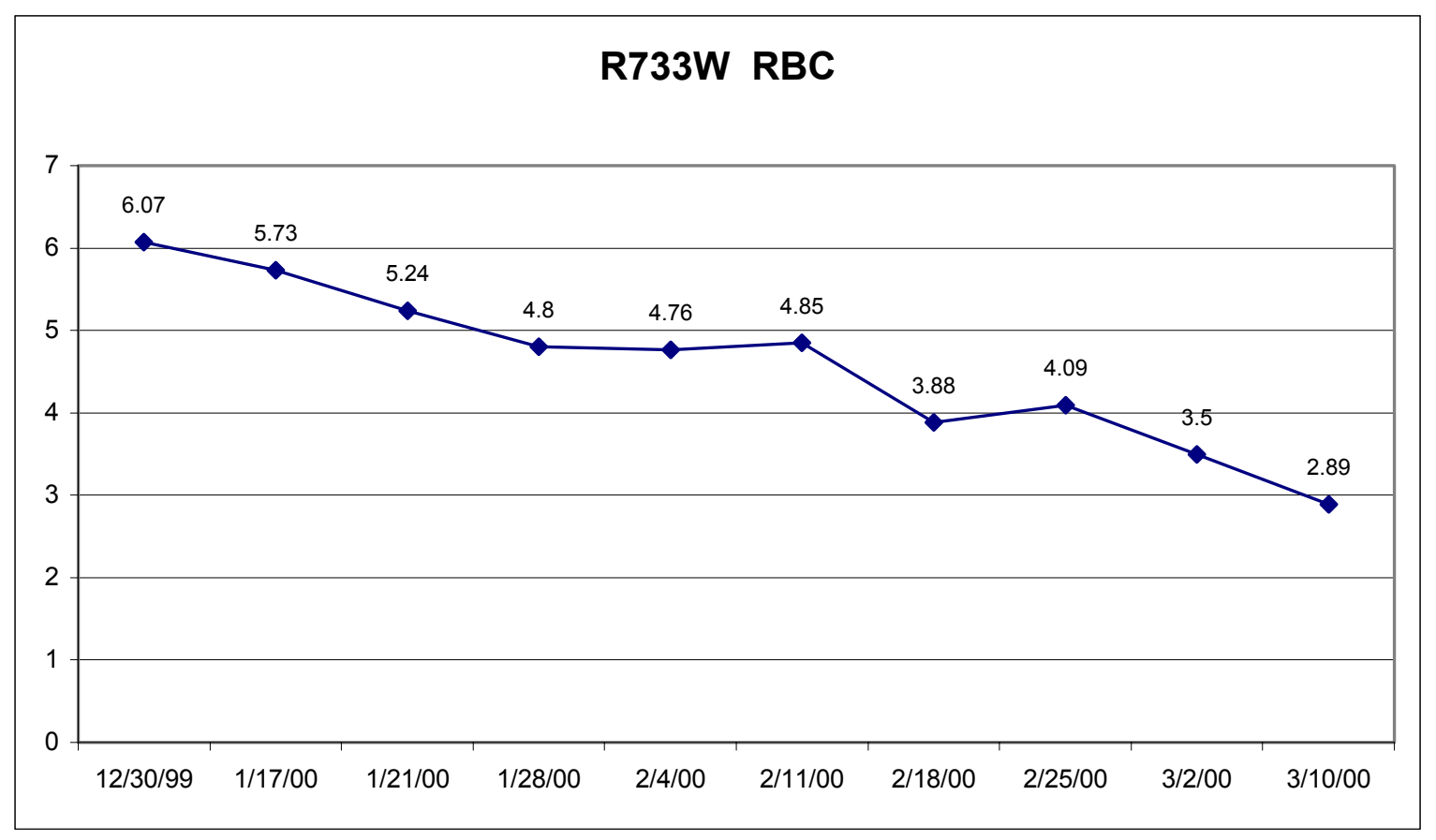

Figure G.39 RBC R733W

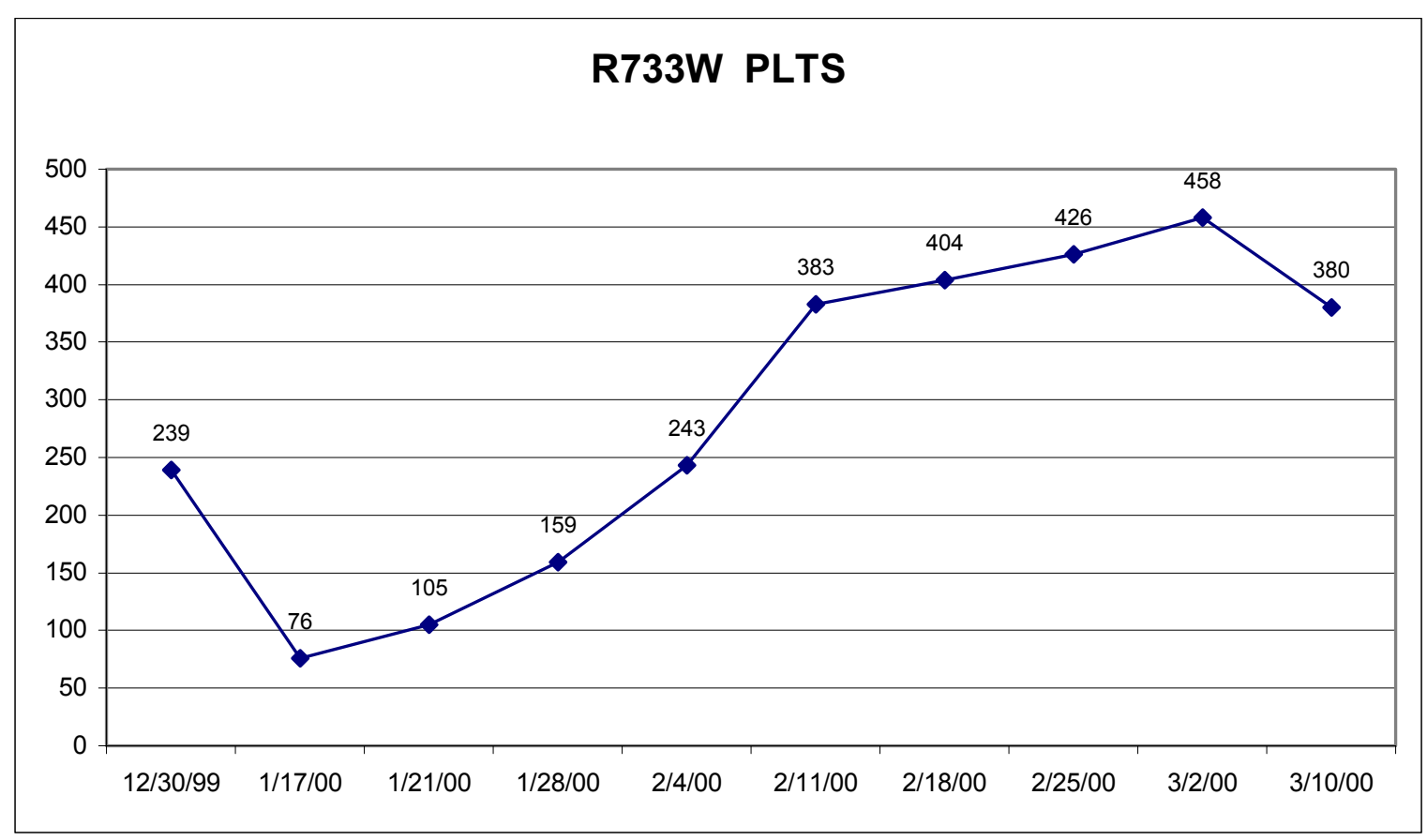

Figure G.40 Platelets R733W 


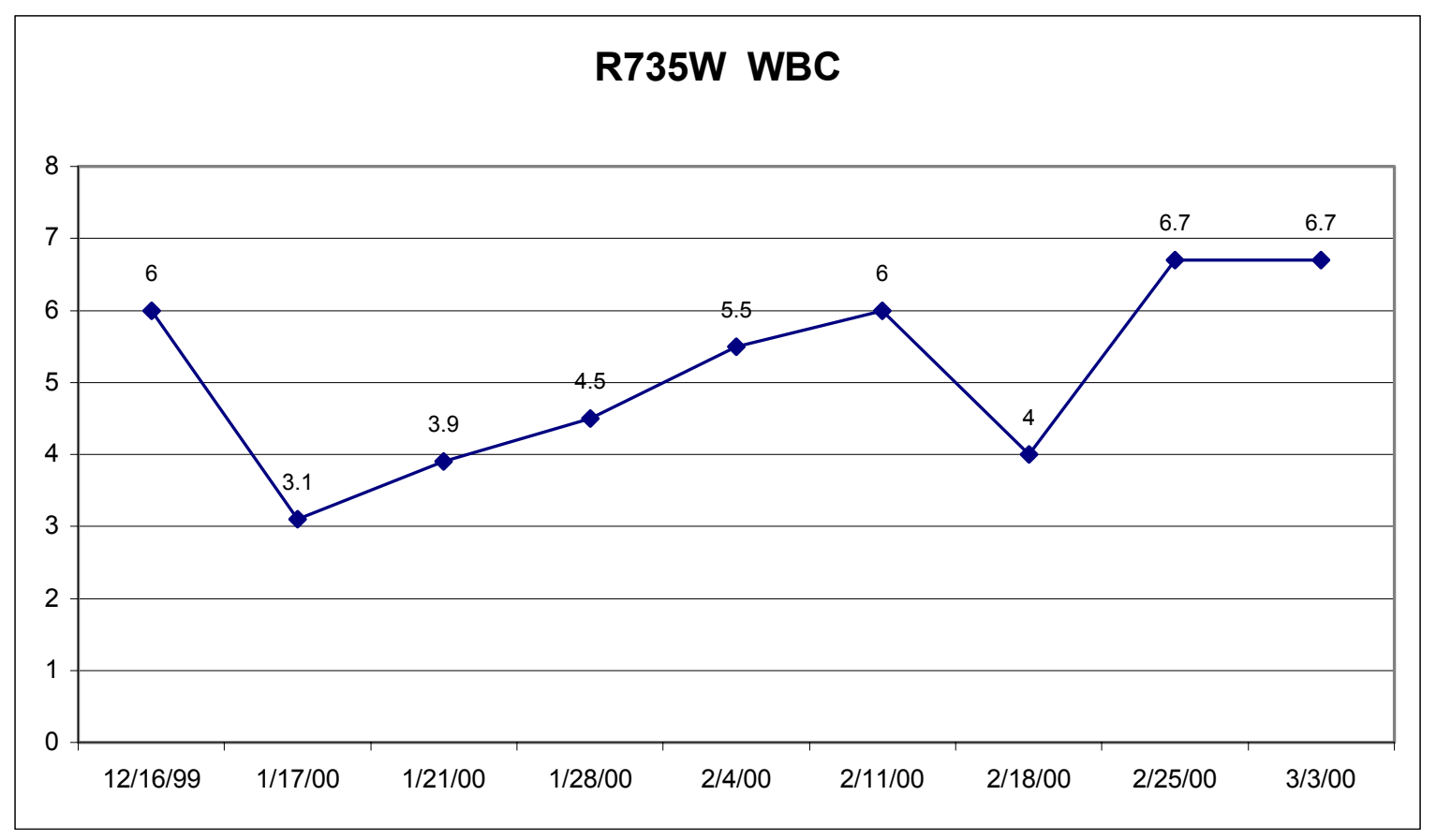

Figure G.41 WBC R735W

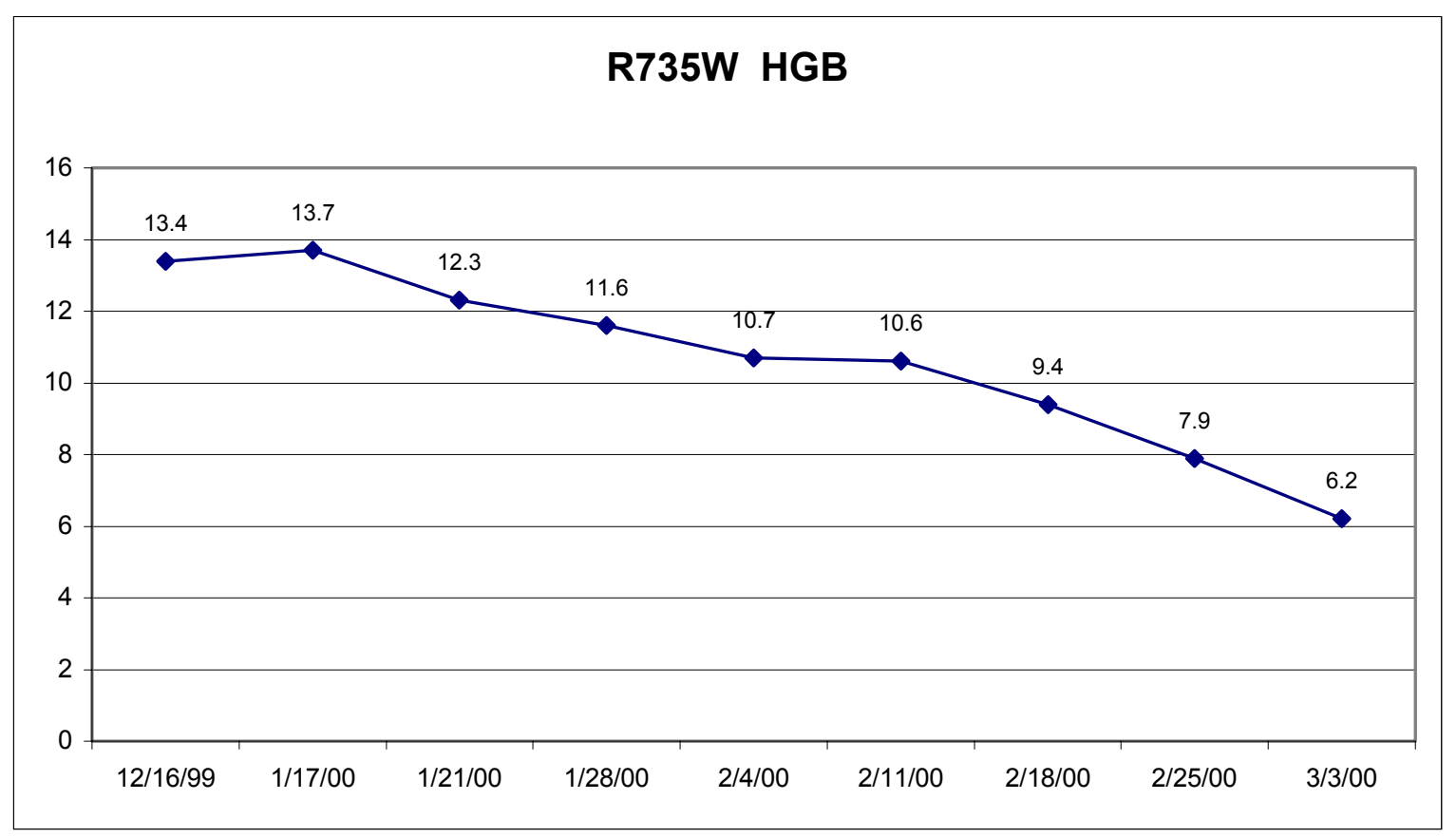

Figure G.42 HGB R735W 


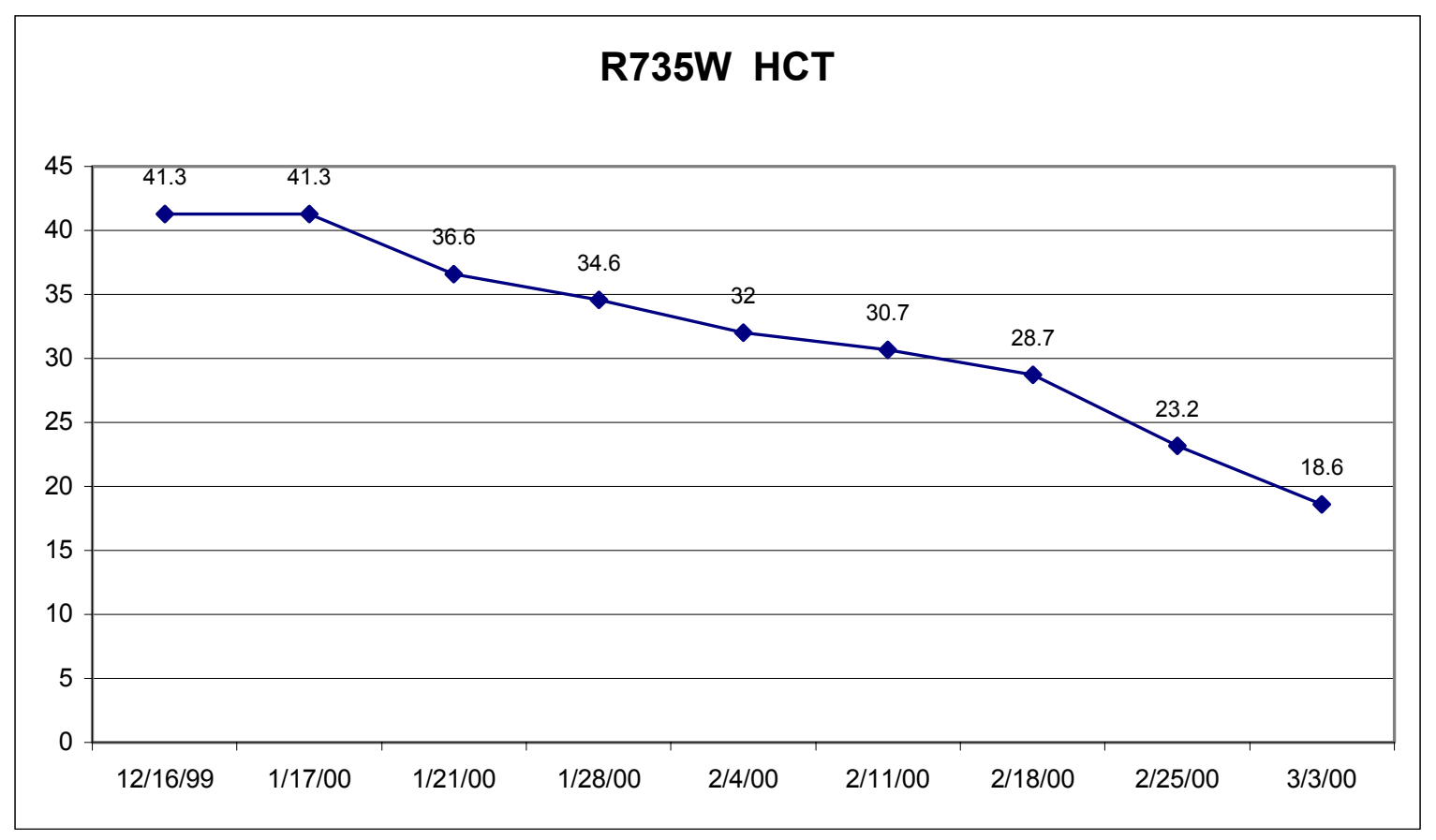

Figure G.43 HCT R735W

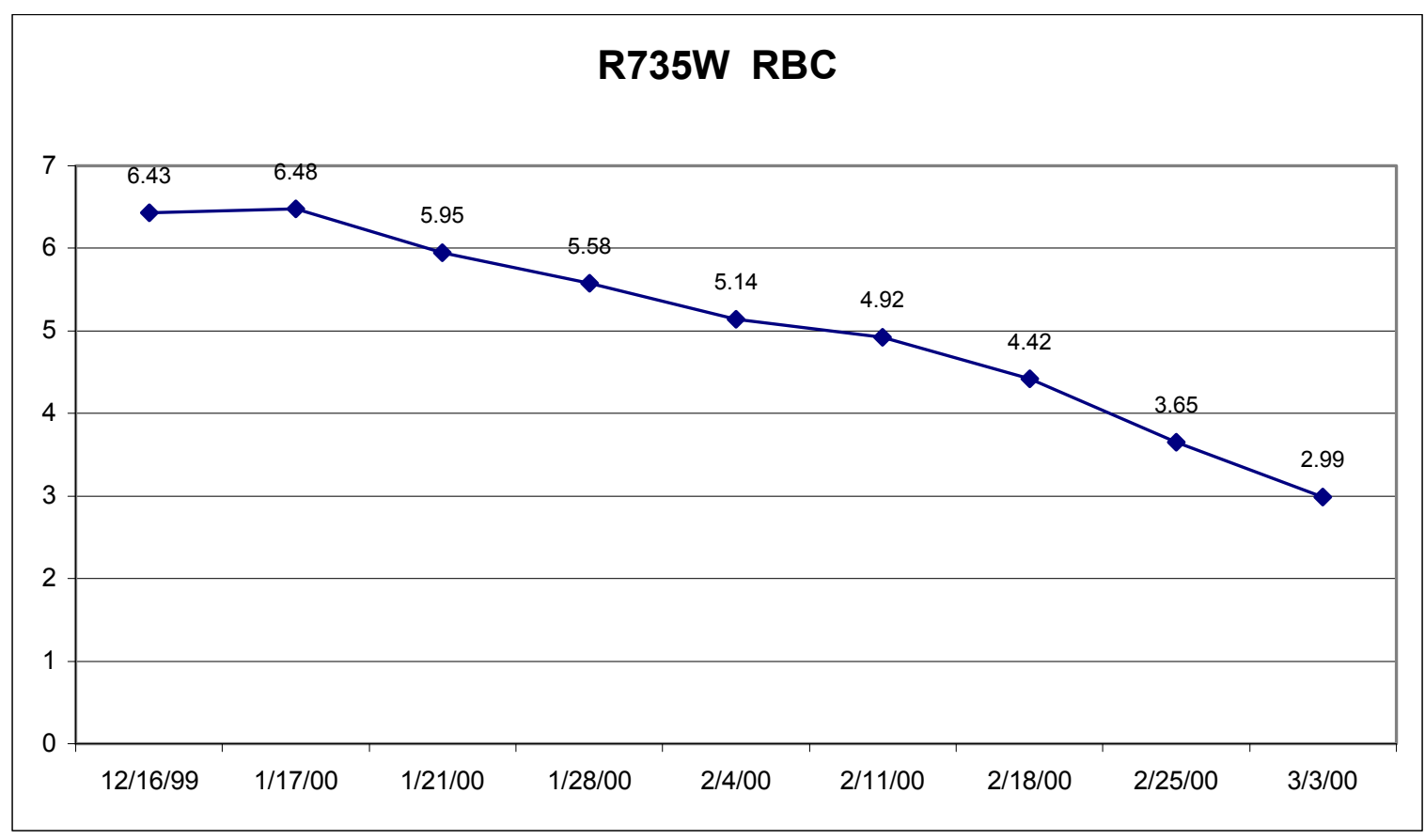

Figure G.44 RBC R735W 


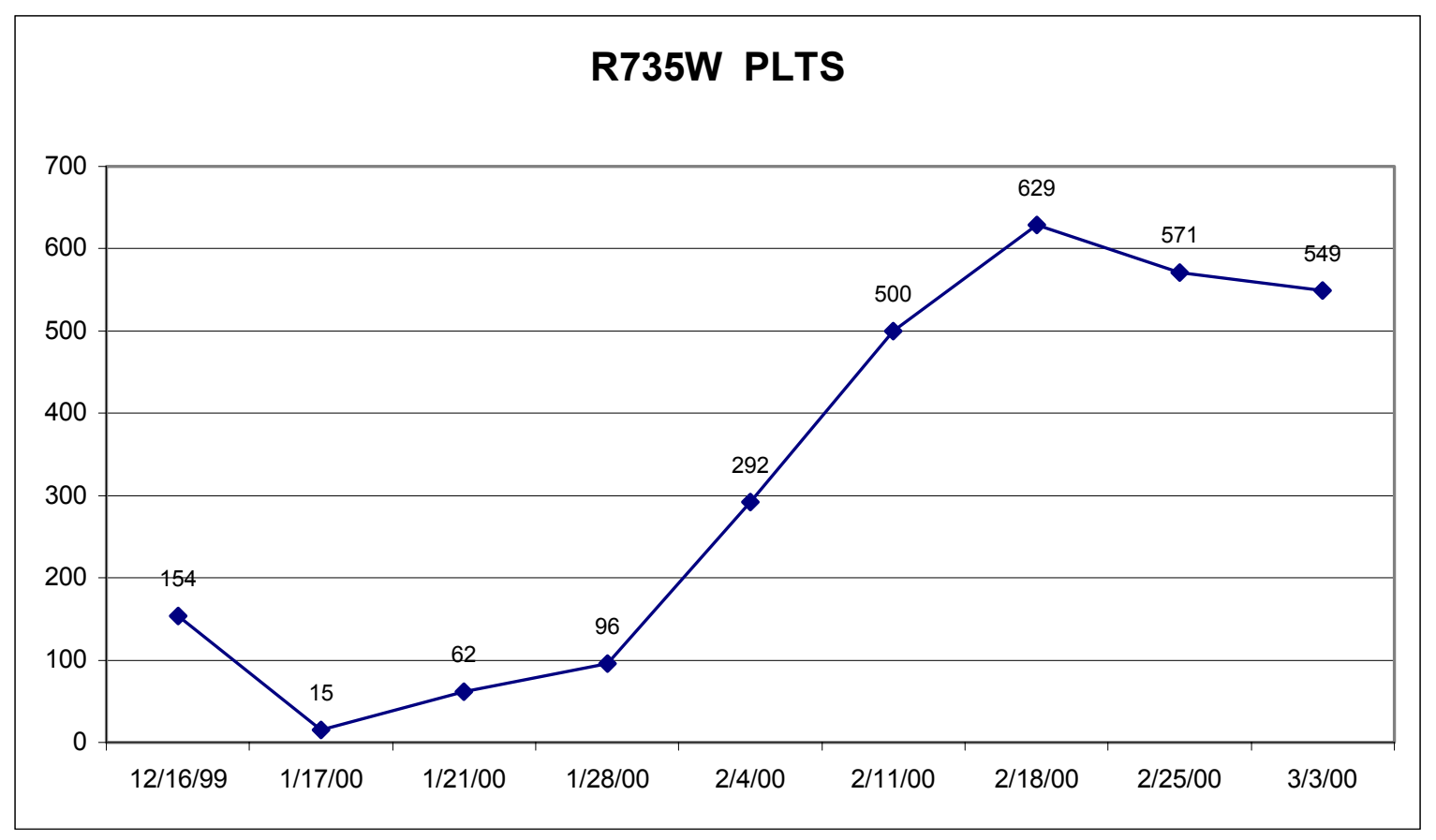

Figure G.45 Platelets R735W

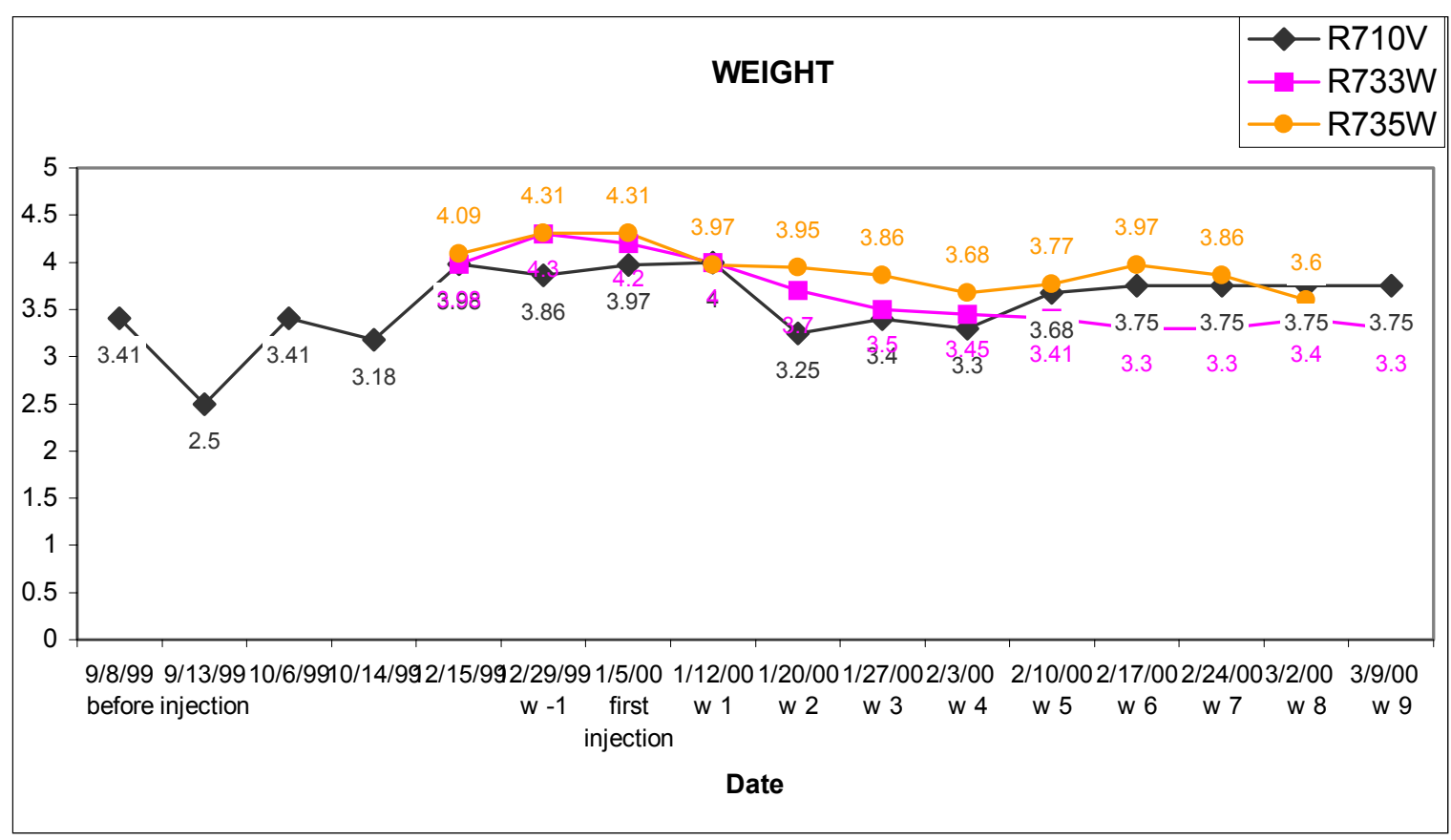

Figure G.46 Weight 


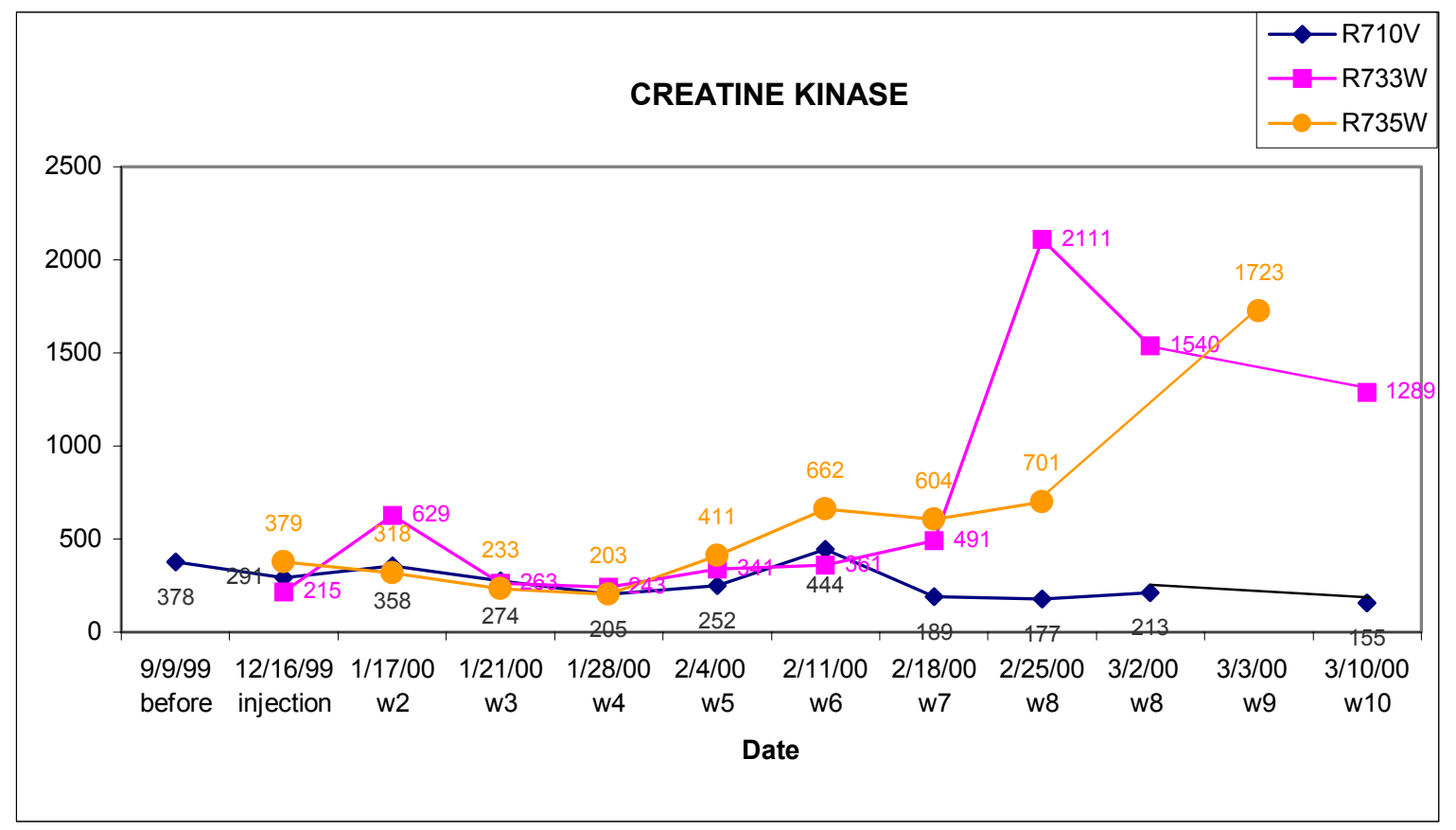

Figure G.47 Creatine kinase

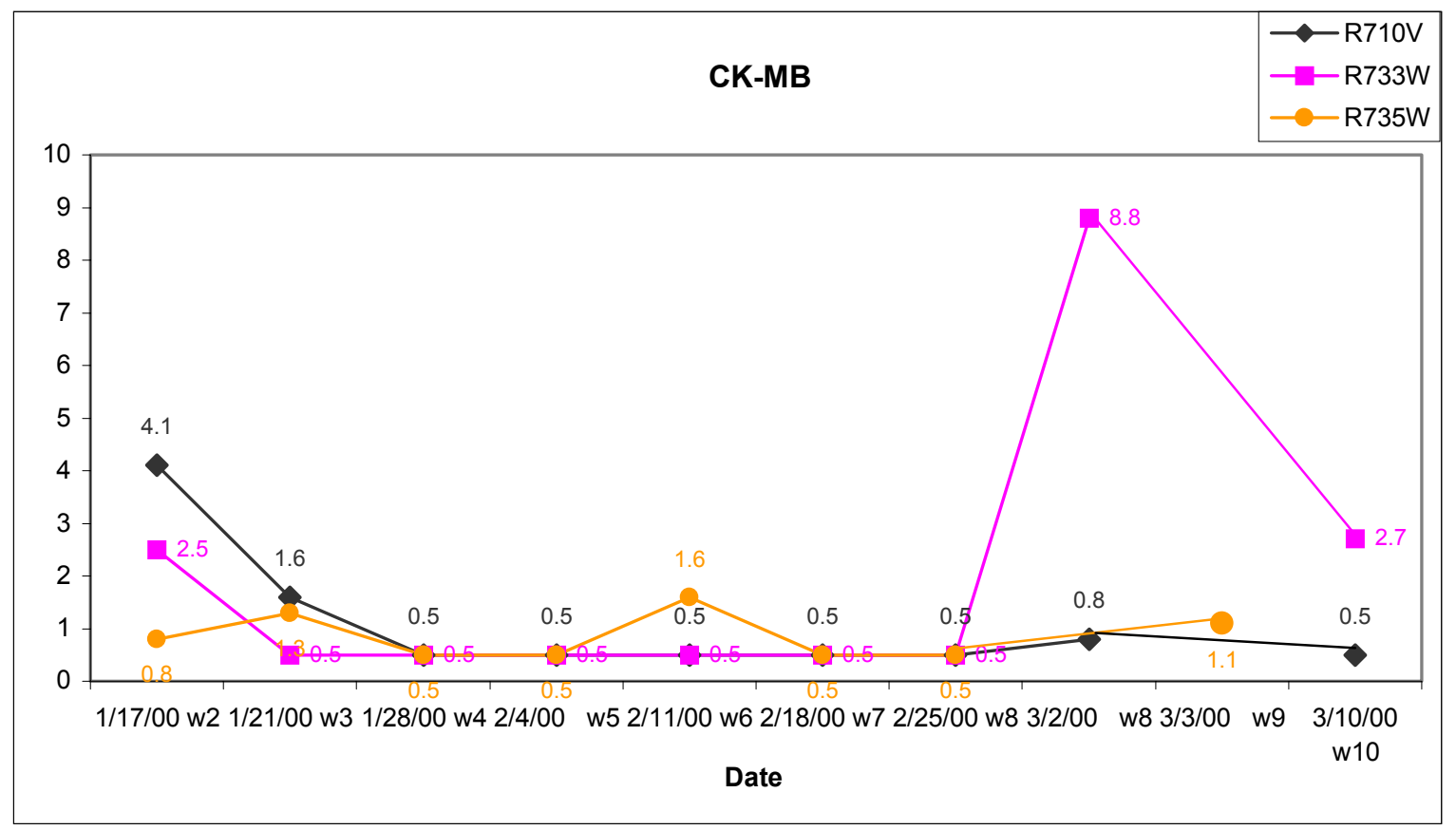

Figure G.48 Creatine kinase - MB 


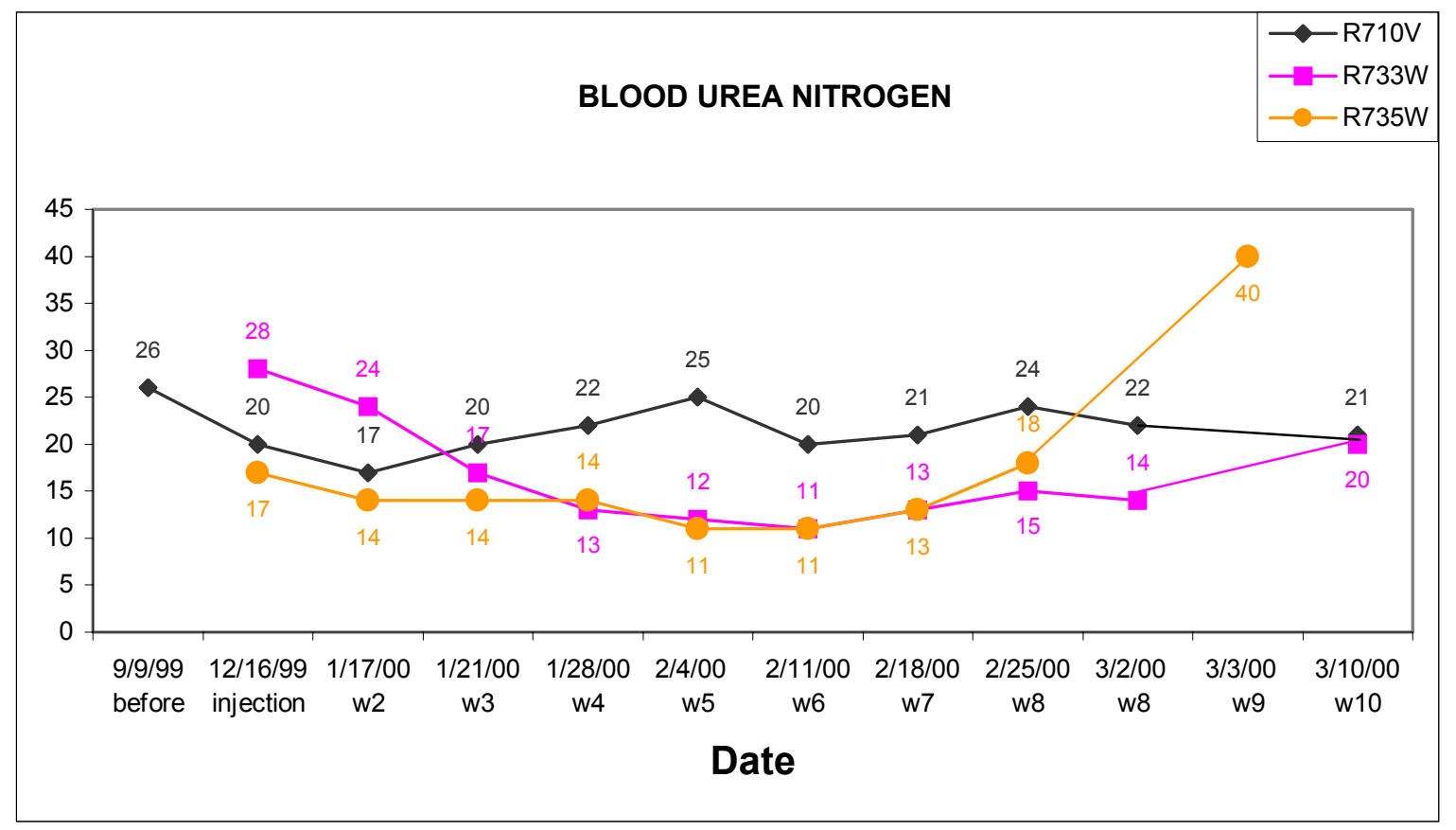

Figure G.49 Blood urea nitrogen

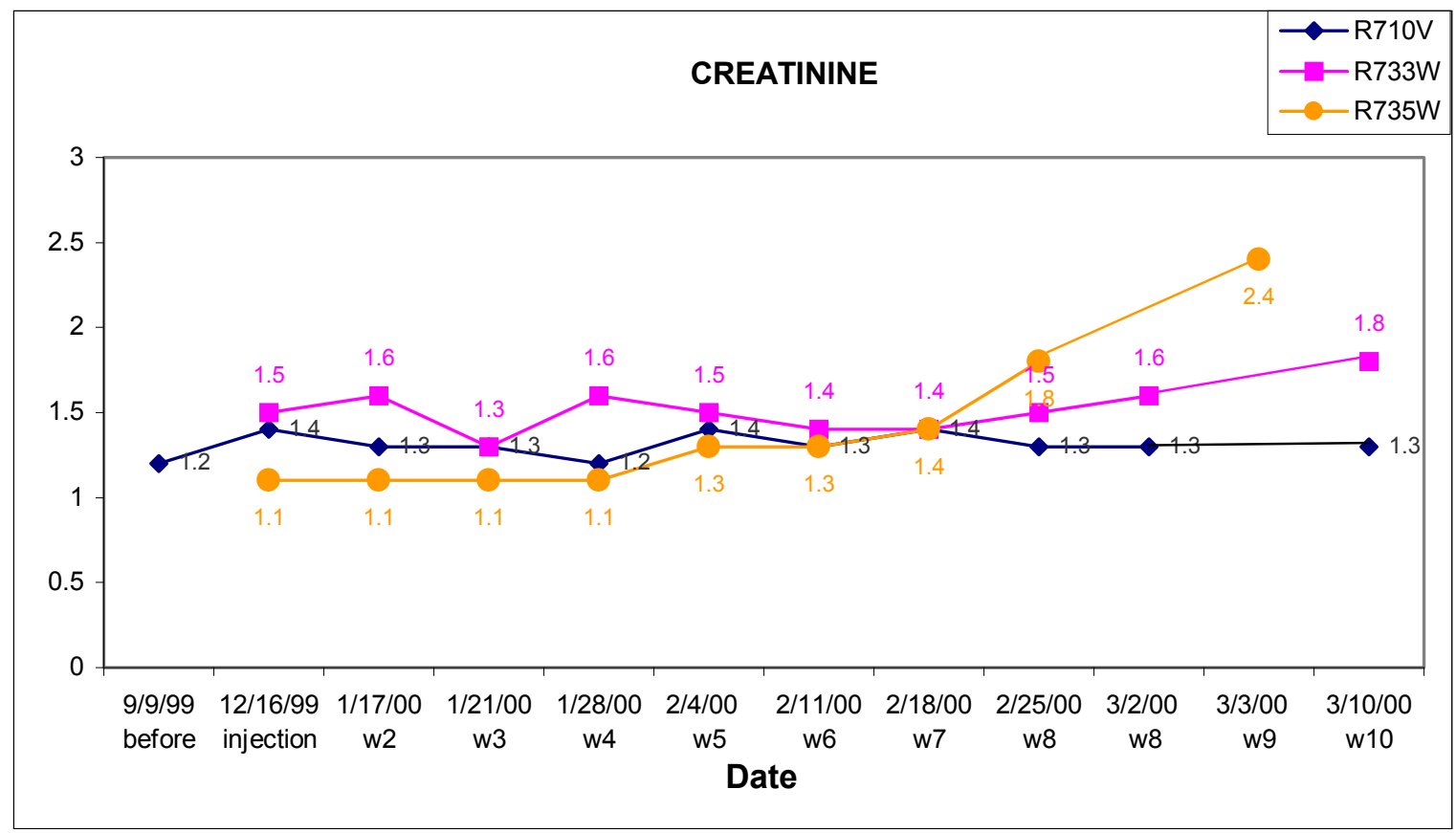

Figure G.50 Creatinine 


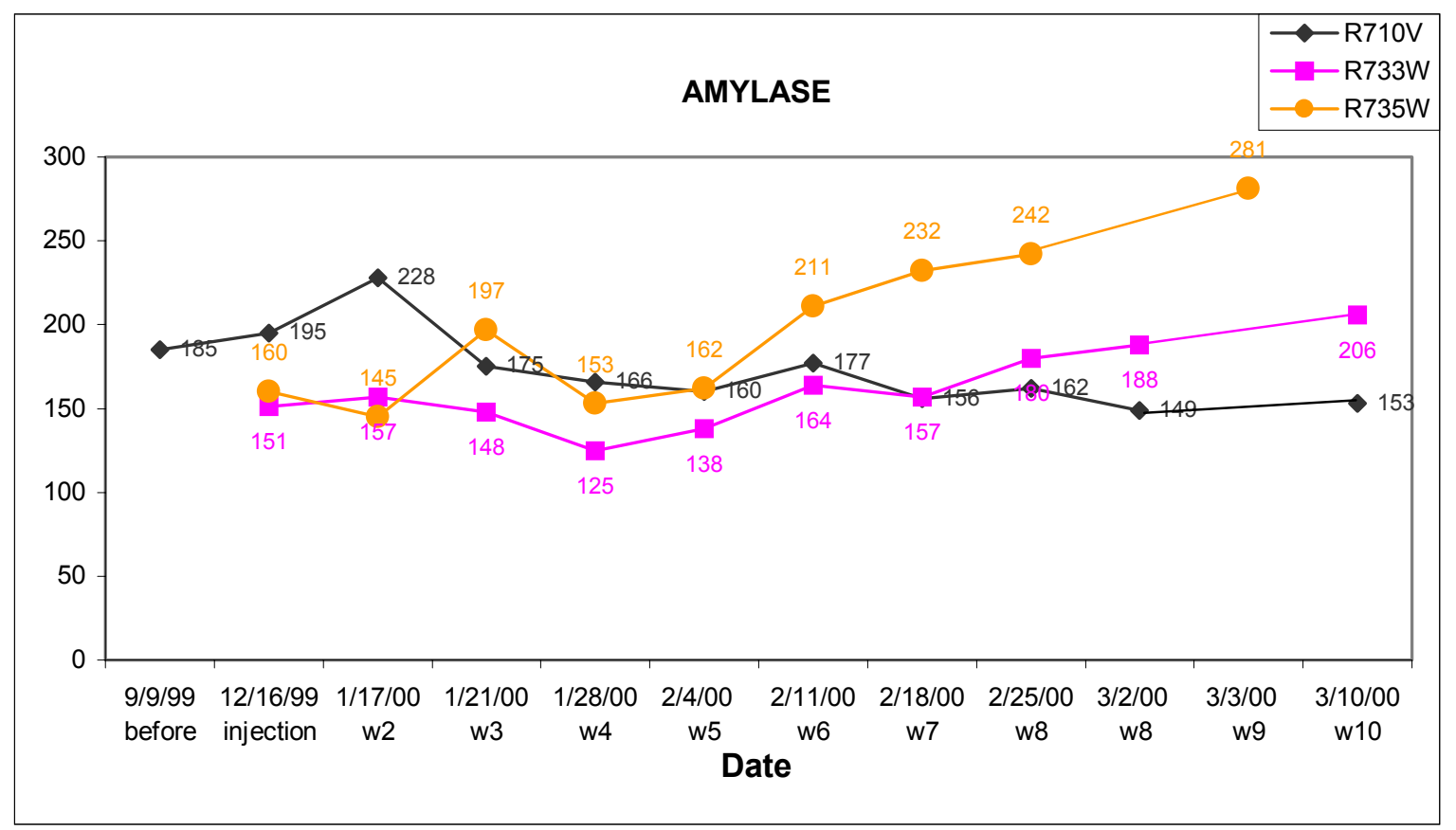

Figure G.51 Amylase

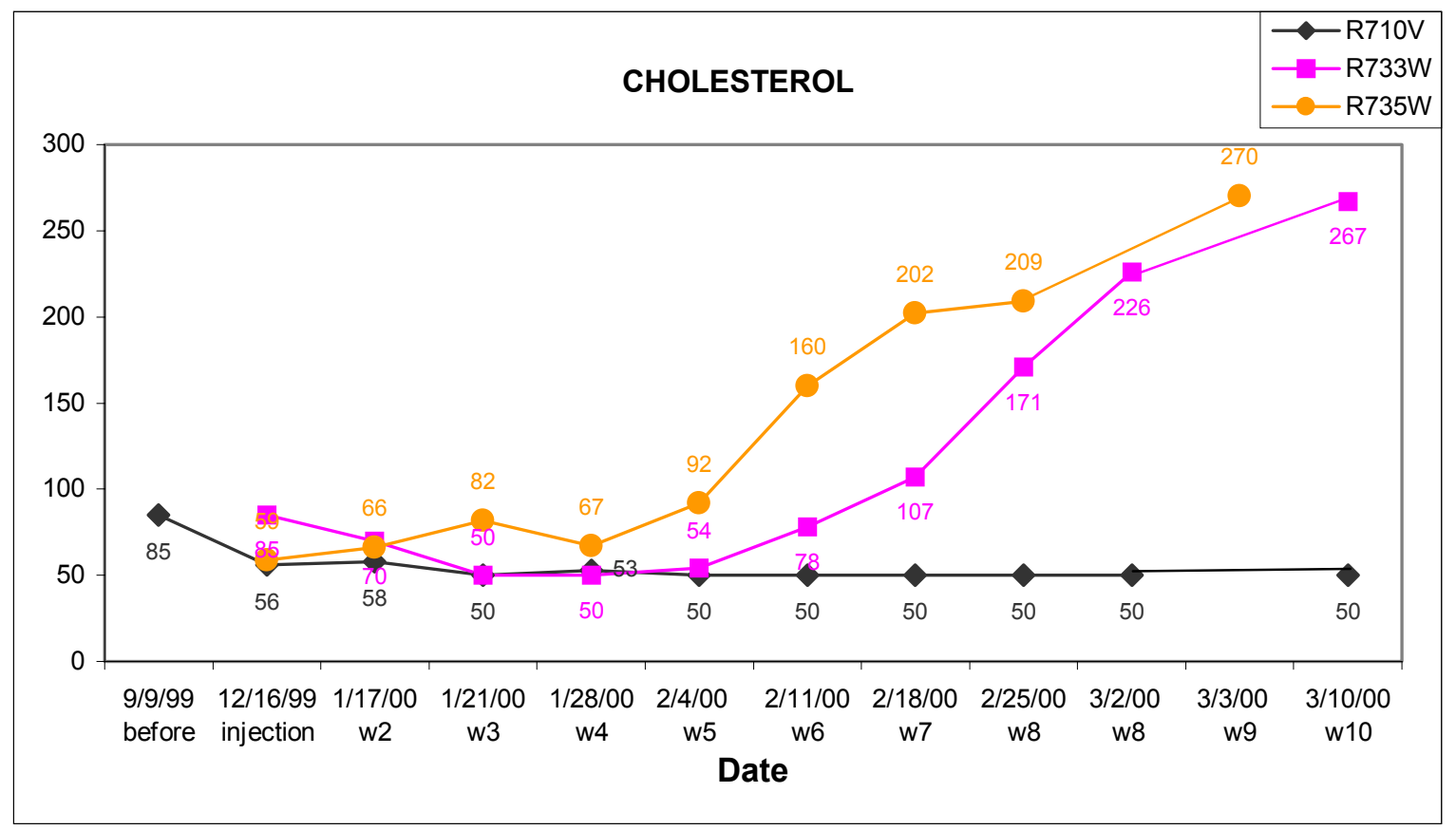

Figure G.52 Cholesterol 


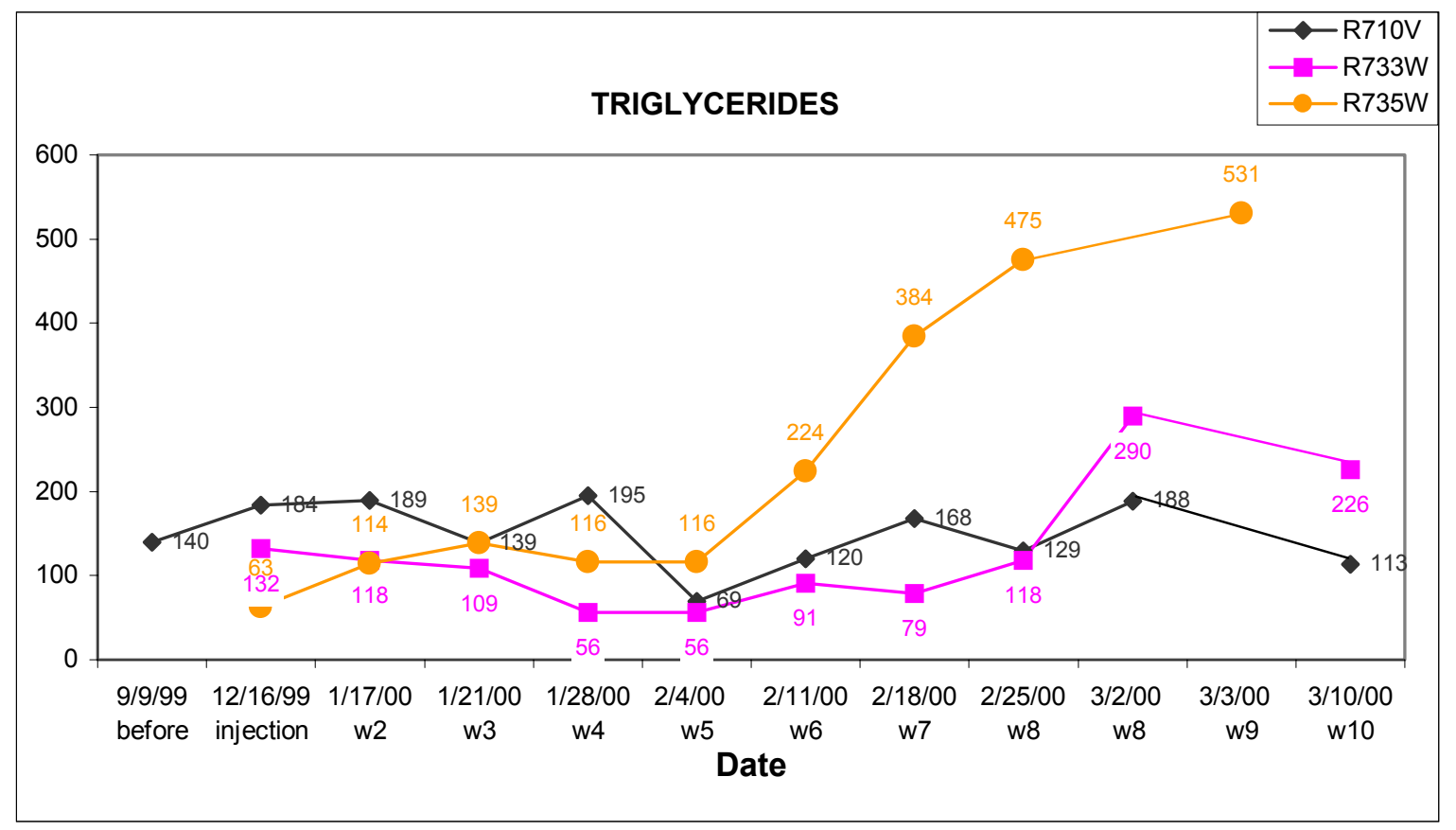

Figure G.53 Triglycerides

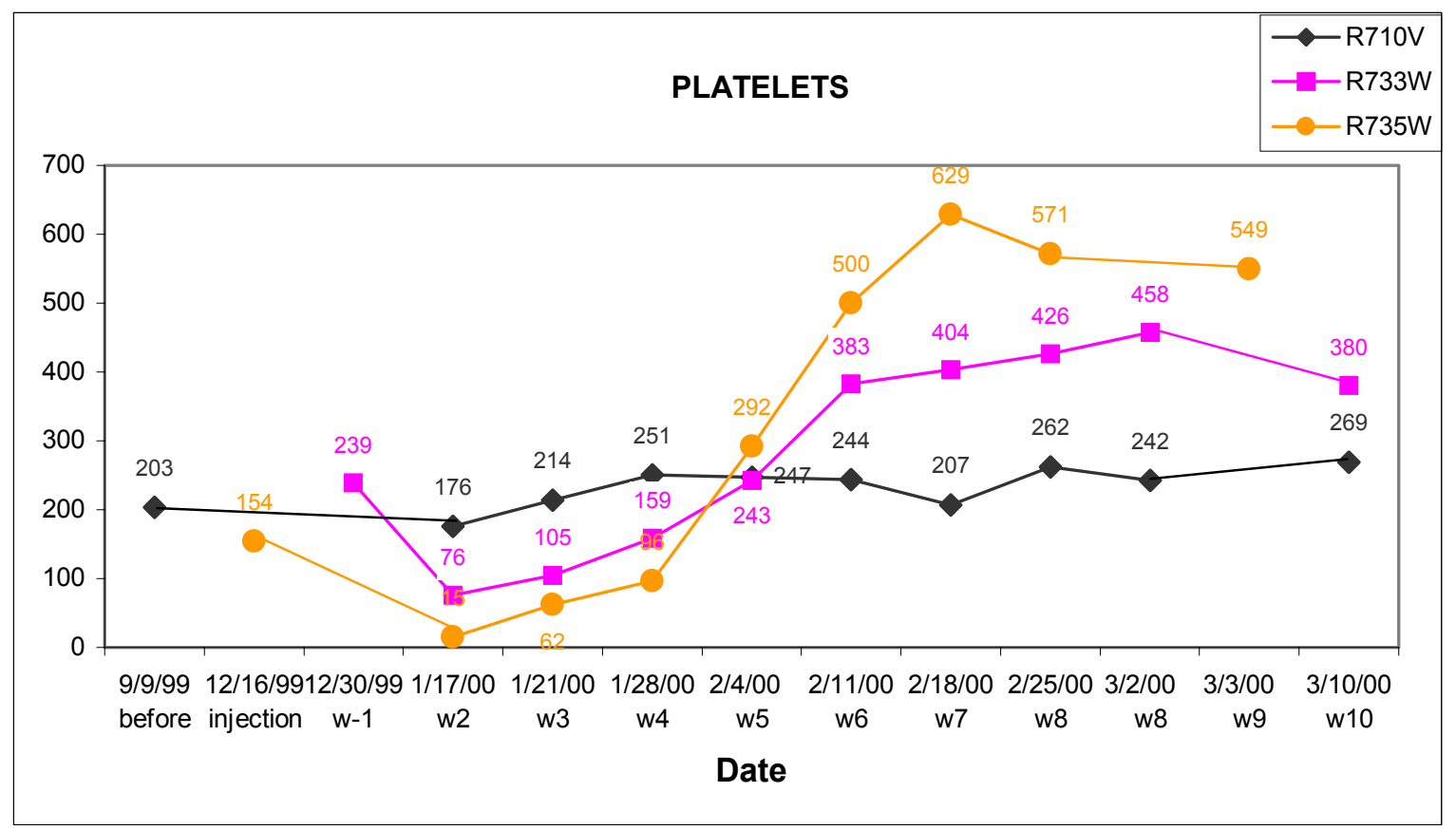

Figure G.54 Platelets 


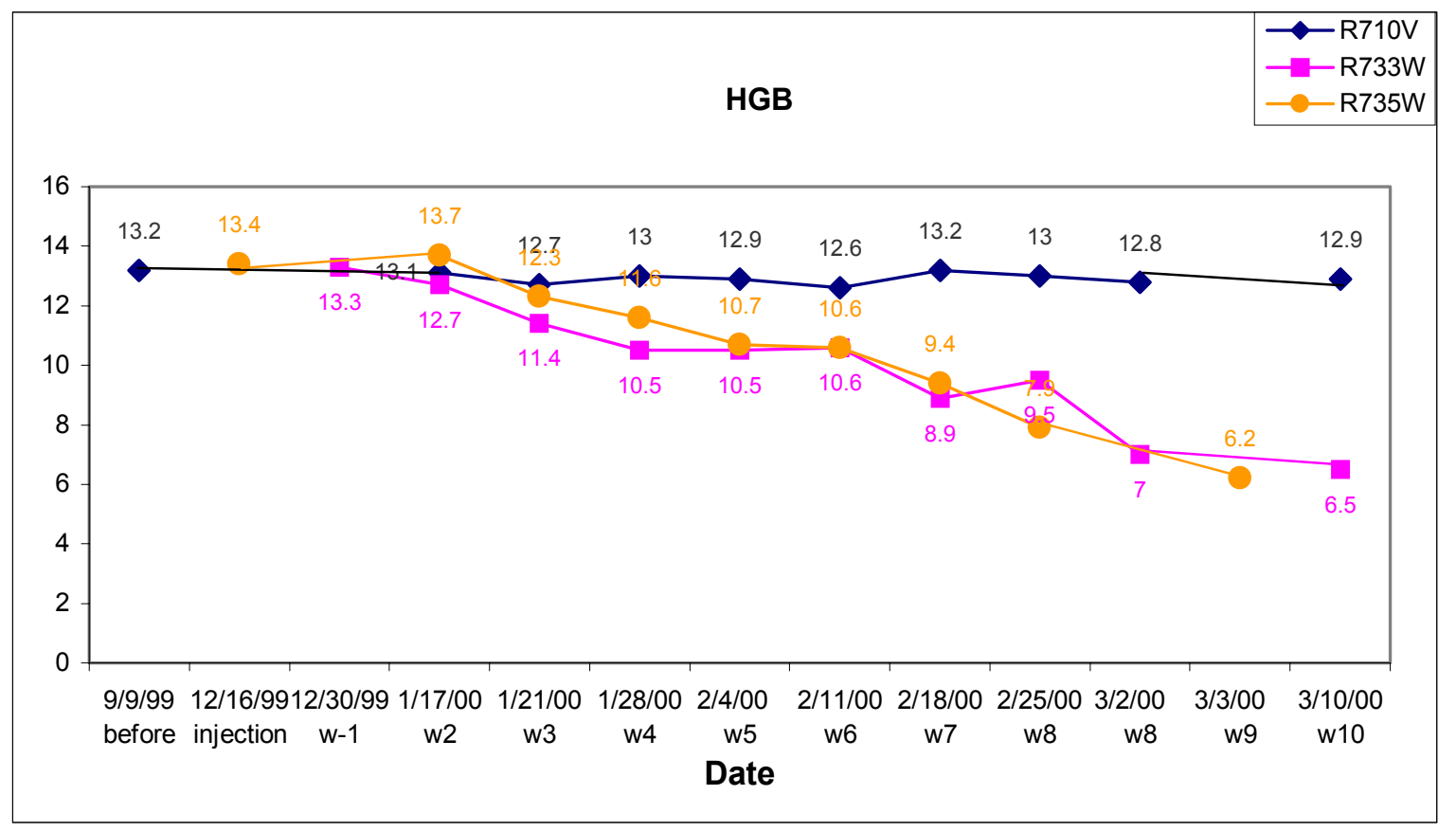

Figure G.55 HGB

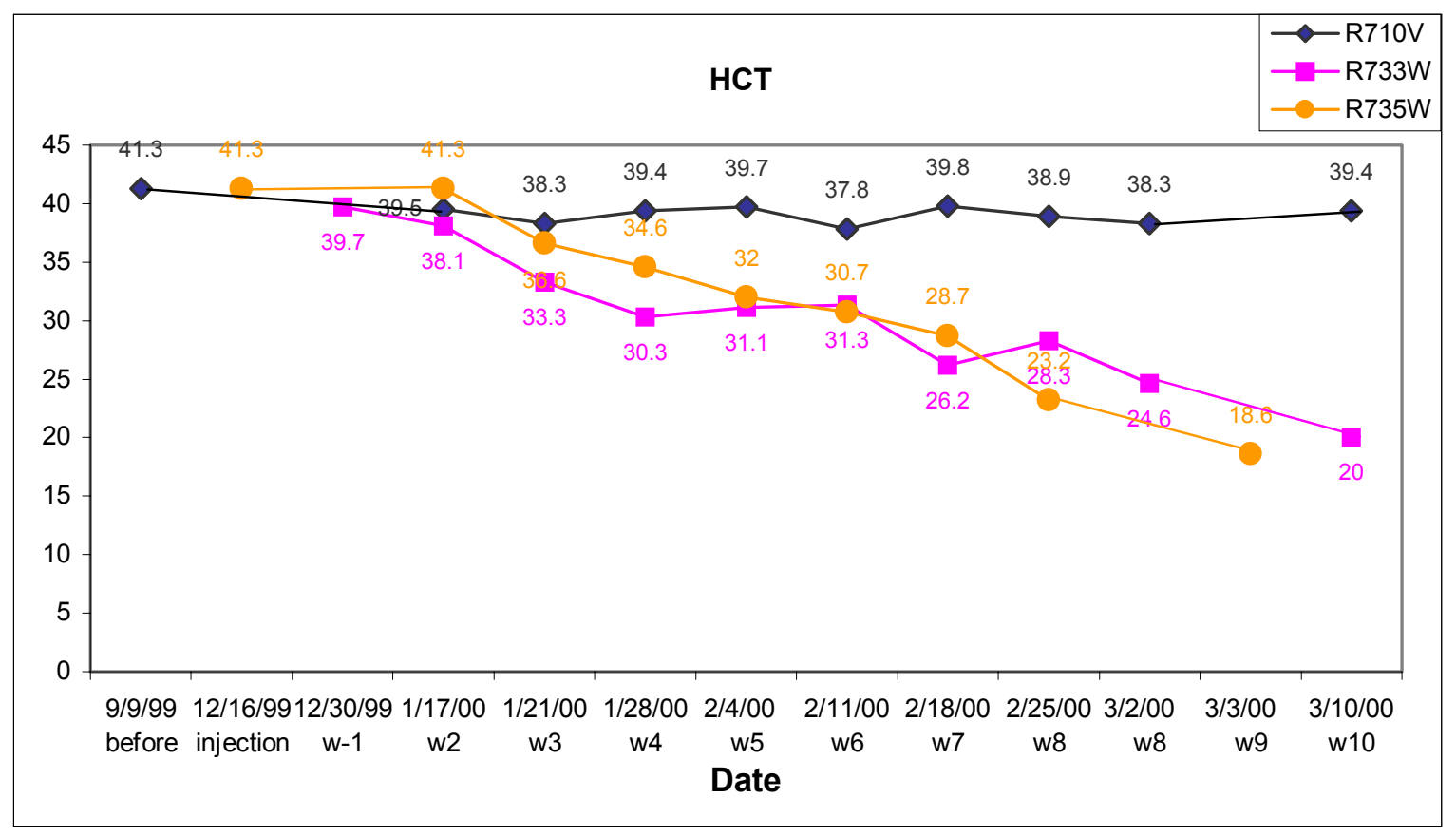

Figure G.56 HCT 


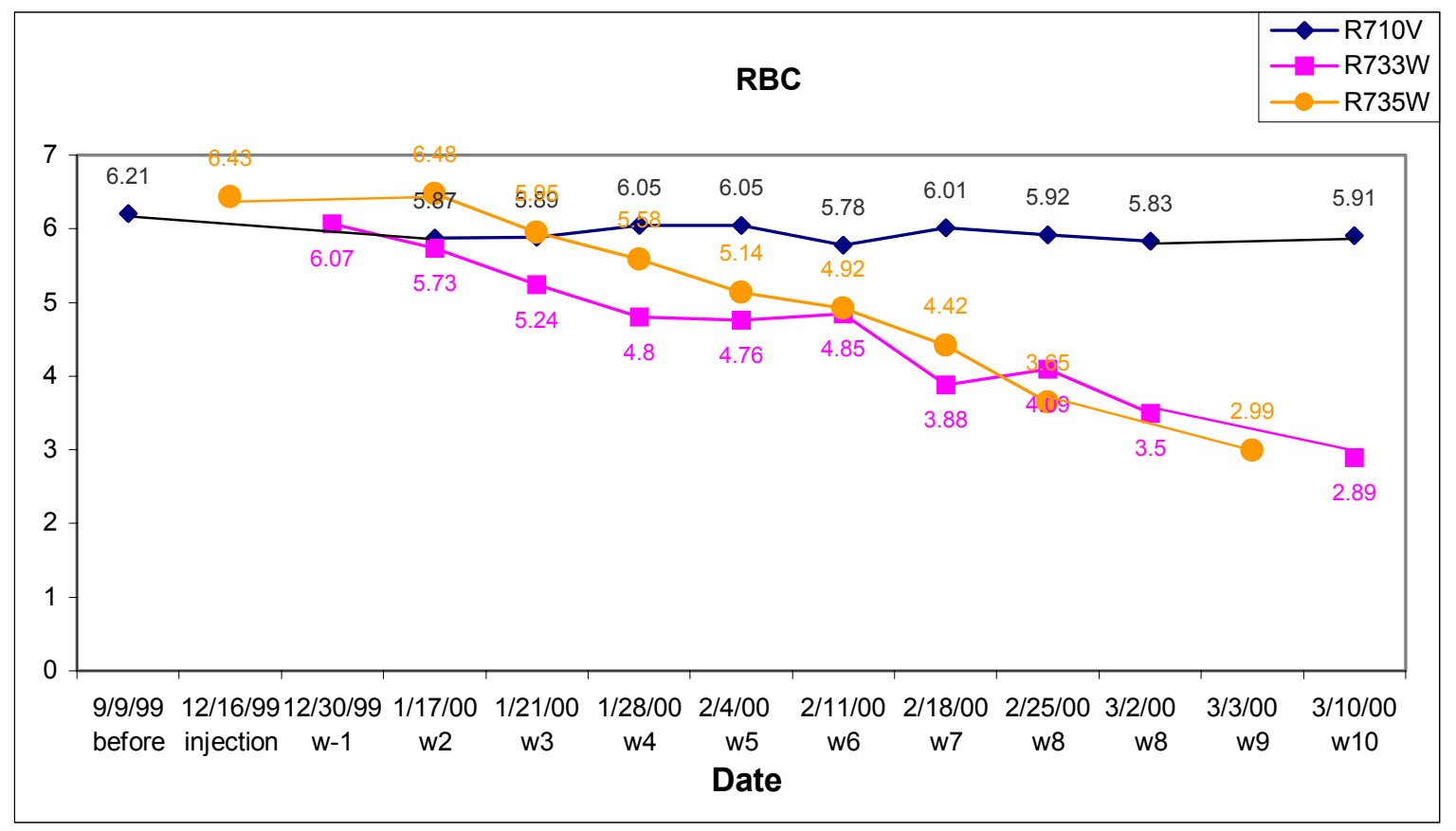

Figure G.57 RBC

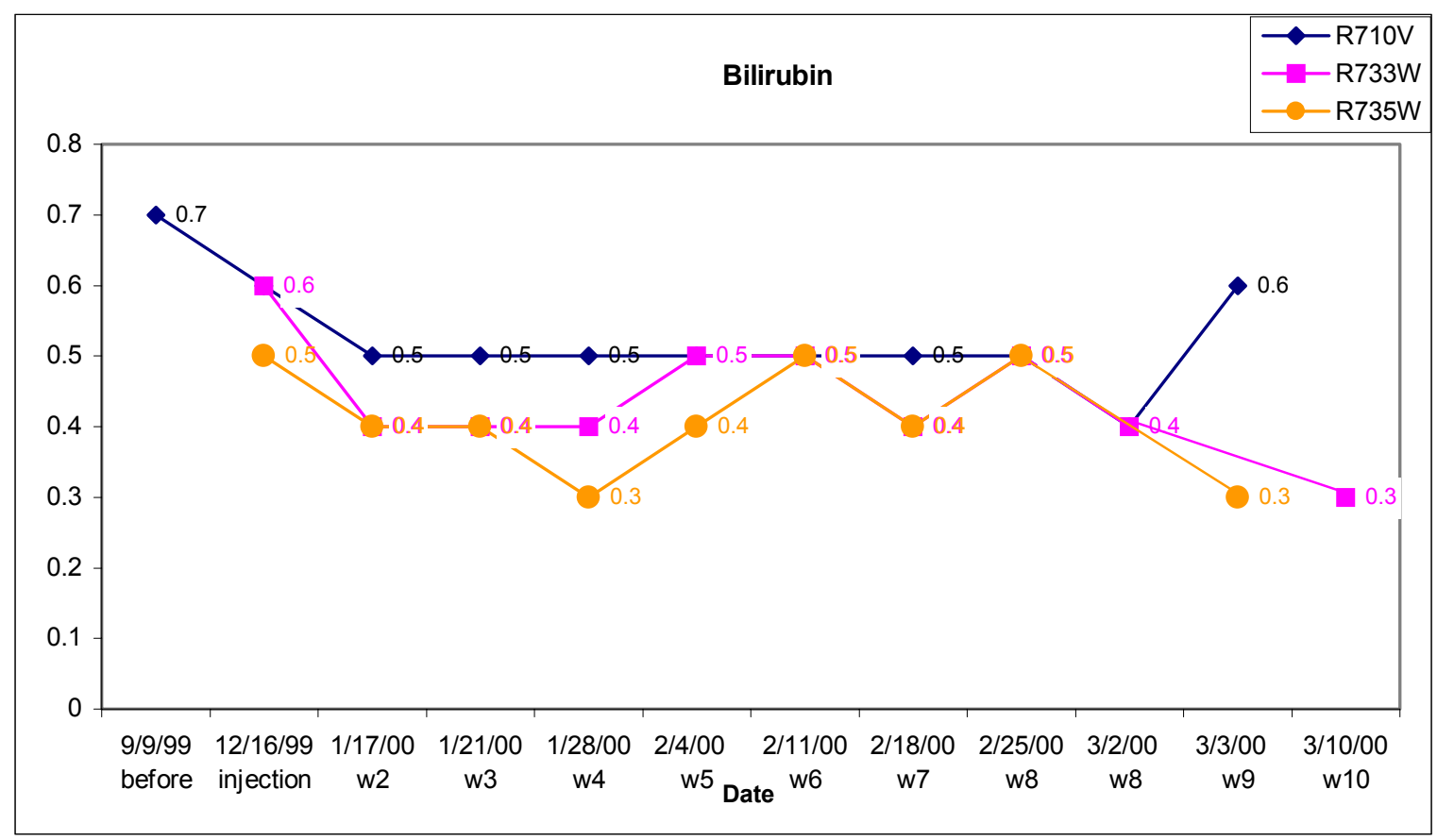

Figure G.58 Bilirubin 
Appendix H ECG signal, injected rabbit R735W, intracardiac channel 


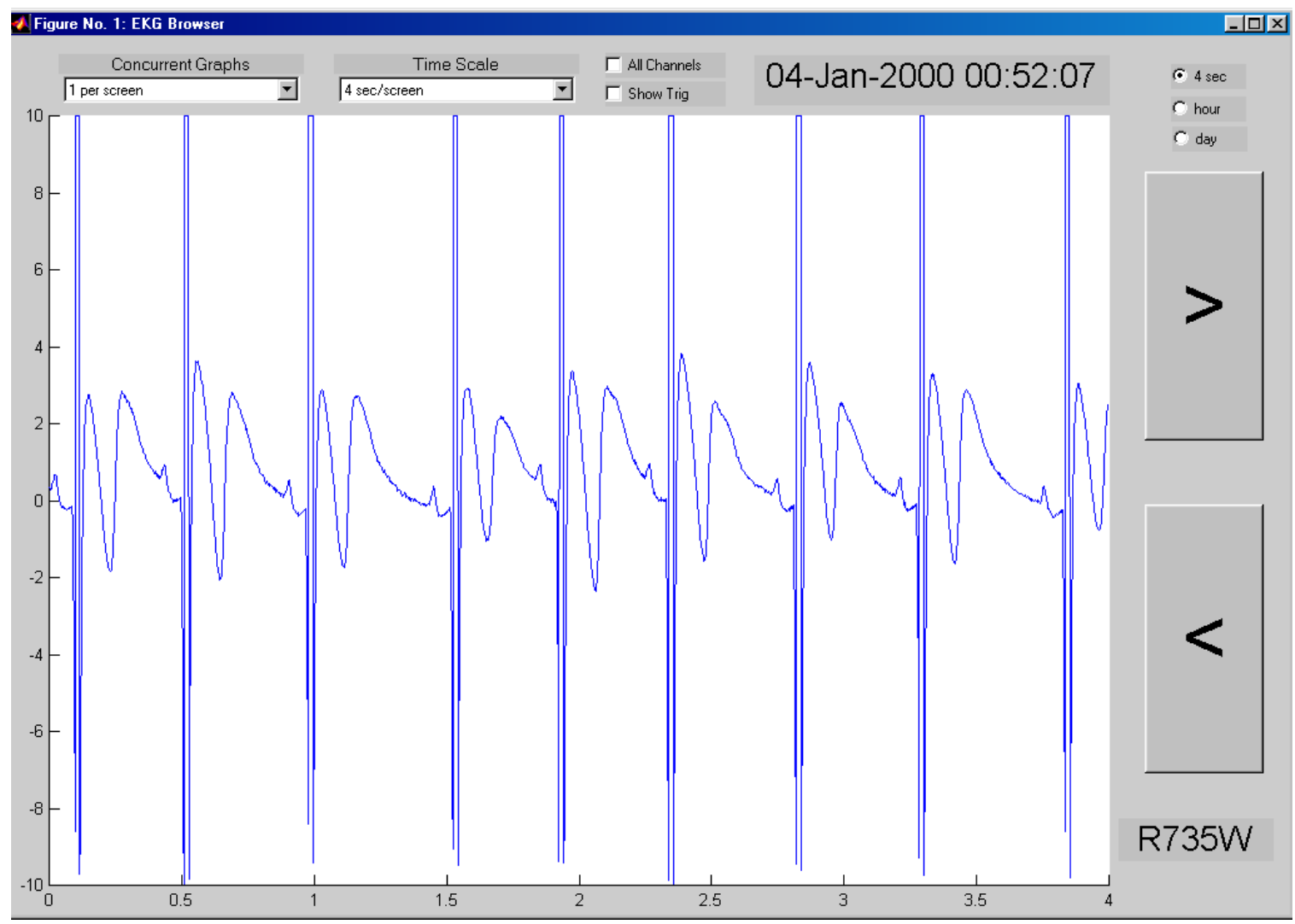

Figure H.1 ECG signal before the first injection, four sec/screen

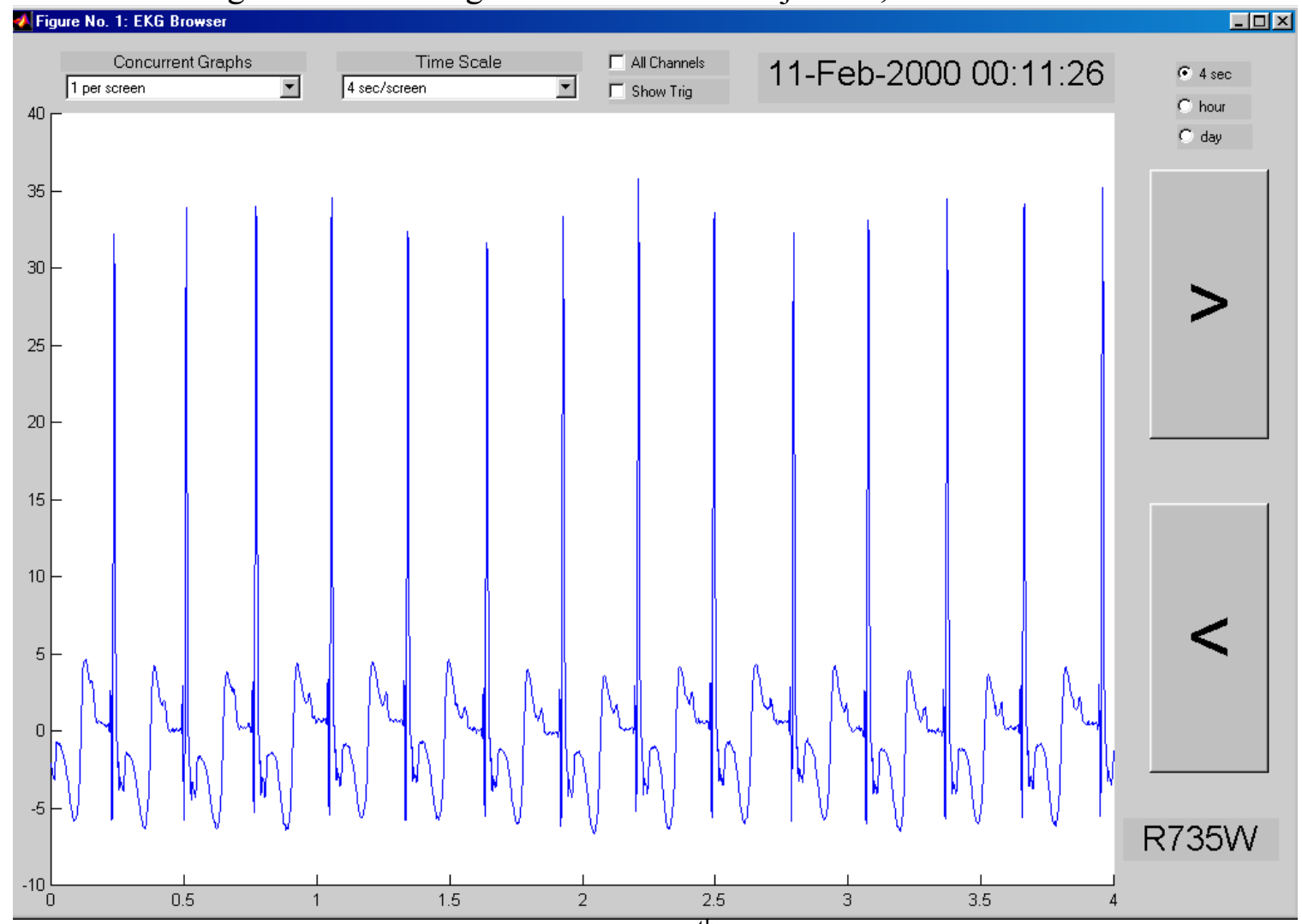

Figure H.2 February $11^{\text {th }}$, four sec/screen 


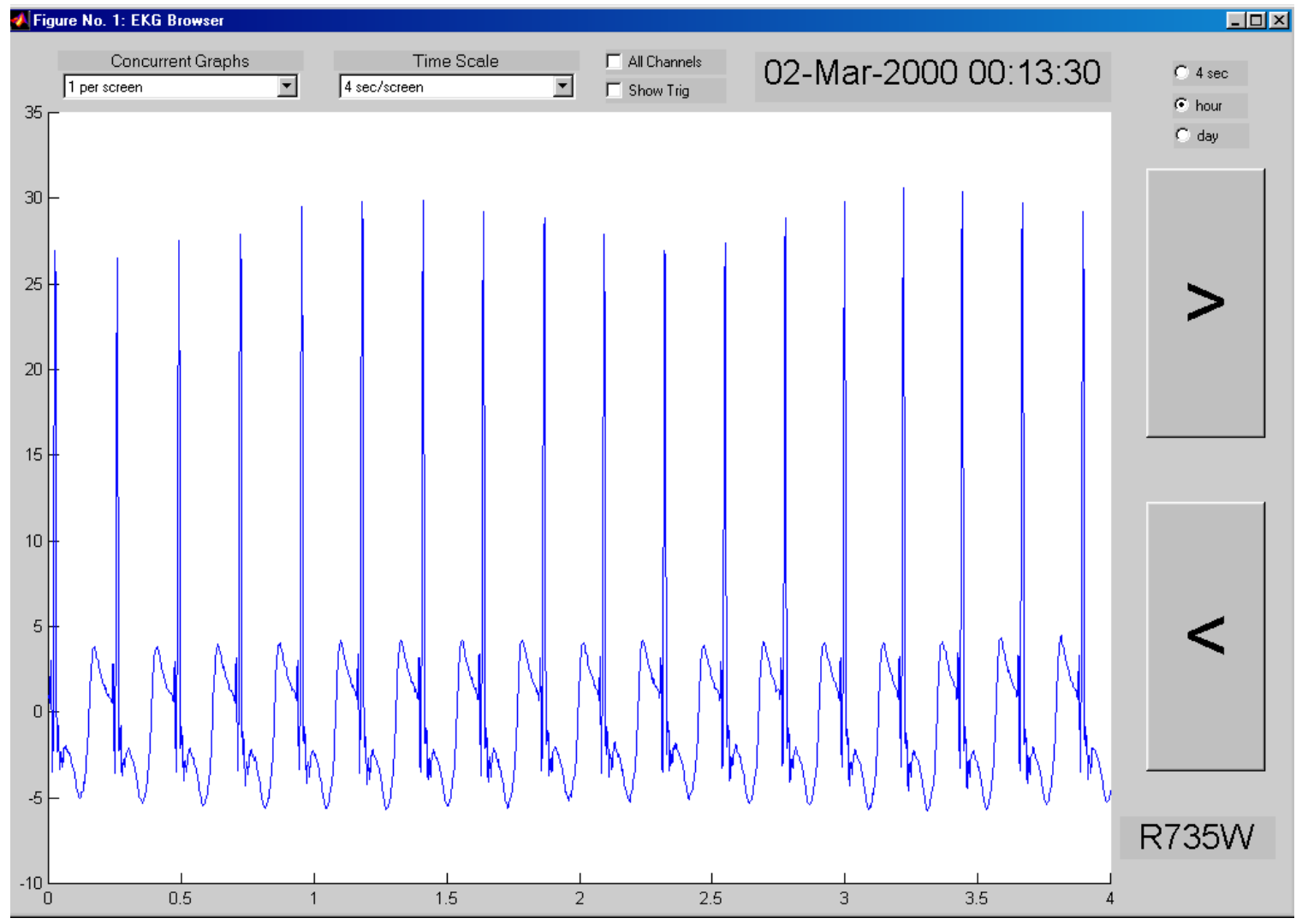

Figure H.3 March second, four sec/screen

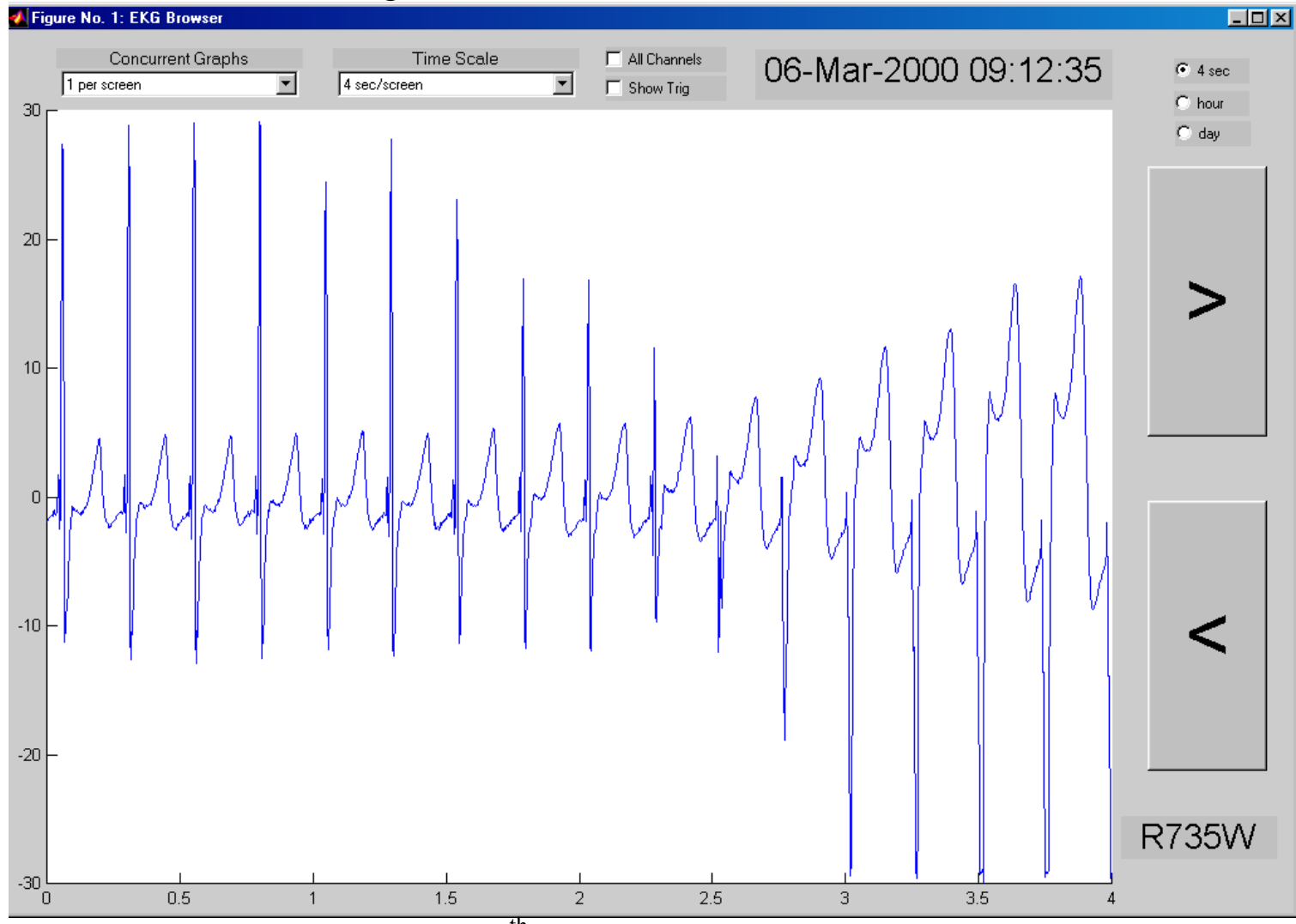

Figure H.4 March $6^{\text {th }}$, myocardial infarction four sec/screen 


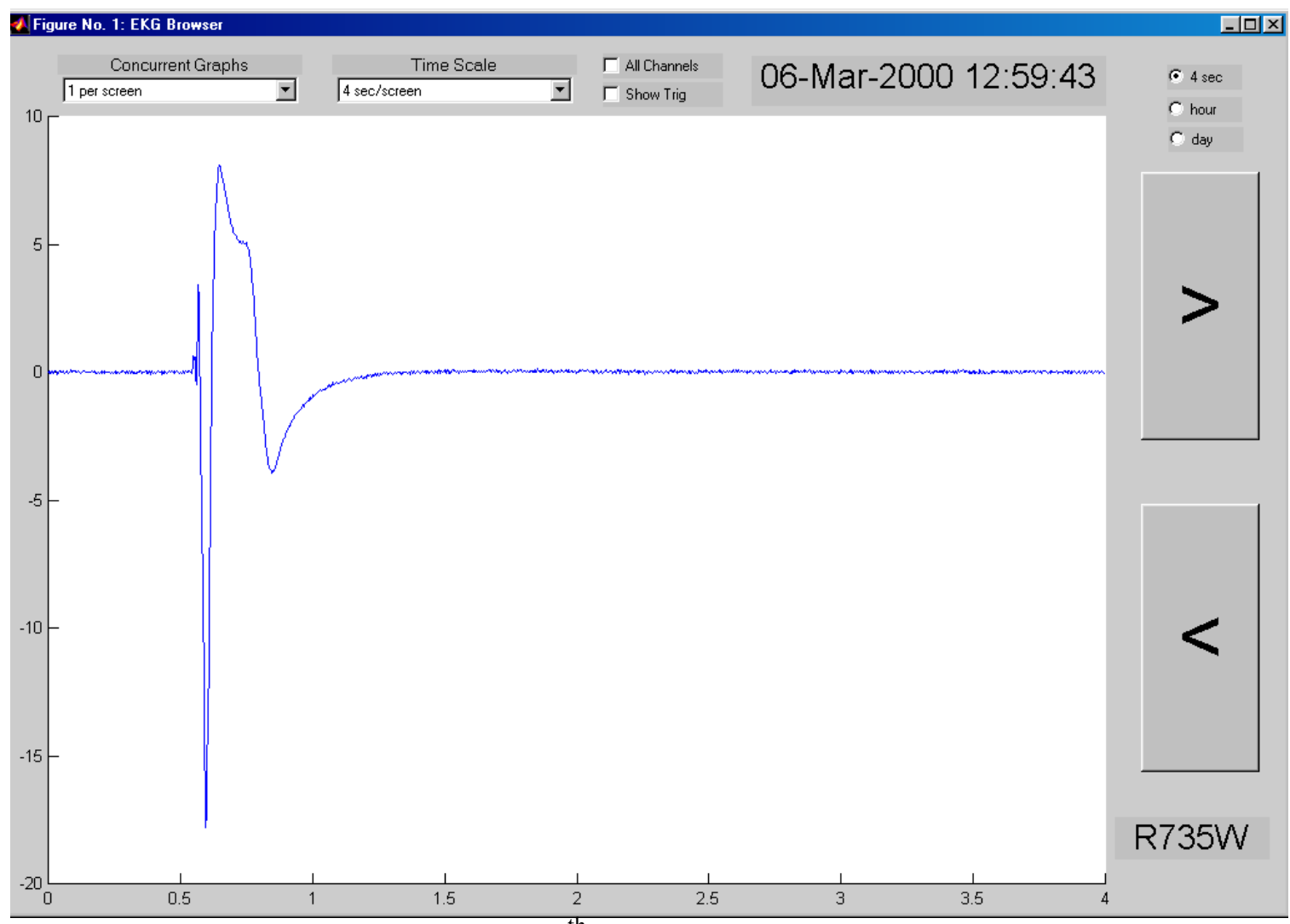

Figure H.5 March $6^{\text {th }}$, bradycardia, four sec/screen

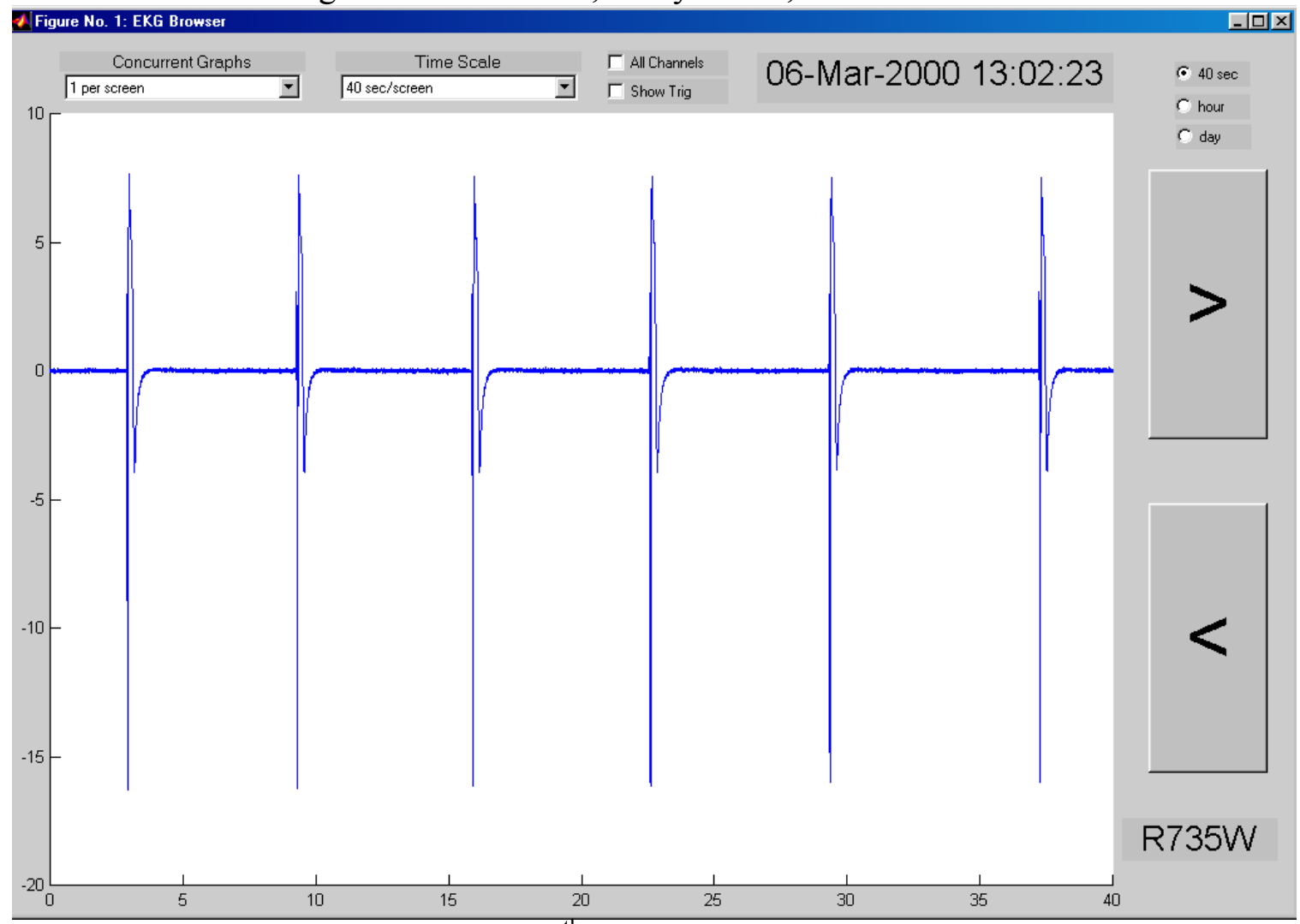

Figure H.6 March $6^{\text {th }}$, bradycardia, fourty sec/screen 


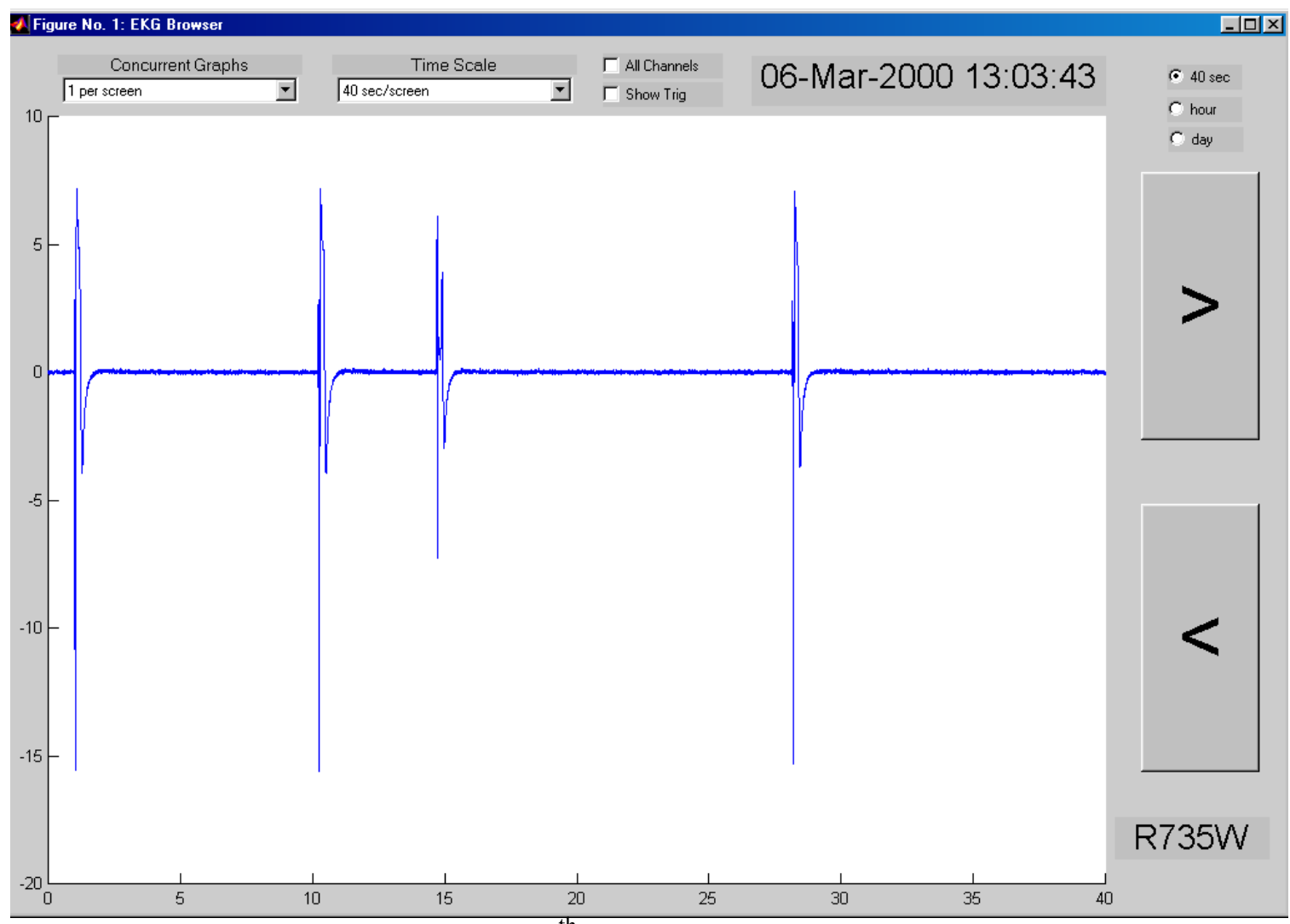

Figure H.7 March $6^{\text {th }}$, bradycardia, fourty sec/screen

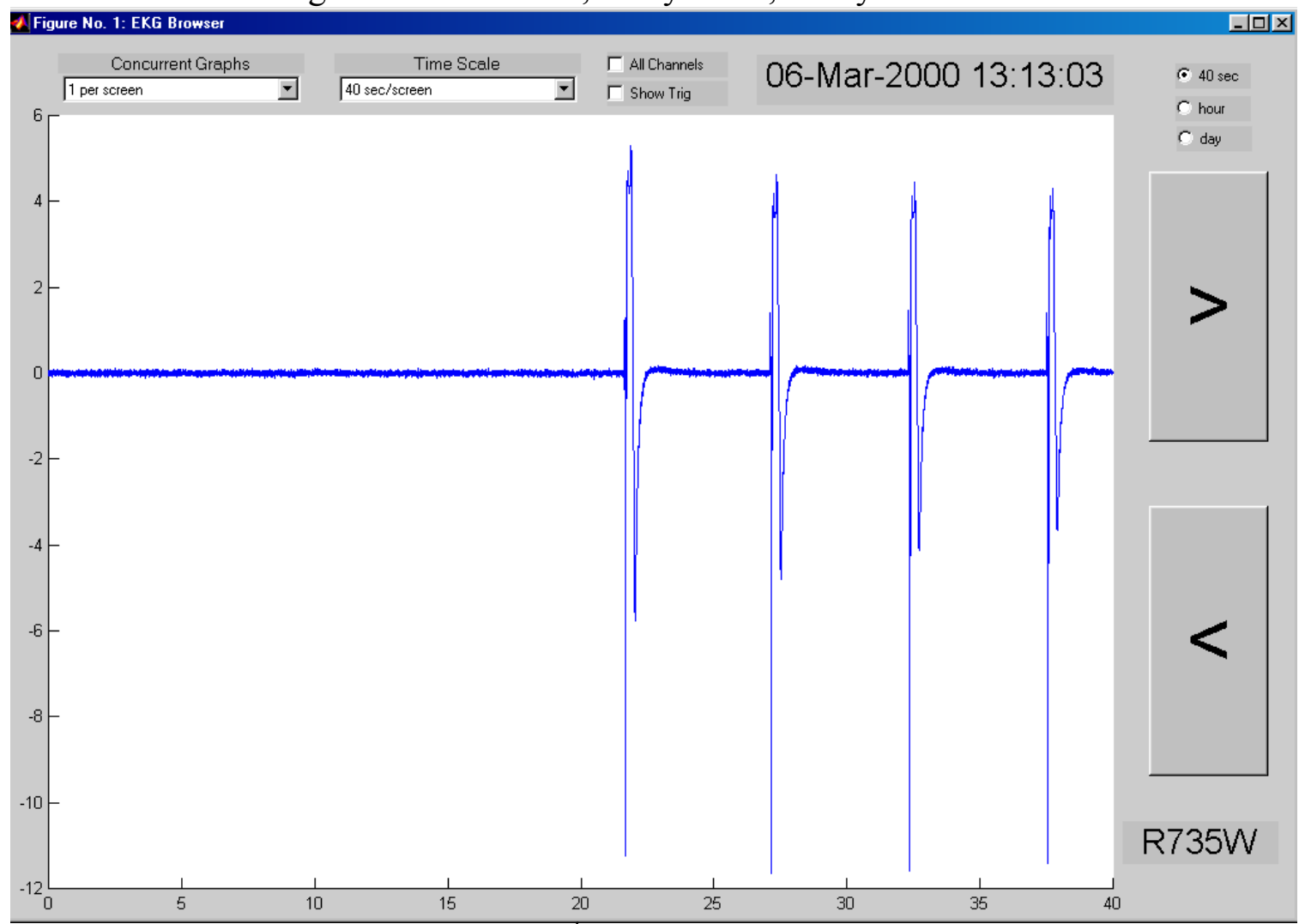

Figure H.8 March $6^{\text {th }}$, exactly before the rabbit died 